\title{
Technical Design Report Second Target Station
}

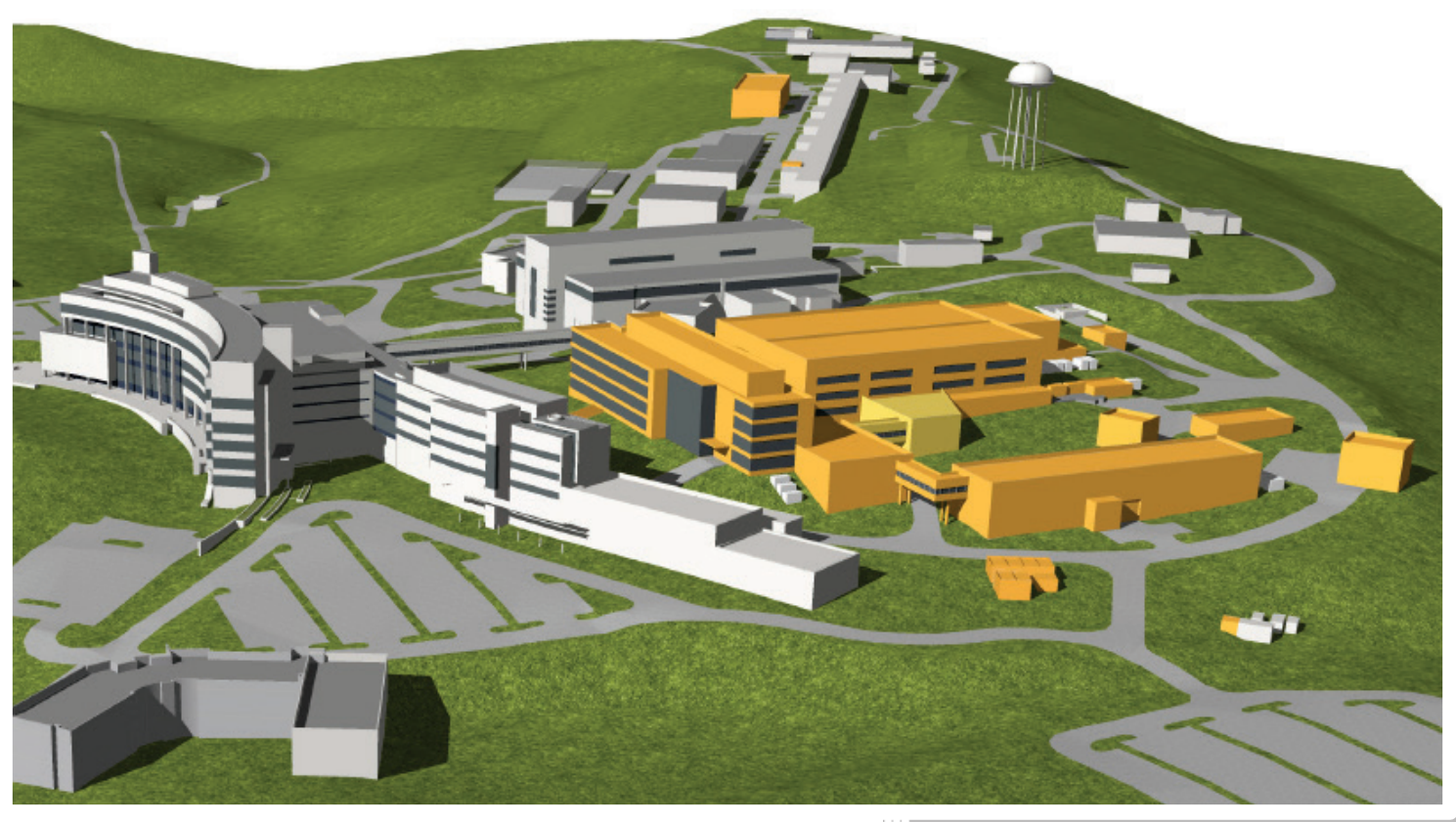

Approved for public release.

January 2015 
This report was prepared as an account of work sponsored by an agency of the United States Government. Neither the United States Government nor any agency thereof, nor any of their employees, makes any warranty, express or implied, or assumes any legal liability or responsibility for the accuracy, completeness, or usefulness of any information, apparatus, product, or process disclosed, or represents that its use would not infringe privately owned rights. Reference herein to any specific commercial product, process, or service by trade name, trademark, manufacturer, or otherwise, does not necessarily constitute or imply its endorsement, recommendation, or favoring by the United States Government or any agency thereof. The views and opinions of authors expressed herein do not necessarily state or reflect those of the United States Government or any agency thereof. 
Neutron Sciences Directorate

\title{
TECHNICAL DESIGN REPORT SECOND TARGET STATION
}

Date Published: January 2015

\author{
Prepared by \\ OAK RIDGE NATIONAL LABORATORY \\ Oak Ridge, Tennessee 37831-6283 \\ managed by \\ UT-BATTELLE, LLC \\ for the \\ US DEPARTMENT OF ENERGY \\ under contract DE-AC05-00OR22725
}





\section{CONTRIBUTORS}

EDITOR: J. Galambos

\section{CONTRIBUTORS}

D. Anderson, D. Bechtol, K. Bethea, N. Brown, F. Carden, S. Chae, A.Clark, K. Craft, D. Counce, M. Crofford, R. Collins, S. Cousineau, D. Curry, R. Cutler, M. Dayton, R. Dean, C. Deibele, M. Doleans, T. Dye, B. Eason, J. Eckroth, C. Erickson, C. Fincrock, S. Fritts, K. Gawne, S. Hartman, K. Herwig, S. Kim, S. Hess, J. Holmes, C. Horak, M. Howell, E. Iverson, L. Jacobs, K. Jones, L. Jones, K. Kasemir, S. Kim, F. Gallmeier, B. Johnson, S. Johnson, G. Laughon, W. Lu, K. Mahoney, J. Mammosser, T. McManamy, M. Michillini, M. Middendorf, E. O’Neal, B. Nemec, C. Peters, M. Plum, G. Regan, I. Remec, M. Rennich, B. Riemer, R. Saethre, J. Schubert, A. Shishlo, C. Smith, H. Strong, K. Tallant, A. Tennant, B. Thibadeau, S. Trumble, S. Trotter, Z. Wang, B. Webb, D. Williams, K. White, J-K Zhao 



\section{CONTENTS}

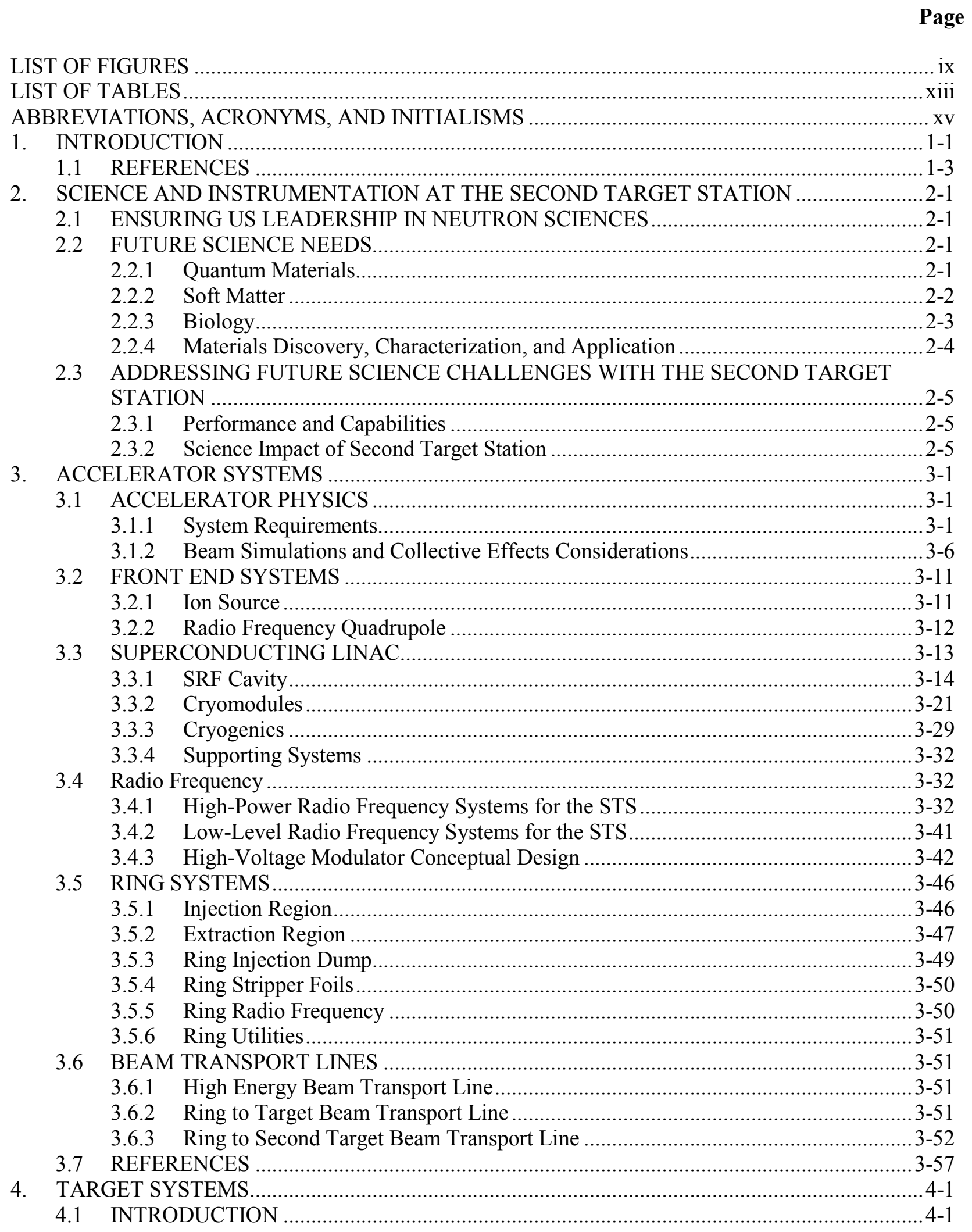


4.1.1 Neutron Production Components............................................................................. 4-1

4.1.2 Monolith Configuration ................................................................................... $4-3$

4.1.3 Target Building Configuration........................................................................... $4-4$

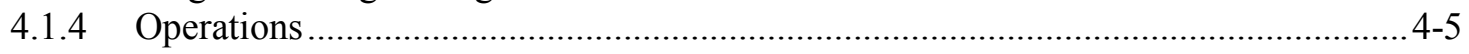

4.1.5 Cost Minimization ...................................................................................... $4-6$

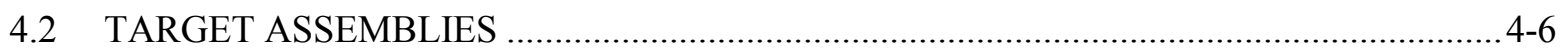

4.2.1 Target Selection Process ................................................................................... 4-6

4.2.2 Target Configuration..............................................................................................4-7

4.2.3 Nuclear Heating Design Requirements................................................................4-10

4.2.4 Target Design Analysis.......................................................................................4-12

4.2.5 Target Decay Heat Removal ................................................................................ 4-14

4.2.6 Target Fabrication....................................................................................... $4-16$

4.2.7 Target Carriage ………………………………............................................. $4-16$

4.3 INNER REFLECTOR PLUG ……………………........................................... 4-18

4.3.1 IRP Configuration.................................................................................... 4-18

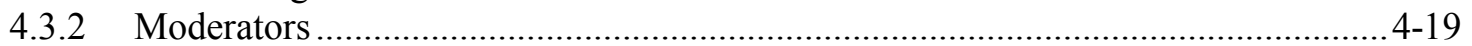

4.3.3 IRP Lifetime Determination ……………………………………………….. 4-21

4.3.4 IRP Alternative Configuration ......................................................................... 4-21

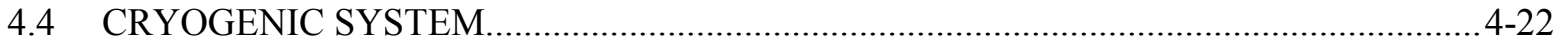

4.4.1 Hydrogen Circulation System ........................................................................... 4-23

4.4.2 Helium Refrigerator System ………………………………………………... $4-26$

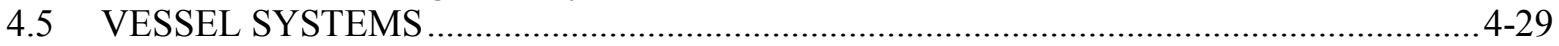

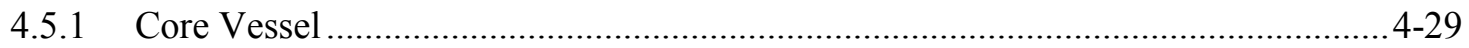

4.5.2 Outer Shield Plug...................................................................................... $4-32$

4.5.3 Proton Beam Window.............................................................................. $4-32$

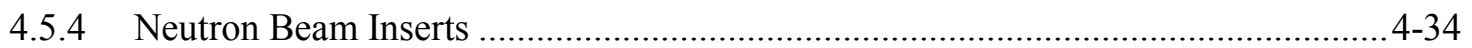

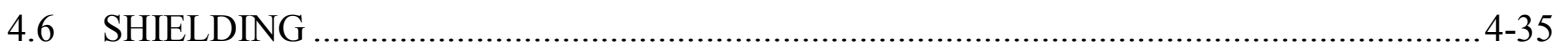

4.6.1 Lower Passive Shielding.................................................................................

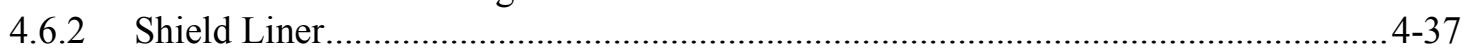

4.6.3 Upper Shielding ...................................................................................... $4-38$

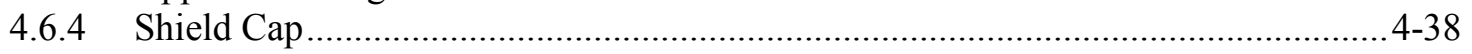

4.6.5 Ventilation and Heat Removal...........................................................................4-38

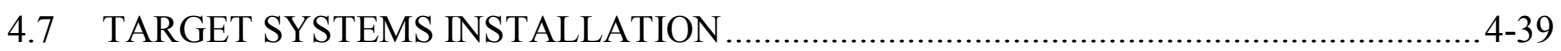

4.7.1 Monolith Installation Requirements..................................................................... 4-39

4.7.2 Monolith Installation Sequence ……………………………………………....4-39

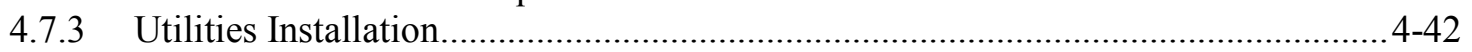

4.7.4 Service Cell Components Installation................................................................... $4-42$

4.7.5 Cryogenic Systems Installation................................................................... 4-42

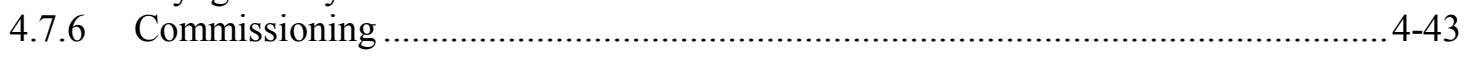

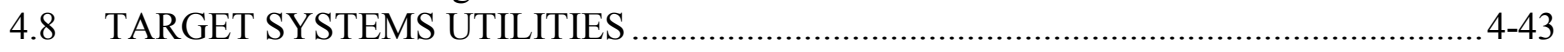

4.8.1 Target Cooling Water System........................................................................... 4-43

4.8.2 Target Utility System Layout....................................................................... 4-45

4.8.3 Inert Gas Systems ...................................................................................... 4-46

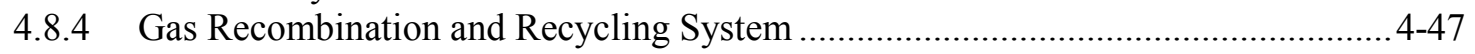

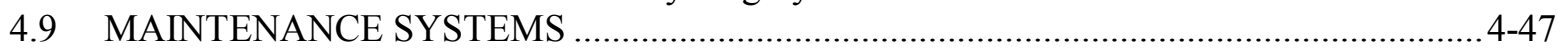

4.9.1 Design Guidelines and Assumptions .................................................................. $4-47$

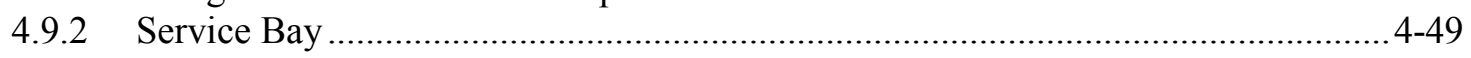

4.9.3 Service Bay Remote Handling Operations …………….......................................... 4-52

4.9.4 Monolith and Beam Line Component Handling ......................................................4-56

4.9.5 IRP Mockup/Storage Vessel ......................................................................... 4-66

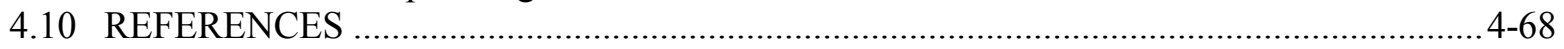




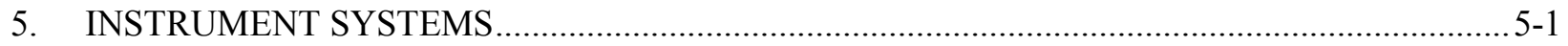

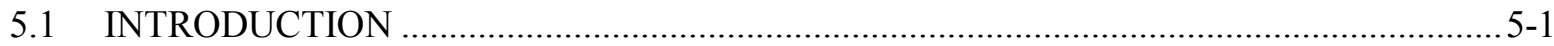

$5.2 \quad$ INSTRUMENT PLANNING SUITE ........................................................................

5.2.1 Zeemans ................................................................................................... $5-3$

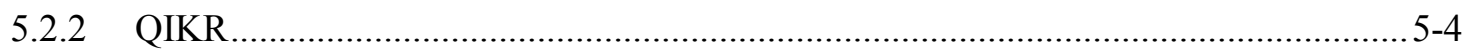

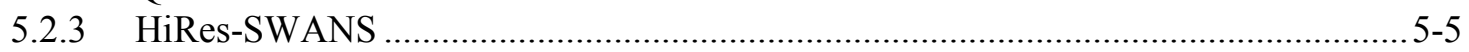

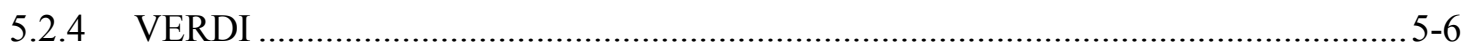

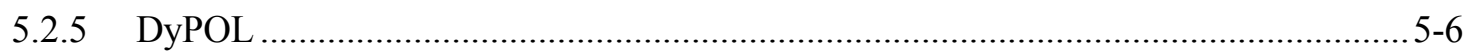

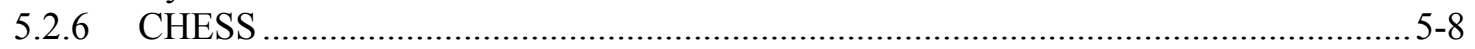

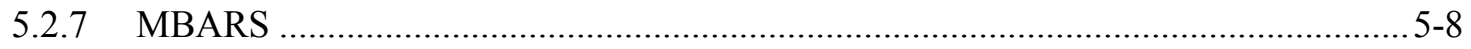

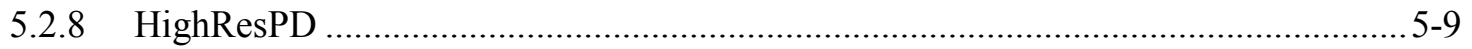

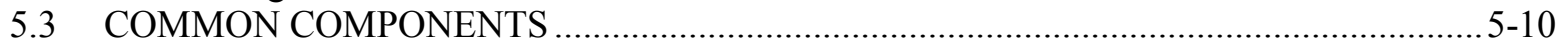

5.3.1 In-monolith Neutron Optics ........................................................................... $5-10$

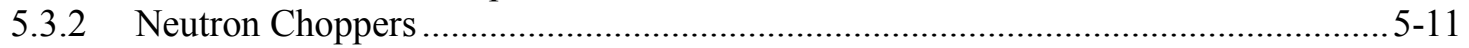

5.3.3 Neutron Guides/Optical Components ...................................................................5-12

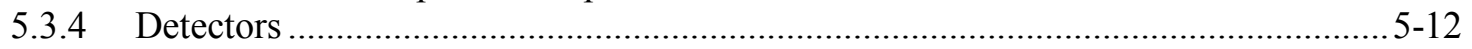

5.3.5 Data Acquisition and Instrument Controls.............................................................5-14

5.3.6 Sample Environment...............................................................................5-14

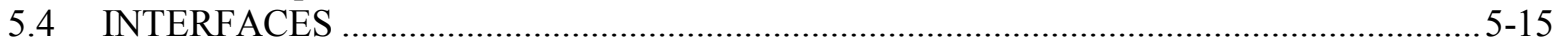

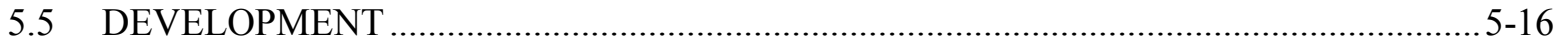

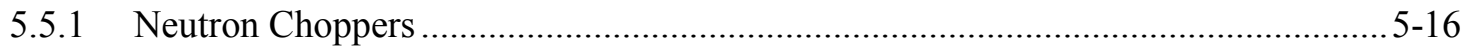

5.5.2 Neutron Detectors .................................................................................... 5-16

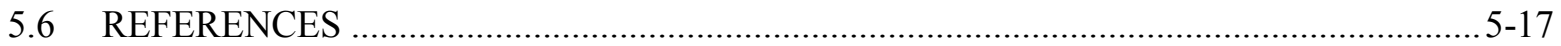

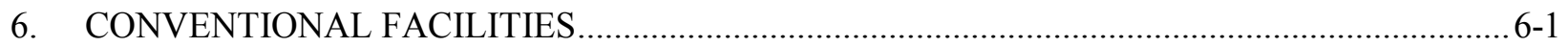

6.1 CF TECHNICAL SUPPORT ................................................................................ $6-1$

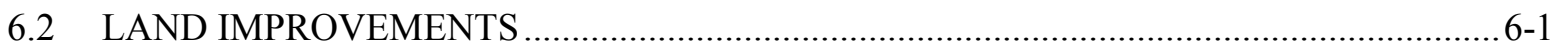

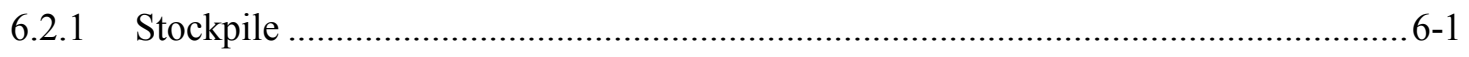

6.2.2 Mass Excavation ........................................................................................... $6-1$

6.2.3 Pond Improvements ......................................................................................6-2

6.2.4 Roads and Parking ……………………………….......................................6-2

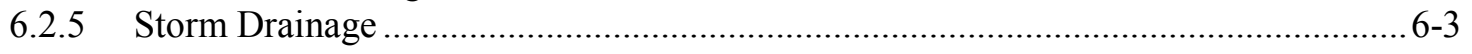

6.2.6 Landscape/Hardscape .......................................................................................6-3

6.2.7 Geotechnical Exploration.................................................................................... 6-3

6.2.8 Temporary Facilities ......................................................................................... $6-4$

6.2.9 Finished Site Grading ................................................................................... $6-4$

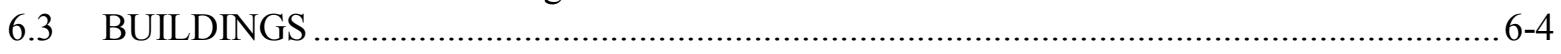

6.3.1 FTS Building Modifications ............................................................................. $6-6$

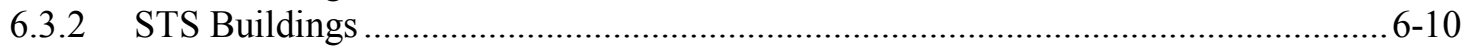

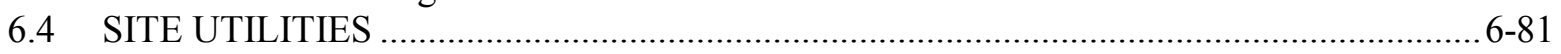

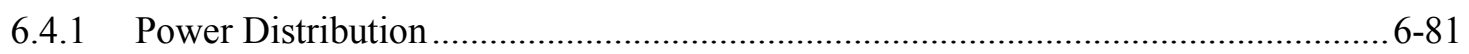

6.4.2 Communications System …………………………....................................... 6-81

6.4.3 Instrument and Control Distribution Description ......................................................6-82

6.4.4 Ventilation Exhaust....................................................................................... 6-82

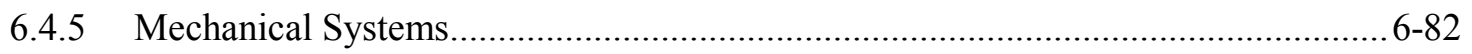

6.4.6 Sanitary Sewer .......................................................................................... 6-83

6.4.7 Fire Protection and Potable Water System ……………………………………....6-83

6.4.8 Zeemans ……………………………………………………………………...6-86

6.5 ASSUMPTIONS/ALTERNATIVES/RISKS/FURTHER DEVELOPMENT ........................6-86

6.5.1 Assumptions............................................................................................. $6-86$

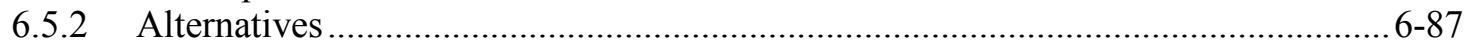

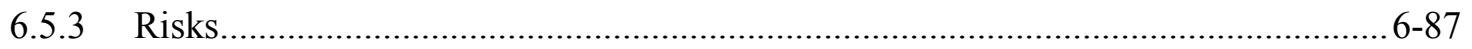


6.5.4 Further Development .................................................................................6-8

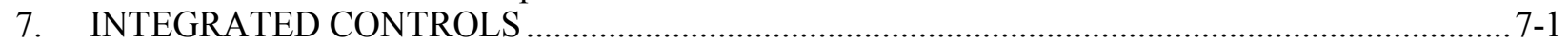

7.1 FIRST TARGET STATION CONTROLS ................................................................

7.1.1 First Target Station Global Control Systems ……………………………………..... 7-1

7.1.2 First Target Station Linac Control System Requirements ………………………...... 7-2

7.1.3 First Target Station Ring Global Control System Requirements................................. 7-7

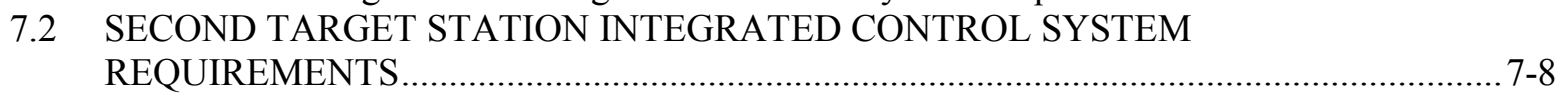

7.2.1 Second Target Station Global Control System Requirements .................................... 7-8

7.2.2 Second Target Station Target Control Systems …………………………………..... 7-10

7.2.3 Personnel Protection Systems ………………………………………………..... $7-11$

7.2.4 Second Target Station Instrument Controls …………............................................. $7-15$

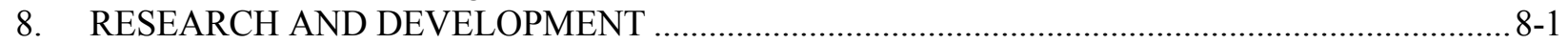

8.1 TARGET SYSTEMS ...........................................................................................

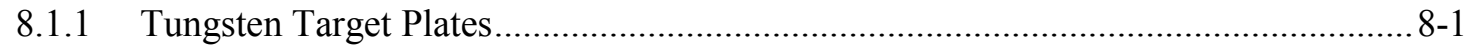

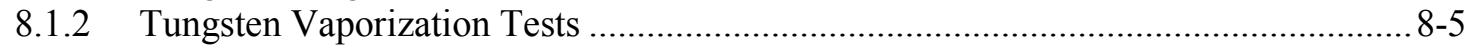

8.1.3 Target Cooling Loop Natural Convection Tests ........................................................ 8-5

8.1.4 Gas Bubble Injection....................................................................................... 8-6

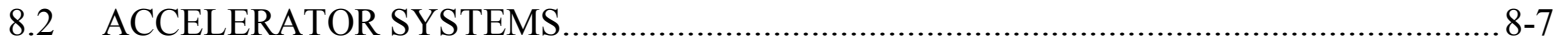

8.2.1 High-Voltage Modulator Systems ...................................................................... 8-7

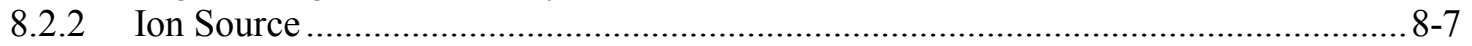

8.2.3 Ring Issues ................................................................................................

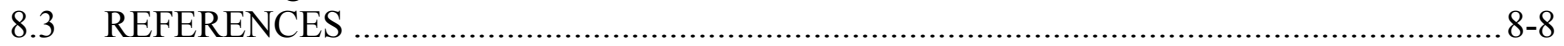

9. FIRST TARGET STATION OPERATION AT 1.3 GEV AND 2 MW ....................................... 9-1

$9.1 \quad$ FTS NEUTRONICS ANALYSES...............................................................................

9.2 FTS TARGET SYSTEMS AT 2 MW AND 1.3 GEV ..................................................... $9-1$

9.3 MERCURY TARGET DESIGN FOR 2 MW AND 1.3 GEV ………................................ $9-2$

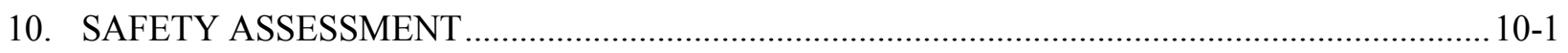

10.1 NATIONAL ENVIRONMENTAL POLICY ACT IMPACT ……………………...............10-1

10.2 RADIOACTIVE CONTAMINATION OF GROUNDWATER ……………………..........10-1

10.2.1 Engineering Controls …………………………….........................................10-1

10.2.2 Groundwater Monitoring Program........................................................................10-2

10.3 RADIOACTIVE EMISSIONS TO THE ATMOSPHERE................................................. 10-4

10.4 WASTE MANAGEMENT .......................................................................................

10.4.1 Disposition Mapping........................................................................................ 10-6

10.4.2 Radioactive Low-Level Waste..........................................................................10-7

10.4.3 Mixed Low-Level Waste …………………………............................................... 10-8

10.4.4 Hazardous Waste .....................................................................................

10.4.5 Sanitary/Industrial Waste .................................................................................... 10-9

10.4.6 Facility Safety …………………………………………………………... 10-9

10.4.7 Natural Phenomenon Hazards.......................................................................... 10-10

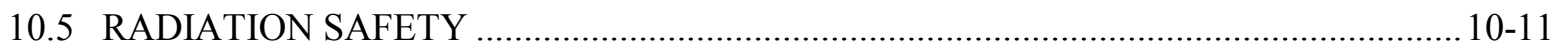

10.5.1 Shielding Policy ………………………………………………………... 10-11

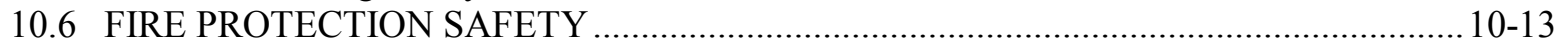

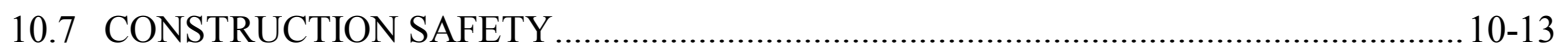

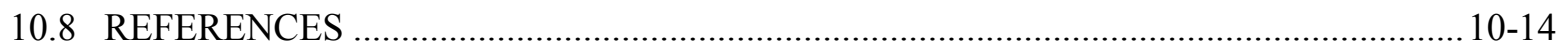

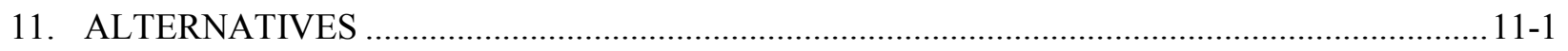

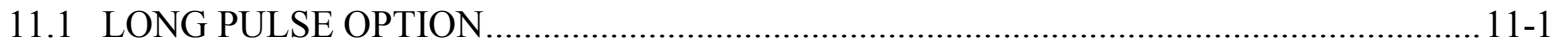

11.1.1 Target/Moderator Optimization ............................................................................11-3

11.1.2 2013 Strategic Plan ..................................................................................11-3

11.1.3 Short Pulse Risk .........................................................................................11-3 


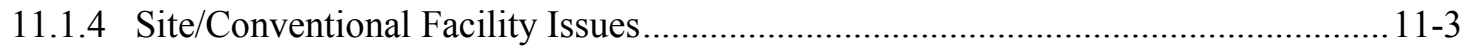

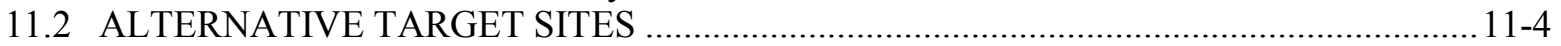

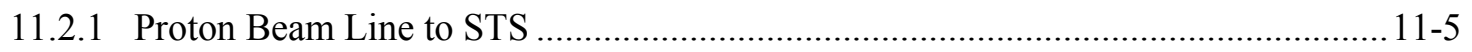

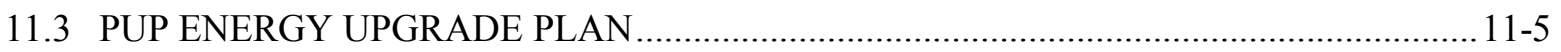

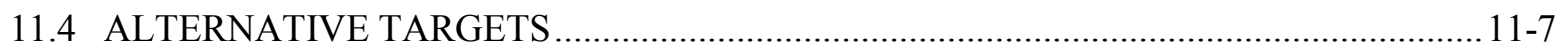

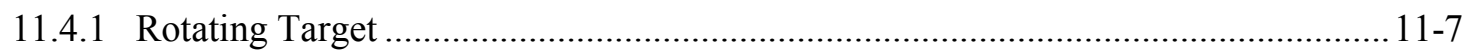

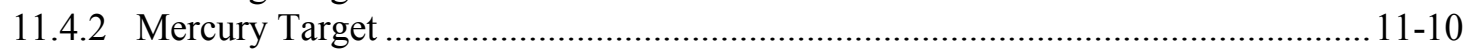

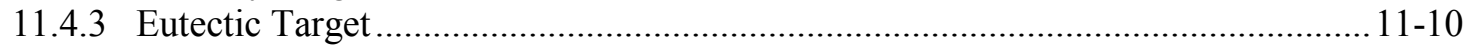

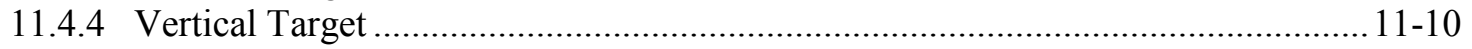

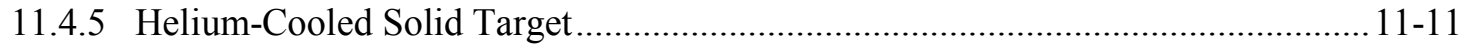

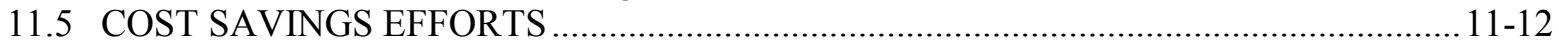

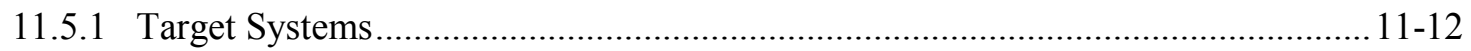

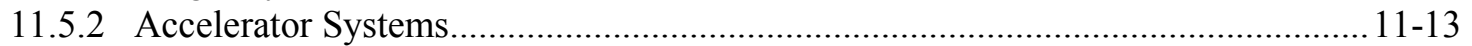

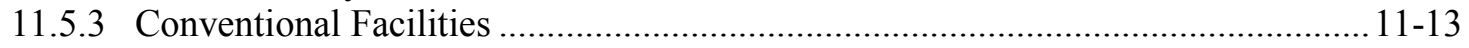

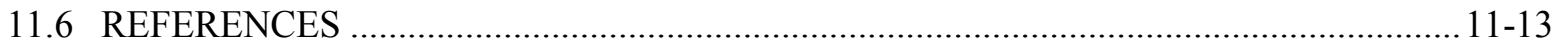





\section{LIST OF FIGURES}

Figures

Page

3.1 Example pattern width variation over the ring injection cycle leading to a clean extraction gap with $11 \%$ higher charge per pulse................................................................

SCL cavity gradients before $($ red $x$ ) and after (blue diamond) the STS upgrade................... 3-4

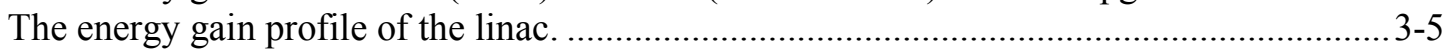

Required SCL klystron output power requirement vs SCL cavity number........................... 3-5

Transverse and longitudinal beam envelopes for the STS linac...................................... 3-6

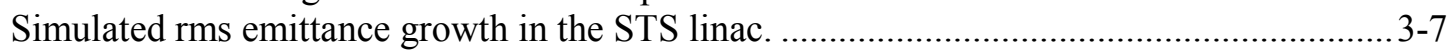

Beam distribution at the HEBT entrance (blue) and at the primary stripper foil in the ring injection area (red).

Beam distribution at the proton beam window upstream of the first target, assuming a

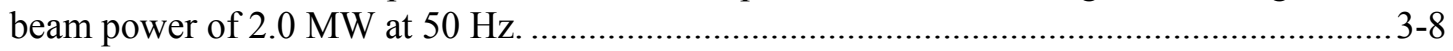

3.11

Beam distribution at the first target assuming a beam power of $2.0 \mathrm{MW}$ at $50 \mathrm{~Hz}$..............3-8

Beam distribution on the second target assuming a beam power of $470 \mathrm{~kW}$.

Recent ion source current, measured at the RFQ entrance on the SNS front-end..................3-12

RFQ beam transmission from simulations for the design value field profile along the RFQ.

End group temperature profiles with (a) $R R R=70$ and (b) $R R R=150$..

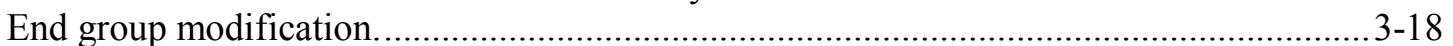

3.16

Completed end group replacement.

$3-18$

Original (left) versus new (right) helium vessel designs.

3-19

3.18

SNS fundamental power coupler (left: schematics, middle: inner conductor assembly,

right: outer conductor assembly).

Comparisons of calculated inner conductor tip temperatures. .......................................... $3-20$

3.19

3.20

3.21

3.22

3.23

3.24

3.25

3.26

3.27

3.28

3.29

3.30

3.31

3.32

3.33

3.34

3.35

3.36

3.37

3.38

3.39

Fundamental power coupler design (left: original design, right: design for the STS)..........3-20

The original high beta cryomodule.

$3-21$

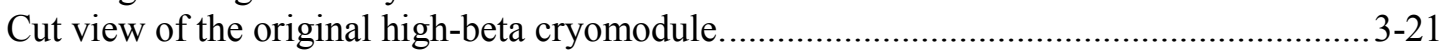

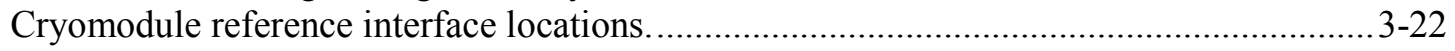

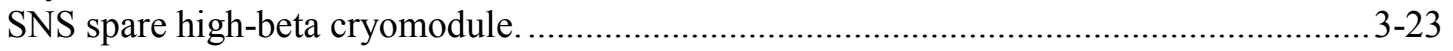

Helium flow schematic and instruments for the spare high beta cryomodule...................... 3-24

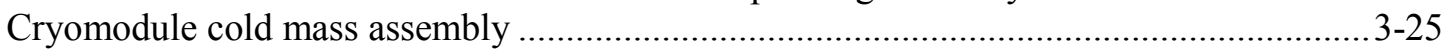

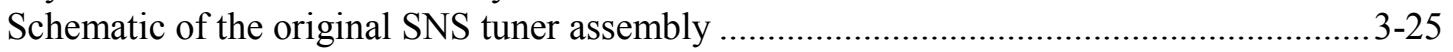

Original (left) versus new (right) vacuum vessel ........................................................ $3-27$

Original (left) versus new (right) vacuum jacket design ................................................ 3-27

JT valve positions for the STS cryomodule (left) and the original (right) cryomodule. ......3-27

Original (left) versus new (right) supply end can........................................................... $3-28$

Original (left) versus new (right) return end can.................................................... $3-28$

Interfaces between end cans and vacuum vessel for the STS cryomodule........................... 3-29

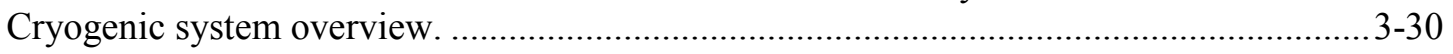

Estimated required RF generator power for the SCL....................................................3-36

Proposed layout of klystron gallery and accelerator tunnel. .........................................3-38

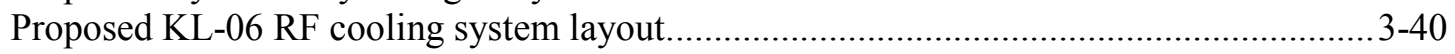

Block diagram of the SNS linac low-level control system.............................................. 3-41

Design considerations for the existing and new SCL HVCM systems. ............................. 3-44

3.40 The impact of the STS on the normal conducting linac klystron forward power and HVCM capacitor bank bus voltages. 


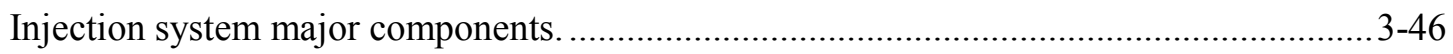

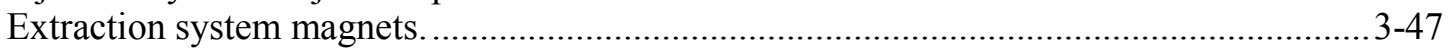

Schematic of the existing extraction kicker tank K1 with magnet types and numbering.....3-48

Schematic of the present extraction kicker tank K2 with magnet types and numbering......3-48

Schematic of extraction kicker tank K1 with annex and renumbered magnets.....................3-48

Schematic of extraction kicker tank K2 with annex and renumbered magnets.....................3-48

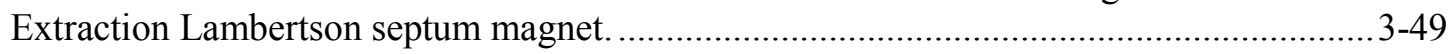

Stripping efficiency vs foil thickness for a $1.3 \mathrm{GeV} \mathrm{H}^{-}$beam. ...............................................

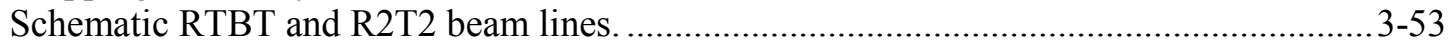

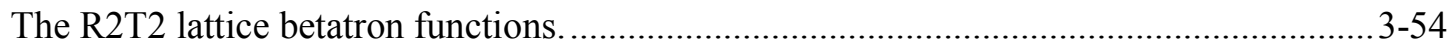

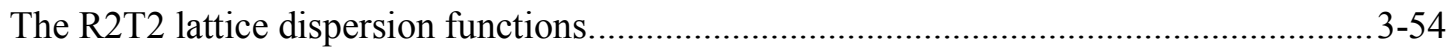

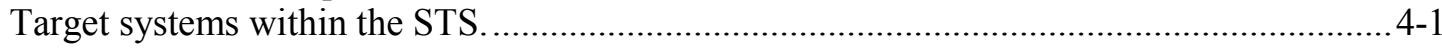

SNS mercury target compared with the STS solid target................................................... 4-2

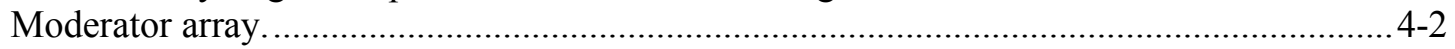

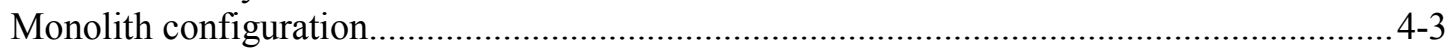

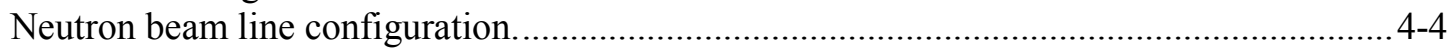

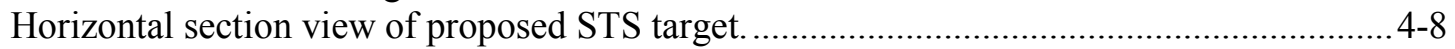

Typical tantalum clad tungsten plate ............................................................................ $4-10$

Volumetric plate heating versus distance from the front plate.........................................4-11

Target decay heat versus time from shutdown............................................................... $4-11$

Side section showing plate and shroud configuration. ................................................. $4-12$

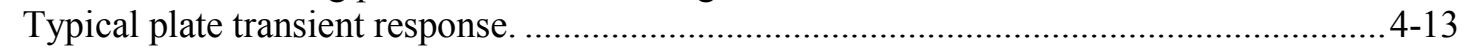

Plate surface and mid-plane temperature for $500 \mathrm{~kW}$ operation...................................... $4-13$

Transient response of highest temperature plate after loss of cooling............................... 4-15

Plate temperatures along beam axis at 40 minutes after loss of cooling. .......................... 4-15

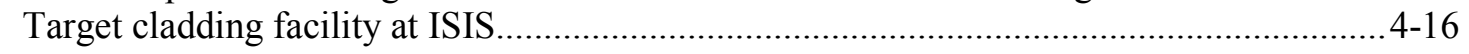

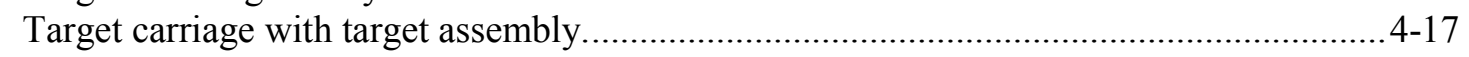

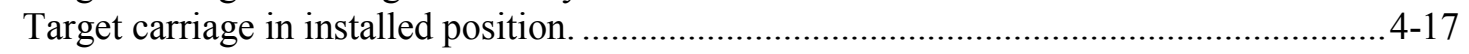

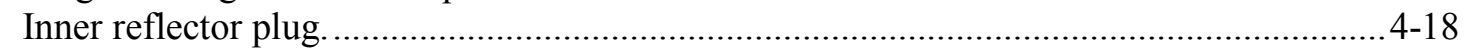

SNS FTS inner reflector plug during installation......................................................... 4-19

Location of moderators along proton beam centerline.................................................. 4-20

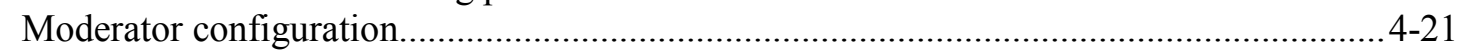

Simplified hydrogen loop diagram for STS. ............................................................. $4-22$

FTS cryogenic heat exchanger and pump modules..................................................... $4-23$

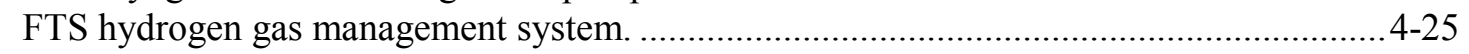

FTS welded pressure relief rupture discs and manifold. .............................................. 4-25

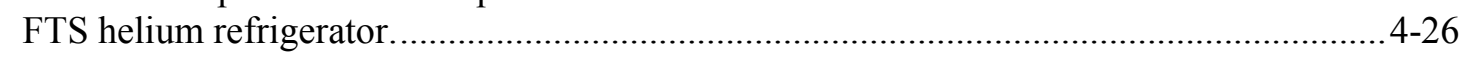

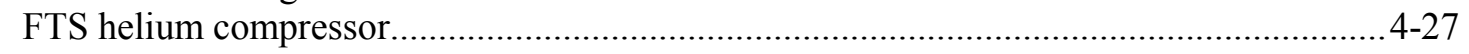

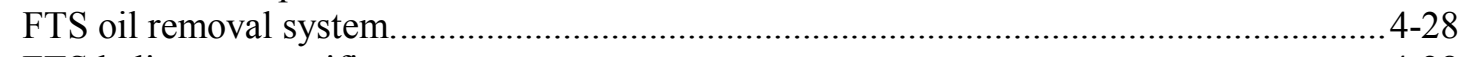

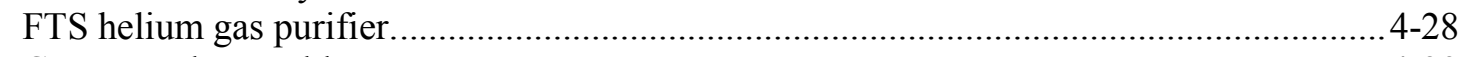

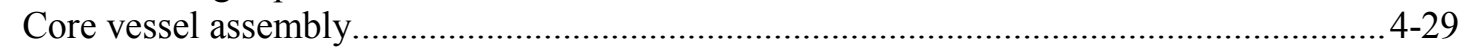

4.31 Nuclear heating (idealized) inside the $40 \mathrm{~cm}$ radius to the target center........................... 4-30

4.32 Core vessel section view showing active heating boundaries. ......................................... 4-31

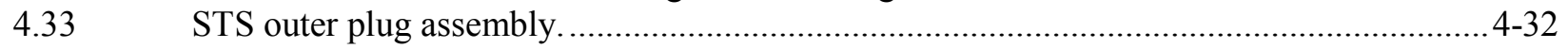

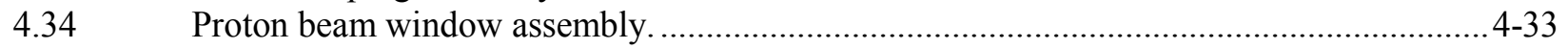

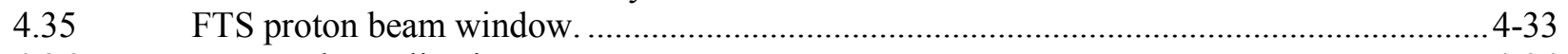

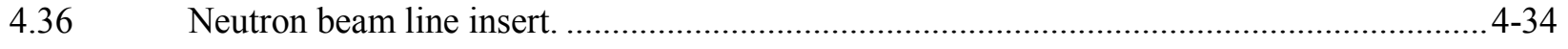

$4.37 \quad$ Neutron beam guides inside vessel........................................................................ 4-35

4.38 Cross-section of target monolith with ideal shield boundaries defined............................... 4-36

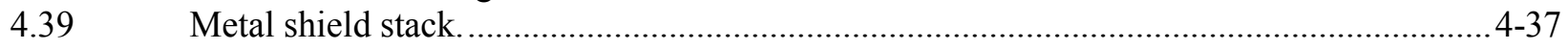

4.40 Recycled steel block placement in FTS beam dump..................................................... 4-37 
Installation of recycled steel in base of monolith.......................................................4-40

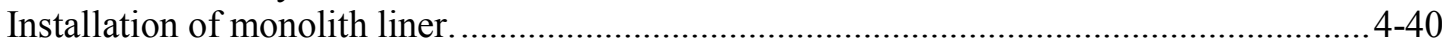

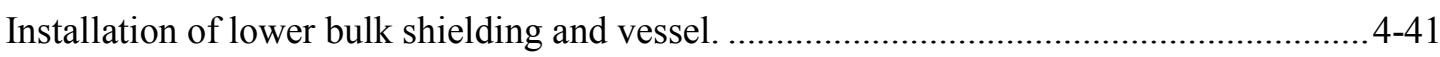

4.44

4.45

4.46

4.47

4.48

4.49

4.50

4.51

4.52

4.53

Installation of upper bulk shielding and proton beam window liner.

4-41

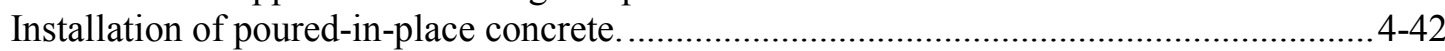

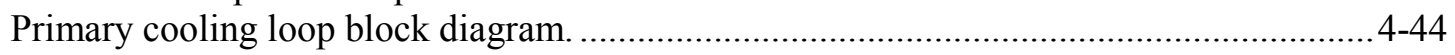

Utility systems in the target building basement. ....................................................... $4-45$

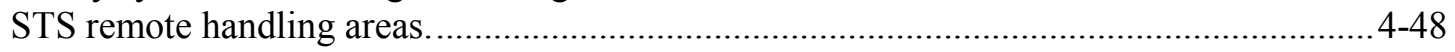

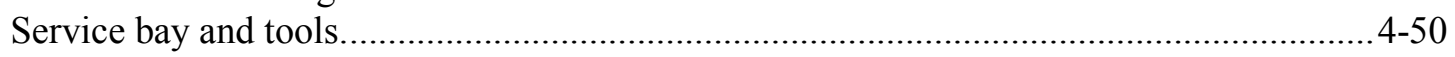

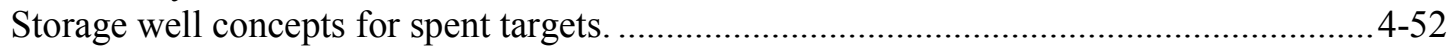

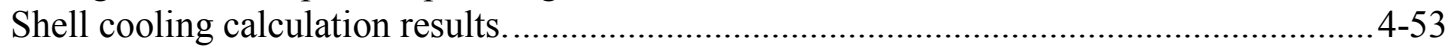

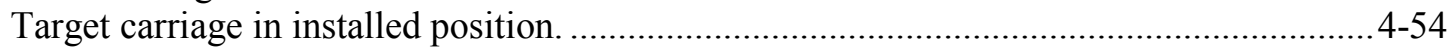

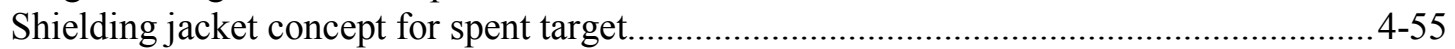

4.54

4.55

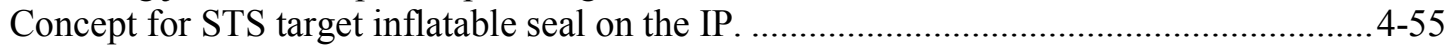

4.56

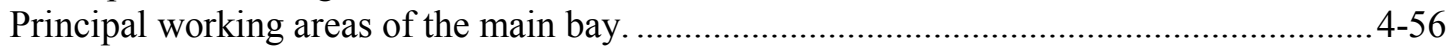

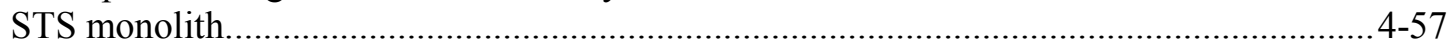

4.57

4.58

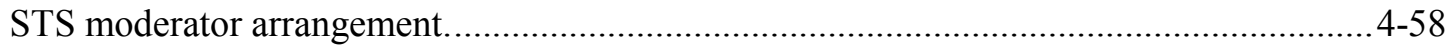

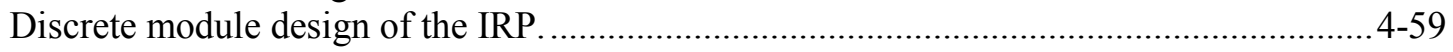

4.59

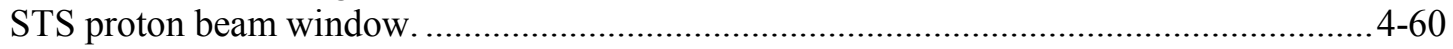

4.60

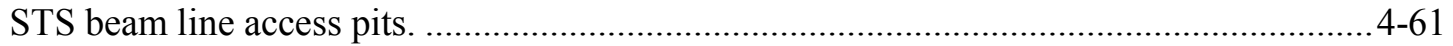

4.61

4.62

STS neutron beam insert (installed in core vessel)...................................................... $4-61$

4.63

4.64

4.65

4.66

4.67

4.68

4.69

4.70

5.1

5.2

5.3

STS neutron beam insert concept.

4-62

STS neutron beam guide handling tool concept............................................................ $4-62$

Comparison of disk size for FTS and STS choppers.........................................................63

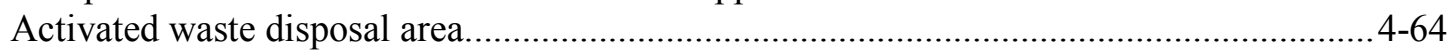

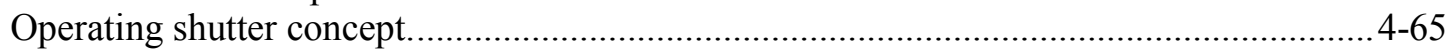

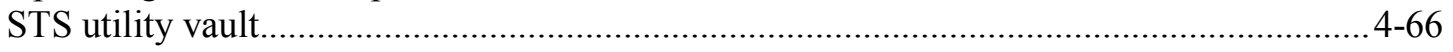

Proposed location of STS IRP mockup vessel. ............................................................ 4-67

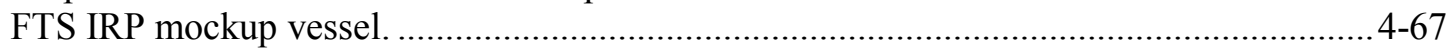

STS IRP mockup vessel (IRP is shown for visual effects)......................................... $4-68$

Zeemans detector vessel and magnet. .......................................................................... 5-4

Hydrogen atom neutron scattering cross-sections as a function of proton polarization.........5-7

Schematic view of the DNP apparatus and its orientation relative to a polarized

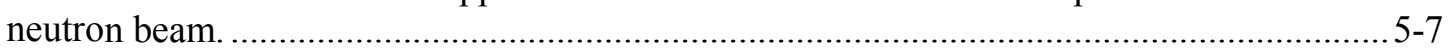

5.4 Schematic view of the MBARS spectrometer as proposed in ref. [6]...............................5-9

5.5 Timing diagram showing contamination at $\lambda=38.6 \AA$ for a single bandwidth chopper located at $10 \mathrm{~m}$ from the moderator. ........................................................................... $5-11$

5.6 Neutron detector types currently in use at the SNS first target station...............................5-13

6.1 Overview of the new buildings planned for the STS effort (orange) relative to existing buildings on the east end of the SNS campus..................................................................... 6-4

6.2 Perspective looking south with new buildings in the foreground. .................................... 6-5

6.3 Perspective looking west, with the new accelerator support buildings in the background.

6.4 Perspective looking east, indicating the connectivity of the present CLO and target building (gray pedestrian bridge) and the new CLO II/ Target II and STS instruments

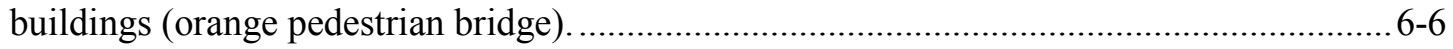

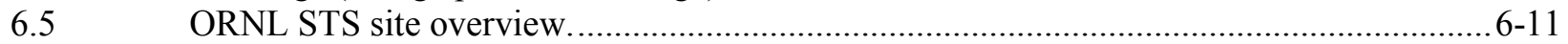

Instrument building locations................................................................................. 6-31

8.1 Photograph of the upper portion of the opened MK 0 Lujan target following service, as viewed from the impinging beam 
8.2 STS tungsten target design concept (left) has similarities to the ISIS TS-1 target design (right).

ISIS shop for tungsten target cladding application................................................... 8 -4

8.4 Photo of a Tantaline treated tungsten specimen (left) with dimensions of $0.9 \times 0.9 \times 0.175 \mathrm{in}^{3}$.

8.5 Schematic of experimental facility used by Greene and Frinfrock .................................. 8-5

8.6 Target 4 inner wall disk specimens show typical heavy central erosion and cross-width

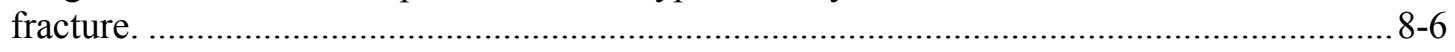

9.1 Target 9 center baffle cavitation erosion at its forward edge is shown on the left; a through-wall crack is apparent in the image on the right, from the mid-length of the center baffle.

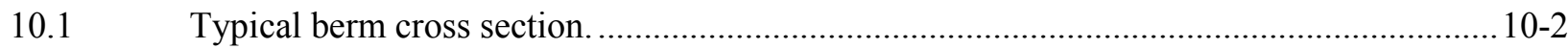

10.2 Groundwater monitoring locations at the SNS site. ..................................................... 10-3

11.1 Comparison of neutron flux between a coupled SP source (left) and an LP source (right) for the same neutron intensity, for $2 \AA$ (left) and $5 \AA$ (right) wavelength neutrons...11-2

11.2 The three sites considered for the STS target/instrument hall.......................................... 11-4

11.3 Proposed alternate paths for the proton beam line to the STS for an LP option................. 11-5

11.4 Power requirements for the superconducting linac from the PUP for different numbers of new cavities and for beam loading similar to the STS requirement .............................. 11-6

11.5 Full-scale 3 MW rotating target mockup........................................................... 11-8

11.6 Conceptual rotating target cross section............................................................... 11-8

11.7 Quasi steady-state rotating target thermal response. ............................................... 11-9 


\section{LIST OF TABLES}

\section{Tables}

Page

1.1 High-level parameters for the STS and the original SNS design .................................... 1-2

3.1

Comparison of required front end parameters for the present STS design and the earlier

STS accelerator upgrade plan.......

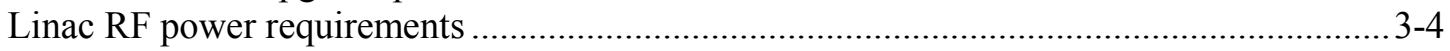

Initial Twiss parameters for the linac simulation .......................................................... $3-6$

Comparison of collective forces in SNS for four operating scenarios: present

production, the original design, first target with intensity upgrade, and second target

with intensity upgrade

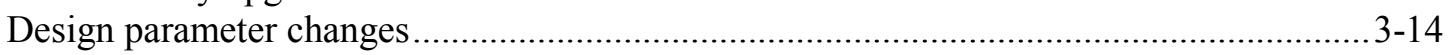

Major parameters of the SNS high-beta cavity .............................................................. 3-14

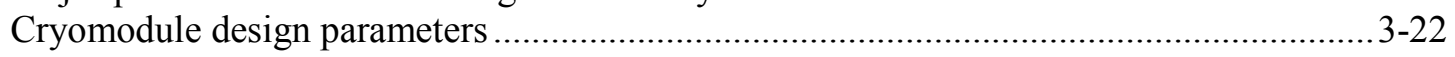

Cryogenic load design values for the high beta cryomodule............................................. 3-24

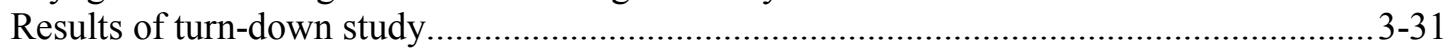

Measured structure parameters for the normal conducting linac .................................... 3-33

Estimated required RF generator power for the normal conducting linac..........................3-34

Normal conducting linac high-power RF systems for the STS ......................................3-35

Superconducting linac high power RF systems for the STS .........................................3-37

Present and projected operating parameters for existing and new HVCM systems ..............3-43

Components included in the new R2T2 beam line..............................................................

Target beam parameter design basis at STS, SNS FTS, LANSCE, and ISIS FTS ................ 4-7

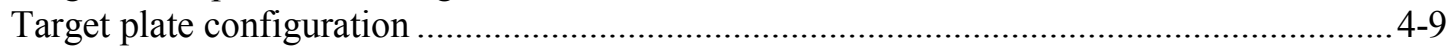

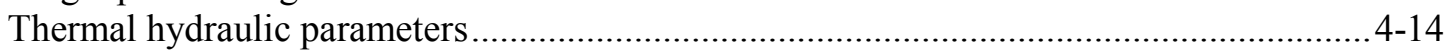

Second Target Station design basis cooling water heat loads ...........................................4-43

Established ORNL limits for personnel exposure ........................................................ $4-48$

SNS Second Target Station remote handling design guidelines .....................................4-49

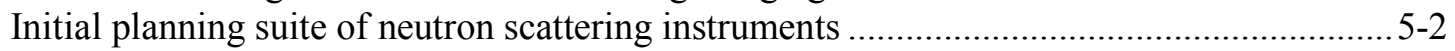

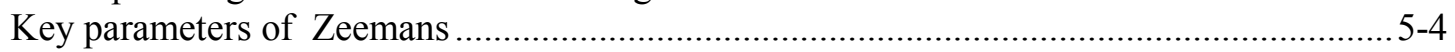

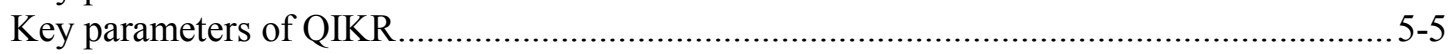

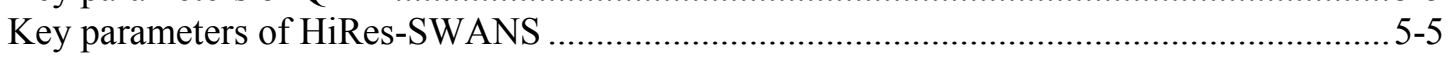

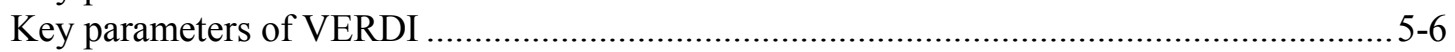

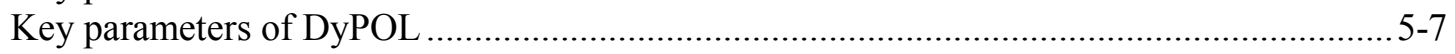

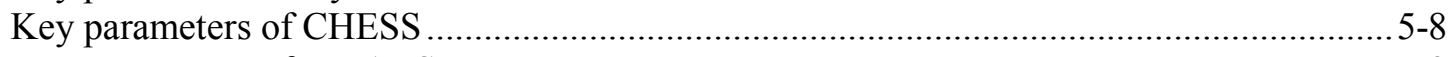

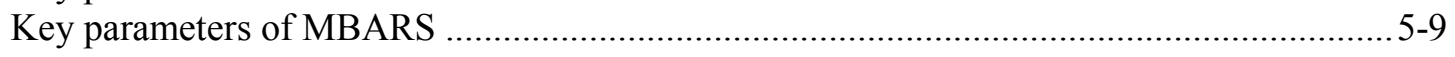

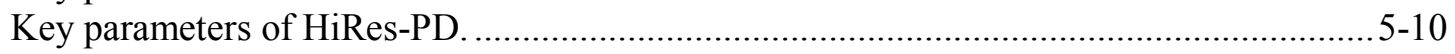

Operating parameters for detectors currently used at SNS first target station .....................5-13

Estimate of the detector requirements for the eight initial instruments...............................5-14

List of sample environment equipment for the eight project instruments......................... $5-15$

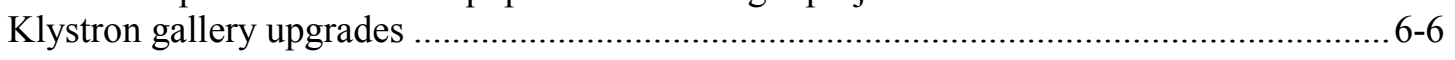

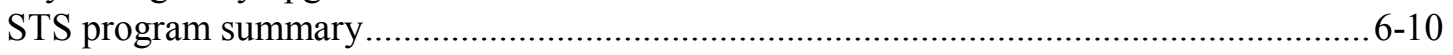

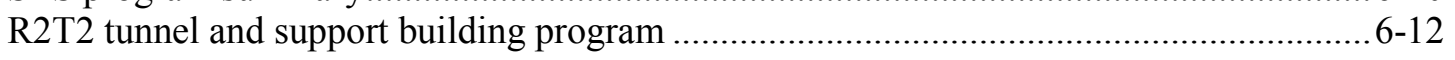

RT2T tunnel and support building mechanical equipment schedules ................................6-18

Target Building II program summary...................................................................... 6-20

Target Building II preliminary mechanical equipment schedules .....................................6-27

Instrument buildings and tunnels program summary .......................................................6-30

Estimated instrument buildings chilled water loads .....................................................6-37 
6stimated instrument buildings heating water loads ...............................................6-38

6.10 Instrument building and tunnels equipment schedules...................................................6-38

6.11 Zeemans building and support buildings program summary ...........................................6-41

6.12 Central Laboratory and Office Building II program summary.........................................6-45

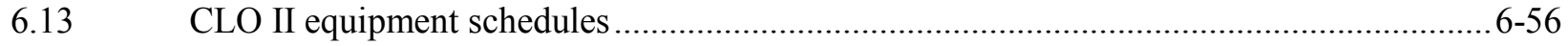

6.14 Central Utility Building II program summary ................................................................ $6-59$

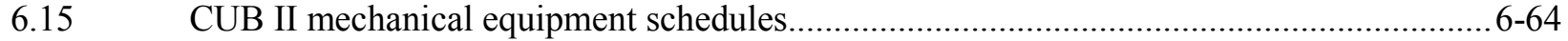

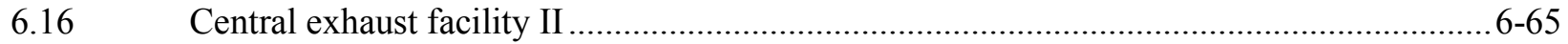

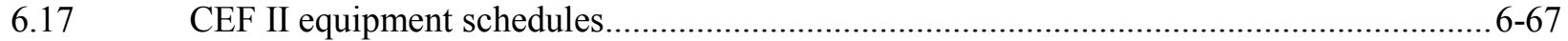

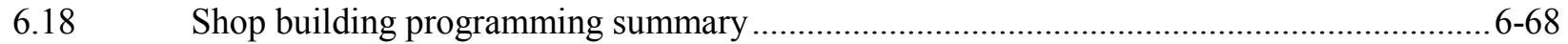

6.19 Shop building preliminary mechanical equipment schedule .......................................6-71

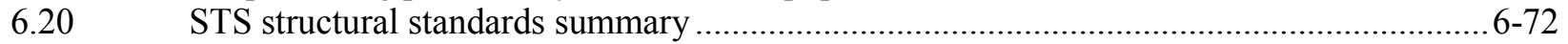

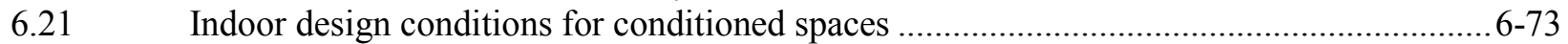

6.22 Indoor design conditions for unconditioned spaces .......................................................

$10.1 \quad$ Seismic design categories used for the STS buildings ............................................. 10-10

11.1 Comparison of high-level machine parameters of the present design and the

11.2 Comparison of high-level SRF cavity characteristics for STS and other ongoing

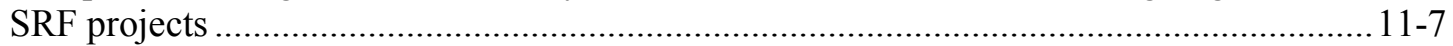

Thermal analysis results for helium cooling of solid target ...................................... 11-11 


\section{ABBREVIATIONS, ACRONYMS, AND INITIALISMS}

\begin{tabular}{|c|c|}
\hline Ac & alternating current \\
\hline ADARA & Accelerating Data Acquisition Reduction and Analysis \\
\hline $\mathrm{AE} / \mathrm{CM}$ & architect engineer/construction manager \\
\hline AFF & adaptive feed-forward \\
\hline AHU & air handling unit \\
\hline ALARA & as low as reasonably achievable \\
\hline ANL & Argonne National Laboratory \\
\hline ARR & Accelerator Readiness Review \\
\hline ASCE & American Society of Civil Engineers \\
\hline ASE & Accelerator Safety Envelope \\
\hline ASO & Accelerator Safety Order \\
\hline ASHRAE & American Society of Heating, Refrigerating and Air Conditioning Engineers \\
\hline ASME & American Society of Mechanical Engineers \\
\hline ASTM & American Society for Testing and Materials \\
\hline ATS & automatic transfer switch (definition to be confirmed by authors) \\
\hline BAS & building automation system \\
\hline BCM & beam current monitor \\
\hline $\mathrm{BCP}$ & buffered chemical process \\
\hline $\mathrm{B} \& \mathrm{PV}$ & Boiler and Pressure Vessel \\
\hline BLI & beam line interface \\
\hline BLM & beam loss monitor \\
\hline BPM & beam position monitor \\
\hline CAV & constant air volume \\
\hline $\mathrm{CCL}$ & coupled cavity linac \\
\hline CEBAF & Continuous Electron Beam Accelerator Facility \\
\hline CEC & Credited Engineering Control \\
\hline CEF & Central Exhaust Facility \\
\hline $\mathrm{CF}$ & conventional facilities \\
\hline CFD & computational fluid dynamics \\
\hline CHL & central helium liquefier \\
\hline $\mathrm{CHW}$ & chilled water system \\
\hline CLO & Central Laboratory and Office Building \\
\hline CLO II & Central Laboratory and Office Building II \\
\hline $\mathrm{CM}$ & cyromodule \\
\hline CNMS & Center for Nanophase Materials Sciences \\
\hline CPU & central processing unit \\
\hline CRL & Central Research Laboratories \\
\hline CUB & Central Uilities Building \\
\hline DAS & data acquisition system \\
\hline $\mathrm{dc}$ & direct current \\
\hline DDC & direct digital control \\
\hline DI & deionized \\
\hline DIWS & deionized water system \\
\hline DNP & dynamic neutron polarization \\
\hline DOE & Department of Energy \\
\hline
\end{tabular}




\begin{tabular}{|c|c|}
\hline DOE-HQ & Department of Energy Headquarters \\
\hline DTL & drift tube linac \\
\hline EIA & Electronic Interface Associates \\
\hline EISA & Energy Independence and Security Act \\
\hline EP & electropolishing \\
\hline e-p & electron-proton \\
\hline EPA & Environmental Protection Agency \\
\hline EPICS & Experimental Physics and Industrial Control System \\
\hline ES\&H & environment, safety, and health \\
\hline ESS & European Spallation Source \\
\hline FASD & Facility Safety Assessment Document \\
\hline FCM & field control module \\
\hline FEB & Front End Building \\
\hline FEIS & final environmental impact statement \\
\hline FPGA & field-programmable gate array \\
\hline FODO & focus-drift-defocus-drift \\
\hline FPC & fundamental power coupler \\
\hline FTS & First Target Station \\
\hline FWHM & full width at half maximum \\
\hline GPM & gallon per minute \\
\hline GPP & General Plant Project \\
\hline GSF & gross square feet \\
\hline $\mathrm{HB}$ & high beta \\
\hline HICM & high-intensity coupled moderator \\
\hline HDPE & high-density polyethylene \\
\hline HEBT & high-energy beam transport line \\
\hline HEBT-SB & high-energy beam transport line service building \\
\hline HEDP & high-energy differential pumping \\
\hline HEPA & high-efficiency particulate air \\
\hline HFIR & High Flux Isotope Reator \\
\hline HIP & hot isotopic pressing \\
\hline $\mathrm{HOG}$ & hot off-gas \\
\hline HOM & higher order mode \\
\hline HPCM & coupled para-hydrogen moderator \\
\hline HPM & high-power protection mode \\
\hline HPRF & high power radio frequency \\
\hline HRB & high-resolution backscattering \\
\hline HRP & high-resolution powder \\
\hline HUR & hydrogen utility room \\
\hline HVAC & heating ventilation air and conditioning \\
\hline HVCM & high-voltage convertor modulator \\
\hline IBC & International Building Code \\
\hline ICS & integrated control system \\
\hline IES & Illuminating Engineering Society \\
\hline IGBT & insulated gate bipolar transistor \\
\hline IGPP & Institutional General Plant Project \\
\hline $\mathrm{I} / \mathrm{O}$ & input/output \\
\hline
\end{tabular}




$\begin{array}{ll}\text { IOC } & \text { input output controller } \\ \text { IP } & \text { intermediate plug } \\ \text { IRP } & \text { inner reflector plug } \\ \text { ISIS } & \text { research center in the UK } \\ \text { ISMS } & \text { Integrated Safety Management System } \\ \text { IT } & \text { information technology } \\ \text { JPARC } & \text { Japan Proton Accelerator Research Complex } \\ \text { JSNS } & \text { Japan Spallation Neutron Source } \\ \text { JT } & \text { Joule-Thomson } \\ \text { KEK } & \text { High Energy Accelerator Research Organization } \\ & \\ \text { LANL } & \text { Los Alamos National Laboratory } \\ \text { LANSCE } & \text { Los Alamos Neutron Science Center } \\ \text { LEBT } & \text { low-energy beam transport line } \\ \text { LED } & \text { light-emitting diode } \\ \text { LHC } & \text { Large Hadron Collider } \\ \text { LEED } & \text { Leadership in Energy and Environmental Design } \\ \text { linac } & \text { linear accelerator } \\ \text { LLRF } & \text { low-level radio frequency } \\ \text { LLLW } & \text { liquid low-level waste } \\ \text { LLW } & \text { low-level waste } \\ \text { LN2 } & \text { liquid nitrogen } \\ \text { LP } & \text { long pulse } \\ \text { LPSD } & \text { linear position-sensitive detector } \\ \text { LSS } & \text { laboratory shift superintendent } \\ \text { MERV } & \text { minimum efficiency reporting value } \\ \text { MCNPX } & \text { Monte Carlo Neutronics PX- Target } \\ \text { MIOC } & \text { MPS IOC } \\ \text { MLLW } & \begin{array}{l}\text { mixed low-level waste } \\ \text { machine protection system }\end{array} \\ \text { MPS } & \text { multi-spectral decoupled moderator-cold } \\ \text { MSDC-C } & \text { multi-spectral decoupled moderator-thermal } \\ \text { MSDC-T } & \text { proton beam window } \\ \text { pulse forming network } & \\ \text { NDE } & \text { nondestructive examination } \\ \text { NEPA } & \text { National Environmental Policy Act } \\ \text { NFPA } & \text { National Fire Protection Association } \\ \text { NNSS } & \text { Nevada National Security Site } \\ \text { NPH } & \text { natural phenomena hazard } \\ \text { NTS } & \text { Nevada Test Site } \\ & \text { oxygen deficiency hazard } \\ \text { outer plug } \\ \text { operator interface } \\ \text { Oak Ridge National Laboratory }\end{array}$




\begin{tabular}{|c|c|}
\hline PID & proportional-integral-derivative \\
\hline PLC & programmable logic controller \\
\hline PPS & personnel protection system \\
\hline PS & power supply \\
\hline PUP & Power Upgrade Project \\
\hline PVC & polyvinyl chloride \\
\hline PW & pattern width \\
\hline $\mathrm{R} 2 \mathrm{~T} 2$ & ring to target two beam transfer line \\
\hline $\mathrm{R} \& \mathrm{D}$ & research and development \\
\hline RCCS & resonance control cooling system \\
\hline RCRA & Resource Conservation and Recovery Act \\
\hline RF & radio frequency \\
\hline RFQ & radio frequency quadrupole \\
\hline RFTF & radio frequency test facility \\
\hline RGA & residual gas analyzer \\
\hline RH & remote handling \\
\hline ROD & record of decision \\
\hline RSB & Ring Service Building \\
\hline RRR & residual resistance ratio \\
\hline RT & rotating target \\
\hline RTBT & ring to target beam transfer line \\
\hline $\mathrm{SAD}$ & Safety Assessment Document \\
\hline SANS & small-angle neutron scattering \\
\hline SBMS & Standards Based Management System \\
\hline SCE & secondary containment exhaust \\
\hline SCFM & standard cubic foot per meter \\
\hline SCL & superconducting linac \\
\hline SCR & silicon controlled rectifier \\
\hline SLPM & standard liter per minute \\
\hline SMACNA & Sheet Metal and Air Conditioning Contractors National Association \\
\hline SNS & Spallation Neutron Source \\
\hline SP & short pulse \\
\hline SSCs & structures, systems, and components \\
\hline STS & Second Target Station \\
\hline SRF & superconducting radio frequency \\
\hline TBDPMS & target bay differential pressure monitoring system \\
\hline TDEC & Tennessee Department of Environment and Conservation \\
\hline TDH & total dynamic head \\
\hline TESLA & Teraelectronvolt Energy Superconducting Linear Accelerator \\
\hline TIA & Telecommunications Industry Association \\
\hline TJNAF & Thomas Jefferson National Accelerator Facility \\
\hline TN-RAM & shipping cask for used targets \\
\hline TPS & Target Protection System \\
\hline TSCA & Toxic Substances Control Act \\
\hline TVA & Tennessee Valley Authority \\
\hline TW & Tower Water system \\
\hline UL & Underwriters Laboratories \\
\hline
\end{tabular}




$\begin{array}{ll}\text { UPS } & \text { uninterruptible power supply } \\ \text { USI } & \begin{array}{l}\text { Unreviewed Safety Issue } \\ \text { ultraviolet }\end{array} \\ \text { UV } & \text { volt alternating current } \\ \text { Vac } & \begin{array}{l}\text { variable air volume } \\ \text { VAV }\end{array} \\ \text { Vdc } & \text { volt direct current } \\ \text { VESDA } & \text { Very Early Smoke Detection Apparatus } \\ \text { VFD } & \text { variable-frequency drive } \\ \text { VOIP } & \text { Voice Over Internet Protocol } \\ & \\ \text { WAVES } & \text { Wide-Angle Velocity Selector } \\ \text { WBS } & \text { Work Breakdown Structure } \\ \text { WOC } & \text { White Oak Creek }\end{array}$

\section{Proposed STS instruments}

$\begin{array}{ll}\text { BWAVES } & \text { Broad-range Wide Angle Velocity Selector } \\ \text { CHESS } & \text { Chopper Spectrometer for Small Samples } \\ \text { DyPOL } & \text { Dynamically Polarized Crystallography instrument } \\ \text { EWALD } & \text { Enhanced Wide-Angle Laue Diffractometer } \\ \text { FLOODS } & \text { Flux-Optimized Order-Disorder SANS } \\ \text { HERTZ } & \text { High Energy Resolution Terahertz spectrometer } \\ \text { HighResPD } & \text { High-Resolution Powder Diffractometer } \\ \text { HiRes-SWANS } & \text { High-Resolution Small/Wide Angle Neutron Scattering } \\ \text { JANUS } & \text { hybrid indirect geometry spectrometer coupled with a low-angle direct geometry } \\ & \text { spectrometer } \\ \text { MBARS } & \text { Mica Backscattering Spectrometer } \\ \text { MSTAR } & \text { Magnetism-Second Target Advanced Reflectomer } \\ \text { M-WASABI } & \text { Magnetism-Wide and Small Angles with Big Intensity } \\ \text { NeSCRY } & \text { Neutron Single Crystal Diffractometer } \\ \text { QIKR } & \text { Quite Intense Kinetics Reflectometer } \\ \text { SPHINX } & \text { Spherical Indirect Inelastic Xtal Spectrometer } \\ \text { VBPR } & \text { Variable Beam Profile Reflectometer } \\ \text { VERDI } & \text { cold neutron diffractometer optimized for magnetic and large cell structures } \\ \text { XTREME-X } & \text { Extreme Multi-Energy Spectrometer with Xtal analyzers } \\ \text { Zeemans } & \text { instrument designed for studies at the highest magnetic fields }\end{array}$





\section{INTRODUCTION}

The Spallation Neutron Source (SNS) was designed from the outset to accommodate a second target station (STS) and an upgrade of the accelerator power. Planning for the new target station began during the initial SNS project stage [1] and has evolved over the past decade [2]. Both long proton pulse and short pulse approaches have been considered and, as explained in Sect. 11, the short pulse option was adopted for the present STS. The $10 \mathrm{~Hz}$ STS source described in this report will provide unparalleled neutron peak brightness and dynamic range for long wavelength cold neutron applications. Applications favoring high, time-averaged neutron flux can be accommodated at the High Flux Isotope Reactor (HFIR), a sister neutron science facility at Oak Ridge National Laboratory (ORNL), whereas instruments that require the highest time resolution are typically located at the SNS first target station (FTS); this arrangement is a key component of the Neutron Sciences Directorate 2014 Strategic Plan [3]. The performance and capabilities of the neutron scattering instrumentation enabled by STS will make it a truly next-generation facility. Projected performance gains of two orders of magnitude and beyond relative to existing capabilities will be transformative for many of the research areas described in Section 2.

In 2013, a Department of Energy (DOE) Basic Energy Science subcommittee [4] reviewed proposed scientific facility upgrades in the United States and noted that the STS will be absolutely central to US world-leading science. However, it was also noted that there remain "scientific/engineering challenges to resolve before initiating construction." This design report addresses the unresolved challenges and describes the technical approach proposed to realize the STS with minimal technical development required. The STS design takes advantage of provisions allocated during the original SNS project and leverages lessons learned during the design, installation, and initial years of operation of the SNS.

The STS concept proposed here is a short pulse, long wavelength configuration driven with a $10 \mathrm{~Hz}, 470$ $\mathrm{kW}$ proton beam. Some design parameters are shown in Table 1.1. Space for 22 neutron beam lines is provided in the present design, and 8 instruments will be delivered for day one operation. The instrument hall approach is different from that used in the FTS and is driven by the longer beam lines associated with the long wavelength instrument applications. Collecting instruments of similar length close to one another - within the constraints of appropriate moderator assignment - to minimize the required building space, is recommended to minimize the impact of accommodating longer beam lines. The STS target and instrument halls are located in unoccupied space east of the existing Experiment Hall. This space has a number of advantages, including direct access from the storage ring and proximity to the existing Experiment Hall and its support facilities. The second target and its associated systems are described in Sect. 4, and the conventional facilities supporting the target systems are described in Section 6.

The $10 \mathrm{~Hz}$ beam will be diverted from the $60 \mathrm{~Hz}$ pulse stream presently delivered to the SNS First Target Station (FTS). The STS proton beam intensity per pulse will be doubled from the present operational 1.4 MW level by increasing the beam energy from $940 \mathrm{MeV}$ to $1.3 \mathrm{GeV}$ and increasing the average beam current by $45 \%$. This proton beam power upgrade will permit the delivery of up to $2.3 \mathrm{MW}$ beam operation at the FTS, even at the reduced repetition rate of $50 \mathrm{~Hz}$. Sufficient space is provided in the linear accelerator (linac) tunnel for the needed additional cryomodules for the energy upgrade, and there is space in the Klystron Gallery for the needed additional radio frequency (RF) support equipment. More than $95 \%$ of the installed ring and transport systems are presently capable of $1.3 \mathrm{GeV}$ operation. The power upgrade leverages operational experience, gained with the fabrication and operation of a spare superconducting RF cryomodule, to reduce the number of required additional cryomodules and supporting RF equipment needed. Also, more effective use of $\mathrm{H}^{-}$beam chopping will reduce the required peak ion source current from previous estimates, obviating the need to develop a dual ion-source frontend system as envisioned in earlier STS plans. 
Table 1.1. High-level parameters for the STS and the original SNS design

\begin{tabular}{lcccc}
\hline & $\begin{array}{c}\text { SNS } \\
\mathbf{1 . 4} \text { MW }\end{array}$ & $\begin{array}{c}\text { Accelerator } \\
\text { upgrade }\end{array}$ & FTS & STS \\
\hline Proton beam power (MW) & 1.4 & 2.47 & 2 & 0.47 \\
Beam energy (GeV) & 0.94 & 1.3 & 1.3 & 1.3 \\
Proton pulse length on target $(\mu \mathrm{s})$ & 0.75 & & 0.75 & 0.75 \\
Average beam current on target (mA) & 1.5 & & 1.5 & 0.38 \\
RFQ output peak beam current (mA) & 33 & & 46 & 46 \\
Average linac chopping fraction (\%) & 22 & & 33 & 18 \\
Pulse repetition rate (Hz) & 60 & & 50 & 10 \\
Ring extraction beam gap (ns) & 250 & & 300 & 200 \\
R2T2 transport line (m) & & 219 & & \\
High-beta cryomodules & 12 & 19 & & \\
Protons per pulse on target & $1.55 \times 10^{14}$ & & $4.92 \times 10^{14}$ & $2.25 \times 10^{14}$ \\
Energy per pulse on target (kJ) & 23 & & 40 & 47 \\
Target material & & & $1 / 3$ & Tungsten \\
Number ambient / cold moderators & & & 18 & $1 / 3^{a}$ \\
Number of neutron shutters & & 3 & 0 \\
Initial instrument suite & & 24 & 8 \\
Number of instrument positions & & 13.75 & 22 \\
Nominal neutron beam line separation (degrees) & & & & 11 \\
\hline
\end{tabular}

$\mathrm{RFQ}=$ radio frequency quadrupole

${ }^{a}$ One moderator assembly has a thermal side and a cold side

A key factor in producing the high-intensity peak neutron flux is use of a compact target. In particular, this STS design incorporates a compact, stationary, water-cooled tungsten target. Surrounding the target will be two para-hydrogen cold moderators and a single cold para-hydrogen/water moderator surrounded by a water-cooled beryllium reflector. The target monolith will not include primary shutters, allowing close placement of the neutron beam line guides to the moderators, which will both save space and maximize the use of neutrons. The target hall will be arranged similarly to the FTS facility, with emphasis on allowing a maximum number of neutron beam lines. A similar approach to that at the FTS will be used for remote maintenance and servicing of the target system components. The design will allow reuse of equipment and resources. Shorter beam lines will be arranged on one side of the target and longer beam lines on the other side. This arrangement will allow for a relatively small instrument hall to house half of the beam lines. The instrument hall will be a separate building from the target hall to minimize instrument building safety requirements.

Site work and additional buildings represent a significant portion of the STS scope. The conventional facilities layout is largely focused on providing a seamless neutron science functional capability between FTS and STS. This seamless design starts with the siting of the STS close to the FTS. Building layout has been aimed at providing the required space for people and equipment while maintaining access between facilities to allow sharing of resources where possible. However, the long beam lines associated with the long wavelength cold neutron applications present challenges. Additional buildings beyond those required to support target, instrument, and accelerator functions include a new Central Utility Building to handle the additional utility load required and additional office, laboratory, and support building space to support a doubling of the number of neutron instruments. Generally the conventional facilities approach is an extension of the approach taken in the original SNS project. The conventional facility cost is a significant 
fraction of the STS cost, and the building requirements will be refined as part of a value engineering exercise.

The design concept presented in this report is a snapshot of the initial consideration of a facility that meets STS requirements, derived from input from SNS instrument and support personnel where possible and from best judgment otherwise. It is expected that there will be iterations, improvements, and cost reductions based on review of these results. Although the present proposal is not intended to be final, it is a useful tool to evaluate the impact of critical choices. It also highlights the areas requiring development and the areas that will be extensions of existing technologies.

\subsection{REFERENCES}

1. J. M. Carpenter and T. E. Mason, Technical Concepts for a Long Wavelength Target Station for the Spallation Neutron Source, ANL-02/16, ORNL/SNS-TM-2001/163, November 2002.

2. A Second Target Station for the Spallation Neutron Source, SNS 100000000-TR0029-R00, Oak Ridge National Laboratory, October 2007.

3. 2014 Neutron Sciences Strategic Plan, Internal SNS report, October 2013.

4. Basic Energy Sciences Facilities Prioritization, US Department of Energy, Washington, D.C., February 2013. Report of the Basic Energy Sciences Advisory Committee Subcommittee on BES Facilities Prioritization, February 26-27, 2013. Available at http://science.energy.gov/ /media/bes/besac/pdf/Reports/BESAC_Facilities_Prioritization_Report 20 13.pdf. (Accessed December 2014) 



\section{SCIENCE AND INSTRUMENTATION AT THE SECOND TARGET STATION}

\subsection{ENSURING US LEADERSHIP IN NEUTRON SCIENCES}

Oak Ridge National Laboratory (ORNL) is a leading center for neutron science worldwide. It has the mission to ensure the United States is served with cutting-edge capabilities for undertaking research that addresses the needs of DOE as well as the broader community. As part of this mission, the Neutron Sciences Directorate has undertaken, in consultation with its sponsors and the research community, an indepth look at the major needs for neutron science and its areas of impact for the next decade and beyond. A central part of this process has been the formation of four high-level workshops to delineate the challenges ahead and the priority areas in which developments in neutron sciences are most needed.

These workshops addressed the future of Quantum Condensed Matter, Soft Matter, Biology, and Frontiers in Materials Discovery and provided a host of compelling scientific and technological challenges for the decade ahead that are inaccessible to other techniques. They also identified areas in which a step change in capabilities is needed. In nearly all cases, the primary capability gaps that must addressed depend on more intense beams in the long wavelength ("cold") regime, and on instrumentation using a large bandwidth. The STS proposed in this technical design report is designed to provide exactly these capabilities and will be the most advanced source of its kind. Indeed, the capabilities envisaged will make this a truly next-generation facility; together with the FTS and the HFIR reactor source, it will ensure US capabilities that go beyond any existing or planned sources worldwide for the next generation. Details of the science case for the STS are provided in a more extensive separate document. ${ }^{1}$

\subsection{FUTURE SCIENCE NEEDS}

The SNS STS was determined to be "absolutely central" to US science in the Office of Science Facilities Prioritization round in 2013. However, that round also identified "scientific/engineering challenges to resolve before initiating construction." The rest of this Technical Design Report presents the resolution of the engineering challenges. This section addresses the science challenges, particularly the identification of emerging science and research community needs, that require the STS.

\subsubsection{Quantum Materials ${ }^{2}$}

Although normally associated with physics at the atomic scale, quantum coherence can give rise to spectacular properties when it transcends the atomic scale through collective behavior in so-called quantum materials. Neutrons provide access to spatial and temporal electronic correlations and have played a pivotal role in our rapidly developing understanding of these materials.

The workshop on Quantum Condensed Matter examined the current state of research on quantum materials, how the field might evolve over the next decade, and the role of neutron scattering in these developments. It also covered the synthesis of new materials, the complementary use of neutrons and xrays, and muons and high magnetic fields. Overall, the scientific field of quantum condensed matter was determined to be uncovering a rich variety of collective phenomena, some of which present exciting opportunities for technological impacts; and neutron scattering was determined to be an absolutely central technique for progress in the field.

\footnotetext{
${ }^{1}$ Instrumentation to Address Emerging and Next Generation Science Challenges, ed. K. W. Herwig and D. A. Tennant, Neutron Sciences Directorate, ORNL 2014 (unpublished).

${ }^{2}$ Based on the report of the Quantum Condensed Matter Workshop December 15-16, 2013, Lawrence Berkeley National Laboratory, Bob Birgeneau, University of California-Berkeley.
} 
A number of crucial problems in quantum materials research were highlighted to which neutrons are indispensable. These range from understanding the exotic ground states that emerge in quantum spin systems, quantum critical phenomena, topological states of matter, and quantum materials out of equilibrium, through the physics underlying unconventional superconductors and itinerant magnets.

Increasingly, quantum materials are poised to have major technological impacts in both information technologies and energy. Progress in new devices and in spintronics, in particular, requires advances in neutron capabilities for structure and dynamics in thin films/heterostructures/nanomaterials. Meanwhile, the importance of the special properties of strongly correlated states in energy storage and conversion is now being recognized and opens up new needs for diffraction and inelastic neutron scattering.

A major trend in the coming years will be the increasing importance of mesoscale phenomena for controlling properties. Studying these will require spatially resolved probes of (especially magnetic) structures of materials on the submicron scale, as well as the determination of the structure of partially ordered materials, including defect structures.

The new challenges identified in this section need the much more advanced capabilities in cold neutrons offered by the STS. Focused beams will be required to measure smaller samples, and the technology to fully exploit polarized beams is necessary to separate the component parts of complex states in materials. Coupling neutrons with materials exploration calls for more versatile measurement capabilities, including the application of multiple characterization techniques; and the manipulation of states in situ means enhanced sample environments are crucial. Neutrons are unparalleled in their direct relevance to theory, and the most advanced data analysis and simulation capabilities are needed to complement measurement interpretation with theory.

\subsubsection{Soft Matter}

One of the goals of modern science is to create materials by design with specific functionalities. Soft matter composed of covalently bound molecular building blocks (including polymers, surfactants, nanoparticles, and gels) provides almost endless complexity and tunability for making new materials to achieve this goal. As the complexity of these systems grows, so do the challenges for developing our fundamental understanding of the materials properties, with the ultimate goal of controlling static and dynamic function.

Areas of compelling scientific need in which neutrons will play a key role have been established. These extend into the response of soft matter to mechanical deformation, soft/hard composite materials, interfaces, and transport in soft matter. Other fundamental needs are capabilities to understand polar solvents other than water, polyelectrolytes, and complex structures in solution, such as hierarchical assemblies. The widespread impact of soft matter technologies will require looking at soft matter under industrial processing conditions, understanding active soft materials, expanding the ability to make quantitative measurements, and gaining insight into the effects of poly-dispersity on physical properties.

Neutrons are an essential tool for studying soft matter today, but in the future, new techniques and new sources will provide even more information on these complex systems The proposed STS will be optimized to provide a high flux of long-wavelength neutrons over a wide bandwidth. It will be ideally suited to simultaneously studying multiple temporal and spatial scales and crucial to understanding complexity in soft systems. To exploit and fully interpret the data, a closer coupling between modeling and experiment will be essential. Significantly increased facilities for selective isotopic labeling

\footnotetext{
${ }^{3}$ Report from Grand Challenges in Soft Matter workshop May 17-18, 2014, University of California-Santa Barbara, Fyl Pincus (University of California-Santa Barbara) and Matt Tirrell (University of Chicago).
} 
(primarily deuteration) will be needed. Soft matter has a growing synergistic relationship with molecular and cellular biology in the area of synthetically reproducing and enhancing the functionality found in living systems. Achieving these goals may involve the marriage of synthetic and biological moieties.

\subsubsection{Biology ${ }^{4}$}

Gaining a predictive understanding of the behavior of complex biological systems is one of the greatest scientific challenges that the scientific community faces over the next decade. This understanding will provide guidance in protecting and repairing physiological systems; will enable researchers to mimic the architectures and processes of living systems to create new biomaterials and bio-inspired technologies; and will provide the information necessary to manipulate microorganisms and their ecosystems to create new biotechnology and biorefinery solutions to emerging energy and environmental challenges.

Neutrons provide several types of unique information that will be important in addressing future problems and that are poised to have a major potential impact, including on membrane-associated biological processes and the dynamic assembly and regulation of large biological complexes. The high sensitivity of neutrons to hydrogen, which drives the chemistry and physics of living systems, in conjunction with deuterium labeling (which enhances the visibility of specific parts of complex biological systems through isotopic substitution) gives neutrons direct access to crucial processes. The application of neutrons is being opened up by high performance computing simulations that allow for the prediction and interpretation of data from systems that are too complex for analytical theory. Neutrons are also complementary to techniques using photons and electrons. While photons and electrons interact with the atomic electric field and are most sensitive to heavy atoms, hydrogen, with just one electron, is all but invisible to them. Neutrons interact with nuclei; light atoms such as hydrogen are highly visible to them. In addition, because they cause little radiation damage and are highly penetrating, neutrons enable the use of complex sample environments.

Despite the advantages in using neutron scattering, significant technical gaps must be bridged not only in neutron scattering instrumentation but also in molecular biology, deuterium labeling, and computational technologies. These gaps include the need for

- more advanced deuterium labeling techniques

- better access to neutron beam lines

- increased neutron flux on available beam lines

- neutron beam lines optimized for membrane diffraction

- innovative techniques for polarizing neutron beams and hydrogen atoms in samples to enhance scattering power and to dynamically control scattering contrast

- new instrumentation that allows simultaneous access to broad regions of time and space

- better integration of high performance computing techniques with neutron scattering experiments

- computational tools that allow the combination of experimental data from multiple complementary techniques to generate more complete models of complex biological systems.

\footnotetext{
${ }^{4}$ Report from Grand Challenges in Biological Neutron Scattering workshop, January 17-18, 2014, University of California-San Diego, Susan Taylor (University of California-San Diego) and Heidi Hamm (Vanderbilt University).
} 
The STS will be able to bridge these gaps and so will allow neutrons to be used in a transformative way to unify structural and dynamical descriptions of biological systems across length and time scales. This will transition the concept of a predictive understanding of biological systems to a reality.

\subsubsection{Materials Discovery, Characterization, and Application ${ }^{5}$}

Materials are at the heart of technologies that will define the future economy and provide solutions to challenges in energy, security, transport, and so on. Predictive modeling of materials holds promise to accelerate the development of new solutions. A prerequisite for such modeling is an understanding of material structures and dynamics from the atomic scale to real-world components and systems. In addition, understanding and modeling synthesis and processing is vital to achieving transformative impact.

The unique physical properties of neutrons make high-intensity beams indispensable to materials discovery, characterization, and application, in which they complement the capabilities of electrons and photons. Among these tools, their nondestructive nature, ability to penetrate real components and materials under working condition, sensitivity to light elements, ability to observe modes and dynamics over virtually all length and time scales, and ability to highlight components using isotope substitution make the contribution of neutrons unique. Areas in which neutrons will be essential for future materials science and engineering include infrastructure stewardship, advanced propulsion systems, advanced materials processing, nuclear fuels and radiation-tolerant materials, energy storage and energy conversion integrated systems, materials by design/integrated computational materials engineering, and materials under extreme environments.

To meet future challenges, new developments are needed in

- monitoring and understanding chemical reactions and catalysis

- analytical chemical spectroscopy, in which neutrons could provide a high-throughput technique providing information not accessible to photons

- understanding the roles of disorder and defects and how to manipulate them

- $\quad$ addressing the fundamental challenges of glasses and liquids, as well as fluid flow and reactivity

- in situ studies, including examination under pumping conditions, kinetics studies, materials growth and synthesis, and materials under extreme conditions

- understanding how components and integrated materials in devices function under realistic conditions, using instruments that can combine multiple techniques such as imaging, diffraction, and spectroscopy

The combination of intensity, dynamical range, and beam focusing at the STS is crucial to provide the transformative capabilities to enable these developments.

\footnotetext{
${ }^{5}$ Report from Frontiers in Materials Discovery, Characterization, and Application workshop, August 2-3, 2014, Schaumburg, Illinois, George Crabtree (Argonne National Laboratory) and John Parise (University of Stony Brook).
} 


\subsection{ADDRESSING FUTURE SCIENCE CHALLENGES WITH THE SECOND TARGET STATION}

A series of 14 working groups was convened to connect these science challenges with the source capabilities that would be provided by an STS at the SNS, as well as explore how they would be complemented by the SNS FTS and HFIR. The groups involved 70 scientists in exploring new concepts and instrumentation for the future. Together, these groups provide a case for neutrons for the coming decade and beyond and the compelling need for an STS at the SNS.

\subsubsection{Performance and Capabilities}

The performance and capabilities of the instrumentation enabled by the STS will make it truly a nextgeneration neutron facility. Gains in performance of two orders of magnitude and beyond over existing capabilities will make it transformative for many of the compelling research areas identified. At the heart of this dramatic step forward in capabilities are a series of advances that, when combined, create new classes of instrumentation. The major drivers for this include, of course, the advances in target and moderator technology highlighted in this Technical Design Report; in addition, high performance computing would allow the reconstruction and analysis of multimodal data and simulation of systems, glue together disparate data sets, and open complex scenarios to analysis. In addition, the big advances in manipulation of the spins of electrons, nuclei, and neutrons will allow control of scattering cross sections and contrast in situ and will revolutionize what can be measured in magnetic and hydrogenous materials. Another area of great advance is in optics, where lensing, guiding, and analysis are all taking major steps forward. Together, these will define the STS as a new generation neutron facility with far-reaching science impact.

\subsubsection{Science Impact of Second Target Station}

Overall, the development of the STS will ensure US leadership in neutron sciences for the foreseeable future. It will impact our understanding of materials by enabling an integrated understanding from the atomic to real-world scales. It will facilitate unprecedented access to quantum and complex matter and ensure that the unique contribution of neutrons to discovery and understanding in these fields continues for decades ahead. The advent of 3-dimensional mapping and micro-spot technologies, as well as the intensities and instrumentation, will allow in situ and in operando studies - not just of exemplary materials but also of real operating systems - in the most challenging problems in technology and engineering. Automation and high-throughput instrumentation, coupled with advanced modeling, databases, and libraries, will put STS center stage in the areas of materials by design and big data. Already, the close relationship between theory and experiment is putting neutrons at the forefront of the revolution in combining simulation and data. The high-intensity beams and advanced handling capabilities will open up the study of kinetics, nonequilibrium dynamics, and chemical reactions. They will facilitate understanding of materials, including the effects of material synthesis and processing. Such capabilities are expected to become all the more important as the prediction of compelling materials makes advances in their synthesis more pressing. Finally, the major step forward in capabilities possible with STS will reach out into new science areas and bring into reach whole areas of science, such as biology and chemistry, in which the unique properties of neutrons can have as important a role as they currently play in the study of quantum condensed matter and soft materials. 



\section{ACCELERATOR SYSTEMS}

A key part of the STS effort is providing the capability to double the beam intensity per pulse delivered to the target in short pulse mode (or double the proton beam power). The SNS accelerator was designed with a power upgrade in mind. Additional empty space was provided in the linac tunnel and in the radio frequency (RF) support building to accommodate the energy increase portion of the power upgrade.

A key aspect of the proposed accelerator upgrade is leveraging lessons learned during the initial 7 years of operation. For example, fewer superconducting RF cryomodules are needed for the energy increase than originally planned, based largely on the experience with the successful spare cryomodule presently in operation. Also, by incorporating intelligent $\mathrm{H}^{-}$beam chopping patterns, ion source requirements have been reduced, obviating the need for a dual source development.

Much of the proposed additional accelerator equipment is of the same type as is presently in use throughout the accelerator. Thus much of the accelerator scope does not involve development effort. One development area concerns the high-voltage convertor modulators (HVCMs), which supply modulated high-voltage waveforms for the RF generating sources. The increased beam loading associated with the higher beam intensity per pulse creates higher loads for this equipment, and some development is needed. Also, in the storage ring, the increased intensity raises some issues with the charge exchange injection process and with space charge, which are discussed later in this section.

Finally, the beam/target interface requires additional study. Efforts to provide as uniform a beam as possible on the target will include optimization of the injection painting and possible incorporation of nonlinear focusing elements. Target protection issues will also need to be addressed.

\subsection{ACCELERATOR PHYSICS}

\subsubsection{System Requirements}

The accelerator systems will be upgraded from the baseline capability of 1.4 to $2.8 \mathrm{MW}$. This will be accomplished by a combination of increased beam energy and increased beam current. The beam energy, presently $0.94 \mathrm{GeV}$, will be increased to $1.3 \mathrm{GeV}$ by plasma-processing of the existing superconducting linac (SCL) cavities and the addition of seven cryomodules. The average (chopped) macropulse beam current will be increased from 25 to $32 \mathrm{~mA}$ for the FTS and to $38 \mathrm{~mA}$ for the STS by improvements to the chopping scheme and to the ion source. Some minor modifications to the ring are also required to accommodate the higher beam energy. The SNS initial baseline and SNS STS design parameters are summarized in Table 3.1.

Table 3.1. Present operational and upgraded parameters

\begin{tabular}{lccc}
\hline \multicolumn{1}{c}{ Parameter } & 1.4 MW operation & Upgraded FTS & Upgraded STS \\
\hline Beam power on target $(\mathrm{MW})$ & 1.4 & 2.0 & 0.47 \\
Beam energy $(\mathrm{GeV})^{\text {Average linac current }(\mathrm{mA})^{a}}$ & 0.94 & 1.3 & 1.3 \\
${\text { Average linac macropulse current }(\mathrm{mA})^{a}}^{a}$ & 1.6 & 1.6 & 0.38 \\
$402.5 \mathrm{MHz}, 2.5 \mathrm{MW}$ klystrons & 25 & 32 & 38 \\
\hline $402.5 \mathrm{MHz}, 3 \mathrm{MW}$ klystrons & 7 & 5 & 5 \\
\hline $805 \mathrm{MHz}, 5 \mathrm{MW}$ klystrons & 0 & 2 & 2 \\
\hline $805 \mathrm{MHz}, 550 \mathrm{~kW}$ klystrons & 4 & 4 & 4 \\
\hline
\end{tabular}


Table 3.1. Present operational and upgraded parameters (continued)

\begin{tabular}{lccc}
\hline \multicolumn{1}{c}{ Parameter } & 1.4 MW operation & Upgraded FTS & Upgraded STS \\
\hline 805 MHz 700 kW klystrons & 0 & 28 & 28 \\
\hline SRF transmitter racks & 14 & 19 & 19 \\
\hline SRF high-voltage converter modulators & 7 & 10 & 10 \\
\hline High-beta cryomodules & 12 & 19 & 19 \\
\hline
\end{tabular}

${ }^{a}$ Assumes $5 \%$ of linac beam power is sent to injection dump due to stripping inefficiency.

\subsubsection{Chopping Requirements}

The radio frequency quadrupole (RFQ) output peak current requirement (46 mA) is substantially lower than the peak current requirement for the original power upgrade planning [1] (59 mA). Some ion source parameters are compared for the present design with the original power upgrade proposal in Table 3.2. The reduction in required peak current is driven primarily by a decrease in the fraction of beam chopped to provide a gap for fast extraction in the ring. In the original design, 30\% of the beam was chopped, whereas in the present design, only $18 \%$ of the beam is chopped. We have demonstrated during highpower operation $(1.4 \mathrm{MW})$ that it is possible to chop only $\sim 21 \%$ of the beam and still maintain low extraction beam loss. Simulations show that it is possible to further reduce the chopping fraction by varying the minipulse width (also referred to as pattern width [PW]) throughout the injection cycle. Figure 3.1 shows one example of a pattern width variation that increases the beam charge per pulse by $11 \%$ beyond the constant $\mathrm{PW}=51$ case presently used in operation, yet achieves good extraction efficiency [2]. Further gains in beam charge should be possible by also varying the RF buncher voltage during the ring injection time. Pursuing these measures greatly decreases the required ion source current, especially because the RFQ transmission decreases sharply above 60-70 mA input. With the present requirements, the existing RFQ design appears to be a feasible approach, and the required ion source output is a modest increase over present operation. The existing low-energy beam transport line (LEBT) chopper system does not allow variations in the minipulse width beyond the initial ramp, so an upgraded chopper system will be designed and installed.

Table 3.2. Comparison of required front end parameters for the present STS design and the earlier STS accelerator upgrade plan

\begin{tabular}{lcc}
\hline \multicolumn{1}{c}{ Parameter } & Original upgrade design & Present STS design \\
\hline Ion source output $(\mathrm{mA})$ & 74 & 54 \\
RFQ output current ${ }^{a}(\mathrm{~mA})$ & 59 & 46 \\
Chopping fraction $_{\text {Average linac macro-pulse current }(\mathrm{mA})}$ & 0.70 & 0.82 \\
\hline
\end{tabular}

${ }^{a}$ Calculated for transmission of a 0.25 pi-mm-mrad emittance input beam with the design field profile along the RFQ. 


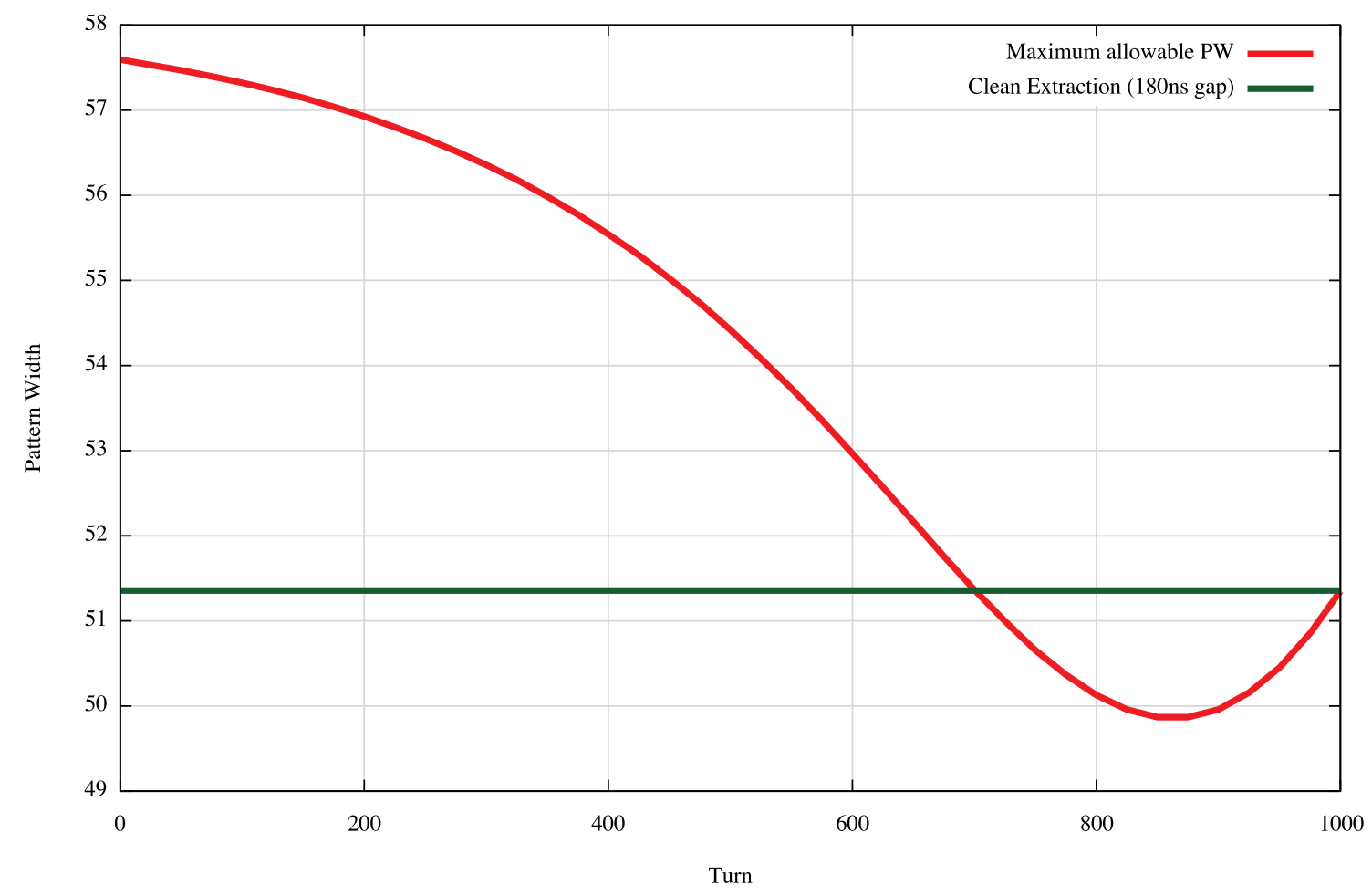

Fig. 3.1. Example pattern width variation over the ring injection cycle leading to a clean extraction gap with $11 \%$ higher charge per pulse. Any pattern width variation that falls beneath the red line is predicted to have equal or lower beam loss at extraction.

\subsubsection{Linac RF Power Requirements}

The linac RF cavities will require more RF power to maintain the same accelerating gradient at the higher STS beam currents. In the warm linac, it is not possible to mitigate the power increase by lowering the gradient because the geometry of the cavities dictates the beam energy for each cavity, and because many cells within the cavities are powered by a single klystron, which fixes the RF phase at each cell. Therefore, the only option is to increase the RF power delivered to each cavity. The warm linac RF systems, in which existing margin is not sufficient, will be upgraded to support acceleration of the $38 \mathrm{~mA}$ average linac macropulse beam current. The linac RF power requirements are summarized in Table 3.3.

In the SCL, there is more flexibility because there are only six cells per cavity (and per klystron). To support acceleration of a $38 \mathrm{~mA}$ beam current (averaged over the macropulse), some SCL gradients will be decreased because the RF power systems are already close to their maximum output powers. Other SCL gradients will be increased because their present gradients are limited by the cavity itself. Plasma processing, which is now being developed, will allow these gradient increases. The seven new cryomodules will take advantage of klystron improvements that raise the maximum output power from 550 to $700 \mathrm{~kW}$. Figure 3.2 shows the SCL RF cavity gradients before and after the STS upgrade. The resultant energy gain profile is shown in Fig. 3.3. The figure shows an output beam energy of 1,358 MeV, but in practice the capability above $1,300 \mathrm{MeV}$ will be used as reserve capacity. The corresponding SCL klystron output power requirements are shown in Fig. 3.4, including the beam loading and a control margin of $15 \%$. 
Table 3.3. Linac RF power requirements

\begin{tabular}{|c|c|}
\hline System & RF power requirement \\
\hline Warm linac $402.5 \mathrm{MHz}$ & Upgrade to support $38 \mathrm{~mA}$ avg. macropulse beam current \\
\hline Warm linac $805 \mathrm{MHz}$ & Upgrade to support $38 \mathrm{~mA}$ avg. macropulse beam current \\
\hline $\begin{array}{l}\text { SCL linac } 805 \mathrm{MHz} \text { - existing } \\
\text { cryomodules }\end{array}$ & $\begin{array}{l}\text { Upgrade to support } 38 \mathrm{~mA} \text { avg. macropulse beam current at cavity } \\
\text { gradients shown in Fig. 3.2. }\end{array}$ \\
\hline $\begin{array}{l}\text { SCL linac } 805 \mathrm{MHz}-\text { new } \\
\text { cryomodules }\end{array}$ & $\begin{array}{l}\text { New RF systems to support } 38 \mathrm{~mA} \text { avg. macropulse beam current at } 16 \\
\mathrm{MV} / \mathrm{m} \text { gradient }\end{array}$ \\
\hline
\end{tabular}

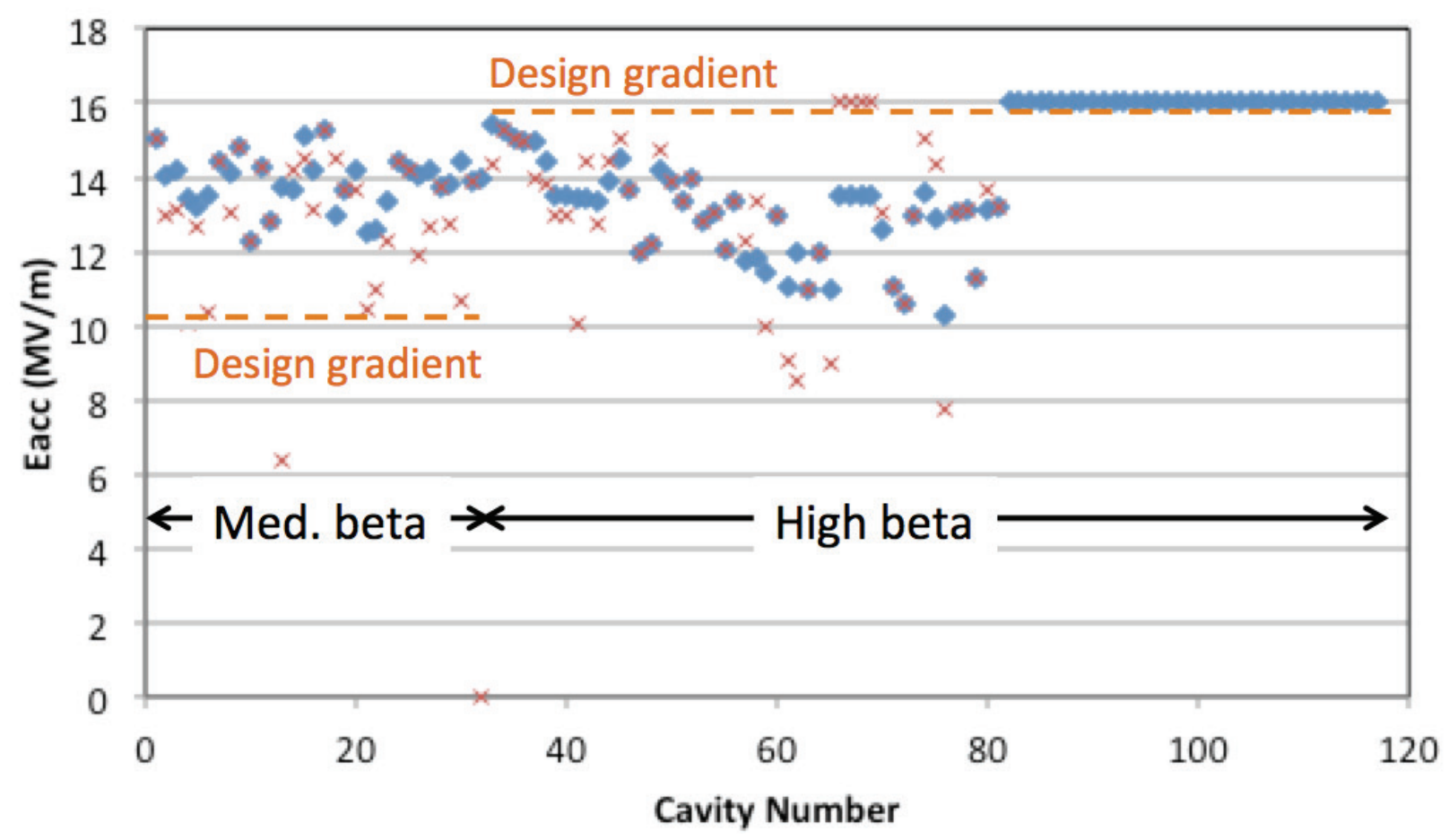

Fig. 3.2. SCL cavity gradients before ( $\operatorname{red} x$ ) and after (blue diamond) the STS upgrade. The orange dashed lines show the original design gradients. 


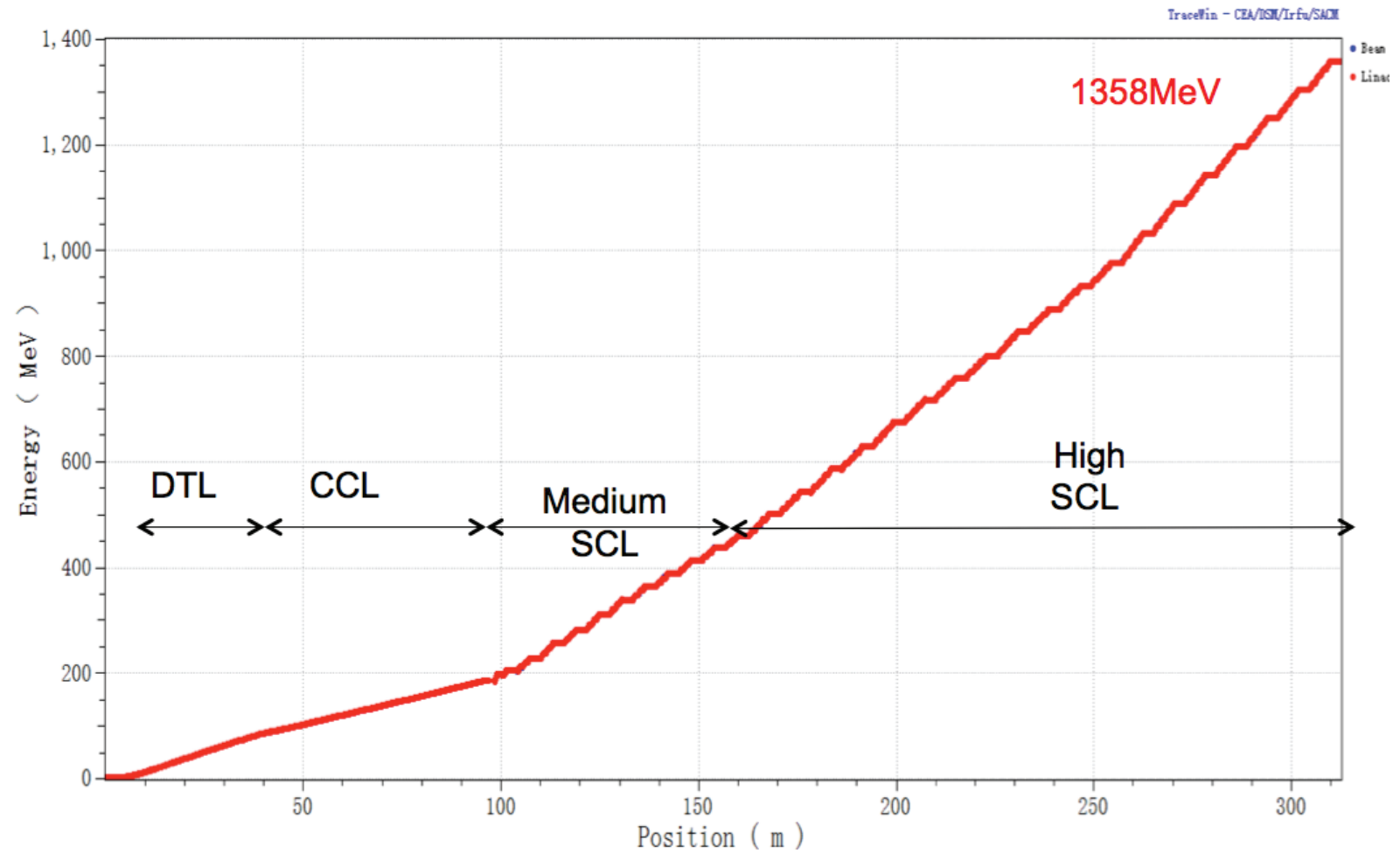

Fig. 3.3. The energy gain profile of the linac. The output energy of $1,358 \mathrm{MeV}$ includes the $58 \mathrm{MeV}$ reserve energy gain from the last three cavities.

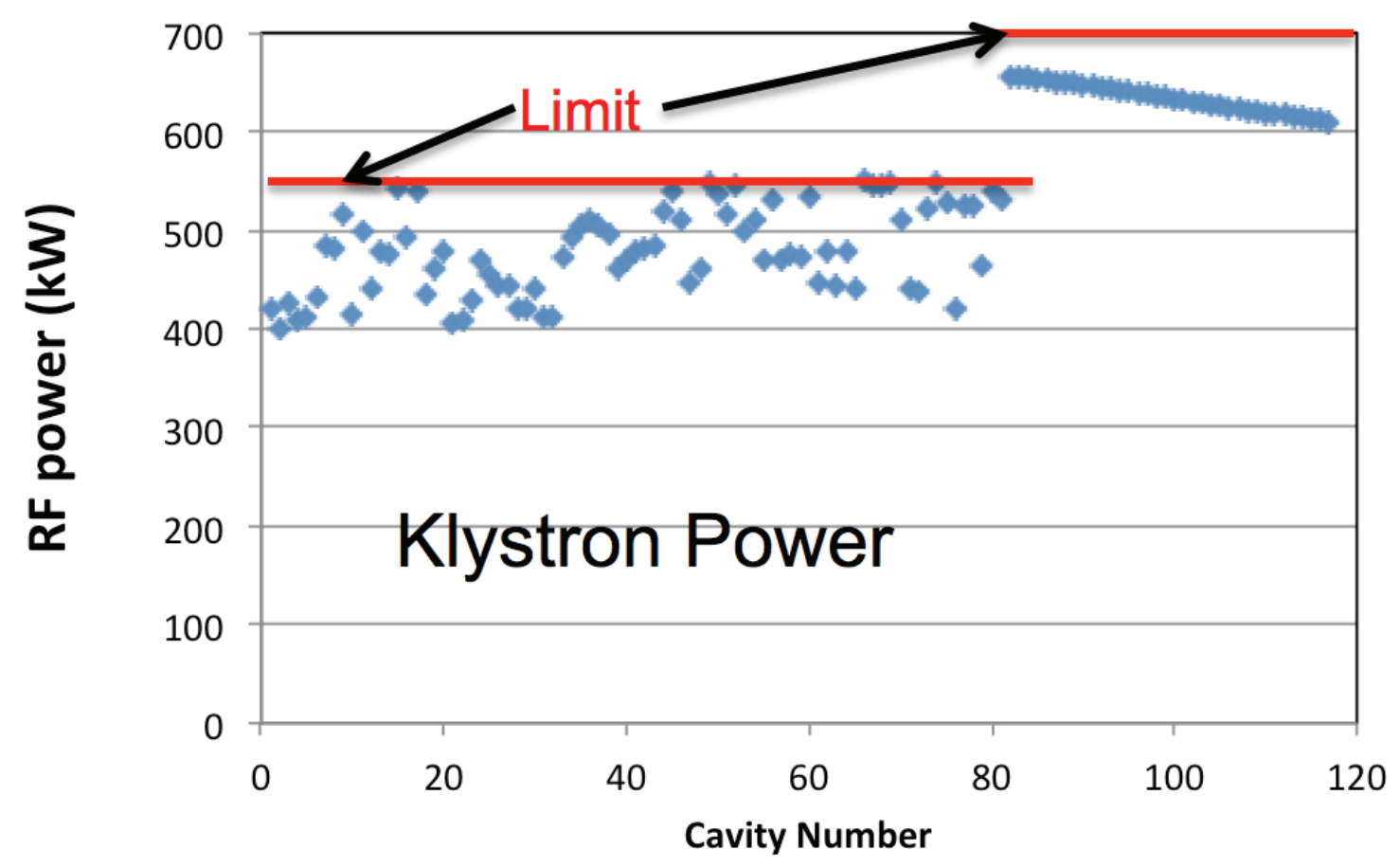

Fig. 3.4. Required SCL klystron output power requirement vs SCL cavity number. 


\subsubsection{Beam Simulations and Collective Effects Considerations}

\subsubsection{Linac Simulations}

Particle tracking simulations [3] have been performed for the STS linac to verify the new linac design [private communication from $\mathrm{Z}$. Wang regarding simulation using TraceWin, May 2014]. One million particles were tracked [3] from the exit of the RFQ to the exit of the SCL. The initial particle distribution was assumed to be a 3-sigma Gaussian with Twiss parameters taken from the standard SNS baseline case and reproduced in Table 3.4. The beam current was assumed to be $50 \mathrm{~mA}$, and the beam energy $2.5 \mathrm{MeV}$ (design output energy of the RFQ).

Table 3.4. Initial Twiss parameters for the linac simulation

\begin{tabular}{lccc}
\hline & Alpha & Beta $(\mathbf{m m} / \mathbf{m r a d})$ & Emittance $(\mathbf{m m} / \mathbf{m r a d})$ \\
\hline $\mathrm{X}$ & -1.9619 & 0.18314 & 0.21 \\
$\mathrm{Y}$ & 1.7681 & 0.16203 & 0.21 \\
$\mathrm{Z}$ & 0.0196 & 0.58 & 0.24153 \\
\hline
\end{tabular}

The resultant transverse and longitudinal beam envelopes are shown in Fig. 3.5, and the rms emittance growth is shown in Fig. 3.6. The simulation results are close to expectations and do not show any problems.
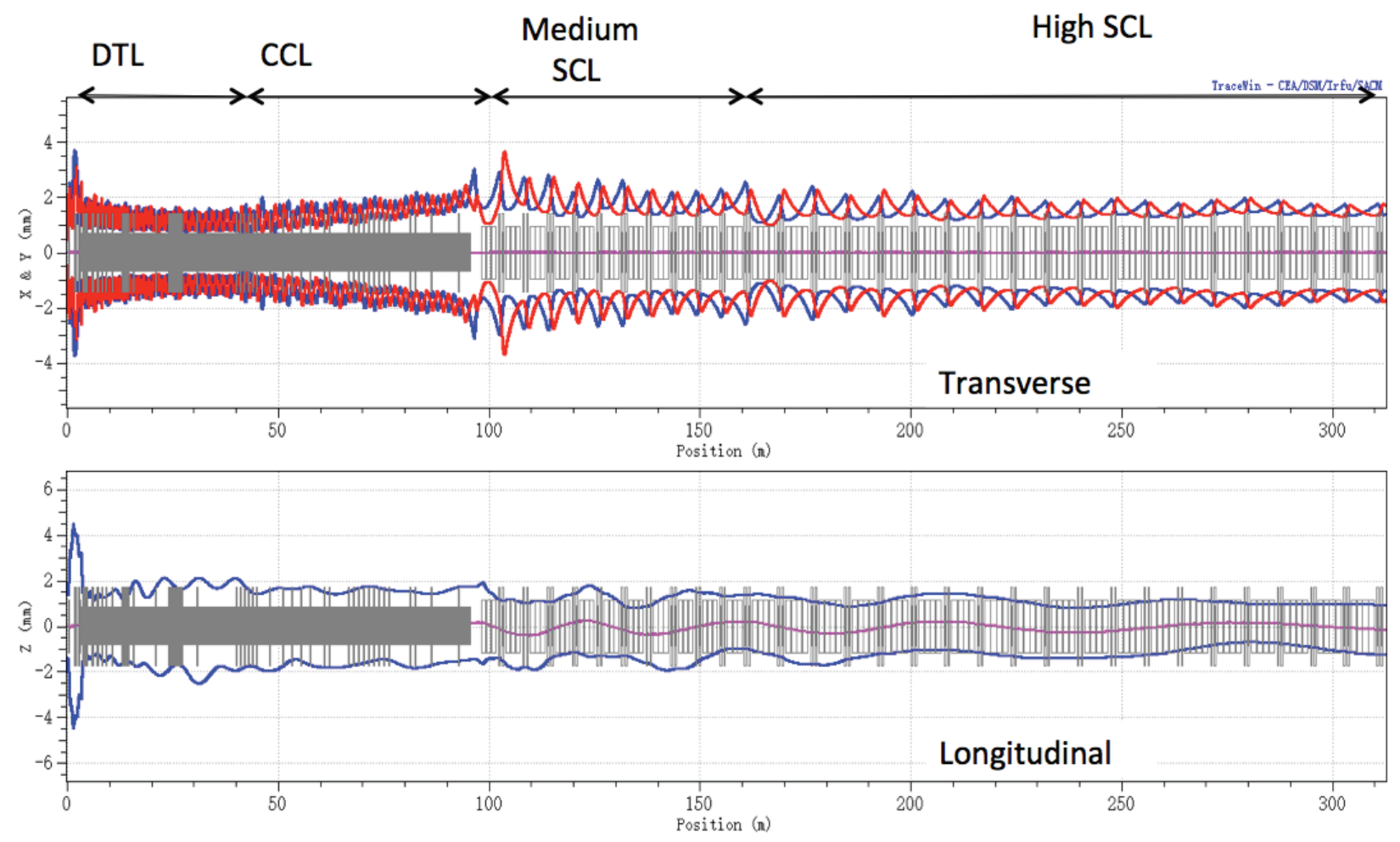

Fig. 3.5. Transverse and longitudinal beam envelopes for the STS linac. 


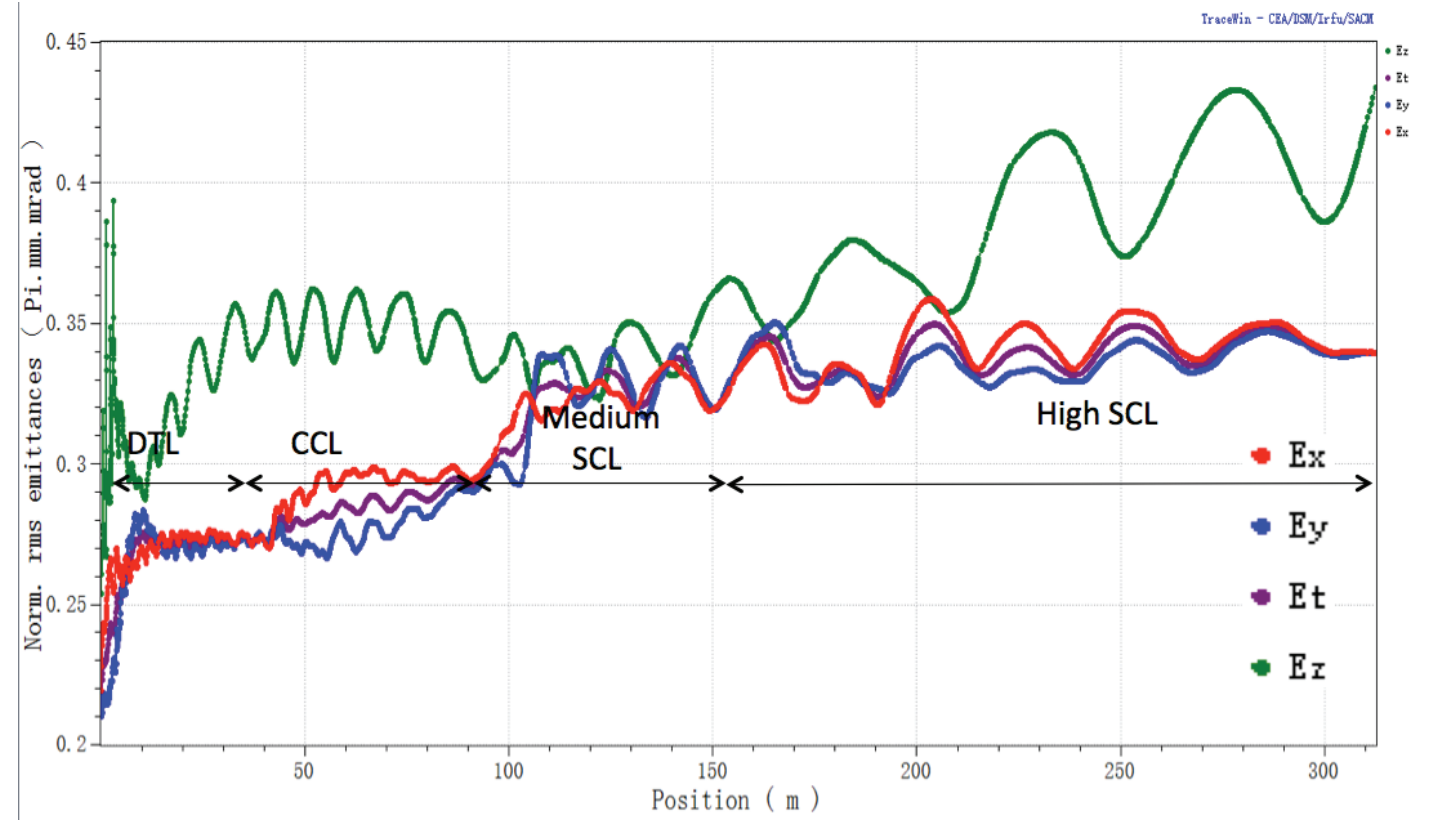

Fig. 3.6. Simulated rms emittance growth in the STS linac.

\subsubsection{HEBT Simulations}

The beam distribution from the linac simulation was used as a starting point for a separate particle-tracking simulation, using the ORBIT particle tracking code [4], of the high-energy beam transport line (HEBT) ending at the primary stripper foil in the ring injection. The results are in good agreement with expectations and similar to the SNS baseline case. Figure 3.7 shows one of the resulting plots. The beam distribution at the foil is suitable for charge-exchange injection into the accumulator ring at the stripper foil.

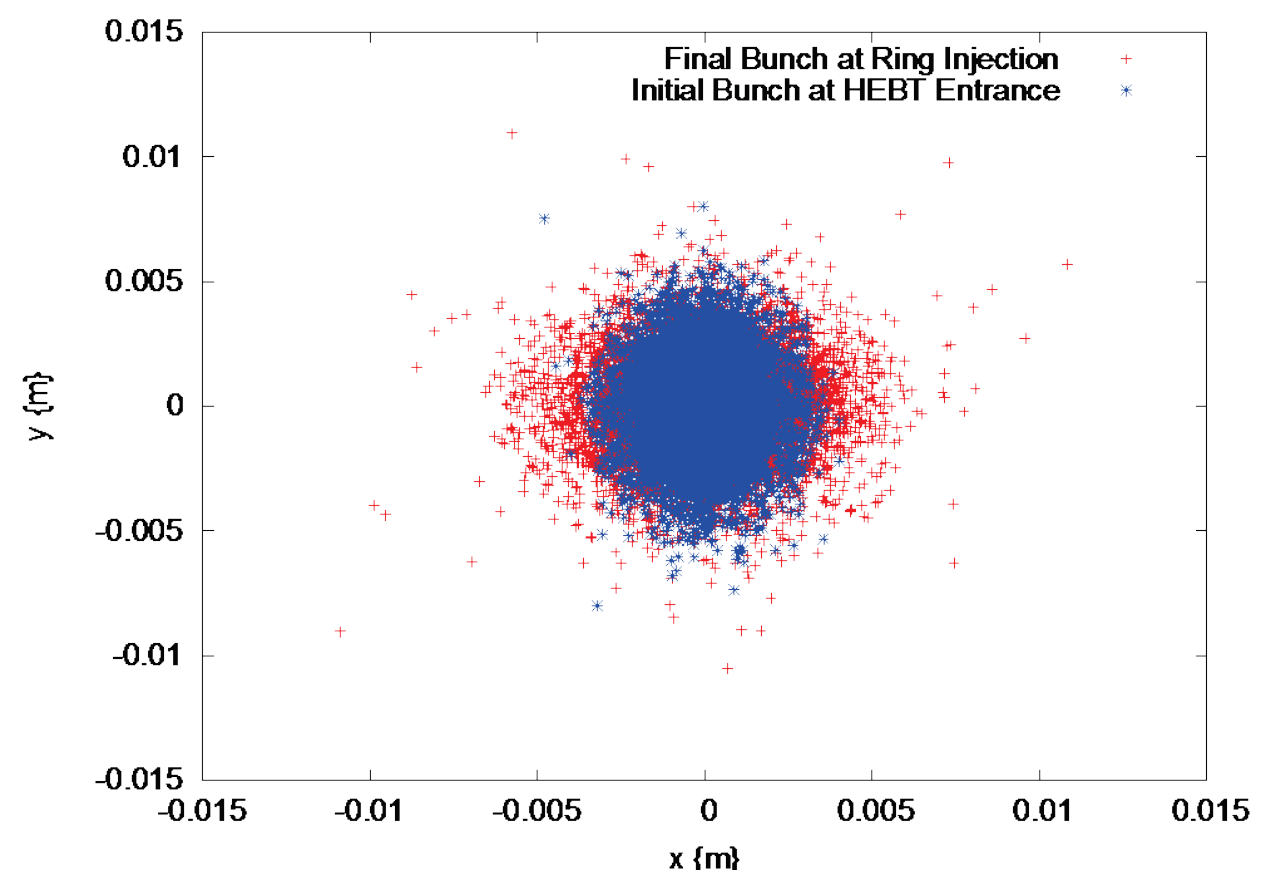

Fig. 3.7. Beam distribution at the HEBT entrance (blue) and at the primary stripper foil in the ring injection area (red). 


\subsubsection{Ring and RTBT simulations}

The ring simulation assumes the original SNS design input beam distribution at the stripper foil. This is acceptable because the STS HEBT simulation mentioned earlier produces essentially the same distribution, and because the input distribution represents a small paintbrush into the much larger ring aperture that is filled by the injection painting process. This simulation checks whether the beam delivery to the FTS will be essentially the same before and after the STS upgrade. Figures 3.8 and 3.9 show the resultant beam distributions at the proton beam window upstream of the first target and at the face of the first target, respectively.

\section{Beam Current at Target Window $\left[\mathrm{mA} / \mathrm{m}^{\wedge} 2\right]$}

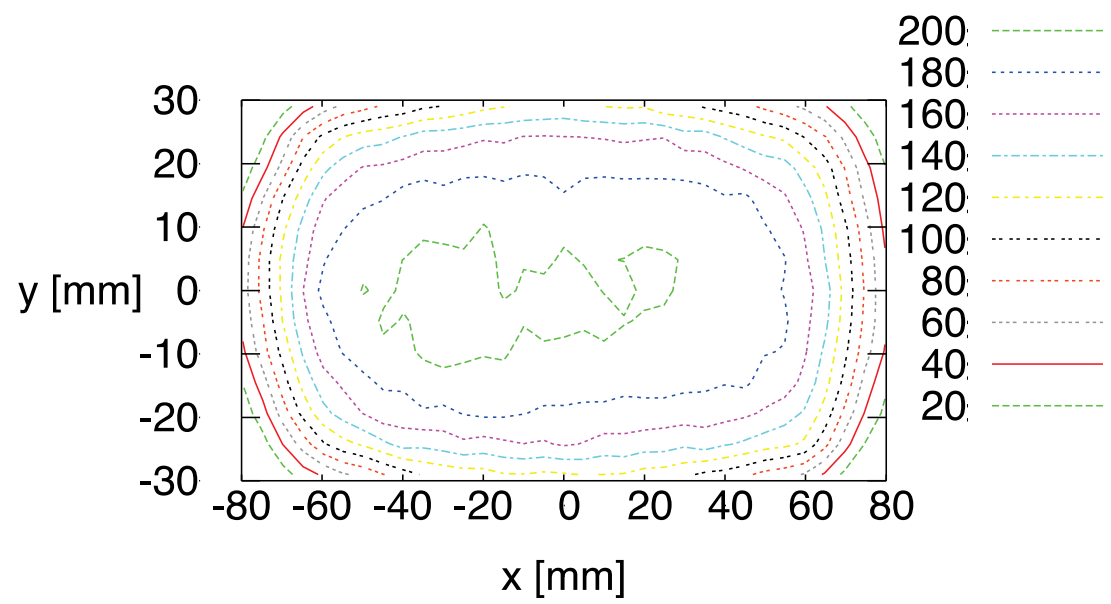

Fig. 3.8. Beam distribution at the proton beam window upstream of the first target, assuming a beam power of $2.0 \mathrm{MW}$ at $50 \mathrm{~Hz}$.

\section{Beam Current on Target $\left[\mathrm{mA} / \mathrm{m}^{\wedge} 2\right]$}

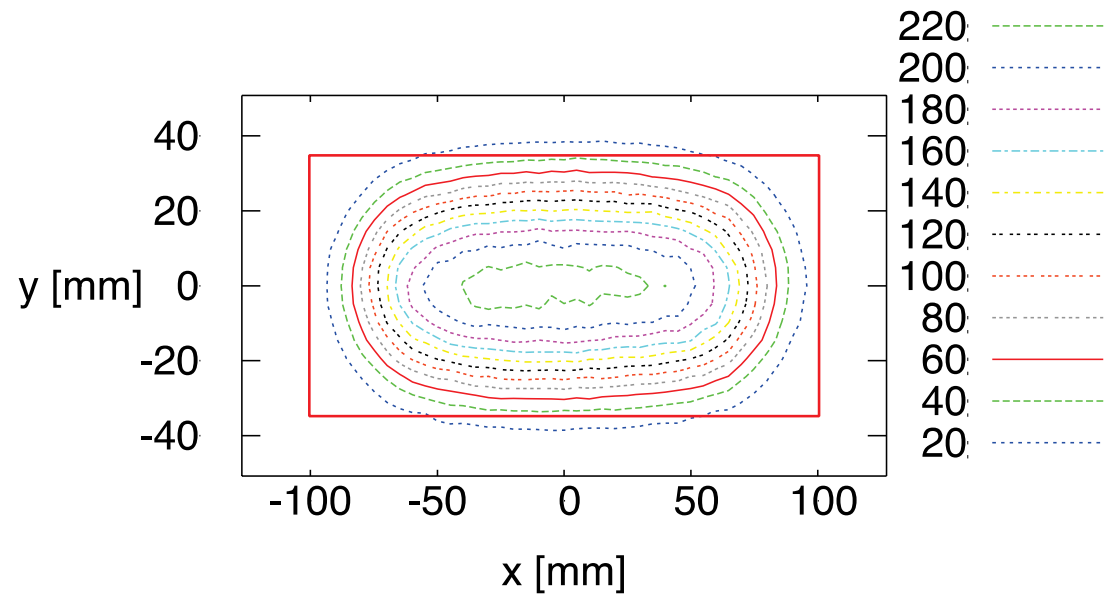

Fig. 3.9. Beam distribution at the first target assuming a beam power of $2.0 \mathrm{MW}$ at $50 \mathrm{~Hz}$. 


\subsubsection{Ring to Second Target Beam Transport Line Simulations}

The beam distribution from the ring simulation was also used to simulate the new beam transport to the STS (ring to second target beam transport line [R2T2]). The lattice for this beam line is described in Sect. 3.6.3. This simulation assumes the desired beam distribution on the target is such that the entire beam will fit within a rectangle $75 \mathrm{~mm}$ wide and $40 \mathrm{~mm}$ tall, as shown in Fig. 3.10. The R2T2 lattice is flexible enough to accommodate changes in the beam size requirement and changes in the starting beam distribution.

The present simulations use the same injection-painting scheme as used for the FTS. Future efforts will include optimizing the STS painting, using a nonlinear lens to provide a more uniform STS beam distribution, and incorporating a collimation system to protect moderators from beam halo and missteered beam.

\section{Beam Current on Target $\left[\mathrm{mA} / \mathrm{m}^{\wedge} 2\right]$}

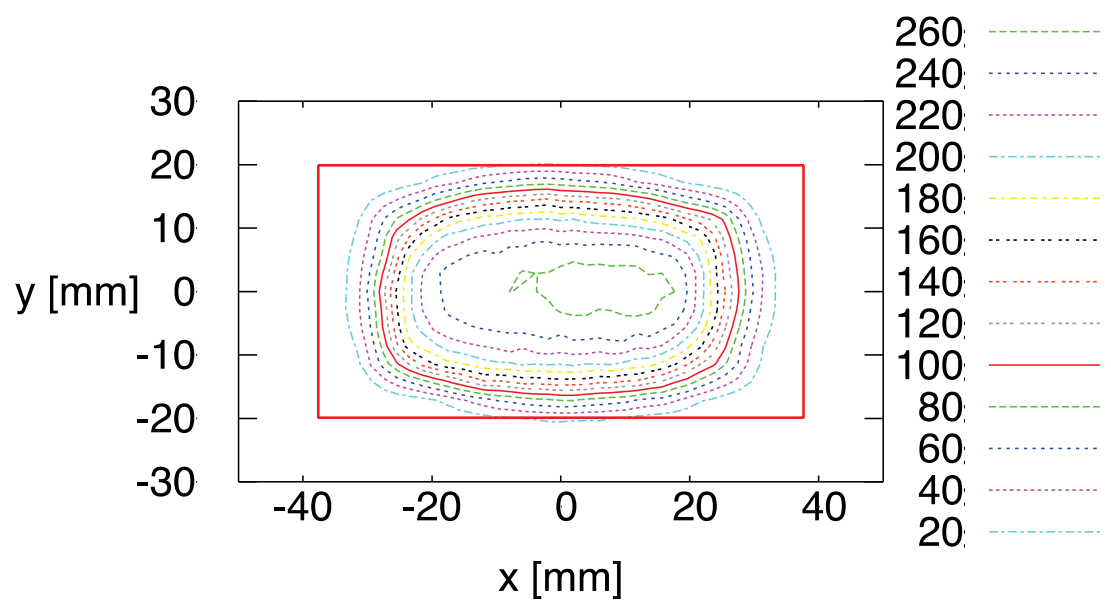

Fig. 3.10. Beam distribution on the second target assuming a beam power of $470 \mathrm{~kW}$.

\subsubsection{Space Charge Effects}

The purpose of this section is to assess the impact of collective effects on beam accumulation and transport at the proposed higher intensities for the STS. Because the allowable losses in SNS are extremely small, the accumulator ring was constructed with a large aperture and the beam pipe was coated with titanium nitride to reduce electron multipacting. The primary collective phenomena that could contribute to losses in the SNS ring are space charge, and extraction kicker impedances or electron clouddriven instabilities. In present operations, none of these phenomena contribute significantly to beam loss. Losses in the SNS ring at this time are dominated by beam scattering from the primary stripper foil [5]. However, it is necessary to assess the impact of collective effects as operation at still higher intensities is considered.

In the longitudinal direction, it is operationally possible to maintain a clean gap for beam extraction to the target with significantly less RF power than is called for in the original SNS design. From this fact, we can infer that longitudinal space charge and impedance effects will be mild. This is consistent with simulations performed during the SNS design that showed a wide margin of stability in the longitudinal plane [6]. In the transverse plane, space charge effects are observed to smooth the beam profiles and to 
introduce some coupling between the horizontal and vertical directions, but these have no effect on losses or the ability to provide the required beam footprint at the target. In one controlled experiment, it was necessary to go to great lengths to induce instability because of the impedance of the extraction kickers. Simulations matched the observed growth rate of this instability under the conditions of the experiment [7], and similar simulations show a wide margin of stability under normal operating conditions. The most likely cause of instabilities at high intensity may be electron cloud phenomena. In anticipation of this, the beam pipe in the ring was coated with titanium nitride to reduce electron multipacting. Even so, there are indications in the broadband transverse spectrum that the SNS ring beam may be at the onset of the electron cloud instability under present operating conditions. However, at present, no losses can be attributed to electron cloud activity.

It is possible to estimate analytically the effects of increased beam energy and intensity on the forces due to space charge and to known impedances. Table 3.5 presents such an estimate for four sets of parameters: present SNS production, the original design, the beam to the FTS with upgraded energy and intensity, and the beam to the STS with upgraded energy and intensity. The latter two cases use the parameters given in Plum et al. [8]. In all cases, the beam distribution sizes and shapes are considered to be the same, and the changes in impedance due to the altered ring and betatron frequencies are neglected. The scaling of the space charge and impedance forces is calculated as functions of beam energy and intensity only. However, an additional factor of $8 / 7$ is included in the impedance-induced forces for the upgrade scenarios, because the number of extraction kickers will increase by 2, from 14 to 16, for the upgrade. The quantities used to compare the effects of the forces under the four scenarios are the induced energy changes for the longitudinal forces and the angular kicks for the transverse forces. The strengths of the forces for all cases are normalized to those for the present production parameters. Under the stated assumptions, the strengths of all forces increase linearly with increasing beam intensity, as measured by number of injected protons. Except for the longitudinal impedance, this increase with intensity is offset by decreasing strength with increasing energy, as a result of relativistic effects and increasing beam stiffness. Table 3.5 shows that, because of these offsetting effects, neither longitudinal nor transverse space charge forces should pose a problem under upgraded operational scenarios. The longitudinal space charge force barely increases with the STS upgrade parameters, and the transverse space charge force actually decreases. The forces associated with the extraction kicker impedance do increase, especially for the STS parameters. Previous simulations [7, 9] show there is a wide margin of stability-a factor of at least five in the longitudinal direction and more than two in the transverse direction - with respect to the extraction kicker impedance. Given these results and the values in Table 3.5, we anticipate that operation at the upgrade parameters should be stable with respect to the space charge and impedance forces. To further support those expectations, we have performed simulations of the first and second target upgrade scenarios with the ORBIT code.

Table 3.5. Comparison of collective forces in SNS for four operating scenarios: present production, the original design, first target with intensity upgrade, and second target with intensity upgrade

\begin{tabular}{lcccc}
\hline \multicolumn{1}{c}{ Case } & Production & Design & Target 1 upgrade & Target 2 upgrade \\
\hline Kinetic energy $(\mathrm{GeV})$ & 0.94 & 1.0 & 1.3 & 1.3 \\
Relativistic $\gamma$ & 2.002 & 2.066 & 2.386 & 2.386 \\
Relativistic $\beta$ & 0.866 & 0.875 & 0.908 & 0.908 \\
$\mathrm{~N}$ protons $\left(\times 10^{14}\right)$ & 1.55 & 1.5 & 1.921 & 2.258 \\
Longitudinal space charge $\left(\Delta \mathrm{E} \sim \mathrm{N} \times \gamma^{-2}\right)$ & 1.0 & 0.909 & 0.873 & 1.026 \\
Longitudinal impedance $(\Delta \mathrm{E} \sim \mathrm{N} \times \beta)$ & 1.0 & 0.977 & $a 1.484$ & 0.745 \\
Transverse space charge & 1.0 & 0.863 & 0.667 & 0.784 \\
$\left(\Delta \theta \sim \mathrm{N} \times \gamma^{-3} \times \beta^{-2}\right)$ & & & & $a$ \\
Transverse impedance $\left(\Delta \theta \sim \mathrm{N} \times \gamma^{-1} \times \beta^{-1}\right.$ & 1.0 & 0.928 & $a 1.134$ & \\
\hline
\end{tabular}

${ }^{a}$ Impedance forces for upgrade scenarios multiplied by 8/7 to account for two additional extraction kickers. 
Simple analytic estimation is not possible for the effect of electron clouds on the accumulating proton beam, as it is for the effects of space charge and known impedances. The specifics of the generation mechanisms, locations, and quantities of ambient electrons in the accelerator are not precisely known. It is generally accepted that ambient electrons are generated by beam collisions with the beam pipe (beam loss), by ionization of the imperfect vacuum by the beam, and by multipacting from existing electrons striking the beam pipe [10]. A possible additional mechanism in SNS is incomplete collection of stripped electrons at the injection point. The behavior of ambient electrons generated in the beam pipe will depend on their location. Electrons in a drift space, dipole field, or quadrupole field have different orbits and will respond to the beam in different ways. Little is known of the location of electrons in the SNS ring, but it may be assumed that they are located in the vicinity of the highest beam losses, downstream of the injection point and in the collimation section.

At the SNS, steps have been taken that mitigate the electron generation mechanisms, although the primary motivation for some of these steps is to minimize activation from beam loss. Fractional beam losses are maintained at a very low level $\left(\sim 10^{-4}\right)$, and low beam loss corresponds to the generation of fewer electrons. The large beam pipe aperture ( 8 in. diameter) helps in loss minimization, and it places the location of electron multipacting further from the beam. A high vacuum of $10^{-8}$ Torr is maintained in the ring, so electron generation by gas ionization is small. The geometry of the magnetic field in the vicinity of the stripper foil has been carefully designed and studied [11] to guide the stripped electrons to a collector. The walls of the SNS ring have been coated with titanium nitride for the specific purpose of reducing the secondary electron yield from multipacting. Additionally, a broadband feedback system has been developed to counter the effect of any observed electron cloud-induced instability [12].

Some simulations of the electron cloud dynamics in SNS have been performed $[13,14]$. According to Cousineau et al. [12], a linearized stability model and more detailed simulations are consistent with one another in predicting that, with $2 \times 10^{14}$ protons, SNS should be stable for first harmonic RF voltages exceeding $15 \mathrm{kV}$. The available first harmonic RF voltage in SNS is $40 \mathrm{kV}$. Overall, we anticipate that SNS can be operated stably with respect to electron clouds at the upgraded parameters. If electron cloud instabilities should arise, additional measures, such as broadband feedback stabilization and longitudinal profile tailoring, can be applied.

\subsection{FRONT END SYSTEMS}

The STS power increase will require a peak current of $46 \mathrm{~mA}$ out of the RFQ. This implies a peak current of about $54 \mathrm{~mA}$ delivered by the source, which corresponds to an $87 \%$ RFQ beam transmission. No increase above the present beam duty factor is planned for STS operation; it will remain at $60 \mathrm{~Hz} \times 1 \mathrm{~ms}$ or $6 \%$. The baseline plan to reach a $54 \mathrm{~mA}$ source output is incremental improvement of the baseline ion source using the existing test stand. The plan for reaching the design RFQ transmission of $87 \%$ at this current level is to use the existing, recently procured new RFQ. Also, a modified chopping pattern waveform procedure will be implemented to produce the required $82 \%$ unchopped beam fraction, as discussed in Sect. 3.1. Each of these developments is discussed in the following sections.

\subsubsection{Ion Source}

The present ion source beam production currents are shown in Fig. 3.11 [private communication from Martin Stockli, May 2014]. These data are measured at the entrance to the RFQ on the front end of the SNS machine. Three primary sources are used in operations, averaging about 45-50 mA with some demonstrating up to $\sim 65 \mathrm{~mA}$ (note that for much of the period before 2014, there was not a demand for higher beam current, as the beam power ramp-up was not pursued). Increasing the reliable source output to $54 \mathrm{~mA}$ will accomplished by 
- increasing the RF power capability

- optimizing the source collar aperture and dumping the magnetic field profile

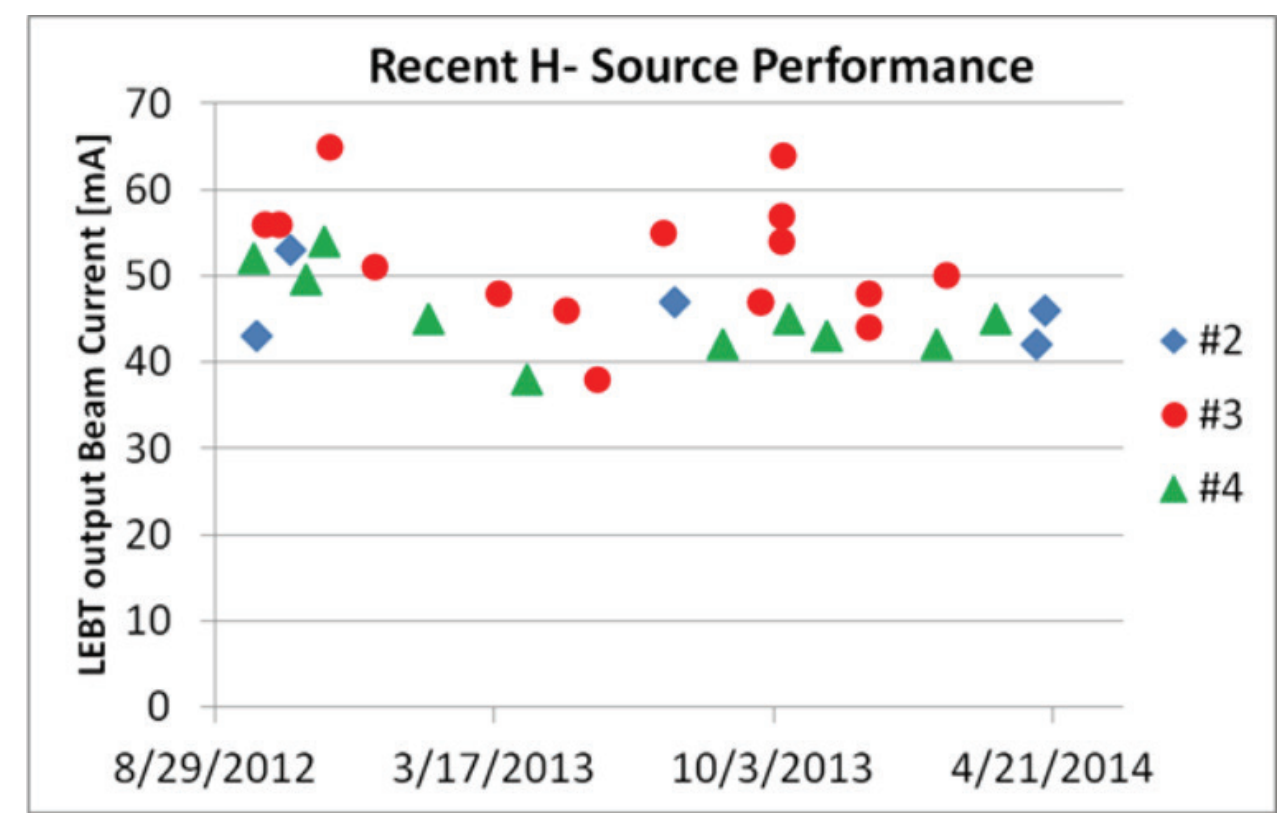

Fig. 3.11. Recent ion source current, measured at the RFQ entrance on the SNS front-end.

\subsubsection{Radio Frequency Quadrupole}

The nominal RFQ beam transmission is shown in Fig. 3.12 for several input beam emittance levels [private communication from A. Aleksandrov, June 2014]. For the nominal input emittance of $0.25 \mathrm{pi}$ $\mathrm{mm}$-mrad, the transmission is $\sim 87 \%$ for an input current of $\sim 54 \mathrm{~mA}$. Above $\sim 70 \mathrm{~mA}$ the transmission efficiency begins to drop quickly, but this current level is well above the STS requirement.

Nonetheless, $87 \%$ transmission is significantly higher than the measured transmission of $70-80 \%$ in the original, presently installed RFQ. There is a known field profile issue with the present RFQ, which was the original equipment item delivered from Lawrence Berkeley National Laboratory. The present operational RFQ, however, has had several detuning incidents, and the field profile at the RFQ entrance is not known (after the last detuning incident the field profile was corrected along most of the RFQ, but measurements were not possible at the RFQ entrance because of access limitations). To address this vulnerability, a new RFQ was manufactured; has been delivered; and is presently being prepared for testing, at high power and with beam, in a test stand. Measurements from this test with beam will provide a basis for the assumptions discussed for the ion source and RFQ performance. 


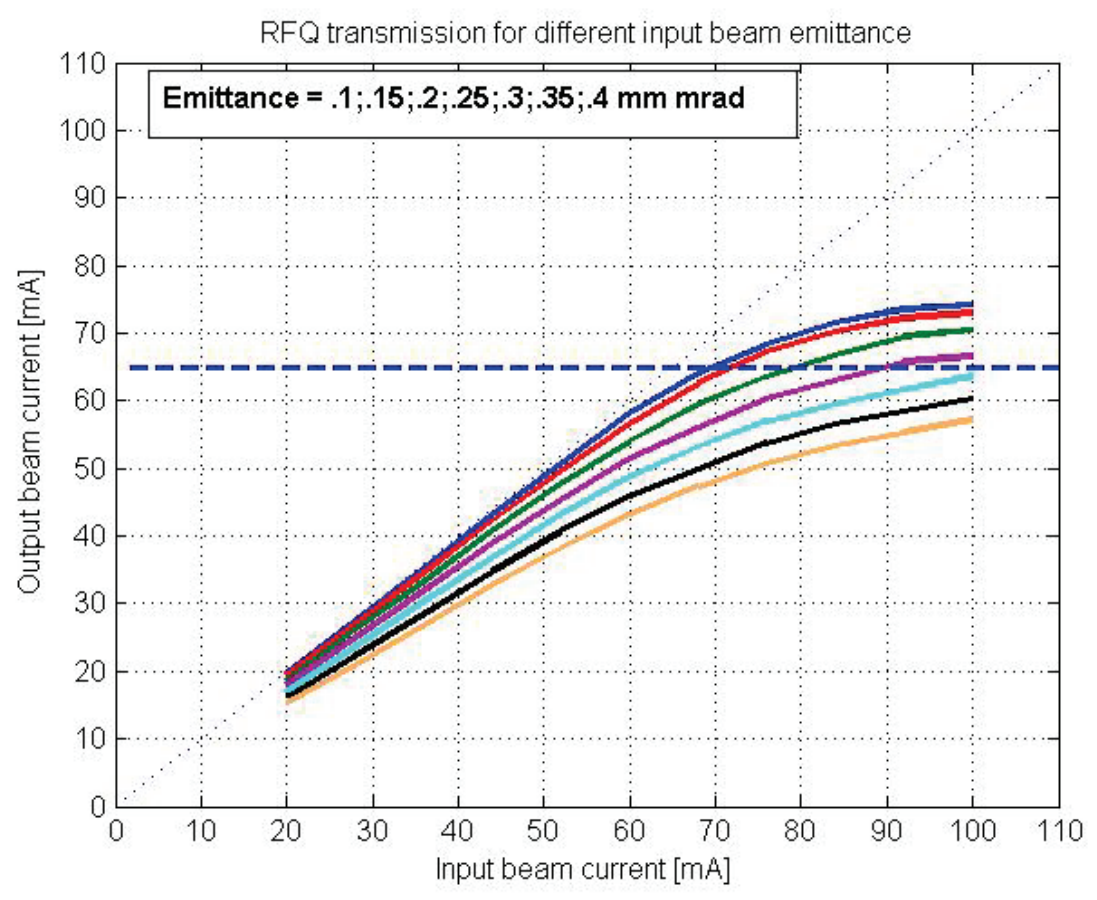

Fig. 3.12. RFQ beam transmission from simulations for the design value field profile along the RFQ.

\subsection{SUPERCONDUCTING LINAC}

Seven cryomodules will be installed in the reserved space at the end of the linac tunnel to produce the linac output energy of $1.3 \mathrm{GeV}$ with an energy margin specified in Sect. 3.1.

The optimum geometric beta of the cavities for STS is higher than that of the original high-beta cavities; but the module length and, accordingly, the cavity geometric beta will be kept the same for economic reasons. All helium transfer lines were already installed during the SNS project with bayonets for the high-beta cryomodules. Waveguide penetrations from the Klystron Gallery to the linac tunnel were also installed.

Some changes, which do not require changes of overall layout, will be made based on the lessons learned from experience over the last 10 years and on the pressure-vessel compliance issue. Table 3.6 summarizes the design changes between the original SNS high-beta cryomodule and the high-beta cryomodule for the STS. Some details for the changes are described in the following subsections. 
Table 3.6. Design parameter changes

\begin{tabular}{|c|c|c|}
\hline Parameters & $\begin{array}{c}\text { Original SNS high-beta } \\
\text { cryomodule }\end{array}$ & STS high-beta cryomodule \\
\hline $\begin{array}{l}\mathrm{E}_{\mathrm{acc}}\left(=\mathrm{E}_{\mathrm{o}} \mathrm{T}_{\mathrm{g}}, \mathrm{T}_{\mathrm{g}}: \text { Transit time factor at }\right. \\
\beta=0.81)(\mathrm{MV} / \mathrm{m})\end{array}$ & 15.8 & 16.0 \\
\hline $\begin{array}{l}\text { Fundamental power coupler (FPC) } \\
\text { rating, peak and average }(\mathrm{kW})\end{array}$ & 550,50 & 700,65 \\
\hline External Q of FPC, $Q_{\mathrm{ex}}$ & $7 \times 10^{5}( \pm 20 \%)$, fixed type & $8 \times 10^{5}( \pm 20 \%)$, fixed type \\
\hline Material of cavity & $\begin{array}{l}\text { High } \mathrm{RRR} \mathrm{Nb}(\mathrm{RRR}>250) \text { for cells, } \\
\text { reactor-grade } \mathrm{Nb} \text { for end groups }\end{array}$ & $\begin{array}{l}\text { High RRR } \mathrm{Nb}(\mathrm{RRR}>250) \text { for } \\
\text { both cells and end groups }\end{array}$ \\
\hline Higher order mode couplers per cavity & 2 (one at each end group) & None \\
\hline Tuner & $\begin{array}{l}1 \text { mechanical tune, } 1 \text { fast piezo } \\
\text { tuner }\end{array}$ & $\begin{array}{l}1 \text { mechanical tuner (no fast piezo } \\
\text { tuner) }\end{array}$ \\
\hline Pressure vessel & Good engineering practice & Code stamp required \\
\hline
\end{tabular}

$\mathrm{RRR}=$ residual resistance ratio

\subsubsection{SRF Cavity}

Cavity RF parameters for the STS are the same as the original ones. Table 3.7 shows the common parameters between the original and STS cavities.

Table 3.7. Major parameters of the SNS high-beta cavity

\begin{tabular}{lc}
\hline Frequency $(\mathrm{MHz})$ & 805 \\
Type & Elliptical \\
Operating mode & pi \\
Geometric beta & 0.81 \\
Equivalent cavity length $(\mathrm{mm})$ & 906 \\
Bore radius (mm) & 48.8 \\
Inter-cell coupling $(\%)$ & 1.6 \\
r/Q at geometric beta $(\mathrm{ohm})$ & 483 \\
Epeak/Eacc & 2.2 \\
Bpeak/Eacc (mT/MV/m) & 4.75 \\
Wall thickness (mm) & $2 \mathrm{~K}$ \\
Operating temperature $(\mathrm{K})$ & $>5 \times 10^{9}$ at $2 \mathrm{~K}$ \\
Qo & 6 \\
Number of cells per cavity & Yes (at $\mathrm{r}=80 \mathrm{~mm})$ \\
Stiffener & 1 \\
Fundamental power coupler per cavity & \\
\hline
\end{tabular}

\subsubsection{Accelerating Gradient}

The accelerating gradients of the STS, shown in Sect. 3.1, are based on the results from the spare highbeta cryomodule and the research and development for performance improvements. 
New cavities for the STS. The high-beta spare cryomodule was developed in-house and has been installed in the tunnel for operation since summer 2012. All four cavities in the spare cryomodule were commissioned at $17 \mathrm{MV} / \mathrm{m}$ or higher at the full duty factor. Only one cavity shows minor x-rays in the tunnel, and all four cavities have been running at $16 \mathrm{MV} / \mathrm{m}$ for the production run $[15,16]$. The available RF power limits the operating gradients at $16 \mathrm{MV} / \mathrm{m}$. The performances of the original SNS cavities in the linac are limited by field emission to lower gradients. Improvement of the cavity performance for the spare cryomodule came from changing the cavity chemical processing recipe from buffered chemical processing $(\mathrm{BCP})$ to electropolishing $(\mathrm{EP})$. EP produces a smoother surface finish on the inside of the cavity and has typically shown higher operational gradients and less field emission at lower gradients. One identified limitation of all original SNS cavities is that the end group surfaces are very rough as a result of high-aspect-ratio deep drawing and the additional heat treatments. These end groups were fabricated from reactor-grade niobium, rather than high-purity or high residual resistance ratio (RRR) material, in an attempt to reduce the material costs of the cavities. The reactor-grade material was then heat-treated in a vacuum furnace to improve its thermal conductivity, but this increased the niobium grain size in the process. The increased grain size coupled with BCP preferentially etches the grain edges, creating a very rough surface finish on all original SNS cavities. This rough surface on the end groups is difficult to clean and has many sharp edges at the grain boundaries. It could also produce trapped volumes for particulates and gases, consequently increasing the secondary emission yield and field emission due to field enhancement at the sharp edges. The EP process that was applied to the spare cryomodule cavities improved the surfaces of both the cells and the end groups and allowed for better cleaning of all surfaces following the chemical processing. The cavity performance observed in the high-beta spare cryomodule offers high confidence that the STS cryomodules will meet the gradient specification by using EP instead of BCP for the final chemistry and by improving the cavity end groups.

Original SNS cavities in the tunnel. Most of the original operational cavities exhibit heavy field emission, which directly or indirectly (through heating of the end groups) limits the gradient achievable in normal operation with beam. In addition to individual cavity field emission, collective effects have been observed that affect neighboring cavities in a cryomodule. These effects, which result in heating of cavity elements, is driven not only by the amplitude but also by the relative phases of neighboring cavities. In the SNS linac, the amplitudes and phases of neighboring cavities are correlated; therefore, operation into heavy field emission must be prevented for the sake of stability, thus limiting the final linac output beam energy. Each cavity is set at a maximum gradient based on the collective limiting gradients achieved through a series of superconducting RF (SRF) cavity/cryomodule performance tests at SNS, rather than by setting uniform gradients as designed [17].

At SNS, in situ processing in the tunnel has been identified as an important area of research to improve the SRF cavity performance while minimizing the machine operational impact and reducing the cost of the improvements. Active research and development for in situ processing using plasma is ongoing at SNS; the results show the potential to increase the accelerating gradients in operating cavities by $2 \mathrm{MV} / \mathrm{m}$ on average. The accelerating gradient of the existing cavities for the STS is an example of gradients after plasma processing for both medium- and high-beta cavities. Accelerating gradients of some high-beta cavities need to be lowered owing to available RF power. In addition, cavities 5a and 1 are known to exhibit problems. These cavities are not candidates for in situ processing and are assumed to be taken out of service and repaired for operation. If performance improvements after repair or in situ plasma processing are less than expected, the linac output energy will still be $1.3 \mathrm{GeV}$, but with reduced energy margin.

Dynamic detuning compensation. Observed dynamic detuning of the original SNS high-beta cavities due to the Lorentz force is in the range of $1-2 \mathrm{~Hz} /(\mathrm{MV} / \mathrm{m})^{2}$ during the whole pulse. The dynamic detuning during the beam is less than $0.5 \mathrm{~Hz} /(\mathrm{MV} / \mathrm{m})^{2}$. Since the cavity bandwidth is relatively large $(\sim 500 \mathrm{~Hz})$ or external Q is relatively low $\left(<10^{6}\right)$ because of the high beam loading, the additional RF 
power needed to compensate for the dynamic detuning is minor. This amount of detuning is well managed by the adaptive feed forward. Fast piezo tuners were installed on the original cavities to mitigate any unexpected mechanical resonance conditions, but these fast tuners have never been used at SNS. The piezo tuner design has proved to be mechanically unreliable and will not be part of the tuners for the STS.

\subsubsection{End Group}

End group thermal stability. The RRR of niobium for the end group of the existing cavities is about 70 after heat treatment. The end group is cooled by indirect conduction to the helium circuit. The thermal processes of the end group are more or less slow, since the surface magnetic fields are relatively small and the cooling relies on conduction. In terms of thermal stability, the end group can allow a fairly large material defect size because the field is low and the total thermal load is small. But the end group is sensitive to heating from a broad range of thermal loads, such as thermal radiation from the inner conductor of the fundamental power coupler (FPC), field emission, and multipacting, because of the long conduction path to the thermal sink. As mentioned earlier, most of the existing cavities show large field emission, which is the major operational limiting condition, leading to end group heating, gas bursts, and cavity quench [18].

If the RRR of niobium is larger than 150, the end group will be stable at larger thermal loads from electron activities and/or local heating at the end group. Figure 3.13 shows temperature profiles of the high-beta cavity end group. Thermal radiation from the FPC is assumed at $3 \mathrm{~W}$ uniformly distributed over the end group surface for a conservative analysis, and the design gradient surface magnetic field is used for the $4 \mathrm{~K}$ helium case. For the $2 \mathrm{~K}$ helium case, the end group temperature profile is about same, except in very close proximity to the helium vessel. Without any other thermal load except thermal radiation from the FPC, the maximum temperature in the end group will be lower by $1 \mathrm{~K}$ for the $\mathrm{RRR}=150$ case. The analysis also indicates that the end group will not be stable with an additional localized external thermal load of 0.2 to $2 \mathrm{~W}$, depending on the magnetic field for the RRR=70 case, whereas it will still be stable with five times larger additional thermal loads for the $R R R=150$ case.

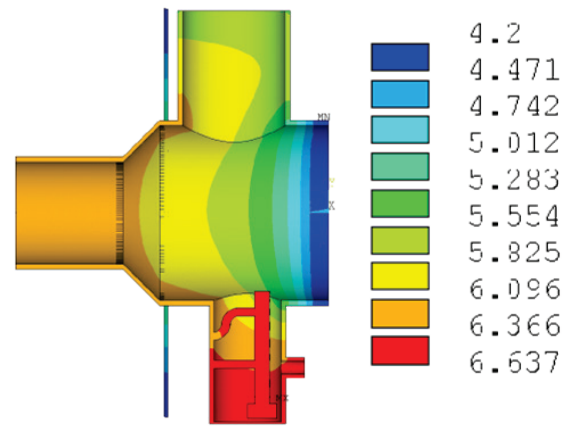

(a) $\mathrm{RRR}=\mathbf{7 0}$

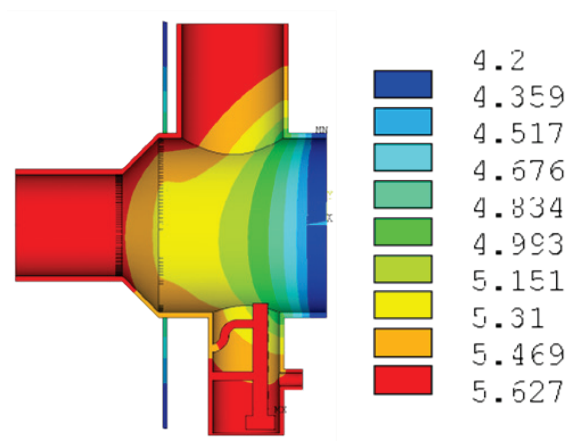

(b) $R R R=150$

Fig. 3.13. End group temperature profiles with (a) $R R R=70$ and (b) $R R R=150$.

The other thermally weak location in the original cryomodules is the cavity end group at the field probe side at the warm-to-cold transitions, as shown in Fig. 3.14. At normal operating conditions, the temperature at this location is about $7-8 \mathrm{~K}$. When there is electron activity, other external thermal loads, such as beam halo or a combination of these around this warm-to-cold transition, the local temperature easily goes to hydrogen evaporation temperature. This can result in a large vacuum burst, in which the electrons interact with the RF field. Sometimes, a vacuum burst changes the cavity condition drastically, and cavities then require serious conditioning or reduction of gradient. For the high-beta spare cryomodule, a thermal cooling block cooled by $5 \mathrm{~K}$ supercritical helium was installed at each side. 


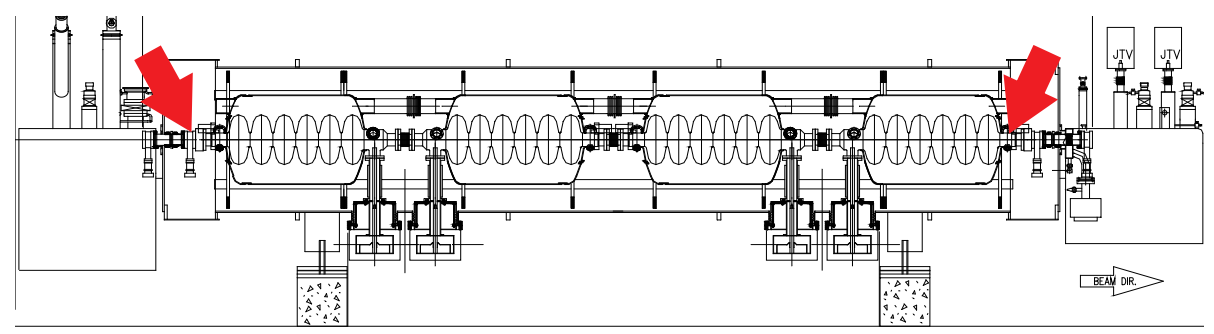

Fig. 3.14. Thermal block locations for the $5 \mathrm{~K}$ boundary.

With these two changes (higher RRR for the cavity end groups and thermal cooling blocks for the warmto-cold transition), the whole system will have a much larger thermal margin against thermal radiation from the FPC, thermal loads from electron activities such as field emission and multipacting, and any other additional external thermal loads. These are all included in the STS design.

Higher order mode (HOM) coupler. When an HOM is near the beam spectral lines, the induced voltage could be harmful to the beam and/or generate large HOM power. To address these two main concerns, systematic studies were carried out. In the case of SNS, bunch tracking simulations for both the transverse and longitudinal directions show that beam instabilities are not a main concern if the external quality factor $\mathrm{Q}_{\mathrm{ex}}$ for each HOM is less than $10^{8}$, the loaded cavity $\mathrm{Q}$ for each non-pi mode fundamental mode has the expected value, and the expected cavity-to-cavity frequency variation is present. Concerning the HOM power issue, general analytic expressions of beam-induced voltages and the HOM power from the multiple beam time-structures were analyzed. From these, the effect of the beam time-structure on the beam-induced voltage and the HOM power development can be fully understood by exploring the parameter space of the HOM properties. The damping requirement of each mode is set up in terms of $\mathrm{Q}_{\mathrm{ex}}$ by taking into account the actual HOM frequency behavior of elliptical SC cavities. The probability that any cavity frequency will fall on a significant beam power spectral line at any instant in time is estimated to be extremely small. During the SNS project, it was decided to include HOM couplers as insurance because of unknown parameters during the design period, such as HOM frequency spread and HOM frequency centroid difference between calculated values and real ones [19, 20].

About $15 \%$ of installed cavities in the SNS SCL are showing abnormal signals through HOM feedthroughs. All attenuators for the HOM signals were damaged during 1 year of operation. Observations and physical conditions near the HOM couplers imply that HOM coupler failures and/or degradations seem to be a result of electron activity originated by combinations of multiple causes such as electromagnetic field at the HOM coupler, multipacting, field emission, and even a gas discharge with fundamental mode. A few cavities were inoperable as a result of large coupling with the fundamental accelerating mode [21]. In 2007, the SNS reevaluated the HOM characteristics, including dangerous HOM modes measurement for all installed cavities. It was concluded that HOM couplers are not needed for the SNS, and it was decided to remove HOM couplers whenever cryomodules were taken out of the tunnel for repairs. Four cryomodules have been taken out of the tunnel, so far and leaks were detected from half of the HOM feedthroughs. Therefore, the STS cavities will not have HOM couplers.

Refurbishment of the spare cavities and new cavities. Consideration was given to using the existing 20 high-beta spare cavities, produced during the SNS project, in STS. However, given the end group design of the original cavities, meeting the gradient specification $(16 \mathrm{MV} / \mathrm{m})$ for the STS project is a large risk. Currently, a study is in progress in which the end groups were replaced on two spare cavities. For these test cavities, the new end groups were constructed of high-RRR niobium, the HOM cans were removed from the design, and high-aspect-ratio deep drawing was not used for manufacturing of parts. These cavities were delivered from the vendor and chemical polishing has been completed. Testing of the 
cavities is scheduled for late in calendar year 2014. Figure 3.15 shows a model of the old cavity versus the modified cavity. Figure 3.16 depicts the completed end group replacement.

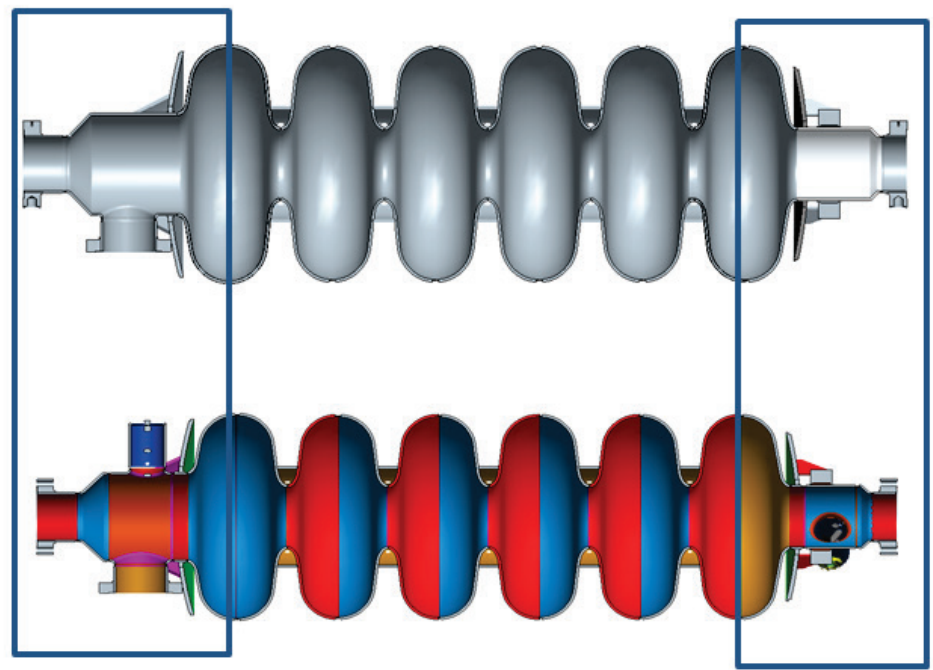

Fig. 3.15. End group modification. (Top: cavity with modified end group for the STS. Bottom: Original SNS highbeta cavity).

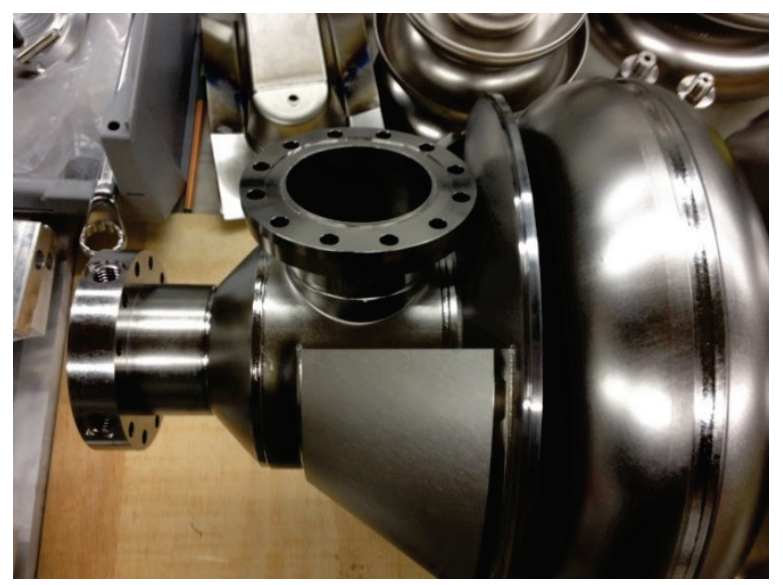

Fig. 3.16. Completed end group replacement.

The helium vessel is constructed of titanium and a niobium/titanium alloy. In the old design, the stiffeners in the head of the vessel were welded to a ring that allowed the length of the vessel to be adjusted. This allowed for flexibility in adjusting the final position of the body of the vessel to the heads of the vessel. Because of the position of this ring, a full-penetration weld was not possible. The new design eliminated this ring and redesigned the stiffener to fit completely with the heads of the vessel. Therefore, the main body of the helium vessel was lengthened to account for the added length from the slip fit rings. The main body of the vessel will be attached to the heads of the vessel via full-penetration welds. This more closely meets the intent of the ASME Boiler and Pressure Vessel (B\&PV) code. Figure 3.17 shows the original helium vessel design versus the new design. This modification was successfully incorporated in the spare high-beta cryomodule. 

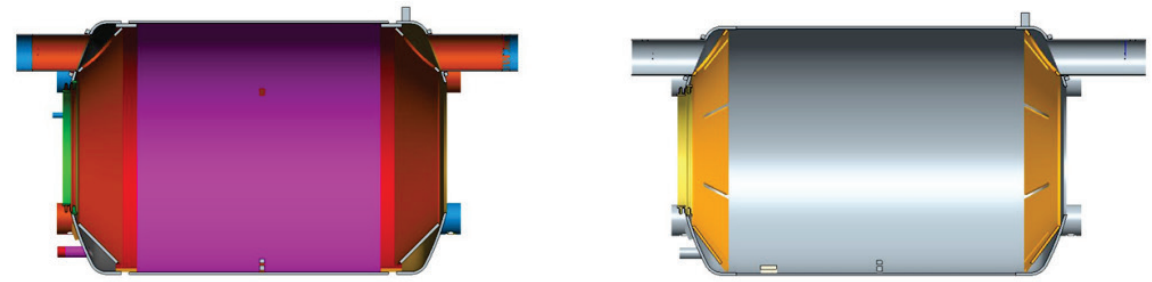

Fig. 3.17. Original (left) versus new (right) helium vessel designs.

\subsubsection{Fundamental Power Coupler}

The original SNS FPC is designed and scaled from the one KEK developed for $508.8 \mathrm{MHz}$. A simple window geometry was chosen to facilitate manufacturing and assembly. The design of the SNS FPC relies on a simple coaxial line at $50 \Omega$. The planar alumina window includes impedance-matching elements, as well as a TiN anti-multipacting coating. The chokes at both sides of the ceramic window have the important role of improving the window matching. The depths of the chokes were optimized in the simulations to allow the lowest return loss and insertion loss [22]. The transition between the WR975 waveguide from the klystron and the coaxial line of the FPC is provided by a doorknob configuration. Even a small mechanical change inside the structure can result in a significant change in the RF performance. Such disturbances include grooves, slits, rounded corners, and others. The FPC includes the possibility of biasing the inner conductor, via a capacitor gap between the doorknob and the inner conductor itself, at variable voltages between -2.5 and $+2.5 \mathrm{kV}$. The gap is filled with Kapton foil, which is capable of withstanding the biasing voltage. The requirement for the maximum allowable thermal radiation from the FPC to the end group is $2 \mathrm{~W}$. The outer conductor of the FPC has a direct conduction path to room temperature. To minimize heat transfer to the end group, the FPC outer conductor is designed to be cooled by supercritical helium at 3 atm and $5 \mathrm{~K}$ with $0.04 \mathrm{~g} / \mathrm{s}$ of mass flow rate, which removes about $30 \mathrm{~W}$ of static and dynamic heat load. The inner surface of the outer conductor is designed to be copper-plated with $15 \mu \mathrm{m}(\mathrm{RRR}=10)$ of copper on stainless steel to reduce wall dissipation. The inner conductor of the FPC is made of copper and does not have active cooling. Figure 3.18 shows a schematic, the inner conductor assembly, and the outer conductor assembly of the FPC.
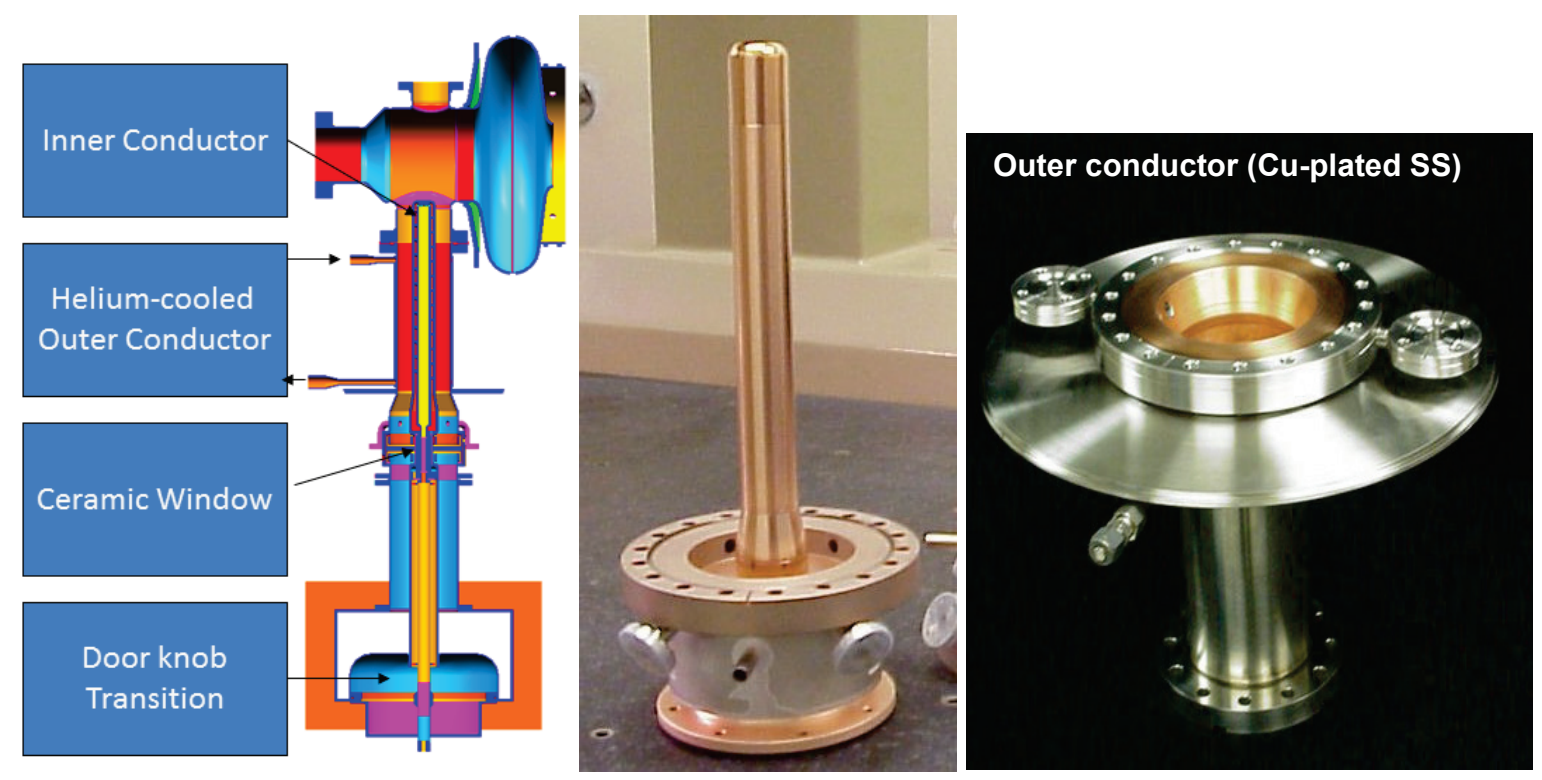

Fig. 3.18. SNS fundamental power coupler (left: schematics, middle: inner conductor assembly, right: outer conductor assembly). 
The original FPCs were tested at up to $2 \mathrm{MW}$ peak for the full traveling wave condition in the test stand and over $550 \mathrm{~kW}$ peak power in real cavity operation at various standing wave ratios limited by the operational envelope. The FPC for the STS cavities must be able to transfer up to $700 \mathrm{~kW}$ peak power over a $1.3 \mathrm{~ms}$ pulse width at a repetition rate of $60 \mathrm{pps}$. Based on the testing and operational experience, the RF performance of the original FPC satisfies the STS requirements. But the thermal radiation from the inner conductor will be higher as a result of the increased average RF power. A thicker inner conductor will be sufficient for the STS requirement, which would be the simplest solution. Active cooling for the inner conductor was also taken into account, if the passive enhancement of cooling with the thicker inner conductor is not adequate. Active cooling will require a more complex design configuration and may result in operational difficulties at an upset condition. The temperatures are calculated for various inner conductor thicknesses at both full traveling and standing wave conditions. During pulsed operation, the actual condition in the FPC is between full standing and full traveling wave condition, closer to the full traveling wave condition. Figure 3.19 shows comparisons of inner conductor tip temperatures. With a $7 \mathrm{~m}$ thick inner conductor, the inner conductor tip temperature at the STS condition can be kept below that of the FPCs presently operating at SNS. The prototype FPC for STS with increased wall thickness was manufactured by the vendor that provided the original FPCs, installed in a horizontal test apparatus, and successfully tested. Figure 3.20 shows the design change for the STS FPC. Except for the wall thickness of the inner conductor, all other designs are exactly same.

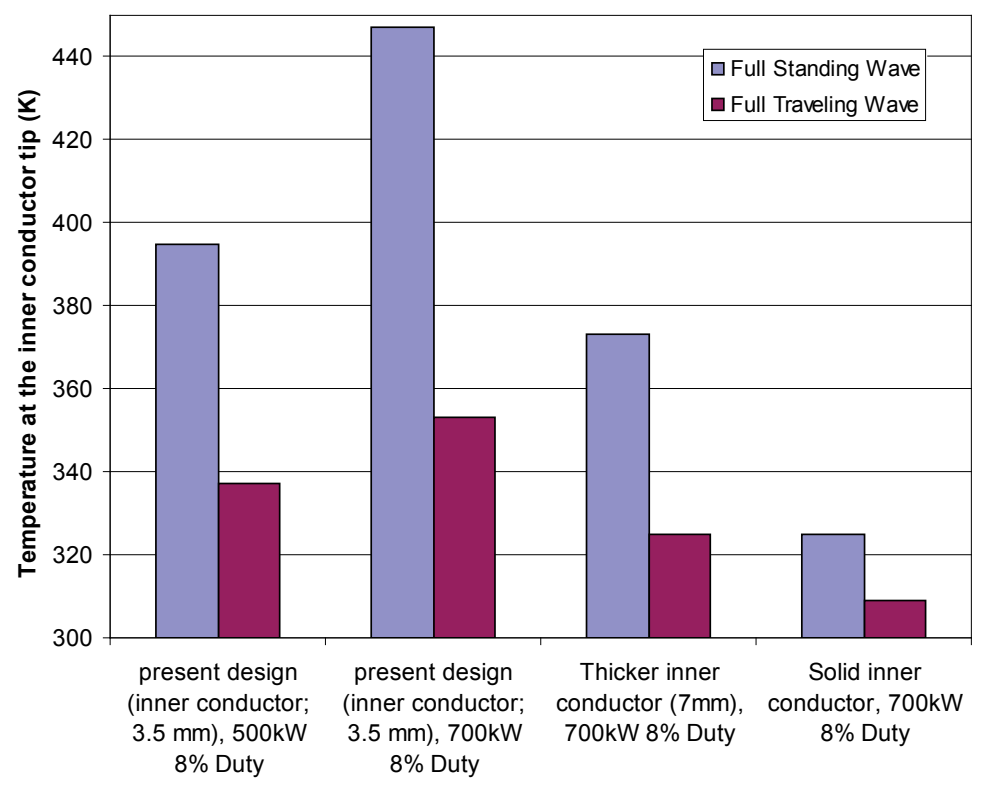

Fig. 3.19. Comparisons of calculated inner conductor tip temperatures.
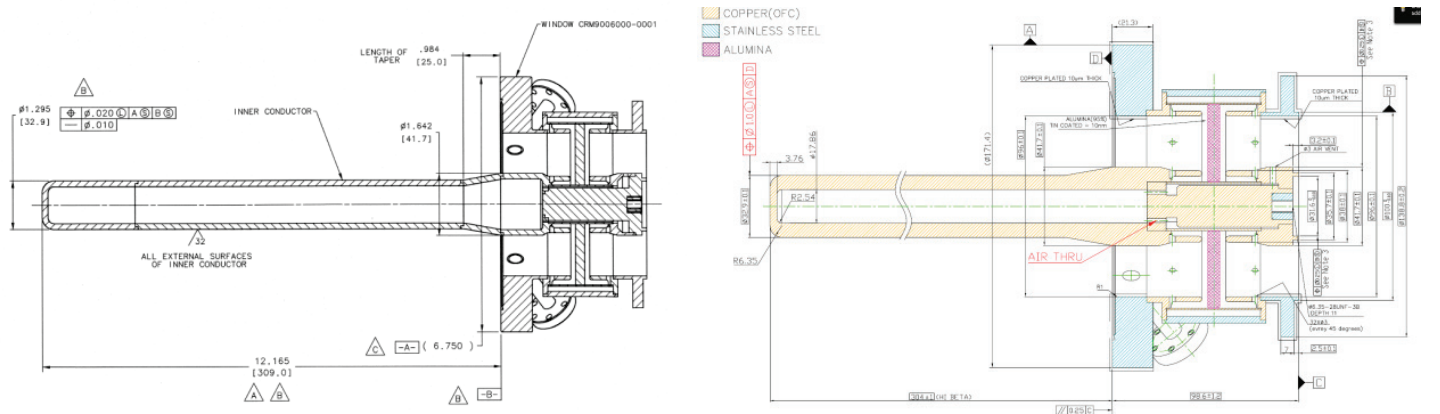

Fig. 3.20. Fundamental power coupler design (left: original design, right: design for the STS). 
The $\mathrm{Q}_{\mathrm{ex}}$ value for the original cavities was set during the early stage of the SNS project, when the beam and the accelerating gradients of the high-beta cavities were different from those in the final design. The $\mathrm{Q}_{\mathrm{ex}}$ value chosen for the STS in Table 3.6 is optimized to reduce the RF power requirement.

\subsubsection{Cryomodules}

The SNS superconducting linac consists of 23 cryomodules, 11 of which are medium-beta and 12 are high-beta. The medium-beta cryomodules each contain three six-cell elliptical cavities, whereas the highbeta cryomodules contain four six-cell elliptical cavities. The cavities are described in Sect. 3.3.1. The design was based on the Continuous Electron Beam Accelerator Facility cryomodule with improvements inspired by the Large Hadron Collider, TESLA (Teraelectronvolt Energy Superconducting Linear Accelerator), and the Jefferson Laboratory $12 \mathrm{GeV}$ upgrade design efforts [23]. Figure 3.21 shows the elevation view of the high-beta cryomodule design by Jefferson Laboratory.

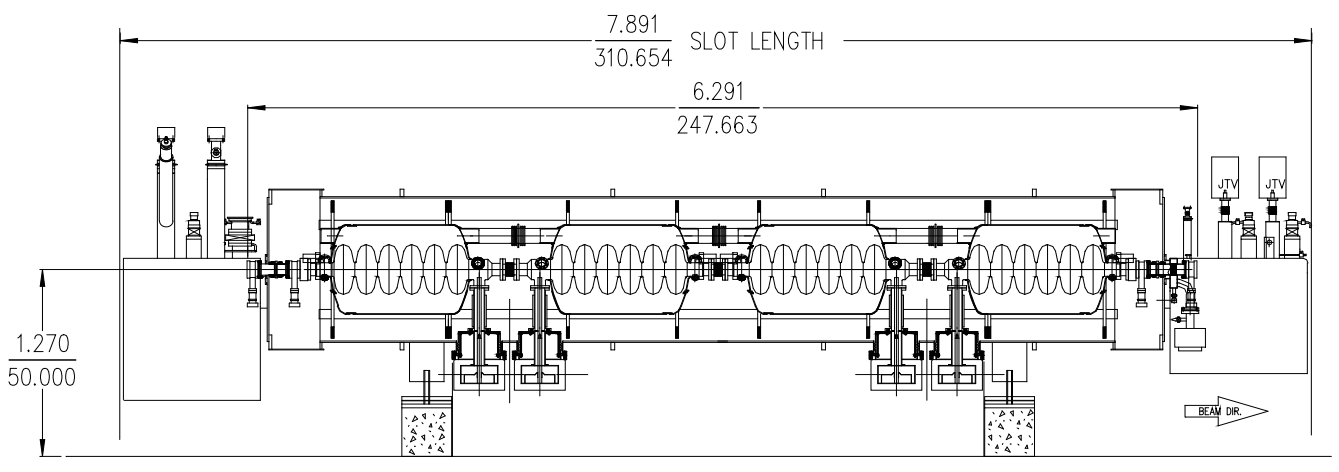

Fig. 3.21. The original high beta cryomodule.

The cryomodule consists of a cavity string, a space frame, a thermal shield, two layers of magnetic shielding, a vacuum vessel, and two end cans. The general arrangement of these components is depicted in Fig. 3.22.

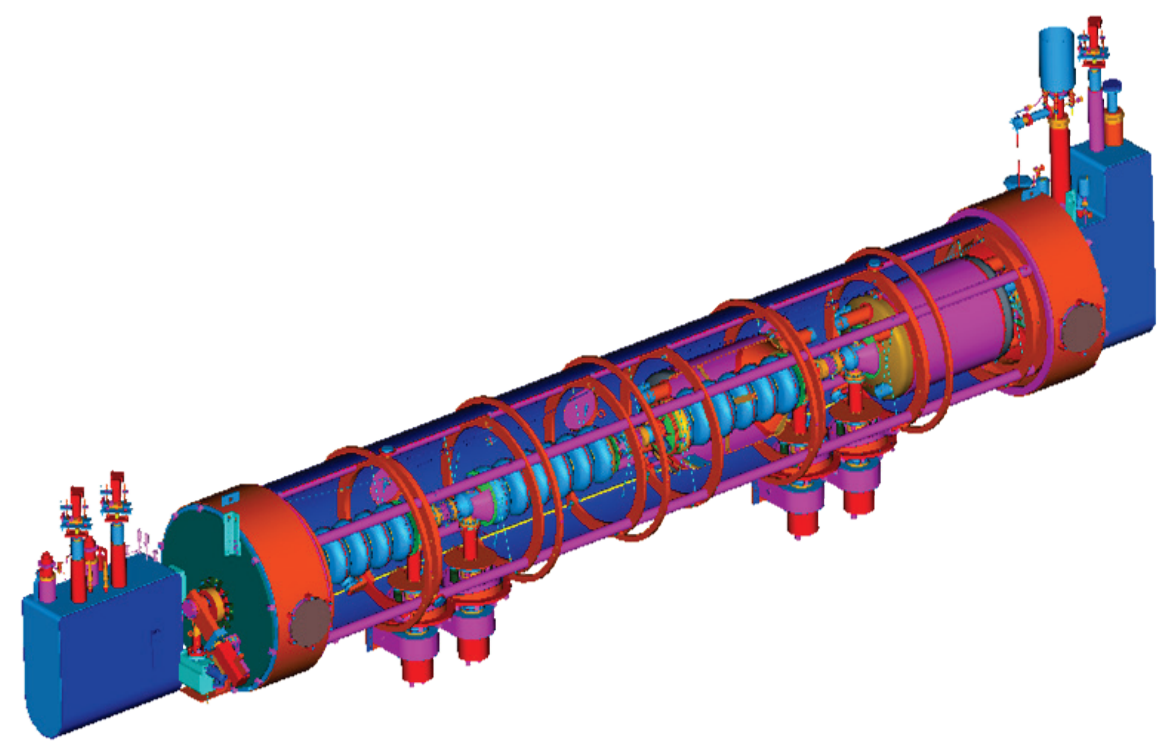

Fig. 3.22. Cut view of the original high-beta cryomodule. 
During the STS project, seven cryomodules similar to the original high-beta cryomodules will be installed in the linac tunnel. The relevant parameters for the mechanical design of the original high-beta cryomodules, which remain as design constraints for the new cryomodules, are listed in Table 3.8.

Table 3.8. Cryomodule design parameters

\begin{tabular}{lc}
\hline \multicolumn{1}{c}{ Parameter } & Value \\
\hline Slot length & $7.891 \mathrm{~m}$ \\
CM length (bore tube) & $6.291 \mathrm{~m}$ \\
Number of bayonets & 4 \\
Number of control valves & 5 \\
\hline
\end{tabular}

In 2012, SNS completed fabrication and testing of a spare high-beta cryomodule. The approach to the engineering design for this cryomodule was to maintain critical features of the original design, such as bayonet positions, coupler positions, cold mass assembly, and overall footprint. The components that were fixed in location and those that were considered movable are shown in Fig. 3.23. However, this new cryomodule design was required to meet the pressure requirements put forth in 10 CFR 851: "Worker Safety and Health Program." The most significant engineering change was applying Sect. VIII of the ASME B\&PV Code to the vacuum vessel of this cryomodule, instead of using the traditional designs in which the helium circuit is the pressure boundary. Applying the B\&PV Code to the helium circuit within the cryomodule was considered. However, it was determined to be schedule-prohibitive because it required a code case for the niobium materials, which is not currently covered by the code. Good engineering practice, however, was applied to the internal components to verify the quality and integrity of the entire cryomodule [15]. Details of the changes to the vacuum vessel and end cans to apply the code to these components are detailed in Sects. 3.3.2.3 and 3.3.2.4, respectively.

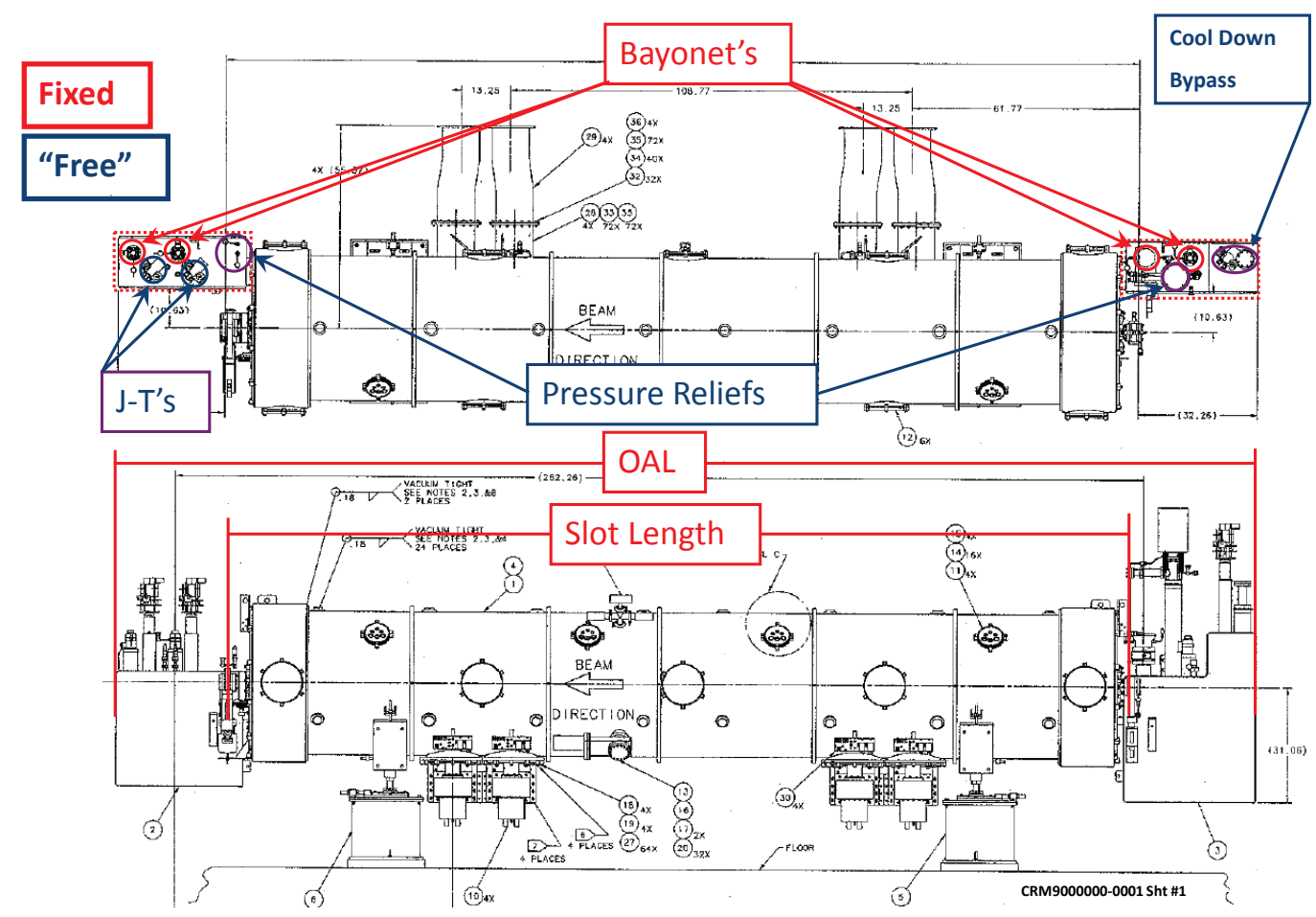

Fig. 3.23. Cryomodule reference interface locations. 
Several other improvements were made to the spare high-beta cryomodule beyond the changes implemented as a result of applying the B\&PV Code. The HOM feedthroughs on the end groups of the cavities were blanked. The helium vessels were upgraded to meet the intent of the code. Cooling blocks were added to the outside end groups of the cavity string to thermally stabilize these regions. Cold instrument feedthroughs were minimized in the design to prevent possible leaks. All of these improvements are discussed in detail in this report. The completed high-beta spare cryomodule is shown in Fig. 3.24.

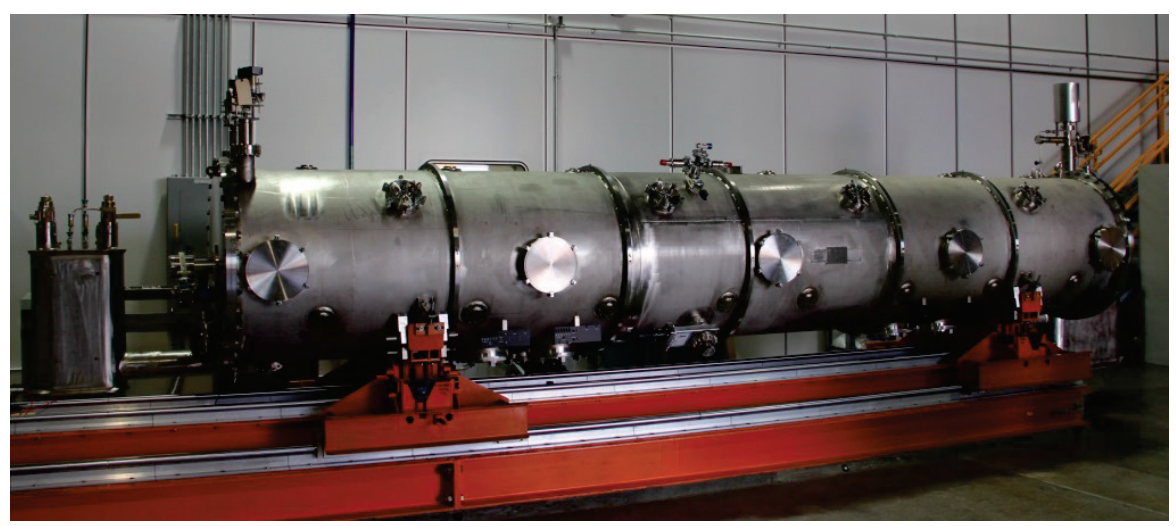

Fig. 3.24. SNS spare high-beta cryomodule.

The spare high-beta cryomodule serves as the baseline design for the STS cryomodules. A couple of modifications to this design will be necessary for the cryomodule to function according to the requirements of STS. The cavities will be upgraded as detailed in Sect. 3.3.1. A higher-power coupler will be used, as discussed in Sect. 3.3.1.3. Other than these two changes, the STS cryomodule is of the same design as the spare high-beta cryomodule. Methodologies for fabrication and assembly were developed and staff expertise was gained during the assembly of the high-beta spare.

\subsubsection{Head Loads and Helium Circuits}

There are three cooling circuits within the cryomodule. Primary cooling provides the liquid helium that cools the cavities within the helium vessels. Secondary cooling provides supercritical helium cooling to the couplers. Because the FPC requires a $5 \mathrm{~K}$ source to cool the outer conductor, as mentioned in Sect. 3.3.1.3, the Large Hardon Collider concept of producing $2 \mathrm{~K}$ helium in the cryomodule rather than in the refrigerator is used [23]. This drives the design of placing individual heat exchangers in each of the cryomodules rather than locating one large heat exchange in the refrigerator. In addition, efficiencies are improved with this heat exchanger placement. Shield cooling provides cooling to the thermal shield located in the cryomodule and transfer lines. Figure 3.25 is the updated flow diagram for the spare highbeta SNS cryomodule. The STS cryomodules will have the same helium circuits as the spare high-beta cryomodule.

The primary helium flow to the cryomodule will be split into two circuits, the primary and the secondary circuits. The primary helium flow will enter the cryomodule in the supply end can and flow through the heat exchanger in the return end can, through the primary Joule-Thomson (JT) valve located on the supply side of the cryomodule, and into the helium vessels. Helium will exit the helium vessels and flow either out the cool-down circuit or through the heat exchanger back into the return transfer line. The cooldown path will be used to imbalance the heat exchanger during the cool-down of the cryomodule so that a desired cool-down rate of $100-150 \mathrm{~K} / \mathrm{h}$ can be achieved. 


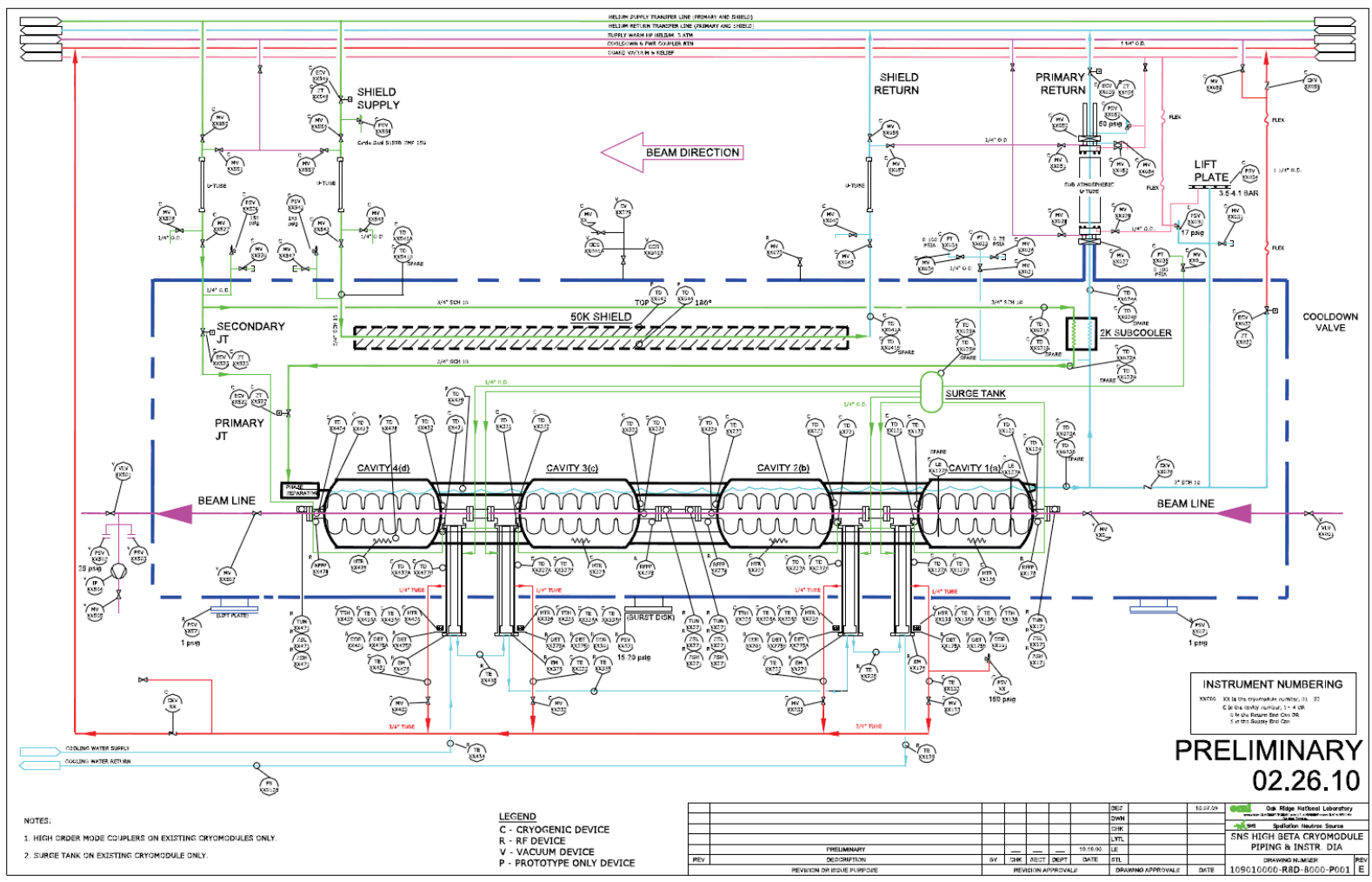

Fig. 3.25. Helium flow schematic and instruments for the spare high beta cryomodule.

The secondary circuit will flow through a secondary JT valve, a cooling block on the end group of the supply side cavity, each of the FPC flanges, a cooling block on the end group on the return side cavity, into a surge tank, and out to four parallel paths to the FPC outer conductors. The return from the couplers will flow though metering valves for each of the couplers. The four flows will be combined and discharged into the cool-down header and act as a liquefaction load on the refrigerator.

The shield flow will be supplied from the turbine 1 outlet in the main cold box at approximately $38 \mathrm{~K}$. It will flow from the turbine outlet, through the transfer line, and into the cryomodules through the shield supply valves. Helium will then flow through the cryomodule and back into the return transfer line, where it will return to the medium-pressure header in the main cold box. The design criteria for the original high-beta cryomodule [24] are listed in Table 3.9, along with those for the STS based on the operating experience of the spare high-beta cryomodule.

The original SNS cryogenic system was designed with a 100\% capacity margin from the primary and secondary circuits and a 35\% margin for the shield passage. This was done to account for the uncertainty of the new design and to mitigate risk to the project. With these margins in place, it is anticipated that the addition of seven cryomodules to the linac tunnel will be well within the capability of the SNS cryogenic system. See Sect. 3.3.3 for more detailed cryogenic system information.

Table 3.9. Cryogenic load design values for the high beta cryomodule

\begin{tabular}{lcc}
\hline \multicolumn{1}{c}{ Parameter } & Original cryomodule & STS cryomodule \\
\hline 2 K heat load (static/dynamic) & $28 / 20 \mathrm{~W}$ & $25 / 40 \mathrm{~W}$ \\
Coupler flow & $0.075 \mathrm{~g} / \mathrm{s}$ & $0.067 \mathrm{~g} / \mathrm{s}$ \\
Shield load including transfer line & $200 \mathrm{~W}$ & $200 \mathrm{~W}$ \\
\hline
\end{tabular}




\subsubsection{Cold Mass}

The cold mass of the cryomodule will consist of the cavity string, space frame, $50 \mathrm{~K}$ thermal shield, magnetic shield, and insulation. The spare high-beta cryomodule cold mass just before the second layer of magnetic shielding was installed is shown in Fig. 3.26.

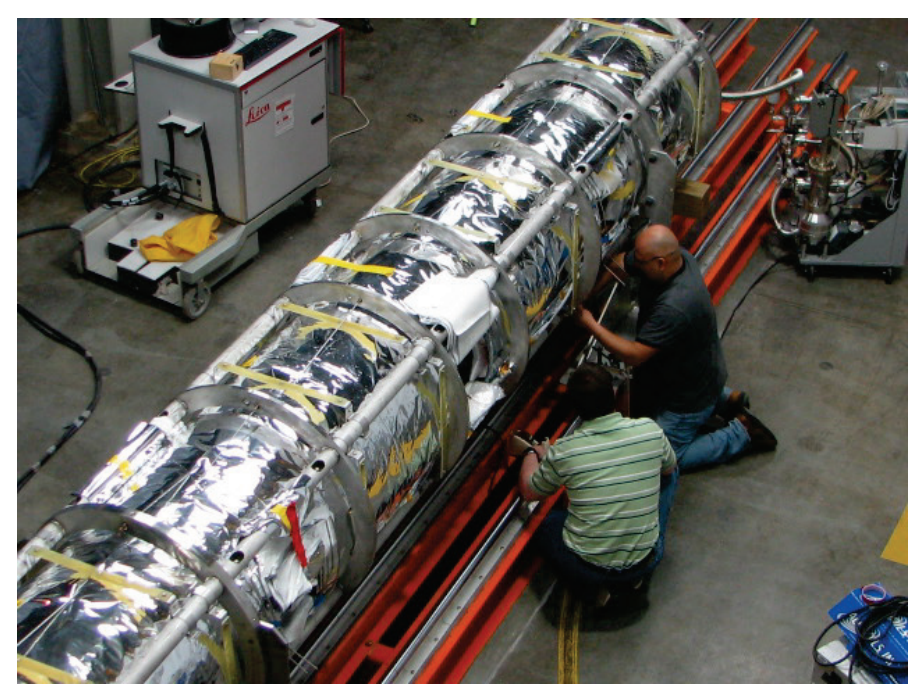

Fig. 3.26. Cryomodule cold mass assembly

The original SNS tuner design shown in Fig. 3.27 has been adapted from a Saclay design for the TESLA Test Facility cavities [25]. It is attached to the cavity at three points, two standard standoffs and one piezo tuner. The tuner is adjusted by a motor and harmonic drive unit. This provides a tuning range of approximately $400 \mathrm{kHz}[26]$.

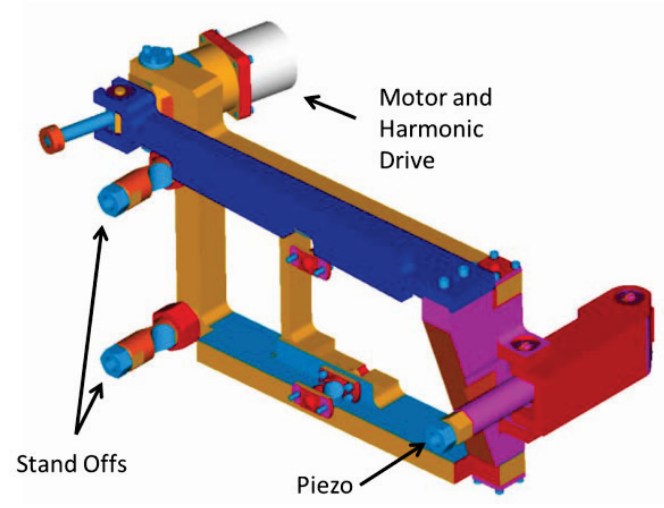

Fig. 3.27. Schematic of the original SNS tuner assembly

One key change to the original cavity string assembly is the removal of the piezo tuner from the tuner assembly. The original design included the piezo tuner to compensate for Lorentz force detuning of the cavities. However, because of a high-gain low-level RF control system and because there are no adverse mechanical resonance conditions, the cavity phase and RF amplitude are within the compensation range of the RF system [27]. Because of pressure changes in the cryomodules either during the 2 to $4 \mathrm{~K}$ transition or during upset conditions, several failures with piezo stacks have occurred. The piezo tuners were replaced with the standard standoffs used on the other two legs of the tuner. The piezo tuners have 
never been actuated in operation at SNS. Because of this history, it was decided to eliminate them from future cryomodules built for SNS.

Thermal and magnetic shielding is incorporated in the design of the cold mass. The cavity string will be surrounded by a thermal shield that operates at approximately $50 \mathrm{~K}$. This shield will be wrapped in multilayer insulation and will provide a thermal radiation barrier between the cavities and the ambient environment. The thermal shield used for the upgrade cryomodules will be very similar to the original shields used for the original cryomodules. Two layers of magnetic shielding will be incorporated into the cold mass assembly. One layer will be installed on the outside of the helium vessels within the cavity string and the other on the outside of the space frame. These layers will reduce the Earth's magnetic fields by a factor of 20 or higher to minimize the effect on cavity operation. The only change to the shields will be to adjust their geometry to properly fit within the new B\&PV Code-complaint vacuum vessel.

Another component of the cold mass is the space frame, which was developed at Jefferson Laboratory to facilitate installation of long cavity strings into cryomodules at a relatively low cost. Because the original cryomodules were assembled at Jefferson Laboratory and shipped to SNS, the space frame was

strengthened to handle the transportation load while maintaining the alignment of the cavities. This same design will be used in the STS cryomodules. The space frame will provide support to the cavity string through nitronic rods. The space frame will be supported from the vacuum vessel. The use of this system to support the cavity string will limit the conduction of heat to the cavities. Nitronic rods were selected to support the transportation loads because of their high strength. An alternative of using stainless steel may be considered for the STS cryomodules.

\subsubsection{Vacuum Vessel and Support Stands}

To meet the new B\&PV Code, the decision was made to define the vacuum vessel and end can envelope as the pressure boundary because of the difficulty in applying the pressure code to the helium circuit materials. The niobium, the titanium, and the niobium-titanium alloy are not code-listed at the operating temperature that is routinely maintained within the cryomodules. The approach of using the vacuum vessel as the pressure boundary made use of an interpretation of VIII-1-89-82 in which it was deemed acceptable to stamp the exterior vessel of a heat exchanger if the tube side exceeded the rated operating pressure, provided the shell and associated relief devices were designed to withstand the highest design pressure associated with the tube side. Moving the pressure boundary from the cavity helium circuit to the vacuum vessel has additional safety benefits. (1) The vacuum shell material is 304 stainless steel, which is one of the best materials for fracture toughness and ease of fabrication. (2) The vacuum shell will never reach the helium operating temperature even with a catastrophic failure of the helium lines because of the thermal mass of the vessel material, which is at room temperature. Therefore, the material properties at LN2 temperature can be used. (3) The vacuum vessel envelope could be easily pressure-tested without the SRF cavity string installed [28].

Enacting the code allowed removal of the bridging ring from the original design concept. That required that the main part of the vacuum vessel in the spare cryomodule be longer than the original cryomodules. Removal of the bridging ring complicated assembly in the warm-to-cold transition region, necessitating design changes to this region of the cryomodule. The changes affected the flexibility of the alignment of the string to the warm beam line flange. Therefore, modeling of the string within the vacuum vessel had to be very precise because the movement of the warm-to-cold transition in the old design was eliminated. The alignment during the spare high-beta cryomodule assembly was performed with a laser tracker and the modeling was successful so that the string aligned with the warm valve within the specification limit of $1 \mathrm{~mm}$ [15]. Figures 3.28 and 3.29 depict changes in the design of the vacuum vessel. 

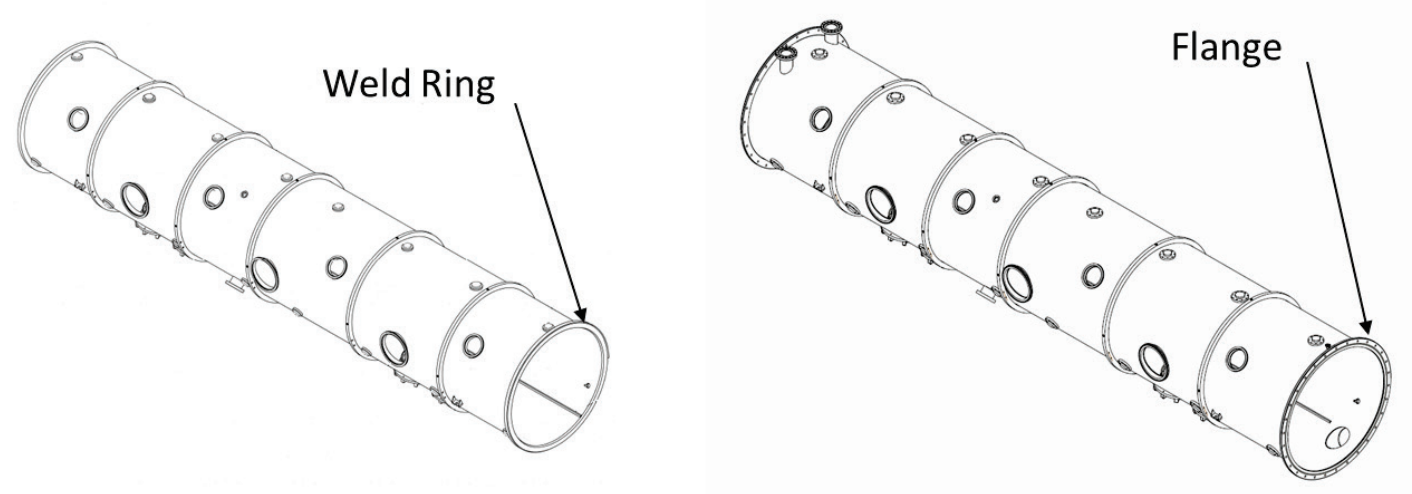

Fig. 3.28. Original (left) versus new (right) vacuum vessel
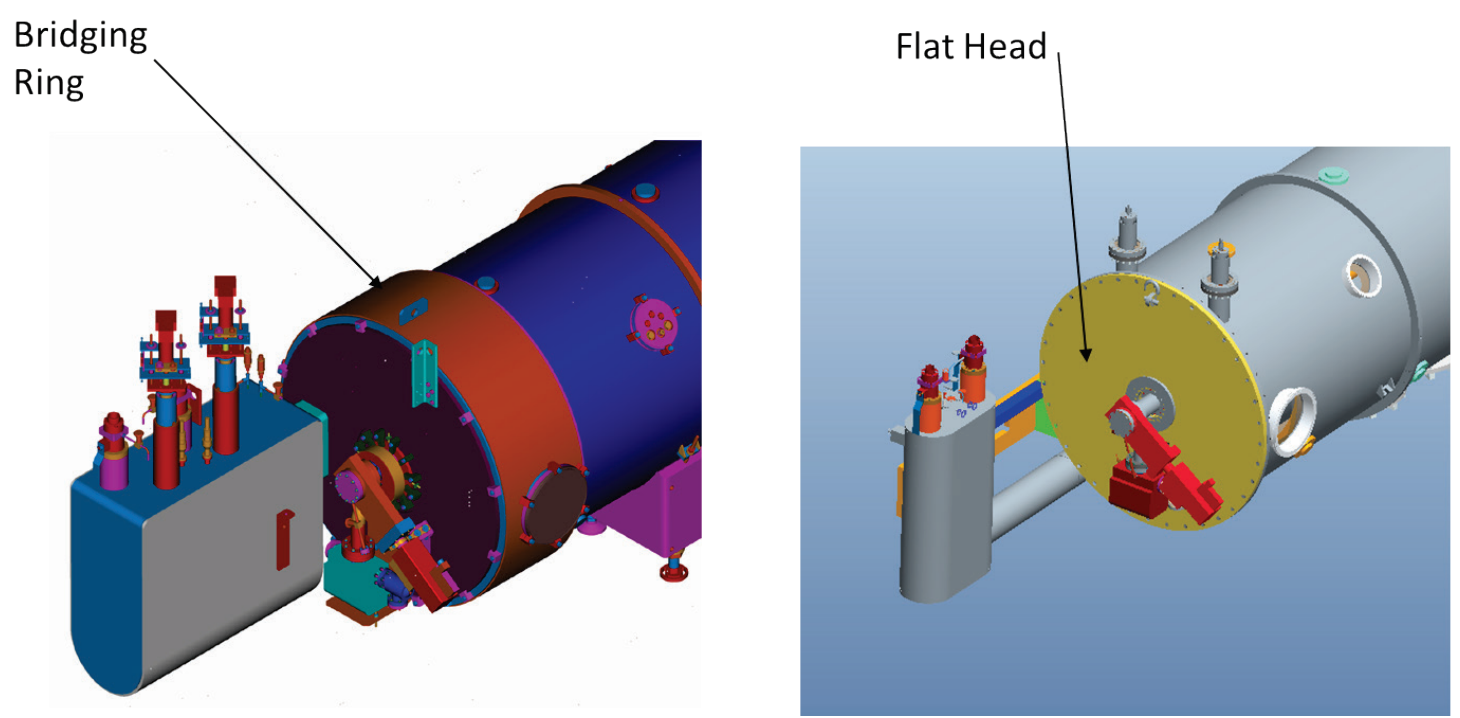

Fig. 3.29. Original (left) versus new (right) vacuum jacket design

To simplify the supply end can as much as possible, the primary and secondary JT valves were moved from the end can to the vacuum vessel. This required a change in the vacuum vessel design, and more piping was added to the main body of the cryomodule. Figure 3.30 depicts this change.
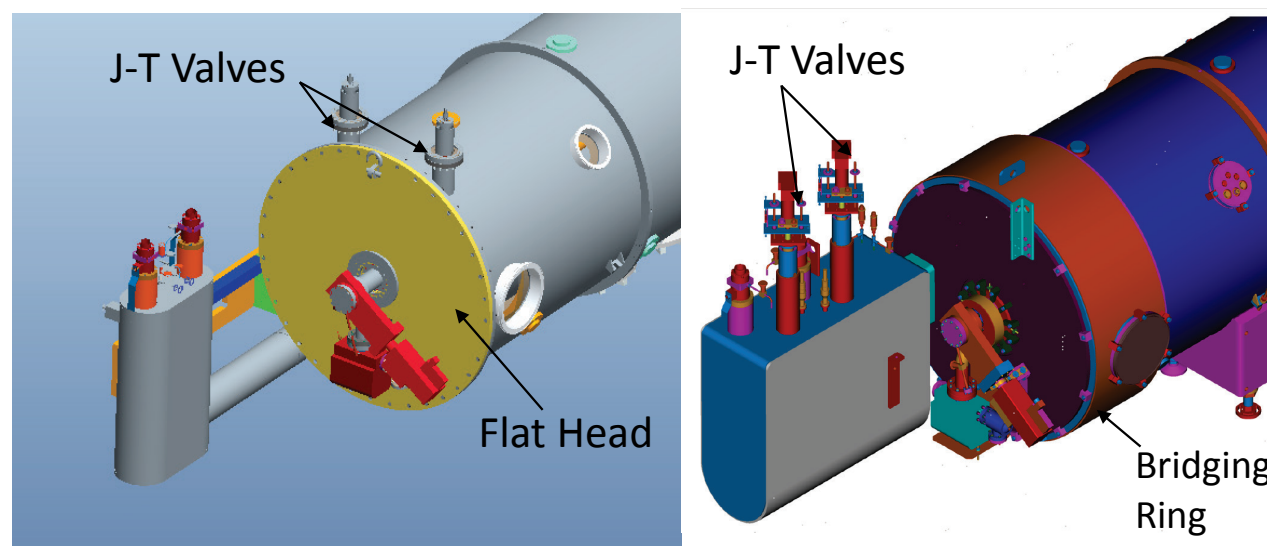

Fig. 3.30. JT valve positions for the STS cryomodule (left) and the original (right) cryomodule. 


\subsubsection{End Cans}

In designing the high-beta spare cryomodule, multiple changes were made to the end cans. Previously, the end cans had not been designed or fabricated in such a way that the vacuum boundary could withstand pressure. Therefore, the shape and thickness of the end cans were modified. Because of the three pressure stamps philosophy, the end cans had to be capable of being pressure-tested individually; the same was true for the vacuum vessel. The end cans will attach to the side of the vacuum vessel by a flanged connection, an arrangement that allows easy pressure testing of all three vessels.

The piping within the supply end can was significantly reduced. The new supply end design is compared with the original design in Fig. 3.31. Similar analysis was conducted to simplify the return end can by moving the heat exchanger into the vacuum vessel. To do so, it would have been necessary to change the heat exchanger. However, the decision was made to keep all of the original equipment in the return end can, since that was a proven, reliable design. The piping in the return can was simplified as much as possible for ease of fabrication (Fig. 3.32). Because the new pressure-rated end cans were connected differently to the vacuum vessel, they required new mechanical support brackets, as shown in Fig. 3.33.
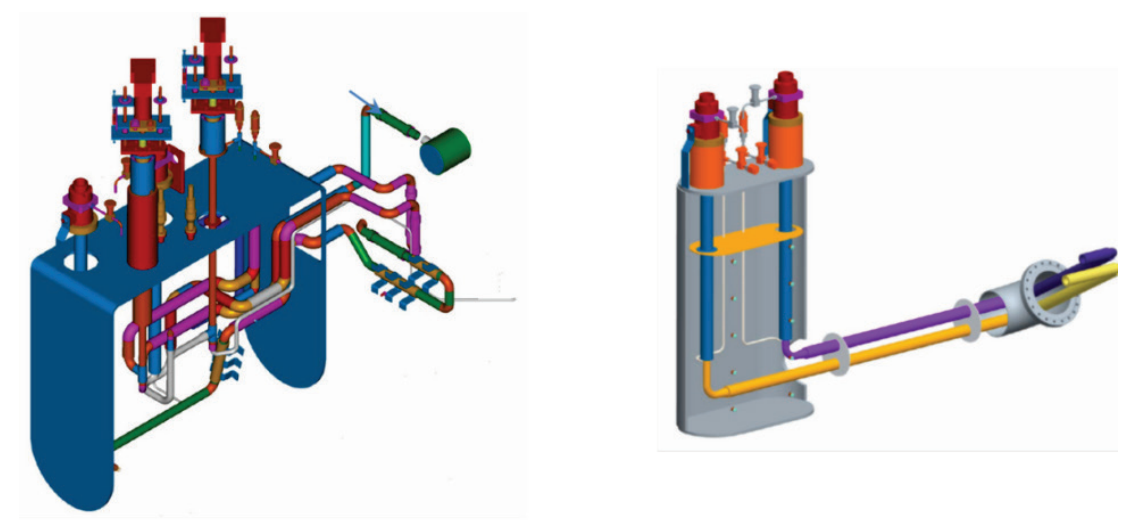

Fig. 3.31. Original (left) versus new (right) supply end can.
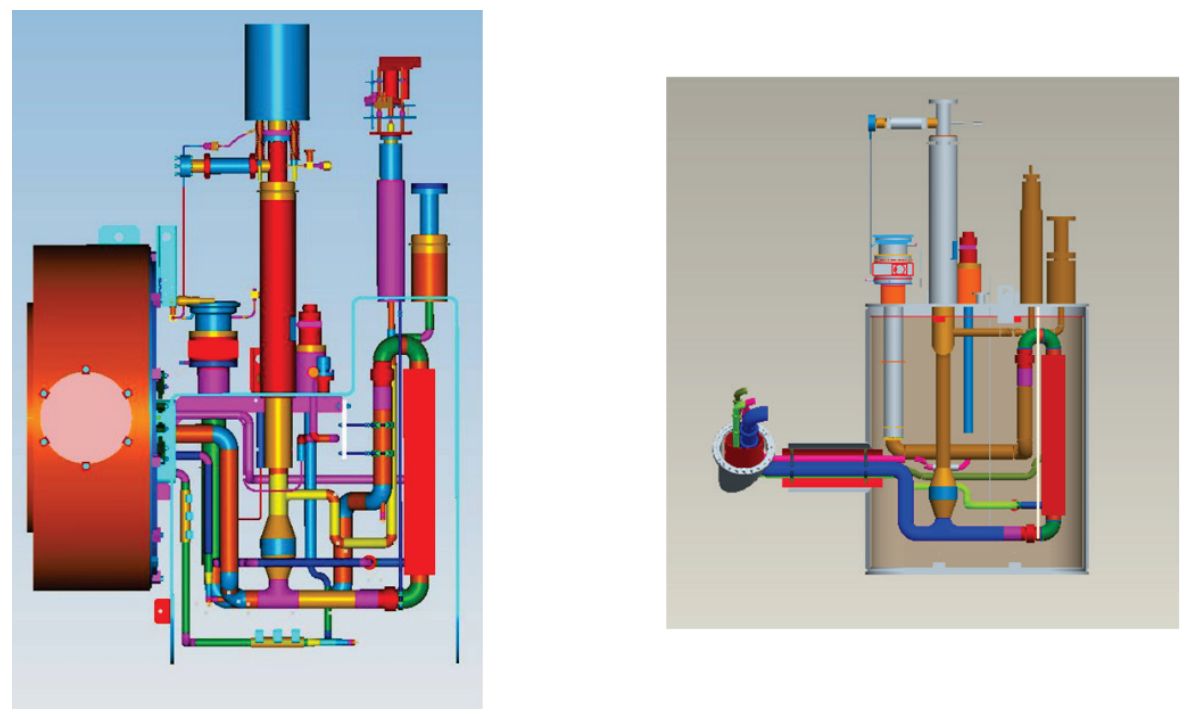

Fig. 3.32. Original (left) versus new (right) return end can. 


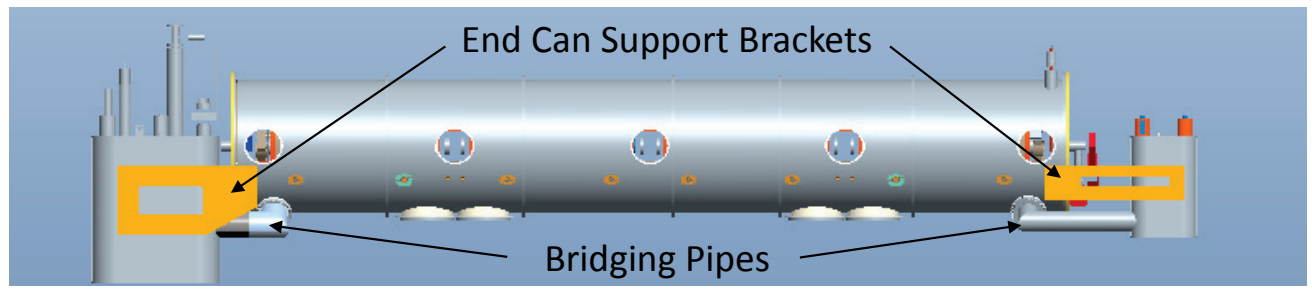

Fig. 3.33. Interfaces between end cans and vacuum vessel for the STS cryomodule.

Although the helium circuit is not code-stamped with this design philosophy, good engineering practice was applied to this portion of the cryomodule for the spare high-beta cryomodule. The helium vessels were modified so that the stiffening in the heads of the vessels was increased. In addition, the design of the cylindrical portion of the helium vessel was modified so that all welds could be full-penetration welds. The fabrication techniques used were consistent with ASME code practices and procedures. The inspection requirements were also consistent with those called out in the ASME B\&PV code. All welds that would eventually be subjected to cryogenic temperatures were made using low-ferrite filler material. Ferrite is known to reduce toughness in cryogenic applications; therefore, filler material had a ferrite number below 5 [29].

\subsubsection{Instrumentation Hardware}

The original cryomodules were equipped with many in-process diodes for accurate temperature measurement of helium streams. The in-process diodes required a cold instrument feedthrough to bring the wires out of the process space into the insulating vacuum space. During welding for the original cryomodule fabrication, the ceramic portion of the some feedthroughs was overheated. Many leaks in the cryomodules were observed before the operation of the SNS linac. Multiple repairs were performed by cutting through the insulating vacuum boundary and thermal shield to access the diodes. The cold feedthroughs were removed and replaced by surface-mounted diodes. These surface-mounted diodes have given reliable temperature feedback, and operations using these readings have been successful. Therefore, in the high-beta spare cryomodule, all diodes were surface-mounted. This eliminated many of the cold feedthroughs from the original design. The diodes located on the cavity surfaces at the top and bottom of a cavity were also relocated to the outside of the helium vessel for the spare high-beta cryomodule. Using these diodes to determine the cooldown rate has been proved to be very similar to using the cryomodule with diodes directly mounted to the cavities.

Because of the high radiation levels within the tunnel, the pressure transmitters used on the original SNS cryomodules failed in a short time. They were replaced with a Honeywell strain gauge with no internal electronics, making them much more reliable in a high-radiation environment. Figure 3.25 shows the locations of instruments on the cryomodule.

\subsubsection{Cryogenics}

The SNS cryogenic system design was based on the Thomas Jefferson National Accelerator Facility (TJNAF) cryogenic system with some modifications and has about half of the TJNAF cryogenic system capacity [30]. The SNS cryogenic system was designed to provide refrigeration capacities of $2850 \mathrm{~W}$ at $2.1 \mathrm{~K}, 8300 \mathrm{~W}$ of shield cooling, and $15 \mathrm{~g} / \mathrm{s}$ of liquefaction flow. A flow diagram of the system is shown in Fig. 3.34. 


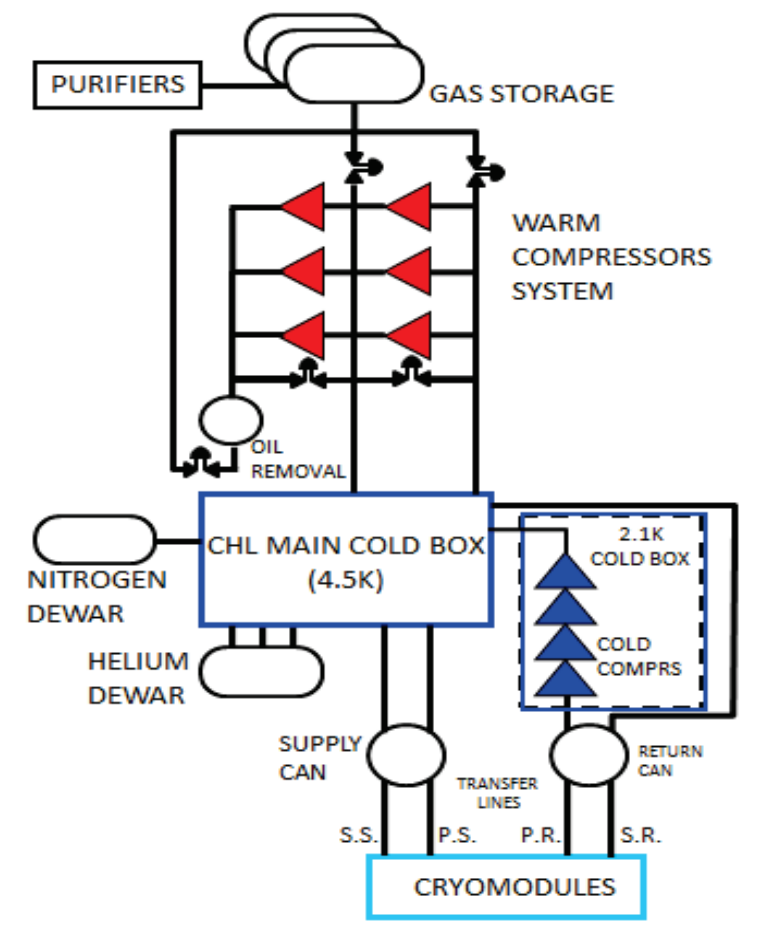

Fig. 3.34. Cryogenic system overview.

The control system is an integral part of the SNS cryogenic system. Within the Experimental Physics and Industrial Control System (EPICS), multiple control sequences run to control the operation. These sequences include the $2 \mathrm{~K}$ pump down, the heater control, the $2 \mathrm{~K}$ trip response, and JT off sequence. Each time a cryomodule is added to the tunnel, these sequences must be amended to include the new cryomodules. Testing of these sequences will be an essential part of ensuring the success of the SCL operation during the STS project. Perhaps the most critical of these sequences to test is the $2 \mathrm{~K}$ pump down sequence. This is an empirically derived sequence that adjusts the gear ratios of the cold compressors as pressure decreases in the return transfer line header. If cryomodules are added to the tunnel, flow dynamics and temperature profiles within the system could change, requiring development and modification of the $2 \mathrm{~K}$ pump down sequence.

Because the initial design of the cryogenic system had a large margin of capacity, turn-down studies of the cryogenic system have been conducted at SNS to enable the system to run more efficiently. The results of these studies showed that the SNS cold compressors could be successfully operated while reducing flow by approximately $25 \%$ from the design flow [31]. Results of the turn-down studies are presented in Table 3.10. Typically, the system is operated with the flow turned town $12.5 \%$ from the design flow. This allows more efficient operation of the system by reducing power consumption and decreasing nitrogen usage while maintaining the robustness of the original design. Even with the system turned down, it is necessary to input approximately $1 \mathrm{~kW}$ of heat into the helium baths of the cryomodules with electric heaters to maintain the minimum flow for the $2 \mathrm{~K}$ cold box. When seven additional cryomodules are added, the turned-down system will be more efficient. The heat load of the additional cryomodules will be less than the heat that is currently added to the system. Therefore, the heater sequence will be adjusted to reduce the external heat presently added, as additional new cryomodules are installed. 
Table 3.10. Results of turn-down study

\begin{tabular}{|c|c|c|c|c|}
\hline & $\begin{array}{c}\text { Design } \\
\text { basis }\end{array}$ & $\begin{array}{c}\text { Maximum } \\
\text { capacity }\end{array}$ & $\begin{array}{l}\text { Nominal } \\
\text { capacity }\end{array}$ & $\begin{array}{r}\text { Minimum } \\
\text { capacity }\end{array}$ \\
\hline First stage compressors (kW) & 608 & & & \\
\hline $\mathrm{C} 1(\mathrm{~kW})$ & & 300 & 250 & 244 \\
\hline $\mathrm{C} 3(\mathrm{~kW})$ & & 300 & 300 & 203 \\
\hline Second stage compressors $(\mathrm{kW})$ & 2074 & & & \\
\hline $\mathrm{C} 4(\mathrm{~kW})$ & & 1456 & 1355 & 762 \\
\hline C5 (kW) & & 1456 & 1355 & 1154 \\
\hline LN2 usage (g/s) & 120 & 200 & 180 & 150 \\
\hline $\begin{array}{l}\text { Total electric input power to } \\
\text { compressors }(\mathrm{kW})\end{array}$ & 2682 & 3512 & 3260 & 2363 \\
\hline $\begin{array}{l}\text { LN2 equivalent power (@35\% } \\
\text { Carnot) (kW) }\end{array}$ & 216 & 360 & 324 & 270 \\
\hline Total input power equivalent $(\mathrm{kW})$ & 2898 & 3872 & 3584 & 2633 \\
\hline HP to cold box (Atm) & 16.8 & 17 & 16.5 & 12.9 \\
\hline MP cold box out (Atm) & 4 & 2.8 & 2.8 & 2.5 \\
\hline Cold box HP flow (g/s) & 1150 & 1077 & 1030 & 829 \\
\hline CC flow $(\mathrm{g} / \mathrm{s})$ & 125 & 140 & 125 & 90 \\
\hline Liquefaction load $(\mathrm{g} / \mathrm{s})$ & 15 & 4 & 4 & 4 \\
\hline Shield load (kW) & 8300 & 5300 & 5300 & 5300 \\
\hline \multicolumn{5}{|l|}{$\begin{array}{l}\text { Carnot work based on } 2.1 \mathrm{~K} \\
\text { operations }\end{array}$} \\
\hline Primary load $(\mathrm{kW})$ & 400 & 448 & 400 & 288 \\
\hline Liquefaction load (kW) & 102 & 27 & 27 & 27 \\
\hline Shield load $(\mathrm{kW})$ & 61 & 39 & 39 & 39 \\
\hline Total load Carnot work & 563 & 514 & 466 & 354 \\
\hline $\begin{array}{l}\text { Carnot eff based on } 2.1 \mathrm{~K} \\
\text { operation }\end{array}$ & 0.194 & 0.133 & 0.130 & 0.134 \\
\hline \multicolumn{5}{|l|}{$\begin{array}{l}\text { Carnot work based on } 4.5 \mathrm{~K} \\
\text { operations }\end{array}$} \\
\hline Primary load $(\mathrm{kW})$ & 542 & 607 & 542 & 390 \\
\hline Liquefaction load $(\mathrm{kW})$ & 102 & 27 & 27 & 27 \\
\hline Shield load $(\mathrm{kW})$ & 61 & 39 & 39 & 39 \\
\hline Total load Carnot work(kW) & 705 & 673 & 608 & 456 \\
\hline $\begin{array}{l}\text { Carnot eff based on } 4.5 \mathrm{~K} \\
\text { operation }\end{array}$ & 0.243 & 0.174 & 0.170 & 0.173 \\
\hline
\end{tabular}

$\mathrm{HP}=$ high pressure; $\mathrm{MP}=$ medium pressure . 


\subsubsection{Supporting Systems}

The support systems for the SCL portion of the STS project include cooling water, beam line vacuum, and insulating vacuum.

The cooling water system will provide cooling to the FPC and is anticipated to be a direct copy of the existing systems. Each of the seven new high-beta cryomodules to be installed in the linac tunnel will have four RF couplers that require water cooling. Similar to the existing high-beta cryomodules, the four RF couplers will be cooled in series with an RTD between each cavity and a flow switch at the return header. The coupler system is designed to remove a maximum of $200 \mathrm{~W}$ per coupler with a $1 \mathrm{gpm}$ minimum flow. The existing quadrupole magnet cooling system will be the source of cooling and was verified during the Power Upgrade Project (PUP) to meet these additional requirements without modification.

The beam line vacuum system will isolate certain sections of the beam line during maintenance or in the event of a leak by actuating beam line valves. Each beam line section will be equipped with an ion pump that maintains a high vacuum.

Originally, SNS cryomodules were not equipped with an insulating vacuum system. Individual pump carts were placed on the cryomodules that had leaks. Because of the elevated radiation levels in the tunnel, the controllers for these pumps did not have a long life. A two-turbo molecular pumping system was manifolded to four cryomodules and the controllers were relocated to the Klystron Gallery. This system has proved effective. However, it is expensive and has a limited pumping capacity. Recently, a cryomodule positioned in the tunnel beyond the manifolded pumping systems was found to leak. A turbo pump was directly connected to the cryomodule with a backing scroll pump. The controller was located in the Klystron Gallery away from the radiation environment. The STS project will maintain provisions for adding this system to each of the seven additional cryomodules.

\subsection{RADIO FREQUENCY}

\subsubsection{High-Power Radio Frequency Systems for the STS}

The existing SNS linac high-power RF (HPRF) systems are well described in references [32,33,34].

The high-level beam parameters for the STS driving design considerations regarding the linac HPRF systems are described in Sect. 3.1. There are two primary RF impacts. (1) The STS will require additional SCL HPRF systems to support the additional accelerating structures required to reach a beam energy of $1.3 \mathrm{GeV}$. (2) Modifications will be required to some of the existing normal conducting linac HPRF systems to support the additional beam loading associated with the increase in the average linac beam current.

\subsubsection{Estimated Required RF Generator Power for the Normal Conducting Linac}

\section{Background}

The baseline system requirements for the existing normal conducting linac are detailed in reference [35]. The design criteria used to size the baseline HPRF systems were based on the estimated required RF generator power for an average linac beam current of $35 \mathrm{~mA}$, calculated using accelerating structure parameters provided by 2-dimensional cavity simulations. 
The HPRF power sources were sized to include a minimum power margin to provide available RF power over and above the estimated required RF generator power. The power margin will provide additional available RF power to accommodate for losses between the klystron and cavity; variations in klystron and HVCM performance; and the low-level RF phase, amplitude, and resonance control required to maintain steady state operation in response to beam-induced and environmentally induced disturbances.

Power margin is stated as a percentage of available RF power, and calculated as

$$
\text { Power Margin }(\%)=\left(\frac{\text { Pklystron-Prf }}{\text { Prf }}\right) * 100 \%
$$

where

$$
\begin{aligned}
& \mathrm{P}_{\text {klystron }} \text { is the rated saturated output power of the klystron } \\
& \mathrm{P}_{\mathrm{rf}}=\mathrm{P}_{\text {copper }}+\mathrm{P}_{\text {beam }}
\end{aligned}
$$

\section{Assumptions}

Coupling factor, unloaded Q, external Q, and loaded Q measured on the normal conducting linac

\begin{tabular}{|c|c|c|c|c|}
\hline Structure & $\boldsymbol{\beta}_{\mathrm{cpl}}$ & $\mathbf{Q}_{\mathbf{0}}$ & $\mathbf{Q}_{\text {ext }}$ & $\mathbf{Q}_{\mathbf{L}}$ \\
\hline DTL1 & 2.324 & 40886 & 17815 & 12144 \\
\hline DTL2 & 1.445 & 43832 & 30274 & 17947 \\
\hline DTL3 & 1.734 & 48279 & 27697 & 17659 \\
\hline DTL4 & 1.301 & 48118 & 36875 & 20911 \\
\hline DTL5 & 1.277 & 48088 & 37654 & 21129 \\
\hline DTL6 & 1.333 & 48139 & 36056 & 20630 \\
\hline CCL1 & 1.554 & 16000 & 10296 & 6265 \\
\hline CCL2 & 1.184 & 18707 & 15800 & 8565 \\
\hline CCL3 & 1.247 & 19332 & 15503 & 8603 \\
\hline CCL4 & 1.22 & 20890 & 17123 & 9410 \\
\hline
\end{tabular}
accelerating structures during installation (Private conversation with C. E. Deibele, July 2014) are summarized in Table 3.11 .

$\mathrm{DTL}=$ drift tube linac; $\mathrm{CCL}=$ coupled cavity linac

Recent measured cavity decay time constants agree closely with those calculated from the measured cavity loaded Q values.

Power margins are calculated assuming the baseline RF power sources.

RF power sources for the STS will have available adequate pulsed dc power; i.e., the HVCM will provide factory-specified klystron cathode voltage and current across the pulse.

Required RF generator power estimates, $\mathrm{P}_{\mathrm{g}}$, are calculated as

$$
P g=V_{a}^{2} \frac{(1+\beta)}{8 \beta} \frac{1}{r_{L}}\left[\left(1+\frac{I_{g} r_{L}}{V_{a}} \cos \theta\right)^{2}+\left(\tan \varphi+\frac{I_{g} r_{L}}{V_{a}} \sin \varphi\right)^{2}\right]
$$


where

$$
\begin{aligned}
& V_{a}=V_{0} T \\
& \beta=Q_{0} / Q_{e x t} \\
& r_{L}=r_{e} /(1+\beta) \\
& r_{e}=r_{s h} / 2 \\
& \theta=\text { synchronous phase } \\
& \varphi=\text { detuning angle } \\
& I_{g}=2 * I_{b}, \quad I_{b} \text { is the average linac beam current, } 38 \mathrm{~mA}
\end{aligned}
$$

Conservative estimates of the required RF generator power for an average linac beam current of $38 \mathrm{~mA}$ specified for the STS are provided in Table 3.12.

Table 3.12. Estimated required RF generator power for the normal conducting linac

\begin{tabular}{lcccccc}
\hline Structure & $\begin{array}{c}\mathbf{P}_{\text {copper }} \\
(\mathbf{k W})\end{array}$ & $\begin{array}{c}\mathbf{P}_{\text {beam }} \\
(\mathbf{k W})\end{array}$ & $\begin{array}{c}\mathbf{P}_{\text {total }} \\
(\mathbf{k W})\end{array}$ & $\begin{array}{c}\mathbf{P}_{\text {klystron }} \\
(\mathbf{k W})\end{array}$ & $\begin{array}{c}\text { PM } \\
\mathbf{( \% )}\end{array}$ & $\begin{array}{c}\mathbf{P M}_{\text {min }} \text { for LLRF } \\
(\mathbf{\%})\end{array}$ \\
\hline DTL1 & 354 & 201 & 555 & 2500 & 350 & 25 \\
DTL2 & 985 & 725 & 1710 & 2500 & 46 & 25 \\
DTL3 & 1238 & 765 & 2003 & 2500 & 25 & 25 \\
DTL4 & 1239 & 893 & 2133 & 2500 & 17 & 25 \\
DTL5 & 1258 & 878 & 2136 & 2500 & 17 & 25 \\
DTL6 & 1191 & 763 & 1954 & 2500 & 28 & 25 \\
CCL1 & 2203 & 1002 & 3205 & 5000 & 56 & 33 \\
CCL2 & 2245 & 1242 & 3487 & 5000 & 43 & 33 \\
CCL3 & 2340 & 1328 & 3667 & 5000 & 36 & 33 \\
CCL4 & 2332 & 1395 & 3727 & 5000 & 34 & 33 \\
\hline
\end{tabular}

Operationally, measurements of the required RF generator power provided through EPICS from dual directional waveguide couplers (forward [FWD] and reflected [REFL]) located after the klystron/circulator indicate substantially higher power readings than those calculated based on measured cavity parameters ( $\sim 20$ to $\sim 30 \%$ difference). This could be due to systematic measurement errors, e.g., non-ideal locations of the directional couplers. Additional contributions could be due to unaccounted resistive losses in the waveguide transmission lines. These discrepancies need to be resolved and any source of error removed or reduced.

\subsubsection{Normal Conducting Linac HPRF Systems for the STS}

Each structure of the drift-tube linac (DTL) is currently driven with RF power from a single $402.5 \mathrm{MHz}$, $2.5 \mathrm{MW}_{\mathrm{pk}}\left(200 \mathrm{~kW}_{\mathrm{ave}}\right)$ klystron. Each klystron is supported by a single transmitter that provides power, utilities, monitoring, control, and personnel and equipment protection. A single HVCM provides pulsed dc power to two DTL klystrons. The RF power is transmitted via a WR2100 rectangular waveguide from the output of the klystron, located in the Klystron Gallery, to an RF vacuum window, ridge-loaded waveguide taper, and coupling iris on the DTL tank located in the accelerator tunnel. Each klystron is protected from reflected power by a waveguide circulator with a water/glycol-cooled load. There are a total of three DTL HVCMs; six DTL high-voltage tanks with klystrons; six circulators with circulator loads; and six waveguide runs, RF vacuum windows, tapers, and coupling irises for the six DTL accelerating structures. 
For the coupled-cavity linac (CCL), RF power from a single $805 \mathrm{MHz}, 5 \mathrm{MW}_{\mathrm{pk}}\left(400 \mathrm{~kW}_{\text {ave }}\right)$ klystron is divided equally and drives bridge couplers at two locations on each structure. Each klystron is supported by a single transmitter that provides power, utilities, monitoring, control, and personnel and equipment protection. A single HVCM provides pulsed dc power to a single CCL klystron. Each klystron is protected from reflected power by a waveguide circulator with a water-cooled load. The RF power is transmitted via a single WR1150 rectangular waveguide from the output of the klystron/circulator to the $3 \mathrm{~dB}$ hybrid power divider, located in the Klystron Gallery. RF power is divided equally and transmitted via two WR1150 waveguide runs to RF vacuum windows and bridge couplers on the appropriate CCL tank located in the accelerator tunnel. There are a total of four CCL HVCMs, four CCL high-voltage tanks with klystrons, four circulators with circulator loads, four $3 \mathrm{~dB}$ waveguide hybrid power dividers with water-cooled loads, and eight waveguide runs and RF vacuum windows.

\section{Warm Linac HPRF Summary}

Assuming factory-specified cathode voltage and current across the HVCM pulse, estimates of RF power required by the DTL suggest that DTL4 and DTL5 klystrons will be operating with reduced available power margin with respect to baseline minimum design requirements. In addition, remaining power margins for DTL4, DTL5 and DTL6 will not be adequate for the current detuning limits.

The increase in the average linac beam current to $38 \mathrm{~mA}$ will require modifying the DTL4 and DTL5 HPRF systems to accommodate a $3 \mathrm{MW}_{\mathrm{pk}}\left(240 \mathrm{~kW}_{\mathrm{ave}}\right)$ klystron. Because of the current configuration of two paired DTL klystrons per HVCM, this would require recabling DTL3 and DTL6 together on one HVCM, and DTL4 and DTL5 on a modified HVCM.

Integrating a $3 \mathrm{MW}_{\mathrm{pk}}\left(240 \mathrm{~kW}_{\text {ave }}\right)$ klystron into the current transmitter configuration is anticipated to be straightforward, but it may require upgrading to compatible filament and magnet power supplies. In addition, the increase to $3 \mathrm{MW}_{\mathrm{pk}}\left(240 \mathrm{~kW}_{\text {ave }}\right) \mathrm{RF}$ power would require additional cooling to remove an estimated maximum incremental increase of $\sim 40 \mathrm{~kW}_{\text {ave }}$ per klystron in collector power.

Assuming factory-specified cathode voltage and current across the HVCM pulse, estimates of RF power required by the CCL suggest that there will remain an adequate minimum power margin $\geq 33 \%$. Therefore, the CCL RF systems will remain unchanged from the baseline with the exception of the HVCM.

Table 3.13 summarizes the baseline warm linac RF equipment and the additional required RF equipment to support the STS.

Table 3.13. Normal conducting linac high-power RF systems for the STS

\begin{tabular}{lccc}
\hline \multicolumn{1}{c}{ Major equipment } & Baseline & STS upgrade & Final configuration \\
\hline DTL klystron & $6(2.5 \mathrm{MW})$ & Upgrade 2 sockets to 3 MW & $4(2.5 \mathrm{MW}), 2(3 \mathrm{MW})$ \\
DTL transmitter & 6 & Modify to support 3 MW tube & $4(2.5 \mathrm{MW}), 2(3 \mathrm{MW})$ \\
DTL circulator & 6 & Upgrade to support 3 MW tube & $4(2.5 \mathrm{MW}), 2(3 \mathrm{MW})$ \\
DTL circulator load & 6 & Upgrade to support 3 MW tube & $4(2.5 \mathrm{MW}), 2(3 \mathrm{MW})$ \\
DTL vacuum window & 6 & Upgrade to support 3 MW tube & $4(2.5 \mathrm{MW}), 2(3 \mathrm{MW})$ \\
CCL klystron & $4(5 \mathrm{MW})$ & - & $4(5 \mathrm{MW})$ \\
CCL transmitter & 4 & - & 4 \\
\hline
\end{tabular}




\subsubsection{SCL HPRF Systems for STS}

Each of the existing 81 superconducting cavities is driven with RF power from a single klystron. Currently, 78 of the 81 klystrons in use are the model VKP-8291A manufactured by CPI. Three of the 81 klystrons in use are the model TH2169, manufactured by Thales. Both klystron models operate at $805 \mathrm{MHz}$ and are specified by their manufacturers to produce $550 \mathrm{~kW}_{\mathrm{pk}}\left(49.5 \mathrm{~kW}_{\mathrm{ave}}\right)$ of RF power.

The RF power is transmitted via rectangular waveguide from the output of the klystron, located in the Klystron Gallery, to the cryomodule located in the accelerator tunnel. Each klystron is protected from reflected power by a waveguide circulator and water-cooled load. Each klystron is provided power, utilities, control, and monitoring by a transmitter. Each transmitter supports a group of 6 klystrons and includes 6 filament power supplies, 12 magnet supplies ( 2 per klystron), an ion-pump vacuum controller and high-voltage power supply, water cooling, flow control and monitoring, 6 solid-state RF amplifiers, 2 high-voltage klystron tanks, klystron cathode overcurrent diagnostics, personnel and equipment safety interlocks, and programmable logic controller monitoring and control. A single HVCM provides pulsed dc power to a grouping of 10 or 11 klystrons spread across multiple transmitters.

The increase in beam energy for the STS will require installing an additional $28 \mathrm{RF}$ systems to provide RF power to 28 superconducting accelerating cavities in 7 cryomodules ( 4 cavities per cryomodule), in the SCL.

The increase in average linac beam current will require additional RF generator power because of the increase in beam loading. The estimated required RF generator power profile, resulting power margin, and baseline power margin are shown in Fig. 3.35.

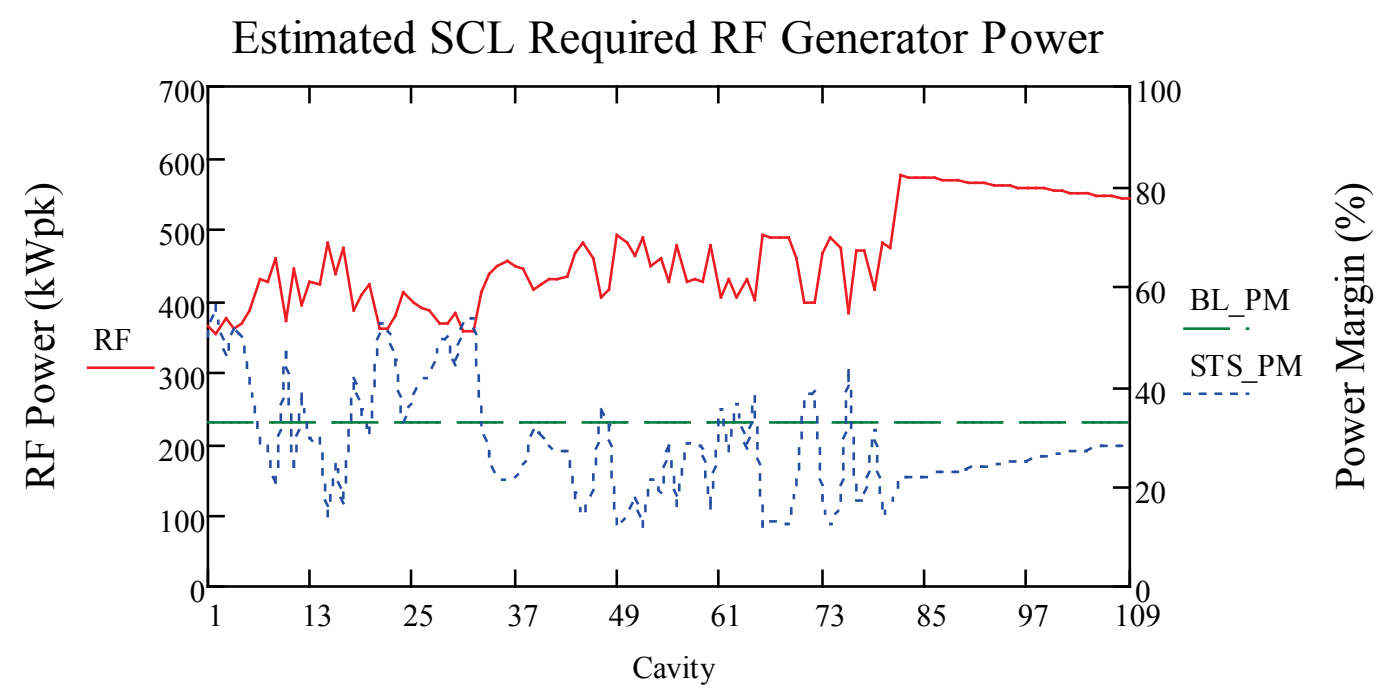

Fig. 3.35. Estimated required RF generator power for the SCL.

The STS upgrade will duplicate the 6 klystron/transmitter configuration for the additional 28 superconducting cavities. This will require an additional 5 transmitters to support the additional 28 klystrons. Two of the six available klystron sockets in one of the transmitters will be unused. To maintain ease of operation and maintenance, these additional transmitters will be physically and functionally identical to the existing SCL transmitters.

The STS upgrade will use 28 CPI VKP-8291B klystrons. The VKP-8291B klystron operates at $805 \mathrm{MHz}$ and is specified by the manufacturer to produce $700 \mathrm{~kW}$ of RF power. The VKP-8291B is physically 
identical to the VKP-8291A klystron except for minor modifications to cavity tuning, output cavity coupling, and increased cooling requirements to allow operation at the higher output power level. The VKP-8219B klystron can also be used as a direct replacement in the existing SCL HPRF klystron sockets.

The current SCL transmitter cooling cart may be redesigned for the STS upgrade to incorporate ultrasonic flow meters, reduce footprint, and enhance access to waveguide components.

\section{SCL HPRF Summary}

The major RF-related equipment for the baseline design and the STS upgrade are summarized in Table 3.14.

Table 3.14. Superconducting linac high power RF systems for the STS

\begin{tabular}{lccc}
\hline \multicolumn{1}{c}{ Major equipment } & Baseline & STS upgrade & Final configuration \\
\hline SRF klystron & $81(550 \mathrm{~kW})$ & $28(700 \mathrm{~kW})$ & 109 \\
SRF klystron magnet & 81 & 28 & 109 \\
SRF circulator & $81(550 \mathrm{~kW})$ & $28(700 \mathrm{~kW})$ & 109 \\
SRF circulator load & $81(550 \mathrm{~kW})$ & $28(700 \mathrm{~kW})$ & 109 \\
SRF waveguide run & 81 & 28 & 109 \\
SRF transmitter & 14 & 5 & 19 \\
SRF HVCM & 7 & 3 & 10 \\
\hline
\end{tabular}

Nine locations are available in the tunnel for additional cryomodules. These locations are identified as CM24 through CM32. For the STS, seven additional cryomodules will be installed in the tunnel in location CM25 and locations CM27 through CM32, leaving locations CM24 and CM26 open. Racks for the 5 transmitters, 5 transmitter cooling carts, 10 high-voltage tanks for the 28 klystrons, and 3 HVCMs will be installed in the Klystron Gallery as indicated in Fig. 3.36. The choice of empty slots to use for the STS cryomodules is driven by considerations to minimize the movement of cables presently in space needed for an upgrade RF waveguide.

The existing SCL HPRF topology consists of modulators that power 9 to 10 klystrons (labeled "SCL_MODXX") and transmitters that service 6 klystrons (labeled "SCLXX"). The topology mapping for the additional five transmitters and three HVCMs considered for the STS is as follows: HVCM SCL_MOD25 will provide pulsed de power to nine klystrons - four in transmitter SCL25 and five in SCL27. HVCM SCL_MOD28 will provide pulsed dc power to nine klystrons - one in SCL27, six in SCL28, and two in SCL30. HVCM SCL_MOD30 will provide pulsed de power to the remaining ten klystrons-four in SCL30 and six in SCL31.

The waveguide layout, including circulators, circulator water loads, and waveguide components, will be identical to the existing installation. Circulators and circulator water loads will be specified to $700 \mathrm{~kW}_{\mathrm{pk}}$ $\left(63 \mathrm{~kW}_{\mathrm{ave}}\right)$.

The five transmitter cooling carts will be installed along the north gallery wall, as indicated in Fig. 3.36. Water connections will be minimized and standardized at the top of the klystron to support ease of replacement and vibration isolation. 


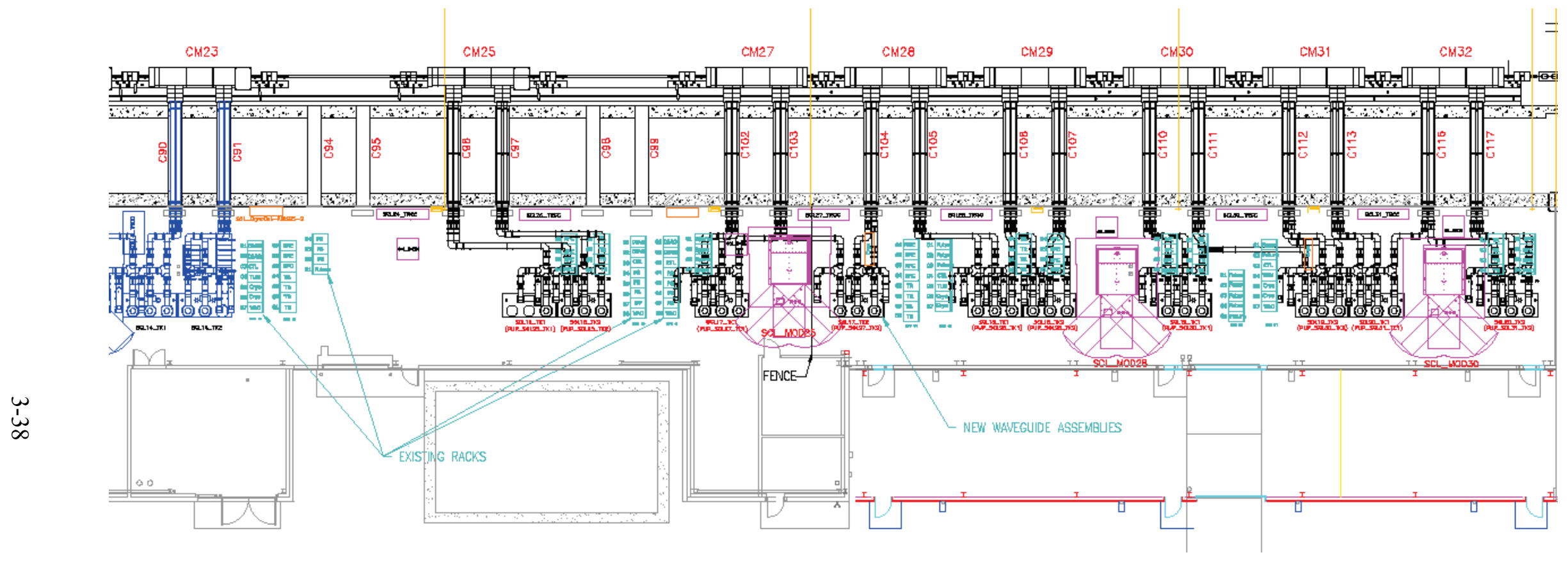

Fig. 3.36. Proposed layout of Klystron Gallery and accelerator tunnel. 


\subsubsection{Cooling for the RF systems}

\section{RF Warm Linac Cooling Systems}

\section{New Front End Building Cooling System (FE-01)}

The current KL-04 cooling system will provide cooling to the Front End Building (FEB) ion source, RFQ, and medium-energy beam transfer equipment, as well as to all the RFQ/DTL klystrons, circulators, HVCM, and silicon-controlled rectifier (SCR) equipment in the Klystron Gallery. The $\sim 20 \%$ higher load on the RFQ and DTL klystrons will require about $125 \mathrm{gpm}$ of additional cooling water to meet the additional STS demands. Currently, the front end equipment requires a $110 \mathrm{gpm}$ load; and the existing KL-04 pumps are currently operating well above their design point, to the point that the desired system pressure cannot be maintained in the Klystron Gallery. To avoid total removal of the DTL cooling system (KL-04) and its replacement with a new larger system, it has been proposed to place the FEB equipment on its own separate cooling system (FE-01) and use the available excess capacity from the FEB for the added STS loads on the RF equipment in the Klystron Gallery. KL-04 would then be decoupled from the FEB.

\section{Upgrade Existing DTL RF Cooling System Upgrade (KL-04)}

The existing DTL klystron cooling system (KL-04) will be upgraded to allow an increase in flow and heat transfer area from the $20 \%$ higher thermal loads on the DTL RF equipment. Increases in pump impeller trim and motor horsepower and additional electrical starters and wiring will be addressed. Heat exchangers will be rebuilt, and additional plates will be added for the needed heat transfer surface area. The temperature control valve will also be resized for the added demand.

If the new FE-01 is not installed, then KL-04 will have to be upgraded a larger pump, heat exchanger, and headers and associated equipment similar in size and cost to that of the new KL-06 system being proposed for the new SCL equipment. Likewise, an accelerator outage of up to 6 to 9 months would be required to install the new system and modify the existing header system.

\section{SCL Radio Frequency Cooling System}

\section{Cooling System for New SCL RF Equipment (KL-06)}

A new Klystron Gallery cooling system (KL-06) will be provided for the cooling of the RF and highvoltage equipment that will be added to the open east end of the Klystron Gallery. Water cooling is required for the RF equipment, the high-voltage equipment, and the existing magnet power supplies at the end of the Klystron Gallery (Building 8300). The RF system components that require water cooling consist of 5 separate transmitter cooling carts that measure and distribute water to 28 circulators, 28 water loads, and 28 klystrons. The three high-voltage modulators and three SCR cabinets will also require water cooling. Finally, the 13 existing magnet power supplies located in the area will be transferred to the new cooling system. Figure 3.37 shows the proposed layout for the KL-06 cooling system. Conventional Facilities will provide the new enclosure and area utilities. The new cooling system and all associated piping are included within this task. 


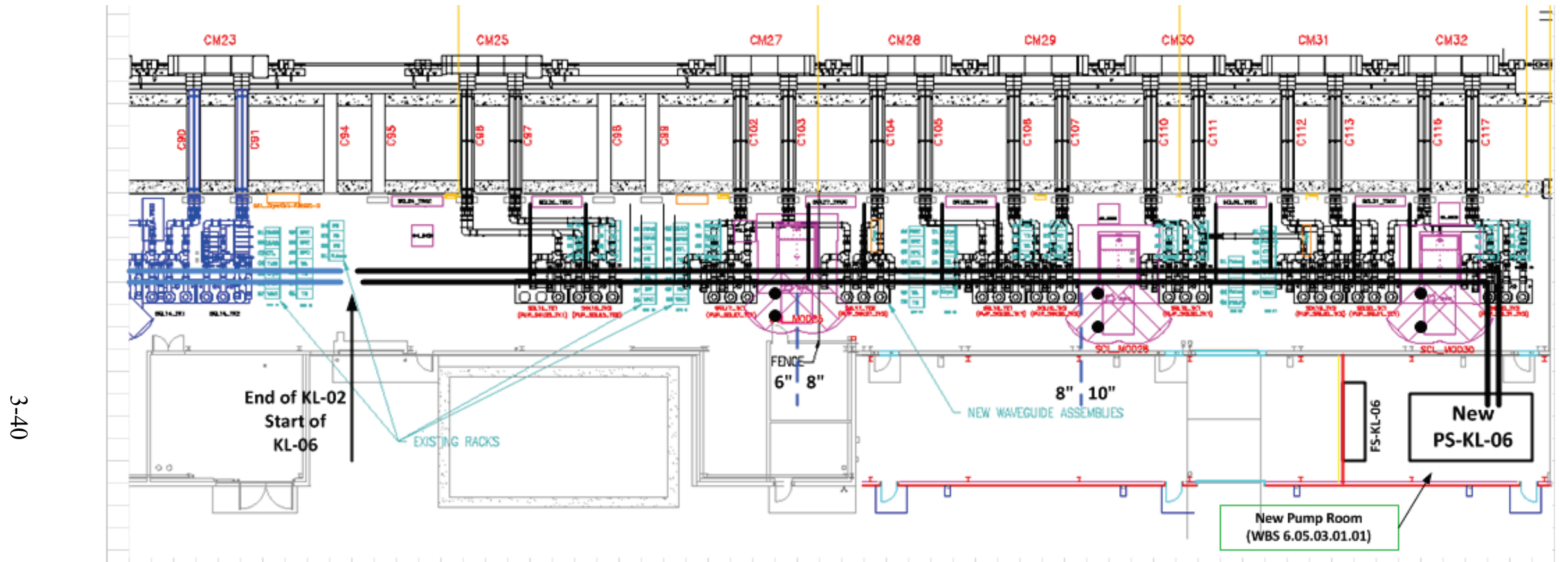

Fig. 3.37. Proposed KL-06 RF cooling system layout. 
Upgrade Existing SCL—RF Cooling Systems (KL-02 and KL-03)

The existing SCL klystron cooling systems (KL-02 and KL-03) will be upgraded to allow increases in flow and heat transfer area due to the $\sim 20 \%$ higher thermal loads on the SCL RF equipment. Increases in pump impeller trim and motor horsepower and additional electrical starters and wiring will be addressed. Heat exchangers will be rebuilt, and additional plates will be added for the needed heat transfer surface area. The temperature control valve will also be resized for the added demand.

\subsubsection{Low-Level Radio Frequency Systems for the STS}

The primary requirement for STS is supplying 28 new units for the 28 new cavities included in the STS scope. The low-level RF (LLRF) systems provide low-power RF drive to the linac RF systems and, most important, control of the phase and amplitude of each cavity. The initial baseline stability requirements for this control are $\pm 1.0^{\circ}$ in phase and $\pm 1.0 \%$ in amplitude. The LLRF control systems on the existing 96 existing systems more than meet this specification, with $\pm 0.5^{\circ}$ in phase and $\pm 0.5 \%$ in amplitude routinely observed. A block diagram for the LLRF system is shown in Fig. 3.38.

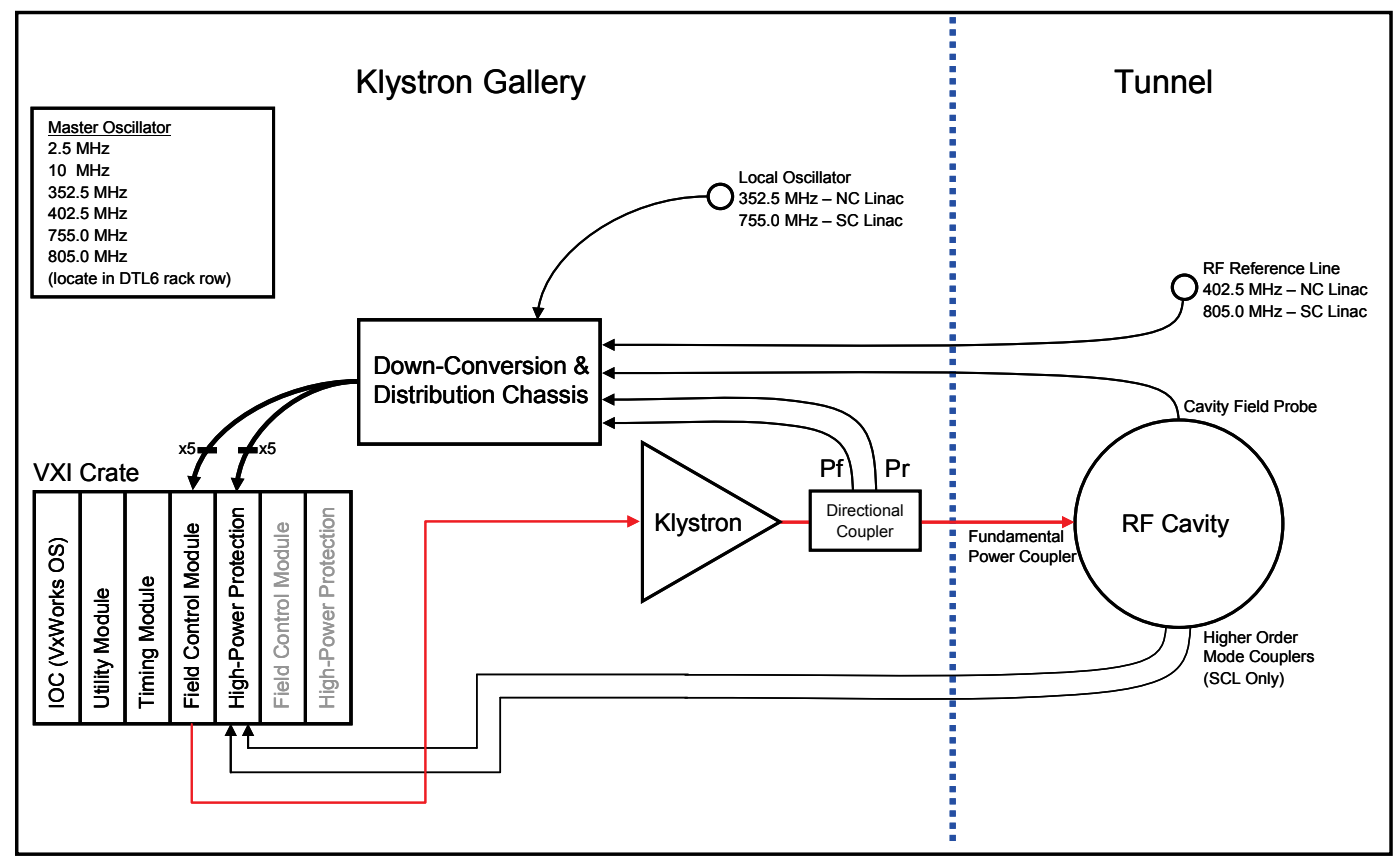

Fig. 3.38. Block diagram of the SNS linac low-level control system.

The current LLRF system is a digital-control system that fundamentally realizes a proportional-integral feedback controller along with adaptive feed-forward to support cavity filling and beam loading compensation. The heart of the system is the field control module (FCM), which digitizes four analog input channels (cavity field, reference, forward, and reflected signals), digitally processes the data stream, and produces an output signal at either 402.5 or $805 \mathrm{MHz}$, depending on the location in the linac. The FCM is a VXI bus module and consists of a motherboard and three daughter boards: the analog front end, digital front end, and RF output. The analog front end takes the analog signals and converts them to a differential pair for input to the ac-dc converters. The digital front end consists primarily of four highspeed, 14-bit A/D converters and a single field-programmable gate array (FPGA) responsible for the realtime processing. The RF output is responsible for receiving the I/Q signals and generating the required RF drive. Communication with the outside world is via the input-output controller running the VxWorks operating system. The LLRF finite state machine is implemented as an EPICS sequencer running on the 
input-output controller for capabilities such as ramping up RF power. The LLRF control system also provides high-power protection via the high-power protection module (HPM), which provides for fast shutdown of the drive in case of RF overpower, cavity quenches, arcs in the distribution system, poor vacuum, or "soft" interlocks such as cryo, coupler cooling, and HPRF permit. Down-conversion of the reference and cavity signals is performed in a temperature-regulated chassis. The master oscillator provides low-noise, phase-coherent reference signals that are distributed throughout the Klystron Gallery and tunnel.

The LLRF control systems are currently installed with two control systems per rack consisting of two FCM/HPM pairs with a shared timing card, utility card, and input-output controller. This configuration will continue to be used for the STS project. This implies splitting systems across two HVCMs, as is done in the existing SNS linac.

STS will require 28 additional SCL LLRF systems to support the increase in beam energy to $1.3 \mathrm{GeV}$ and the increase in average linac beam current to $38 \mathrm{~mA}$. With the plan of a $50 \mathrm{~Hz} / 10 \mathrm{~Hz}$ split in the beam pulses to support two target halls and different beam energy and chopping styles, an additional beam loading adaptive feed-forward (AFF) buffer will be required in the LLRF systems. Adding the additional AFF buffer will require significant development time and effort for implementation.

The current FCM has several obsolete parts, including the FPGA that performs the real-time processing. Along with the obsolescence issue, the gate resources that are available in the current FPGA are marginal for supporting the second AFF buffer. If the resources are found to be insufficient for the new buffer, a replacement FCM will be required for the project. The replacement FCM would need to be deployed for the entire 124 LLRF systems needed for the 96-system baseline RF installation plus the additional 28 systems for the STS. The development time for new hardware would add approximately 1 year to the LLRF effort.

The remaining installed LLRF infrastructure will support the STS without modification. Except for the possibility of a replacement FCM, the 28 new STS systems will be identical to the systems supporting the presently installed RF systems. The remaining subsystems, to include the arc detectors, down-converters, and reference and local oscillator distribution systems, are still available for purchase or manufacture and will not require additional development effort.

\subsubsection{High-Voltage Modulator Conceptual Design}

Modulator design for the STS involves several tradeoffs necessary to ensure that system reliability is at least equivalent to the current HVCM operational performance. The system requirements are dictated by the cavity power requirements and the klystron performance characteristics, which are defined elsewhere. The increased and / or new cavity power requirements demand the following from the modulator systems:

- increased operating voltage levels to drive the $700 \mathrm{~kW}$ klystrons supplying the 28 new cavities

- increased operating voltage levels to allow the existing SCL cavities to operate above current levels

- increased operating voltage levels in the warm section of the linac to support the higher beam current loading

Each of these situations will be considered separately in the subsections below. Options will be presented where new modulator installations are required, but those options are by no means the only ones available. However, wherever possible, the modulators will be similar to the existing HVCMs to minimize spare parts inventories, optimize operational efficiency, and minimize the amount of training 
necessary to support modulator operation. Table 3.15 summarizes the major parameters for the new and upgraded current modulator systems required to support the STS.

Table 3.15. Present and projected operating parameters for existing and new HVCM systems

\begin{tabular}{lccrcc}
\hline HVCM system & $\begin{array}{c}\text { Present output } \\
\text { voltage } \\
\text { (V) }\end{array}$ & $\begin{array}{c}\text { Present } \\
\text { output } \\
\text { current } \\
\text { (A) }\end{array}$ & $\begin{array}{c}\text { STS output } \\
\text { voltage } \\
\text { (V) }\end{array}$ & $\begin{array}{c}\text { STS output } \\
\text { current } \\
\text { (A) }\end{array}$ & $\begin{array}{c}\text { STS } \\
\text { modulator } \\
\text { average power } \\
\text { (kW) }\end{array}$ \\
\hline Existing DTL HVCMs & 126 & 66.9 & 134 & 76.2 & 760 \\
Existing CCL HVCMs & 135 & 69.8 & 141 & 73.8 & 740 \\
Existing SCL HVCMs & 74 & 116 & 77 & 122 & 750 \\
New SCL HVCMs & N/A & N/A & 79 & 115.5 & 730 \\
\hline
\end{tabular}

Modulator tradeoffs primarily revolve around the main active component of the system, the insulated gate bipolar transistor (IGBT) switches. Currently, 3300 V, 1200 A IGBT switches are used since they represent the highest-power modules available that are capable of operating reliably at $20 \mathrm{kHz}$. Other higher-power switches have been studied throughout the years of HVCM development, but they either have unacceptably high switching losses or long delay and/or transition times in turning on or off. Since no external data exist for these switches at the chosen switching frequency, the reliability of the power electronics is based on operational experience and engineering scaling. The SNS HVCMs are able to operate reliably at $70 \%$ of their voltage rating without snubbers and at $\sim 75 \%$ with snubbers. Experience has also shown that turning off an IGBT while it is conducting $1 \mathrm{kA}$ or greater is detrimental to its longterm survival. Likewise, maintaining switching losses below approximately $5 \mathrm{~kW}$ is desirable to maintain system reliability. It is also desirable to maintain these operating limits so as to permit use of existing SCR controllers and $13.8 \mathrm{kV}: 2100 \mathrm{~V}$ dry cast distribution transformers.

\subsubsection{New and Existing SCL Cavities}

To provide $805 \mathrm{MHz}$ RF power to the additional 28 cavities required for the STS, twenty-eight $700 \mathrm{~kW}$ peak power klystrons will be installed in the existing Klystron Gallery. As is typically the case, the ratio of klystrons to modulators is based on optimization of the modulator performance parameters. However, two viable options exist for powering these new klystrons.

The first, and baseline, option is to use the existing HVCM topology and increase the voltage to accommodate the higher required output voltage. The new STS klystrons and cavities will require a minimum operating voltage of $79 \mathrm{kV}$ from the modulators. Figure 3.39 summarizes the issues associated with employing the current HVCM design to power the existing and the new SCL cavities for the STS. The orange and pink horizontal lines represent the minimum required cathode voltage for the current and new SCL klystrons, respectively, to generate the required RF power levels. Current HVCM operation is limited to $\leq 1140 \mathrm{~V}$ on the capacitor bank corresponding to the region labeled "current operating levels," indicated to the left of the vertical blue line. However, initial tests of the HVCM with the snubbers for multiple 1 week continuous run periods suggest that operation in excess of $1200 \mathrm{~V}$ is possible and may be as reliable as current HVCM operation. PSpice simulations produce a family of curves for each klystron/modulator combination, with the relationship between input capacitor voltage and output cathode voltage indicated by lines corresponding to each klystron/modulator ratio. Assuming reliable operation up to $1200 \mathrm{~V}$ on the capacitor bank, the optimum operating point that does not exceed any empirical limits is indicated in the figure by the red and green dots for the existing and new SCL modulators. Unfortunately, 9 klystrons per modulator still leaves 1 klystron unpowered if 3 modulators are selected to power the required 28 new cavities. However, by operating the last 10 new cavities at a power level reduced by 
$\sim 10 \%$, which is consistent with the beam loading requirements in Sect. 3.1, it is still possible to maintain the required cavity gradients and provide sufficient phase and amplitude control margins for klystron operation. This solution of 9 klystrons to one modulator for the first 2 additional modulators, with the last modulator in the gallery powering 10 klystrons at reduced power levels, represents the current STS modulator baseline design to power the required 28 cavities.

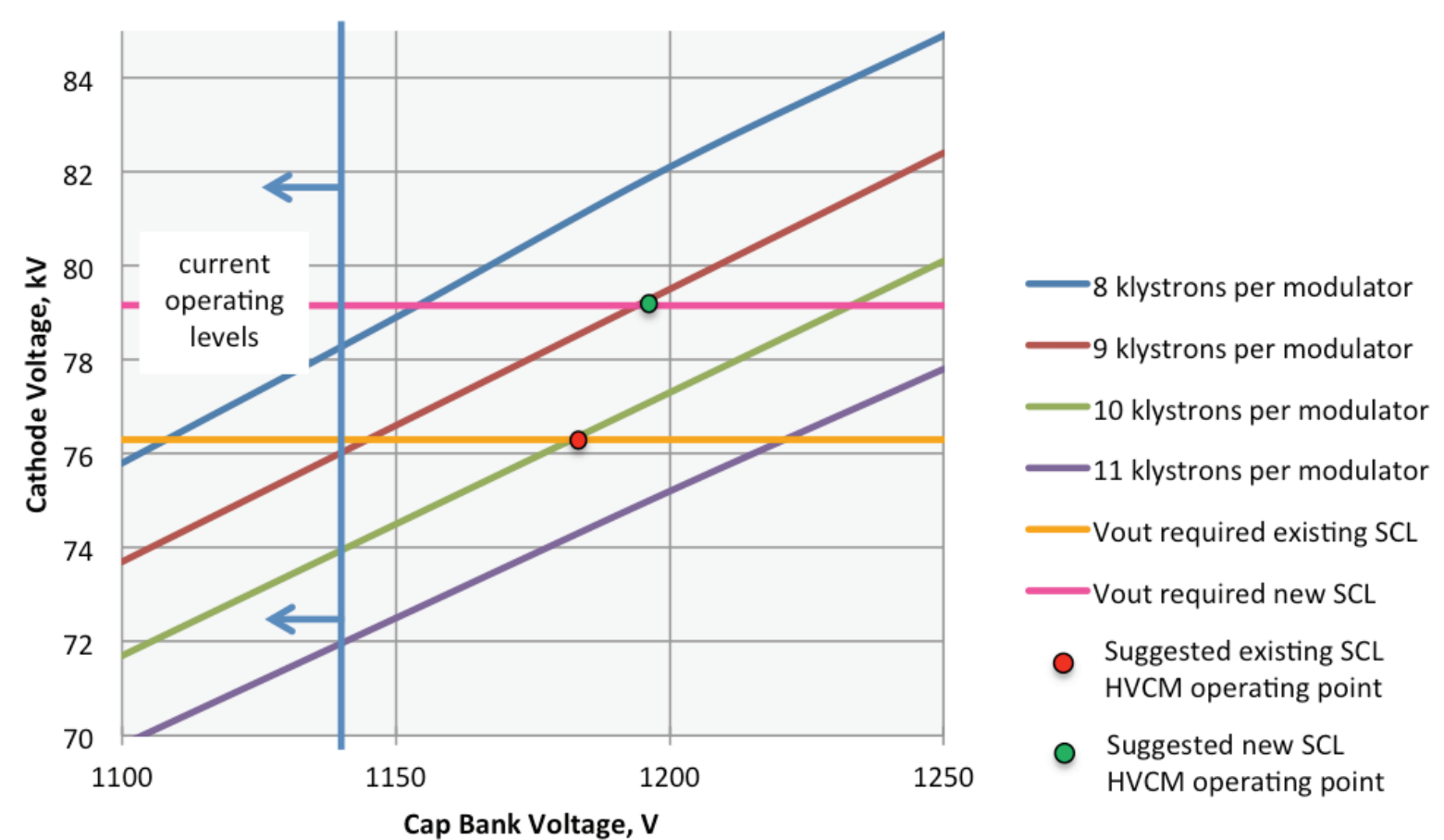

Fig. 3.39. Design considerations for the existing and new SCL HVCM systems. Curves shown represent simulation results of modulator output voltage for a given capacitor voltage for different ratios of klystrons to modulators. Horizontal lines represent the required modulator output voltage for the existing and new SCL HVCMs. The vertical line indicates the present cap bank voltage limitation, which produces high reliability.

The second, backup, option is to use a proposed alternate topology for the HVCM. This design would eliminate the least reliable component in the HVCM, the boost capacitor. The output voltage would also be insensitive to load variations, and this topology would result in soft switching of the IGBTs. This would result in lower losses in the switches and hence a lower heat load and higher reliability. This option requires additional prototyping and testing that is scheduled to begin in the autumn of 2014. There is some concern that the higher IGBT currents necessary would compromise system reliability; therefore, extensive reliability testing would be required before implementing this design in the existing SNS system and/or the STS upgrade. If this option is determined to be reliable, it will become the new baseline design for the STS upgrade because of the identified benefits.

\subsubsection{Existing Normal Conducting Linac Cavities}

The additional beam current required for the STS upgrade does have implications in the warm section of the machine as well. Some of this detail can be found in Sect. 3.4.1. The CCL structures should be capable of being operated at higher beam current with sufficient control margin, assuming minimal modulator changes, provided the new controllers are installed and pulse flattening is implemented. The new controllers, in addition to adding enhanced data acquisition and troubleshooting features to the 
modulators, provide frequency and phase shift modulation capabilities to the IGBT switches. These capabilities allow for compensation of the capacitor bank voltage droop and has been shown to be capable of reducing the output cathode voltage droop to less than $0.5 \%$ on all current modulator configurations. Current plans are for the new controller installation to be complete at the time of STS commissioning. Figure 3.40 details the situation for the current and STS-upgraded normal conducting linac HVCM systems.

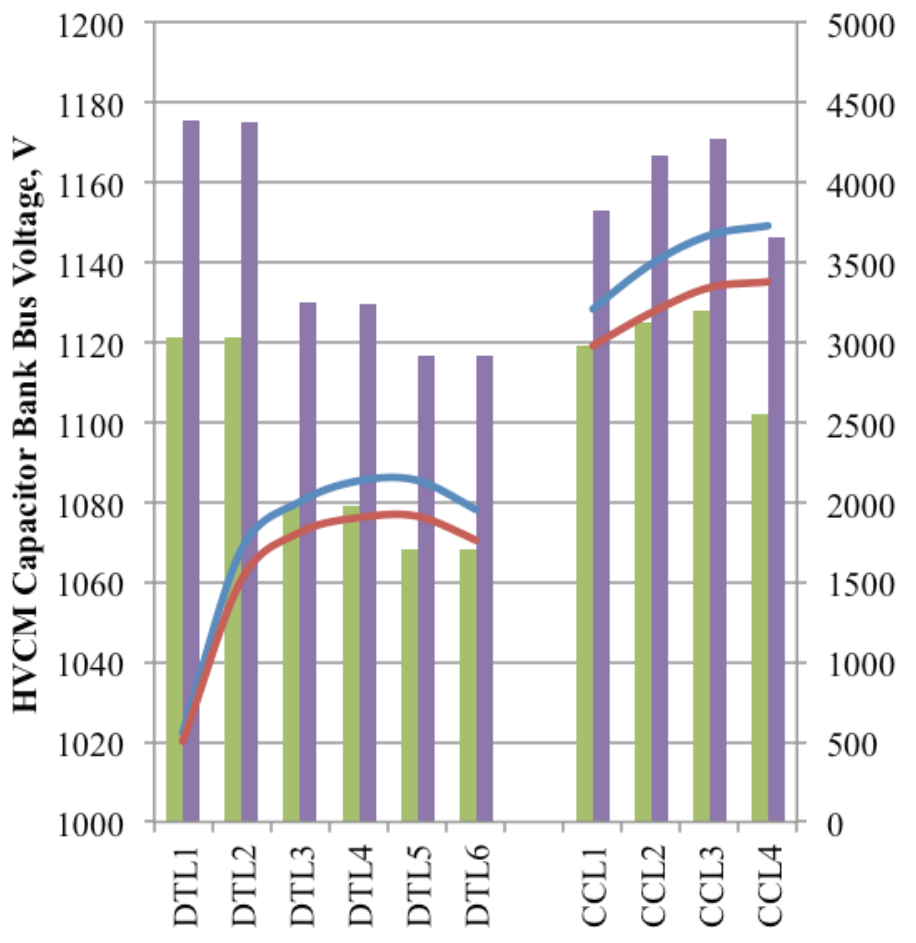

Current HVCM Bus Voltage, V

Required STS HVCM Bus Voltage, $\mathrm{V}$

STS Forward Klstron Power, $\mathrm{kW}$

Current Forward Klystron Power, $\mathrm{kW}$

Fig. 3.40. The impact of the STS on the normal conducting linac klystron forward power and HVCM capacitor bank bus voltages. The additional forward power needed will require higher HVCM bus voltages.

The data shown in Figure 3.40 are for the existing HVCM systems without any further modifications. Based on measurements on other HVCM systems employing snubbers, the operating capacitor bank bus voltages shown should be achievable with no impact on system reliability. Minor modulator changes in the CCL section may be required, depending on how much control margin is required once pulse flattening is achieved. Changing the boost transformers' turns ratios to increase the output voltage and implementing thermal upgrades on affected components should achieve the required results while simultaneously reducing the capacitor bank bus voltage.

In the DTL section of the accelerator, it may be necessary to replace the existing $2.5 \mathrm{MW}$ klystrons with 3.0 MW klystrons. Doing so would reestablish adequate control margins with a marginal cost, should the control margin with the existing klystrons be insufficient. Figure 3.40 does not address this possibility, showing only the necessary increase in voltage to allow the current klystrons to produce the required forward power. However, the modulator modifications similar to those mentioned in the previous paragraph would become mandatory for the existing DTL HVCMs should higher-power klystrons be deployed. Unfortunately, without klystron performance parameters, it is difficult to quantify the changes 
precisely; but reasonable estimates can be made for costing purposes by linearly scaling with the ratio of new required forward power to existing forward power. The cost estimates presented are based on this power scaling.

\subsection{RING SYSTEMS}

The ring will need some relatively minor modifications to the injection and extraction areas. The ring RF system may also need minor modifications to accommodate the higher beam loading effects. All the other areas in the ring are already able to support $1.3 \mathrm{GeV}$ beams. The upgrade plan is consistent with that developed for the earlier Power Upgrade Study [36].

The particle tracking simulations and the space charge effects described earlier show that the ring should perform according to expectations at the higher beam power levels. The following sections describe the modifications to the ring hardware.

\subsubsection{Injection Region}

The purpose of the ring injection system is to inject particles from the HEBT into the accumulator ring and transport the waste beams to the ring injection dump. The key components of the injection system are shown in Fig. 3.41. The injection chicane magnet currents cannot be simply scaled up to accommodate the increased injection energy of $1.3 \mathrm{GeV}$, since this would cause excessive $\mathrm{H}^{-}$stripping. Two of the four chicane magnets will be replaced with a new design, and the injection dump septum magnet will require a new design.

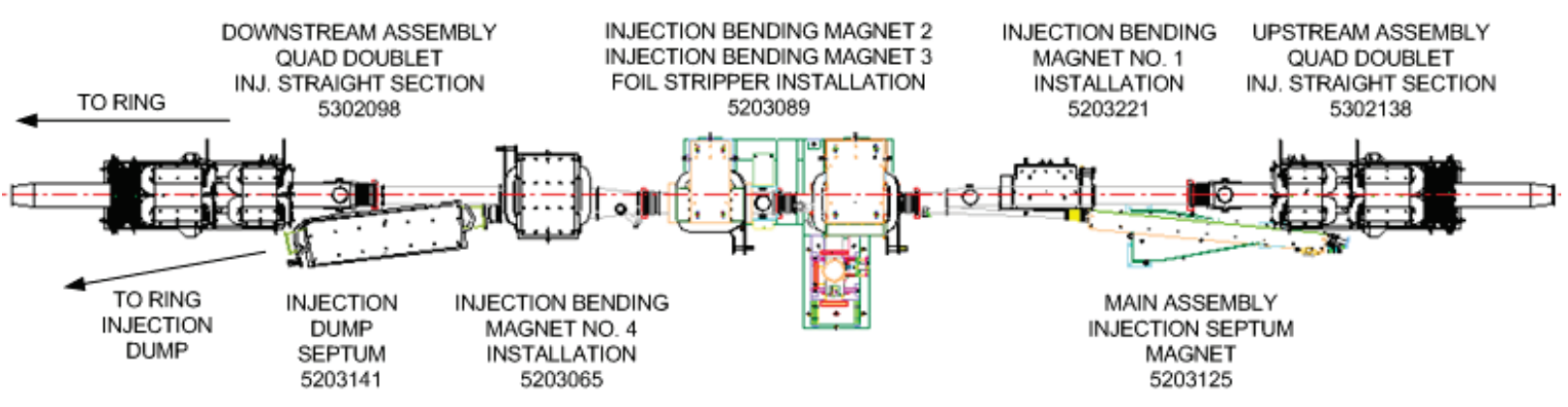

Fig. 3.41. Injection system major components.

Chicane magnets. The modifications required for the bending (chicane) magnets are as follows:

- move magnet 5203221 (DH_A10 Chicane 1) upstream

- redesign and replace magnet 5203072 (DH_A11 Chicane 2)

- redesign and replace magnet 5203104 (DH_A12 Chicane 3)

Both redesigned magnets will retain the basic configuration and mechanical design of the existing units. New vacuum vessels will be required to accommodate the new magnets and to allow the stripper foil mechanism to be relocated.

Injection dump septum magnet. The magnet steel in the existing injection dump septum magnet 5203141 would be driven into the highly saturated region to achieve the magnetic fields required for $1.3 \mathrm{GeV}$ operation, exceeding the current available from the present power supply. This magnet will therefore be replaced with a new design that will occupy the same approximate installation footprint. As 
with the chicane magnets, this magnet will also retain the basic configuration and mechanical design as the existing unit. The vertical aperture through the magnet will be at least as large as in the present design, and the horizontal aperture will accommodate the waste beams with appropriate clearance on each side. A new vacuum vessel for this magnet is also required.

Injection kicker system. The injection kicker magnets by themselves are capable of operating at the higher currents necessary to deflect the $1.3 \mathrm{GeV}$ beam. Empirical field tests at high duty factors show that the thermal performance of the magnets is adequate for the required STS operational parameters. However, the power supplies for these kicker magnets will require some upgrades. All eight injection kicker power supplies will be upgraded from 1400 to 1550 A to give the extra field required to deliver the same kick angles at the higher $(1.3 \mathrm{GeV})$ beam energy.

\subsubsection{Extraction Region}

The purpose of the extraction system is to redirect the trajectory of accumulated proton bunches from the ring toward the target via the ring to target beam transport line (RTBT). The magnets making up the extraction system are shown in Fig. 3.42.

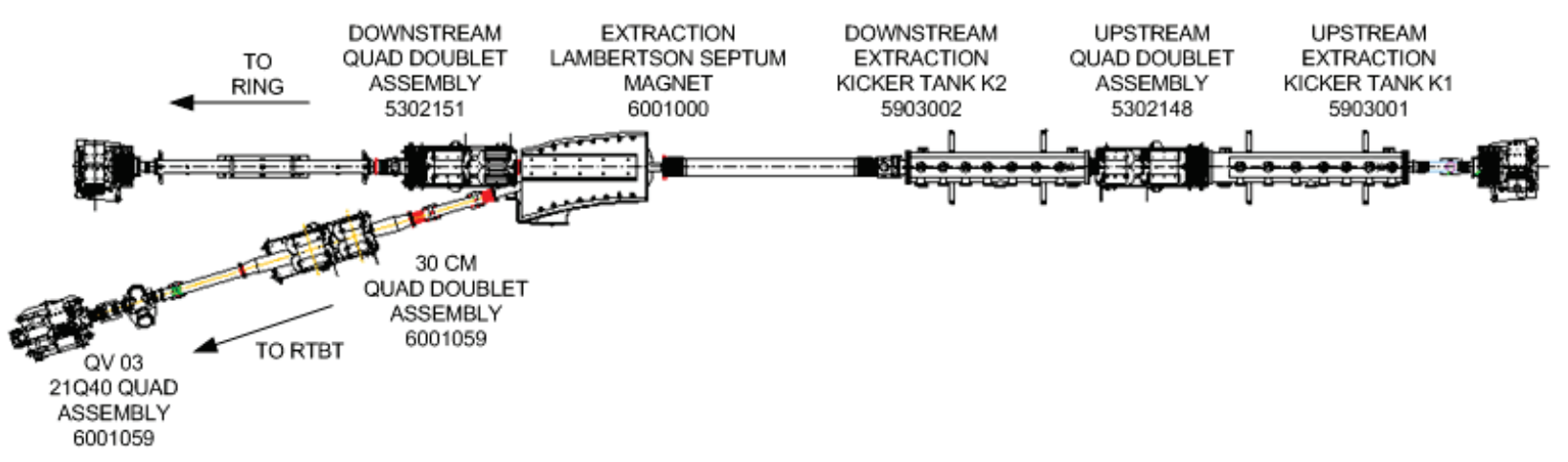

Fig. 3.42. Extraction system magnets.

Each extraction kicker vacuum "tank" contains seven single-turn ferrite magnets. There are six slightly different magnet designs, distinguished primarily by vertical aperture size. The identification and installation arrangements for the present kicker magnets are shown in Figs. 3.43 and 3.44.

To accommodate the $1.3 \mathrm{GeV}$ STS beam energy, two more kickers will be added to this system (for a total of 16 magnets). A vacuum tank extension or "annex" will be added to the upstream end of K1 and to the downstream end of K2. One additional kicker of the same type as the first and last magnet in the respective main tanks will be housed within each annex. The spacing between the upstream annex magnet and K1-1 must match the spacing between K1-1 and K1-2. Likewise, the spacing between the downstream annex magnet and K2-7 must match the spacing between K2-6 and K2-7. The new magnet arrangements will be renumbered as shown schematically in Figs. 3.45 and 3.46. 
BEAM DIRECTION

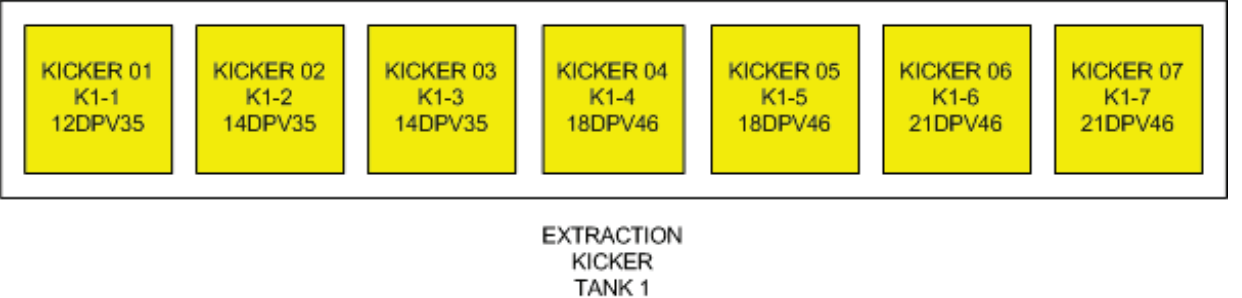

Fig. 3.43. Schematic of the existing extraction kicker tank K1 with magnet types and numbering.

BEAM DIRECTION

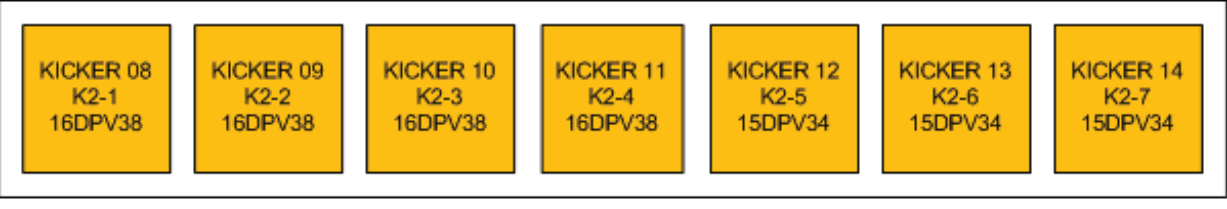

EXTRACTION

KICKER

Fig. 3.44. Schematic of the present extraction kicker tank K2 with magnet types and numbering.

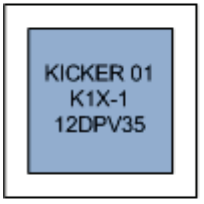

UPSTREAM

ANNEX

TANK

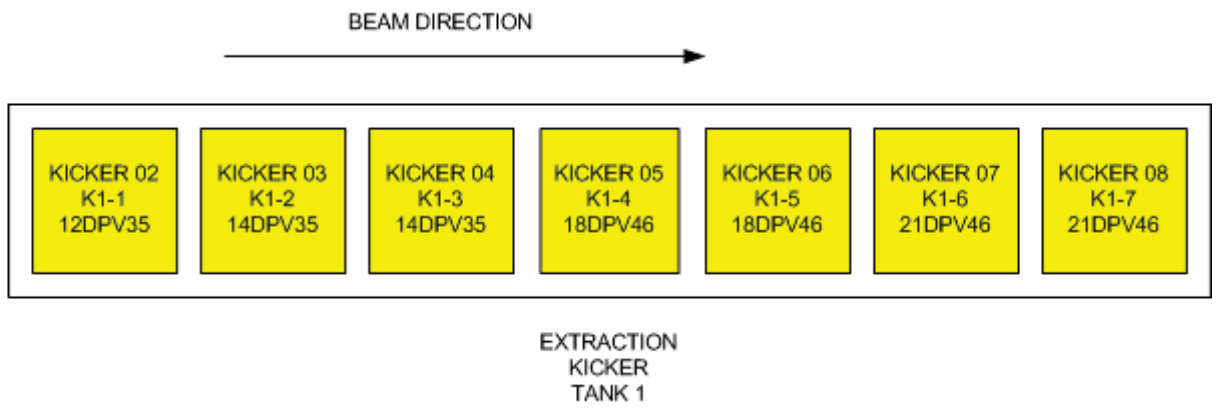

Fig. 3.45. Schematic of extraction kicker tank K1 with annex and renumbered magnets.

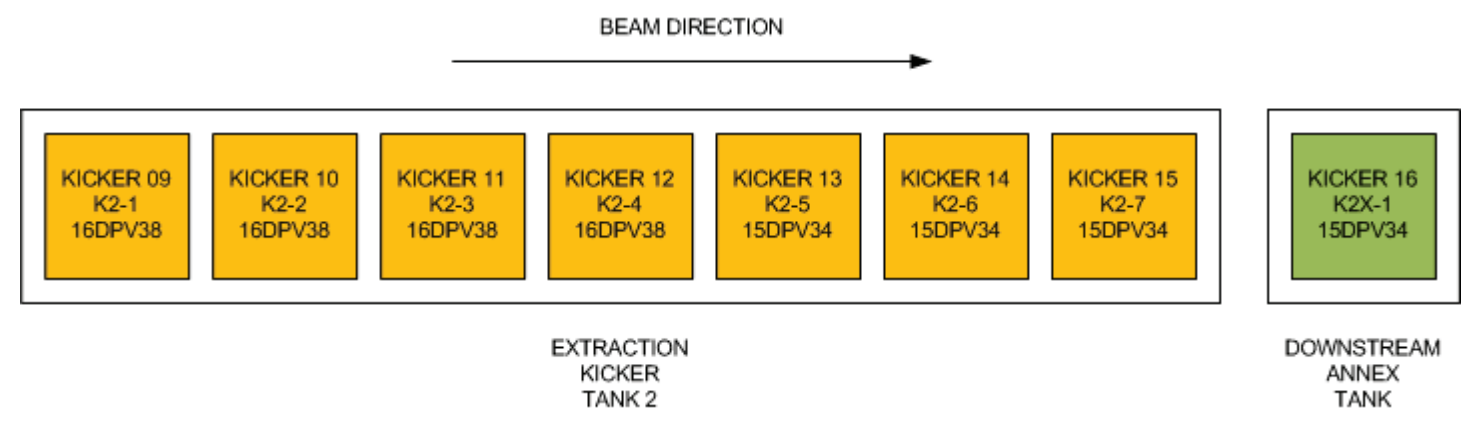

Fig. 3.46. Schematic of extraction kicker tank K2 with annex and renumbered magnets 
The new extraction kicker magnets system summary is as follows:

- The upstream annex magnet will be a type 12DPV35.

- The downstream annex magnet will be a type 15DPV34.

- Both magnets will require titanium nitride coating.

- The eddy current strips on the existing magnets within both main tanks will be carried through to the new magnets.

- The eddy current strips will be grounded at the upstream and downstream ends of the respective K1 and $\mathrm{K} 2$ annex tanks.

Each of the two additional kicker magnets will require a new pulse forming network (PFN) with the requisite power supply. Also, each of the two new PFNs will require a new oil-cooling skid with heat exchanger connections to the existing tower water system. New sets of kicker magnet power cables will be pulled for the additional magnets. The required rack space in the Ring Service Building is already available, since it was reserved for this purpose during SNS construction.

Extraction Lambertson septum magnet. At beam energies higher than $1.0 \mathrm{GeV}$, the power setting of the extraction Lambertson septum magnet, shown in Fig. 3.47, will require differently designed pole tip shims, optimized for $1.3 \mathrm{GeV}$. This is needed to cancel the skew quadrupole component in the magnetic field.

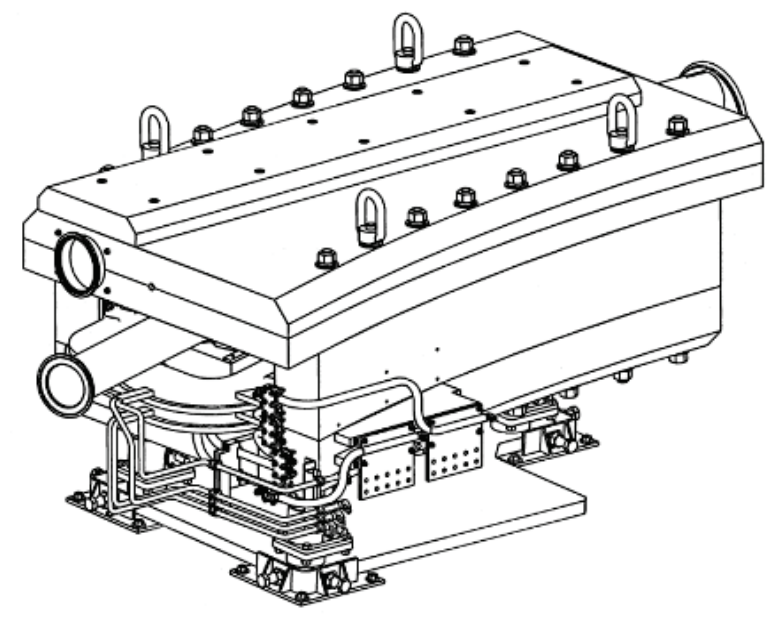

$\frac{\text { ISOMETRIC VIEW }}{\text { FOR REFERENCE ONLY }}$

Fig. 3.47. Extraction Lambertson septum magnet.

\subsubsection{Ring Injection Dump}

The ring injection dump is presently rated for $1.3 \mathrm{GeV}, 150 \mathrm{~kW}$. This power rating is based on thermodynamic simulations of the water-cooled dump, the concrete that surrounds the dump, and the earth that cools the concrete. During normal operating conditions, about $5 \%$ of the linac beam power is delivered to the injection dump, and so the dump power rating of $150 \mathrm{~kW}$ is well matched to $3 \mathrm{MW}$ linac 
operation. However, it can occasionally be desirable to operate the accelerator with lower stripping efficiency, for example, because of short-term stripper foil issues. In these cases, it could be desirable to deliver more than $150 \mathrm{~kW}$ to the dump. A series of thermocouple sensors mounted at various positions around the dump have been periodically read out since initial operation of the SNS. For the STS project, these data will be analyzed to determine if it is practical to increase the dump power rating.

\subsubsection{Ring Stripper Foils}

To maintain stripping efficiency at $1.3 \mathrm{GeV}$, the stripper foil thickness must be increased. Operational experience at the present injection energy of $0.94 \mathrm{GeV}$ has shown a preference for $0.36 \mathrm{mg} / \mathrm{cm}^{2}$ thick nanocrystalline diamond foils. As illustrated in Fig. 3.48, scaling this energy to $1.3 \mathrm{GeV}$ gives a thickness requirement of $0.39 \mathrm{mg} / \mathrm{cm}^{2}$. The thicker foil, combined with the higher beam currents, will increase the foil temperature. Simulations [37] predict the temperature will be about $300 \mathrm{~K}$ higher. To ensure the foil will continue to survive, a series of tests is planned. Some tests will be based on increasing the number of foil hits on the foils installed in the ring, to mimic the heat load expected for the STS beams. Other tests will use the foil test stand, which comprises a $30 \mathrm{keV}, 5 \mathrm{~mA}$ peak pulsed electron beam designed to produce STS-level heat loads, although with relatively smaller beam spots.

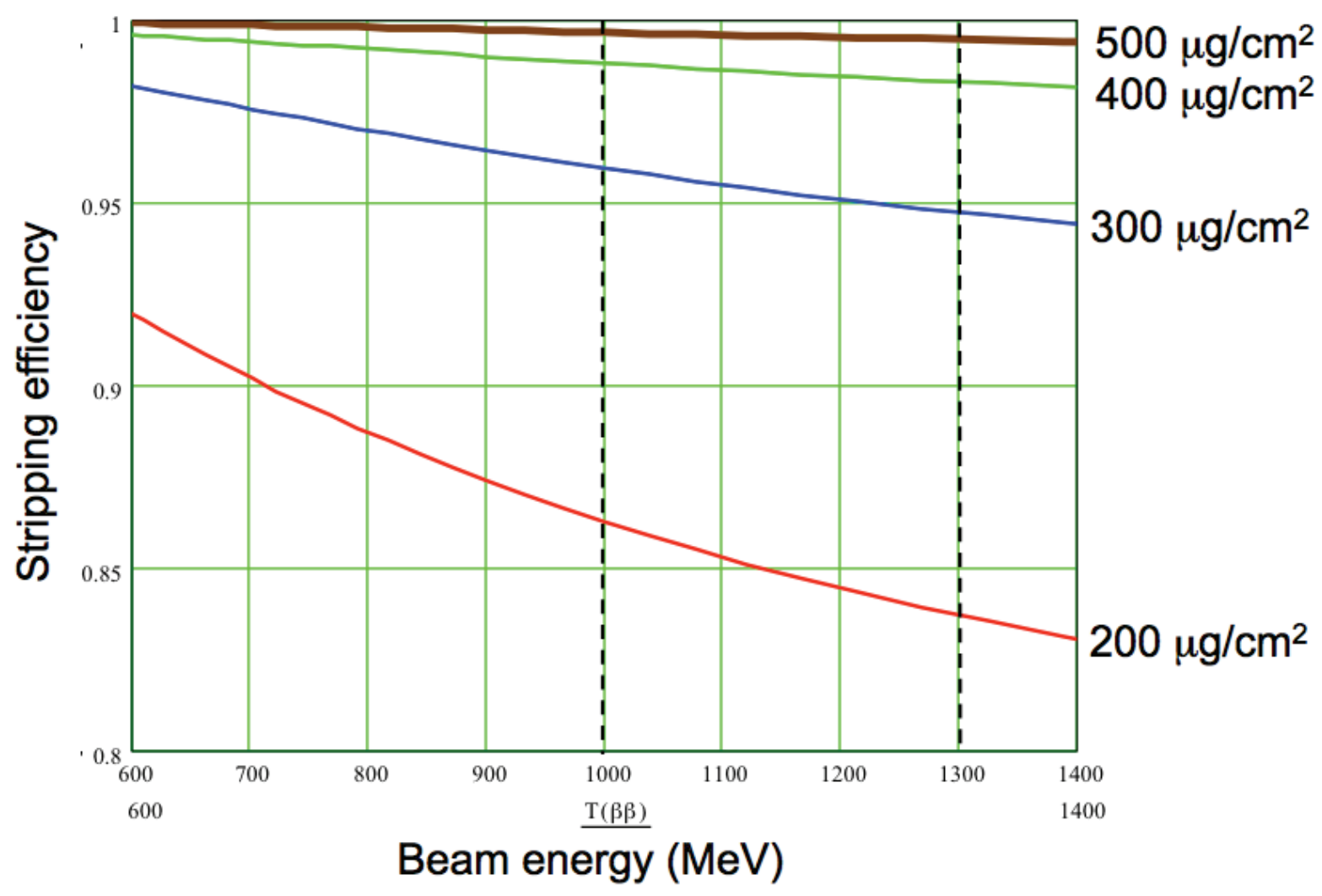

Fig. 3.48. Stripping efficiency vs foil thickness for a $1.3 \mathrm{GeV} \mathrm{H}^{-}$beam.

\subsubsection{Ring Radio Frequency}

The purpose of the ring RF systems is to keep the approximately $250 \mathrm{~ns}$ gap clear of any beam to allow time for the extraction kickers to reach their full fields. Any beam in the gap has the potential to be lost, causing radioactivation of beam line components. Three RF stations operate at the fundamental frequency of the ring, and one operates at the second harmonic. These systems were designed to support $1 \mathrm{GeV}$, 
1.4 MW operations. There is some excess capacity, but it may not be sufficient for the STS. A careful evaluation of the ring RF systems will be required to determine if any upgrades are necessary for the STS.

\subsubsection{Ring Utilities}

Ring injection magnet cooling water system. Several of the ring injection magnets will be replaced with new magnets. The existing lines will be disconnected and then reconnected to the new magnets once the magnets are installed. Flow and flow requirements for the new magnets will be the same as or similar to those for the original magnets. The existing ring magnet cooling systems (RN-01) have excess flow and heat capacity under currently operation. The cooling water system will need to be rebalanced once the changes are made.

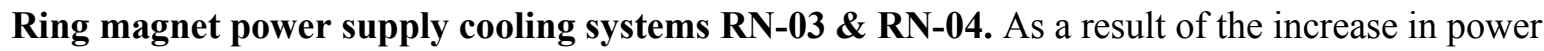
required of all the ring magnet power supplies, additional heat load and flow will be required to cool the existing or replacement power supplies. Based on the PUP power and thermal tests performed on the ring magnet system, it was determined that the existing Ring Service Building power supply cooling system (RN-03) is not adequate to cool the supplies for the PUP or STS power level requirements. Therefore, the existing system will be split into two separate cooling systems (RN-03 and new RN-04). The existing RN-03 system will be modified to cool only the west side of the Ring Service Building, and the new RN04 system will tie into the existing east side header and cool the remaining magnet and RF power supplies.

Ring extraction magnet power supply (PFN) cooling system. The two new extraction kicker magnets in the ring will require two new PFN power supplies, oil cooling skids, and connection to the tower water system for cooling the power supply oil in the Ring Service Building-PFN area. Two new oil cooling skids will be fabricated and installed beside each of the two new PFN oil tanks. New piping will be installed on each oil tank and connected to the pump skid for recirculating the oil from the tank through the skid's heat exchanger and filters. One-inch tower water connections will be extended from the existing tower water headers to each of the new heat exchangers. The system is designed to circulate 20 gpm of oil through each heat exchanger, and the tower water flow will remove up to $5 \mathrm{~kW}$ of heat at 10 gpm tower water flow.

Electrical utilities. Field tests of ring magnet operation at $1.3 \mathrm{GeV}$ levels have shown that the main ring dipole magnet power supply will require that cooling fans be added to the main ring dipole substations. All the other power supplies have sufficient capacity, and no additional electrical power will be required for the Ring Service Building.

\subsection{BEAM TRANSPORT LINES}

\subsubsection{High Energy Beam Transport Line}

No hardware modifications will be needed to the HEBT line that transports the beam from the linac to the accumulator ring, since the original design anticipated the $1.3 \mathrm{GeV}$ upgrade.

\subsubsection{Ring to Target Beam Transport Line}

The RTBT was designed for $1.3 \mathrm{GeV}$, so no changes are needed to accommodate the higher beam energy. The new R2T2 beam line will start about halfway along the RTBT, after kicking the beam horizontally into a septum magnet. The beam line is designed so that when all the R2T2 magnets are powered off, the RTBT will perform as it does now. New pulsed R2T2 magnets will be added in the available spaces between the RTBT magnets, and the positions of the RTBT magnets will not change. Thus when the 
R2T2 magnets are not pulsed on at $10 \mathrm{~Hz}$ to deflect the beam into the R2T2, the RTBT optics will remain unchanged. One RTBT quadrupole magnet (QV11) will be replaced by a quadrupole magnet with a bigger aperture (a $26 \mathrm{~cm}$ vs a $20 \mathrm{~cm}$ aperture).

\subsubsection{Ring to Second Target Beam Transport Line}

Beam to the second target will be deflected from the RTBT at $10 \mathrm{~Hz}$. Pulsed kicker magnets will first deflect the beam horizontally to beam left. The following quadrupole magnet will further deflect the beam to the left, and then two septum magnets will complete the initial separation of the RTBT and R2T2 beam lines. The remainder of the R2T2 beam line is standard achromatic bends and FODO (focus-driftdefocus-drift) lattices until just before the target, where four quadruple magnets will tailor the beam size to the target requirements.

\section{R2T2 Physics Design}

Two short kicker magnets, each about $1 \mathrm{~m}$ long, were chosen rather than one long magnet for the initial deflection of the beam destined for the second target. This allows easy fabrication of the ceramic vacuum chambers. The kickers will deflect the beam to beam-left, and this will cause a shift in the beam position large enough to require the following quadrupole magnet to be replaced with a larger-aperture version. The SNS already has a standard 26Q40 magnet, with 26-cm aperture, that will nicely satisfy this requirement. The next two magnets will be septum magnets. The first magnet will have a very thin septum, which limits the practical magnetic field strength; and the second magnet can then have a thicker septum and a higher magnetic field. These will be challenging magnets to design, and the choice to use two different septum magnets is still preliminary.

Once the beam exits the second septum magnet, it will be well separated from the RTBT beam line. Standard dipole magnets and quadrupole magnets will then transport the beam to the second target. The quadrupole magnets will be clones of existing 21Q40 SNS magnets. The dipole magnets will be a new design. After the first set of dipole magnets, the following long straight section will be used to lower the beam elevation to match the second target. Vertical dipole magnets at each end of the sloped section will take the beam from one horizontal plane to the next. The second set of dipole magnets will point the beam toward the target, and then four quadrupole magnets at the end of the beam line will tailor the beam distribution to the target requirements. A schematic of the beam line is shown in Fig. 3.49.

The quadrupole magnet spacing in the R2T2 beam line has been chosen to be close to that of the RTBT, since it is essentially the same beam in each beam line. In the downstream portion of the RTBT, the quadrupole magnet spacing will be $5.8 \mathrm{~m}$; and in the R2T2, it will vary from 5.8 to $6.3 \mathrm{~m}$. The same type of quadrupole magnet (21Q40) will be used in each beam line. The beam line lattice functions are shown in Figs. 3.50 and 3.51. 


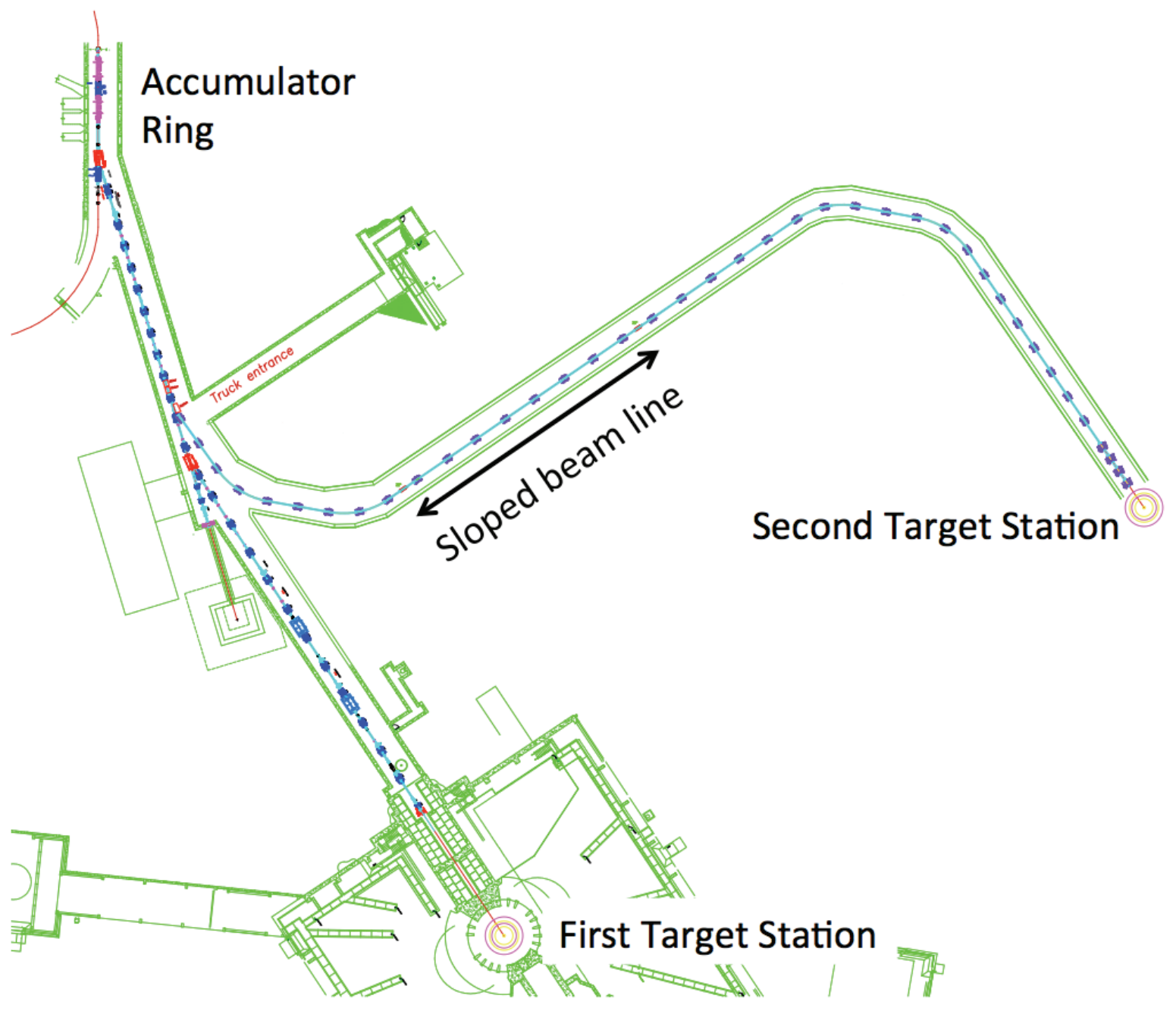

Fig. 3.49. Schematic RTBT and R2T2 beam lines. 


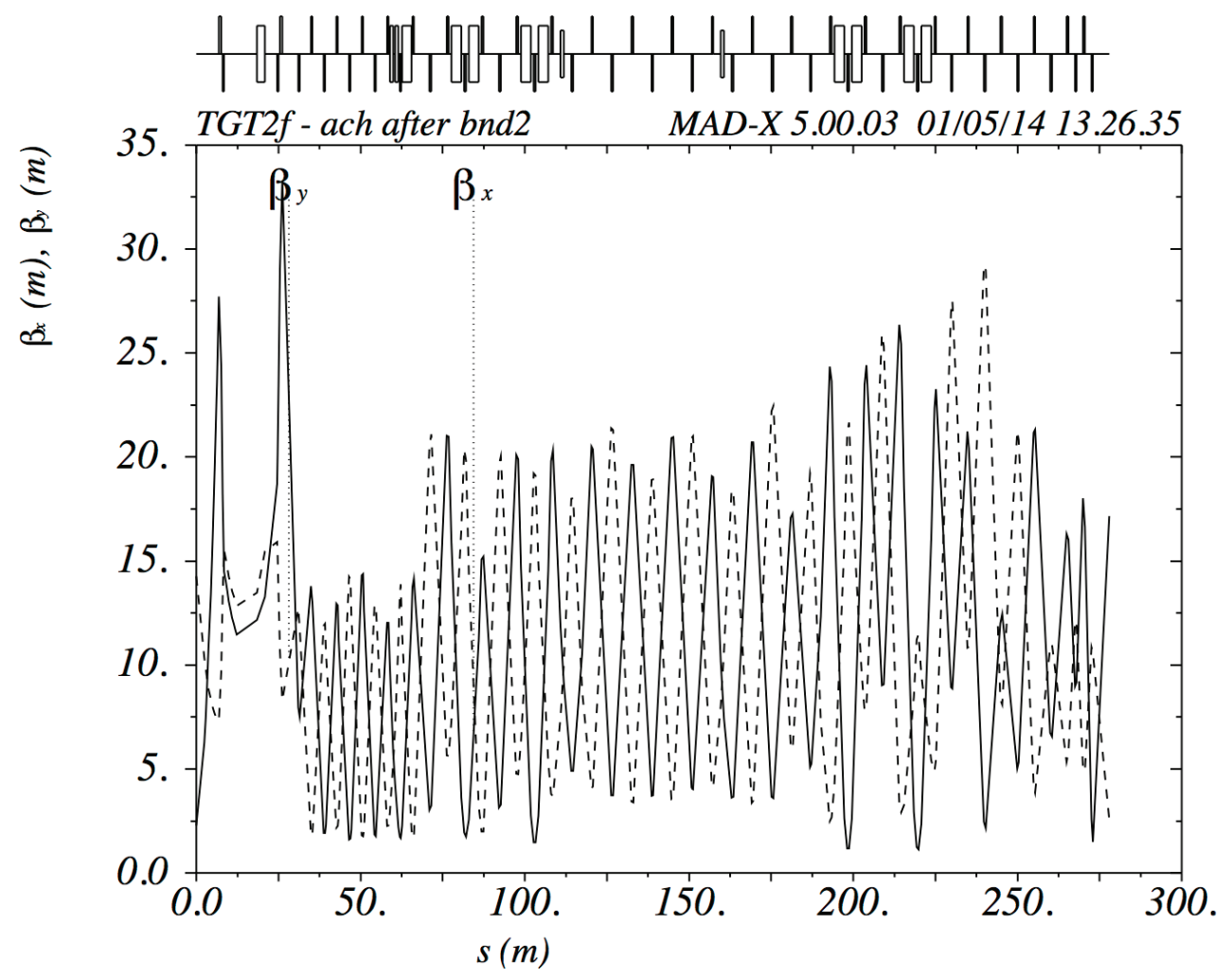

Fig. 3.50. The R2T2 lattice betatron functions.

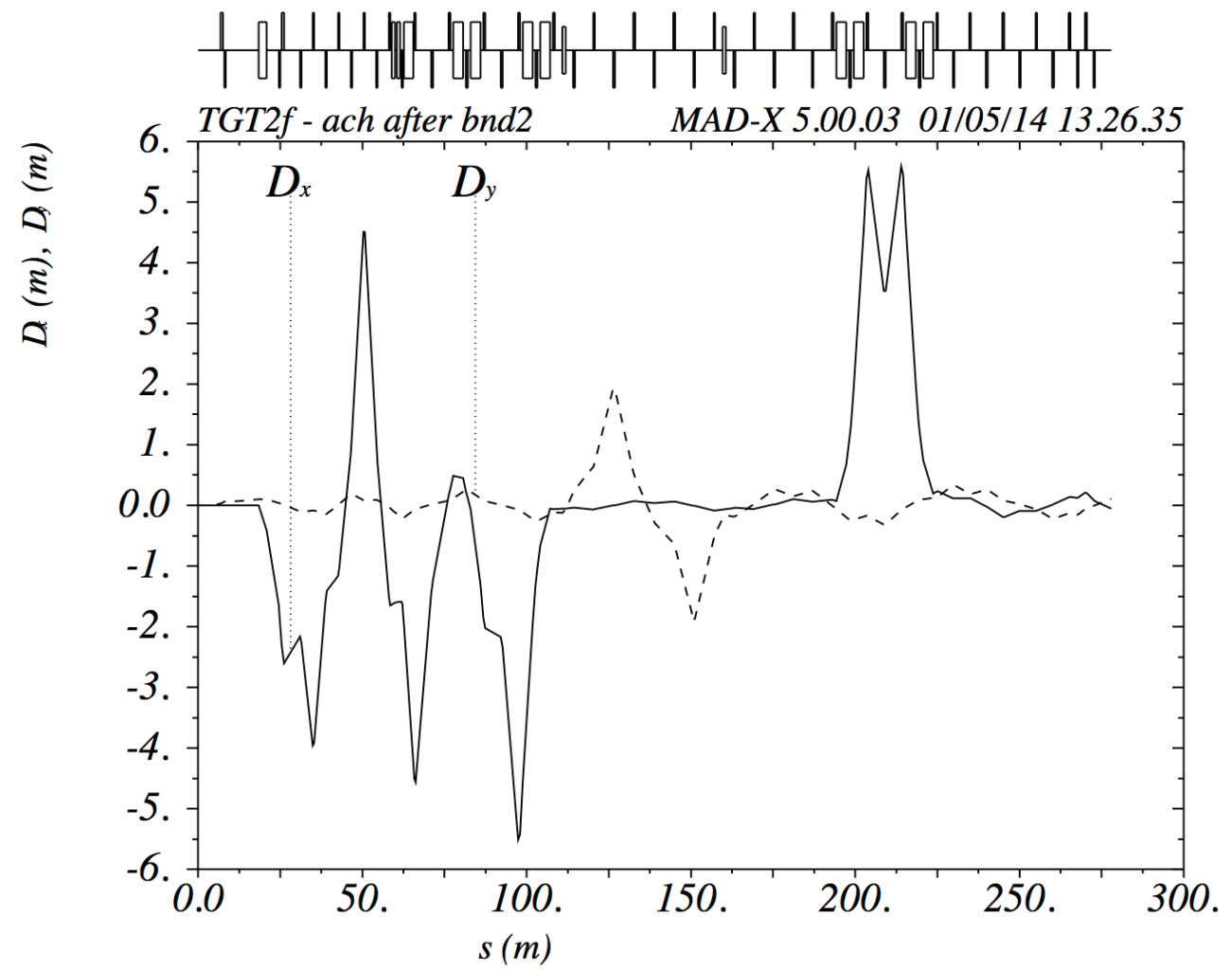

Fig. 3.51. The R2T2 lattice dispersion functions. 
Table 3.16 lists the equipment included in the R2T2 beam line. The items of equipment are, in general, copies of the counter components in the existing beam line, to minimize development and maintenance issues. One notable difference from the RTBT beam line is the absence of a collimation system.

Table 3.16. Components included in the new R2T2 beam line

\begin{tabular}{|c|c|c|c|c|}
\hline Category & Type & $\begin{array}{l}\text { Number } \\
\text { installed }\end{array}$ & $\begin{array}{l}\text { Water } \\
\text { cooled }\end{array}$ & Comments \\
\hline \multirow[t]{7}{*}{ Magnets } & 21Q40 quadrupole magnet & 40 & Yes & Total number of new quads is 40 \\
\hline & 26Q40 quadrupole magnet & 1 & Yes & Quad between kickers and septum \\
\hline & Septum magnet & 2 & Yes & Two consecutive \\
\hline & Kicker magnets & 2 & Yes & Similar to ring injection kickers, 1.4 \\
\hline & Horizontal dipole magnets & 8 & Yes & $3 \mathrm{~m}$ long, $22 \quad \square$ bend \\
\hline & Vertical dipole magnets & 2 & Yes & $1 \mathrm{~m}$ long, $6 \quad \square$ bend \\
\hline & Dipole corrector magnets & 14 & No & Similar to existing RTBT dipole correctors \\
\hline \multirow[t]{9}{*}{$\begin{array}{l}\text { Power } \\
\text { supplies }\end{array}$} & $\begin{array}{l}\text { Power supplies (PS) for } \\
\text { individual quadrupole } \\
\text { magnets }\end{array}$ & 20 & Yes & \\
\hline & PS for 2-quad strings & 2 & Yes & \\
\hline & PS for 3-quad strings & 2 & Yes & \\
\hline & PSs for 5-quad strings & 2 & Yes & \\
\hline & PS for horizontal dipoles & 2 & Yes & 1 PS per set of 4 dipoles \\
\hline & PS for vertical dipoles & 1 & Yes & 1 PS for both vertical dipoles \\
\hline & PS for septa & 2 & Yes & 1 PS per septum magnet \\
\hline & PS for kickers & 2 & Yes & $\begin{array}{l}1 \text { PS per kicker, similar to ring injection } \\
\text { kickers }\end{array}$ \\
\hline & $\begin{array}{l}\text { PS for dipole corrector } \\
\text { magnets }\end{array}$ & 14 & No & Dual polarity, $\pm 20 \mathrm{~A}$ \\
\hline \multirow[t]{7}{*}{ Diagnostics } & Wire scanners & 4 & & Similar to existing RTBT wire scanners \\
\hline & BPMs & 27 & & $\begin{array}{l}\text { Existing RTBT has about } 2 \text { BPMs for every } \\
3 \text { quadrupole magnets }\end{array}$ \\
\hline & $\mathrm{BCM}$ & 1 & & Similar to existing RTBT BCMs \\
\hline & BLMs & 40 & & One for every quad \\
\hline & Harp & 1 & & Downstream as far as possible \\
\hline & Halo monitor & 1 & & Mounted to proton beam window? \\
\hline & Fast valve & 1 & & \\
\hline \multirow[t]{8}{*}{ Vacuum } & Vacuum gate valves in-line & 7 & & \\
\hline & Ion pumps & 22 & & \\
\hline & Turbo pumps (700 lps) & 2 & & \\
\hline & $\mathrm{CCG}$ & 8 & & \\
\hline & TCG & 8 & & \\
\hline & IP controller & 11 & & \\
\hline & Fast valve & 1 & & \\
\hline & Beam pipe $(\sim 216 \mathrm{~m})$ & & & \\
\hline Misc & Gamma blocker & 1 & & \\
\hline
\end{tabular}

$\mathrm{BCM}=$ beam current monitor; $\mathrm{BLM}=$ beam loss monitor; $\mathrm{BPM}=$ beam position monitor. 


\subsubsection{R2T2 Utilities}

\section{R2T2 Tunnel Magnet Cooling System (R2-01)}

A new magnet cooling system will be installed in the R2T2-SB basement (provided by conventional facilities) with supply and return headers extended into the new R2T2 to cool the new magnets in the tunnel to the STS. Once the magnets are installed, the final connections to each magnet assembly will be made and the system will be balanced for optimum operation.

\section{R2T2-SB Magnet Power Supply Cooling System (R2-02)}

A new magnet power supply cooling system will be installed in the R2T2-service building basement (provided by conventional facilities) with supply and return headers extended up to the new R2T2-service building main level to cool the new magnet power supplies in the building. Once the magnet power supplies are installed, the final connections to each power supply assembly will be made and the system will be balanced for optimum operation.

\section{RTBT Tunnel Magnet Addition}

Several quadrupole magnets and extraction septum magnets will be added to the existing RTBT tunnel lattice to provide beam to the R2T2 and the STS. These magnets will be cooled by the existing RN-01 ring magnet cooling system. Once the magnets are installed, the final connections to each magnet assembly will be made and the system will be balanced for optimum operation. The existing ring magnet cooling system (RN-01) has sufficient excess flow and heat capacity under current operation. The system will need to be rebalanced once the changes are made.

\section{RTBT Power Supply Cooling System}

Several additional magnet power supplies will be added to and/or included as modifications to the building to support the new magnets in the RTBT tunnel. The power supplies will be cooled by the existing RS-01 RTBT magnet power supply cooling system. The existing RS-01 systems will be upgraded to allow increases in flow and heat transfer area to satisfy the $20 \%$ higher thermal loads from the additional power supplies. Pump impeller trim increase, motor horsepower increase, and additional electrical starters and wiring will be addressed. Heat exchangers will be rebuilt, and additional plates will be added for the needed heat transfer surface area. The temperature control valve will also be resized for the added demand. 


\subsection{REFERENCES}

1 S. Henderson et al., "Status of the SNS Beam Power Upgrade Project," pp. 345-347 in Proceedings of EPAC 2006, Edinburgh, Scotland (2006). http://accelconf.web.cern.ch/AccelConf/e06/PAPERS/MOPCH129.PDF.

2 B. Erickson and S. Cousineau, Summer 2014 intern study on simulations of variable chopper pattern widths.

3 Particle tracking code TraceWin, http://irfu.cea.fr/Sacm/logiciels/index3.php.

4 J. A. Holmes, S. Cousineau, V. V. Danilov, S. Henderson, A. Shishlo, Y. Sato, W. Chou, L. Michelotti, and F. Ostiguy, "ORBIT: Beam dynamics calculations for high-intensity rings," ICFA Beam Dynamics Newsletter 30, pp. 100-107, International Committee for Future Accelerators, April 2003.

5 J. A. Holmes and M. A. Plum, "Beam loss due to foil scattering in the SNS accumulator ring," in Proceedings of the ICFA High Brightness Workshop HB2012, Beijing, 2012.

6 K. Woody, J. A. Holmes, V. Danilov, and J. D. Galambos, "Longitudinal impedance simulations in ORBIT: Benchmarking and application to the SNS extraction kicker," pp. 2057-2059 in Proceedings of the 2001 Particle Accelerator Conference (PAC 2001), Chicago, June 18-22, 2001.

7 J. A. Holmes, S. Cousineau, V. Danilov, and L. Jain, "Comparison between measurements, simulations, and theoretical predictions of the extraction kicker transverse dipole instability in SNS," Phys. Rev. Special Topics-Accelerators and Beams 14, 074401 (2011).

8 M. A. Plum, J. Galambos, and S.-H. Kim, “Accelerator systems modifications for a Second Target Station at the Oak Ridge Spallation Neutron Source," in Proceedings of the International Particle Accelerator Conference (IPAC14), Dresden, 2014.

9 J. A. Holmes, V. Danilov, and L. Jain," Transverse stability studies of the SNS Ring," in Proceedings of the Particle Accelerator Conference (PAC05), Knoxville, Tennessee, 2005.

10 M. A. Furman and M. Pivi, "Probabilistic model for the simulation of secondary electron emission," Phys. Rev. Special Topics-Accelerators and Beams 5, 124404 (2002).

11 S. Cousineau, J. A. Holmes, M. A. Plum, and W. Lu, "Dynamics of uncaught foil-stripped electrons in the Oak Ridge Spallation Neutron Source accumulator ring," Phys. Rev. Special TopicsAccelerators and Beams 14, 064001 (2011).

12 C. Deibele, et al., "Status and implementation of a wideband feedback system for E-P instabilities in the SNS," p. 462 in Proceedings of the High Brightness (HB2008) conference, Nashville, 2008.

13 M. Blaskiewicz, M. A. Furman, M. Pivi, and R. J. Macek, "Electron cloud instabilities in the Proton Storage Ring and Spallation Neutron Source," Phys. Rev. Special Topics-Accelerators and Beams 6, 2003, 014203.

14 A. Shishlo, S. Cousineau, V. Danilov, S. Henderson, J. Holmes, and M. Plum, "Electron cloud selfconsistent simulations for the SNS ring," pp. 2832-2834 in Proceedings of the European Particle Accelerator Conference (EPAC06), Edinburgh, Scotland, 2006. 
15 M. Howell, et al., "The First ASME Code stamped cryomodule at SNS," pp. 2465-2467 in Proceedings of the 3rd International Particle accelerator Conference (IPAC2012), New Orleans, May 20-25, 2012.

16 S. Kim, et al., "The status of the Superconducting Linac and SRF activities at the SNS," pp. 83-88 in Proceeding of the 16th International Conference on RF Superconductivity (SRF2013), Paris, France, September 23-27, 2013.

17 S. Kim, "SNS Superconducting Linac operational experience and upgrade path," pp. 11-15 in Proceedings of 24th International LINAC conference (LINAC08), Victoria, Canada, September 29October 3, 2008.

18 S. Kim, "Simulation of quench dynamics in SRF cavities under pulsed operation," pp. 1365-1367 in Proceedings of the Particle Accelerator Conference 2003 (PAC2003), Portland, Oregon, May 12-16, 2003.

19 R. Sundelin, et al., "SNS HOM damping requirements via bunch tracking," pp. 1984-1986 in Proceedings of Particle Accelerator Conference 2001 (PAC2001), Chicago, June 18-22, 2001.

20 S. Kim, et al., "Higher-order-mode (HOM) power in elliptical superconducting cavities for intense pulsed proton accelerators," Nuclear Instruments and Methods in Physics Research A, 492(1-2), 1-10 (October 2002).

21 S. Kim, I. E. Campisi, D. Jeon, F. Casagrande, D. Stout, Y. Kang, M. Crofford, M. McCarthy, and M. Champion, "Study on fault scenarios of coaxial type HOM couplers in SRF cavities," pp. 770-772 in Proceedings of Linear Accelerator Conference 2006, Knoxville, Tennessee, August 21-25, 2006.

22 Y. Kang, et al., "Electromagnetic simulations and properties of the fundamental power couplers for the SNS superconducting cavities," pp.1122-1124 in Proceedings of Particle Accelerator Conference 2001 (PAC2001), Chicago, Illinois, June 18-22, 2001.

23 W. J. Schneider, et al., "Design of the Spallation Neutron Source (SNS) cryomodule," pp. 1160-1162 in Proceedings of Particle Accelerator Conference 2001 (PAC2001), Chicago, June 18-22, 2001.

24 C. Rode, "The SNS Superconducting Linac system, pp. 619-623 in Proceedings of Particle Accelerator Conference 2001 (PAC2001), Chicago, June 18-22, 2001.

25 D.A. Edwards, TESLA Test Facility Linac Design Report, DESY Print TESLA Rep. 95-01, 1995.

26 J. Preble, et al., "SNS cryomodule performance," pp. 457-461 in Proceedings of the Particle Accelerator Conference 2003 (PAC2003), Portland, Oregon, May 12-16, 2003.

27 H. Ma, et al., "Low-level RF control of Spallation Neutron Source: System and characterization," Phys. Rev. Special Topics-Accelerators and Beams 9, 032001 (March 2006).

28 J. Mammosser, "Spallation Neutron Source status and upgrade plans," pp. 62-65 in Proceedings of the 14th International Conference on RF Superconductivity (SRF2009), Berlin, Germany, September 20-25, 2009.

29 ASME Boiler and Pressure Vessel Code, Section VIII, Division 1-2010 UHA 51(a)(4)(a)(1), American Society of Mechanical Engineers. 
30 F. Casagrande, et al., "Status of the cryogenic systems commissioning at SNS," pp. 970-972 in Proceedings of Particle Accelerator Conference 2005, Knoxville, Tennessee, May 16-20, 2005.

31 F. Casagrande, et al., "SNS $2.1 \mathrm{~K}$ cold box turn-down studies," pp. 514-516 in Proceedings of 10th European Particle Accelerator Conference (EPAC2006), Edinburgh, Scotland, June 26-30, 2006.

32 M. Lynch, W. Reass, and P. Tallerico, "SNS Linac RF system overview," Proceedings of PAC99, New York, 1999.

33 M. Champion, "SNS RF system performance and operation," Proceedings of PAC07, Albuquerque, New Mexico, 2007.

34 T. Hardek, M. Crofford, Y. Kang, M. Middendorf, M. Piller, A. Vassioutchenko, "Status of the Oak Ridge Spallation Neutron Source (SNS) RF systems," Proceedings of PAC2011, New York, 2011.

35 Design Criteria for Linac RF, SNS-104010000-DC001, Oak Ridge National Laboratory, November $30,2000$.

36 SNS Power Upgrade Project Conceptual Design Report, PUP0-100-DC0001, R04, Oak Ridge National Laboratory, 2010.

37 Y. Takeda, Heat Calculation by ANSYS at SNS: Interim Report, Oak Ridge National Laboratory, April 11, 2013. 



\section{TARGET SYSTEMS}

\subsection{INTRODUCTION}

Target systems encompass the technical components and support facilities necessary to convert the proton beam into cold neutrons and direct the neutrons into the instrument guides. The two primary neutron production components will be the target and moderators; however, most of the target systems will support the operation and maintenance of these components, for example, a service bay to maintain and change out the target, shielding to protect personnel, water cooling utilities, vacuum and gas utilities, and the cryogenic hydrogen and helium systems for the moderators. The locations of the principal target systems are indicated in Fig. 4.1.

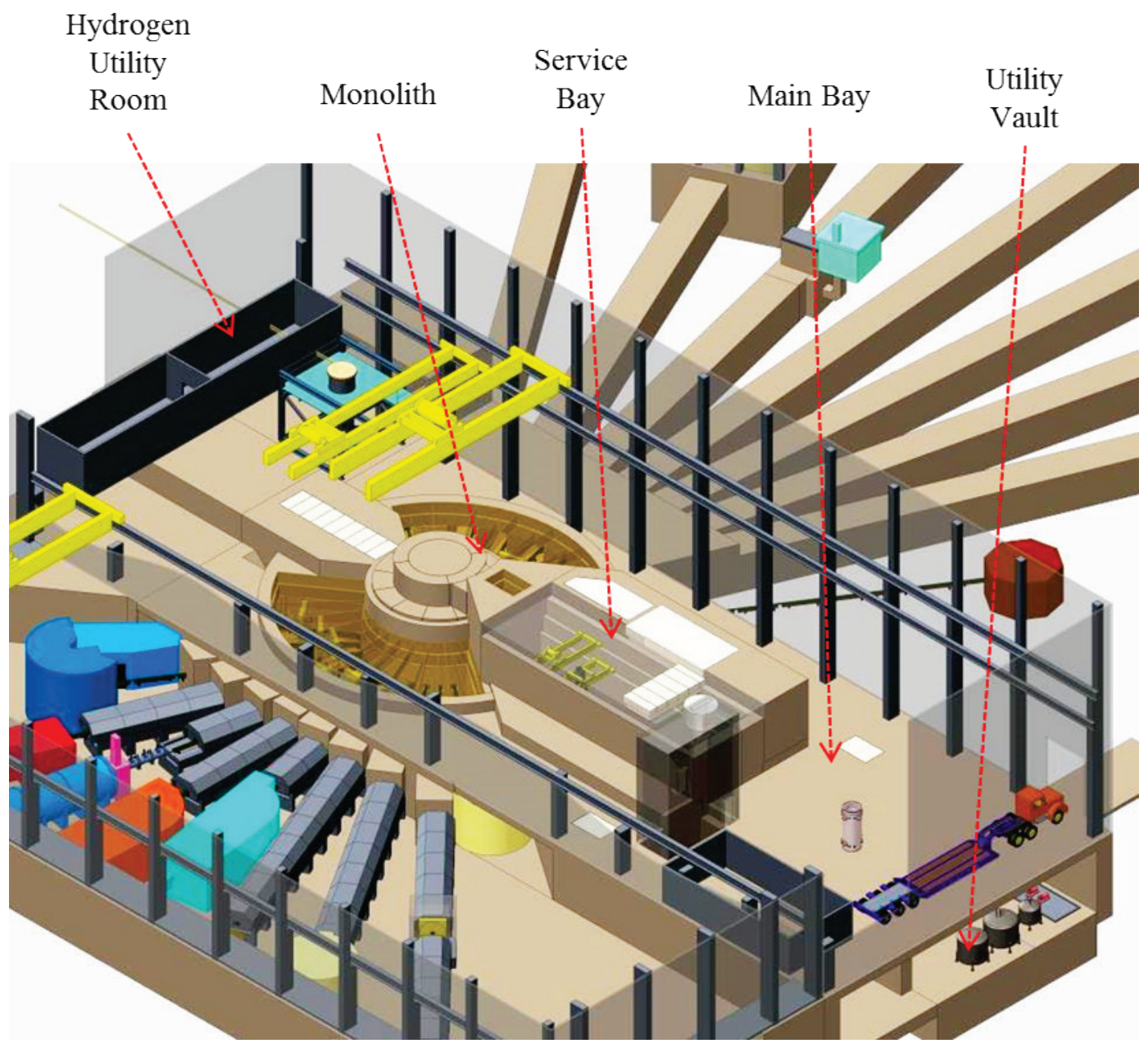

Fig. 4.1. Target systems within the STS.

\subsubsection{Neutron Production Components}

Neutronic studies show that a compact target and para-hydrogen moderators meet the STS goal of providing small, bright neutron beams. As proposed, the target will be driven with a relatively uniformprofile proton beam with an area of $30 \mathrm{~cm}^{2}$. This ensures that the moderators will be well coupled to the peak neutron generation region. Based on the operating experience at several facilities around the world and a preliminary performance analysis of the proposed system, a water-cooled tungsten target 
configuration has been selected as the best alternative. The compact nature of the STS system can be seen in the comparison of the proposed STS solid target with the existing SNS mercury target shown in Fig. 4.2.

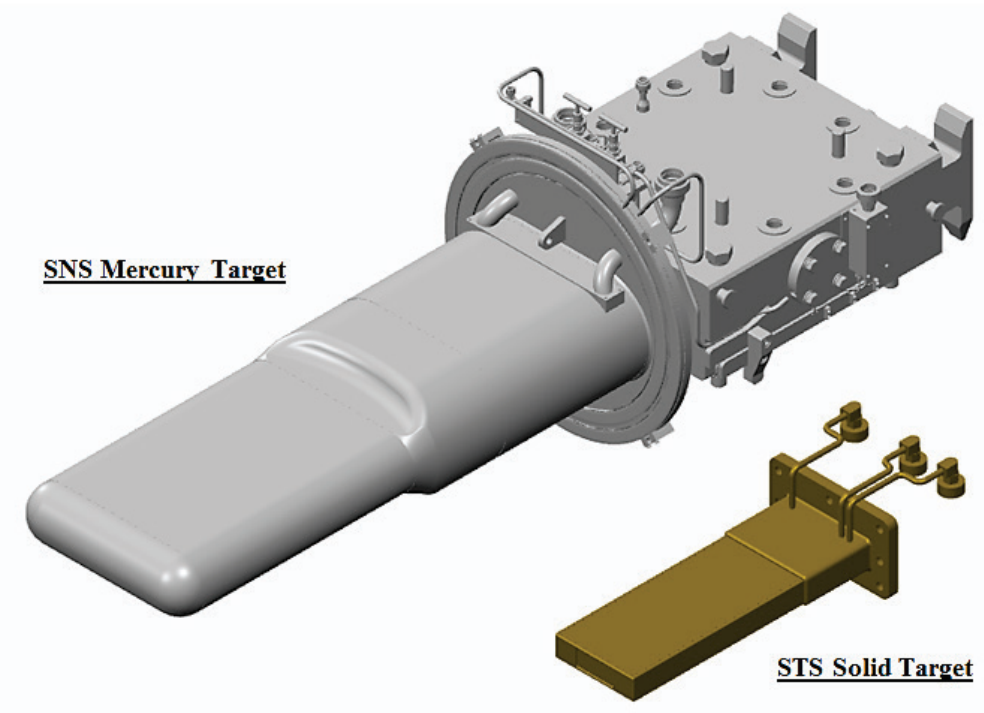

Fig. 4.2. SNS mercury target compared with the STS solid target.

Three moderators are required to service the proposed baseline STS instrument suite (Fig. 4.3). Two coupled moderators will be located near the peak neutron production zone, and a decoupled moderator will be positioned downstream of the upper coupled moderator. The moderators will be surrounded by a water-cooled beryllium reflector with a nominal radius of $35 \mathrm{~cm}$.

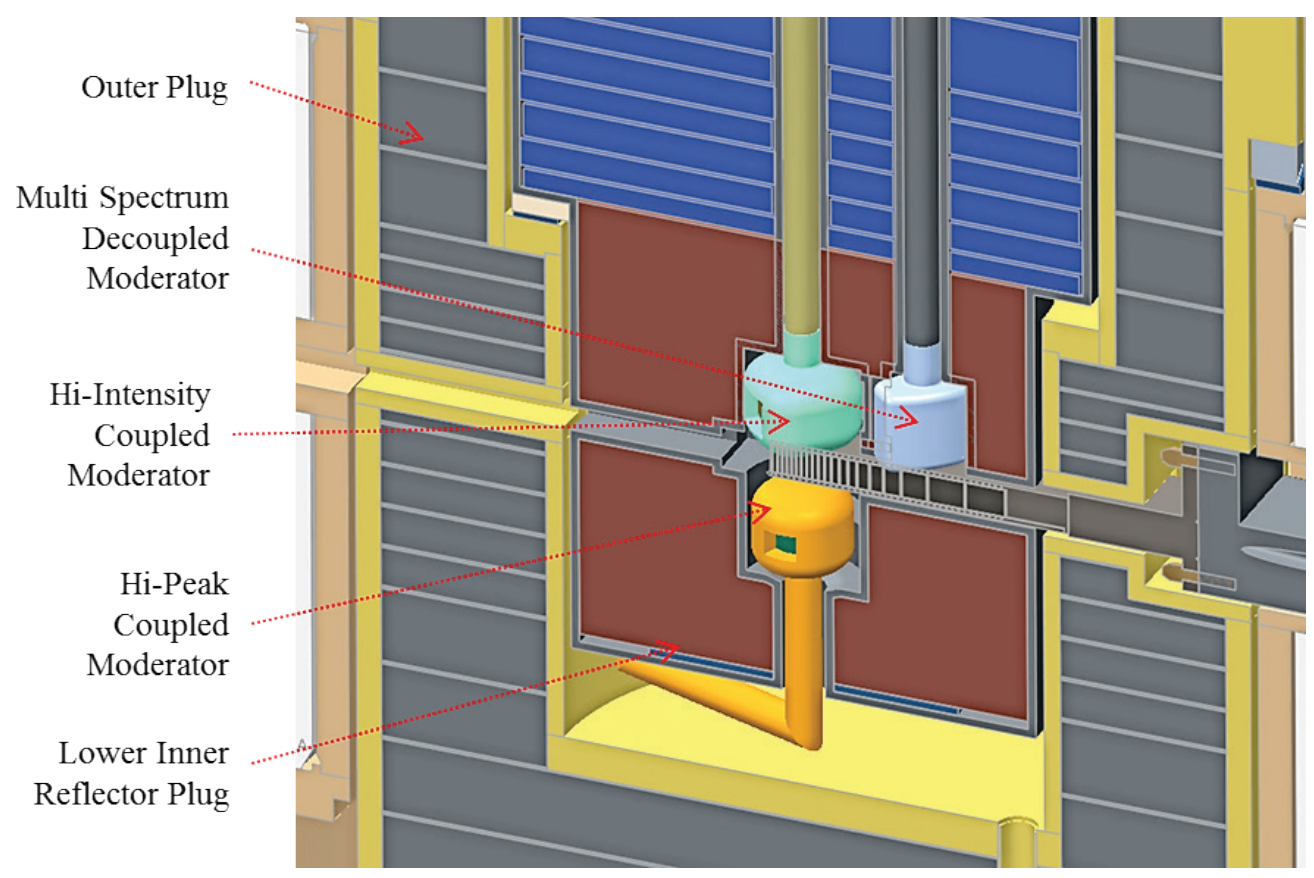

Fig. 4.3. Moderator array. 


\subsubsection{Monolith Configuration}

The STS monolith will be similar to that of many accelerator-based spallation sources. The proton beam will enter horizontally and strike a target with "wing" moderators that face neutron guides emanating on either side of the shielding. In the STS target building, the beam lines and monolith will be recessed approximately $2.8 \mathrm{~m}$ below the main operating floor. This will enable the neutron beam lines to be routed through shielded pits immediately outside the monolith. The pits will provide personnel access to the beam lines during shutdowns both for component maintenance and to monitor and adjust the alignment of guides. Placement of all the beam line shielding during construction also will eliminate foundation shifts resulting from the installation of individual shield stacks as new beam lines come online. Substantial savings also will be realized from the reduced requirements for fixed shielding in the target building and the simplified beam line shield assemblies.

In accordance with the STS physics requirements and the operating experience in FTS, operational shutters will be located outside the monolith. Eliminating shutters in the monolith substantially reduces the cost and simplifies the complexity of the target station. Without shutters inside the monolith, the neutron beam guides will be installed in long $(\sim 3.7 \mathrm{~m})$ inserts installed from outside the bulk shielding perimeter, as shown in Fig. 4.4. The inserts will be mounted in vessel nozzles which will extend radially to the exterior of the monolith shielding. As defined in the baseline instrument array, the neutron beams will range in size from $3 \times 3 \mathrm{~cm}$ to $7 \times 7 \mathrm{~cm}$. However, to provide the flexibility to install different instruments in the future, the vessel nozzles and beam guide inserts will be designed to accommodate larger beams.

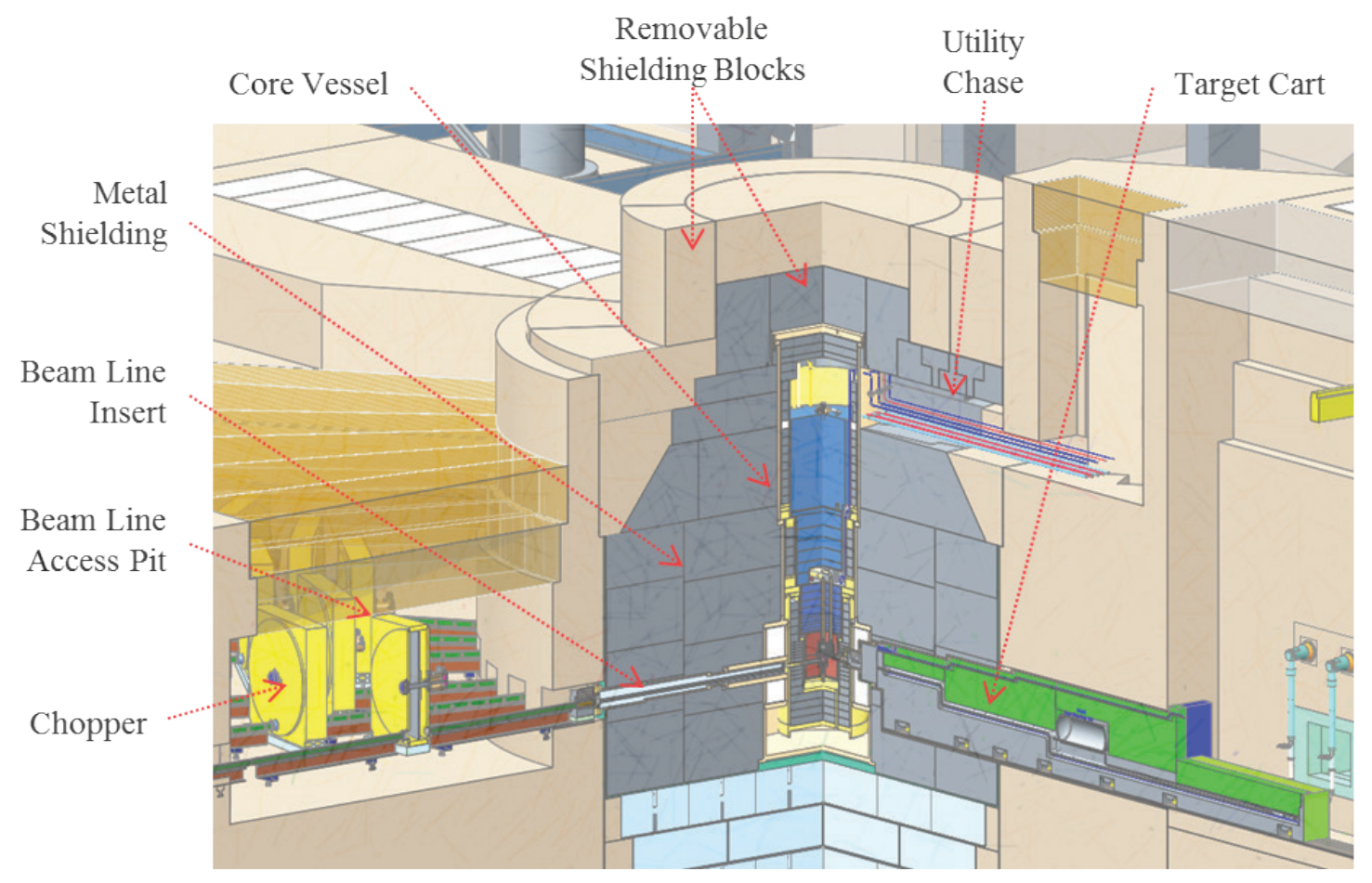

Fig. 4.4. Monolith configuration.

Following the basic design of similar, existing facilities, the target will be mounted on a horizontal cart that will position it inside the monolith during beam-on operation and in the service cell for periodic replacement. The cart will be mounted inside a tunnel installed with and mounted to the core vessel. The 
vessel environment will be separated from the tunnel and service bay by an all-metal inflatable seal. Similarly, the proton beam line environment will be isolated from the target vessel by a replaceable window assembly equipped with an inflatable seal. The proton beam window (PBW) will be mounted as closely as possible to the target to reduce beam scatter.

\subsubsection{Target Building Configuration}

Generally, the basic target station beam line layout is very similar to the FTS configuration (Fig. 4.5). Because the beam energy is the same, it is assumed that the proton beam line requirements will be virtually the same as for the FTS; therefore an identical $\pm 35^{\circ}$ is reserved to accommodate the beam magnets and shielding. Likewise, in the forward direction, the service bay requirements are also nearly identical to those of the FTS. Factors of importance are the ideal manipulator work distance, the thickness of the cell wall, the work area required to operate and maintain the manipulators, and the amount of shield required between the manipulator gallery and the neutron beam lines. The accumulation of these factors results in the $\pm 35^{\circ}$ angular space requirement for the service cell. This leaves two $110^{\circ}$ sectors on either side of the monolith for neutron beam lines.

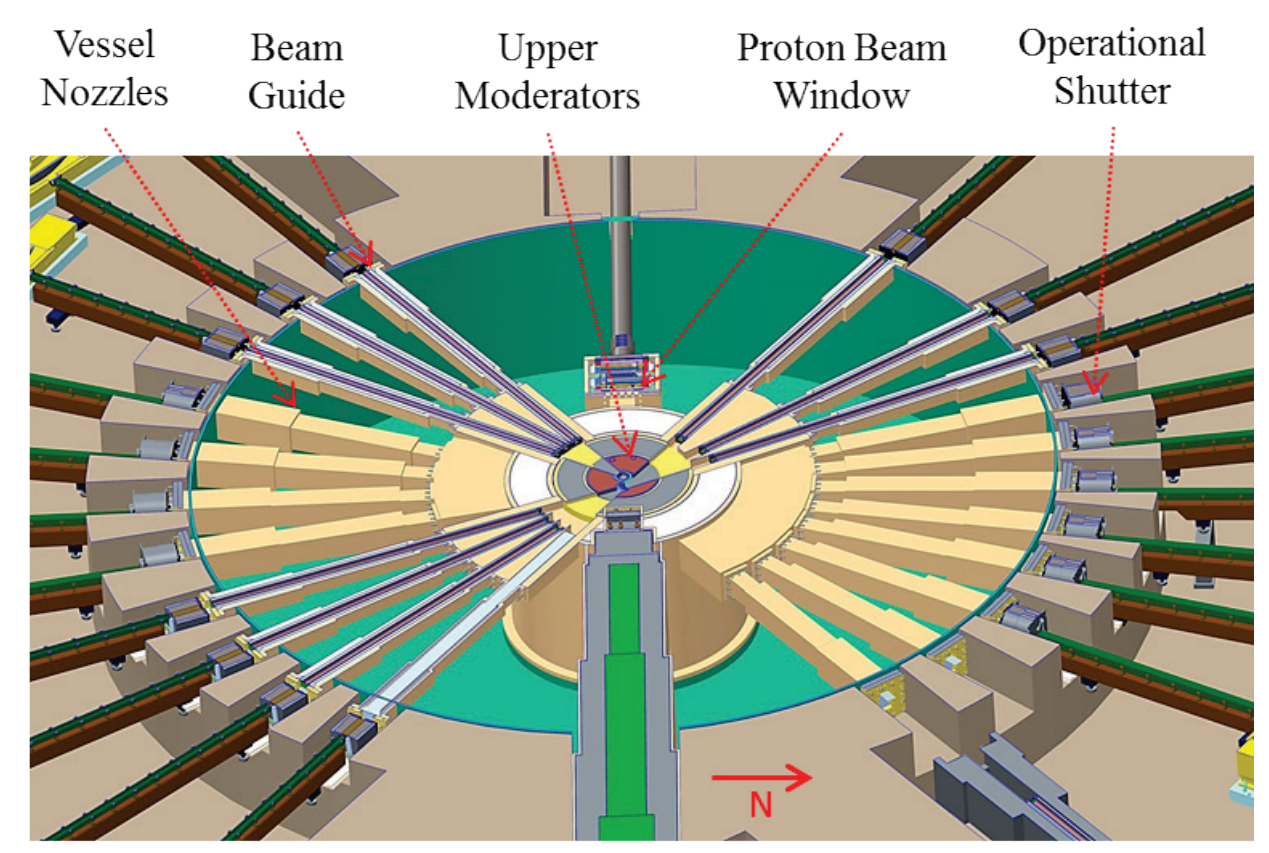

Fig. 4.5. Neutron beam line configuration.

The proposed layout of 11 beam lines on either side of the monolith was determined in conjunction with Conventional Facilities (CF) and the science teams. The "short" instruments (less than $40 \mathrm{~m}$ ) on the south side will be located in a dedicated attached building and equally spaced at $11^{\circ}$. The "long" instruments will be located in dedicated buildings north of the Target Building; the largest of these buildings will house five instruments with a length of about $90 \mathrm{~m}$ each. Because of the difference in lengths and to efficiently locate instruments in the buildings, the angular spacing between the long beam lines will range from 9 to $19^{\circ}$.

Water cooling loops will remove all $500 \mathrm{~kW}$ of proton beam power deposited in the target station. Four separate loops are required, including those for the heavy water required in the target and reflector and the light water used in pre-moderators. The loops are also required to satisfy certain safety requirements for redundancy. The relatively large size and activation of the water cooling system dictates a location in a 
basement vault. Further, the activation of the returning water requires substantial shielding (up to $1.5 \mathrm{~m}$ of high-density concrete) that limits the locations of pipe chases and the head tank to positions adjacent to the service cell.

Cryogenic hydrogen systems are designed with safety as the foremost consideration. This is most apparent in the location of the hydrogen utility room (HUR) at an outside building wall where a specially designed venting system and blowout panels ensure that hydrogen leaks and other failure events will not affect the entire target building. Similarly, the transfer line from the core vessel to the HUR will be located in a shielded chase designed to protect it from crane drops and other physical hazards so that a safe venting path will always be maintained. The supporting helium refrigeration system compressor and oil removal systems will be located in a separate building located near the target building, and the cold box will be located inside the target building near the HUR.

The operating shutters and choppers are not included in target systems; however; they are fully integrated into the target building design. Operating shutters will be located inside the concrete shield perimeter (Fig. 4.5). The shutters will serve to protect users at instruments during beam-on sample changes and to protect maintenance personnel in the beam line access pits during beam-off operation. The shutters are expected to be about $20 \mathrm{~cm}$ long and will include ample perimeter shielding.

Choppers will be relatively large, with a maximum possible diameter approaching $200 \mathrm{~cm}$, to match the $10 \mathrm{~Hz}$ STS pulse rate. Instruments will generally have one or two choppers, and most of these will be located within $10 \mathrm{~m}$ of the moderators. Thus the neutron beam pits have been sized accordingly (Fig. 4.4). Choppers may also be located outside the target building (Section 6, Conventional Facilities).

\subsubsection{Operations}

There are two important aspects to the operation of the target systems. First, the systems have two distinctly different operating modes depending on whether the proton beam is on or off. Second, the target systems are a single-point failure for the overall STS system.

\section{Beam-on Operation}

Only the utility and cryogenic systems will operate during beam-on operation. Both will be controlled by the STS integrated control system (Section 7). Because of the high levels of radiation, personnel will not be able to enter the utility vault, beam line access pits, or service cell while the proton beam is on. Consequently, high reliability, redundancy, and remote monitoring are key elements in the design of these two systems.

\section{Beam-off Operation}

Regular outages will be incorporated into the STS operating schedule to allow personnel to upgrade and maintain equipment. Two types of outages are common: weekly 8 hour shutdowns designated for modest fixes and 1-2 two month biannual maintenance periods designed to allow for significant equipment maintenance.

\section{Hour Shutdown Operations}

Certain equipment has been identified as requiring maintenance or change-out during an 8 hour shutdown. This includes the choppers and the utility system valves, pumps, and sensors. Most of this work will be performed hands-on with limited special tooling. Because choppers are generally repaired only off-line, their maintenance involves change-out. Experience in SNS has shown that exchanging 
choppers can be performed in a few hours and that removing and replacing shielding has been the limiting factor. Consequently, the beam line access pit shield covers will be configured to provide rapid access to keep the total change-out period to less than 8 hours.

Access to the utility vaults will be limited by the half-life of the radiation in the activated water. Experience at SNS with the beam power at $\sim 1 \mathrm{MW}$ has shown that personnel can enter the target utility vaults after 2-3 hours. Local radiation areas are roped off as required to control exposures (e.g., shielded ion exchange columns).

\section{Extended Shutdown Operations}

Maintenance or upgrade of large target system components will have to be performed during extended outages inside the service cell or using special handling equipment because of the high levels of activation.

Targets will be changed and maintained in the shielded cell. All target handling operations will be performed remotely because of the high level of residual radiation. When targets are not exposed in the cell and the target cart is positioned in the monolith, personnel will be able to enter the cell to perform many routine maintenance functions.

Other target system components such as the PBW, beam line inserts, operational shutters, and inner reflector plug (IRP) will require dedicated, shielded handling tools. To simplify operations and reduce costs, much of this work will be based on existing SNS tooling. However, new tooling will be required for the neutron beam line inserts and operating shutters.

\subsubsection{Cost Minimization}

Throughout the target systems design, every effort has been made to reduce both capital and operating costs. Maintenance operations savings include the use of existing SNS remote handling tools and procedures for the IRP and PBW (Section 4.9). The utility systems are based on the proven SNS system and the use of existing filter and ion exchange column designs and tooling (Section 4.8). The PBW design is a virtual copy of the SNS design (Section 4.5.3). Perhaps the most significant savings are realized by the incorporation of the building foundation into the neutron beam line shielding. This configuration reduces shielding cost, improves alignment, simplifies operations, and will ultimately decrease the cost of instruments.

\subsection{TARGET ASSEMBLIES}

The STS is based on the implementation of designs and techniques proved at operating spallation sources. Central to this system is a heavy water-cooled solid target mounted on a shielded horizontal cart. The target is a stainless steel-shrouded stack of tantalum-clad tungsten plates. The cart enables the target to be retracted into a service cell for efficient maintenance and change-out.

\subsubsection{Target Selection Process}

Neutronic studies performed by ORNL in 2013 show that the proposed STS compact solid target with close coupled para-hydrogen moderators performs well with a beam power on the order of $500 \mathrm{~kW}$. At this power level, solid targets with water cooling obtain an average neutron density equivalent to or higher than that provided by liquid mercury, with a relatively simple cooling system and maintenance design. A good deal of operating experience with solid tungsten targets has been obtained at the ISIS facility[1] and at the Los Alamos National Laboratory (LANL) LANSCE facility [2]. Both facilities have 
used tantalum cladding on the tungsten to minimize corrosion by water in the beam. A higher-power solid target upgrade for ISIS has been proposed, and the Chinese Spallation Neutron Source [3] currently being constructed will also use tantalum-clad tungsten.

It is proposed that the STS use a small beam with an area of $30 \mathrm{~cm}^{2}$ and a relatively uniform profile. This configuration will allow the moderators to be well coupled to the peak neutron generation region. A comparison of the beam-on-target parameters at STS with the SNS FTS, the Luan facility at LANL, the ISIS first target station, and the Chinese Spallation Neutron Source is shown in Table 4.1.

Table 4.1. Target beam parameter design basis at STS, SNS FTS, LANSCE, and ISIS FTS

\begin{tabular}{|c|c|c|c|c|c|c|}
\hline Parameter & STS & FTS (SNS) & $\begin{array}{l}\text { Luan } \\
\text { MKIII }\end{array}$ & ISIS TS1 & $\begin{array}{l}\text { ISIS TS1 } \\
\text { upgrade }^{a}\end{array}$ & $\operatorname{CSNS}^{b}$ \\
\hline Energy $(\mathrm{GeV})$ & 1.3 & 1.0 & 0.8 & 0.8 & 0.8 & 1.6 \\
\hline Frequency (Hz) & 10 & 60 & 20 & 50 & 50 & 25 \\
\hline Power $(\mathrm{kW})$ & 500 & 1400 & 160 & 160 & 440 & 100 \\
\hline $\begin{array}{l}\text { Average current } \\
(\mathrm{mA})\end{array}$ & .385 & 1.4 & 0.2 & 0.2 & 0.55 & 0.063 \\
\hline Protons/pulse $\left(10^{14}\right)$ & 2.4 & 1.46 & 0.624 & 0.25 & 0.69 & 0.16 \\
\hline $\begin{array}{l}\text { Semi-uniform beam } \\
\text { area }\left(\mathrm{cm}^{2}\right)\end{array}$ & 30 & NA & NA & NA & NA & NA \\
\hline $\begin{array}{l}\text { Gaussian horizontal } \\
\sigma_{x}(\mathrm{~mm})\end{array}$ & NA & NA & 15 & 16.3 & 16.3 & 50 \\
\hline $\begin{array}{l}\text { Gaussian vertical } \\
\sigma_{\mathrm{y}}(\mathrm{mm})\end{array}$ & NA & NA & 15 & 16.3 & 16.3 & 25 \\
\hline $\mathrm{J}$ peak $\left(\mu \mathrm{A} / \mathrm{cm}^{2}\right)$ & 12.8 & 17.5 & 14.1 & 12.0 & 38.9 & 0.8 \\
\hline $\begin{array}{l}\text { Peak } \\
\text { protons } / \mathrm{m}^{2} / \text { pulse } \\
\left(10^{16}\right)\end{array}$ & 8.0 & 1.82 & 4.4 & 1.5 & 4.1 & 0.2 \\
\hline $\begin{array}{l}\text { Peak heating } \\
\left(\mathrm{W} / \mathrm{m}^{3} / 10^{8}\right)\end{array}$ & 7.5 & 5.6 & & 4.55 & 12.5 & 0.6 \\
\hline
\end{tabular}

The high peak protons $/ \mathrm{m}^{2}$ per pulse for STS compared with the FTS, ISIS, or the Luan MKIII target is one of the factors resulting in improved moderator performance. It would be difficult to accommodate with a liquid metal target because of the much higher pulse pressures in the liquid and enhanced cavitation damage potential. Rotating solid target configurations had been previously considered at 1.5 to $3 \mathrm{MW}$ [4], but at $500 \mathrm{~kW}$, the more conventional fixed compact target design appears to be able to meet all requirements with a less complex arrangement. A more detailed discussion of target alternatives is included in Section 12.3.

\subsubsection{Target Configuration}

A horizontal section view of the target concept is shown in Fig. 4.6. The target cross-section is based on the $8.6 \mathrm{~cm}$ wide by $3.5 \mathrm{~cm}$ high proton beam used in the neutronics model. An additional $0.5 \mathrm{~cm}$ is added on all sides to account for beam spread and misalignment. The target will be composed of layers of rectangular plates of tungsten, each sealed inside a tantalum liner to prevent a damaging water-tungsten interaction. The plates will be stacked normal to the proton beam with small fixed gaps between them to 
allow cooling water to pass. The total tungsten zone length will be on the order of $300 \mathrm{~mm}$. Roughly $50 \%$ of the total beam energy will be absorbed by the target, and the remaining energy by the surrounding reflector and structures and a long-lived downstream water-cooled stainless steel beam stop assembly. Heavy water for cooling will flow horizontally across each plate face from three supply channels on one side to a single return channel on the other side. This arrangement will allow the moderators to be closely coupled above and below the target.

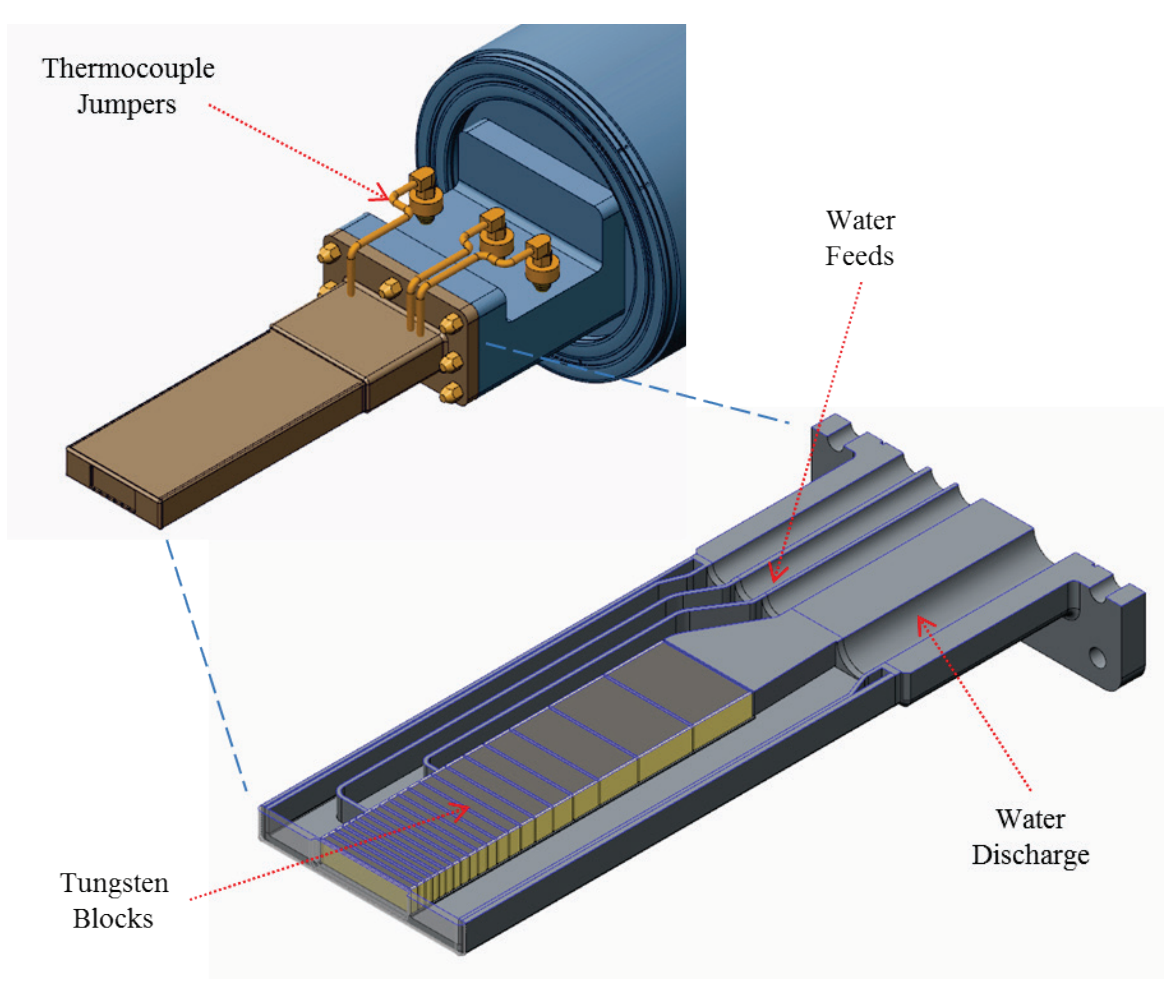

Fig. 4.6. Horizontal section view of proposed STS target.

A second independent cooling channel will be incorporated into the target shroud to remove residual heat in the event of a primary cooling loop failure and during target change-out operations. This loop will be entirely independent and will be served by an uninterruptible power source.

To develop a realistic neutronic model for the 2014 STS design studies, an accurate engineering model was made. Using existing designs, notably ISIS Target Station 1, as a starting point, a water flow velocity of $10 \mathrm{~m} / \mathrm{s}$ was assumed through $1.5 \mathrm{~mm}$ thick flow channels. The flow velocity is relatively high but close to the peak flow velocity in the Luan MKIII design $(9.3 \mathrm{~m} / \mathrm{s})$, close to what was assumed for the ISIS upgrade $(9 \mathrm{~m} / \mathrm{s})$, and less than the $14.5 \mathrm{~m} / \mathrm{s}$ water flow velocity between the HIFR fuel plate elements. This velocity gives high heat transfer coefficients with acceptable pressure drops below 1 bar. For the initial design, an estimate of the axial volumetric heat generation profile was used, based on scaling an analytic function generated for an earlier SNS rotating tungsten target design [4]. Plate thicknesses were computed based on limiting the peak surface temperature for the plates to $110^{\circ} \mathrm{C}$. This surface temperature was found to provide a reasonable compromise between the number and thickness of the plates and the operating pressure required to avoid boiling. The internal plate temperature limit was set at $250^{\circ} \mathrm{C}$ to limit thermal stress though the plate. The plate thicknesses, including clad and mid-plane locations used as input to the refined neutronic calculations, are given in Table 4.2. Note that the $1.5 \mathrm{~mm}$ cooling channel between each plate reduces the metal fraction as a function of distance from the front, as shown in column 4. 
Table 4.2. Target plate configuration

\begin{tabular}{cccc}
\hline $\begin{array}{c}\text { Plate } \\
\text { number }\end{array}$ & $\begin{array}{c}\text { Thickness } \\
(\mathbf{m})\end{array}$ & $\begin{array}{c}\text { Z midplane } \\
(\mathbf{m})\end{array}$ & $\begin{array}{c}\text { Metal } \\
\text { fraction }\end{array}$ \\
\hline 1 & 0.007 & 0.0035 & 1.000 \\
2 & 0.006 & 0.0115 & 0.870 \\
3 & 0.0057 & 0.01885 & 0.841 \\
4 & 0.0057 & 0.02605 & 0.827 \\
5 & 0.0057 & 0.03325 & 0.820 \\
6 & 0.0065 & 0.04085 & 0.816 \\
7 & 0.007 & 0.0491 & 0.817 \\
8 & 0.007 & 0.0576 & 0.818 \\
9 & 0.008 & 0.0666 & 0.820 \\
10 & 0.0083 & 0.07625 & 0.823 \\
11 & 0.009 & 0.0864 & 0.826 \\
12 & 0.011 & 0.0979 & 0.831 \\
13 & 0.0125 & 0.11115 & 0.838 \\
14 & 0.014 & 0.1259 & 0.845 \\
15 & 0.018 & 0.1434 & 0.854 \\
16 & 0.02 & 0.1639 & 0.863 \\
17 & 0.026 & 0.1884 & 0.873 \\
18 & 0.04 & 0.2229 & 0.886 \\
19 & 0.057 & 0.2729 & 0.901 \\
\hline
\end{tabular}

A typical plate with tantalum clad to protect the tungsten from corrosion is shown in Fig. 4.7. A single plate failure could result in the need to change an entire target. Potential failures include cracks in the tantalum clad that would allow corroded tungsten to contaminate the cooling water, and clad delamination, which would result in cooling channel blockage and excessive plate heating. Achieving the high reliability necessary for a target requires very high levels of manufacturing control; consequently, a significant development effort is proposed to ensure an appropriate supply of plates for STS targets (see Section 4.2.6). 


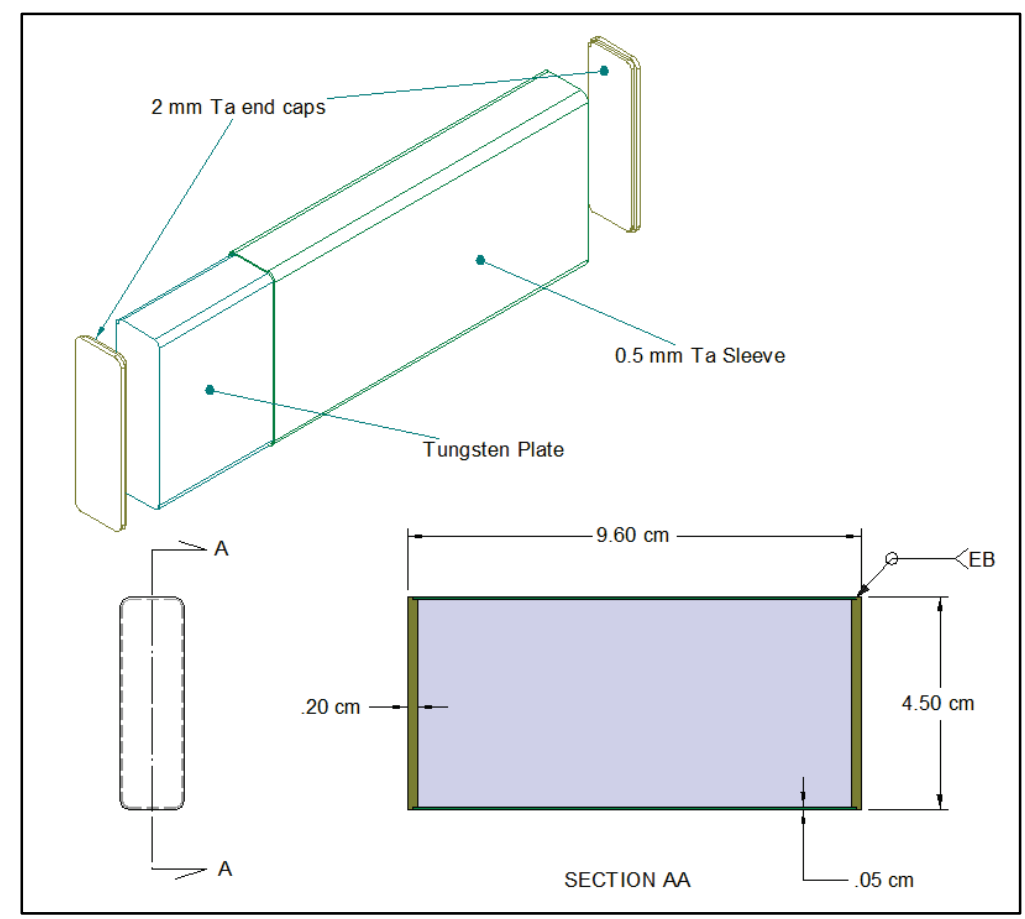

Fig. 4.7. Typical tantalum clad tungsten plate.

\subsubsection{Nuclear Heating Design Requirements}

MCNPX neutronic calculations of performance and heating were performed for the proposed design geometry [I. Remec, Oak Ridge National Laboratory, private communication with T. McManamy, consultant, ORNL, February 12, 2014). The beam was given as $1.3 \mathrm{GeV}$ and $500 \mathrm{~kW}$. Heat loads for the tantalum and tungsten were found for operation and the decay heat after shutdown [I. Remec, Oak Ridge National Laboratory, private communication with T. McManamy, consultant, ORNL, March 6, 2014). The decay heat was based on 6 month operation, similar to present SNS operation. The peak damage in the steel window was estimated at $7.5 \mathrm{dpa}$ for 2,500 hours of beam exposure (6 months). Thermal hydraulic calculations were then done using these heating results. Equivalent volumetric heating for a plate with both tantalum and tungsten was used. Figure 4.8 shows a comparison of the heating rates in the plates from the MCNPX calculation with the heating rates used to develop the initial plate thickness distribution (RT scale). The agreement was fairly good. An analytic function of the heat generation rate is shown in the figure. 


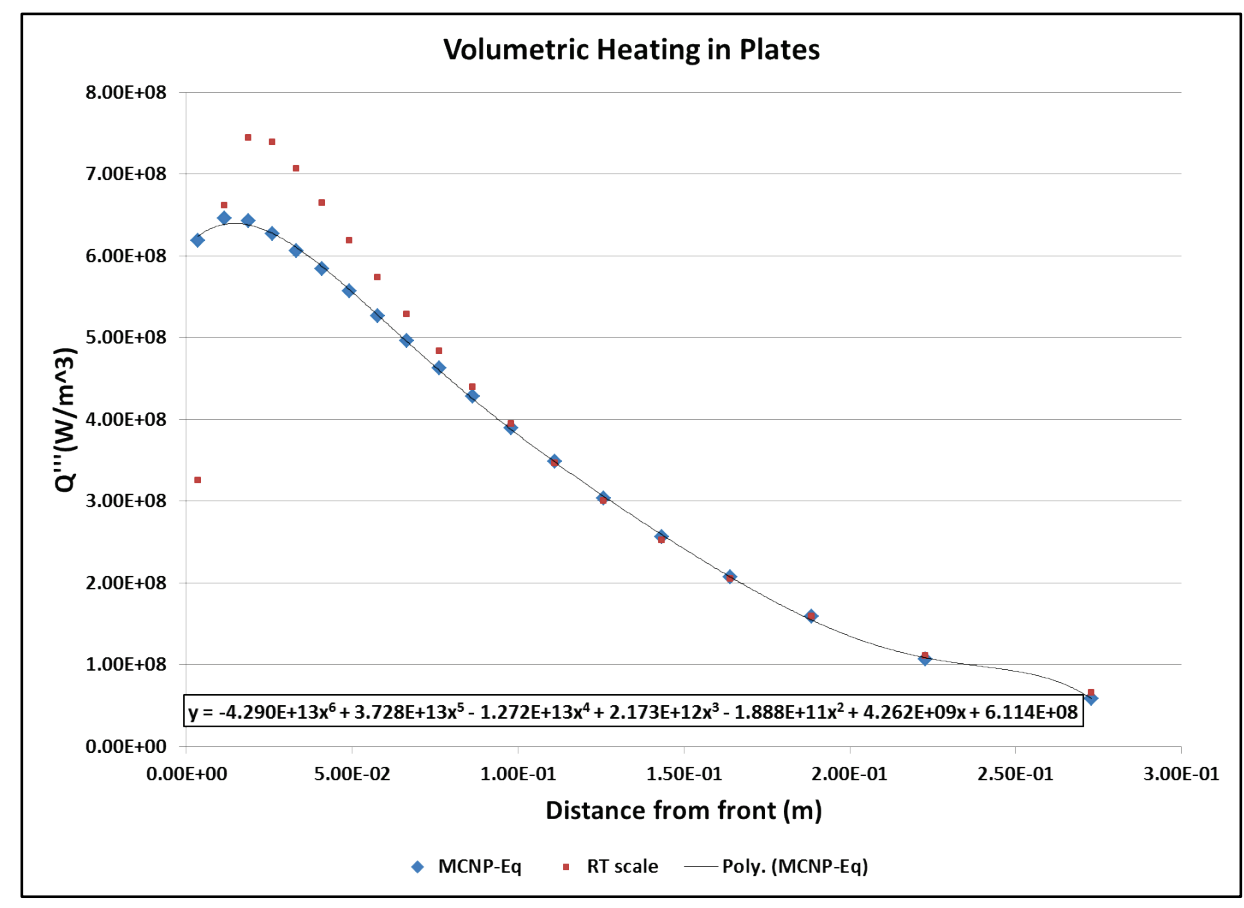

Fig. 4.8. Volumetric plate heating versus distance from the front plate.

The total decay heat for the tungsten and tantalum plates at beam shutdown is $3.1 \mathrm{~kW}$ [I. Remec, Oak Ridge National Laboratory, private communication with T. McManamy, consultant, ORNL]. This is shown as a function of time from shutdown in Fig. 4.9 for the total and for the tungsten and tantalum components. As can be seen, after a couple of hours, tantalum is the principal contributor. Of particular interest is the total heating value of approximately $1,500 \mathrm{~W}$ after a week of cool-down. This will be the amount of heat that must be managed during a typical target change-out.

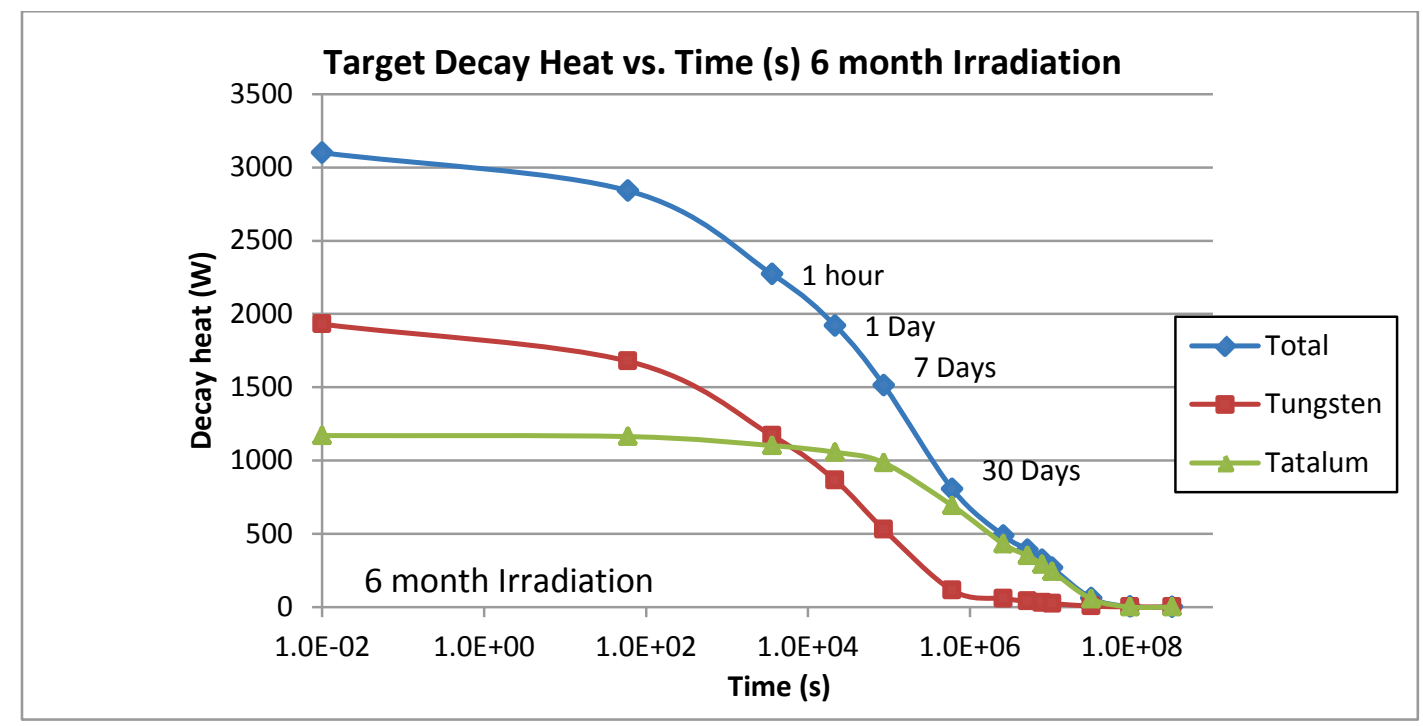

Fig. 4.9. Target decay heat versus time from shutdown. 


\subsubsection{Target Design Analysis}

The primary target cooling scheme assumes parallel flow for all plates. An option for series flow through three zones was initially considered to reduce the volumetric flow, but it was not selected because of the concern that an internal leak between zones could go undetected and cause flow distribution problems that would result in overheating and premature failure. Consequently, three parallel supply zones, each monitored independently, are proposed to allow better flow control. A side section is shown in Fig. 4.10. The top and bottom 316L stainless steel shells include cooling holes for a secondary cooling system that can remove decay heat during shutdown without the primary system. The front $316 \mathrm{~L}$ window is approximately $2 \mathrm{~mm}$ thick.

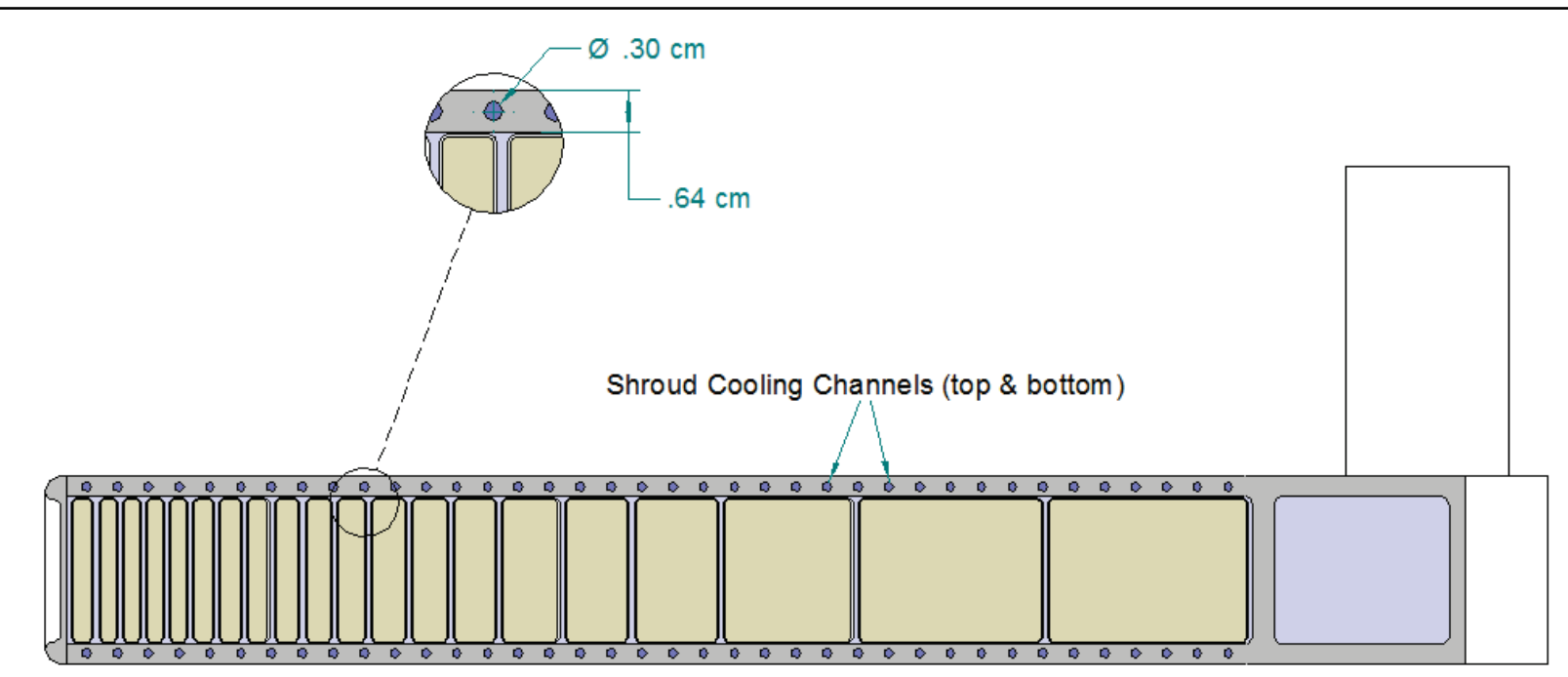

Fig. 4.10. Side section showing plate and shroud configuration.

A $10 \mathrm{~m} / \mathrm{s}$ flow velocity with heavy water with $1.5 \mathrm{~mm}$ flow channels gives a heat transfer coefficient on the plate surfaces of $4.5 \times 10^{4} \mathrm{~W} / \mathrm{m}^{2} \cdot \mathrm{K}$, using the Dittus-Boelter correlation. To estimate the peak plate surface temperatures, the effect of the pulse structure must be considered in addition to the film and bulk water temperature increases. An example of the magnitude of this effect is shown in Fig. 4.11, in which the average heating rate is assumed to be $7 \times 10^{8} \mathrm{~W} / \mathrm{m}^{3}$ in a $6 \mathrm{~mm}$ thick plate with a $10 \mathrm{~Hz}$ pulse structure. The temperature rise from a pulse is about $26^{\circ} \mathrm{C}$, and the quasi-steady state goes from $87^{\circ} \mathrm{C}$ just before a pulse to $112^{\circ} \mathrm{C}$ after the pulse with an approximately linear drop before the next pulse. This assumes a $50^{\circ} \mathrm{C}$ coolant temperature and a heat transfer coefficient of $4.5 \times 10^{4} \mathrm{~W} / \mathrm{m}^{2} \cdot \mathrm{K}$. A reasonable estimate of the peak temperature can be made by adding the steady state surface temperature and half the adiabatic rise per pulse, as shown.

The peak surface and mid-plane temperatures for the plates were calculated using the MCNPX heating rates with the $10 \mathrm{~m} / \mathrm{s}$ parallel flow and $43^{\circ} \mathrm{C}$ inlet temperature, including the film temperature drop, bulk temperature rise in a channel, and half the temperature rise per pulse. As shown in Fig. 4.12, the surface temperatures generally ranged from about 98 to $108^{\circ} \mathrm{C}$, except for the thick plate near the rear of the target where the thickness was limited to keep the mid-plane temperature below $250^{\circ} \mathrm{C}$. A water pressure of 3.5 bar would have a saturation temperature of $140^{\circ} \mathrm{C}$ and give a margin of over $30^{\circ} \mathrm{C}$ to boiling. 


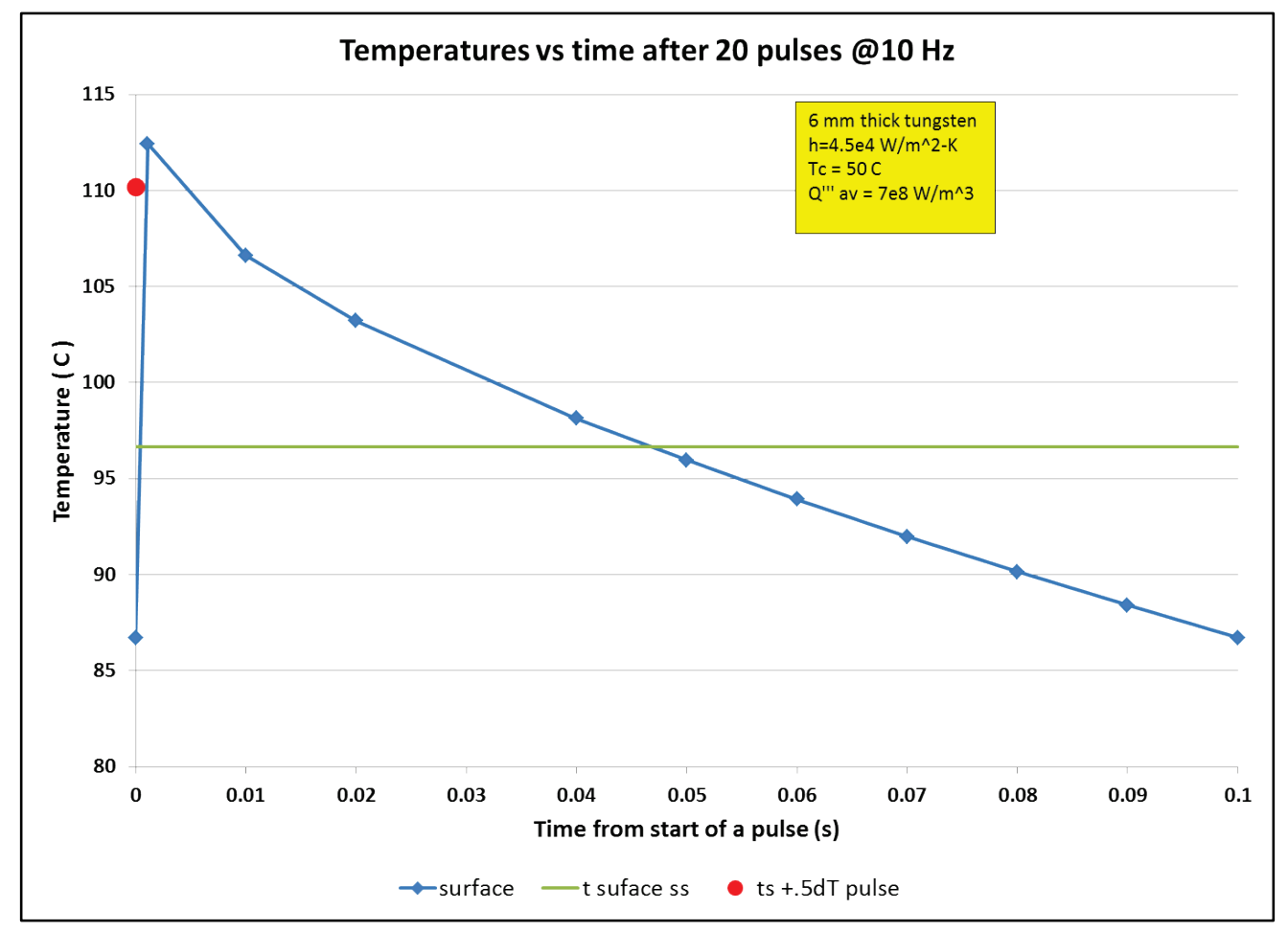

Fig. 4.11. Typical plate transient response.

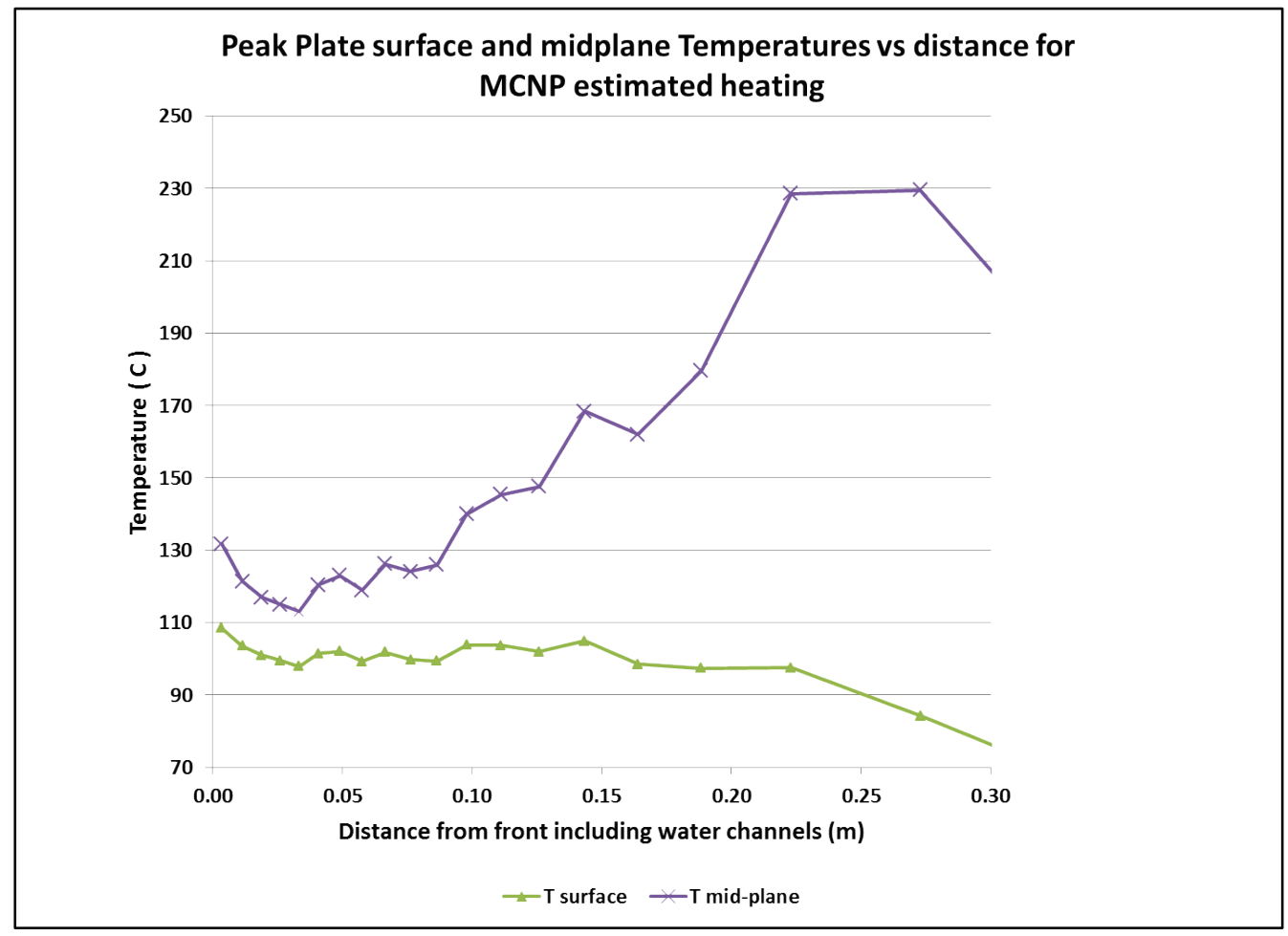

Fig. 4.12. Plate surface and mid-plane temperature for $500 \mathrm{~kW}$ operation. 
During detailed design, the target design will be better matched to the actual beam profile. A preliminary look using the MCNP heating rates with thicknesses selected to give surface temperatures of $110^{\circ} \mathrm{C}$ showed the number of plates could increase as a result of beam peaking. A summary of thermal hydraulic parameters is given in Table 4.3.

Table 4.3. Thermal hydraulic parameters

\begin{tabular}{lc}
\hline Flow velocity $(\mathrm{m} / \mathrm{s})$ & 10 \\
Inlet temperature $\left({ }^{\circ} \mathrm{C}\right)$ & 43 \\
Flow channel height $(\mathrm{mm})$ & 45 \\
Flow channel width $(\mathrm{mm})$ & 1.5 \\
Flow channel length $(\mathrm{mm})$ & 100 \\
Reynolds number & $4.3 \times 10^{4}$ \\
Flow per channel ( liter $/ \mathrm{s})$ & 0.675 \\
Total flow for 20 channels $(\mathrm{gpm})$ & 215 \\
Heat transfer coefficient $\left(\mathrm{W} / \mathrm{m}^{2} \cdot \mathrm{K}\right)$ & $4.5 \times 10^{4}$ \\
Typical surface heat flux $\left(\mathrm{W} / \mathrm{m}^{2}\right)$ & $2 \times 10^{6}$ \\
Typical channel bulk temperature rise $\left({ }^{\circ} \mathrm{C}\right)$ & 5 \\
Typical pressure drop per channel $($ bar $)$ & 0.3 \\
\hline
\end{tabular}

\subsubsection{Target Decay Heat Removal}

A simplified thermal model of the target was analyzed using the decay heat per plate. The transient response of the plate with the highest temperature is shown in Fig. 4.13. For this model, the target was assumed to view horizontal surfaces held at $100^{\circ} \mathrm{C}$ and located $8 \mathrm{~mm}$ above and below the target shell with water vapor in the gap. All surfaces were assumed to have an emissivity of 0.6 . This is an optimistic case assuming pre-moderator and reflector cooling to be intact. The transient response is shown for a rapid beam trip or after a 2 second delay following total loss of cooling. For both cases, the temperature peaks at about $950^{\circ} \mathrm{C}$ at 40 minutes.

For this case, the temperature profile along the beam axis when the temperature peaks is shown in Fig. 4.14. The temperatures in a given plate are quite uniform, with most changes in the $1.5 \mathrm{~mm}$ gas gaps. 


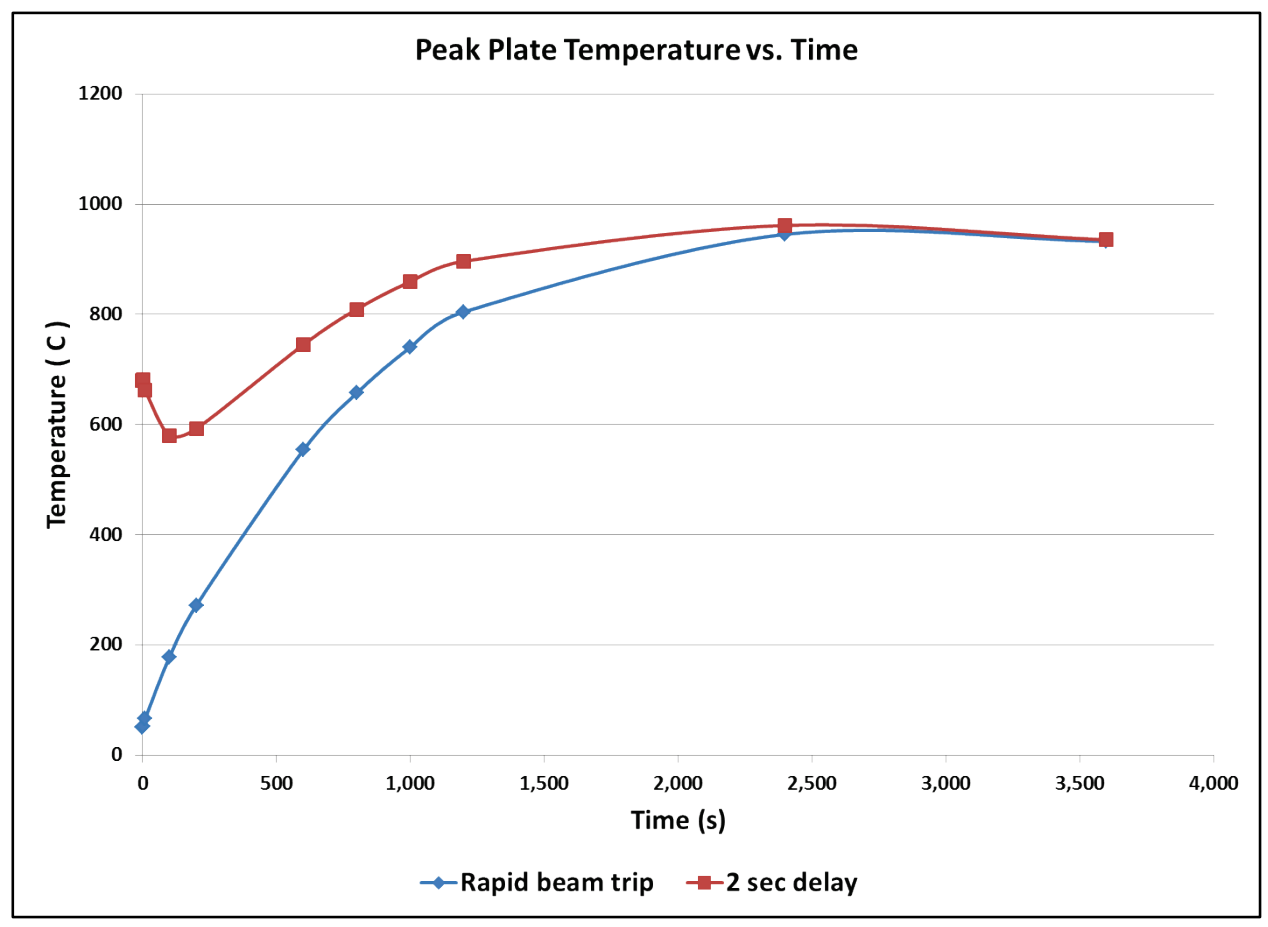

Fig. 4.13. Transient response of highest temperature plate after loss of cooling.

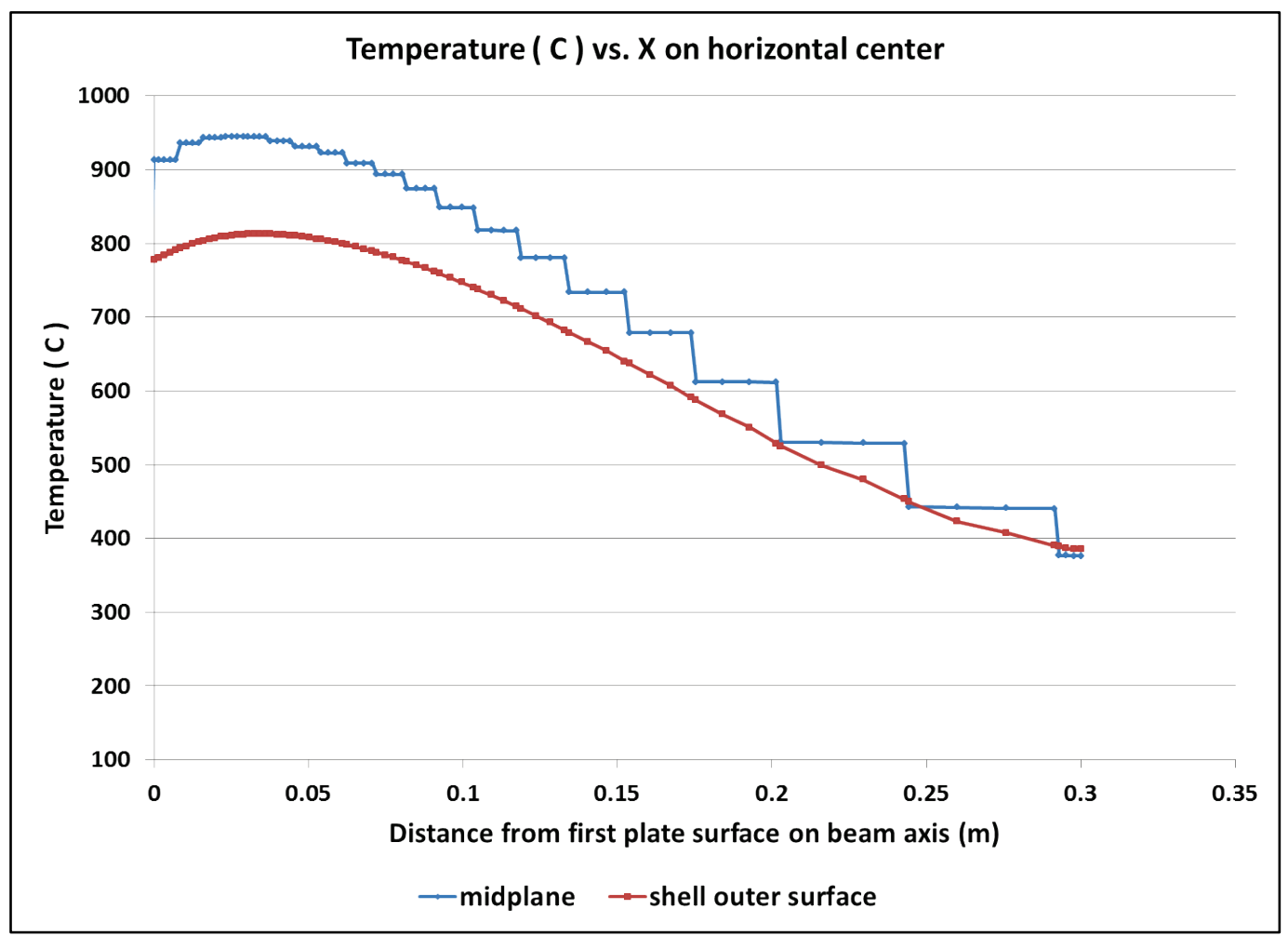

Fig. 4.14. Plate temperatures along beam axis at 40 minutes after loss of cooling. 


\subsubsection{Target Fabrication}

Fabrication of the tungsten/tantalum plates is a critical step in the fabrication of the target. Facilities using such targets have all developed fabrication processes based on hot isotopic pressing (HIP). The basic processes arrived at by all the laboratories is similar. From a mechanical standpoint, the proposed STS plates will have four components (Fig. 4.7). Sealing the tungsten inside the tantalum sleeve before the HIP process is fundamental. Consequently, this design attempts to provide ample material at the end cap joints to provide for reliable assembly welds. Note that the $0.5 \mathrm{~mm}$ sleeve will require a longitudinal butt weld, which is undesirable. However, it can be made before assembly and therefore can be carefully examined by several methods, including radiography.

It may be difficult to find vendors with the capability to fabricate the plates. The ISIS plate cladding is now done in-house in a facility with co-located equipment for e-beam welding, cleaning, HIPing, and nondestructive evaluation of the plates (Fig. 4.15).

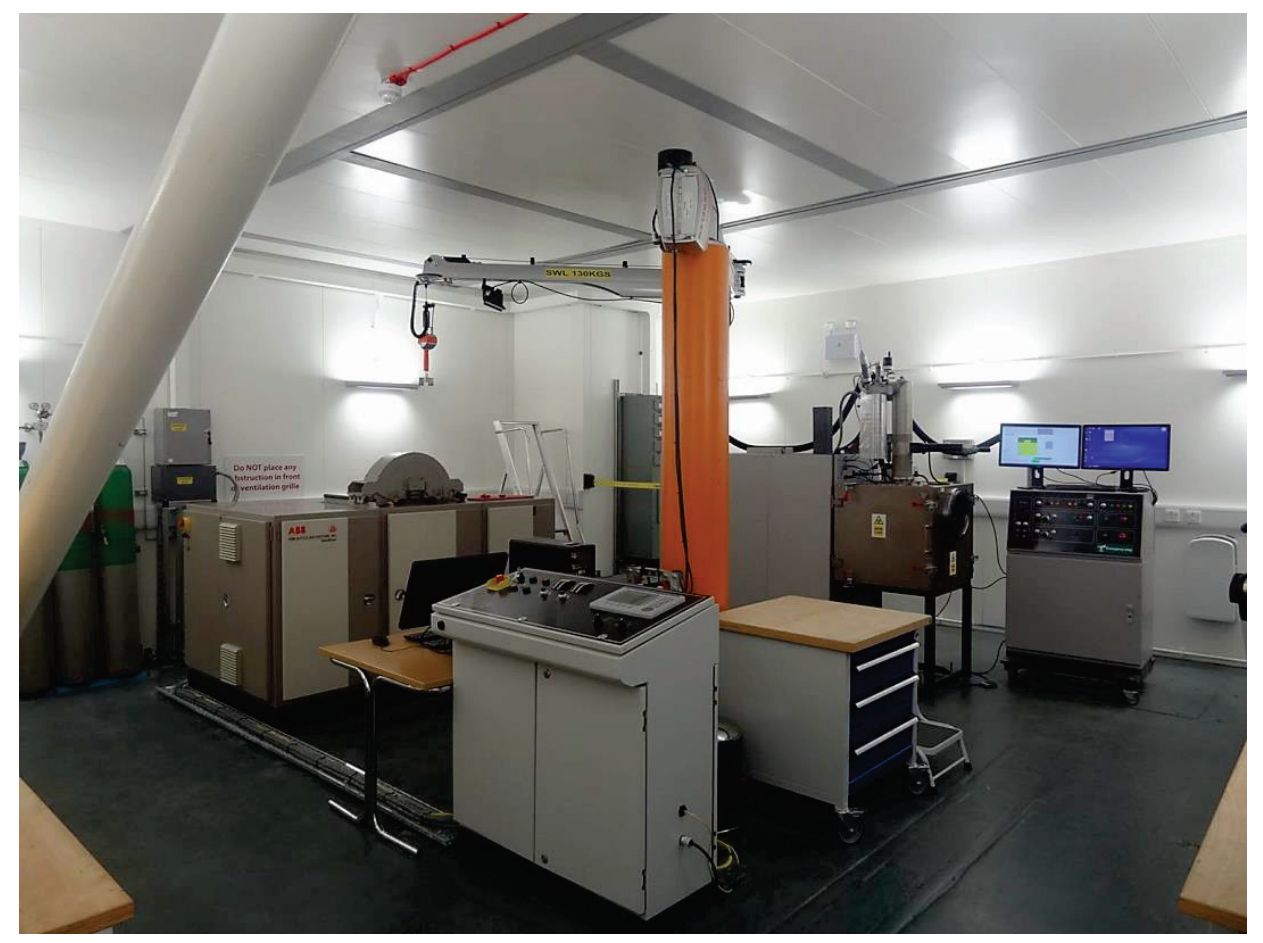

Fig. 4.15. Target cladding facility at ISIS.

A LANSCE target was clad using a commercial HIPing facility, but the company does not want to repeat the process in the future. The Chinese Spallation Neutron Source plans to do the tantalum cladding for its target "in house" similarly to ISIS. Arrangements for a collaboration with the ISIS facility to provide developmental plates are promising; however, ISIS is not interested in providing production plates. Consequently, the STS project will be required to work with industry to develop a dedicated facility capable of producing high quality plates for the life of the facility.

\subsubsection{Target Carriage}

The same basic handling scheme as used at the SNS FTS is planned, with the target mounted on a shielding carriage for horizontal insertion into a core vessel (Fig. 4.16). The primary heavy water cooling lines will be connected to the carriage at installation and disconnected before retraction, similar to the process for the FTS mercury loop system. This will allow the primary loop system component to be 
located outside the cell. A separate loop for shell cooling is provided, which can remain connected during carriage retraction to provide decay heat removal capacity.

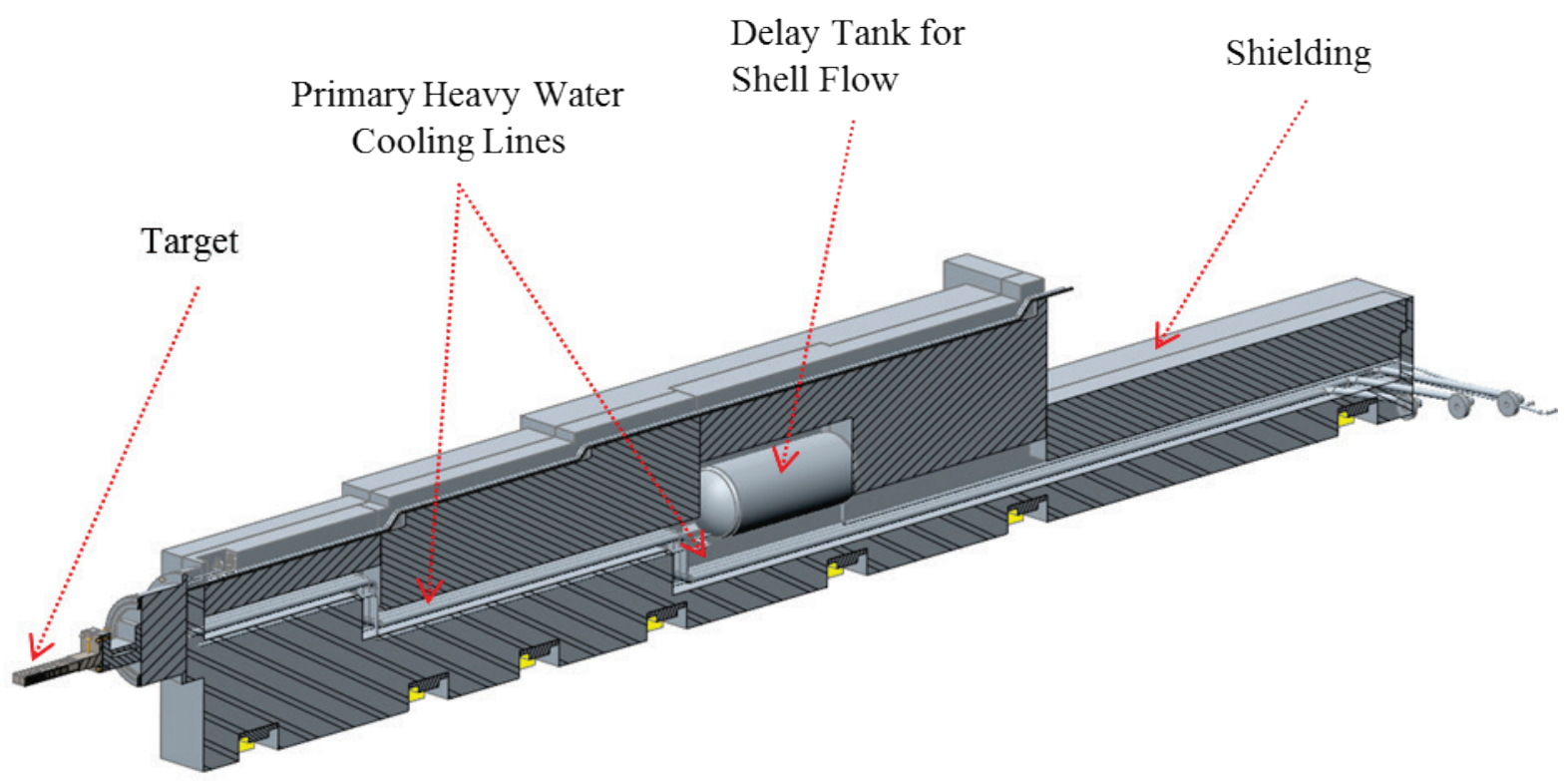

Fig. 4.16. Target carriage with target assembly.

A track-and-wheel system similar to that at the FTS will be used. The carriage will be about 100 tons, $10.6 \mathrm{~m}$ long, and $0.76 \mathrm{~m}$ wide at the front and stepped out for shielding. Included within the carriage will be a delay tank for the separate shell cooling loop. The delay tank will reduce the radiation exposure of hot cell equipment during operation. Figure 4.17 shows a section view of the carriage in the installed position with side connections to the primary cooling system and to a flexible loop for the shell cooling, which can remain connected during target retraction.

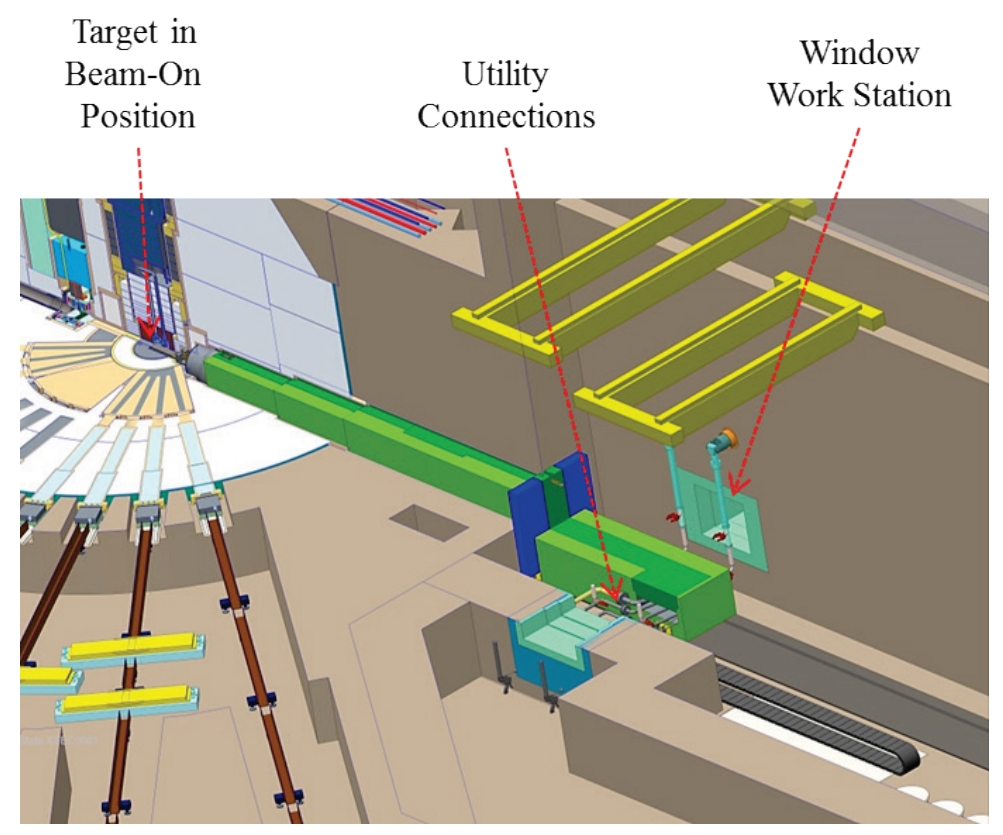

Fig. 4.17. Target carriage in installed position. 


\subsection{INNER REFLECTOR PLUG}

Containment and positioning of the moderators and beryllium reflector is the primary function of the IRP. However, because these components have relatively short lives, the overall plug design is dominated by the need for periodic replacement.

\subsubsection{IRP Configuration}

The plug will have three segments, as shown in Fig. 4.18. The lower IRP will surround the target and include the moderators and reflector components. This section will contain a beryllium reflector with an aluminum shell and will require heavy water cooling. The intermediate section will provide only shielding and will require a limited amount of water cooling. The upper shield will not contain water and will be cooled by conduction to surrounding structures. Approximately $70 \mathrm{~kW}$ of power will be removed by several discrete water cooling channels with a total flow rate of approximately $1.9 \mathrm{l} / \mathrm{s}$. The intermediate and upper IRPs will be stainless steel, as required for compatibly with the vessel environment. The complete IRP assembly will weigh approximately 29 tons.

Five to six IRPs will be required in the 40 year STS operating life; therefore, it is configured for reasonably efficient remote change-out and disposal using existing SNS tooling. This process is described in Section 4.9.

The STS IRP configuration is based on the existing SNS design (Fig. 4.19).

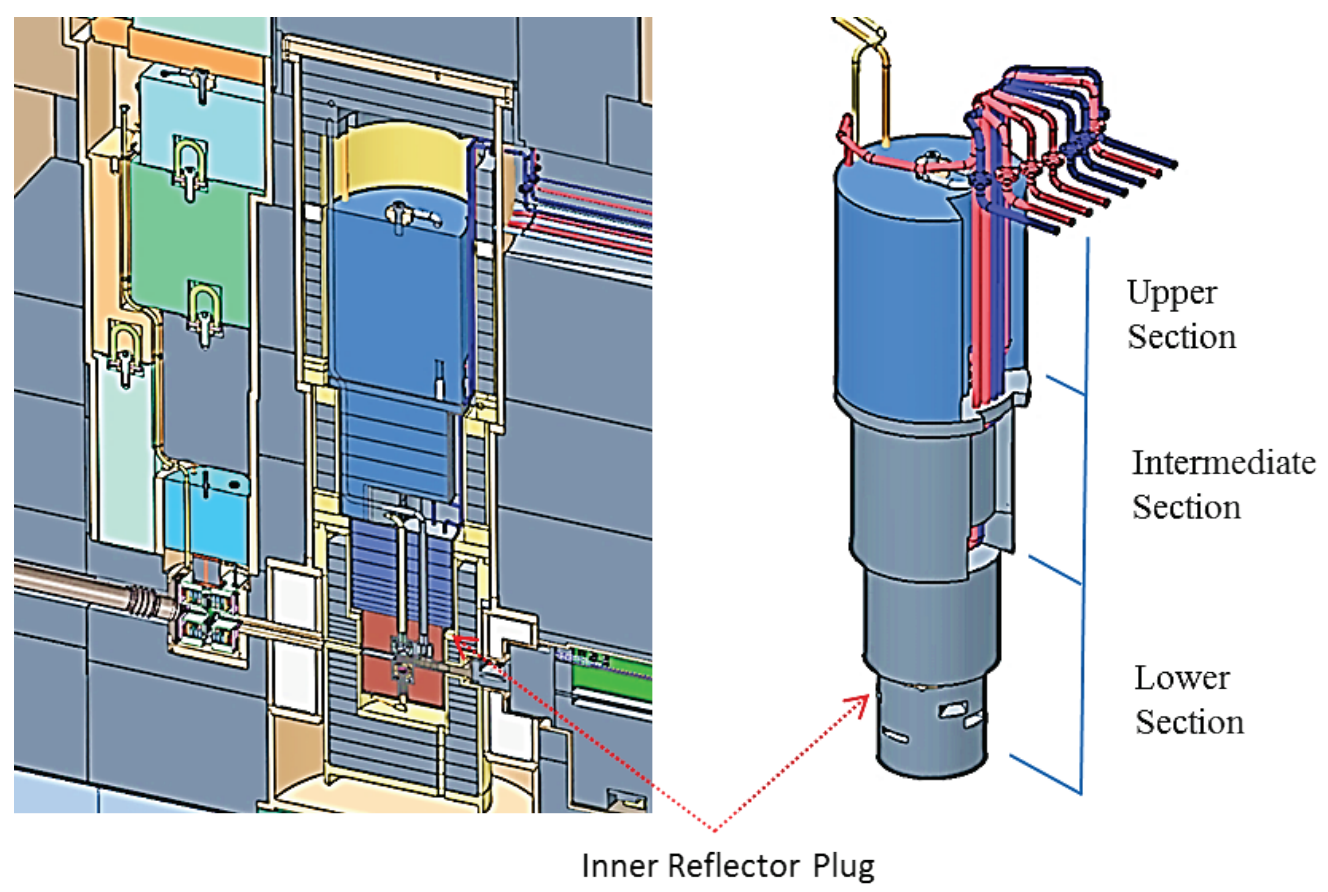

Fig. 4.18. Inner reflector plug. 


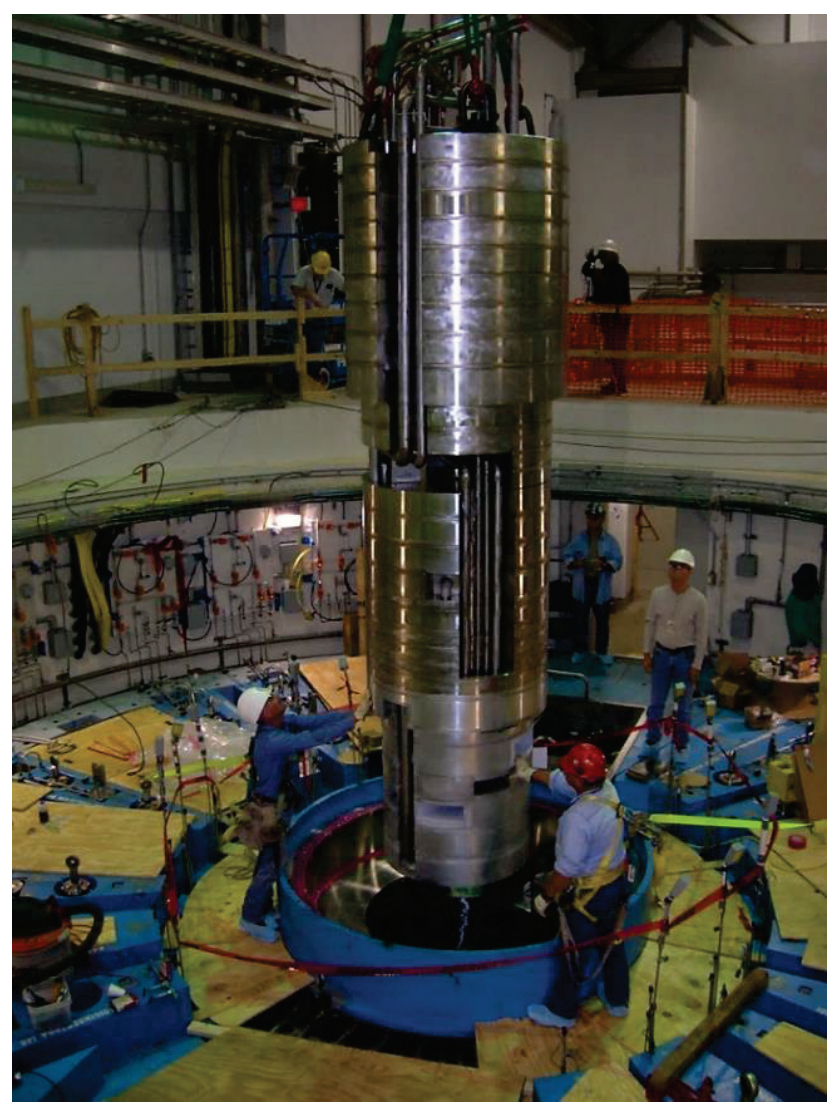

Fig. 4.19. SNS FTS inner reflector plug during installation.

The outer diameter of the lower portion of the IRP is determined by the diameter of the reflector and the dpi damage limit of the outer plug. The preliminary neutronics analysis estimates the diameter to be approximately $70 \mathrm{~cm}$. With the addition of cooling gaps and structure, the result is a lower section diameter of $72.6 \mathrm{~cm}$. The sections above the lower IRP increase in diameter in two $8.9 \mathrm{~cm}$ steps to prevent radiation shine.

Machined beryllium is a unique material that will be procured from a specialty vendor for the lower portion of the IRP. The reflector will be formed from blocks designed to surround the moderators and neutron beam line ports while providing cooling channels for adequate heat removal with heavy water and contained within an aluminum vessel. The vessel will be designed to the ASME Boiler and Pressure Vessel (B\&PV) Code and rated for the water system maximum pressure on the order of 4 bar.

The IRP will be fully assembled at the vendor site. This will facilitate pressure and leak testing before shipment. A dedicated vessel will be located in the target building for storage and testing of replacement IRPs (see Sect 4.9.5). This vessel will be designed to match the interfacing features inside the functional STS vessel. This will include the mounting points and piping connection points. Full vacuum pump-down capability will enable the complete leak testing of the IRP after it arrives on site from the vendor. It will also minimize handling during the change-out process.

\subsubsection{Moderators}

Three moderators will be positioned in "wing" geometry, two above and one below a slot designed to receive the target. Each moderator will contain 0.2 to 0.61 of supercritical para-hydrogen nominally operating at approximately 15 bar and a temperature of $20 \mathrm{~K}$. The two coupled hydrogen moderators will 
be located near the peak neutron production point in the target. The upper moderator will have one viewed face and the bottom will have two viewed faces. Both will include $2 \mathrm{~cm}$ of light water premoderator on all unviewed surfaces. Approximately $1 \mathrm{~mm}$ of cadmium will be applied to the inside of the decoupled moderator neutron beam ports. The lining will be applied by plasma spray to the completed lower IRP by a specialty vendor.

The decoupled and internally poisoned downstream moderator will be located roughly $13 \mathrm{~cm}$ from the peak production position and will be viewed on two faces. This configuration was selected to match the proposed set of instruments and is shown in Figs. 4.20 and 4.21.

Each moderator will be serviced by an independent transfer line from the HUR. To maintain the cryogenic operating temperature in the moderators, the supply piping has four layers: hydrogen (feed and supply), vacuum, helium, and water jacket on the piping. The cryogenic hydrogen utility, which services the cold moderators, is discussed in Section 4.4.

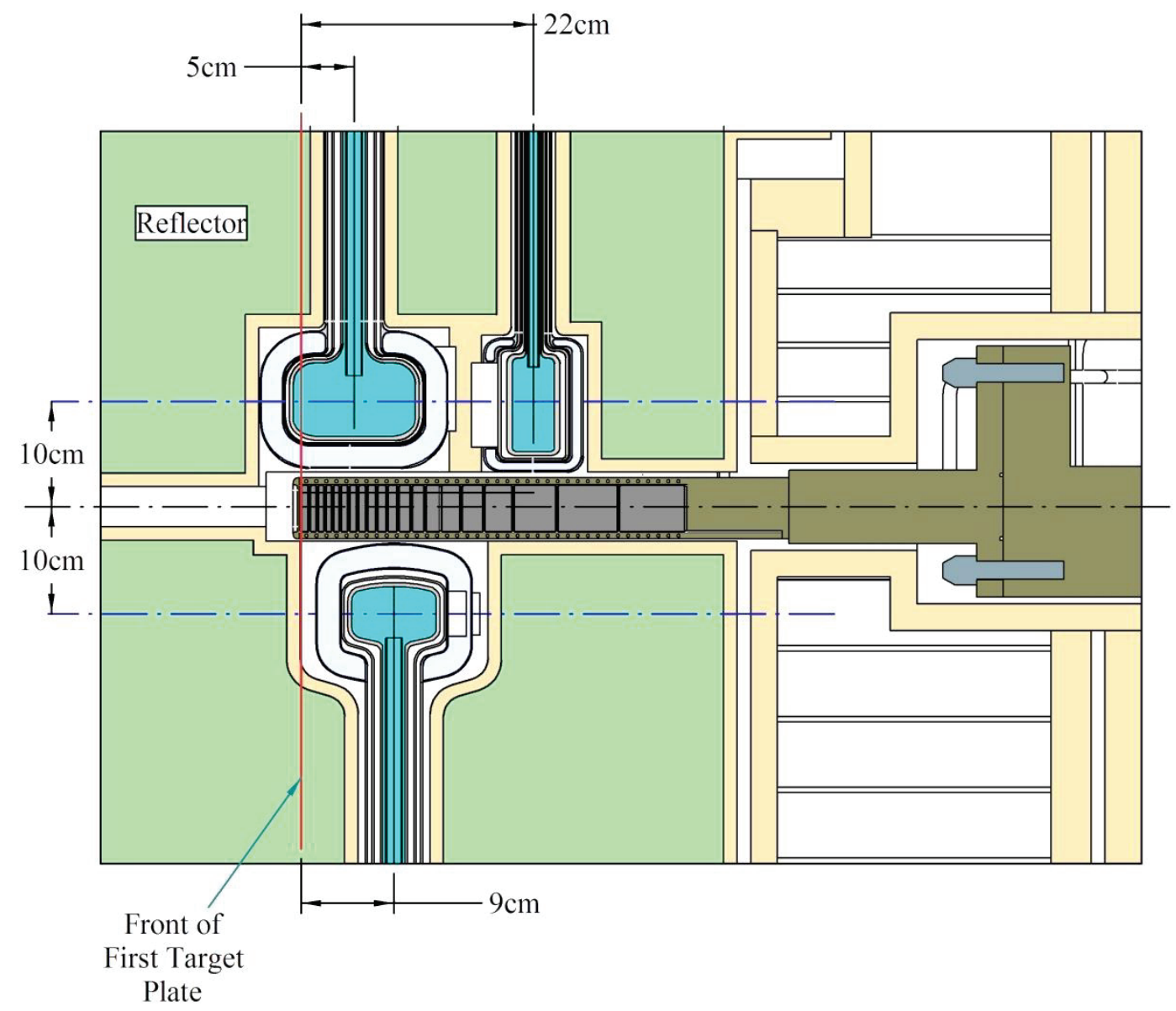

Fig. 4.20. Location of moderators along proton beam centerline. 


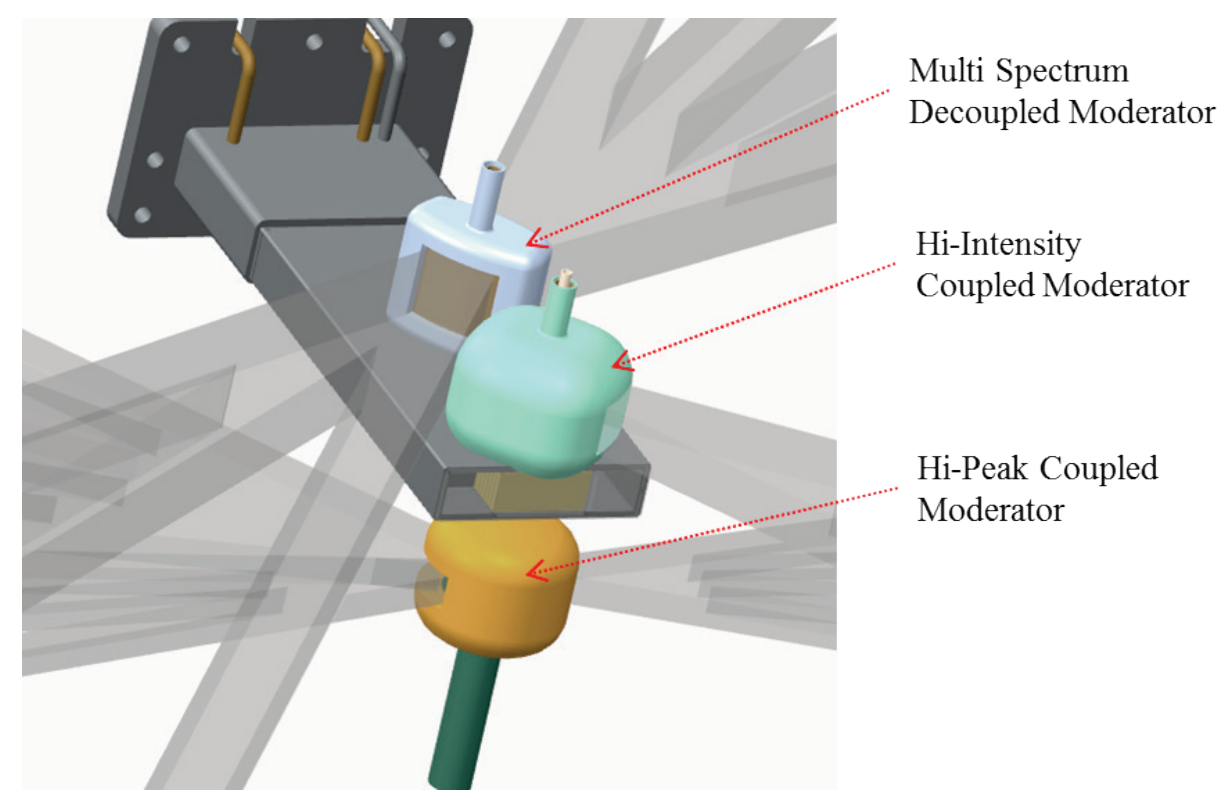

Fig. 4.21. Moderator configuration.

As the moderators will essentially be buried within the IRP, it is imperative that their design be robust. To that end, the moderator vessels, including the surrounding vacuum vessel, will be designed and fabricated according to Section VIII of the ASME B\&PV Code, although they may not be Code stamped. Typical transfer line systems that serve neutron moderators are often equipped with expansion bellows on the warm sections to accommodate the thermal expansion and contraction. To minimize the possibility of a bellows failure in an inaccessible area, Invar36 was used in the fabrication of the hydrogen supply and return layers of the transfer lines for the FTS and will be used for the STS. Friction-welded joints will be used between the Invar and the aluminum moderator vessel.

\subsubsection{IRP Lifetime Determination}

The predicted lifetime of the IRP is determined by the burn-up rate of the cadmium poison plates in the decoupled moderator and the cadmium decoupler coatings. The radiation damage of the $6061 \mathrm{Al}$ structure is not expected to be the limiting factor. If the decoupled moderator were eliminated, then aluminum radiation damage would be the limit. Thicker poison plates could extend the life of the moderators; however, this would result in a significant reduction in the initial neutronic performance. The poison plate and decoupler thicknesses that were selected result in an estimated 6 year lifetime.

\subsubsection{IRP Alternative Configuration}

An alternative IRP design configuration based on individual moderator replacement was considered. The obvious advantage of this arrangement is that it allows for the reuse of large portions of the IRP, potentially saving fabrication cost and reducing waste. However, the special tooling required to change the long, delicate moderator assemblies inside the service cell would likely more than offset those savings. As proposed, the unified IRP design is based on the same segmentation as the FTS assembly so that the existing FTS tooling set and procedures can be used. The individual moderator change-out configuration would require new, more complex handling tools designed to preserve the extended, vertical pipe sections attached to the reusable middle and lower plug sections. 


\subsection{CRYOGENIC SYSTEM}

The cryogenic system provides the target moderator system with cryogenic hydrogen. Forced-flow circulation is required to maintain the temperature of the moderators at $20 \mathrm{~K}$ with a small temperature variation within the moderators. Based on the neutronics analysis of the STS system, the moderators will experience nuclear heating on the order of 2.5 to $4 \mathrm{~kW}$. A refrigeration capacity of approximately $6 \mathrm{~kW}$ will be installed to accommodate additional system heat leaks and provide adequate capacity contingency. Ortho-to-para converters will provide a para-hydrogen concentration of $99 \%$.

The cryogenic system will be physically and functionally similar to the existing SNS FTS hydrogen system. This system was fundamentally based upon the designs successfully used for many years at other spallation neutron sources, namely LANL's Lujan Center and Rutherford Appleton's ISIS. The FTS is a proven design that has operated successfully for over 8 years. Using key design aspects of this known system will help ensure successful operation of the STS cryogenic moderators.

The cryogenic system will consist of two primary subsystems: hydrogen circulation and helium refrigeration. The hydrogen circulation system will consist of a series of three independent cryogenic loops, each containing a circulator, an accumulator, a heat exchanger, and an ortho-para convertor. Figure 4.22 illustrates one of these loops. The system will also include a hydrogen gas management system, pressure safety relief system, and associated interconnecting cryogenic piping. The helium refrigeration system will include a helium refrigerator, helium compressors, oil removal systems, a helium gas management system, helium buffer tank storage, a helium purifier, and associated interconnecting cryogenic piping. Both the hydrogen circulation system and helium refrigeration system will be supported by insulation vacuum systems.

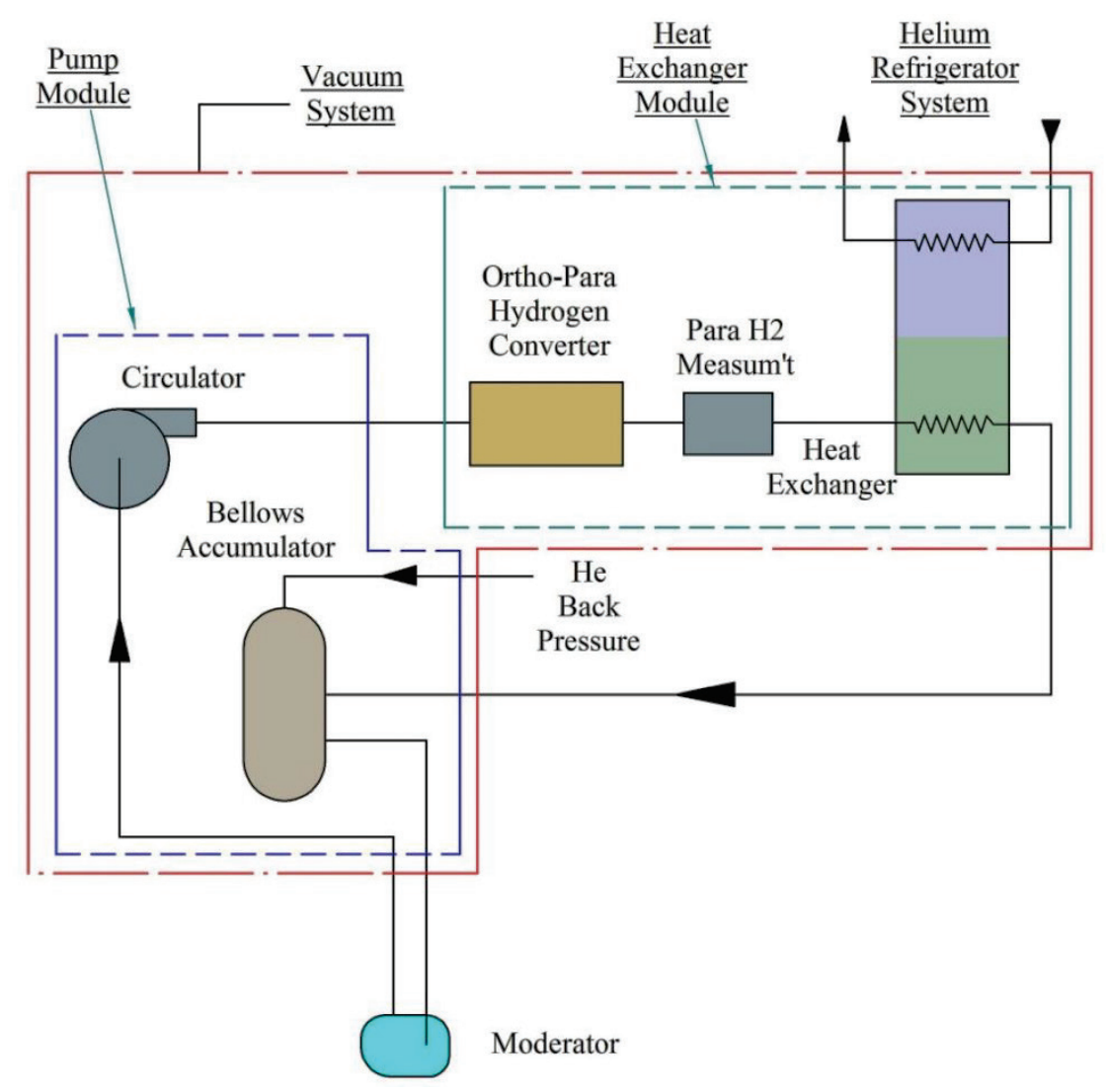

Fig. 4.22. Simplified hydrogen loop diagram for STS. 


\subsubsection{Hydrogen Circulation System}

The goal of the hydrogen circulation system is to safely and reliably provide stable $20 \mathrm{~K}$ hydrogen to the three cryogenic moderators within the IRP. The general control philosophy is to accomplish this goal in as simple a manner as possible. A basic diagram of a hydrogen loop is shown in Fig 4.22.

The hydrogen circulating system will be a forced-flow, nonrecycling, closed-loop system filled from pressurized cylinders of research-grade hydrogen. Since hydrogen in the hydrogen circulation system will be maintained at approximately $20 \mathrm{~K}$ and above the critical pressure, it is technically correct to refer to it as "supercritical" rather than liquid hydrogen. The system will operate at supercritical conditions at all times, eliminating phase-change effects. The base pressure of the system will be maintained at 14 bar, providing a 1 bar margin above the critical pressure.

\section{Hydrogen Cryogenic Equipment}

The cryogenic hydrogen equipment will be contained in two modules designed to maintain the necessary cryogenic temperatures. These are the heat exchanger module and the pump module (see Fig. 4.23). The heat exchanger module will be a vacuum vessel housing the three helium-to-hydrogen heat exchangers and the three ortho-para converters - one for each of the independent hydrogen loops - and flow control and isolation valves on the associated helium piping. The heat exchanger module will also contain three para-hydrogen concentration measurement instruments using Raman spectroscopy, one for each hydrogen loop. The pump module will be a vacuum vessel containing the three variable speed circulators, three cryogenic accumulators, and various flow, temperature, and pressure sensors.

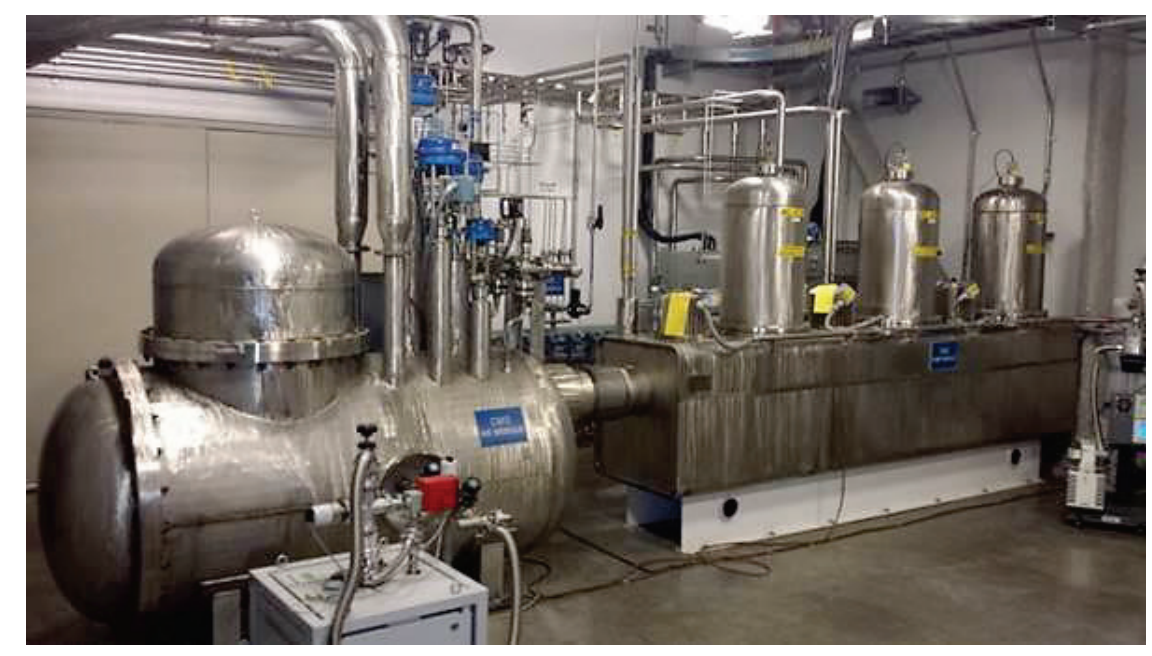

Fig. 4.23. FTS cryogenic heat exchanger and pump modules.

The circulator will pump the supercritical hydrogen around the loop between the heat exchanger and the moderator vessel. Cryogenic helium circulated through the heat exchanger will remove heat that the cryogenic hydrogen absorbs from neutron heating, as well as static heat loads from the related cryogenic equipment.

The pump module and heat exchanger module vacuum systems will be separate from the vacuum for the transfer line and moderator vessel. Thus a hydrogen leak inside the pump or heat exchanger module could not flow through the vacuum layer down into the core vessel. 


\section{Hydrogen Accumulator}

The accumulator accommodates volume changes, which result from operational swings in temperature due to beam heating changes, without adding or subtracting hydrogen to or from the system. The STS accumulator will be the same as the welded metal bellows units used in the FTS cryogenic system. The primary purpose of the accumulator is to control pressure fluctuations by providing each circulating supercritical hydrogen loop with an adequate variable volume for a constant hydrogen mass. This volume allows the system to passively accommodate the density fluctuations that accompany each beam power variation or beam trip-induced temperature transient. Metal bellows have historically been implemented in various systems as accumulators not only for thermal expansion but also for pulsation dampers. Edgewelded bellows with a sufficient number of convolutions theoretically have an infinite cycle life. This is because the bellows movement is kept well within the elastic range. For a given loop, the total system volume was calculated and the necessary expansion bellows volume derived from the density difference at the minimum and maximum operating temperatures.

The expansion bellows must be backed with a compressible gas. As the system will be operating at nominally $20 \mathrm{~K}$, helium was a natural choice. The helium volume backing the expansion bellows governs the overall pressure rise that will result from beam heating. Once an acceptable pressure rise was determined, the helium volume was set and the overall size of the unit established. The accumulator was designed to allow a 1 bar system pressure rise due to the beam heating expected from the proton beam. This would result in a 14-15 bar pressure with no beam and a 15-16 bar pressure with a full-power beam. The expansion bellows was designed with additional margin so as to provide sufficient stroke to passively respond to varying heat loads between the pressures of 13 and 17 bar, thus addressing both overheating and overcooling conditions.

Although it is not used in operation, the cryogenic accumulator will be equipped with a laser-based measuring system to indicate the relative position of the bellows. The actual position of the bellows will not be used for any control inputs, as the system will be completely passive. Knowing the actual bellows position and trending this data over time will assist with monitoring the health of the system. In a constant mass system, the pressure cannot be maintained if inventory is lost. A decrease in the loop base pressure would be the result of either a helium leak on the backside of the expansion bellows or a hydrogen leak. Monitoring the bellows position, however, will make it very clear which system is leaking. A loss of helium in the accumulator would result in bellows expansion and thus pressure decay at constant temperature. A loss of hydrogen would result in bellows compression and thus pressure decay at constant temperature.

\section{Vacuum System}

A major key to reliable operation of the cryogenic system will be the ability to maintain a high-vacuum envelope to provide effective thermal insulation around all parts of the system that contain cryogenic hydrogen. The ability to hold an $\sim 20 \mathrm{~K}$ hydrogen temperature will be very sensitive to the heat input and therefore to leakage of gases into the vacuum insulating layer. The vacuum utilities are designed to provide pump-down to the required $\left(\sim 10^{-6}\right.$ torr $)$ vacuum range. Engineered barriers with all-welded connections will minimize the chance of leakage. Any significant leakage of hydrogen or helium into the vacuum barrier will allow greater than normal heat transfer and cause the hydrogen temperature to increase. If sufficient leakage brought the vacuum into the range of $10^{-2}$ torr or greater, rapid temperature and pressure increases would occur, requiring venting to control the pressure. System pressure would be controlled without operator intervention by the 18 bar spring-loaded relief valves and/or the 19 bar rupture discs, discharging into the inert-gas-purged vent line. 


\section{Hydrogen Gas Management System}

The hydrogen gas management system (see Fig. 4.24) will consist of equipment and instrumentation supporting a purge vacuum pump and various remotely actuated isolation and pressure control valves controlling the warm gas distribution and venting operations. To minimize the potential for hydrogen leaks, piping welded with mechanical couplings will be used only where absolutely necessary. All interconnecting piping that contains the supercritical hydrogen will be vacuum-jacketed to maintain cryogenic temperature and to contain the hydrogen in the event of a leak. All vacuum-jacketed piping will be evacuated with a portable dry vacuum pumping station and then sealed.

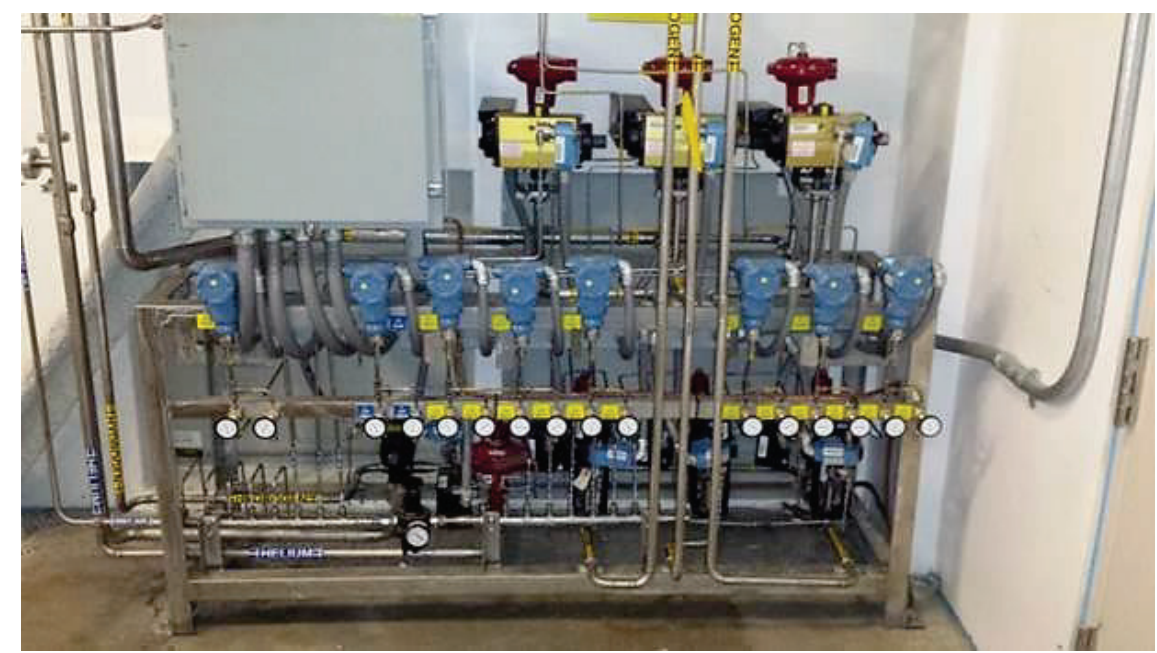

Fig. 4.24. FTS hydrogen gas management system.

The pressure safety relief system will use spring-loaded relief valves and rupture discs welded into the hydrogen fluid lines. Figure 4.25 illustrates the rupture disc assemblies welded into their associated manifolds. The discharge from these rupture discs will be plumbed to a common manifold so that efflux can be safely exhausted.

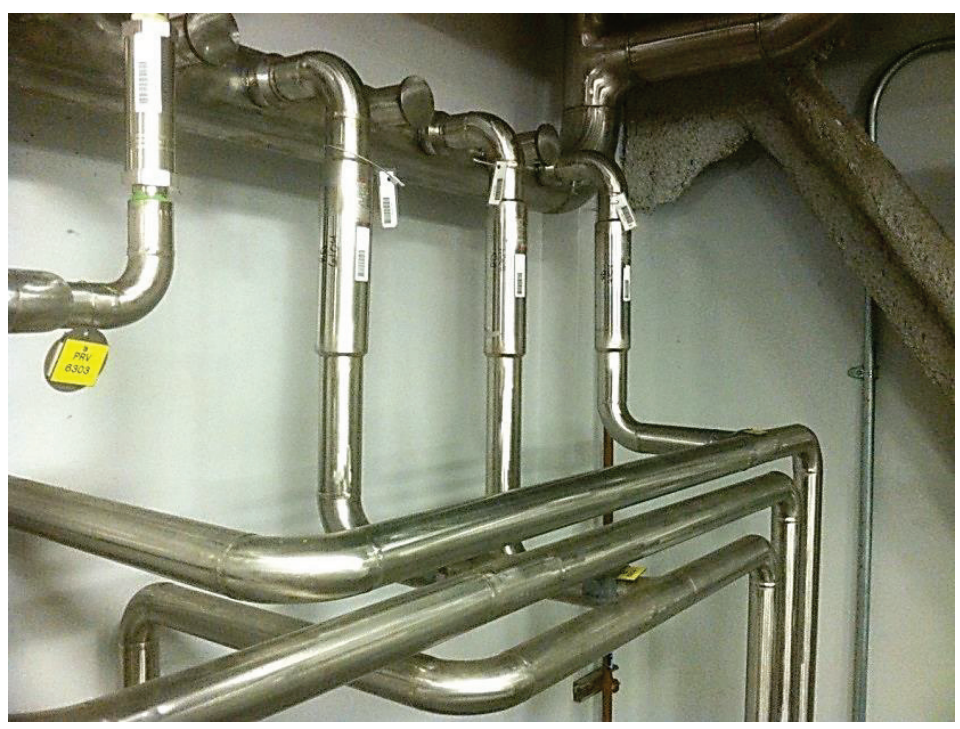

Fig. 4.25. FTS welded pressure relief rupture discs and manifold. 


\section{Hydrogen System Control and Operation}

The hydrogen circulation system equipment will be located in the HUR, except for the portion of the transfer lines that exit the HUR and connect to the moderators within the IRP. The HUR houses active components of the cryogenic system, including the pump module, heat exchange module, gas management system, and rupture discs with associated manifolds. The HUR has its own ventilation system, including redundant active exhaust paths. The normal exhaust path and blower operate continuously during normal operation. The two emergency vent paths remain in standby for actuation on detection of excessive hydrogen in the HUR. Loss of exhaust air flow will be indicated in the target control rooms.

\subsubsection{Helium Refrigerator System}

The helium refrigeration system provides active cryogenic cooling to the hydrogen circulation system by directing a closed-loop stream of cryogenic helium gas to the helium-to-hydrogen heat exchangers in the heat exchanger module described in Section 4.4.1.1.

\section{Helium Refrigerator}

The primary component of the helium refrigeration system is the helium refrigerator (see Fig. 4.26). The refrigerator will have a capacity of $6 \mathrm{~kW}$ to accommodate the calculated $4 \mathrm{~kW}$ moderator heat load plus normal operating loads. The refrigerator will expand and cool gas from the helium compressors to approximately $16 \mathrm{~K}$. Discharged helium will be distributed to the three helium-to-hydrogen heat exchangers where heat from the moderators and hydrogen system equipment will be absorbed. The returning gas stream will flow back through the low-pressure side of the refrigerator to the suction side of the helium compressors.

Note that the helium refrigerator will not have liquid nitrogen precooling.

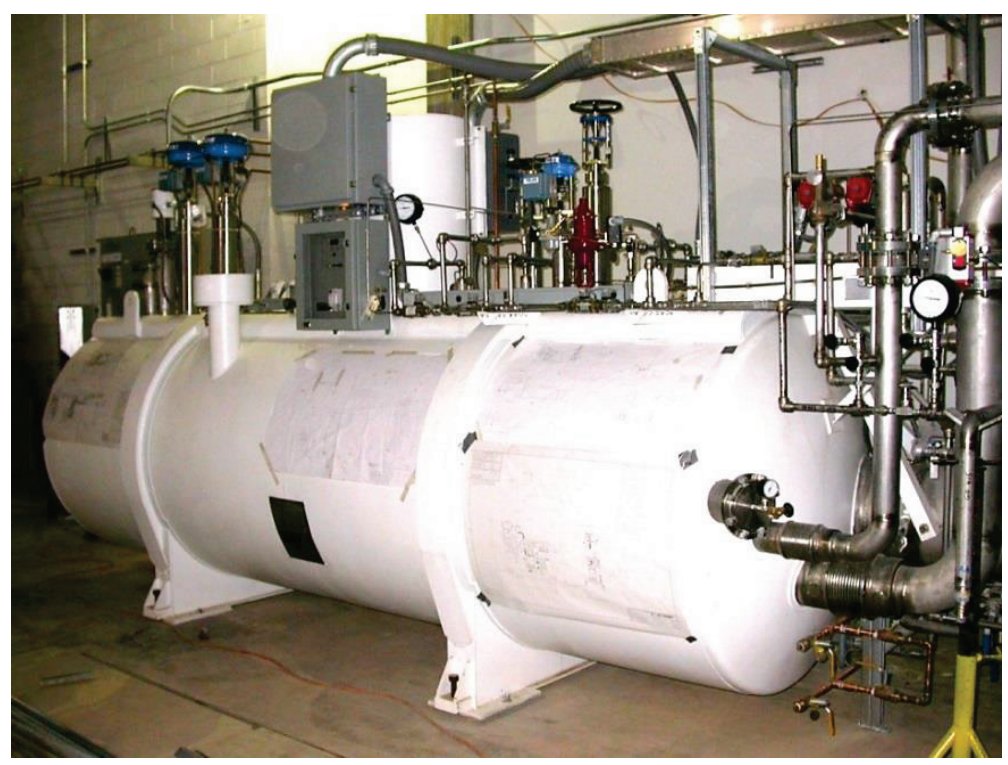

Fig. 4.26. FTS helium refrigerator. 


\section{Helium Compressors}

The helium compressors are oil-injected, water-cooled, screw-type compressors (see Fig. 4.27). Each compressor will be skid-mounted with heat exchangers, relief valves, and an integral bulk oil removal system. The helium refrigeration system will have three compressors, each capable of providing $50 \%$ of the maximum flow needed to operate the refrigerator. This will allow for two compressors to be operating while the third is on standby as a spare. If one compressor should fail, the refrigerator could continue to operate at a reduced capacity until the spare compressor was brought on-line.

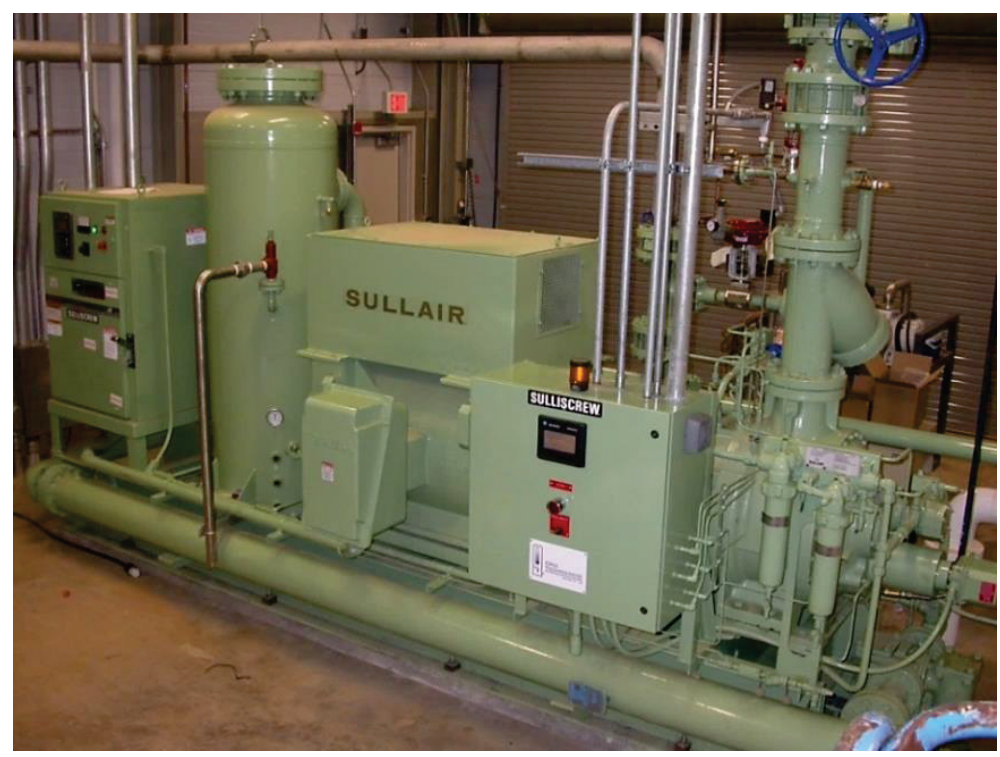

Fig. 4.27. FTS helium compressor.

\section{Helium Purification System}

An oil removal system will maintain the purity of the helium gas by reducing the entrained oil to less than $5 \mathrm{ppb}$. The system will use a series of coalescing filtration units in line with a regenerable charcoal absorber (see Fig. 4.28). The helium refrigeration system will have two identical oil removal systems for redundancy. In addition, a liquid nitrogen-cooled helium purifier sized at $150 \%$ of total compressor capacity will be used to further improve helium purity (see Fig. 4.29). The purifier will be capable of accepting the entire gas flow from the compressors, and its associated piping will be designed to allow the purifier to be isolated from the helium system while it is operating. A pyrolizing multi-component impurity detection system will continuously monitor helium gas purity.

\section{Helium Gas Management System}

The helium gas management system continuously adjusts the amount of helium gas in the system to account for load changes by withdrawing gas from or discharging it to the helium buffer tanks. The thermal stability of the helium refrigeration system will be provided by an integral control heater capable of generating control heat equivalent to the total moderator system load. 


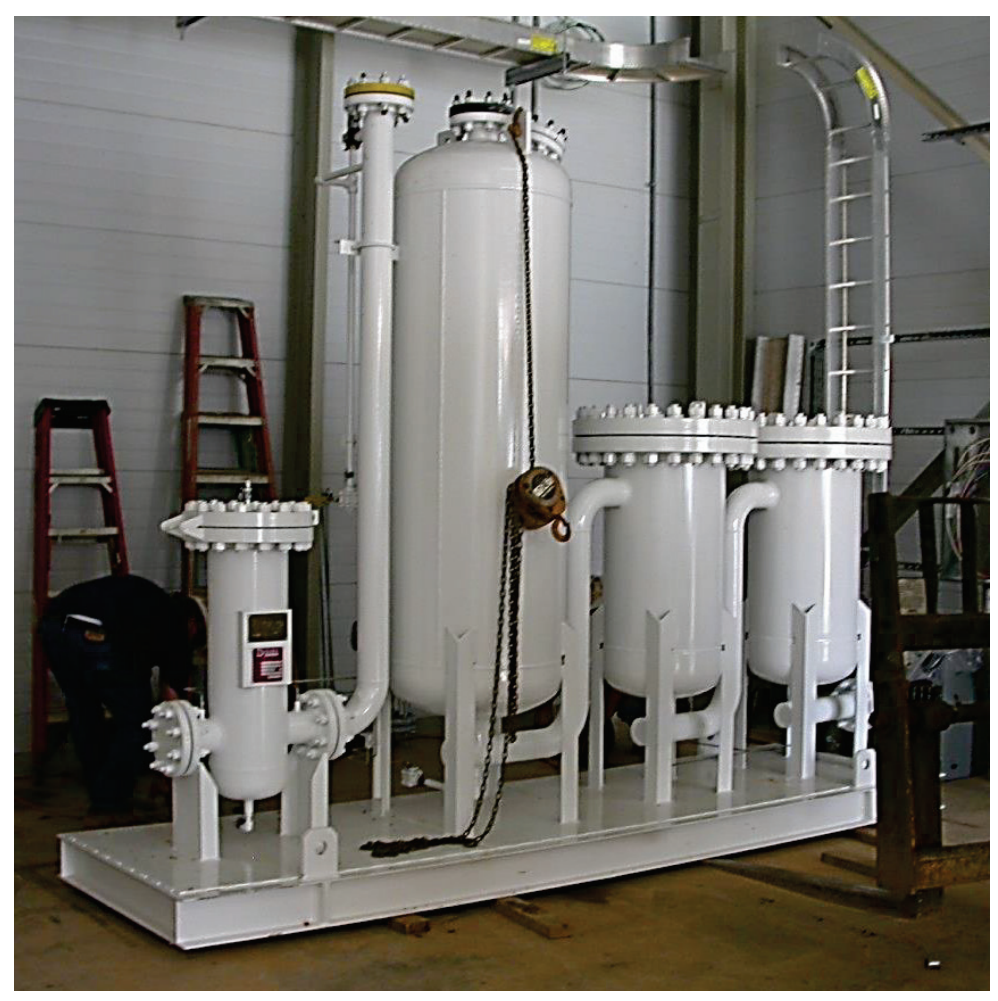

Fig. 4.28. FTS oil removal system.

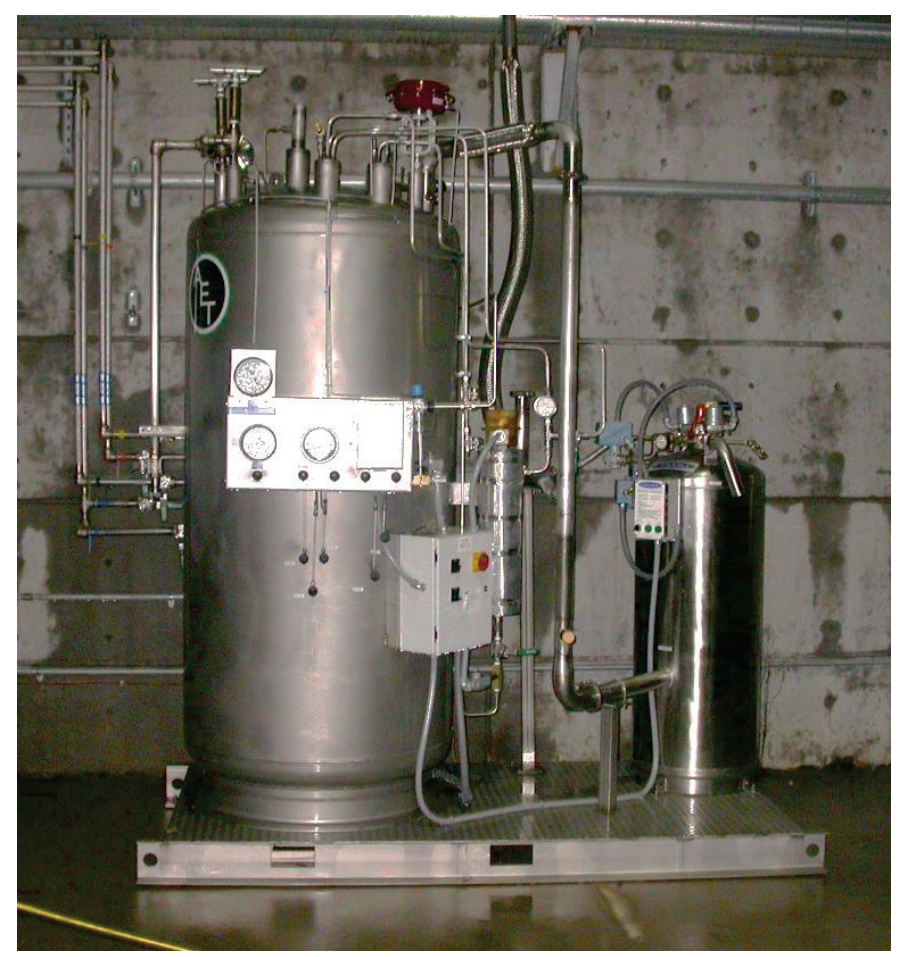

Fig. 4.29. FTS helium gas purifier. 


\subsection{VESSEL SYSTEMS}

Components surrounding the IRP and target assemblies will be grouped into the core vessel assembly as shown in Fig. 4.30. These components will combine to provide several critical functions. The fixed positioning features in the vessel will determine the alignment of the target, moderators, and neutron beam guides. All actively cooled heat removal components and shielding will be contained in the core assembly, and the partial helium or vacuum operating environment near the target will be contained by the vessel. Mechanically, the core assembly will be divided into the vessel, outer reflector plug, PBW, and neutron beam inserts. Although it is possible to construct these components independently, fabrication of the vessel and the outer reflector plug at a single vendor would be desirable to ensure proper fit-up and assembly.

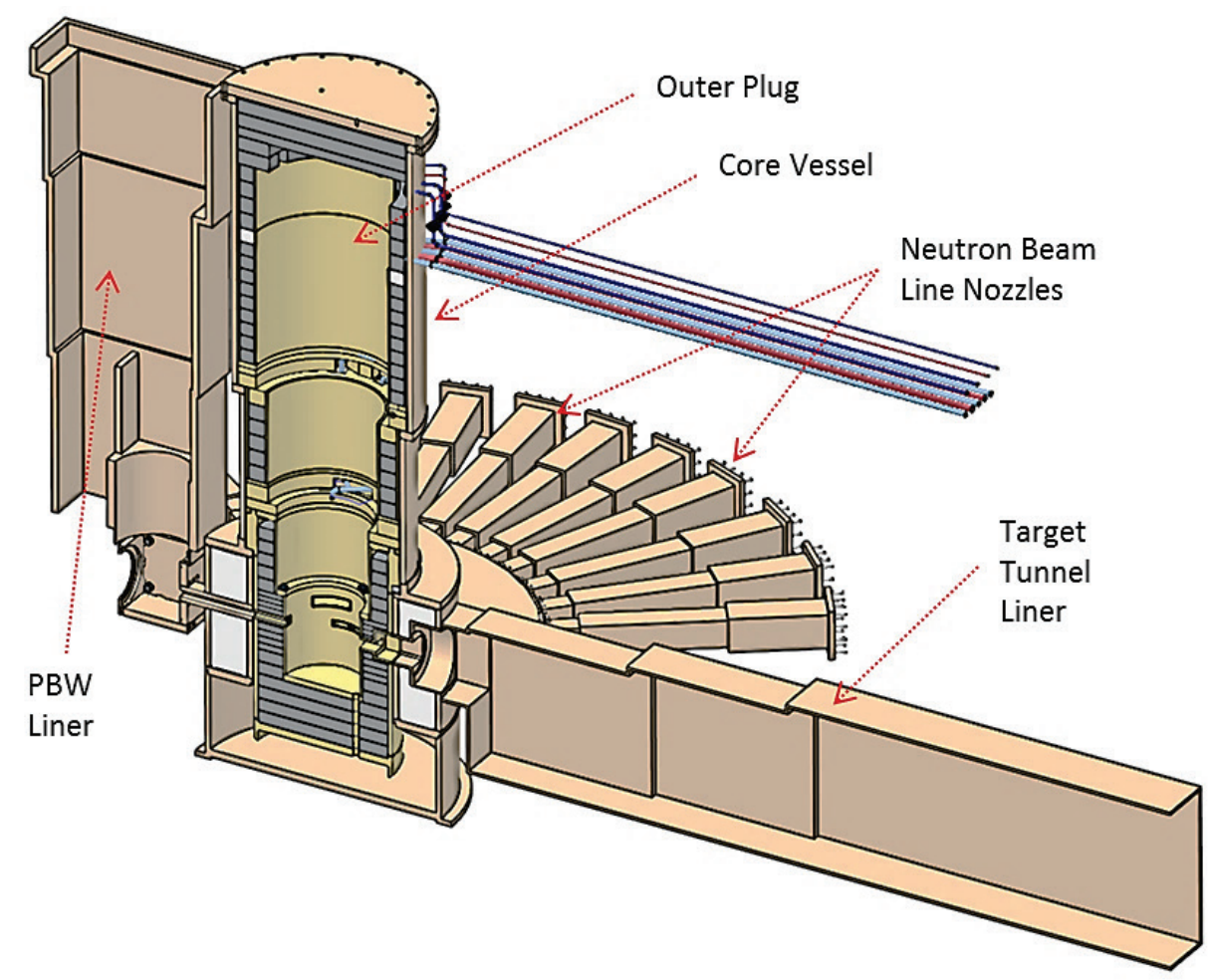

Fig. 4.30. Core vessel assembly.

\subsubsection{Core Vessel}

The core vessel will normally operate with a vacuum atmosphere to limit interference with the neutron beams and eliminate the generation of unwanted isotopes from residual moisture or gas inside the vessel. It will also be possible to operate the vessel with a partial helium atmosphere if doing so is required for functional reasons. A safety relief device (rupture disk) will limit overpressure to somewhat less than $10 \mathrm{psig}$. The safety vent will be designed to discharge outside the target building to minimize personnel exposure. The vent size will be based on the requirements determined in the facility safety analysis planned for the next phase of design.

The size and shape of the core vessel are determined by the active cooling boundary and the requirement for vertical access to the IRP. IRP change-out access requirements necessitate a tall assembly with a flange at the top. Requirements for cooling and for space for nozzle attachment demand a relatively large "waist" at the neutron beam line level. 
The thermal distribution results from the preliminary, idealized neutronics analysis are shown in Fig. 4.31.

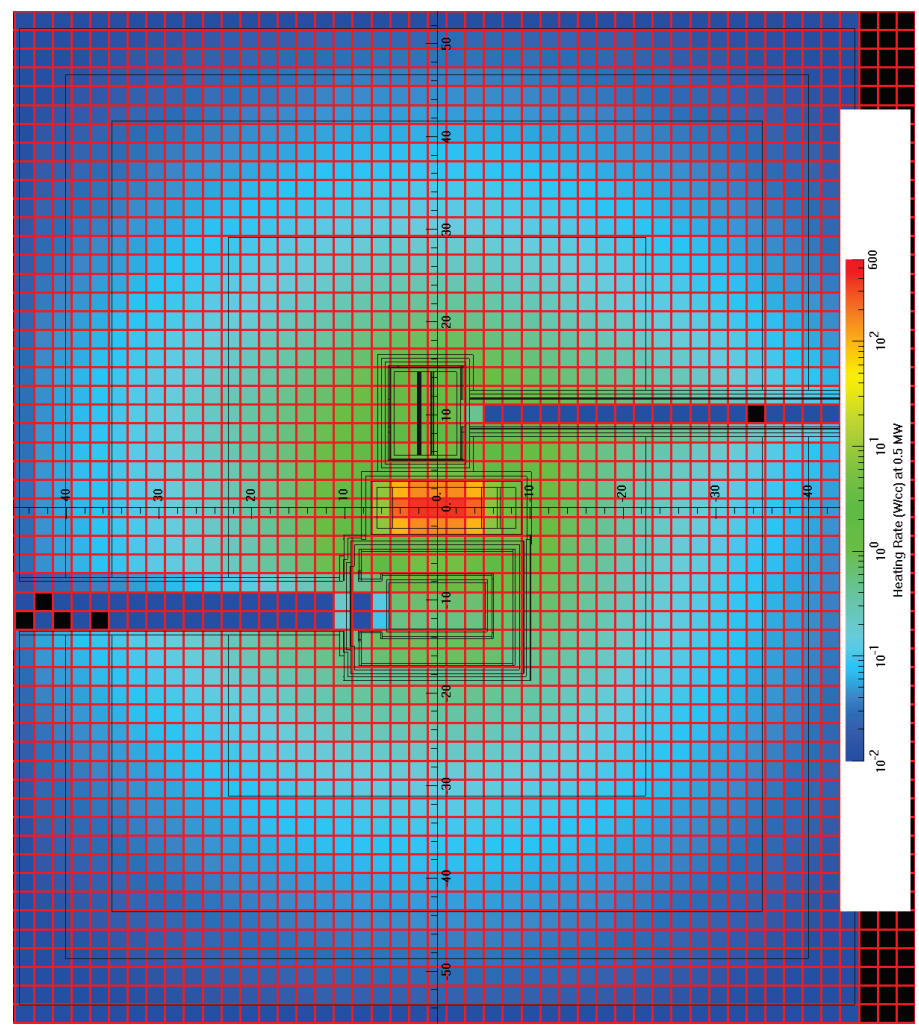

Fig. 4.31. Nuclear heating (idealized) inside the $40 \mathrm{~cm}$ radius to the target center.

The overall active cooling goal is to limit the total energy escaping to the passively cooled shield stack surrounding the vessel to less than $4,000 \mathrm{~W}$. This is to be achieved by extending the active cooling to a boundary at which heating will be significantly below $0.01 \mathrm{~W} / \mathrm{cm} 3$ (see Fig. 4.32). An air cooling path through the shield stack will discharge this heat and vent to the hot off-gas. Excessive heat in the bulk shield could result in unacceptable thermal movements and necessitate a relatively complex forced-air cooling system. Heating along the horizontal plane near the target due to component clearances and neutron beam guides will be accounted for with an actively cooled extended waist and neutron beam nozzles.

The joint between the vessel body and the neutron beam insert extension nozzles must be made during installation. These will be permanent bolted connections with double metal O-ring seals. The vessel extensions will be sized to provide adequate space for the flanges and for personnel access to make the connection during assembly. 


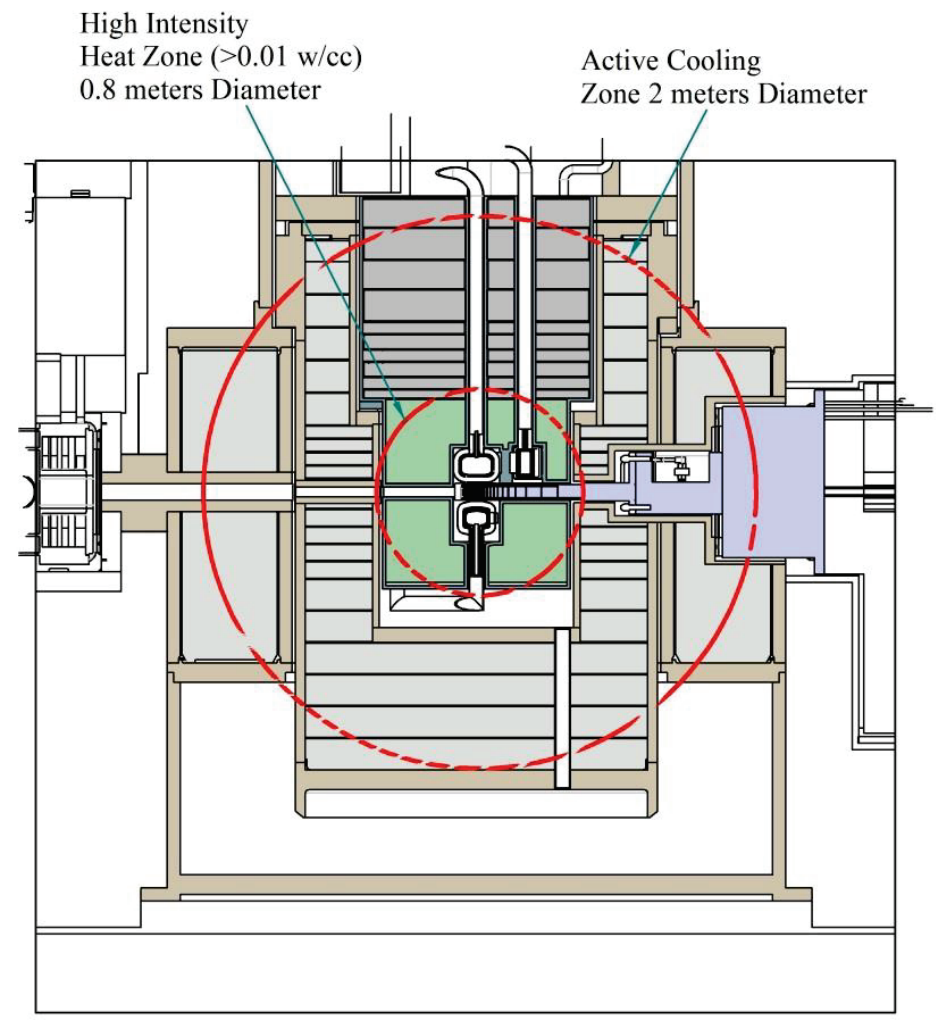

Fig. 4.32. Core vessel section view showing active heating boundaries.

A large array of cooling water pipes, instrument sensors, and gas feedthroughs will be located at the top of the vessel. All the feedthroughs will have flanged joints on the outside of the vessel. IRP cooling water pipes will also be flanged on the inside to facilitate change-out. Cooling water pipes to the outer reflector plug pipes will be welded inside the vessel because this subassembly is permanent. Vessel gas supply and vent pipes will also be permanently installed inside the vessel.

Three transfer lines will carry supercritical hydrogen between the moderators and the pump module in the HUR (see Sect. 4.4, Cryogenic System). These transfer lines will penetrate the HUR wall, traverse the length of the facility to the target area, and enter the IRP at the top of the core vessel. There will be one transfer line for each moderator. Each transfer line will be constructed of six concentric tubes facilitating hydrogen supply, hydrogen return, helium inert gas blanket, pre-moderator water supply, and associated insulation vacuum. The lines will be of all-welded design. The transfer line sections at the top of the vessel will have the capability of being cut and rewelded to facilitate removal and reinstallation of the IRP.

An ullage volume of approximately $1.5 \mathrm{~m}^{3}$ will be located at the bottom of the vessel. This volume is designed to act as a final safety measure to contain a major cooling water break anywhere inside the vessel. The volume is sufficient to hold all the spilled water from any one of the cooling loops should the backflow and shutoff devices fail to function. The ullage will be drained via a syphon line running from the top of the vessel to the cooling water drain tanks in the basement.

The vessel will be fabricated from type 316L stainless steel and it will weigh less than 50 tons, including internal shielding; therefore, it can be handled and placed with the STS overhead bridge crane. It can also be shipped on an over-the-highway truck as a single unit. 
It is assumed the vessel will be fabricated by a specialty vendor capable of machining the precision alignment features with a minimum number of machining setups. Key features include the neutron beam insert mounting faces on each of the nozzles, PBW mounting frame, target mounting port, and IRP mounting plane inside the vessel. The vessel will be fully assembled at the vendor facility for vacuum and pressure testing. This general approach successfully provided satisfactory tolerances in the FTS.

\subsubsection{Outer Shield Plug}

The outer shield plug (Fig. 4.33) will contain the actively cooled shielding between the IRP and the inside wall of the vessel. It is currently assumed that the inner diameter of the outer plug will be beyond the 1020 dpa life-of-facility boundary, making this subassembly a permanent installation. Alignment of the IRP will depend on precision placement of the outer plug relative to the nozzles and other interface features in the vessel. Since the outer plug is not expected to require replacement, it may be possible to incorporate it into the vessel structure, thus improving this alignment and reducing the number of connections in the system. This alternative will be evaluated in the next design phase.

As currently envisioned, the outer plug will be a double walled annular container filled with stainless steel shield segments. The container will be fabricated from either aluminum or type 316L stainless steel and it will be light water cooled. To ensure correct fit-up, the assembly will preferably be fabricated in conjunction with the vessel by the same vendor. The outer plug will be installed at STS to reduce the handling and shipping weight of the vessel.

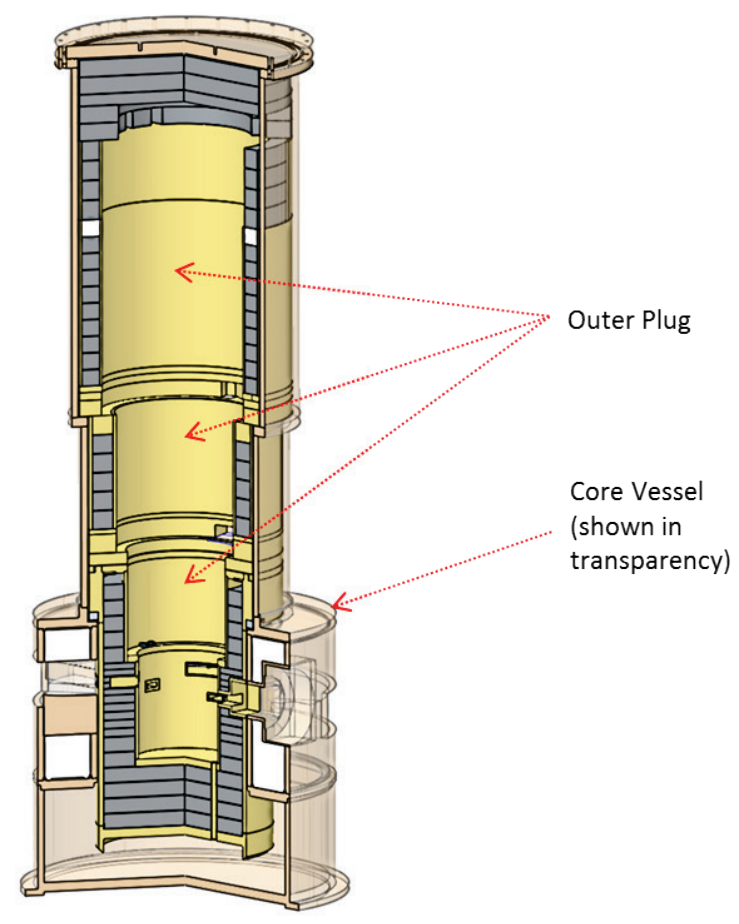

Fig. 4.33. STS outer plug assembly.

\subsubsection{Proton Beam Window}

The STS PBW assembly will mirror the successful FTS assembly (Figs. 4.34 and 4.35). This will reduce the need for new engineering analysis and design. Equally important is that the new assembly will be able to use the existing change-out handling tools and procedures. 


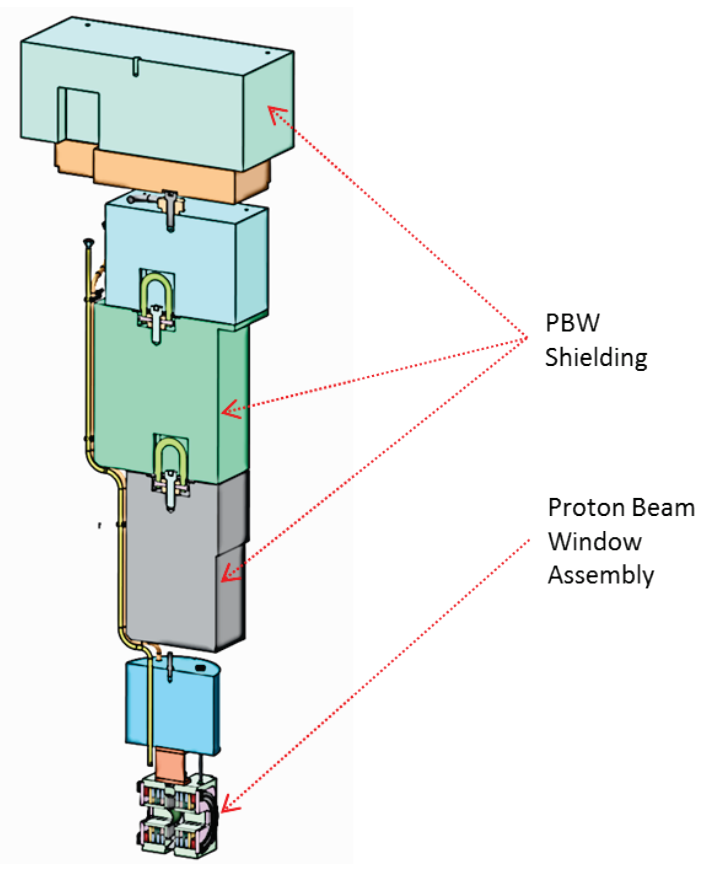

Fig. 4.34. Proton beam window assembly.

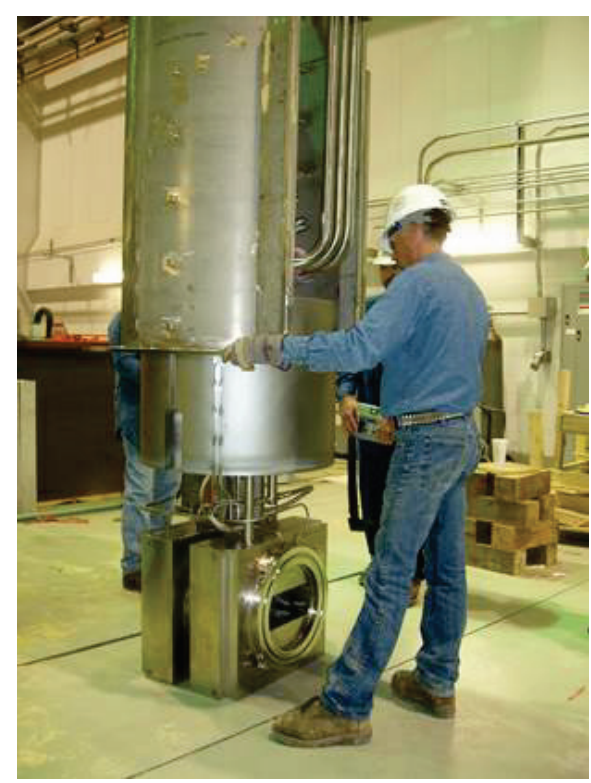

Fig. 4.35. FTS proton beam window.

The size of the PBW itself is the only significant difference between the FTS and STS assemblies. The smaller STS window has not been designed, but it can be assumed that the smaller area will allow thinner window walls. The smaller size may also simplify the fabrication process. A possible life-extending alternative design in which the window is moved in the beam rather than changed will be evaluated in the next phase of design. 


\subsubsection{Neutron Beam Inserts}

The neutron beam inserts (Fig. 4.36) will contain the mirrored neutron guides required for virtually all the STS instruments. The insert assemblies will have a rigid outer frame that will accurately and stably contain the mirrored guide sections. A flange at the outer face will seal the insert to the vessel extension nozzle. Aluminum windows on both ends will contain a helium atmosphere inside the assembly. Precision alignment features mating with the vessel will accurately position neutron beam guides relative to the moderators.

Assuming the use of metal guides near the monolith to avoid radiation damage and adequate cooling, the current goal is to approach to within approximately $60 \mathrm{~cm}$ from the face of the moderators, resulting in a total insert length of up to $3.7 \mathrm{~m}$. Since monolith inserts are custom designed for each beam line, small guides (e.g., $3 \mathrm{~cm}$ wide) can potentially be mounted closer if it is determined that the guide mirrors will be reliably durable.

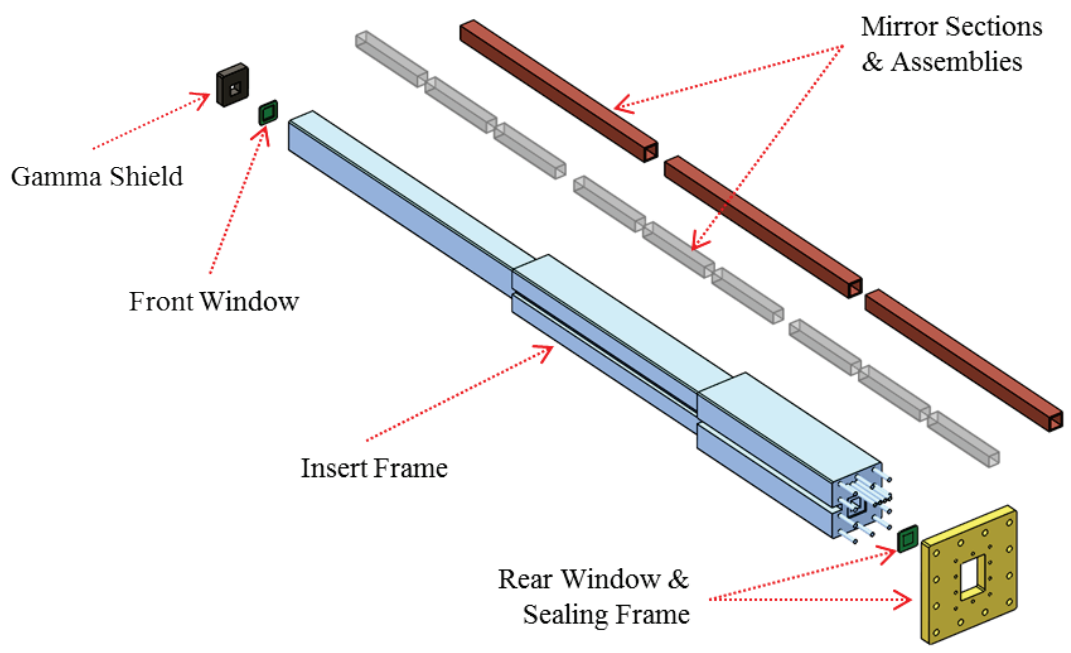

Fig. 4.36. Neutron beam line insert.

Proper alignment of beam line inserts will be critical to achieving the STS performance goals. Precision alignment features will mate the inserts inside the vessel and at the outside of the nozzle extension. As currently conceived, the insert and vessel will have fixed guide features that will engage as the insert moves into its final position. The guide features will be located by precision survey and alignment tooling after assembly of the monolith. It will be possible to make small adjustments to the ends of the nozzles; however, the vessel features will be permanent, as they are required to maintain a fixed relationship with the moderators.

Neutron beam guide inserts will be sealed at the ends of the core vessel nozzles with O-ring seals. These seals will constitute the outer perimeter of the vessel environment. The seal flange will be hands-on accessible for change-out and maintenance. Connections to the insert utilities (helium, cooling water, and instrumentation) will also be located at the flange.

Nuclear heating will be significant in the inner 1-2 $\mathrm{m}$ of the insert assembly; therefore, active water cooling will be required in this region. The mirrored guide sections will be cooled convectively with a helium gas atmosphere inside the cooled structure. This is particularly important since the guide mirror coating will deteriorate at temperatures above $\sim 100 \mathrm{C}$. The design goal is to maintain the temperature of 
the guides below $80^{\circ} \mathrm{C}$. The helium gas will be contained inside the insert structure with aluminum windows. The front window will be cooled conductively by the water-cooled structure. To prevent radiation damage to the guides, at least the first meter of the mirror guide will have an aluminum or copper substrate. The remaining sections will be a combination of metal, float, and borated glass.

A low-carbon steel shield extension will be installed on the front of the insert structure to absorb gamma radiation. The shield will have the same aperture as the front of the guide; however, it will not be included as part of the guide since it will not reflect neutrons. The shield extension will be about $2 \mathrm{~cm}$ long (Fig. 4.37) and it will have to be cooled to keep the maximum temperature below $120^{\circ} \mathrm{C}$.

At startup, STS will have eight instruments with fully functional guides. The active guides will be included as part of the instrument installations. Target systems will provide blank inserts for unoccupied beam lines. The blank inserts will weigh up to $2,700 \mathrm{~kg}$, whereas fully functional inserts will weigh less, depending on the size of the guide.

Replacement of neutron beam line inserts requires a significant amount of remote handling tooling. The tooling and operations involved are described in Sect. 4.9.

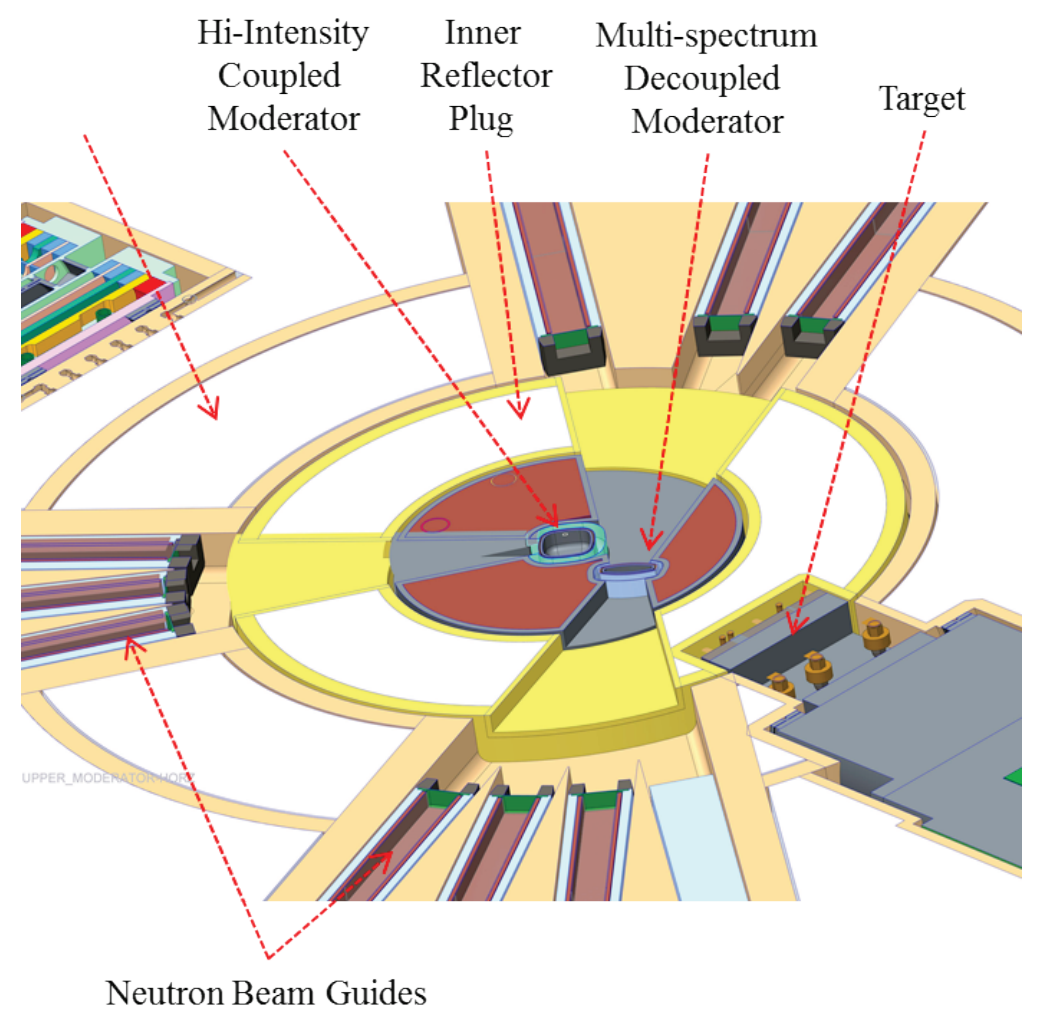

Fig. 4.37. Neutron beam guides inside vessel.

\subsection{SHIELDING}

A combination of metal and concrete shielding will protect personnel from the high-energy gammas and neutrons during beam-on operation throughout the STS facility. The target systems provide only the shielding which surrounds the core components. This shielding will be contained inside the conventional target building structure, which is also part of the shielding system. CF will also provide heavy concrete 
enclosures around the proton beam line, service cell, and other activated utilities. Individual neutron beam lines will provide local shielding as necessary.

The basic monolith shielding boundaries shown in Fig. 4.38 are based on an extrapolation of the FTS design information. The inner layer of actively cooled stainless steel shielding will be enclosed in the water-cooled core vessel. Stacked, passively cooled low-carbon steel will extend to a radius of approximately $4.4 \mathrm{~m}$ and a depth of approximately $1.5 \mathrm{~m}$ below the target, which will be largely contained within an iron liner. Additional layers of steel will be incorporated into the monolith foundation to a depth of around $3.5 \mathrm{~m}$. A detailed shielding analysis performed in the next design phase will refine this configuration.

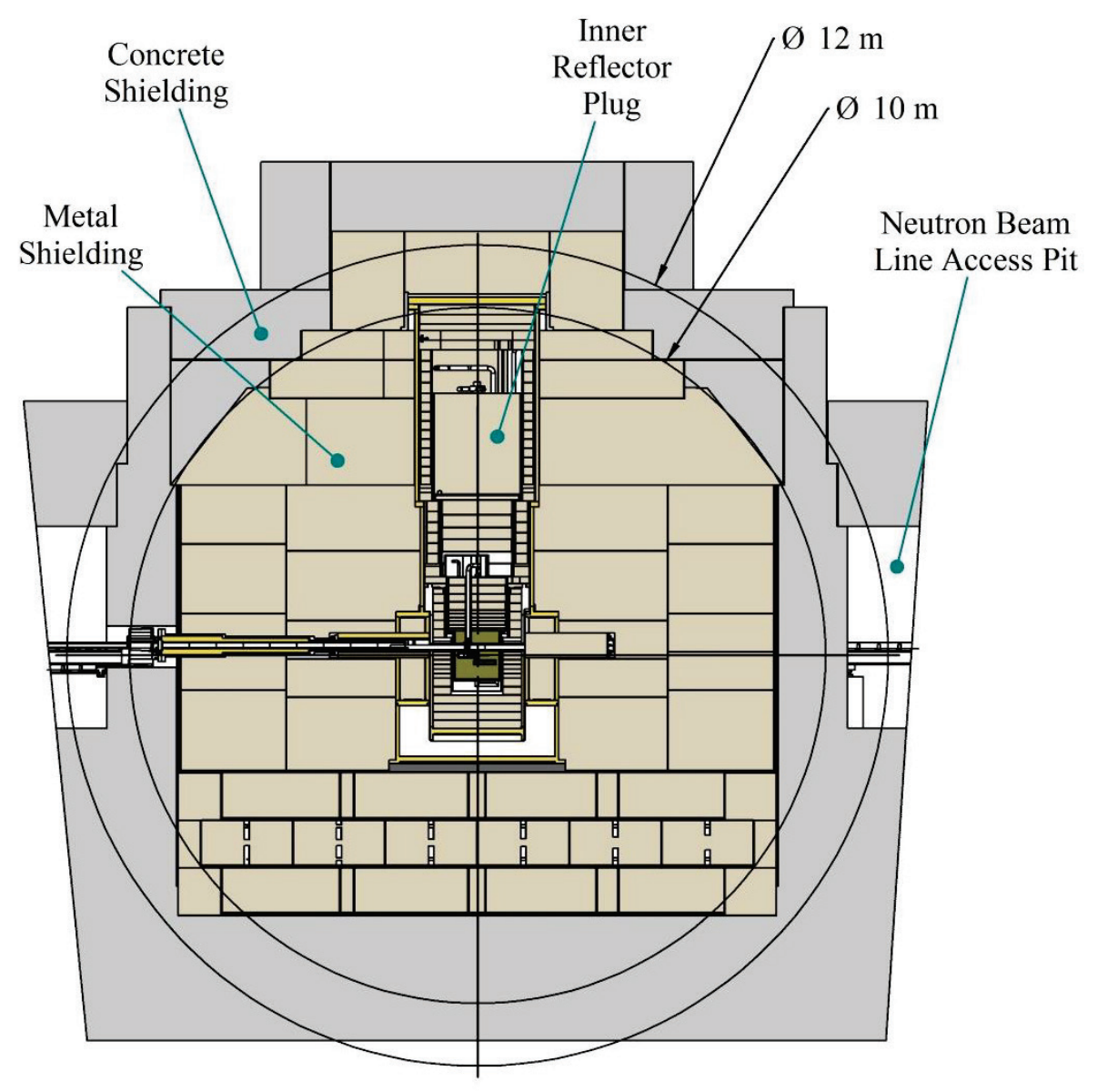

Fig. 4.38. Cross-section of target monolith with ideal shield boundaries defined.

The shielding configuration will surround but not touch the core vessel, the neutron beam line insert nozzles, target carriage tunnel, or proton beam tube. This will allow the shielding to shift as a result of thermal expansion and settling without adversely affecting the alignment of the critical systems.

\subsubsection{Lower Passive Shielding}

Approximately $3,000,000 \mathrm{Kg}$ of passively cooled metal shielding will be required. The shield stack effectively will have four layers (Fig. 4.39). The lowest layer will be composed of $650,000 \mathrm{Kg}$ of grouted recycled steel blocks (Fig. 4.40). These blocks will measure roughly $52 \times 52 \times 26$ in. and an estimated 72 will be required. Since the material may be contaminated, sizing and drilling will not be permitted. A 
tolerance of $\pm 1 / 2$ in. means the spacing will be irregular and a significant amount of high-density concrete grout will be required. This layer will serve primarily to prevent groundwater activation; therefore, use of the relatively inexpensive recycled material is appropriate. Note that this layer must be protected from significant nuclear heating, as cooling it will be difficult.

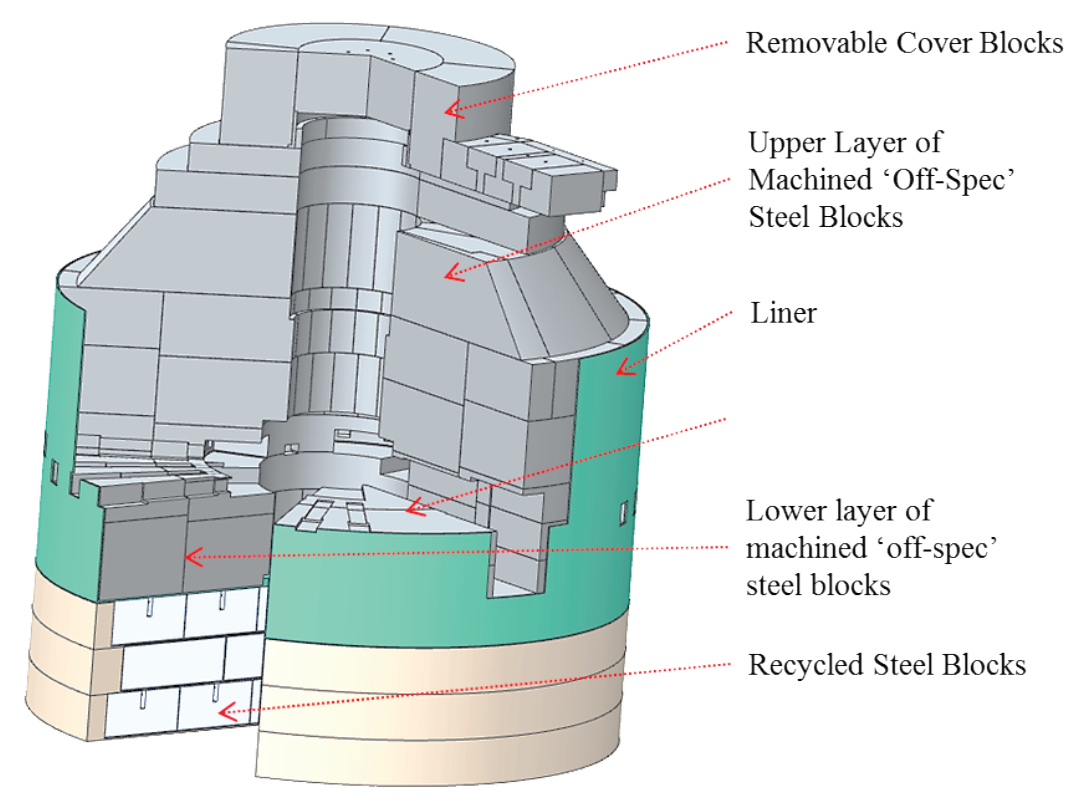

Fig. 4.39. Metal shield stack.

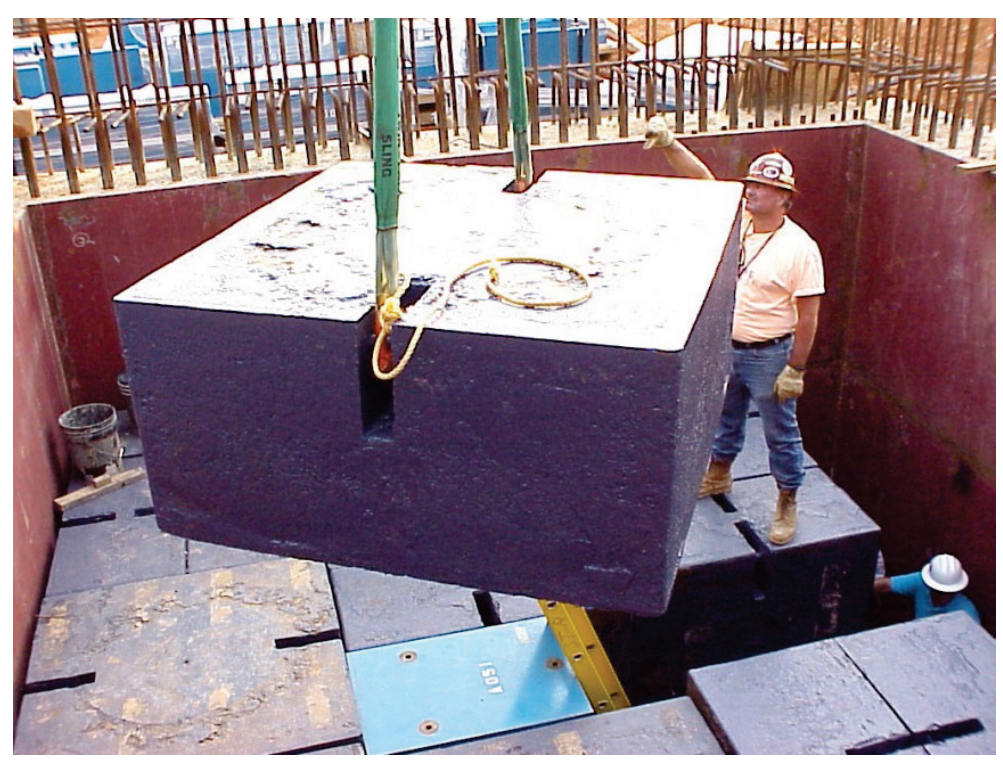

Fig. 4.40. Recycled steel block placement in FTS beam dump.

\subsubsection{Shield Liner}

The bulk iron shielding will be contained within an iron liner (Fig. 4.39). The liner will provide a vapor barrier and will be surrounded by high-density concrete with a minimum thickness of approximately $1 \mathrm{~m}$. Water leaks from the core vessel will be contained by this watertight barrier to a level of $0.5 \mathrm{~m}$ below the 
proton beam line. The liner will include features to channel spilled water to a low-point drain with a valved connection to the utility room drain tanks.

The shield liner will be assembled on the grouted shielding layer and along the inside of the surrounding concrete walls (Note: it is assumed that the building will have been partially completed before the shielding is installed). The core vessel mounting baseplate will be placed before the liner is installed. The baseplate must be precisely positioned and anchored, as it determines the ultimate location of the target, moderators, and neutron beam lines. After the baseplate is anchored, the liner will be seal-welded in a manner designed to prevent disturbing the location.

\subsubsection{Upper Shielding}

Shielding above the liner $(2,270,000 \mathrm{Kg})$ is designed to protect personnel. Therefore, a higher-quality machined fit will be required to prevent radiation shine. Consequently, the blocks in this section will be manufactured by a vendor with large milling capability. To contain costs, the blocks will be composed of welded plates of continuous rolling mill scrap formed during transitions between runs of materials with different specifications. This type of material is readily available and was used successfully in the SNS FTS.

After the second layer of shielding is installed, the vessel will be installed and aligned. At this point the vessel extensions (nozzles, PBW tube, and target tunnel) and shield will be installed in combination until all the extensions are covered. Clearances of approximately $1 \mathrm{~cm}$ will be required near the vessel and extensions to ensure the iron shield does not make contact, as thermal movement and foundation settling will occur over the life of the facility. The resulting gaps will be accounted for with streaming steps along the ports.

Once the relatively complex vessel interface shielding layer has been placed, the stack will be continued to the top of the monolith using relatively simple blocks with offsets designed to prevent radiation shine. The amount of shielding required for this layer will determine the basic height of the shield stack.

Additional removable shielding will be required above the vessel and cooling water piping chase where significant shine paths will be opened by the large number of cooling water pipes necessary to cool the core components.

\subsubsection{Shield Cap}

A $1 \mathrm{~m}$ (minimum) thick layer of concrete shielding will installed on top of the iron shield stack. While most of this layer will be permanent, removable sections will be placed above the vessel, PBW, and cooling water chase.

\subsubsection{Ventilation and Heat Removal}

Virtually all the process energy will be absorbed and removed inside the vessel and target assembly; however, a small amount ( 1 to $4 \mathrm{~kW}$ ) will deposit in the iron shield stack. Consequently, ventilation passages will be built into the stack to allow for convective air cooling. For a $10^{\circ} \mathrm{C}$ temperature rise, several hundred cubic feet/minute of air will be required to limit the temperature rise in the shield stack. Since there is a small chance the air could become contaminated, the discharge will be vented to the primary containment exhaust system. Heating of the lower iron shield layer and foundation concrete will be evaluated later in the design process. A temperature rise of more than few degrees in the grouted shielding could damage the closely packed assembly. 


\subsection{TARGET SYSTEMS INSTALLATION}

The target systems will be closely integrated into the target building; therefore, to ensure efficient coordination and accurate fit-up, a single contractor will be charged with coordinating the installations of all the target systems components. In general, the target systems installations can be segregated into four packages: monolith components, utilities, cryogenic systems, and service cell.

\subsubsection{Monolith Installation Requirements}

All monolith components are being designed to be handled by the either a mobile construction crane or the target building 50 ton crane. This design will enable installation of the target systems to begin before the target building is complete, although a temporary roof will be required to prevent direct exposure to rain. Before placement of the IRP, more stringent environmental control requirements will have to be in place; therefore, the building structure will have to be complete.

The building is assumed to have a maximum settlement tolerance of approximately $1 \mathrm{~cm}$ as it is loaded. This is greater than the expected tolerance for the alignment of the neutron beam line interfaces. Therefore, it will be necessary to complete the shielding stack-up as soon as possible to fully load the target building foundation before installation of the neutron beam lines. This will make possible precise alignment of the neutron beam ports and other vessel extensions without the need to compensate for any building movement.

There are several other alignment considerations that were successfully handled in the SNS FTS and are therefore assumed to be manageable for the STS. The horizontal clocking angle of the vessel and tilt of the vessel will be limited to about $\pm 0.01^{\circ}$. This deviation will result in an offset at a $90 \mathrm{~m}$ instrument of about $16 \mathrm{~cm}$. Angular misalignments of this magnitude will be corrected with the beam line guide elements. "Construction drop-outs" have continued to be a settling problem for the FTS. This is particularly a concern if it will ultimately cause the target building to settle nonuniformly. It is assumed that the drop-outs will be adequately reinforced to prevent a significant problem in the stability of the monolith.

\subsubsection{Monolith Installation Sequence}

The general installation sequence for the monolith core components illustrates the timing and nature of the operations involved (see Fig. 4.41). Installation of the target systems begins once the conventional construction contractor has completed the placement of the monolith foundation and begun to form the perimeter walls. The first step will be to place three layers of recycled steel blocks on the foundation and fix them in place using a high-density grout. The blocks will be fitted with offsets to eliminate gaps through the full stack. The grout will be a flowable, nonshrinking, high-density concrete.

Installation of the monolith liner inside the concrete foundation can begin once the CF installation has progressed to the placement of the concrete for the service cell and proton beam shields, as shown in Fig. 4.42. The liner base will be anchored as necessary to act as a seismic restraint for the upper shield. The vessel interface plate will also be precisely positioned and leveled, since its position will determine the ultimate alignment of the vessel. Because the size of the liner, it will be shipped in sections and weldassembled in place. 


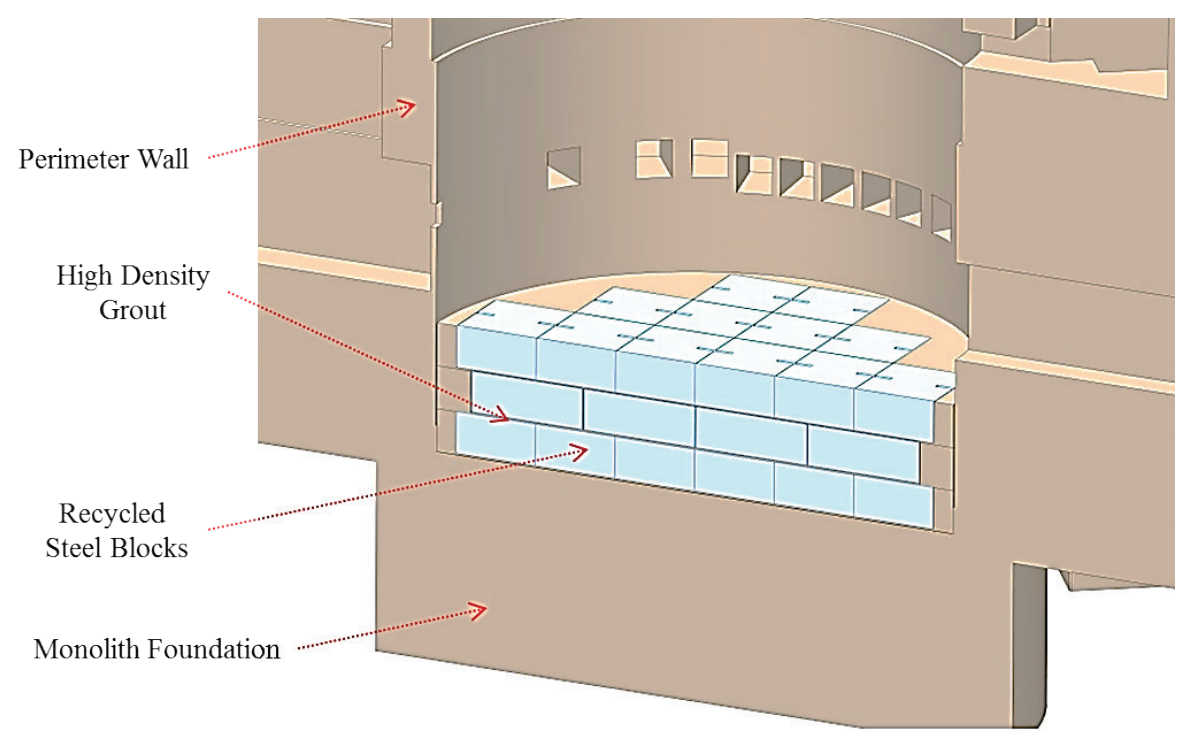

Fig. 4.41. Installation of recycled steel in base of monolith.

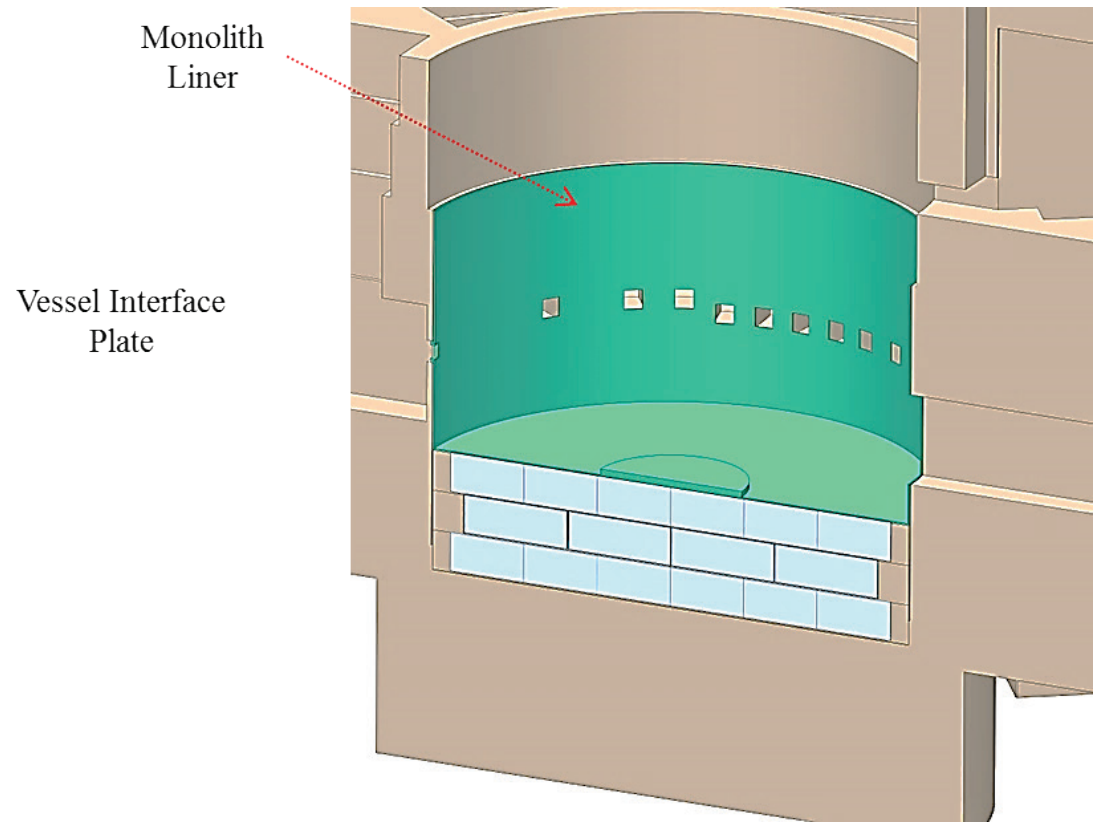

Fig. 4.42. Installation of monolith liner.

The bulk shielding and vessel require concurrent assembly because of the nozzle overlaps. Initially, machined shielding will be added to just below the vessel nozzle level. The shielding blocks will be seismically anchored to the liner and heat removal ventilation features will be added as required. Note that the shielding will have approximately 0.5 in. of clearance to the liner to facilitate installation and account for tolerance stack-ups. With the first layer of shielding in place, the vessel can be placed and anchored to the liner base. Alignment of the vessel will have been established during placement of the liner base plate; however, it will be checked and corrections made at this time. Vessel nozzles, target tunnel, and proton beam pipe will be installed at this point (Fig. 4.43). The remaining upper shield blocks 
will then be added symmetrically as shown in Fig. 4.44 (PBW cavity, shield ventilation, and monitoring features added as required). The upper shield blocks must be seismically anchored.

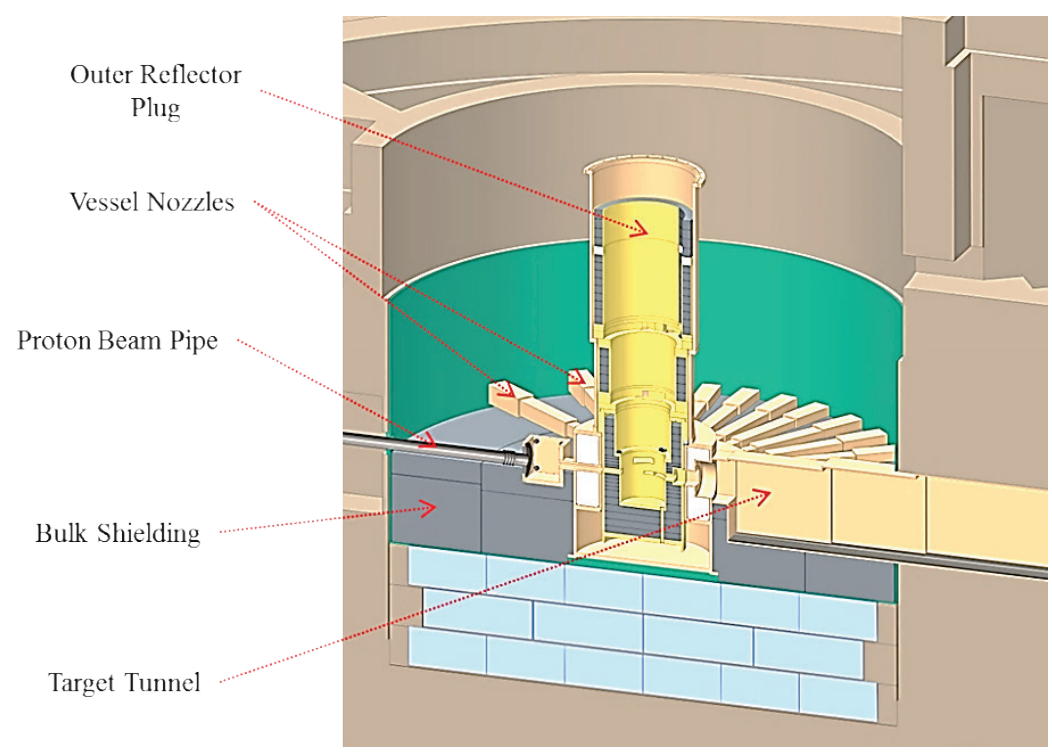

Fig. 4.43. Installation of lower bulk shielding and vessel.

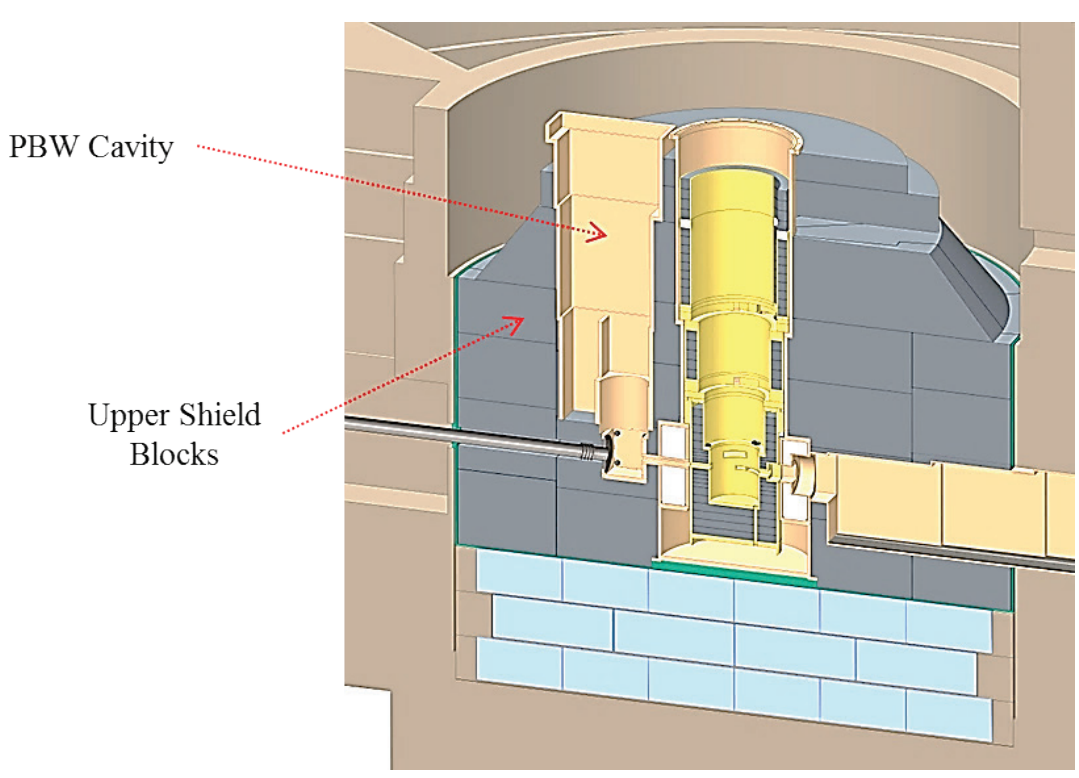

Fig. 4.44. Installation of upper bulk shielding and proton beam window liner.

The utility tray will be installed on top of the completed bulk shield stack. The tray will be configured to drain toward the pipe chase. The fixed bulk shield will then be completed with the placement of the in place concrete shielding on top of the iron shield (Fig. 4.45). Shield ventilation pipes and ducts will be integrated into the concrete cap as necessary. 


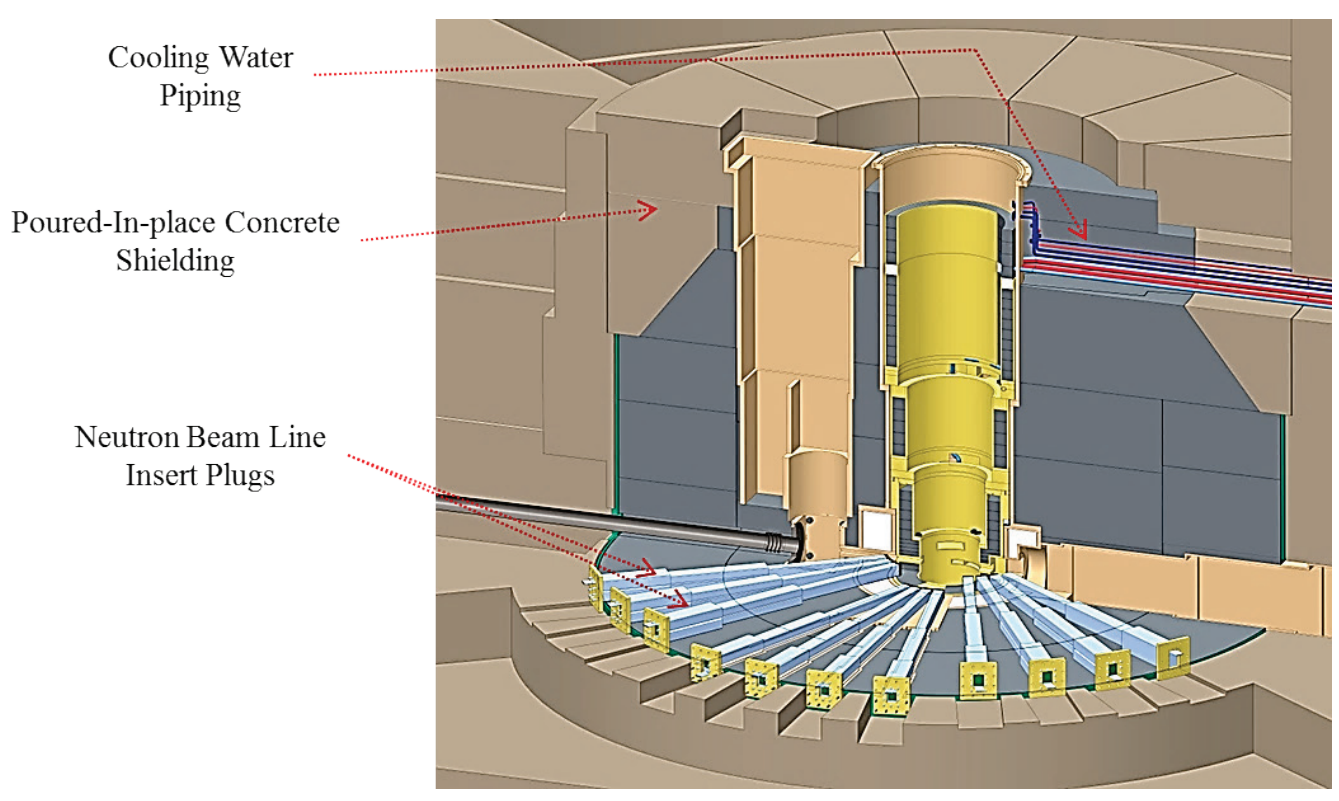

Fig. 4.45. Installation of poured-in-place concrete.

Installations after the completion of the fixed shielding will become more diverse and will generally require that the building protect the components from environmental exposure. This work will include the installation of the cooling water utility piping and the placement of the blank neutron beam line inserts (see Fig. 4.45). Final alignment of the nozzles will also take place at this time to account for shifts in the building due to the placement of the shielding.

\subsubsection{Utilities Installation}

The target systems coordinating contractor will ensure the conventional unities systems are closely integrated into the target building. Components such as piping mounting brackets, tank floor mounting plates, and chase supports will be installed directly into the building structure. The building general contractor will provide services such as electrical power and controls networks.

Installation of the target systems utilities can begin with the start of the target building, because the pumping systems will be located in the basement. The water storage tanks are likely to be the lowest components in the building and will be installed in cavities in the basement floor.

\subsubsection{Service Cell Components Installation}

Technical components provided by target systems for installation in the service cell will be placed as the cell is constructed. For example, many permanent features will be cast into the concrete cell walls. These include the shield window liners, shielded utility pass-throughs, wall brackets, target cart rail bases, and bases for the bridge rails. After the service cell and target building are nearly complete, installation of the target systems technical components will begin. Owing to the nature of the remote components, this will involve extensive electrical and controls interfaces.

\subsubsection{Cryogenic Systems Installation}

The cryogenic systems will be installed after the target building has been enclosed. The hydrogen system components will be installed in the HUR. The helium refrigerator will be installed in the target building near the HUR similar to the arrangement in the FTS (see Fig. 4.26). The major components of the 
cryogenic system (helium refrigerator cold box, helium compressors, oil removal systems, gas storage tanks, heat exchanger module, and pump module) are being designed to be lifted and placed via crane and/or forklift. The helium compressors, oil removal systems, and gas storage will be installed in a separate compressor building adjacent to the target building. Cryogenic transfer lines will be partially assembled and then craned into position. Transfer line fluid connections, including some welded portions and flexible sections, will be made in situ. The gas management systems and safety relief systems for the hydrogen and helium systems will also be partially assembled and then finished in the HUR and compressor building, respectively. Non-cryogenic gas lines, cooling water lines, and oil return lines will be assembled and connected after the major components are installed.

\subsubsection{Commissioning}

ORNL operators will perform the final commissioning of the functional target systems (utilities, cryogenics, and remote handling). This work will begin after the systems have been inspected and accepted as complete.

\subsection{TARGET SYSTEMS UTILITIES}

There will be four primary activated cooling water loops servicing target components, three heavy water stations, and one light water station. Included in these systems will be components such as drain tanks, piping assemblies, and drain lines that will be fabricated by specialty shops.

\subsubsection{Target Cooling Water System}

Pre-conceptual design basis activated cooling water heat loads are summarized in Table 4.4. The design basis heat loads for the target and target shroud were estimated for the STS design based on the preliminary neutronics analysis. The remaining heat loads were scaled based on the heat loads for comparable technical components in the FTS operating at a beam power of $1.32 \mathrm{MW}$. Design safety factors were applied to all the heat loads. The factors applied were loosely based on the design safety factors used in the design of the FTS.

Table 4.4. Second Target Station design basis cooling water heat loads

\begin{tabular}{cccc}
\hline $\begin{array}{c}\text { Activated cooling } \\
\text { water loop }\end{array}$ & $\begin{array}{c}\text { Design basis heat } \\
\text { load (kW) }\end{array}$ & Water type & $\begin{array}{c}\text { Ultimate } \\
\text { heat sink }\end{array}$ \\
\hline Loop 1 & 309 & $\mathrm{D}_{2} \mathrm{O}$ & $\mathrm{TW}$ \\
Loop 2 & 28 & $\mathrm{D}_{2} \mathrm{O}$ & TW \\
Loop 3 & 69 & $\mathrm{D}_{2} \mathrm{O}$ & CHW \\
Loop 4 & 190 & $\mathrm{H}_{2} \mathrm{O}$ & TW \\
\hline
\end{tabular}

\section{Primary Cooling Water Loops}

A simplified block flow diagram representing the primary components in an activated target station primary cooling water loop is depicted in Fig. 4.46. The four primary loops are described in the following paragraphs.

Heavy water cooling loop 1 will remove heat from the target and the PBW. Water in loop 1 will be directly exposed to the proton beam and neutron radiation and will be significantly activated. Combining the target and PBW loops serves a safety function wherein a flow failure will result in a mechanical 
failure of the window, which will ensure a proton beam shutdown. This feature will act as a backup to the normal target protection system.

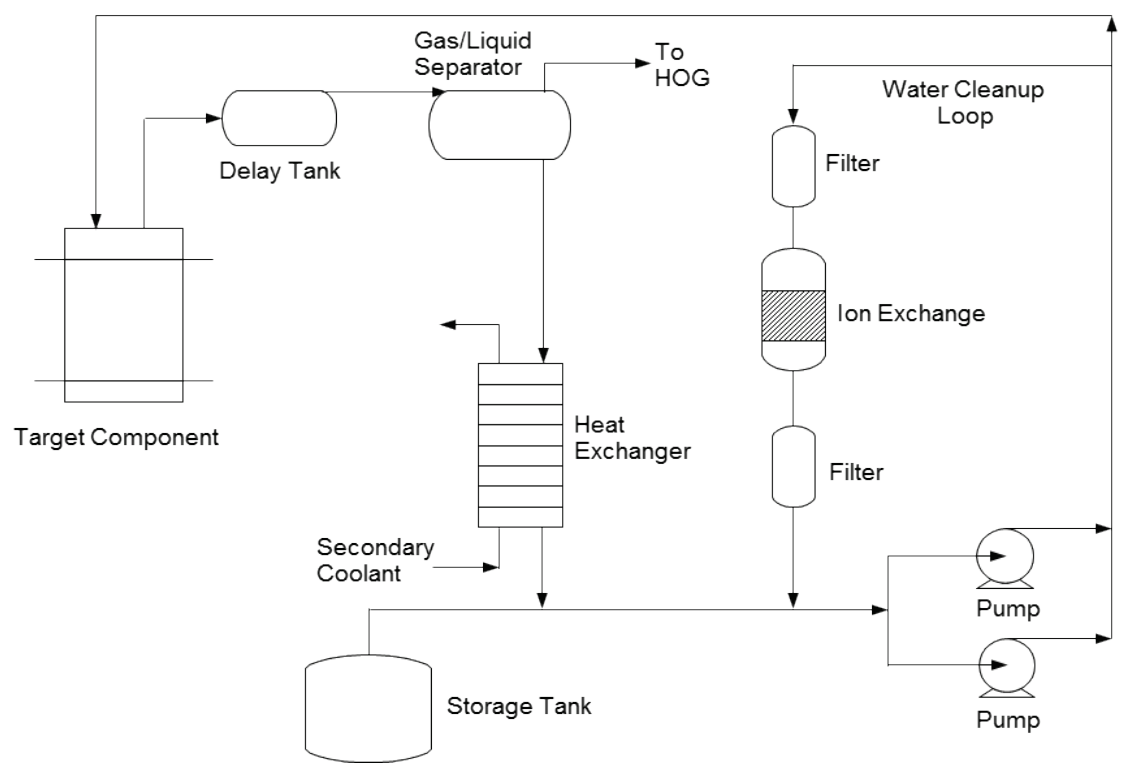

Fig. 4.46. Primary cooling loop block diagram.

Heavy water cooling loop 2 will remove heat from the target shroud. Water in loop 2 will be directly exposed to the proton beam and neutron radiation and will be significantly activated. This loop will serve as an independent decay heat removal system for the target with the proton beam off. It will have an uninterruptible power source backup.

Heavy water cooling loop 3 will remove heat from the lower IRP. The loop 3 water will be primarily exposed to a neutron radiation environment. It was assumed that this loop would be cooled by a chilled $70^{\circ} \mathrm{F}$ deionized (DI) water loop, but it may instead be cooled by a secondary $87^{\circ} \mathrm{F}$ loop that rejects heat directly to the tower water system.

Light water cooling loop 4 will remove heat from the vessel wall, outer plugs, intermediate inner plug, target beam stop, PBW box, pre-moderators, and neutron beam line inserts. Loop 4 water will be primarily exposed to a neutron radiation environment.

Components or features not included in or unique to individual loops are not identified. During normal operation, water circulates in a closed loop independent of the storage tank. It is expected that discharge to the storage tank will be performed only if required (e.g., because of an abnormal occurrence or to facilitate maintenance of loop equipment). Each of the primary target cooling water loops will reject heat to a secondary cooling water system. Delay tanks will be employed in the return line of each activated water loop to facilitate localized decay of some of the short-lived gamma and beta activity. The delay will also reduce the potential for neutron activation of components located in areas to which access must be provided for maintenance. Localized shielding will be provided as required to address the anticipated deposition of the longer-lived activation product radionuclides such as Be-7 in system components (e.g., heat exchangers, ion exchange units, and filters). Separation of gases generated in the water loops as a result of spallation and/or the radiolytic decomposition of water will be achieved in a gas/liquid separation tank to be located at the high point in the cooling loop. The deuterium and oxygen produced by radiolysis of the heavy water will be recombined to reduce heavy water losses to the hot off-gas system. The activation gases will be vented to the hot off-gas system provided by CF. 
Components successfully used in the FTS water loops will be considered for applicability in the STS to reduce spare parts inventory and required maintenance diversity (e.g., ion exchange columns, filters, valves, pumps, instruments).

\section{Secondary Cooling Water Loops}

Primary cooling loops 1,2 , and 4 will reject their heat to a secondary $87^{\circ} \mathrm{F}$ DI water cooling loop, which in turn will reject heat to the $82^{\circ} \mathrm{F}$ tower water system. Cooling loop 3 will reject its heat to a chilled $70^{\circ} \mathrm{F}$ DI water cooling system, which will in turn reject heat to the $45^{\circ} \mathrm{F}$ chilled water system. If a chilled $70^{\circ} \mathrm{F}$ DI water loop is not needed, Loop 3 may be cooled by either the main secondary $87^{\circ} \mathrm{F}$ DI loop or an independent secondary $87^{\circ} \mathrm{F}$ DI loop that may be provided for operational reliability. Additionally, a $55^{\circ} \mathrm{F}$ sensible chilled water system will be provided to cool neutron beam line components and associated equipment. This cooling loop will reject heat to the $45^{\circ} \mathrm{F}$ chilled water system. These secondary loops will be provided by CF.

\subsubsection{Target Utility System Layout}

The primary and secondary cooling loops will be located in a shielded room in the basement of the target building (Fig. 4.47). The delay tank and gas/liquid separator tank cavities and the core vessel insert pipe pan will drain by gravity to the top of the vertical chases that run from the top of the shield stack to the basement. As liquids accumulate in the double-wall drain line, they will drain to a collection tank located in the basement, where the verification will be made as to whether the water is heavy or light or a combination of both.

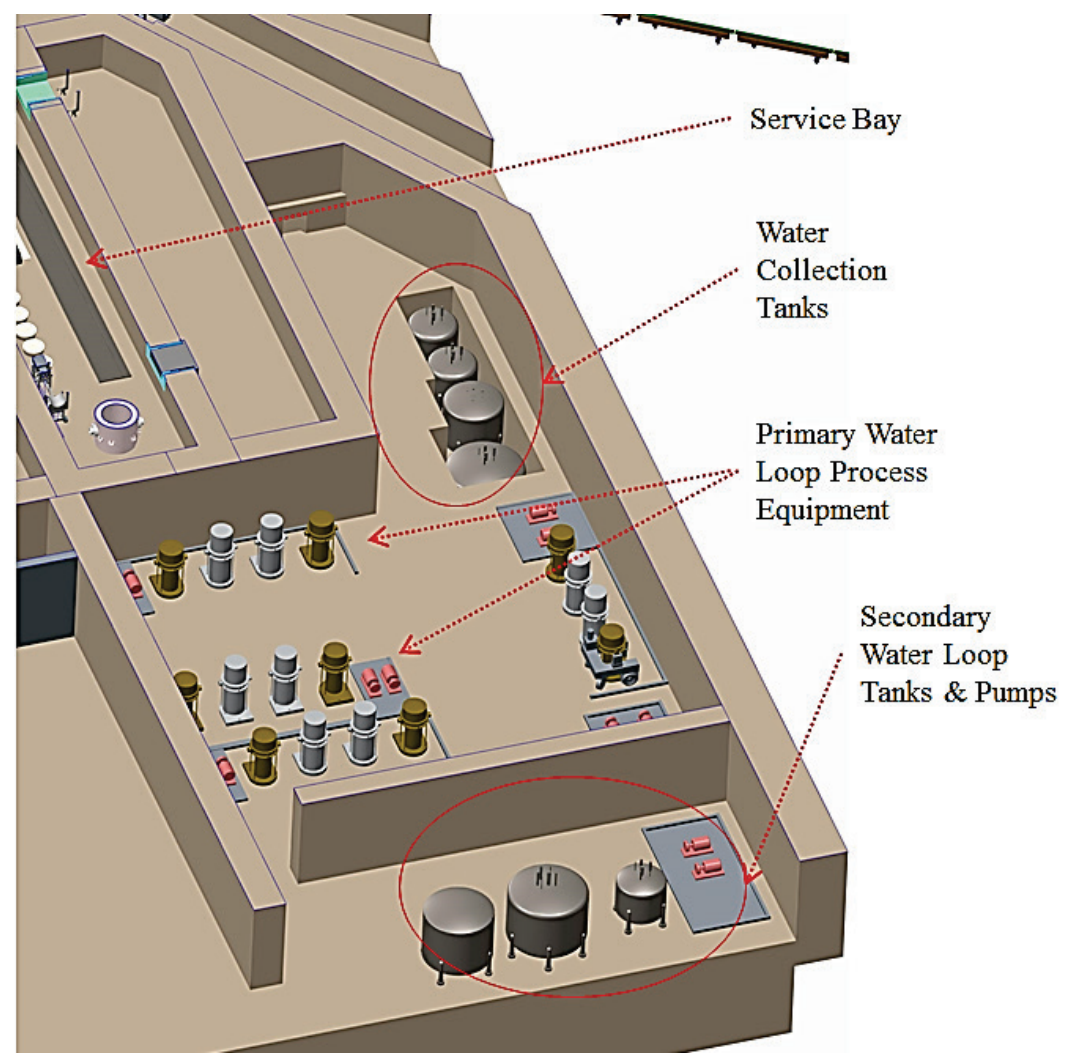

Fig. 4.47. Utility systems in the target building basement. 
The core vessel will be equipped with a double-wall drain pipe used to direct water that drains into the core vessel to a collection tank for detection and disposition.

The bulk shielding liner will also be equipped with a double-wall drain pipe used to collect any liquids that drain from the bulk shielding liner. Liquid detection and a quick disconnect fitting will be provided on the end of the drain line to facilitate detection and transfer of the accumulated liquid to an alternate location.

Drain tanks will be provided in pits in the target basement to facilitate draining of the corresponding water loops for technical component removal or maintenance.

Prefabricated pipe chases will be provided to accommodate the routing of utilities between the basement and the top of the shield stack and between the tank cavities and the top of the shield stack. Because of the high levels of short-lived radiation, the return cooling water lines and delay tanks require approximately $1.25 \mathrm{~m}$ of high-density concrete shielding.

\subsubsection{Inert Gas Systems}

The core vessel will normally operate with a vacuum atmosphere to limit interference with neutron beams while providing a limited ability to eliminate or remove harmful gases or liquids that can be generated by small water leaks. It will also be possible to operate the vessel with a low-pressure helium atmosphere. If the normal operating mode shifts to the helium mode, a helium recycling system may be considered.

\section{Helium Distribution}

Helium gas will be supplied to the STS by CF (WBS 6.2.2.3). WBS 6.2.2.3 shall provide the regulation/metering, the main gas supply line into the target building, and the main distribution headers within the target building. The gaseous helium supply from the tube trailers will have a minimum purity of $99.995 \%$.

Target utility systems (WBS 6.3.8.3) will distribute the helium to various end users within the target building (e.g., core vessel, inflatable seals, core vessel inserts).

\section{Nitrogen Distribution}

Nitrogen gas will be supplied to the STS by CF (WBS 6.2.2.3). WBS 6.2.2.3 shall provide the liquid nitrogen tank and regulation, vacuum-jacketed supply lines, gas flowmeter, main supply lines, and main distribution headers within the target building. The gaseous nitrogen supply from the liquid nitrogen storage tank and vaporizer will have a minimum purity of $99.998 \%$. The capability to refill liquid dewars at an outdoor refilling station adjacent to the liquid nitrogen storage tank also will be provided by $\mathrm{CF}$ WBS 6.2.2.3.

Target utility systems will distribute the nitrogen to various target group end users within the target building (e.g., gas/liquid separation tanks). Nitrogen will be used as a purge-and-cover gas in areas exposed to high-energy radiation to minimize the production of activated and corrosive gases and to prevent flammable gas mixtures from reaching the lower explosive limit concentration. Nitrogen will also be used to purge and backfill process systems before the cooling water loops are drained and filled with water, purge trapped volumes of cooling water from target components, and purge hydrogen safe vent stacks to minimize the potential for air ingress. 


\section{Core Vessel Inerting System}

The normal operating environment of the core vessel will range from full vacuum $\left(\sim 10^{-3}\right.$ torr $)$ to a helium environment at slightly below atmospheric pressure (approximately $-30 \mathrm{in}$. water gauge). The core vessel inerting system will consist of a regulated helium supply and an air ejector controlled to maintain a slight vacuum within the core vessel during the helium mode of operation. Before a transition to the helium mode of operation, the core vessel vacuum pumps will be used to remove air and to make a general assessment of seal integrity. The exhaust from the vacuum pumps and the ejector will be routed to the primary confinement exhaust system provided by WBS 6.2.2.3.

A residual gas analyzer will be provided in the core vessel vent line to monitor the composition of the gas leaving the core vessel in either vacuum or helium mode.

In the event that water is detected in the core vessel, the core vessel will be isolated and, in some cases, the vessel off-gas will be redirected to the hydrogen safe vent stack. A rupture disk will also be provided on the core vessel by WBS 6.3.5 to protect the vessel against overpressurization.

\subsubsection{Gas Recombination and Recycling System}

The preliminary conceptual design of a recombination/recycling system involves using a catalyst bed recombiner to recombine the deuterium and oxygen formed by radiolysis in the heavy water loops. An inert gas recycling system will be employed to further reduce losses to the stack. As with the FTS, gas will be vented from the loop to maintain the loop pressure within an acceptable operating range. To be conservative, the recombination/recycle equipment sizing was based on the maximum observed " $\mathrm{G}$ factor" calculated from FTS operating data, scaled to $0.5 \mathrm{MW}$ beam power and with the gas concentration controlled to $1.5 \%$ D2 (with "G factor" being g-moles D2 produced per $100 \mathrm{eV}$ of energy deposited in the water). Recycling the purge gas has a side benefit of reducing the total gas flow through the hot off-gas decay tank, thereby increasing residence time. A significant beneficial consequence is that the increased residence time associated with recycling the gas will result in lower stack emissions. Refer to CF Sect. 6.2.2.8 for details on the hot off-gas decay system.

\subsection{MAINTENANCE SYSTEMS}

\subsubsection{Design Guidelines and Assumptions}

SNS has an overall operating availability factor of $90 \%$. To achieve this level of availability, the STS target systems design is focused on reliability, accessibility, and remote handling. Several factors assist with this effort. The most important of these is the solid metal target cooled by heavy water. As has been demonstrated at several facilities worldwide, personnel are able to accomplish many maintenance tasks on the process systems for solid targets within hours of a beam shutdown. This eliminates many remote handling functions that would be required for alternative target designs (e.g., mercury or eutectic). To simplify handling and maintenance, virtually all activated equipment and remote handling tasks will be located inside the target building with access only by operational personnel. The target building will be equipped with full-span bridge cranes to simplify and speed material transfers. The reduced handling is also expected to improve safety. Finally, neutron beam line access has been simplified by minimizing the complexity of the shielding. This will provide fast, efficient access to the choppers and shutters.

Although personnel access is allowed to most areas when the beam is off, four main areas will require (or are likely to require) remote handling, or at least long-handled tools, to perform tasks because of expected high radiation levels at the time of certain operations (see Fig. 4.48). Those areas are (1) the service bay which is designed mainly for handling the target change-out and storage operations; (2) the main bay for 
handling the IRP, PBW, monolith insert plugs, secondary shutters, and choppers; (3) the basement, which will house activated utilities; and (4) the activated waste disposal area for packaging of activated wastes for transport to permanent storage sites.

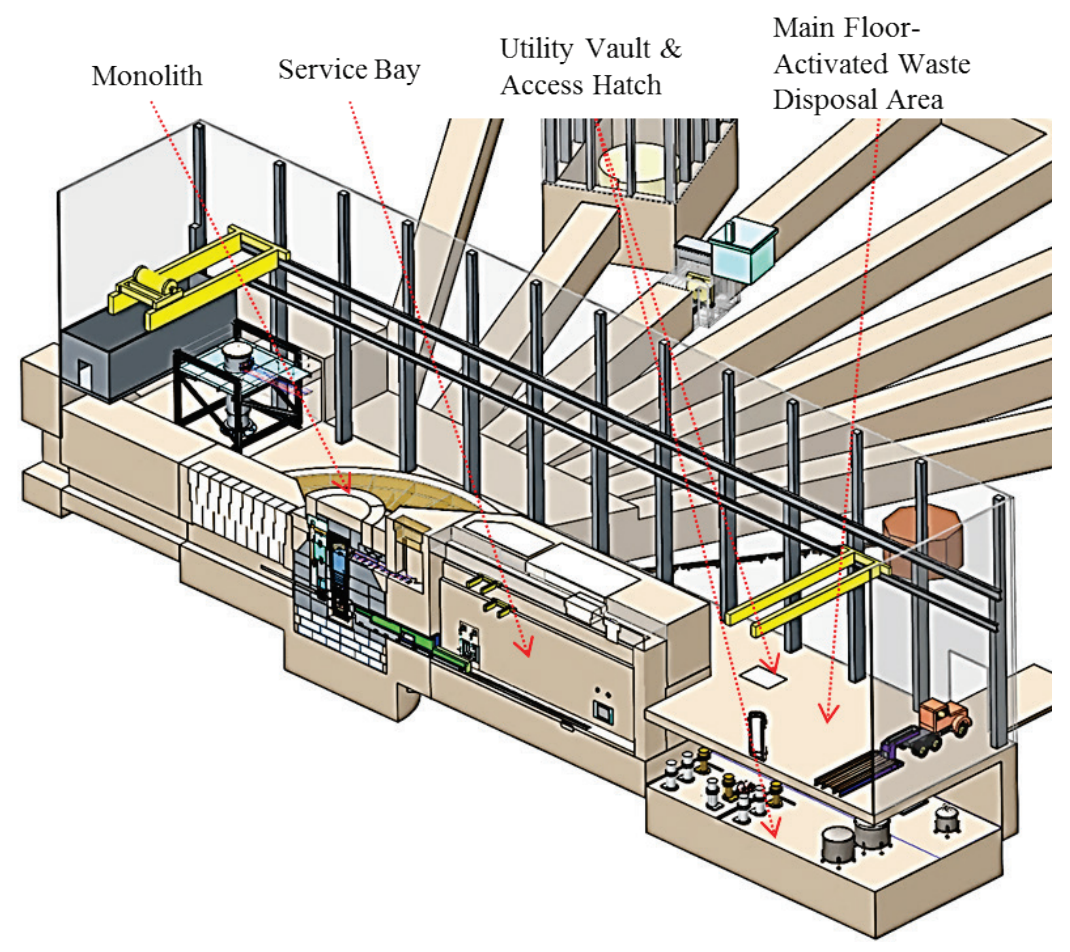

Fig. 4.48. STS remote handling areas.

All work performed in STS will be controlled so that the radiation exposure to personnel will not exceed the established ORNL limits shown in Table 4.5. To meet this goal, shielded tooling is generally designed to limit personnel doses to less than $5 \mathrm{mrem} / \mathrm{h}$ at $30 \mathrm{~cm}$. Where possible, proven FTS tooling will be used and shielding for newly designed tooling will incorporate the experience gained from FTS remote handling operations.

Table 4.5. Established ORNL limits for personnel exposure

\begin{tabular}{rcc}
\hline \multirow{2}{*}{ Parameters } & \multicolumn{2}{c}{ ORNL limits } \\
\cline { 2 - 3 } & mrem / year & mrem/hour \\
\hline Employee time unlimited & 500 & \\
Employee time limited & \\
$<500$ hours & 0.50 \\
$<200$ hours & 1.25 \\
$<100$ hours & 2.50 \\
\hline
\end{tabular}

Guidelines have also been established for the level of remote tooling development expected (Table 4.6). As can be seen in the table, only likely or critical operational needs will be met at startup. Tools and operations of lower priority will be developed to an appropriate level at which cost and risk are balanced. 
Table 4.6. SNS Second Target Station remote handling design guidelines

\begin{tabular}{|c|c|c|c|c|}
\hline $\begin{array}{c}\text { Failure } \\
\text { consequence }\end{array}$ & $\begin{array}{c}\text { Expected } \\
\text { component life }\end{array}$ & RH tooling design & $\begin{array}{c}\text { RH tools and fixtures status } \\
\text { at startup }\end{array}$ & Mockup testing \\
\hline \multirow[t]{4}{*}{ High } & $<1$ year & Complete & Fabricated before SNS startup & $\begin{array}{l}\text { Functional cold } \\
\text { test }\end{array}$ \\
\hline & 1 to 5 years & Complete & Determined in review & $\begin{array}{l}\text { Determined in } \\
\text { review }\end{array}$ \\
\hline & 5 to 10 years & Preliminary & Limited fabrication & None \\
\hline & $>10$ years & Concept only & No fabrication expected & None \\
\hline \multirow[t]{3}{*}{ Moderate } & $<1$ year & Complete & Determined in review & $\begin{array}{l}\text { Determined in } \\
\text { review }\end{array}$ \\
\hline & 1 to 5 years & Preliminary & Limited fabrication & None \\
\hline & $>5$ years & Concept only & No fabrication expected & None \\
\hline \multirow[t]{2}{*}{ Low } & $<1$ year & Preliminary & Limited fabrication & None \\
\hline & $>1$ year & Concept only & No fabrication expected & None \\
\hline Very Low & - & Concept only & No fabrication expected & None \\
\hline
\end{tabular}

\subsubsection{Service Bay}

The service bay is primarily required for the remote change-out and storage of spent targets, so its cell design is focused on those operations. Significantly, the bay will also be accessible for hands-on operations when the proton beam is off and the target is in the monolith.

\section{Service Bay Layout}

The service bay will have four general areas: target change-out station, target cart retraction zone, storage, and overhead tooling systems. The target change-out window workstations will be located at the west end of the cell as close to the monolith as possible while providing adequate space to remove/install the through-wall manipulators. This position will also be optimized in conjunction with the amount of cart retraction to maximize manipulator maintenance, operator access for remote operations, and crane access for remote handling at the west end of the bay. The overall length of the bay is determined by the cart retraction zone and the storage area at the back end of the cell. The width is determined by the window workstation access requirements and space required for the storage wells. The height of the bay is determined by the overhead tooling systems (bridge crane and servo) clearance over the in-cell components, particularly the TN-RAM shipping cask. STS targets are expected have a contact dose level similar to that for the FTS. This level requires a personnel shield thickness of approximately $1 \mathrm{~m}$ of highdensity concrete. The roof will have two access openings, one for shipping casks such as TN_RAM and other shielded casks and the other for the cranes.

\section{Target Change-out Station}

The STS service bay will have two window workstations at the west end of the bay, one on each wall, and a provision for future installation of a third window workstation near the center of the bay (Fig. 4.49). Each workstation will consist of a lead-shielded window, two through-the-wall manipulators, and utility feedthroughs. The third window workstation will include a utility tray similar to window workstation 3 in the FTS service bay. The reach of the manipulators will be limited to the immediate vicinity of the stations. Therefore, the two primary window workstations will be dedicated to target change-out operations and are designed to provide the following main functions: 
- connect and disconnect the utility jumpers/flanges

- work in conjunction with the 5 ton crane (see Fig. 4.49) to position, align and operate change-out carts

- work in conjunction with the bridge-mounted power arm to remove, install and torque fasteners

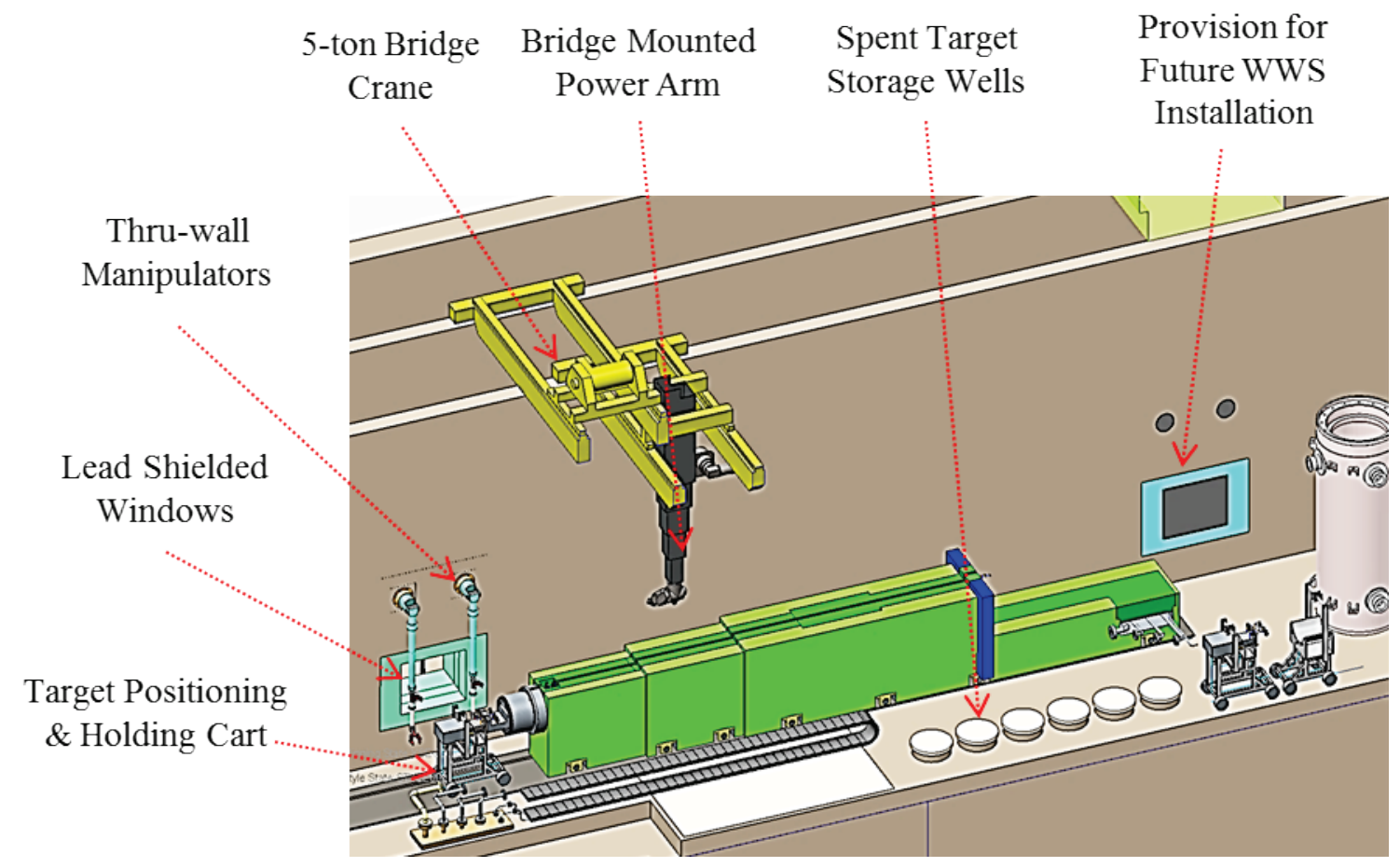

Fig. 4.49. Service bay and tools.

The through-the-wall manipulators will be operated by workers occupying the manipulator gallery. The ORNL de facto standard, Central Research Laboratories Model F mechanically linked master-slave manipulator pairs, will be specified. The manipulators will be useful for tasks requiring high dexterity, such as handling wrenches and small fixtures and performing inspections with swipes or closed-circuit cameras. The maximum capacity of the manipulators will be $\sim 50 \mathrm{lb}$.

\section{Overhead Tooling Systems}

In addition to the through-the-wall manipulators, a bridge crane-mounted telerobotic servomanipulator system will be provided for remote handling tasks inside the service bay. A telerobotic servomanipulator system (PaR system M3000/TR4350 power arm or equivalent) will be mounted on the end of a telescopic boom attached to a two axis bridge. The power arm will have a vertical travel, allowing activities between floor level and approximately $13 \mathrm{ft}$ above the bay floor over the full floor area of the bay. The power arm will be rated for a maximum load of $100 \mathrm{lb}$ for M3000 (225 lb for TR4350). The system will be used throughout the bay to handle tools and to lift fixtures and other loads. The manipulator can be fitted with conventional parallel pinch grips, a hook, and tool-holding hands.

Above the servomanipulator system, a 5 ton overhead bridge crane will provide coverage to handle most loads inside the service bay area. It will have a powered rotation feature to enable hook placement. Generally, this feature will involve only components with lifting attachments designed to mate with the crane hook. Identified loads include shielding components, used process components, change-out carts associated with target replacement, new target and intermediate plug assemblies, waste-handling TN- 
RAM liners with and without loads, and spent target assemblies. The vertical hook height of the crane will be approximately $22 \mathrm{ft}$ above the floor level. The overall hook travel will be approximately $28 \mathrm{ft}$ to enable the crane to reach below the target service bay floor level to allow access inside the storage tank pit. The hook will be able to approach to within $\sim 12$ in. of all of the service bay walls.

The crane, servomanipulator, and video systems will be controlled from a small control station in the manipulator gallery. Other systems operated from the gallery will include the service bay access door, air coolers, and lights. Owing to the size of the target service bay, video cameras will be required to provide a view for the operators. Video cameras (five will be installed initially) will be mounted on the bridge systems and walls of the service bay. The cameras will provide viewing throughout the inside of the service bay and supplement direct viewing though the three window workstations. Video systems may be operated and viewed from the three window workstations. A video recording system will be included to capture video of critical operations.

\section{Storage Area}

Numerous target handling tools will be regularly required for normal service bay operations. Adequate space will be provided in the cell for storage and staging of these tools. In addition, six shielded wells will be provided to hold spent targets until the level of residual heating has decreased to a level acceptable for a standard waste shipping container.

The following tools will be provided for the required functions in the service bay:

- target positioning and holding cart

- dedicated change-out carts for target and IRP

- specialized tooling for handling of change-out carts and activated components

- special target fastener removal wrenches

- manipulator adapted hand tools

- portable lights and cameras

The spent targets removed from the plug must be stored in storage wells until the total decay heating in the tantalum-tungsten plates is below $400 \mathrm{~W}$. Calculations indicate that cooling will take about 120 days from the shutdown (Fig. 4.9). During this time, the spent targets will be radioactive and thermally hot. Therefore, the storage wells will be designed to provide sufficient shielding from the spent target and to provide a natural convection cooling path for passive cooling of the spent target while it is stored. Figure 4.50 shows the current concept for storage wells. 


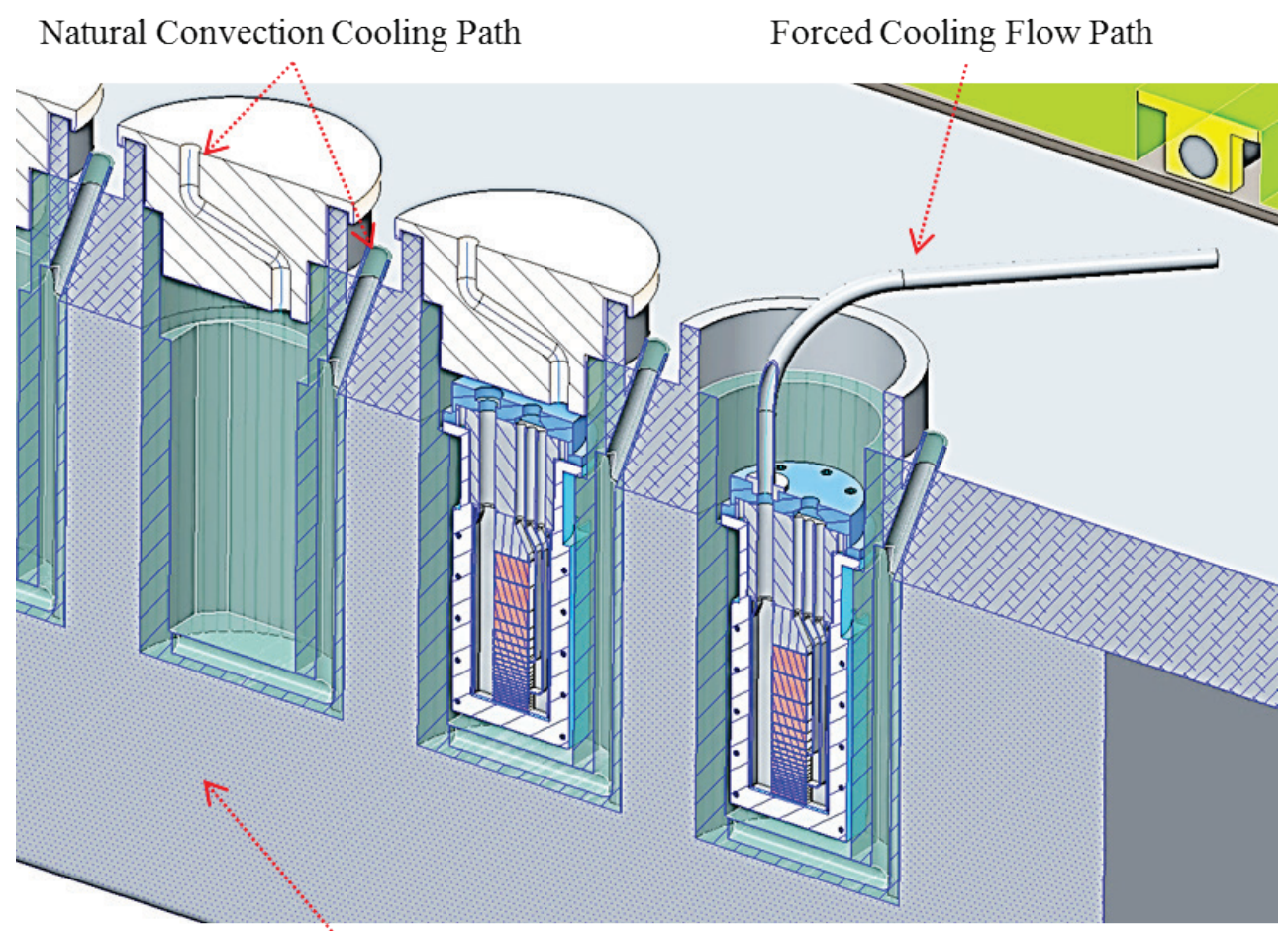

HD Concrete Shielding

Fig. 4.50. Storage well concepts for spent targets.

\subsubsection{Service Bay Remote Handling Operations}

Remote handling will be required for operations such as installing a shielding jacket on the spent target after it is retracted from the core vessel, establishing a temporary cooling area (for the spent target) for the duration of change-out operation, unbolting the spent target from the plug, transporting and inserting the spent target into the storage well, disconnecting the temporary cooling, establishing storage well cooling, and placing the storage well covers. Installation of a new target and pre-installation testing of the new target will also be accomplished remotely. The complex target post-irradiation examination operations will not be performed in the STS service bay.

\section{Spent Target Change-out Operations}

The target will be mounted on a cart that also contains the utility lines that service the target during operation, as described in Sect. 4.2. The primary heavy water cooling lines will be connected to the carriage at installation and disconnected before retraction. This will allow the primary loop system component to be located out of the bay. A separate loop for the shell cooling will be provided which can remain connected during carriage retraction to provide decay heat removal capacity. The target decay heating will rapidly decrease following beam shutdown; but it will remain high enough to require active cooling to maintain the target temperature at a desirable level during change-out operations, which are planned for one week after shutdown. Calculations indicate the shell cooling will be adequate for such a purpose (Fig. 4.51). 


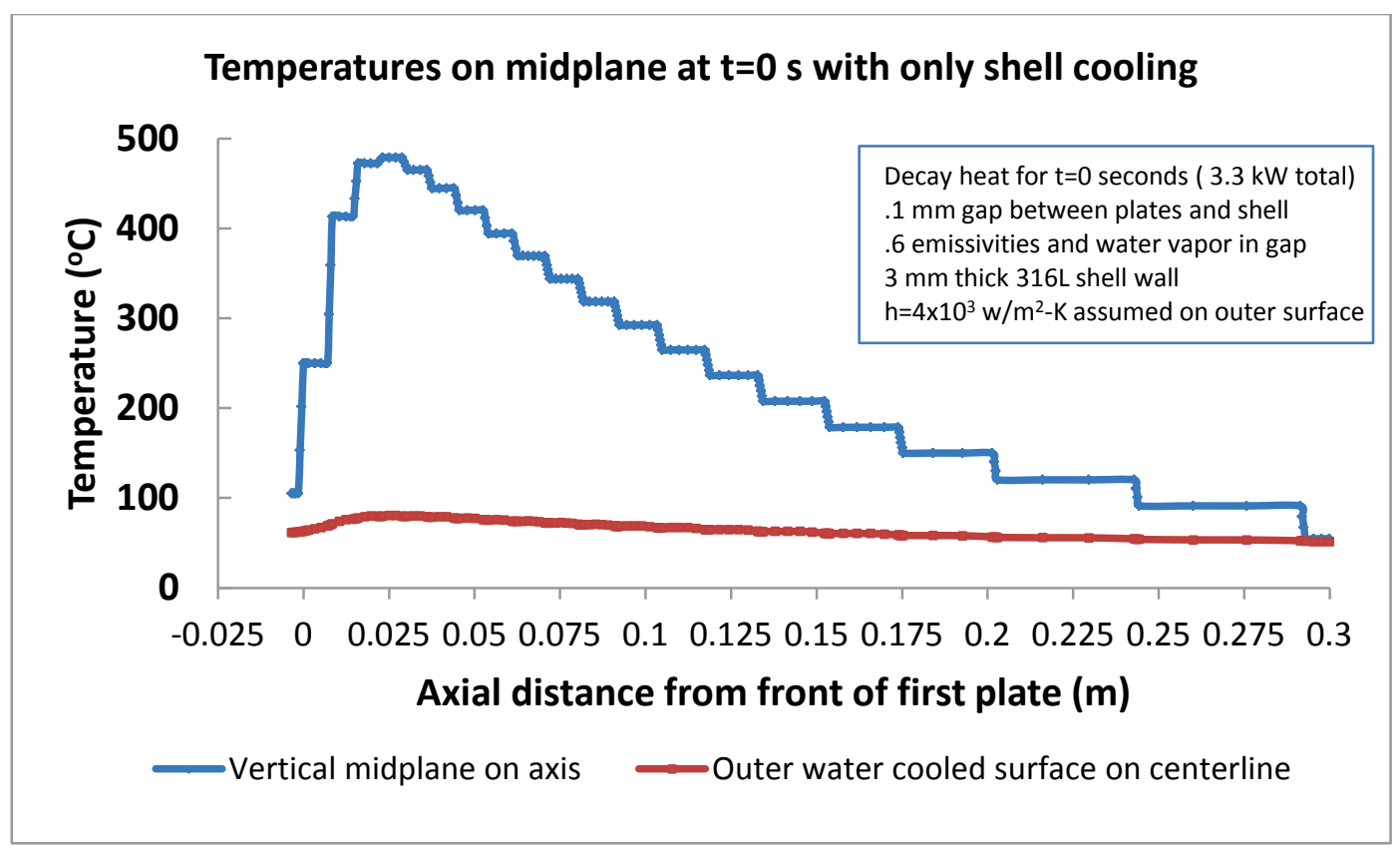

Fig. 4.51. Shell cooling calculation results.

The target plug will be mounted on a guided track and wheel carriage. With shielding included, the moving assembly will weigh about 100 tons and have an overall length of $10.6 \mathrm{~m}$ and a width of $0.76 \mathrm{~m}$ at the front. There will be two radiation streaming steps along the length of the plug. Included within the carriage will be a $0.187 \mathrm{~m}^{3}$ delay tank for the separate shell cooling loop. The delay tank will reduce the radiation exposure of service bay equipment during operation. Figure 4.52 shows the carriage in the installed position with side connections to the primary cooling system and to a flexible loop for the shell cooling. Because the service bay will be accessible with the beam off, a simple drive system using an electric motor with a gear and rack system is envisioned. This system could then be maintained manually. A backup system would also be provided which could insert the carriage remotely if the drive should fail to enable personnel entry into the bay for repairs. The drive system would also include a provision to disengage the drive and to lock the carriage in the inserted position to react to axial loads resulting from inflatable seal operation. This provision would also accommodate thermal expansion in the carriage using a spring preload system similar to the FTS carriage design. 


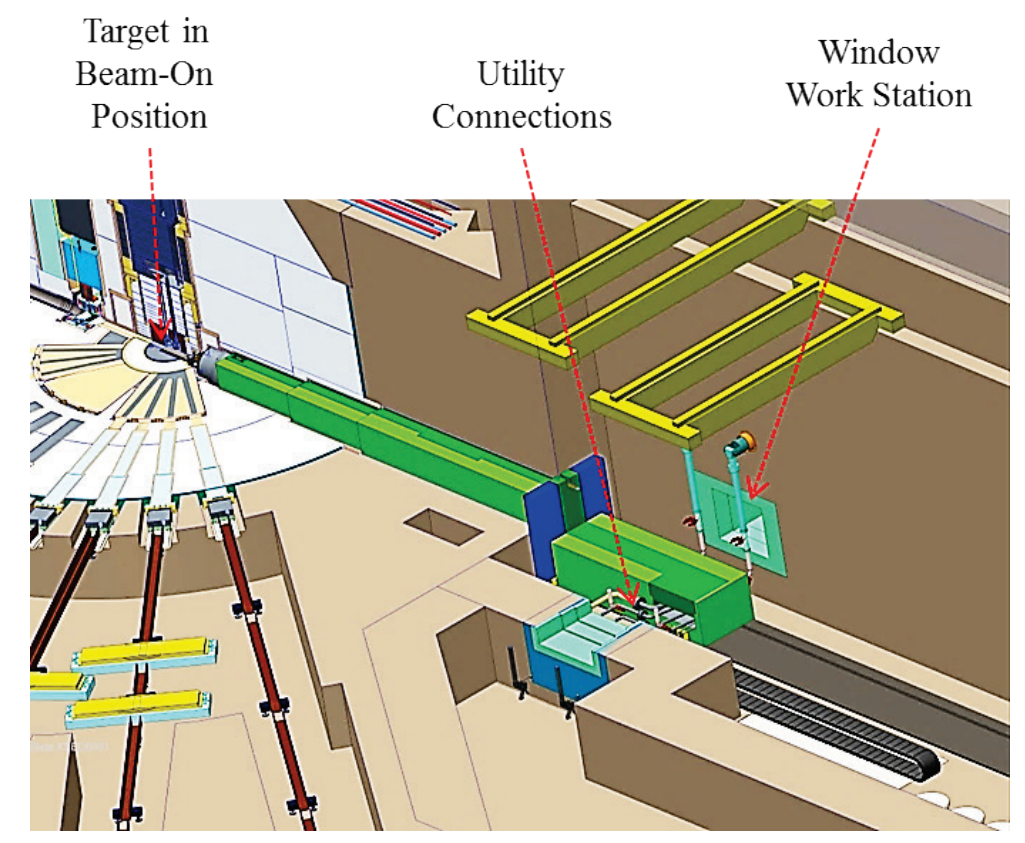

Fig. 4.52. Target carriage in installed position.

\section{Spent Target Shielding Jacket Handling Operations}

When the target is retracted into the service bay for the target change-out operation, a shielding jacket will be installed on the target immediately to reduce the radiation level in the bay. This shielding jacket will be designed to provide sufficient shielding to significantly reduce the radiation level in the bay, thus protecting the in-bay equipment. At the same time, it will be designed to provide optimum heat transfer capacity for removing heat from the spent target while it is in the storage well.

The current shielding jacket concept includes two bolted halves that will allow application of the highest contact surface pressure obtainable, which in turn will minimize the thermal contact resistance between the target shell and the shielding jacket. The shielding jacket will be made of either copper or tungsten, materials with excellent shielding and thermal properties. A preliminary calculation by the SNS Neutronics Group indicates tungsten shielding of 2.76 in. thickness is adequate to limit the radiation dose level to $100 \mathrm{R} / \mathrm{h}$ at a $12 \mathrm{in}$. distance a day after shutdown. The shielding jacket may or may not include cooling fins to improve the heat transfer characteristics. The cap for the shielding jacket will include features to facilitate remote handling of the transport of the spent target from the plug to the storage well, as well as easy installation and removal of the conduits (e.g., air duct, tube, or pipe) for the cooling fluid (air or water) that can be delivered either passively or actively as needed. A change-out cart will be designed to align the lower portion of the shielding jacket to a retracted target. The upper portion of the shielding jacket will then be lowered into position via alignment pins using the 5 ton crane. The manipulators at the primary window workstations will then install and torque the fasteners securing the shielded jacket. The shielded jacket will support the spent target module and enable removal of the fasteners securing the target module to the intermediate plug. Following fastener removal, the shielded jacket and target module will be moved away from the intermediate plug, enabling installation of the "cap" portion of the shielded jacket. The cap will include lifting interfaces for rotation of the shielded spent target assembly to a vertical, nose-down orientation required for installation into a storage well. Figure 4.53 shows one of the current shielding jacket concepts. 


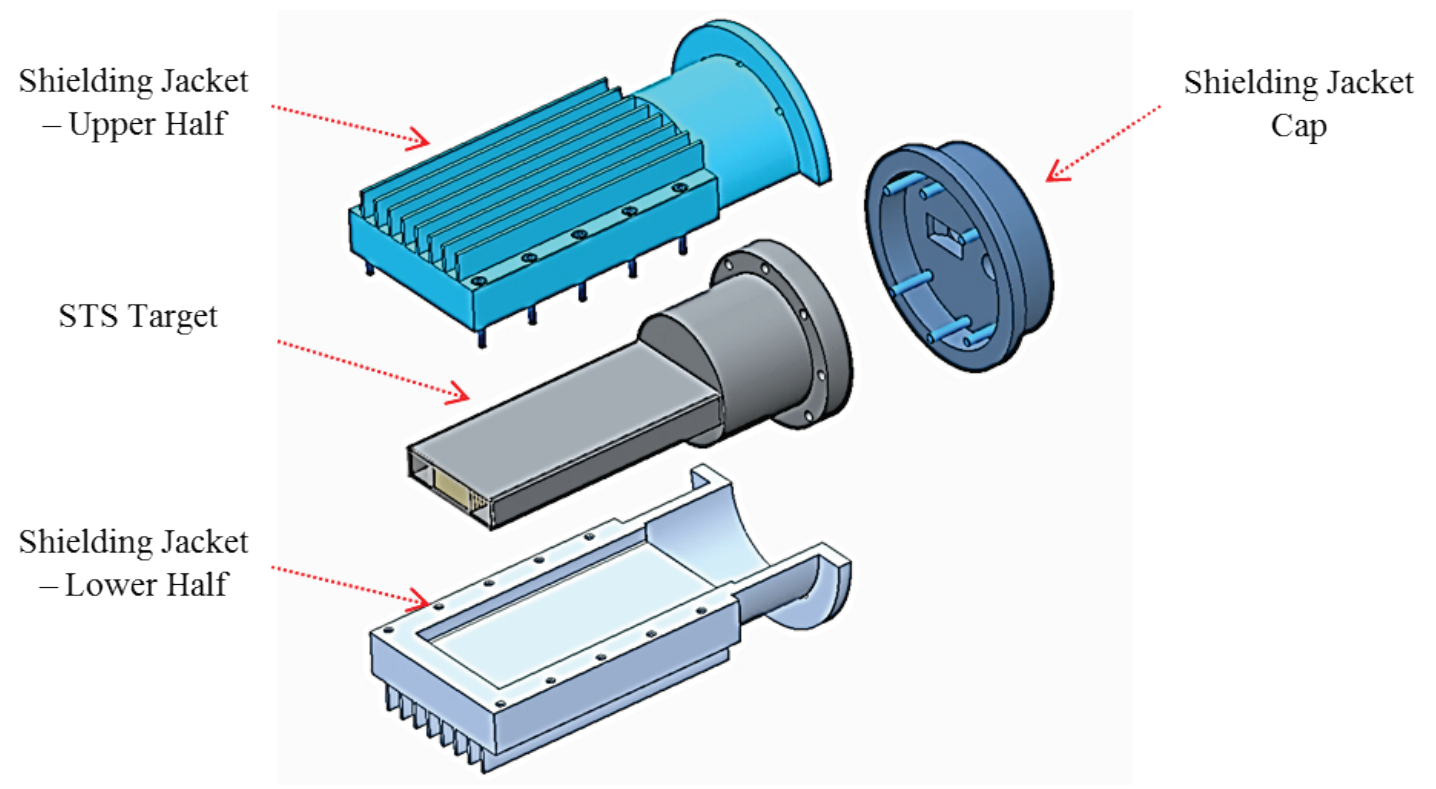

Fig. 4.53. Shielding jacket concept for spent target.

\section{Intermediate Plug Change-out Operation}

The target plug assembly will have a transition section known as the intermediate plug (IP) that is designed to mount relatively long-lived components such as the inflatable metal seal and the target sealing face. (Fig. 4.54) It is currently estimated that the IP should have a life of about 10 years. The current design of the IP includes features to facilitate periodic maintenance/replacement of the section. Also, it is expected that the IP will be replaced using a dedicated change-out cart. In a similar manner to target module removal, the IP will use a change-out cart to support the component during fastener removal. Following removal, the IP will be translated away from the carriage and rotated vertically for removal from the cart.

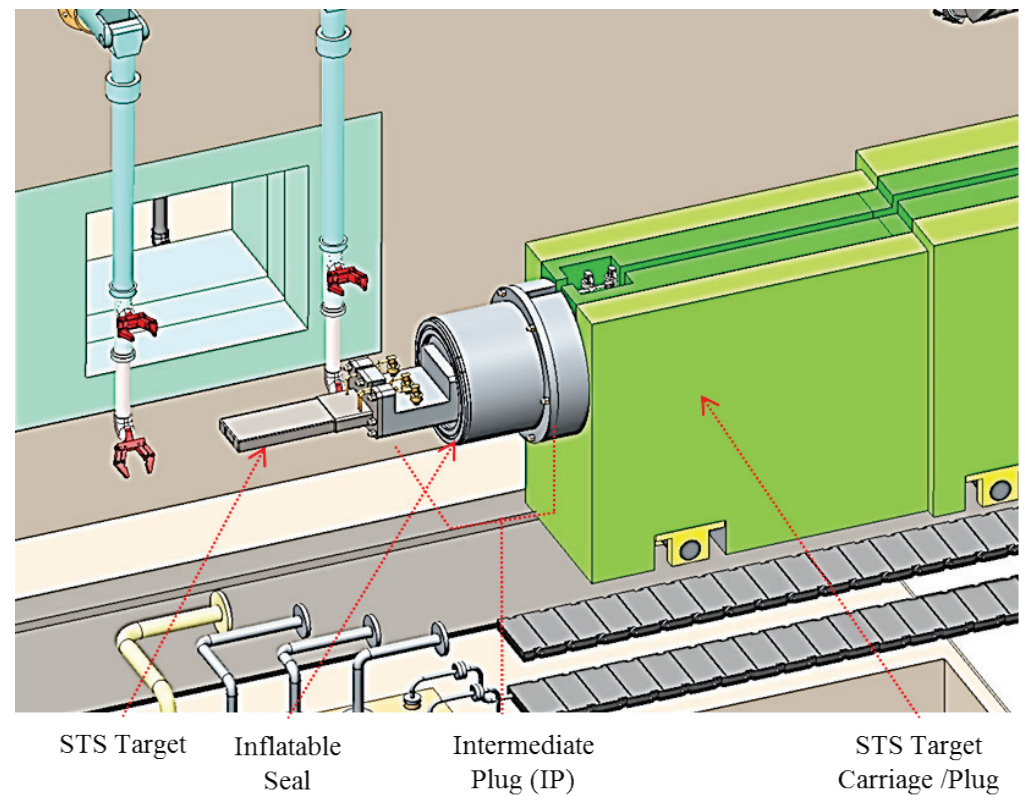

Fig. 4.54. Concept for STS target inflatable seal on the IP. 
The current concept for the IP includes a bolted joint for easy installation and removal of the IP from the target plug. The change-out cart will be designed to establish and maintain alignment of the IP to the carriage and will maintain this alignment during fastener installation. The spent/used IPs are expected to be active and therefore must be remotely handled for removal and disposal operations. The decay heating in the spent/used IPs is expected to be low enough that a spent/used IP will not require interim storage in the storage wells, but it will required shielded storage in the service bay before disposal to enable personnel access. The installation of a new IP will be performed remotely because of the high radiation level expected at the mounting face of the STS target carriage plug. The change-out cart used for removal of the spent IP will be used to align and install the new IP onto the carriage.

\subsubsection{Monolith and Beam Line Component Handling}

Component handling outside of the service bay will be done in three principal working areas of the main bay: (1) top of monolith, (2) access pits, and (3) main floor. The top of the monolith area will be used for servicing core vessel components such as the moderators, the IRP, and the PBWs. The beam line access pits will be used for servicing choppers, operating shutters, and neutron beam inserts and for handling tools. The main floor will consist of the basement access hatches, chopper maintenance area, truck access area, and laydown and waste shipping areas (Fig. 4.55).

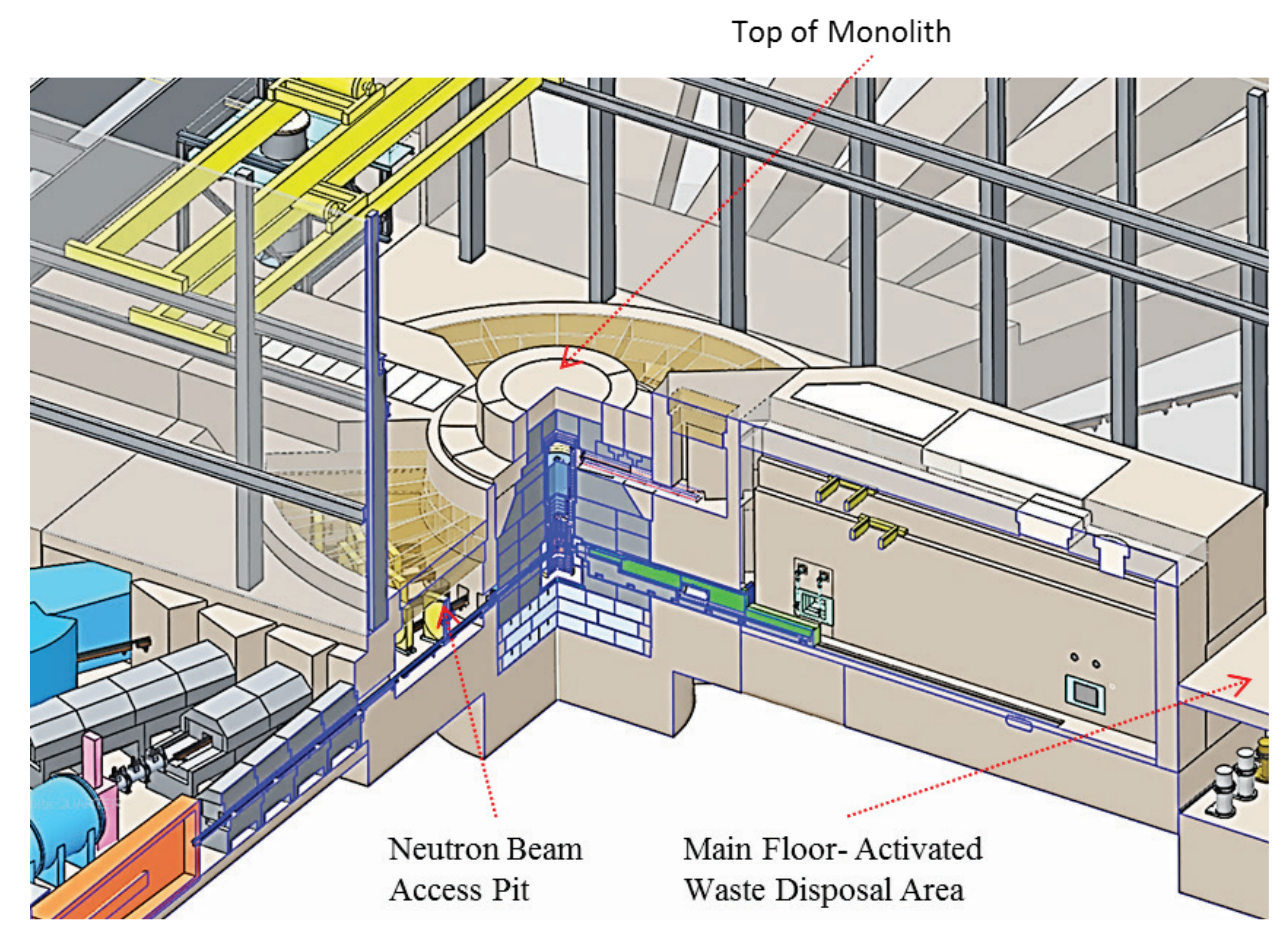

Fig. 4.55. Principal working areas of the main bay.

\section{Top of Monolith}

The monolith will include the primary shielding, core vessel assembly, and neutron beam line guides. The STS monolith will not have primary shutters. The top of monolith will provide access for change-out of the following core vessel components (Fig. 4.56):

- IRP

- outer plug 
- moderators

- PBW

- utility piping

The expected radiation level at the top of the monolith in the STS during change-out of these components is predicted to be low enough to eliminate the need for the pedestal manipulator used in the FTS high bay. Instead, long-handled tools and purpose-built open-bottom shield containers will be used for handling the mildly activated core vessel components. Local shielding will be used as needed for all change-out operations undertaken at the top of the monolith.

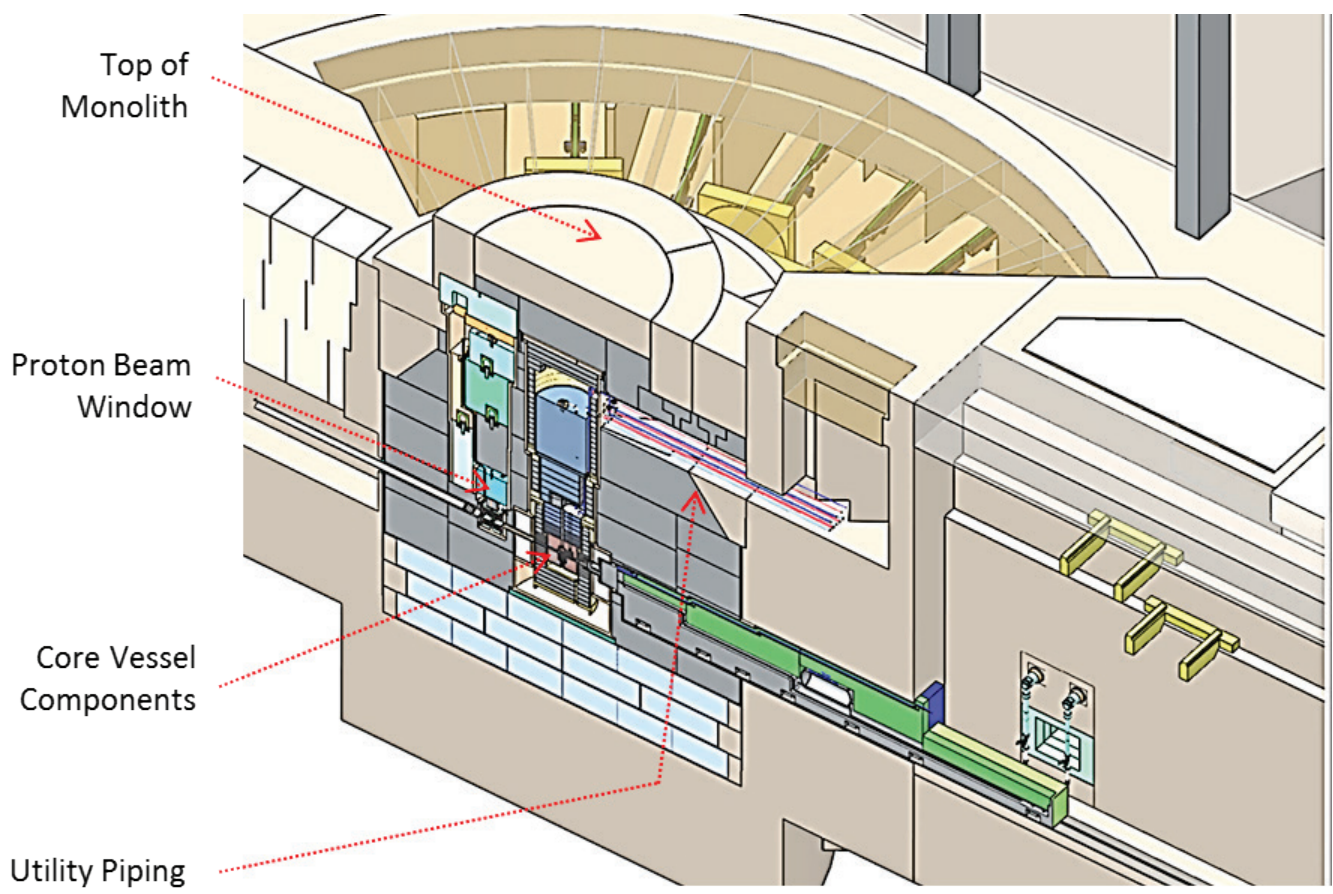

Fig. 4.56. STS monolith.

Two overhead bridge cranes will be provided to handle most loads in the top of the monolith area-one 50 ton and one 10 ton bridge cranes. These cranes will be the primary maintenance tools used for loading, transporting, and handling virtually all the shield containers within the main target building area. The FTS pneumatic hoist will be used to interface with the top of the shield containers to lift activated components into the containers. The pneumatic hoist has several safety controls, such as lift capacity regulations, that should limit possible damage to the monolith components.

\section{Moderators, IRP and Outer Plug}

The moderator design is still in the development stage; therefore, it has not been determined how frequently the moderators will have to be replaced. However, based on the lifetime limit of the SNS FTS moderators and the current design parameters of the STS moderators, it appears that the change-out frequency for the STS moderators is likely to be on the order of 6 years (Fig. 4.57). 


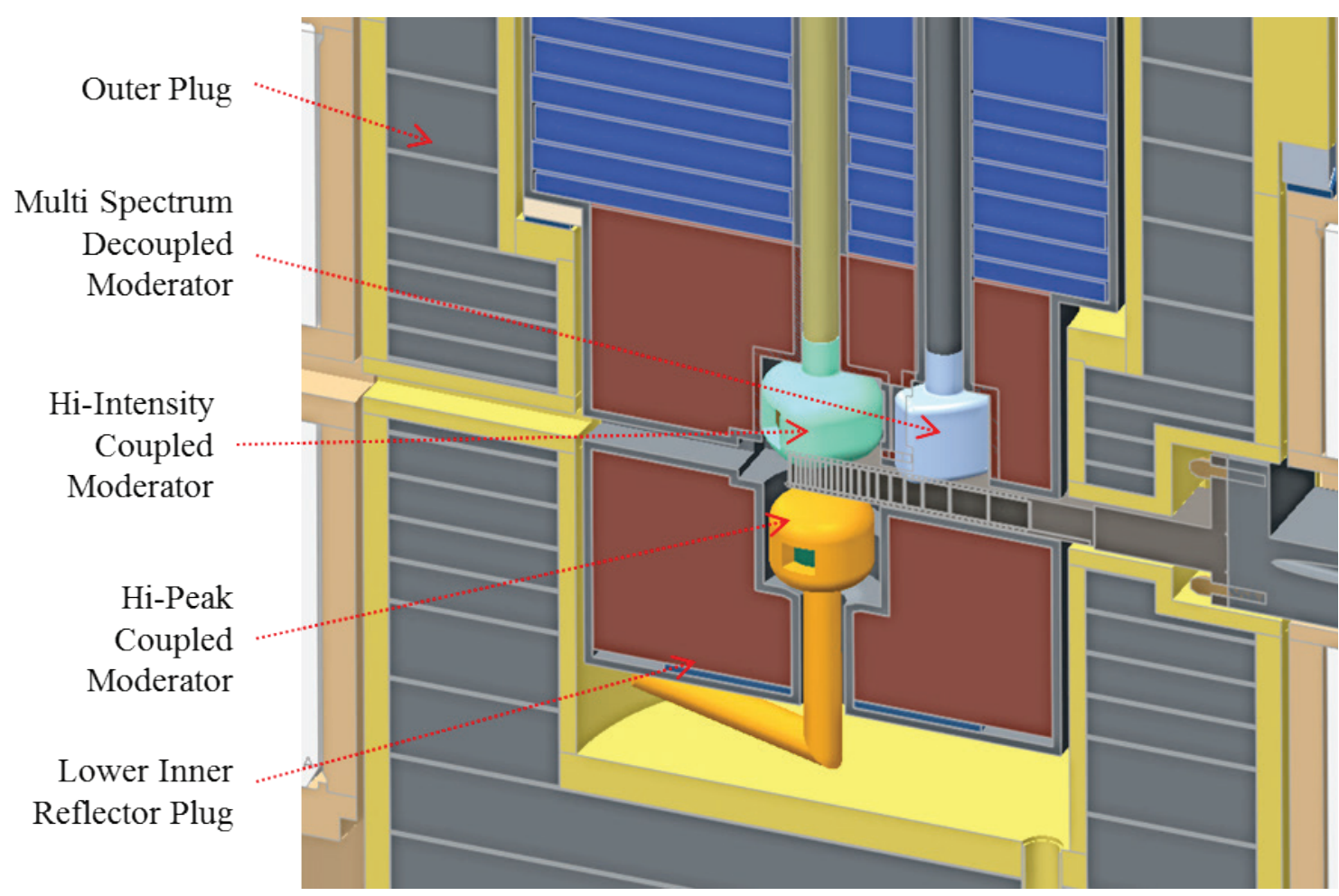

Fig. 4.57. STS moderator arrangement.

Periodic removal/replacement of the core vessel components such as the moderators and the IRP will be done without true remote handling tools like the servomanipulators. Instead, the remote handling operational philosophy employed in the FTS - based on the distance between the activated components and workers aided by long-handled tools and the use of shield containers with a built-in pneumatic liftwill be employed for change-out operations. Like the FTS, the STS monolith and the core vessel components are designed for shield container access. Therefore, all the active components such as the IRP are segmented into discrete modules to permit in situ disassembly and reassembly, similar to the FTS PBW maintenance procedure (Fig. 4.58). Passive shielding components are expected to be reused, whereas the actively cooled components are expected to be replaced with new ones. Some cutting of utility piping is expected for the IRP and moderator change-out operations. The outer plug is not expected to be removed or replaced and is considered a "life of the facility" component. 


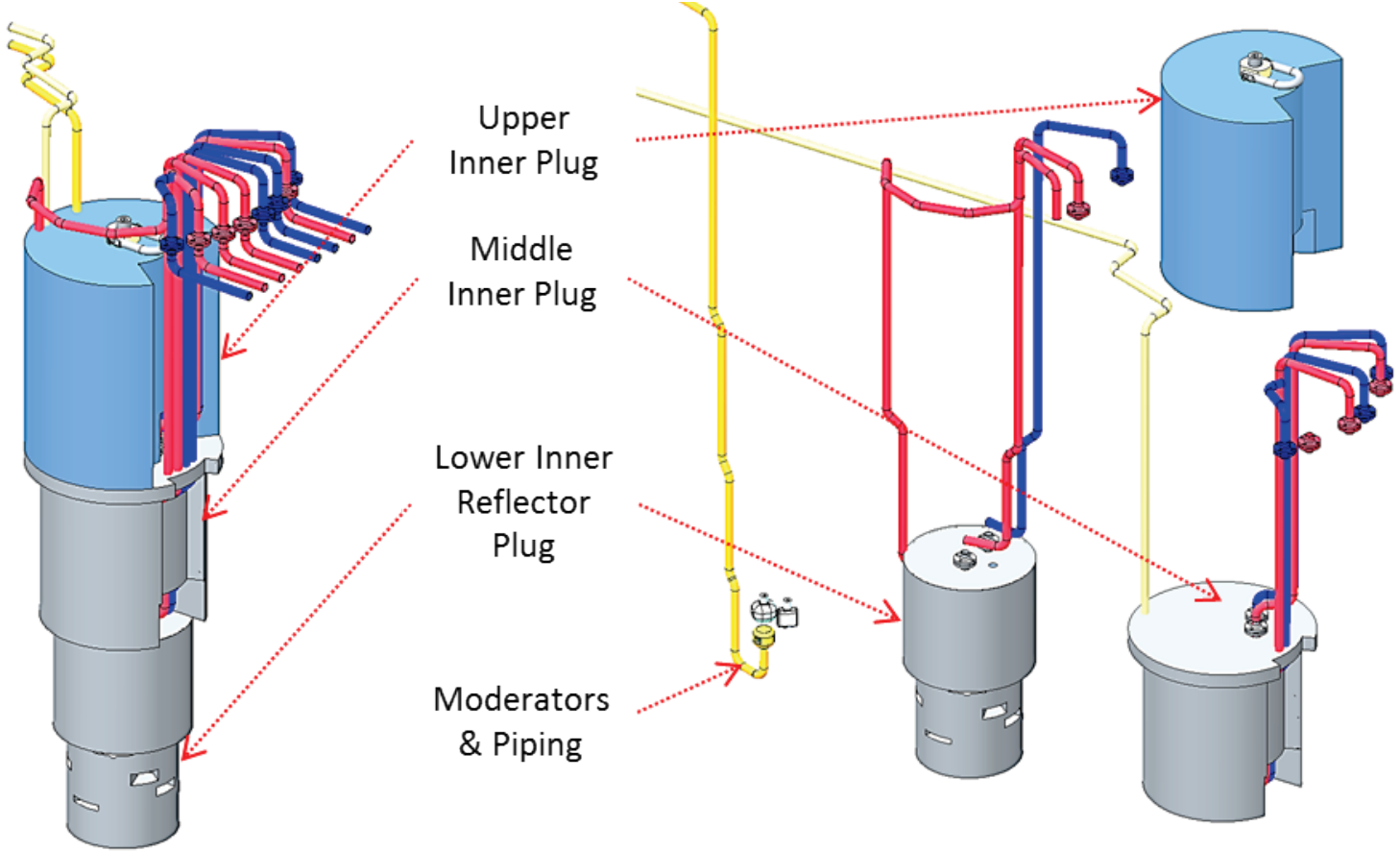

Fig. 4.58. Discrete module design of the IRP.

\section{IRP Maintenance Procedure}

The IRP maintenance procedure is based on proven FTS methods. The preliminary outline of the STS IRP maintenance procedure is as follows:

- Dry components to remove cooling water.

- Remove external passive shielding (hands-on).

- Remove vessel lid (hands-on).

\section{All further operations will require stand-back/long-handled tools}

- Disconnect necessary pipe fittings (designed for remote operation).

- Remove upper, passive shield with overhead bridge crane.

- Remove middle shield (pipe stem presents a handling problem) into dedicated shield container using pneumatic hoist.

- Shear moderator pipe stems (remove and segment for disposal).

- Remove lower reflector plug dedicated shield container using pneumatic hoist.

- Carry out replacement operations in reverse order. New components will be lowered into the IRP cavity using the overhead crane and hook extenders as required. 


\section{Proton Beam Window}

The STS PBW will mirror the FTS PBW design and configuration. The only significant difference between the FTS and STS PBWs is the size of the windows. The window for the STS PBW is expected to be much smaller. It is expected that change-out of an STS PBW will be very similar to that for the FTS. The current plan is to use the FTS change-out tooling for the STS PBW. (Fig. 4.59)

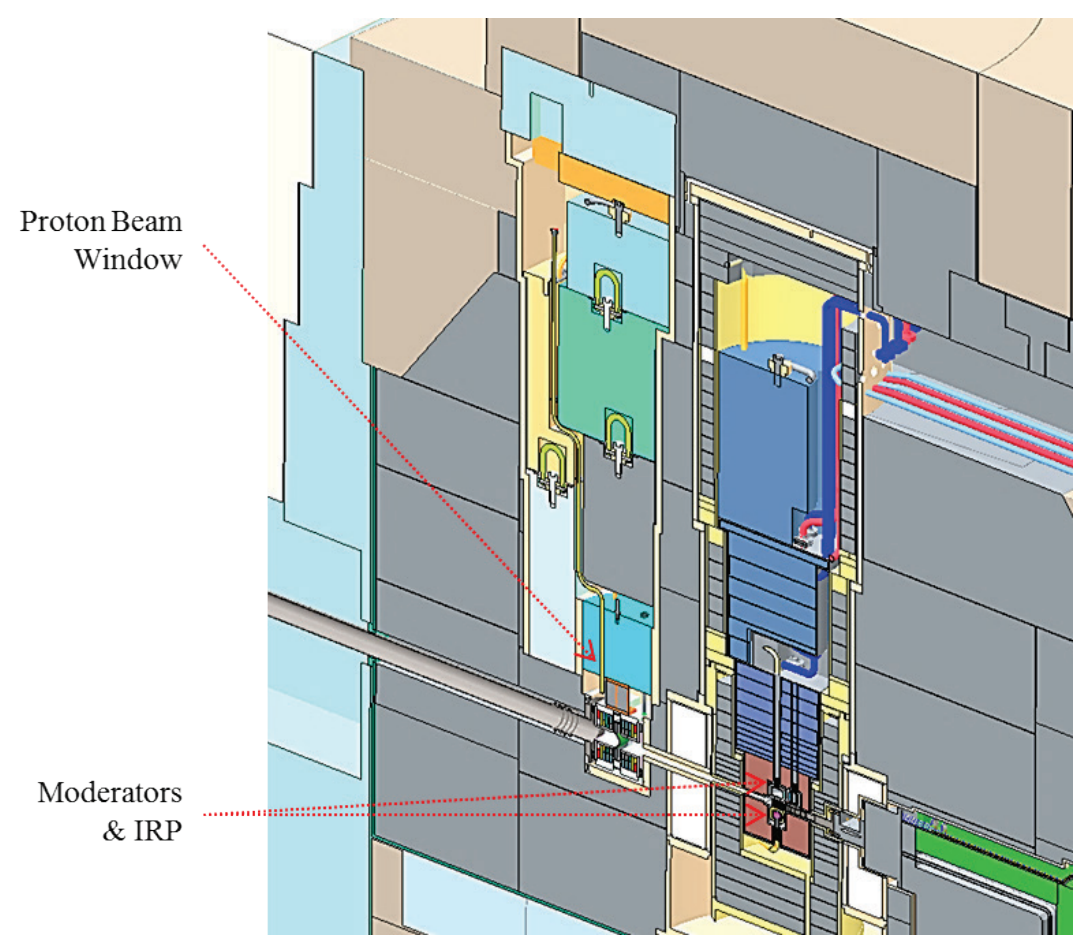

Fig. 4.59. STS proton beam window.

\section{Beam Line Access Pits}

Shielded pits will be located on either side of the monolith to accommodate the neutron beam insert handling tool, choppers, and shutters. The pits will be capped with the equivalent of approximately $2 \mathrm{~m}$ of high-density shielding (see Fig. 4.60). They will cover the full beam line arc of $110^{\circ}$ on both sides of the monolith and extend to a beam radius of $11 \mathrm{~m}$.

\section{Maintenance in Beam Line Access Pits}

The pits are primarily designed to accommodate choppers and the insert removal tooling. Access platforms and stairs will be required because personnel will not reasonably be able to cross lines in the pit. The pit cover blocks will to be designed in a " $T$ "/inverted " $T$ " arrangement so that they can be removed in sections without sequential removal of all the blocks. 


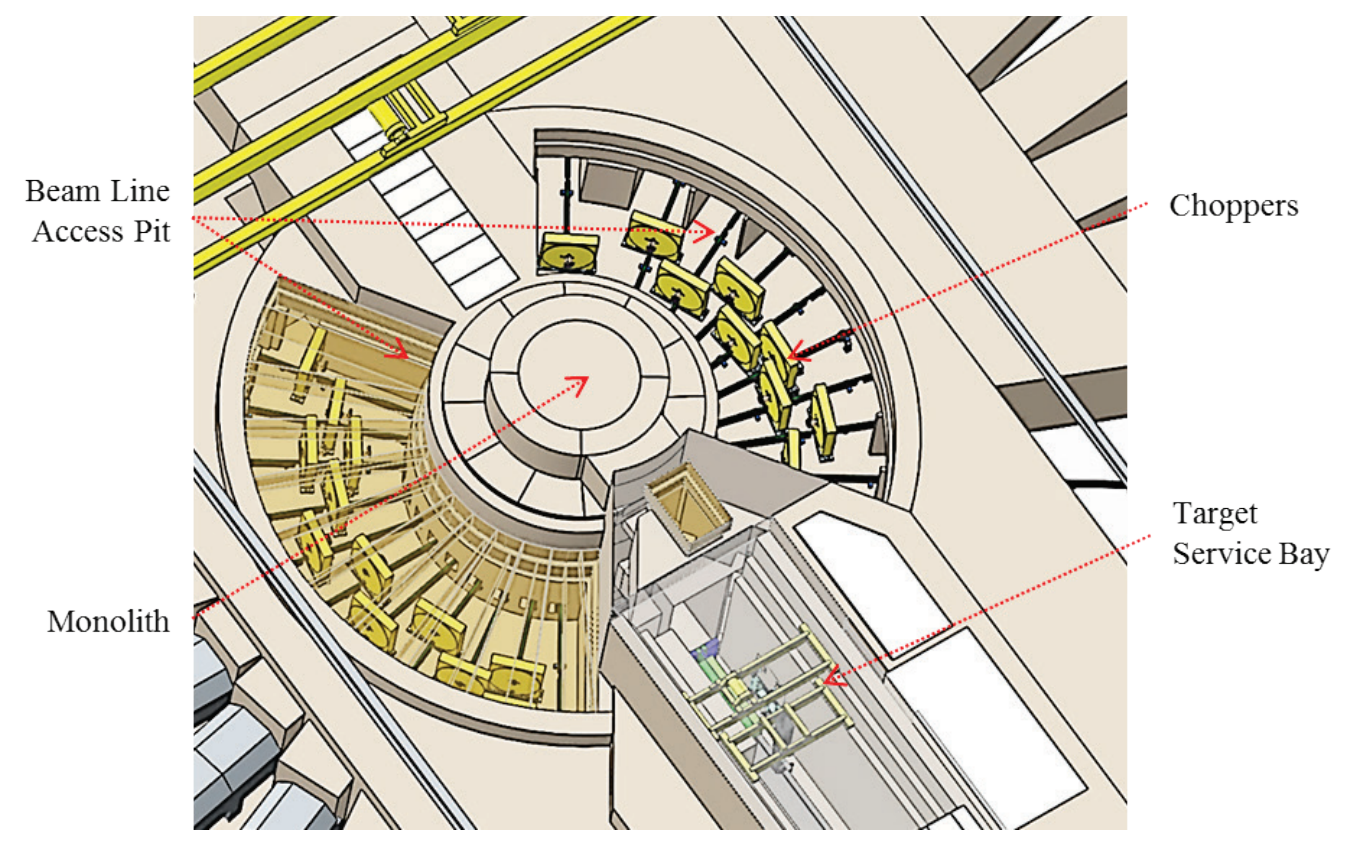

Fig. 4.60. STS beam line access pits.

Neutron beam line (solid/blank) inserts are designed for flexibility and stability. All unused core vessel nozzles will have solid plugs inserted until they are replaced with neutron guides. (Fig. 4.61) Blank inserts will eliminate the need for temporary shielding during the installation of new instruments after startup (Fig. 4.62). Blank inserts will be as long as over $4.15 \mathrm{~m}$ (163.58 in.) and will weigh up to 6,000 lb. The insert structures will be water cooled within $2 \mathrm{~m}$ of the moderator.

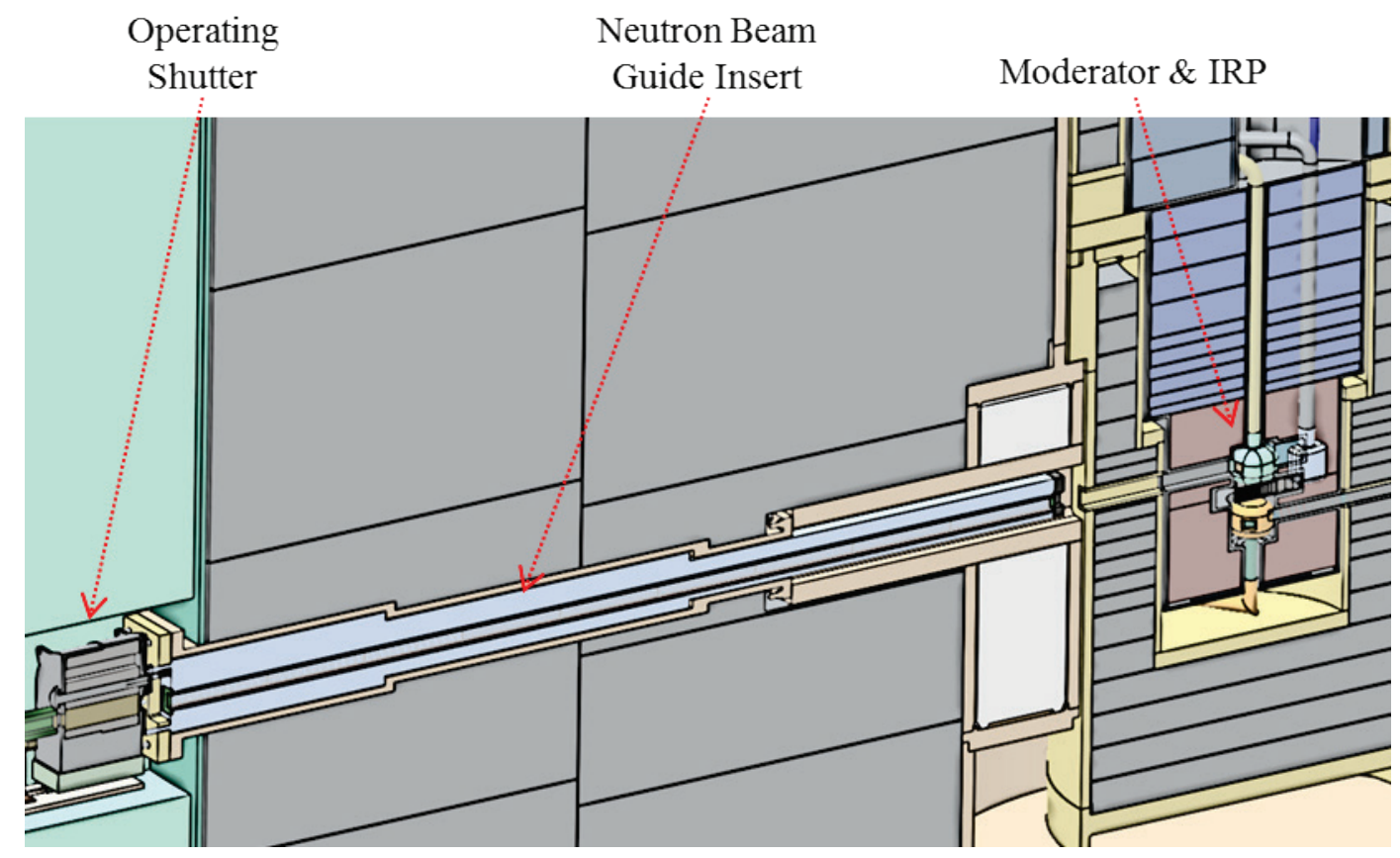

Fig. 4.61. STS neutron beam insert (installed in core vessel). 


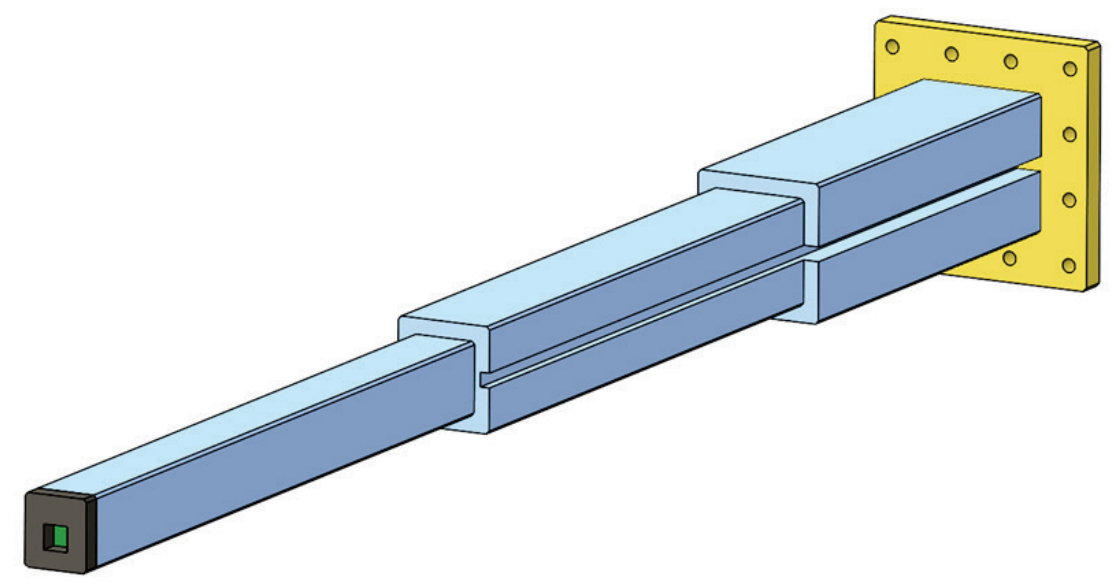

Fig. 4.62. STS neutron beam insert concept.

The neutron guide inserts to replace the blank ones will be custom built for each beam line. However, all guides have common features such as a stainless steel structural enclosure (insert frame), insertion rails, cooling and gas connections, and precision alignment features. (Fig. 4.36)

\section{Neutron Guide Insert Handling Tool}

The neutron guide handling tool shown in Fig. 4.63 will be used for retracting activated inserts into a shield container for handling and temporary storage. The tool will be designed to be used both to retract activated inserts and to insert new ones. Following removal of the activated insert, the tool will be removed from the pit and mated to a shielded storage cask. The activated insert will then be loaded into the storage cask, and a new insert will be loaded into the tool. Finally, the tool with a new insert will be lowered into the pit to allow insertion of the new insert. The beam line components in the pit must be removed to make room for the tool during such operations. The neighboring choppers may also have to be removed for the operation.

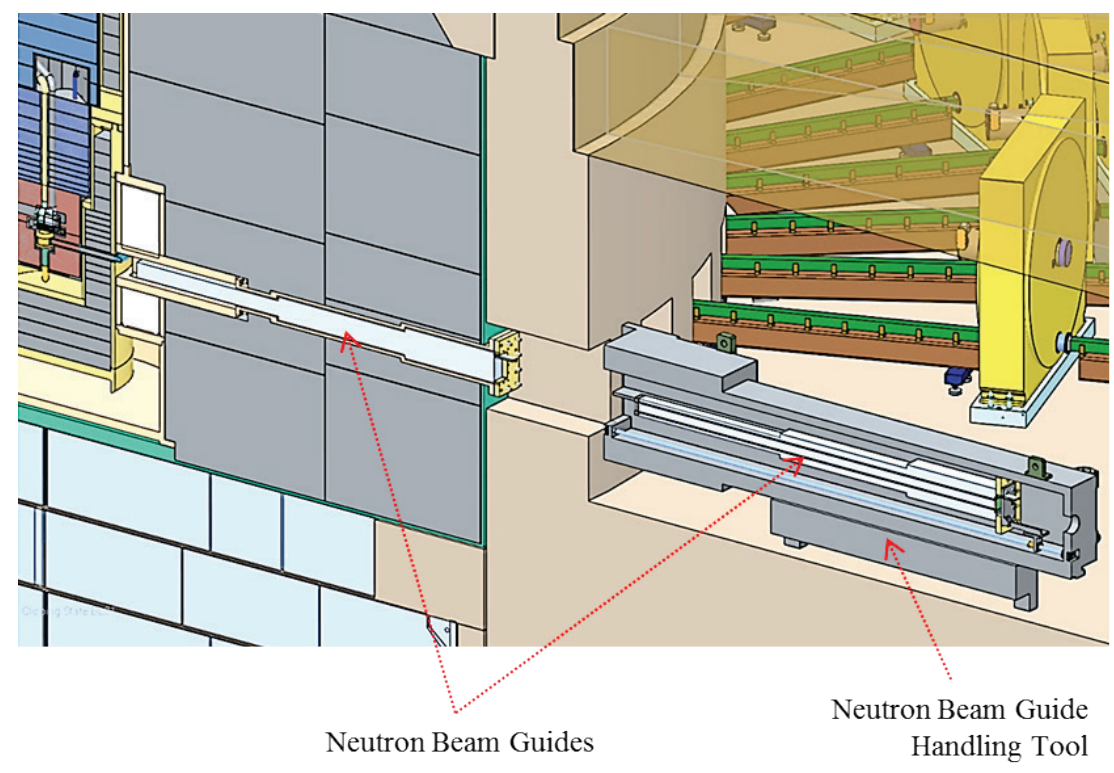

Fig. 4.63. STS neutron beam guide handling tool concept. 


\section{Insert Change-out Procedure}

The insert change-out procedure will be similar to the current FTS and Paul Scherrer Institute methods. The preliminary outline is as follows:

- Remove enough pit shielding to permit access to insert (hands-on).

- Remove necessary beam line components - e.g., chopper, guide (hands-on).

- Disconnect necessary service fittings on operating shutter (hands-on).

- Retract and remove operating shutter.

Note: At this point streaming radiation will become significant. Additional design effort is required to understand and overcome this problem.

- Disconnect insert service fittings.

- Disconnect insert attachment/sealing flange.

- Install insert handling tool, including power and control cabling.

- Attach tool to insert and remove insert from monolith.

- Close insert tool shield door and disconnect service cabling.

- Move tool with insert inside to main bay for disposition.

- Carry out replacement operations in reverse order.

\section{Chopper Handling}

Each STS beam line will require choppers for operation of the instrument. The STS choppers will be larger and slower than those for the FTS. Their larger diameter, $1.7 \mathrm{~m}$, will provide the same aperture sweep time through the neutron beam as the $60 \mathrm{~Hz}$ of the FTS (Fig. 4.64). The required operating speed of the STS choppers will be $10 \mathrm{~Hz}$ to match the slower neutron production rate of the STS. A $1.7 \mathrm{~m}$ disk is within the manufacturing capabilities of a current disk vendor. Existing drives and bearings can also be used.

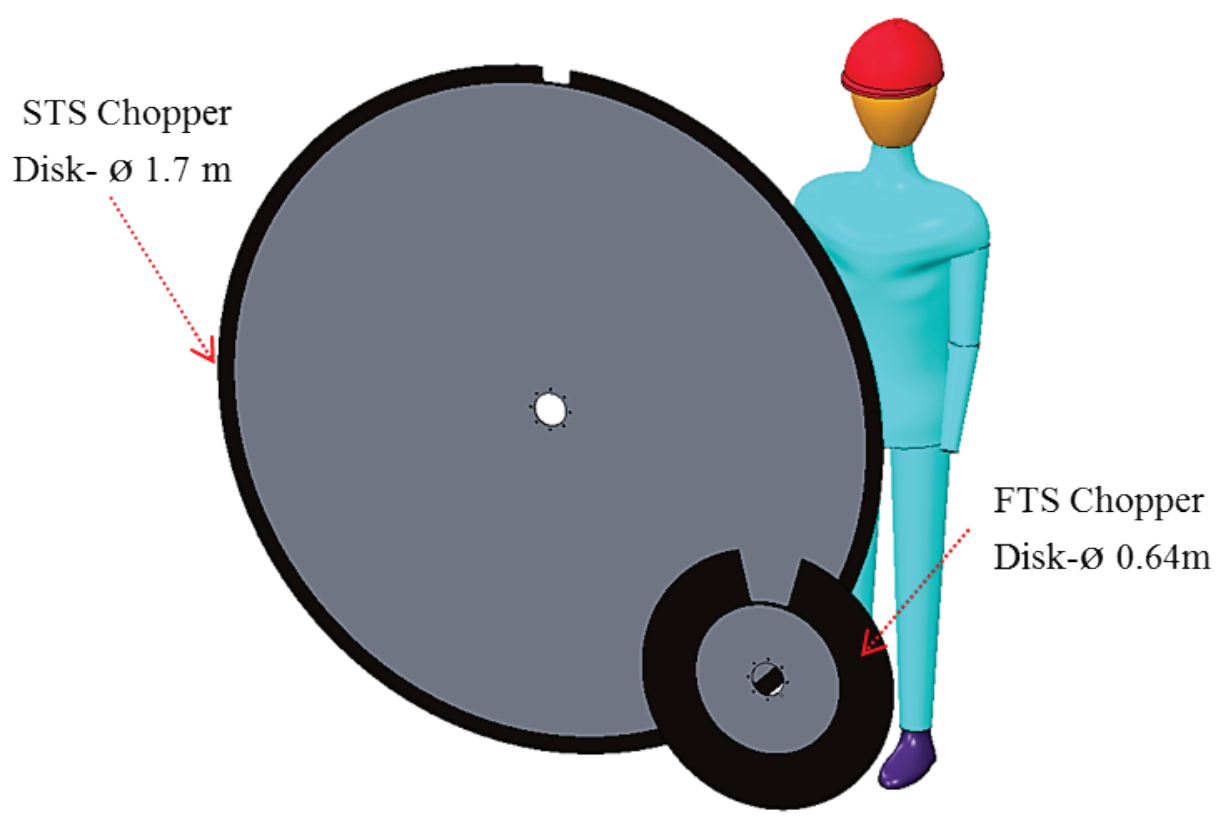

Fig. 4.64. Comparison of disk size for FTS and STS choppers. 
Operating failures of the STS choppers are expected to be rare $(<1 /$ year). However, regular maintenance will require removal/replacement of approximately six choppers per year. STS choppers will be handled based on FTS experience. The goal is to be able to change each chopper within a single shift. Most lines will have bandwidth choppers located in the shielded pit and will be directly accessible to the overhead bridge cranes. Transfer carts and accommodations for lifting will be required for servicing choppers outside the shielded pits. The shielded pits and tunnels are designed to accept a maximum chopper diameter of $\sim 2 \mathrm{~m}$. The choppers will be serviced and maintained hands-on in a dedicated chopper maintenance area. The current STS building layout concept shows the chopper maintenance area setup in the main floor, adjacent to the truck access area of the target building (Fig. 4.65).

\section{Operating Shutter Handling}

Each STS beam line will require an operating shutter to prevent streaming during beam-off maintenance operations and to shield instrument areas during sample exchanges. Shutters will generally have thick steel gates or drums faced with $\mathrm{B}_{3} \mathrm{C}$ absorber. (Fig. 4.66) Ideally, all operating shutters will be identical, but that design issue has not been finalized. Since shutters will not be directly accessible to the overhead crane, they will be mounted on horizontal rails for movement into the beam line pit. Shutters will be handled hands-on. The drive components (pneumatic cylinder and limit switches) may be maintained inplace, hands-on. The concept is currently in development pending additional radiation source information.

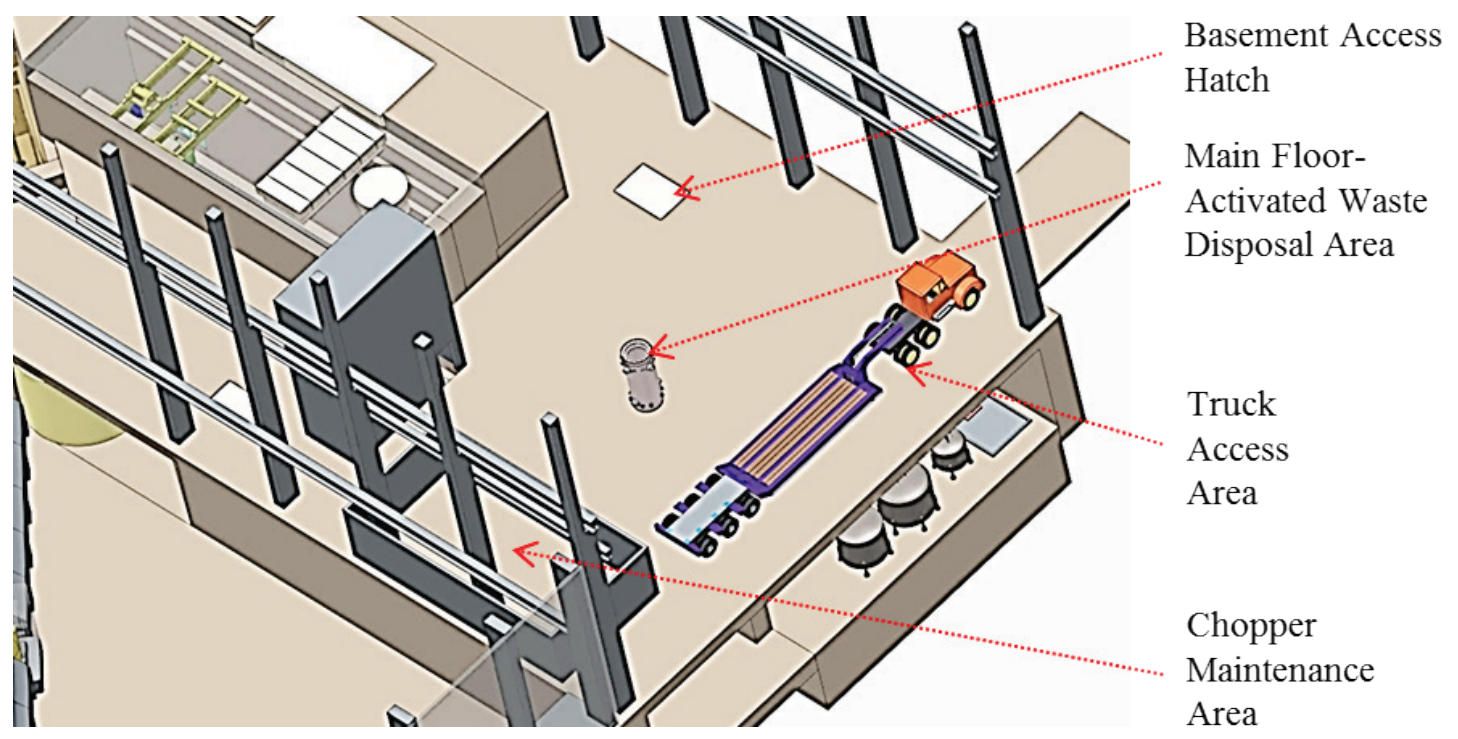

Fig. 4.65. Activated waste disposal area. 


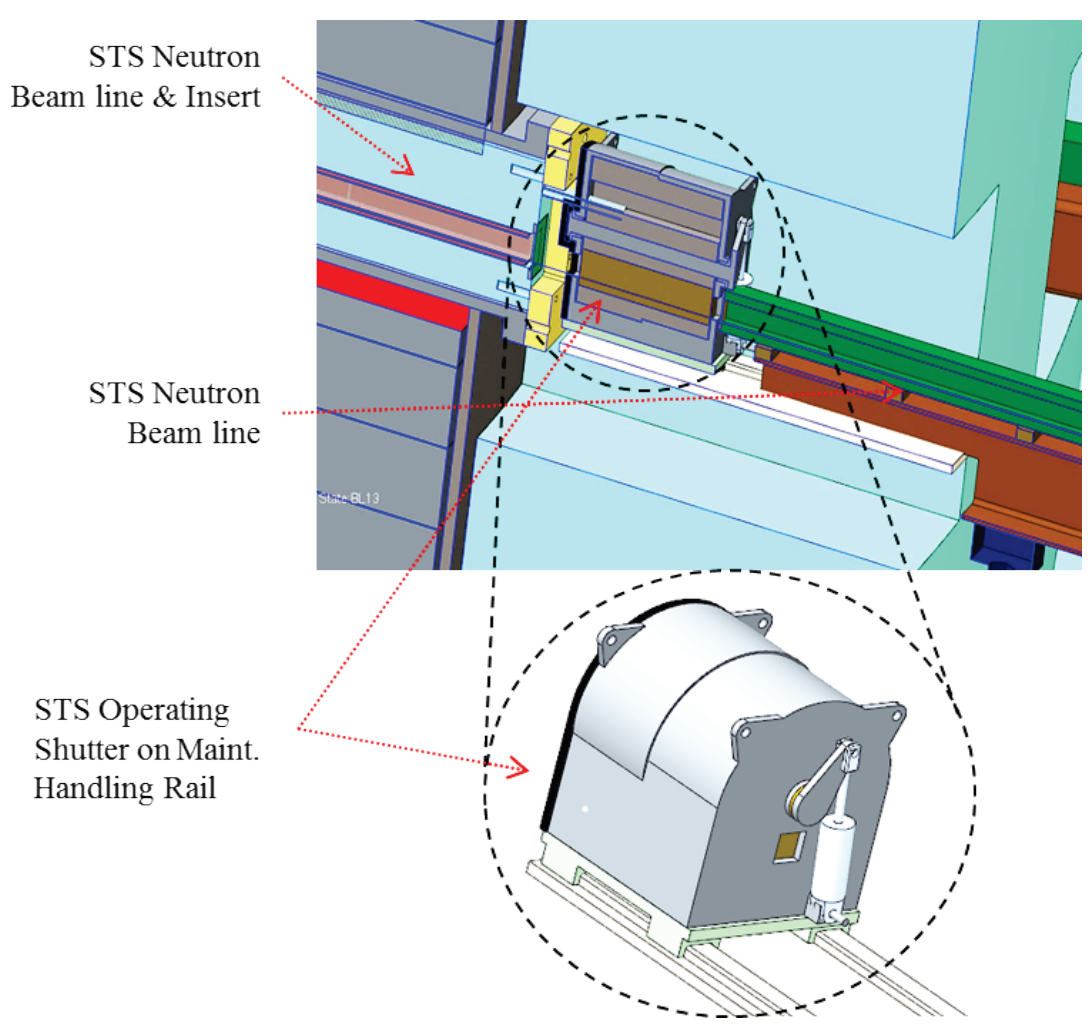

Fig. 4.66. Operating shutter concept.

\section{Main Floor}

The main floor will consist of the basement access hatches, chopper maintenance area, truck access area, and laydown and waste shipping areas. (Fig. 4.67) It is assumed that the TN-RAM cask will be used for high-dosage waste. Shielded casks will also be used for onsite transfer as needed. Direct crane access to the basement via floor hatches will enable ease movement of utility waste to the main floor for packaging and handling. The two bridge cranes will be the primary maintenance tools providing the necessary capabilities for loading, transporting, and handling virtually all the casks and containers within the main floor.

\section{Basement}

The target building basement will house all activated utilities (Fig. 4.67). These will include the cooling water utilities and hot off-gas process. All maintenance will be hands-on within days after the beam is shut down. Integrally shielded filter and ion exchange columns will be maintained using FTS procedures and tools. The hatch in the target building floor will provide bridge crane access to target system components. It will also house personnel operating areas such as change rooms, local control, and electrical distribution. 


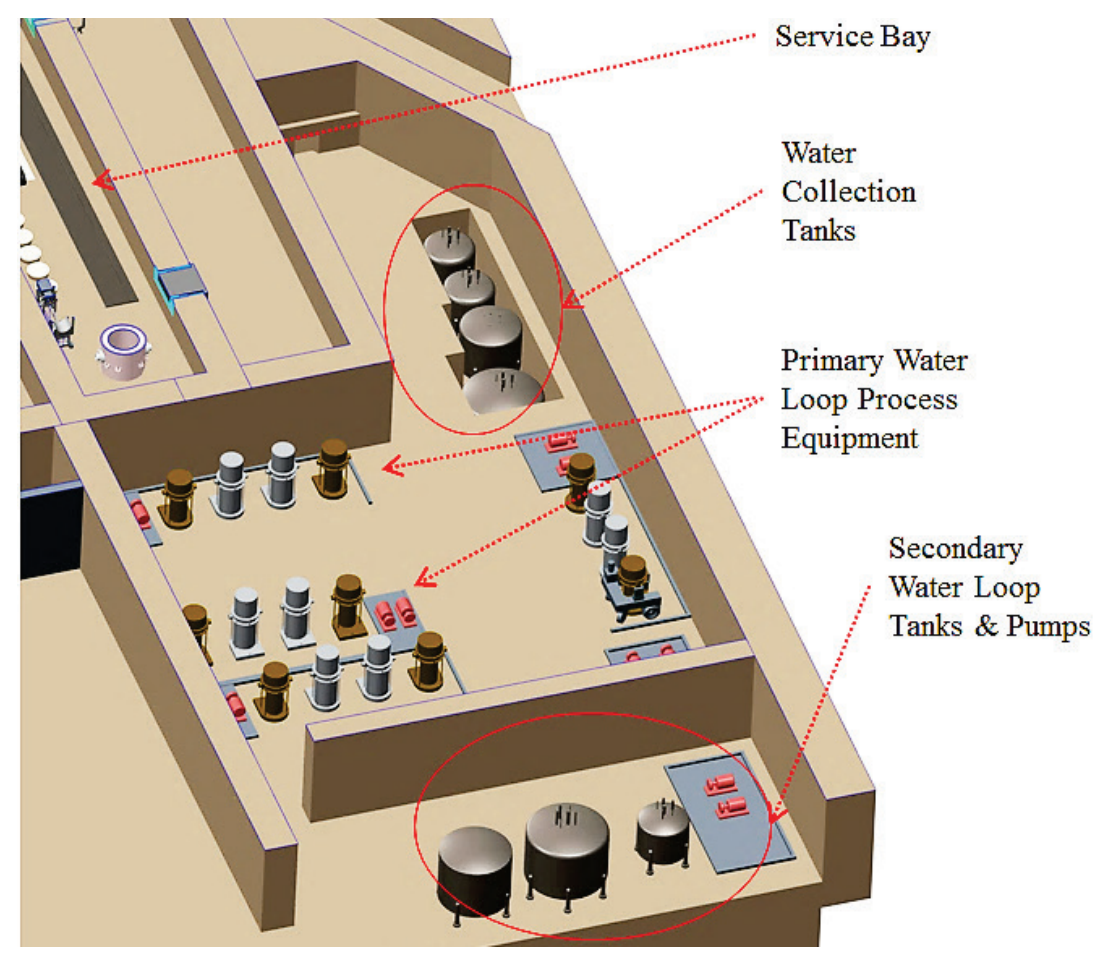

Fig. 4.67. STS utility vault.

\subsubsection{IRP Mockup/Storage Vessel}

An IRP mockup/storage vessel will be installed in the northwest corner of the STS building adjacent to the HUR (Fig. 4.68). The mockup vessel will be used mainly for three tasks: (1) mock-up testing of procedures for IRP installation and removal operations, (2) acceptance testing of replacement IRPs, and (3) storage for replacement IRPs before their installation. The STS mockup vessel will be very similar to the existing FTS mockup vessel shown in Fig. 4.69.

The STS mockup vessel design will match the interfacing features inside the functional STS vessel. (Fig. 4.70), including the mounting points and piping connection points. In addition, full vacuum pumpdown capability will be included in the mockup vessel design for complete leak testing of the replacement IRPs after they arrive on site from the vendor. Unlike the FTS mockup vessel, the STS mockup vessel will not match the core vessel elevation, since the STS building will provide enough headroom over the core vessel for routine maintenance work without concerns about tight space. The building cranes (50 ton and 10 ton) will provide full coverage for handling the tasks associated with the mockup. 


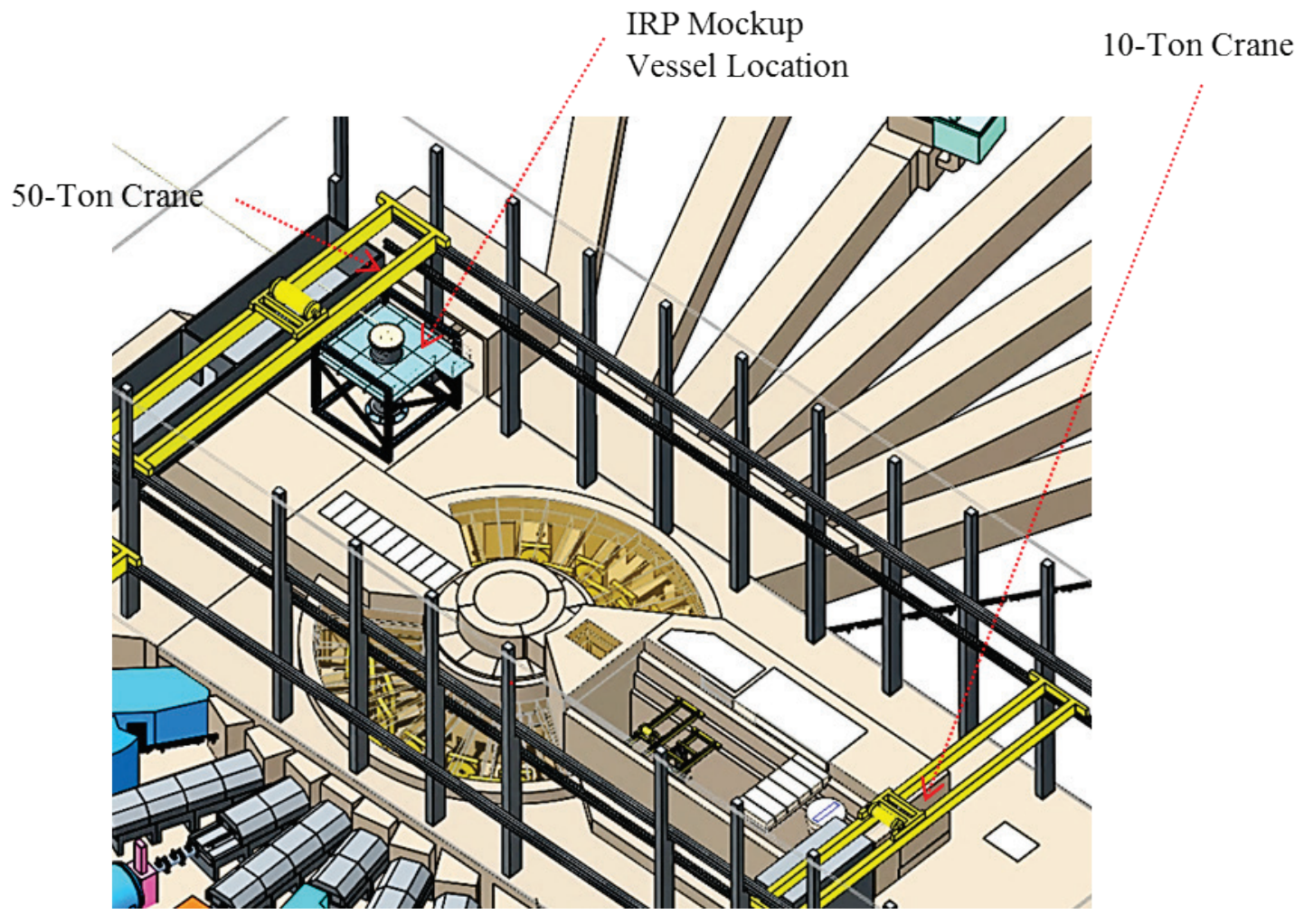

Fig. 4.68. Proposed location of STS IRP mockup vessel.

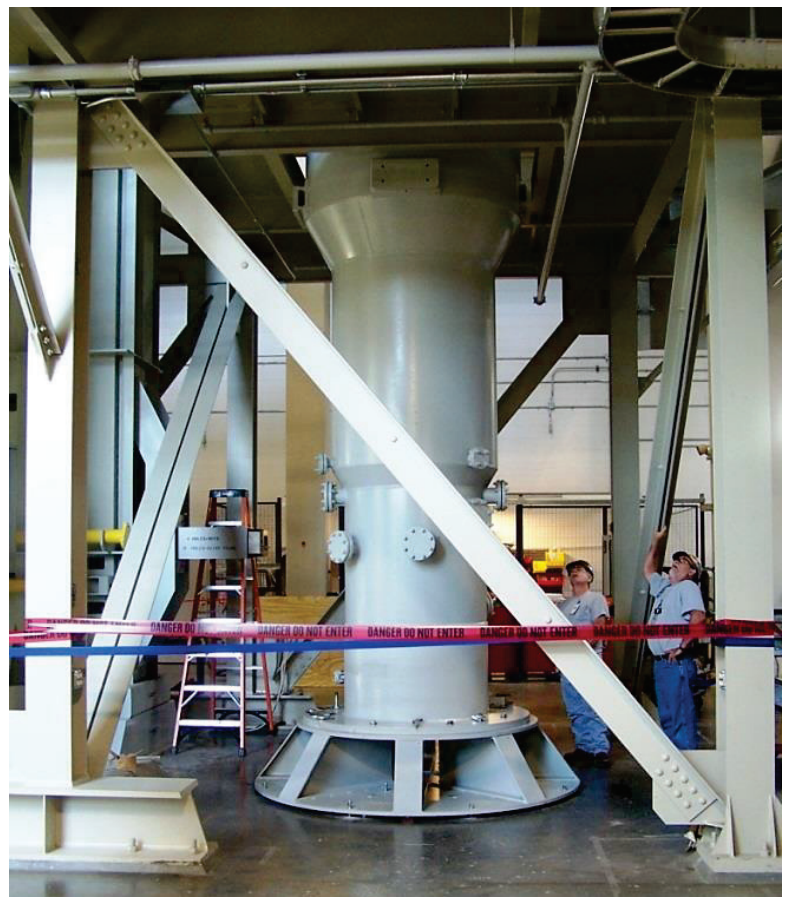

Fig. 4.69. FTS IRP mockup vessel. 


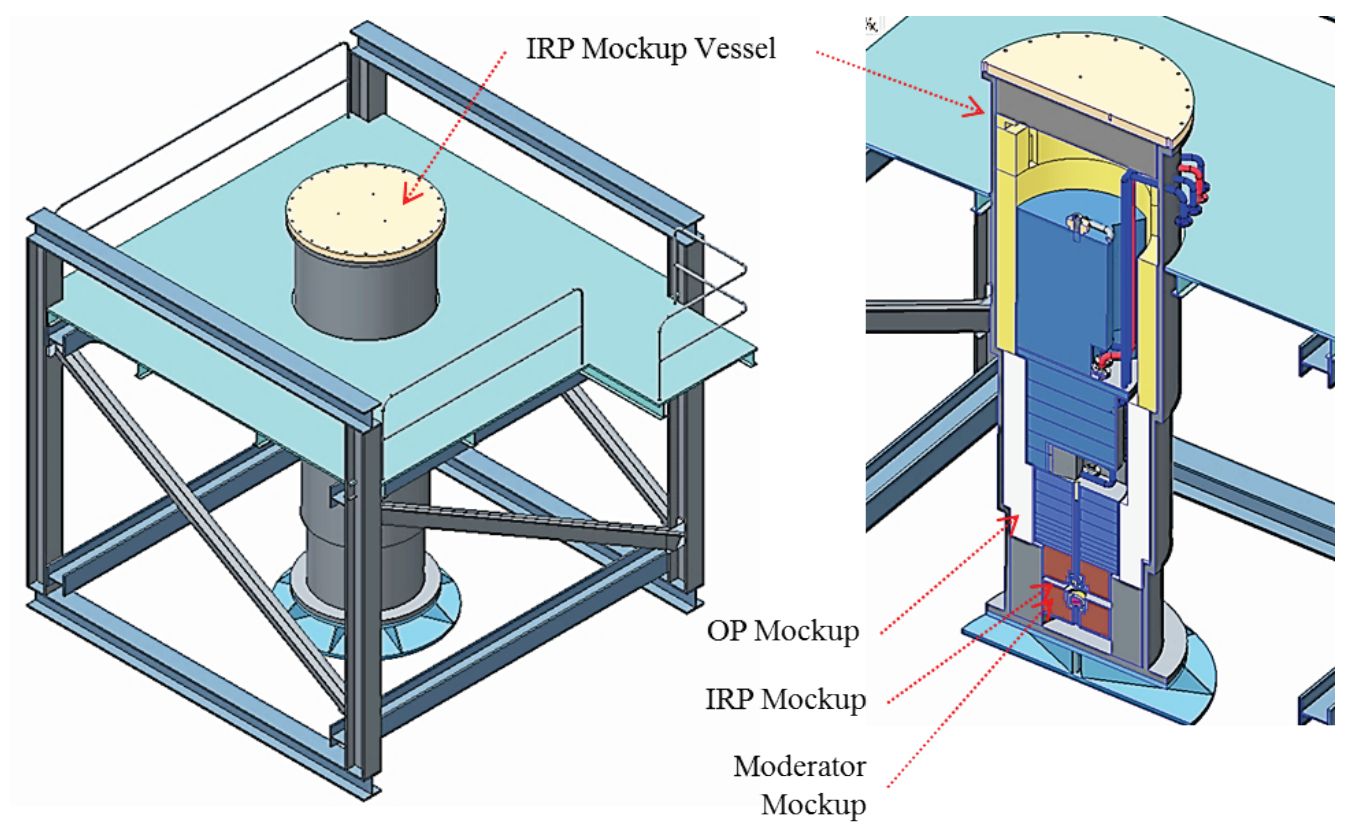

Fig. 4.70. STS IRP mockup vessel (IRP is shown for visual effects).

Additionally, a mock-up testing facility will be required for the neutron guide insert and operating shutter replacement activities. This facility will replicate the nominal core vessel nozzle configuration and support all phases of the replacement operation. This facility will be used to enable testing of the tooling operation, technician training, and storage of the tooling when it is not in use.

\subsection{REFERENCES}

1. D. Haynes, "Introduction and Overview of the ISIS Target Systems," June 25, 2013, ISIS and SNS Bilateral Workshop, Oak Ridge, Tennessee, June 25-27, 2013.

2. A. T. Nelson, et.al., "Fabrication of a tantalum-clad tungsten target for LANSCE," Journal of Nuclear Materials 431(1-3), 172-184 (2012). j.jnucmat.2011.11.041

3. F. W. Wang, et. al., "Physical design of target station and neutron instruments for China Spallation Neutron Source," Science China 56(12), 2410-2424 (December 2013).

4. T. McManamy, et.al., "Rotating Target Development for SNS Second Target Station," ICANS XIX, Grindelwald, Switzerland, January 1, 2010. 


\section{INSTRUMENT SYSTEMS}

\subsection{INTRODUCTION}

Instrument Systems will deliver the technical scope required to produce eight neutron scattering instrument end stations. This includes (1) the infrastructure to transport the neutrons from the moderator face to the sample position and to shape, manipulate, and shield the neutron beam as required along the incident flight path and (2) the instrument end station and its associated shielding, mechanical components, neutron detectors, data acquisition system, and initial suite of sample environment equipment. By the conclusion of the project, these eight instruments will be ready to accept neutron beams and proceed with their commissioning.

\subsection{INSTRUMENT PLANNING SUITE}

A number of instruments have been included in the initial STS scoping exercise to explore the diversity of source parameters and geometries required, particularly as regards required moderator performance. These initial instrument concepts represent the outcome of a number of working groups seeking to address scientific challenges that were identified in four major workshops (2014). Further details can be found in Ref. [1]. This exercise led to the three-moderator configuration described in Sect. 4.3.2. Table 5.1 lists these instruments, critical design parameters, and initial moderator requirements. The requirements span a larger number of moderators than can be supported by STS; therefore, those listed in the table represent something of a compromise across this instrument suite. The three moderators are

1. A high-intensity coupled moderator (HICM), nominally a $5 \times 5$ to $7 \times 7 \mathrm{~cm}^{2}$ viewed face, coupled parahydrogen moderator viewed from a single side.

2. A high-peak-brightness coupled para-hydrogen moderator (HPCM) with three viewed faces. Faces -a and $-\mathrm{c}$ are $3 \times 3 \mathrm{~cm}^{2}$ viewed surfaces, and $-\mathrm{b}$ is a $3 \times 6 \mathrm{~cm}^{2}$ viewed surface.

3. A multi-spectral decoupled moderator with $7 \times 7 \mathrm{~cm}^{2}$ viewed surfaces. One side is ambienttemperature water (MSDT, T for thermal) and the second side cold para-hydrogen (MSDC, $\mathrm{C}$ for cold).

As instrument concepts mature, the selection of the optimum moderator is likely to change in some cases. Iteration between instrument needs and moderator design will probably modify the characteristics of the current moderator suite.

Instrument Systems will provide eight initial instruments as its project baseline, although STS will support a final instrument complement of 22. Eight instruments have been selected from the list in Table 5.1 (indicated by ${ }^{b}$ ) as representative of the highest performance and scientific impact. Note that final instrument selection will be a continuing process that engages the neutron scattering user community through the conceptual design phase of the project. Detailed science cases for these instruments have been described in Ref. [1]. This section presents additional details for these eight illustrative instruments. 
Table 5.1. Initial planning suite of neutron scattering instruments

\begin{tabular}{|c|c|c|c|c|}
\hline $\begin{array}{l}\text { Beam } \\
\text { line }\end{array}$ & Name & Description & $\begin{array}{l}{ }^{a} \text { Nominal } \\
\text { length } \\
\text { (m) }\end{array}$ & $\begin{array}{l}\text { Moderator } \\
\text { type }\end{array}$ \\
\hline 1 & ${ }^{b}$ Zeemans & $\begin{array}{l}\text { Versatile instrument designed for studies at the highest } \\
\text { magnetic fields (integrated high-field magnet) }\end{array}$ & 60 & HICM \\
\hline 2 & ${ }^{b} \mathrm{QIKR}$ & $\begin{array}{l}\text { Kinetics Reflectometer-horizontal sample } \\
\text { reflectometer optimized for rapid measurements }\end{array}$ & 20 & HICM \\
\hline 3 & $\begin{array}{l}{ }^{b} \text { HiRes- } \\
\text { SWANS }\end{array}$ & $\begin{array}{l}\text { High Resolution Small/Wide Angle Neutron } \\
\text { Scattering-SANS/diffractometer optimized for length } \\
\text { scales from the molecular to tens of nanometers }\end{array}$ & 17 & HICM \\
\hline 4 & VBPR & $\begin{array}{l}\text { Variable Beam Profile Reflectometer optimized to } \\
\text { illuminate millimeter-size samples }\end{array}$ & 30 & HРCM-a \\
\hline 5 & FLOODS & $\begin{array}{l}\text { Flux-Optimized Order-Disorder SANS for fast kinetics } \\
\text { and out-of-equilibrium behavior }\end{array}$ & $25-40$ & HPCM-a \\
\hline 6 & M-WASABI & $\begin{array}{l}\text { Magnetism-Wide and Small Angles with Big } \\
\text { Intensity-optimized for complete polarized } \\
\text { reflectometry (specular, off-specular, GISANS) }\end{array}$ & $38-40$ & HPCM-a \\
\hline 7 & M-STAR & $\begin{array}{l}\text { Magnetism-Second Target Advanced Reflectomer- } \\
\text { optimized for magnetic studies of small samples }\end{array}$ & 32 & HPCM-a \\
\hline 8 & SPHIINX & $\begin{array}{l}\text { Spherical Indirect Inelastic Xtal Spectrometer- } \\
\text { optimized for broad-band inelastic measurements of } \\
\text { small samples }\end{array}$ & 40 & MSDT \\
\hline 9 & XTREME-X & $\begin{array}{l}\text { Extreme Multi-Energy Spectrometer with Xtal } \\
\text { analyzers-optimized for measurements restricted to the } \\
\text { horizontal plane by extreme sample environments }\end{array}$ & 45 & MSDT \\
\hline 10 & JANUS & $\begin{array}{l}\text { A hybrid indirect geometry spectrometer coupled with a } \\
\text { low-angle direct geometry spectrometer optimized for } \\
\text { inelastic measurements of irreversible phenomena and in } \\
\text { situ manipulation of samples }\end{array}$ & 40 & MSDT \\
\hline 11 & TBD & & TBD & MSDT \\
\hline 12 & BWAVES & $\begin{array}{l}\text { Broad-range Wide Angle Velocity Selector-an indirect } \\
\text { geometry spectrometer that provides very high } \\
\text { resolution coupled to a broad dynamic range in energy } \\
\text { transfer }\end{array}$ & 16 & HPCM-b \\
\hline 13 & HERTZ & $\begin{array}{l}\text { High Energy Resolution Terahertz spectrometer - } \mathrm{a} \\
\text { cold neutron chopper spectrometer optimized for large } \\
\text { samples and relatively high energy resolution }\end{array}$ & $25-30$ & HPCM-b \\
\hline 14 & ${ }^{b}$ VERDI & $\begin{array}{l}\text { Versatile Diffractometer-a diffractometer optimized } \\
\text { for magnetic structure studies of both powders and } \\
\text { single crystals }\end{array}$ & $40-60$ & HPCM-b \\
\hline 15 & ${ }^{b}$ DYPOL & $\begin{array}{l}\text { Dynamically Polarized crystallography instrument-- } \\
\text { optimized for study of small hydrogen-containing single } \\
\text { crystals, particularly proteins }\end{array}$ & 91 & HPCM-b \\
\hline 16 & NeSCry & $\begin{array}{l}\text { Neutron Single Crystal diffractometer-optimized for } \\
\text { study of small single crystals and high, low-Q resolution } \\
\text { with an emphasis on magnetic structure }\end{array}$ & $30-50$ & HPCM-b \\
\hline 17 & TBD & & TBD & HPCM-c \\
\hline
\end{tabular}


Table 5.1. Initial planning suite of neutron scattering instruments (continued)

\begin{tabular}{|c|c|c|c|c|}
\hline $\begin{array}{l}\text { Beam } \\
\text { line }\end{array}$ & Name & Description & $\begin{array}{l}{ }^{a} \text { Nominal } \\
\text { length } \\
(\mathbf{m})\end{array}$ & $\begin{array}{l}\text { Moderator } \\
\text { type }\end{array}$ \\
\hline 18 & EWALD & $\begin{array}{l}\text { Enhanced Wide-Angle Laue Diffractometer-a single- } \\
\text { crystal diffractomter optimized for the study of small } \\
\text { protein crystals }\end{array}$ & 91 & HPCM-c \\
\hline 19 & ${ }^{b} \mathrm{CHESS}$ & $\begin{array}{l}\text { Chopper Spectrometer for Small Samples-a cold } \\
\text { neutron chopper spectrometer optimized for the study of } \\
\text { very small samples }\end{array}$ & $25-30$ & HPCM-c \\
\hline 20 & ${ }^{b} \mathrm{MBARS}$ & $\begin{array}{l}\text { Mica Backscattering Spectrometer-an indirect } \\
\text { geometry, ultra-high-resolution spectrometer optimized } \\
\text { for dynamics study of biological and soft matter samples }\end{array}$ & 75 & MSDC \\
\hline 21 & ${ }^{b}$ HighResPD & $\begin{array}{l}\text { High Resolution Powder Diffractometer—optimized for } \\
\text { the highest resolution }\end{array}$ & 120 & MSDC \\
\hline 22 & TBD & & TBD & MSDC \\
\hline
\end{tabular}

\subsubsection{Zeemans}

Zeemans is a versatile neutron scattering instrument with an integrated, horizontal high-field magnet and dedicated sample environment equipment specific to use with the magnet. It will be a facility reaching fields beyond 35 Tesla and offering a wide suite of neutron scattering techniques including spectroscopy, diffraction, reflectometry, and small-angle neutron scattering (SANS). The goal is to create the world center for high-magnetic-field neutron scattering.

The Zeemans instrument is envisioned as occupying neutron beam line 1 viewing the HICM. The beam line will be curved and may include a bender to avoid a direct line-of-sight view of the moderator. It will incorporate a number of neutron choppers and adjustable optical components (the last $5 \mathrm{~m}$ of the guide system) so as to tailor the neutron beam for individual scattering techniques. The instrument will include the capability to provide a polarized neutron beam and to perform analysis of the scattered beam polarization. A previous National Science Foundation-funded project resulted in a conceptual and engineering design for the superconducting magnet system that provides a head start for Zeemans.[2-4] Figure 5.1 shows the concept for the detector vessel and magnet that was developed in that project.

Zeemans requires significant infrastructure, including a dedicated building, helium liquification plant, dc power potentially up to $12 \mathrm{MW}$ for the hybrid form of the magnet (highest field conditions), and sufficient cooling water (a dedicated cooling tower). The Zeemans building and associated utilities are described in Sect. 6, Conventional Facilities. The Zeemans instrument will require the development of specialized sample environment equipment that is compatible for use with high magnetic fields, matches the specific geometry of the instrument, and is capable of spanning the temperature range of $50 \mathrm{mK}$ to $1000^{\circ} \mathrm{C}$ (likely with multiple devices). Table 5.2 lists key Zeemans instrument parameters. 


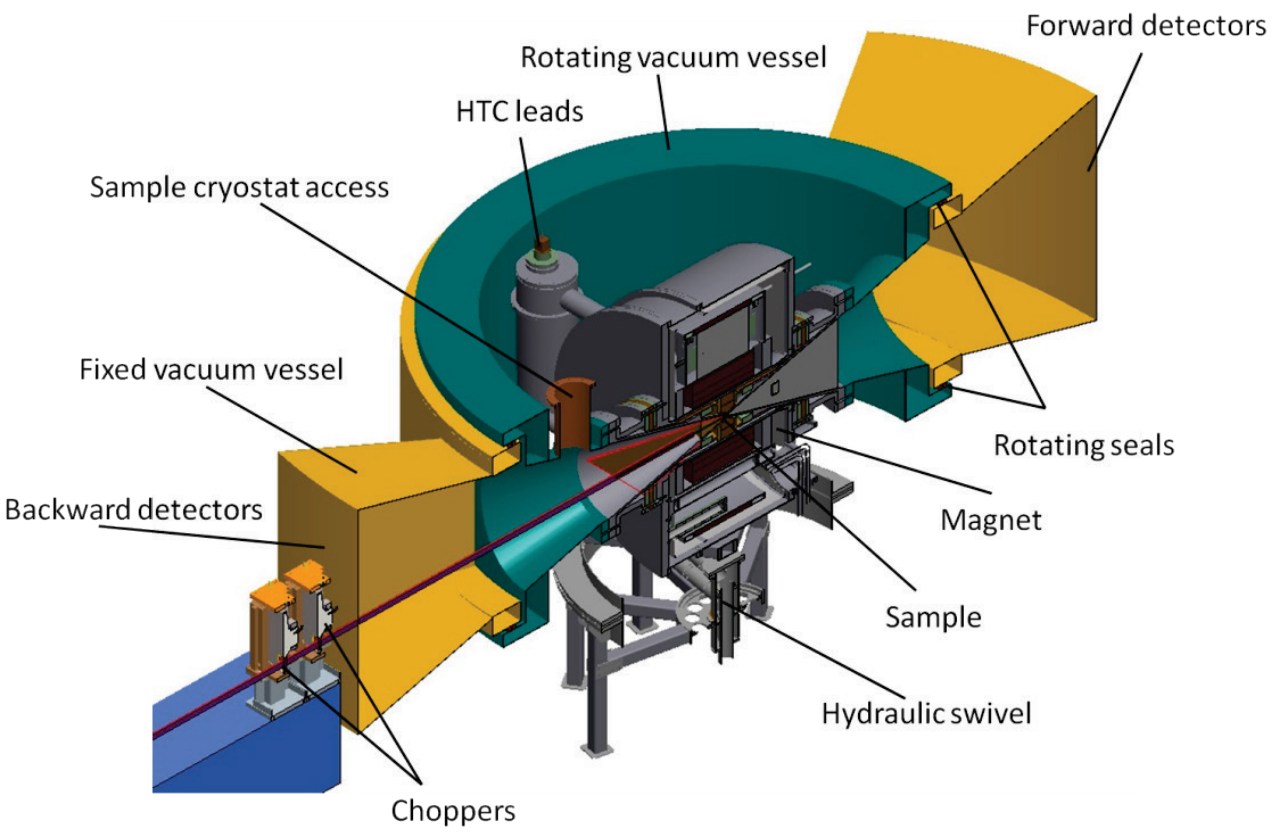

Fig. 5.1. Zeemans detector vessel and magnet.

Table 5.2. Key parameters of Zeemans

\begin{tabular}{cl}
\hline Parameter & \multicolumn{1}{c}{ Description } \\
\hline Moderator & High-intensity coupled moderator \\
Sample size & Maximum: $2 \times 2 \mathrm{~cm}^{2}$ \\
Moderator-sample distance & $60 \mathrm{~m}$ \\
Sample-detector distance & $5 \mathrm{~m}$ \\
Inelastic: Energy range & $10 \mathrm{meV} \leq \mathrm{E}_{\mathrm{i}} \leq 200 \mathrm{meV}$ \\
SANS and diffraction: $\lambda$-range & $1 \AA \leq \lambda \leq 10 \AA$ \\
Inelastic resolution & $\Delta \mathrm{E} / \mathrm{E}_{\mathrm{i}}$ range between 1 and $15 \%$ depending \\
on $\mathrm{E}_{\mathrm{i}}$ and chopper settings \\
Detector & $8 \mathrm{~mm}$ linear position-sensitive detector, ${ }^{3} \mathrm{He}$ \\
\hline
\end{tabular}

\subsubsection{QIKR}

The Quite Intense Kinetics Reflectometer (QIKR) features horizontal sample geometry and a broad "single-shot" Q-range for structural and kinetic studies of solid, liquid/solid, and free liquid surfaces and interfaces. The instrument will be placed as close as possible to the moderator, capitalizing on the STS low-repetition rate to collect specular reflectivity data at a single incident beam angle and in a single frame over a full decade of $\mathrm{Q}$ (e.g., $0.02 \AA^{-1} \leq \mathrm{Q} \leq 0.2 \AA^{-1}$ ). Collecting data in a single setting of the instrument greatly simplifies both data collection and reduction and eliminates the 60-90 s needed to change instrument settings between Q-bands on the FTS reflectometers. Broad-Q specular reflectivity will be collected in seconds or less.

This instrument will be installed at beam line 2 viewing the HICM. The sample position will be placed as close to the moderator as possible to maximize the Q-range sampled in a single frame. It requires a $20 \times 20 \mathrm{~cm}^{2}$ detector with $1 \mathrm{~mm}$ pixel resolution capable of a sustained count rate of $0.5-1 \mathrm{M}$ counts/s and a peak count rate of 5-10 M counts/s. As the entire reflectivity curve is captured in a single instrument setting, the detector 
must have a dynamic range of $10^{7}$. The neutron guide will be curved or possibly will incorporate a multichannel bender and may employ elliptical focusing in the horizontal direction. One bandwidth neutron chopper (or possibly two) will define the incident neutron wavelength band. Table 5.3 lists key QIKR parameters.

Table 5.3. Key parameters of QIKR

\begin{tabular}{cl}
\hline Parameter & \multicolumn{1}{c}{ Description } \\
\hline Moderator & High-intensity coupled moderator \\
Sample Size & $1.5 \times 3 \mathrm{~cm}^{2}$ \\
Moderator-sample distance & as short as $13.5 \mathrm{~m}$ \\
Sample-detector distance & $1.5 \mathrm{~m}$ \\
Wavelength range & $2.5 \AA \leq \lambda \leq 30 \AA$ \\
Resolution & $\Delta \lambda / \lambda<0.01$ \\
& ${ }^{3} \mathrm{He} 2$-dimensional position-sensitive detector \\
Detector & (if possible) \\
\hline
\end{tabular}

\subsubsection{HiRes-SWANS}

High-Resolution Small/Wide Angle Neutron Scattering (HiRes-SWANS) is single instrument that combines the features of a modest-resolution neutron diffractometer and a SANS instrument to probe length scales from the interatomic out to tens of nanometers simultaneously. The instrument will cover a broad, simultaneous Q-range by taking advantage of the low STS repetition rate and being located as close to the moderator as possible. The optics system will be flexible, enabling grazing incidence geometry as well.

HiRes-SWANS will be built on beam line 3 viewing the HICM. (It is likely that instrument optimization will identify the high-brightness moderator as a better match.) The instrument will have a broad neutron bandwidth able to probe structures from sub-1 $\AA$ to $600 \AA$ simultaneously. The instrument will include a multi-channel bender, a focusing optics system, and flexible collimation. The optics design will also be capable of deflecting the beam downward to support grazing-incidence geometry. The low-angle SANS detector will be located 3-5 $\mathrm{m}$ from the sample, and the diffraction detectors at angles up to two-theta $=45^{\circ}$ will be located approximately $1-1.5 \mathrm{~m}$ from the sample. The lowest-scattering angles will require relatively high spatial resolution and must be able to achieve high count rates. Table 5.4 lists key HiRes SWANS parameters.

Table 5.4. Key parameters of HiRes-SWANS

\begin{tabular}{cl}
\hline Parameter & \multicolumn{1}{c}{ Description } \\
\hline Moderator & High-intensity coupled moderator \\
Sample size & up to $1 \times 1 \mathrm{~cm}^{2}$ \\
Moderator-sample distance & $15-17 \mathrm{~m}$ \\
Sample-detector distance & $1.5-5 \mathrm{~m}$ \\
Wavelength range & $0.25 \AA \leq \lambda \leq 20 \AA$ \\
Resolution & $\Delta \mathrm{Q} / \mathrm{Q}=0.01$ \\
& $0.8 \mathrm{~cm}$ diameter linear position-sensitive \\
& detector, ${ }^{3} \mathrm{He}$ \\
Detector & Low-angle Anger camera $(1.2 \mathrm{~mm}$ \\
& resolution) \\
\hline
\end{tabular}




\subsubsection{VERDI}

VERDI is a cold-neutron diffractometer optimized for studies of magnetic and large unit cell structures. It benefits from the $10 \mathrm{~Hz}$ repetition rate giving a large wavelength band per frame, allowing the instrument to be built at 40-60 $\mathrm{m}$ from the moderator. This long flight path allows VERDI to use the broader time pulses of the STS coupled moderators and the significantly higher peak intensity of the neutron pulses, compared with the de-coupled, poisoned moderators typically used on diffraction instruments.

VERDI will be installed on beam line 14 and view the $3 \times 6 \mathrm{~cm}^{2}$ face $\mathrm{b}$ of the HPCM. It will operate as either a powder or single-crystal diffractometer as desired and will have an integrated polarization capability. The instrument will have a neutron bandwidth of 6.5-9.8 $\AA$ so that the desired Q-range can typically be accessed in a single frame. The elliptical guide system will provide sufficient flexibility to cover a wide range of incident beam divergences, depending on the mode of operation (powder diffraction: horizontal beam divergence of 0.2 to $1^{\circ}$ and vertical beam divergence of 2 to $3^{\circ}$; single-crystal diffraction: symmetric beam divergence of up to $1^{\circ}$ ). This large range of desired divergence will likely require translating guide components. The instrument will include an oscillating radial collimator for background reduction. The ${ }^{3} \mathrm{He}$ detector array will cover $175^{\circ}$ in the horizontal plane and extend to $\pm 20^{\circ}$ in the vertical. In addition to bandwidth neutron choppers, the beam line is envisioned as incorporating a Fermi chopper that can be translated into and out of the beam to provide a monochromatic beam for studies of diffuse scattering. Table 5.5 lists key VERDI parameters.

Table 5.5. Key parameters of VERDI

\begin{tabular}{cl}
\hline Parameter & \multicolumn{1}{c}{ Description } \\
\hline Moderator & High-peak-brightness coupled moderator, side b \\
Sample size & $2 \times 2 \mathrm{~mm}^{2}$ to $1 \times 2 \mathrm{~cm}^{2}$ \\
Moderator-sample distance & $40-60 \mathrm{~m}$ \\
Sample-detector distance & $2.0-3.5 \mathrm{~m}$ \\
$\lambda$ Range & $1.2 \AA \leq \lambda \leq 14 \AA$ \\
Resolution & $\Delta \mathrm{Q} / \mathrm{Q} 0.2-0.4 \%$ \\
& $0.8 \mathrm{~cm}$ diameter linear positive-sensitive \\
Detector & detector, ${ }^{3} \mathrm{He}$ \\
\hline
\end{tabular}

\subsubsection{DyPOL}

The Dynamically Polarized Crystallography instrument (DyPOL) is a single-crystal diffractometer optimized for macromolecules, with the integrated capability to dynamically polarize the spins of hydrogen atoms in the sample. Polarizing the neutron beam and aligning the proton (hydrogen) spins in a polarized sample drastically changes the coherent and incoherent cross sections of hydrogen, amplifying the coherent signal by almost an order of magnitude and suppressing the incoherent background to zero, as illustrated in Figure 5.2. Its instrument parameters are essentially identical to those of the proposed Enhanced Wide-Angle Laue Diffractometer (EWALD) instrument. It multiples the intensity gains enabled at STS by the gains enabled by dynamic neutron polarization (DNP). Development of DNP is ongoing at ORNL and at other locations in the world. Figure 5.3 illustrates the essential elements of a DNP apparatus that includes a low-temperature dilution refrigerator, a 2.5-5 T superconducting magnet, and a microwave source $(2-4 \mathrm{~mm})$. The instrument exploits the low-repetition rate of STS by retaining the ideal bandwidth per frame at an instrument length that provides sufficient wavelength resolution using the neutron pulse widths produced by an STS coupled moderator. 


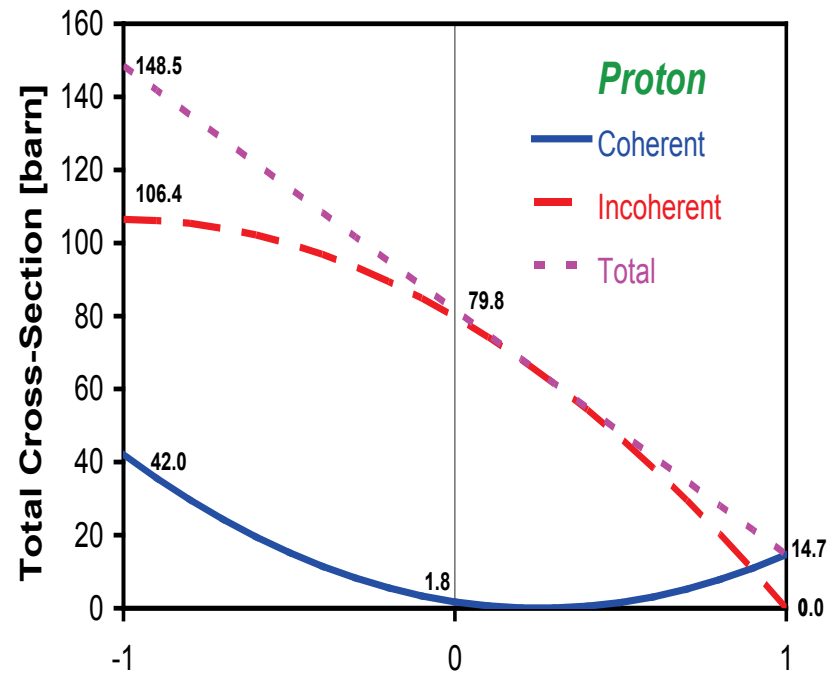

Proton Polarization

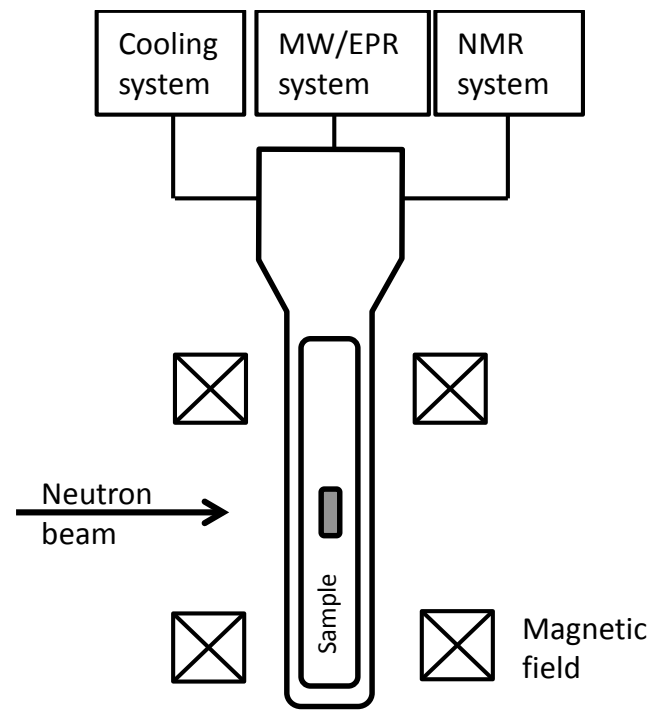

Fig. 5.3. Schematic view of the DNP apparatus and its orientation relative to a polarized neutron beam. sections as a function of proton polarization. When protons are fully polarized parallel to the polarization of neutrons, the incoherent cross section decreases from 79.8 to 0 barns, while the coherent cross section increases from 1.8 to 14.7 barns.

DyPOL will be installed on beam line 15 viewing the $3 \times 6 \mathrm{~cm}^{2}$ face of the HPCM, side b. At a $90 \mathrm{~m}$ moderator-sample distance, DyPOL will have the same wavelength resolution as the much shorter MaNDi instrument that views a decoupled, poisoned hydrogen moderator at FTS. The bandwidth per frame, $4.4 \AA$, is perfectly matched to the most common MaNDi operating mode. The guide system will be curved to avoid the fast neutrons and gammas produced as the protons strike the neutron production target. At least one and possibly two bandwidth choppers will define the wavelength band incident on the sample. Anger camera neutron detectors will be used to measure the scattered neutrons.

Table 5.6. Key parameters of DyPOL

\begin{tabular}{cl}
\hline Parameter & \multicolumn{1}{c}{ Description } \\
\hline Moderator & High-peak-brightness coupled moderator, side b \\
Sample size & $1 \times 1 \mathrm{~mm}^{2}$ \\
Moderator-sample distance & $90 \mathrm{~m}$ \\
Sample-detector distance & $0.45 \mathrm{~m}$ \\
Wavelength range & $2 \AA \leq \lambda \leq 8 \AA$ \\
Resolution & $\Delta \mathrm{d} / \mathrm{d}=0.0015$ \\
Detector & Anger Camera with $1.2 \mathrm{~mm}$ spatial resolution \\
\hline
\end{tabular}




\subsubsection{CHESS}

The Chopper Spectrometer for Small Samples (CHESS) is a direct-geometry, cold-neutron chopper spectrometer optimized for sample sizes between $1 \mathrm{~mm}^{3}$ and $1 \mathrm{~cm}^{3}$ and medium-energy resolution. CHESS will take full advantage of the peak brilliance of the high-peak-brightness STS moderators. It will include polarized beam capabilities to enable the 3-dimensional polarization analysis required to measure individual components of the $\mathrm{S}_{\alpha \beta}(\mathrm{Q}, \omega)$ tensor.

The low repetition rate of STS will enable simultaneous measurement with a range of incident energies using repetition rate multiplication methods. The $10 \mathrm{~Hz}$ repetition rate coupled with a $25-30 \mathrm{~m}$ incident flight path means that all incident energies down to $1 \mathrm{meV}$ can be accessed in the first frame. This extremely broad incident energy range requires development of a special pulse suppression chopper that allows long-wavelength neutron sub-frames more counting time at the detector. Such a chopper has been prototyped as part of the European Spallation Source T-REX project.[5]

This instrument will occupy beam line 19 and view the $3 \times 3 \mathrm{~cm}^{2}$ HPCM face ' $\mathrm{c}$ '. The beam line will be straight and will incorporate a number of choppers to shape the neutron pulse, block the high-energy prompt pulse to minimize background, control the sub-frame durations to support repetition rate multiplication, and monochromate the incident neutron energy. Particular attention must be paid to sample environment equipment so as to maximize sample illumination of the large detector array covering $2 \pi$ ster of solid angle. The neutron beam delivery system must be adaptable to increase beam divergence for small samples to as large as $\pm 4^{\circ}$ in either the horizontal or the vertical direction as required. Relaxed energy resolution allows relatively close placement of the detectors to the sample.

Table 5.7. Key parameters of CHESS

\begin{tabular}{cl}
\hline Parameter & \multicolumn{1}{c}{ Description } \\
\hline Moderator & High-peak-brightness coupled moderator, \\
& side c \\
Sample size & $1 \times 1 \mathrm{~mm}^{2}$ to $1 \times 1 \mathrm{~cm}^{2}$ \\
Moderator-sample distance & $25-30 \mathrm{~m}$ \\
Sample-detector distance & $2.5-3.5 \mathrm{~m}$ \\
Energy range & $0.5 \mathrm{meV} \leq \mathrm{E}_{\mathrm{i}} \leq 100 \mathrm{meV}$ \\
& $2.5-4.5 \%$ variable $\Delta \mathrm{E} / \mathrm{E}_{\mathrm{i}}$ range; \\
Resolution & $\Delta \mathrm{Q}<0.05 \AA^{-1}$ \\
& $1.5 \mathrm{~cm} \mathrm{diameter} \mathrm{linear} \mathrm{position-sensitive}$ \\
Detector & detector, ${ }^{3} \mathrm{He}$ \\
\hline
\end{tabular}

\subsubsection{MBARS}

The Mica Backscattering Spectrometer (MBARS) is a mica-based crystal analyzer spectrometer operating in near-backscattering conditions achieving an energy resolution of $\approx 200 \mathrm{neV}$ full width at half maximum (FWHM) operating at an elastic neutron wavelength of $20 \AA$. This instrument will provide unparalleled energy resolution coupled with a very broad dynamic range in energy transfer of $\pm 50 \mu \mathrm{eV}$ ( \pm 200 resolution widths). MBARS requires sharp neutron pulses at a low-repetition rate; therefore, it is ideally matched to a high timing resolution (de-coupled) cold moderator at STS. This concept is nearly identical to an early proposal [6] (Figure 5.4) but proposes an innovative means to filter out high-order contamination from the analyzer crystals using a novel device, the Wide-Angle Velocity Selector (WAVES), under development at ORNL.[7] 
This instrument will be installed on beam line 20 viewing the cold, para-hydrogen $7 \times 7 \mathrm{~cm}^{2}$ face of the multi-spectral decoupled moderator. The neutron guide will be curved to avoid line-of-sight of the moderator and reduce the background from prompt neutrons and gammas. The analyzer will be synthetic fluorophlogopite with a $0.3^{\circ}$ mosaic (FWHM) in near-backscattering geometry covering a solid angle of 3.7 ster. The neutron guide will deliver at least $\pm 3^{\circ}$ of beam divergence in the horizontal direction (higher is acceptable). Divergence in the vertical direction may be limited by use of the WAVES device but will be at least $\pm 0.5^{\circ}$. The beam line will incorporate one or maybe two bandwidth choppers (if sharper open/close transition is required).

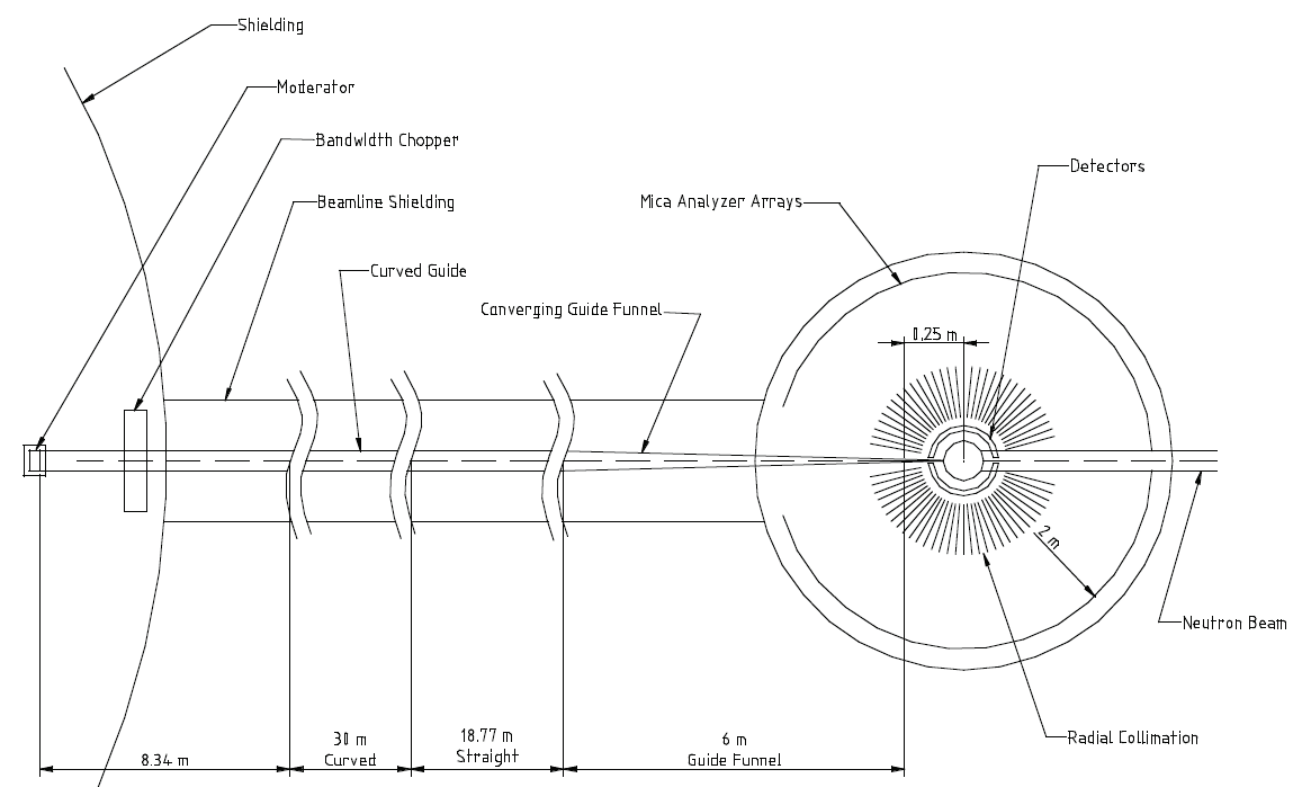

Fig. 5.4. Schematic view of the MBARS spectrometer as proposed in ref. [6]. The radial collimator may be replaced with the WAVES device. The instrument length proposed here is somewhat longer than the original.

Table 5.8. Key parameters of MBARS

\begin{tabular}{cl}
\hline Parameter & \multicolumn{1}{c}{ Description } \\
\hline Moderator & Multi-spectral decoupled moderator -cold \\
Sample size & $5 \times 5 \mathrm{~mm}^{2}$ \\
Moderator-sample distance & $75 \mathrm{~m}$ \\
Sample-analyzer distance & $2 \mathrm{~m}$ \\
Analyzer-detector distance & $1.75 \mathrm{~m}$ \\
Wavelength range & $17.9 \AA \leq \lambda \leq 23.1 \AA$ \\
Q-range & $0.03 \AA^{-1} \leq \mathrm{Q} \leq 0.6 \AA^{-1}$ \\
Resolution & $200 \mathrm{neV}(\mathrm{FWHM})$ at the elastic peak \\
Detector & $0.8 \mathrm{~cm} \mathrm{diameter} \mathrm{linear} \mathrm{position-sensitive}$ \\
& detector, ${ }^{3} \mathrm{He}$ \\
\hline
\end{tabular}

\subsubsection{HighResPD}

The High-Resolution Powder Diffractometer (HighResPD) is specified to complement the existing POWGEN and proposed RAPID instruments on FTS. It will provide a $\Delta \mathrm{d} / \mathrm{d}$ resolution of $0.035 \%$ 
matching the world's highest-resolution neutron diffraction instruments at JPARC and ISIS. This instrument will be the longest instrument at the SNS site and will require the low repetition rate of the STS to give it a sufficiently broad wavelength band to cover a broad range of d-spacings.

This instrument will be located on beam line 21 viewing the cold, para-hydrogen $7 \times 7 \mathrm{~cm}^{2}$ face of the multi-spectral decoupled moderator. This beam line views the moderator at normal incidence, and the site geography allows the instrument to be as long as $120 \mathrm{~m}$. Both of these elements are required to achieve the high-wavelength resolution required by this instrument. The moderator is anticipated to provide a moderator pulse width of $6.8 \mu \mathrm{sec}$ at a neutron wavelength of $1 \AA$, giving $\Delta \lambda / \lambda$ of $2.2 \cdot 10^{-4}$ at $120 \mathrm{~m}$, which is sufficient to achieve the desired resolution. The beam line will be straight to provide uniform illumination across the width of the sample. Optimization might result in a slightly shorter instrument at $100 \mathrm{~m}$, providing a bit more bandwidth in a single frame, $4 \AA$ vs $3.3 \AA$, to cover a broader range in dspacing at a correspondingly reduced wavelength resolution of $\Delta \lambda / \lambda=2.6 \cdot 10^{-4}$. One or possibly two bandwidth choppers will define the incident neutron wavelength band and a T0 chopper will be required. The instrument must preserve the ability to operate effectively at $5 \mathrm{~Hz}$ when the broadest wavelength band in a single frame is required.

Table 5.9. Key parameters of HiRes-PD.

\begin{tabular}{cl}
\hline Parameter & \multicolumn{1}{c}{ Description } \\
\hline Moderator & multi-spectral decoupled moderator-cold \\
Sample size & 0.5 to $1.0 \mathrm{~cm}$ diameter; 1 to $2.5 \mathrm{~cm}$ tall \\
$\begin{array}{c}\text { Moderator-sample } \\
\text { distance }\end{array}$ & $120 \mathrm{~m}$ \\
$\begin{array}{c}\text { Sample-detector } \\
\text { distance }\end{array}$ & Backscattering and $90^{\circ}: 2 \mathrm{~m}$ \\
Wavelength range & $0.5 \AA \leq \lambda \leq 8 \AA$ \\
Resolution & $\Delta \mathrm{d} / \mathrm{d}=0.035 \%$ \\
Detector & Low-angle: $0.8 \mathrm{~cm}$ diameter linear position-sensitive detector, ${ }^{3} \mathrm{He}$ \\
& Backscattering and $90^{\circ}:$ TBD \\
\hline
\end{tabular}

\subsection{COMMON COMPONENTS}

The neutron scattering instruments have many components in common. Common designs and standardization of requirements will result in lower cost and efficiency.

\subsubsection{In-monolith Neutron Optics}

The smaller, high-brightness STS moderators will enable better optimization of neutron optics systems, but probably at the cost of increased mechanical complexity and possibly active alignment systems. Particularly in the near moderator regions, care must be taken in the mechanical design of the target monolith to support the higher precision required. It will be important to support a close approach to the moderator of neutron optical systems, $\approx 60 \mathrm{~cm}$ or less, to minimize the effects of under-illumination of the neutron optics system. Instrument Systems will provide the designs and internal components and will be responsible for installation of the eight beam line inserts that support the baseline instrument suite. As noted in Section 4.5.4, Target Systems will provide the remaining 14 "blank" inserts required to close the empty beam line ports. 


\subsubsection{Neutron Choppers}

Neutron choppers are rotating mechanical devices that interrupt the neutron beam to modify the wavelength and/or time distribution of neutrons in the incident (and possibly in the scattered) neutron flight path. The three general types of neutron choppers used on the SNS FTS will certainly find application at STS beam lines, with the possible exception of the high-speed Fermi choppers (vertical rotation axis) used on the Cold Neutron Chopper Spectrometer for pulse-shaping and on the Wide Angular-Range Chopper Spectrometer (ARCS), the Fine-Resolution Fermi Chopper Spectrometer (SEQUOIA), and the Hybrid Spectrometer (HYSPEC) for monochromatization. As instrument concepts mature, the lower repetition rate of STS relative to FTS may require modifications to both the bandwidth and the T0 choppers.

\section{Bandwidth choppers}

Bandwidth choppers are devices that rotate aluminum or carbon fiber disks coated with neutron adsorbing material. One or more apertures are cut out of the disk, which is phased with the neutron source to allow neutrons within defined wavelength ranges to be transmitted while the remainder are removed. At the STS $10 \mathrm{~Hz}$ repetition rate, fewer bandwidth choppers will be required than is typical at FTS, to prohibit long-wavelength neutrons originating from a previous frame to transmit through a subsequent open state of the chopper (see the timing diagram in Figure 5.5). The low repetition rate also reduces the need to place a chopper close to the face of the target monolith. For many instruments, a single bandwidth chopper might be sufficient. In some cases, a sharper transition between the open and closed states may be desired, requiring a larger disk than in use at FTS. These types of choppers are already being developed by vendors for the European Spallation Source and should be readily available for STS instruments. Every neutron scattering beam line is anticipated to require at least one or perhaps two bandwidth choppers.

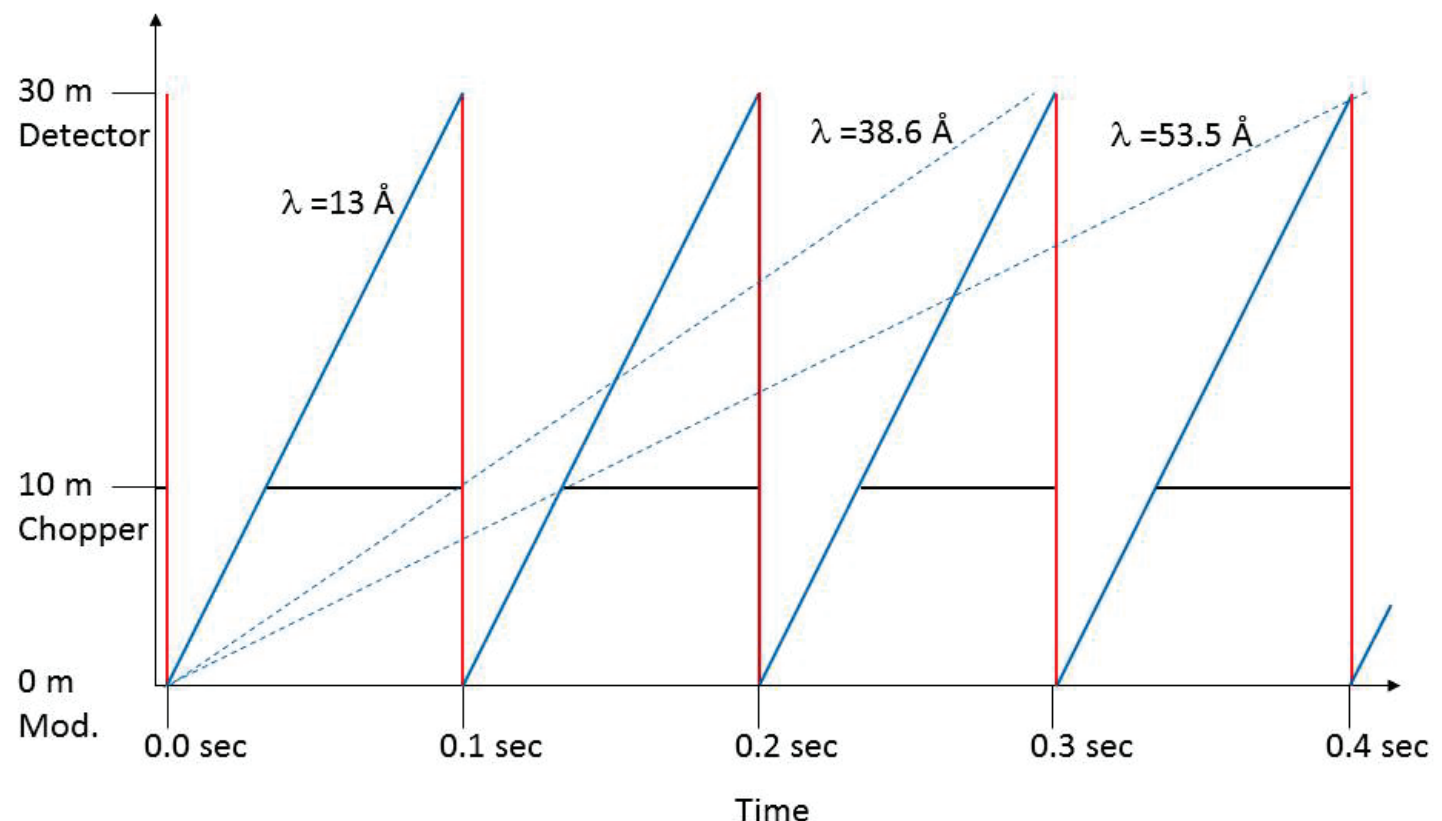

Fig. 5.5. Timing diagram showing contamination at $\lambda=38.6 \AA$ for a single bandwidth chopper located at $10 \mathrm{~m}$ from the moderator. At these detector and chopper distances, contamination occurs in the third and fourth frame at $38.6 \AA \leq \lambda \leq 53.5 \AA$. Placing the chopper closer to the moderator and target monolith shifts the contamination band to longer wavelengths. 


\section{T0 choppers}

Each short beam line, of which there are likely to be several, will require a bender (a multi-channel curved guide), a T0 chopper, or perhaps both to minimize the gamma rays and high-energy neutrons produced as the proton beam strikes the neutron production target. This chopper rotates a block of Inconel through the beam path at the instant protons reach the target, and it is large enough to continue to block the beam for some short period thereafter; but it must move out of the beam fast enough to allow the minimum desired neutron wavelength to pass by unimpeded. For the canonical case of $10 \mathrm{~Hz}$ operationa disk radius of $25 \mathrm{~cm}, 8 \mathrm{~m}$ from moderator to chopper, and a $5 \mathrm{~cm}$ neutron beam width - the chopper requires $0.0032 \mathrm{~s}$ to move from a fully closed to a fully open state, which at this distance corresponds to a wavelength band of $1.6 \AA$. This is unlikely to be acceptable for most beam lines requiring a T0 chopper. Either the chopper will have to operate at a higher harmonic of the source frequency, which will have the undesirable effect of blocking the beam for one or more periods in the middle of the frame, or a new T0 chopper will need to be designed with a larger radius and hence a larger footprint adjacent to the beam line.

\section{Fast Disk Choppers}

Fast disk choppers rapidly rotate neutron absorbing disks, often two counter-rotating disks used in tandem, through the neutron beam to produce a degree of monochromatization or pulse shaping. Open times of as little as $10 \mu \mathrm{sec}$ are desired. These choppers will be very similar to those used on FTS but may require more flexibility in the distance between disks and in the aperture design. Instruments that need to use repetition rate multiplication will require more choppers of this type than their counterparts at FTS.

\subsubsection{Neutron Guides/Optical Components}

The average anticipated length of an STS neutron scattering instrument is $\approx 45 \mathrm{~m}$ (see Table 5.1) which is considerably longer than the average FTS length of $28 \mathrm{~m}$. This additional length puts a premium on standardizing both the mechanical housing and the alignment systems deployed. Novel neutron optics systems delivering highly optimized beams (in size, divergence, and polarization) will likely require much better alignment systems and stability than the guides currently used at FTS. In some cases, active or at least periodic adjustment and alignment may be required.

\subsubsection{Detectors}

Instruments at the SNS currently use one of four detector technologies (see Fig. 5.6): (1) linear position sensitive ${ }^{3} \mathrm{He}$ tube (LPSD), (2) multi-wire ${ }^{3} \mathrm{He}$ gas chamber, (3) lithium-glass scintillator-based Anger camera, or (4) $\mathrm{LiF} / \mathrm{ZnS}$ wavelength-shifting fiber detector. Several of these technologies will be used in STS neutron scattering instruments. Table 5.10 summarizes the key operating parameters of this current generation of detectors. The higher peak brightness (as great as $17 \times$ relative to the coupled moderators on a 2 MW FTS at a neutron wavelength of $2 \AA$ ) will challenge or exceed the limits of current SNS detector and electronics technologies. Table 5.11 provides an estimate of the detector requirements for the eight instruments described above. If Anger camera technology is deployed as indicated in the table, development will be required in two areas: HiRes-SWANS requires a vacuum-compatible low-angle detector; and DyPOL and QIKR require a detector that is insensitive to stray magnetic fields, which would likely require replacing the current photomultiplier tubes with solid state technology. The global shortage of ${ }^{3} \mathrm{He}$ has driven a large international effort to develop alternate technologies, some of which may provide viable and desirable alternatives to the detectors listed in Table 5.11. The maturity of these technologies and their cost, capabilities, and maintainability will all be considered in final detector choices. ORNL is the custodian of sufficient ${ }^{3} \mathrm{He}$ to meet the needs described in Table 5.11. 

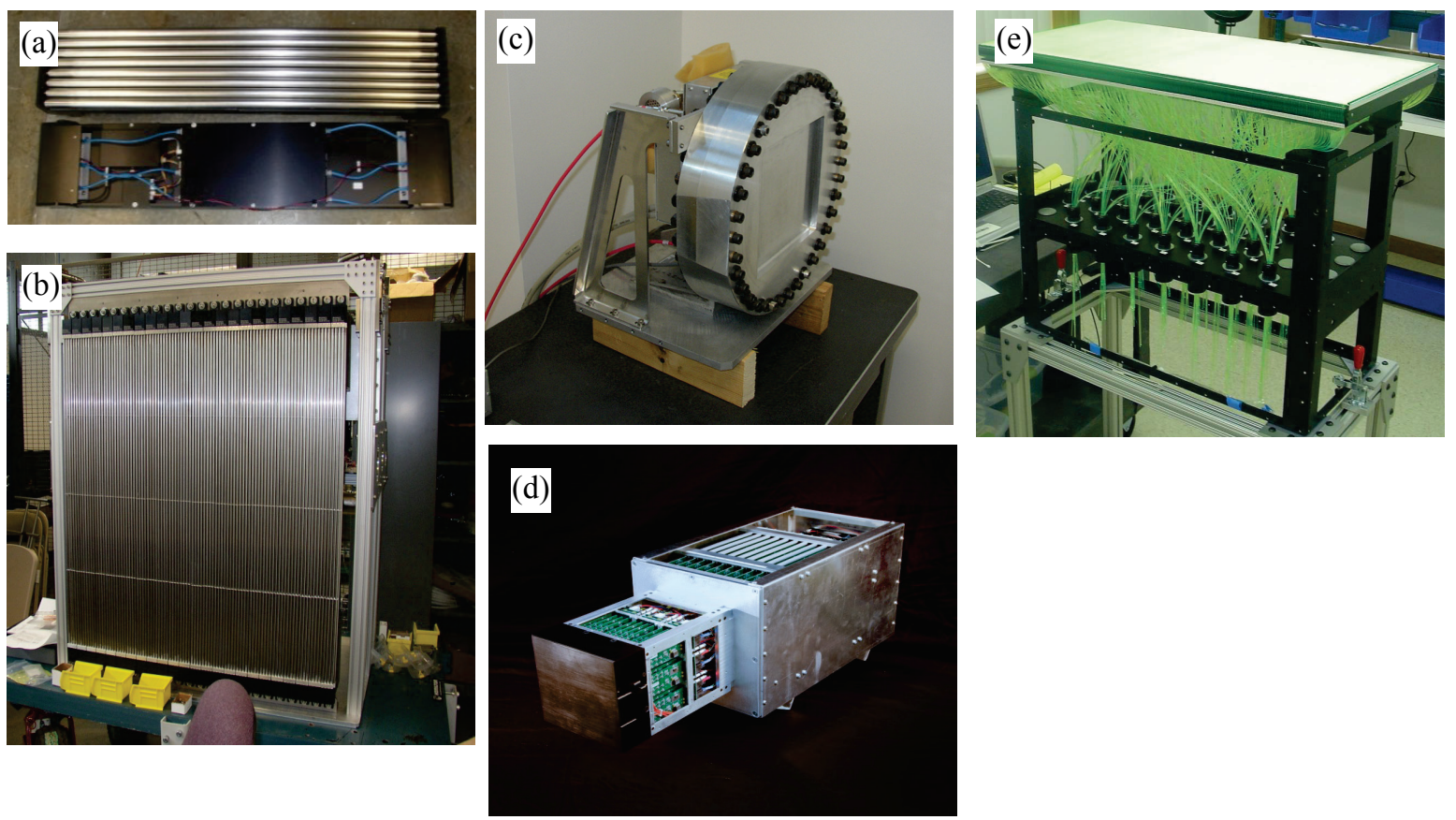

Fig. 5.6. Neutron detector types currently in use at the SNS first target station. Panels (a) and (b) show ${ }^{3} \mathrm{He}$ tubes; (c) is a multi-wire ${ }^{3} \mathrm{He}$ gas detector; (d) is a lithium glass-based Anger camera; (e) is an $\mathrm{LiF} / \mathrm{ZnS}$ wavelength-shifting fiber detector.

Table 5.10. Operating parameters for detectors currently used at SNS first target station

\begin{tabular}{|c|c|c|c|c|c|c|}
\hline Technology & Resolution & $\begin{array}{c}\text { Efficiency at } \\
2 \AA\end{array}$ & $\begin{array}{c}\text { Local rate } \\
\text { counts } / \mathbf{c m}^{2} / \mathbf{s}\end{array}$ & $\begin{array}{l}\text { Full detector } \\
\text { rate counts/s }\end{array}$ & $\begin{array}{c}\text { Gamma }^{a} \\
\text { sensitivity }\end{array}$ & $\begin{array}{c}\text { Magnetic field } \\
\text { sensitivity }\end{array}$ \\
\hline LPSD & $5-25 \mathrm{~mm}$ & $80-90 \%$ & $10-20 \mathrm{~K}$ & $100 \mathrm{~K}$ & $10^{-7}-10^{-8}$ & None \\
\hline Multi-wire & $1.5-2 \mathrm{~mm}$ & $70 \%$ & $20 \mathrm{~K}$ & $20 \mathrm{~K}$ & $10^{-7}-10^{-8}$ & None \\
\hline $\begin{array}{l}\text { Anger } \\
\text { Camera }\end{array}$ & $1.2 \mathrm{~mm}$ & $80-90 \%$ & $40 \mathrm{~K}$ & $40 \mathrm{~K}$ & $10^{-5}-10^{-6}$ & $\begin{array}{c}\text { Requires } \\
<25 \mathrm{G}\end{array}$ \\
\hline $\begin{array}{l}\text { Wavelength } \\
\text { shifting } \\
\text { fiber }\end{array}$ & $5 \mathrm{~mm} \times 5 \mathrm{~cm}$ & $50 \%$ & $10 \mathrm{~K}$ & $10 \mathrm{~K}$ & $10^{-6}-10^{-8}$ & $\begin{array}{l}\text { Requires } \\
<100 \mathrm{G}\end{array}$ \\
\hline
\end{tabular}

${ }^{a}$ Gamma sensitivity is defined as the fraction of boron energy gammas reported as counts by the detector system. 
Table 5.11. Estimate of the detector requirements for the eight initial instruments

\begin{tabular}{|c|c|c|c|c|}
\hline Instrument & $\begin{array}{l}\text { Requirements (resolution, } \\
\text { average count rate) }\end{array}$ & Coverage & $\begin{array}{l}\text { Possible detector } \\
\text { technology }\end{array}$ & Notes \\
\hline Zeemans & $1 \mathrm{~cm}, \mathrm{TBD}$ & $\approx 10 \mathrm{~m}^{2}$ & $8 \mathrm{~mm}{ }^{3} \mathrm{He}$ LPSD & $\begin{array}{l}\text { High stray } \\
\text { magnetic fields }\end{array}$ \\
\hline QIKR & $1 \mathrm{~mm}, 0.5-1 \mathrm{M}$ & $20 \times 20 \mathrm{~cm}^{2}$ & $\begin{array}{c}{ }^{3} \mathrm{He} 2 \mathrm{D} \text { if possible } \\
\text { Anger camera } \\
\text { (alternate) }\end{array}$ & $\begin{array}{l}\text { Possible polarized } \\
\text { beam }\end{array}$ \\
\hline HiRes-SWANS & $\begin{array}{l}\text { Low-mid angle: } 8 \mathrm{~mm}, 500 \mathrm{~K} \\
\text { Small angle: } 1-2 \mathrm{~mm}, 500 \mathrm{~K}\end{array}$ & $\begin{array}{c}\approx 2 \mathrm{~m}^{2} \\
20 \times 20 \mathrm{~cm}^{2}\end{array}$ & $\begin{array}{l}8 \mathrm{~mm}{ }^{3} \mathrm{He} \text { LPSD } \\
\text { Anger camera }\end{array}$ & $\begin{array}{l}\text { Curved detector } \\
\text { possible }\end{array}$ \\
\hline VERDI & $1 \mathrm{~cm}, 100 \mathrm{~K}$ & $17 \mathrm{~m}^{2}$ & $8 \mathrm{~mm}^{3} \mathrm{He}$ LPSD & \\
\hline DyPOL & $0.5-1 \mathrm{~mm}, 100 \mathrm{~K}$ & $1.3-1.7 \mathrm{~m}^{2}$ & Anger camera & \\
\hline CHESS & $1.5 \mathrm{~cm}, 20-40 \mathrm{~K}$ & $50 \mathrm{~m}^{2}$ & $1.5 \mathrm{~cm}^{3} \mathrm{He}$ LPSD & $\begin{array}{l}>100 \text { kcounts } / \mathrm{sec} \\
\text { peak rate }\end{array}$ \\
\hline MBARS & $1 \mathrm{~cm}, 20 \mathrm{~K}$ & $0.2 \mathrm{~m}^{2}$ & $1 \mathrm{~cm}^{3} \mathrm{He}$ LPSD & \\
\hline HighResPD & $\approx 1-3 \mathrm{~mm}, 50 \mathrm{~K}$ & TBD & $\begin{array}{l}\mathrm{TBD},{ }^{3} \mathrm{He} \text { at low } \\
\text { angles }\end{array}$ & $\begin{array}{l}\text { May require new } \\
\text { geometry for } \\
\text { backscattering }\end{array}$ \\
\hline
\end{tabular}

\subsubsection{Data Acquisition and Instrument Controls}

Data acquisition and instrument controls will build on systems and standards developed for FTS to facilitate common operational support for the instruments across both facilities. These systems are in a state of transition for FTS instruments as new methods, algorithms, and frameworks are deployed; these include Accelerating Data Acquisition Reduction and Analysis (ADARA), which provides near-real-time access to reduced data sets during an experiment and handles data transfer and management. Instrument control is transitioning to an Experimental Physics and Industrial Control System (EPICS) -based framework. Instrument Systems will provide the necessary hardware and programming support to deploy these systems on the eight project instruments.

\subsubsection{Sample Environment}

The neutron scattering instruments require a suite of equipment to control the environment around samples. Sample environments typically include control of temperature, pressure, magnetic field, or some combination thereof, as well as more specialized environments that may be customized to particular instruments. Some of this equipment can be used across multiple instruments, but much will be customized to the particular geometries of individual instruments. This is especially true for Zeemans, which will require the development of customized sample environment equipment that is compatible with both the high-magnetic-field environment and atypical instrument geometry. Table 5.12 lists some of the sample environment types and requirements needed by the eight instruments described. Instrument systems will provide the sample environment equipment required to commission the experiment and deliver its initial science program. 
Table 5.12. List of sample environment equipment for the eight project instruments.

\begin{tabular}{|c|c|c|}
\hline Instrument & Equipment description & Notes \\
\hline Zeemans & Temperature control from $50 \mathrm{mK}$ to $1000^{\circ} \mathrm{C}$ & $\begin{array}{l}\text { All sample environment } \\
\text { equipment will be unique to } \\
\text { Zeemans }\end{array}$ \\
\hline QIKR & $\begin{array}{ll}- & \text { Langmuir trough } \\
\text { - } & \text { Pressure cell } \\
\text { - } & \text { Humidity cell }\end{array}$ & $\begin{array}{l}\text { Range of equipment - probably } \\
\text { compatible with FTS Liquids } \\
\text { Reflectometer suite }\end{array}$ \\
\hline HiRes-SWANS & $\begin{array}{l}\text { - } \quad \text { Automatic sample changer: }-2-80^{\circ} \mathrm{C} \\
\text { - } \quad \text { Furnace with auto-changer: } 30-500^{\circ} \mathrm{C}\end{array}$ & $\begin{array}{l}\text { May require additional } \\
\text { customized apparatus for grazing } \\
\text { incidence geometry }\end{array}$ \\
\hline VERDI & $\begin{array}{l}\text { - } 12 \mathrm{~T} \text { superconducting cryomagnet } \\
\text { - } \quad \text { Cryostat with mK inserts } \\
\text { - Closed-cycle refrigerator with automatic } \\
\text { sample changer }\end{array}$ & \\
\hline DyPOL & $\begin{array}{ll}\text { - } & \text { Cryostream : } \mathrm{T}<100 \mathrm{~K} \\
\text { Integrated dynamic nuclear polarization } \\
\text { device }\end{array}$ & \\
\hline CHESS & $\begin{array}{ll}\text { - } & \text { Low temperature: } \mathrm{T} \leq 30 \mathrm{mK} \\
\text { - } & 12 \mathrm{~T} \text { superconducting cryomagnet } \\
\text { - } & \text { Furnace }: 800^{\circ} \mathrm{C} \\
\text { - } & \text { Integrated device to measure } \mathrm{S} \\
& \text { Closed-cycle refrigerator with automatic } \\
\text { - } & \text { Pample changer } \\
\text { Pressure cells }\end{array}$ & $\begin{array}{l}\text { May require custom apparatus to } \\
\text { ᄃ take full advantage of nearly } 2 \pi \\
\text { detector solid angle }\end{array}$ \\
\hline MBARS & $\begin{array}{ll}\text { - } & \text { Closed-cycle refrigerator: } 5-800 \mathrm{~K} \\
\text { - } & \text { Furnace: } 30-1600^{\circ} \mathrm{C} \\
\text { - } & \text { Cryostat: } 1.5-300 \mathrm{~K}\end{array}$ & \\
\hline HighResPD & $\begin{array}{ll}\text { - } & \text { Closed-cycle refrigerator with automatic } \\
\text { - } & \text { Fample changer } \\
\text { - } & \text { Cryo-furnace: } 1100^{\circ} \mathrm{C} \\
\text { - } & \text { Cryostat: } 2-300 \mathrm{~K}\end{array}$ & \\
\hline
\end{tabular}

\subsection{INTERFACES}

Instrument Systems relies on a number of the other work packages for certain areas of support and has a number of interfaces across the project as delineated:

- Conventional Facilities: Instrument Systems relies on Conventional Facilities to provide the buildings that house the instrument end stations and transport lines, as well as to provide standard utilities routed to designated locations inside the buildings. As instrument designs mature, requirements for floor loading and stability will be developed. Routing utilities from these locations to the instrument end stations as required will be the responsibility of Instrument Systems.

- Target Systems: Instrument Systems interfaces with Target Systems both within and external to the target monolith. Final selection of both the types and geometries of neutron moderators must support the instrument requirements. Instrument Systems will provide the beam line inserts that house neutron optics and neutron beam extraction equipment for the eight project instruments. STS will have "beam 
line access pits" outside the target monolith that provide a common shielded bunker, which will house instrument components from the edge of the target monolith to an approximately $11 \mathrm{~m}$ radius.

Instrument Systems will install choppers, neutron optical components, and local instrument shielding in the interior of this area.

- Integrated Controls: Instrument Systems relies on Integrated Controls for distribution of the Ethernet communications network for the instrument hardware and data acquisition systems, and the appropriate timing signals required to synchronize them to accelerator proton pulse production. These systems are anticipated to be similar to those currently in use for instruments at FTS.

\subsection{DEVELOPMENT}

Development efforts specific to individual beam lines, such as the unique sample environment equipment associated with Zeemans, are described under each specific instrument. This section outlines anticipated development that is common to multiple beam lines.

\subsubsection{Neutron Choppers}

The modifications possibly required for bandwidth choppers (larger disks) and high-speed, fast choppers (disk size, aperture arrangement, and distance between disks) are also required by European Spallation Source instruments. It is anticipated that chopper vendors will be able to provide these choppers without further development effort under the STS project. However, it is important that specifications be iterated with these vendors in a timely fashion.

Changes to the $\mathrm{T} 0$ chopper require development, primarily in the form of new engineering designs, tuning of control parameters, and testing, will be the responsibility of the STS project.

\subsubsection{Neutron Detectors}

Selection of the final instrument suite may require a different set of detector requirements from those described earlier. Following are some known and expected areas in which detector development will be required.

- Current and proposed reflectometers are underserved by the current 2-dimensional, multi-wire ${ }^{3} \mathrm{He}$ gas detectors; and, although it is desirable, existing gas detector technology may not meet the needs for speed and resolution. These instruments could be served by a scintillating detector that is insensitive to magnetic fields or possibly by an ionization mode or resistive plate detector. Faster electronic encoding of neutron position is likely to be a key requirement to keep up with the high peak count rate of QIKR.

- Improving the spatial resolution of the current Anger camera from 1.2 to $0.5 \mathrm{~mm}$ would benefit DyPOL in its ability to study very small samples on the order of $0.03 \mathrm{~mm}^{3}$ or smaller. Progress in this area requires a brighter scintillator than the GS20 lithium glass currently used.

- There will be a strong preference for ${ }^{3} \mathrm{He}$ detectors for a number of the instruments, particularly for the large-detector-area inelastic instruments like CHESS. Improvements are needed in the methods for linking these large systems together, including addressing methods of powering and communicating with these units.

- It may be impractical to provide sufficiently high spatial resolution for the backscattering and $90^{\circ}$ detectors of HighResPD using ${ }^{3} \mathrm{He}$ detector technology. In particular, the backscattering detector 
would be best served by a segmented, annular detector geometry that follows the Debye-Scherrer rings.

- The higher peak and sustained count rates on many of the STS instruments will require development in support of higher-speed communications and streaming of the data from the detector through the data acquisition system and associated processing and into high-speed data storage.

\subsection{REFERENCES}

1. Instruments for Emerging Science: A Science Case for the Second Target Station (2014). A. T. Savici and G. E. Granroth, SNS-NSSD-TOF-TR-0002-R00, Oak Ridge National Laboratory, 2009.

2. G. E. Granroth, A. T. Savici, M. D. Bird, L. Santodonato, Y. Lee, and C. L. Broholm, "Zeemans: A high magnetic field beamline for the SNS, ICANS XIX," 19th meeting on Collaboration of Advanced Neutron Sources, Grindewald, Switzerland, March 18-22, 2010.

3. A. T. Savici et al., "Zeemans - A new facility to probe matter at high magnetic field through neutron scattering," J. Phys.: Conf. Ser. 251, 0120573 (2010).

4. J. Voigt et al., "Chopper layout for spectrometers at long pulse neutron sources," Nucl. Instrum. Meth. A 741, 26-32 (2014).

5. H. N. Bordallo, K. W. Herwig, and G. Zsigmond, "Analytical calculations and Monte-Carlo simulations of a high-resolution backscattering spectrometer for the long wavelength target station at the Spallation Neutron Source," Nucl. Instrum. Meth. A 491, 216-225 (2002).

6. E. Mamontov, "Wide-angle mechanical velocity selection for scattered neutrons in inelastic neutron spectrometers," Nucl. Instrum. Meth. A, 759, 83-91 (2014). 



\section{CONVENTIONAL FACILITIES}

Conventional facilities (CF) consist of site improvements, buildings, tunnels, cooling systems, plantwide control systems, waste handling systems, maintenance systems, fire protection, business computing, and all other needed support services to the accelerator, target, and neutron instruments. Necessary electrical and mechanical utilities will be provided. Approximately $380,000 \mathrm{ft}^{2}$ of additional buildings will be provided to meet the specified technical requirements. Mechanical utilities will be provided by a new Central Utilities Building (CUB) located on Chestnut Ridge on the Oak Ridge Reservation adjacent to the existing SNS site. No alternative sites are under investigation.

\subsection{CF TECHNICAL SUPPORT}

Technical support will be provided for CF during design, construction, and commissioning phases of the project. Activities will include preliminary design, final design, design support, construction support, project management, external reviews, construction management, field support, subcontract administration, LEED (Leadership in Energy and Environmental Design) certification, commissioning and support for tie-ins, security, and other laboratory interfaces. Participants will include ORNL staff, architect, engineers, the construction manager, and the commissioning agent. The optimum execution strategy will be developed during the conceptual design phase. These efforts are required to ensure that the CF scope is performed so as to achieve a fully functional facility and that interfaces are managed while minimizing the impact to ongoing operations.

\subsection{LAND IMPROVEMENTS}

\subsubsection{Stockpile}

A stockpile consisting of excess materials and spoils from construction of the existing SNS facility remains at the proposed location for STS. This stockpile will have to be removed to allow proposed tunnel, target, and instrument building construction. The estimated volume of the existing stockpile is approximately $200,000 \mathrm{yd}^{3}$. Much of this material will not be initially usable for structural fill because of accumulated moisture; therefore, it will have to be sorted, spread in lifts, and allowed to dry before compaction. The mass excavation plan allows for this material to be spread and dried in nonstructural fill areas to the west in the proposed shop building location. Alternatively, unsuitable structural material may be placed in fill areas in the east area proposed for construction laydown, trailers, and parking. Geotechnical observation and testing services during construction will be required to manage proper placement of any unsuitable materials.

\subsubsection{Mass Excavation}

The mass excavation plan includes the construction excavation required for proposed tunnel, target, and instrument building construction; the eastern temporary facility area; the construction access road from Bethel Valley Access Road east of the ORNL Guest House; and the western haul road and spoil area. The overall cut volume for the mass excavation plan, including the existing stockpile volume removal, is estimated at 700,000 $\mathrm{yd}^{3}$. The cut materials will be placed as fill, as shown on the mass excavation plan. The primary fill areas will consist of the west spoil area, the eastern temporary facility area, and the target area cover stockpile.

Conflicts with ongoing operations will be mitigated by constructing new isolated haul roads on the east and west ends of the site as shown on the mass excavation plan and by using North Perimeter Drive. The haul roads will be maintained as gravel roads during construction. 
The haul road connection from Argonne Drive (the elliptical road surrounding the Experiment Hall) to the temporary facility area will be graded to maintain less than $9 \mathrm{ft}$ of fill over the existing $24 \mathrm{in}$. water line to avoid the expense and logistics of relocation or protection of the existing water line, which feeds all of ORNL.

The temporary facility area consists of 7.1 acres that is graveled and graded with suitable slopes for architect-engineer/construction manager $(\mathrm{AE} / \mathrm{CM})$ trailers, contractor laydown, and construction craft parking. This area will be repurposed for facility parking after construction, as described in Sect. 6.2.4, "Roads and Parking."

\subsubsection{Pond Improvements}

The existing East Pond has a drainage watershed of approximately 35 acres. Currently, about 5.5 acres in the watershed are impervious surfaces, with an additional small amount of gravel improvements. Based on the current site layout, the amount of impervious surface may increase to 10 acres. The existing pond will be required to maintain the existing stormwater flow rates for various storm events (10-100 years). The pond will also have to retain the volume of the process cooling water for $24 \mathrm{~h}$ and detain the runoff from the 95th percentile storm (1.5 in.) for compliance with Sect. 438 of the Energy Independence and Security Act (EISA).

Based on a preliminary review of construction drawings, the existing East Pond has a volume of between 150,000 and 180,000 $\mathrm{ft}^{3}$ below elevation 1020.57 (the top of the overflow structure). It is anticipated, based on preliminary hydrology modeling, that the pond outlet can be reconfigured to handle the process cooling water and the EISA requirements. However, the pond may have to be enlarged to meet these requirements and limit the peak discharges. Because the existing water line runs along the eastern edge of the pond, it is anticipated that the enlargement would be to the north. The amount of silt accumulation in the pond will need to be evaluated as well. One concern is that the only outlet to the pond is through the piping system; there is no emergency overflow from the pond.

The main detention pond serves the Experiment Hall and the area along the north of Spallation Drive. The pond has sufficient size for a small amount (approximately 0.5 acre) of additional building development (impervious surface). However, it is not anticipated that the STS development would have any impact on the main pond, and some of the additional development runoff could be accommodated in the main pond if necessary.

\subsubsection{Roads and Parking}

The permanent roads and parking for the STS are depicted on the finished site grading plan. A portion of the temporary facilities area east of the STS will be developed as a permanent, 250-space parking lot for facility staff. Additional small parking areas are proposed adjacent to the central exhaust systems II and remote instrument buildings. All parking areas are proposed to be asphalt with curbing for drainage and traffic control.

Several new roadways will be built as part of the finished site plan:

- extension and connection of Argonne Drive and Spallation Drive around the east end of STS

- connection from the Center for Nanophase Materials Sciences (CNMS) to the new parking facility and the Spallation Drive extension

- a fire access road between the FTS and Target Building II 
- a truck and service access road from Spallation Drive under Central Laboratory and Office Building II (CLO II) to Target Building II basement

- an access road to the southeast corner of Target Building II

- roadway access service and parking areas near the central exhaust system

All of these roadways are planned as asphalt. Curbing will be included when needed for traffic control and drainage collection. All new roads, including the access road between the FTS and Target Building II, will provide fire department vehicle access in accordance with ORNL Fire Department requirements.

\subsubsection{Storm Drainage}

Most of the stormwater runoff from the STS, CLO II, CUB II, Zeemans instrument facility, and other constructed improvements, will be collected in the existing and new stormwater piping and inlets and conveyed to the existing East Pond. Part of the area along Spallation Drive and the area between Experiment Hall and Target Building II currently flows to the main detention basin. A few of the existing drainage inlets and piping associated with the Experiment Hall and the area to the north of Spallation Drive will have to be relocated because they are in the footprint of the proposed buildings. Some of the existing piping to the north of the facility may also have to be replaced because of construction activities.

\subsubsection{Landscape/Hardscape}

\section{Landscape}

The landscape for the site will be in keeping with the current character of the SNS site. Plant palette selections will be in accordance with ORNL and SNS's commitment to the use of native plants to limit maintenance and water use. Landscape focus areas will be around building entrances, along heavily used pedestrian pathways, and at key roadway intersections. Trees will be incorporated into parking lot designs to reduce the heat island effect. Where possible, rain gardens and bioswales will be used in the landscape to reduce stormwater runoff per EISA guidelines.

\section{Hardscape}

Hardscape elements will include sidewalks connecting parking areas and CLO II entrances and plaza spaces at key building entrances. All sidewalks will be concrete and will be a minimum of $4 \mathrm{ft}$ wide $(6 \mathrm{ft}$ wide in areas of heavier pedestrian traffic). Plaza space materials will be a mix of concrete and concrete paver bands. Where possible, the use of permeable concrete pavers will be used for larger plaza spaces.

\subsubsection{Geotechnical Exploration}

The geotechnical exploration of the STS site will be based on plans developed jointly by geotechnical and structural engineers from the proposed site layout and loading(s) of the facility structures. The investigation will consist of borings of varying depths to determine the physical properties and design parameters of the soil and rock; in-situ and laboratory testing will be conducted as recommended by the engineers. The borings will typically be located within the footprint of the various structures. The data will be used to determine recommended foundation systems, settlement estimates, bearing capacities, slope stability, soil reuse suitability information, site-specific seismic response spectra, and other parameters as required for building and site design. 


\subsubsection{Temporary Facilities}

The temporary facilities area will be developed at the beginning of the mass excavation process by clearing and grading a 7.1 acre area east of Argonne Drive and the STS area. It is proposed that this area will be graveled and designated for $\mathrm{AE} / \mathrm{CM}$ trailers, 350 construction craft parking spaces, and construction laydown. Temporary sewer, water, and electrical utilities will be routed to this area to serve the temporary facilities. A smaller area of 0.2 acres to the north of the existing CNMS and east of the Ring Road is available for CM trailers.

\subsubsection{Finished Site Grading}

The STS area cover stockpile to the east will consist of $200,000 \mathrm{yd}^{3}$ of suitable cover material that will be excavated and stored during mass excavation. The stockpiled material will be used as backfill and cover materials to establish finished grades. As grading is completed, the finished grades will be seeded or paved as shown in the finished grading plan. See Sect. 6.2.6 (Landscape/Hardscape) for a description of other finishing procedures. The construction stockpile area will be stabilized and seeded when the grading activities are complete.

\subsection{BUILDINGS}

The STS effort will involve significant CF effort, particularly for new buildings for the new target, instruments, offices, and other support activities. Figure 6.1 shows an overview of the new buildings in the STS area. Some perspective views of these buildings are also shown in Figs. 6.2-6.4. The requirements for these buildings and their characteristics are discussed in this section.

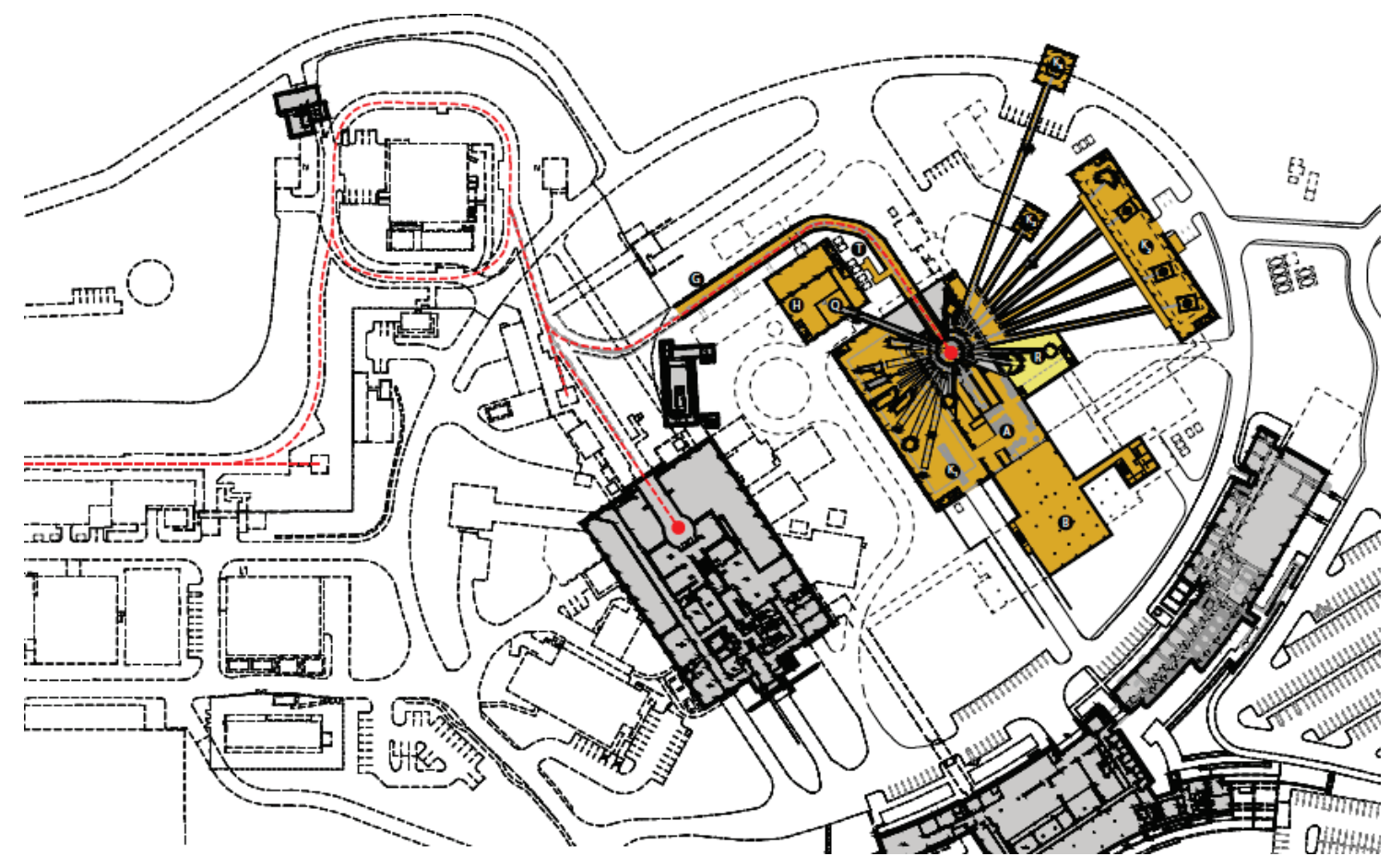

Fig. 6.1. Overview of the new buildings planned for the STS effort (orange) relative to existing buildings on the east end of the SNS campus. 


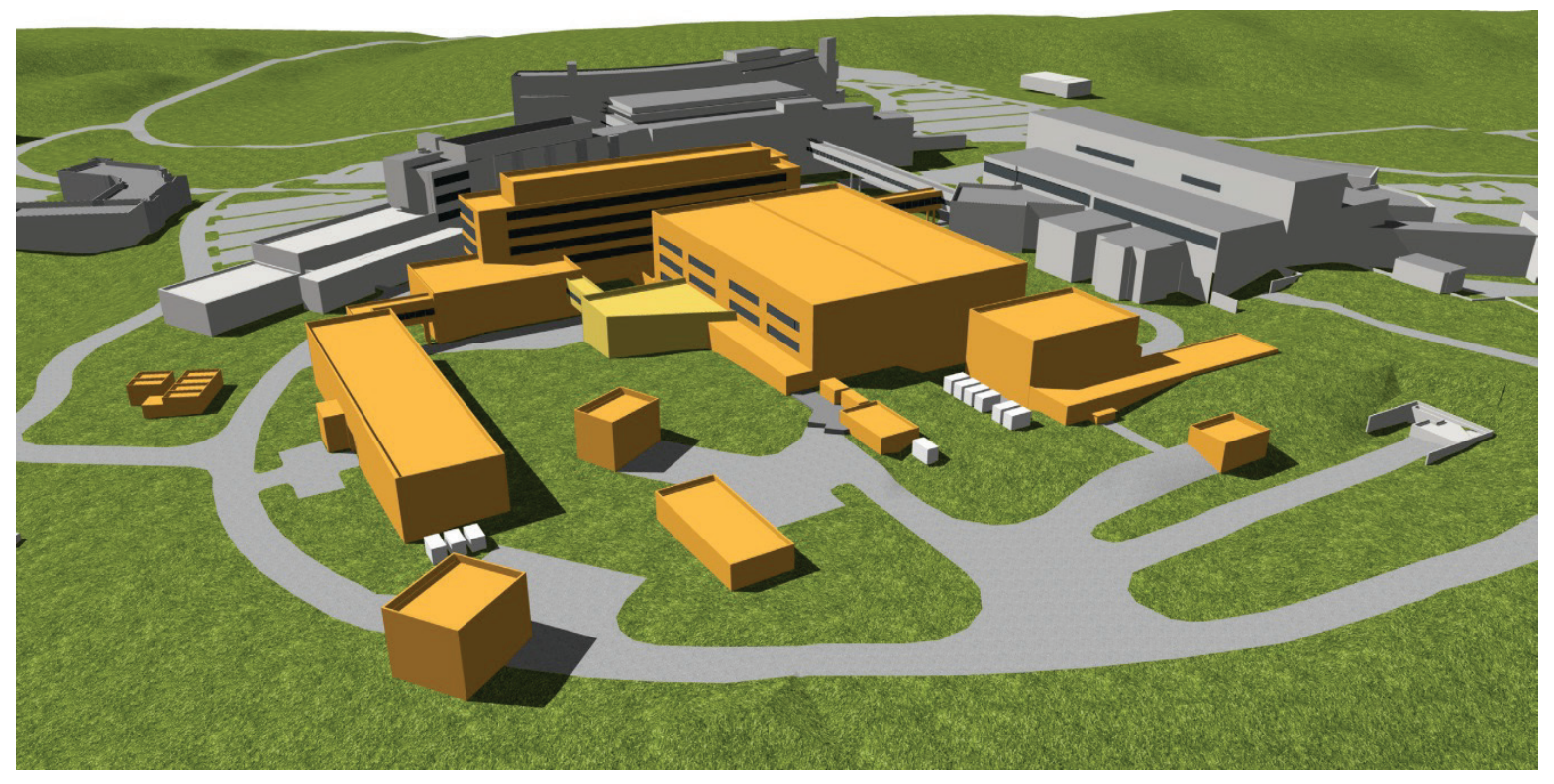

Fig. 6.2. Perspective looking south with new buildings in the foreground.

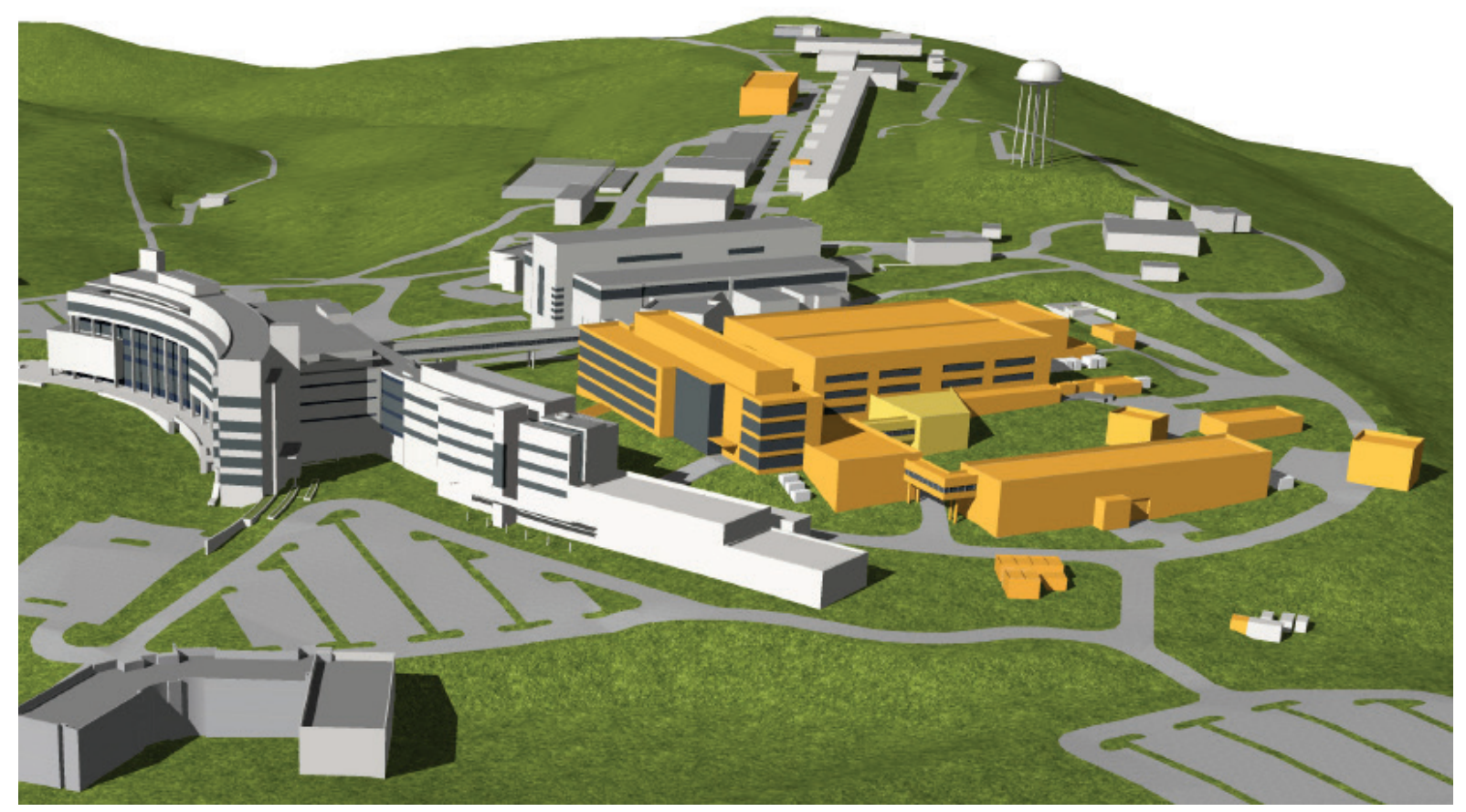

Fig. 6.3. Perspective looking west, with the new accelerator support buildings in the background. 


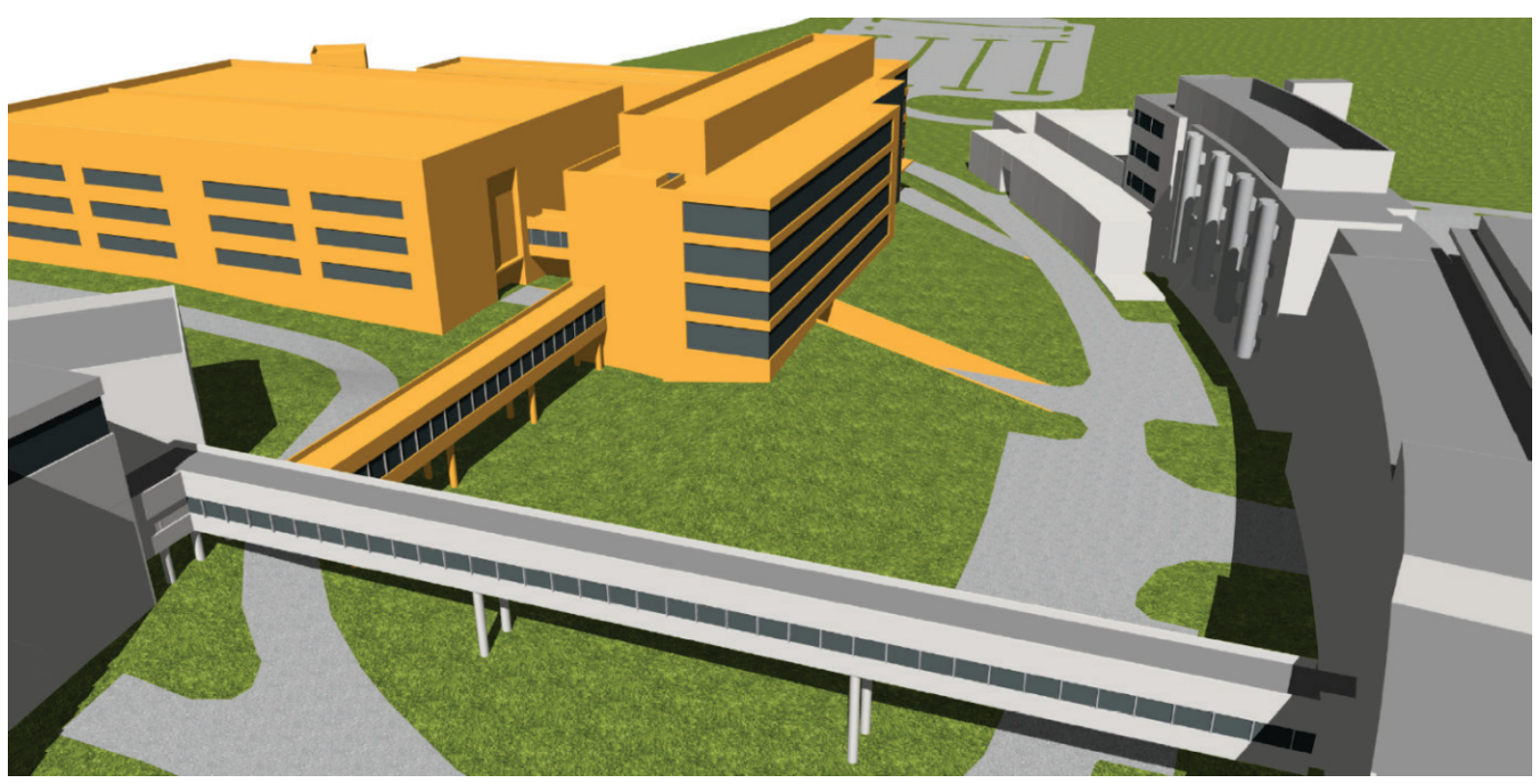

Fig. 6.4. Perspective looking east, indicating the connectivity of the present CLO and target building (gray pedestrian bridge) and the new CLO II/ Target II and STS instruments buildings (orange pedestrian bridge).

\subsubsection{FTS Building Modifications}

\subsubsection{Klystron gallery}

\section{Programming}

The Klystron Gallery will need to be modified to support the new accelerator equipment at the east end of the existing space to increase the beam energy to $1.3 \mathrm{GeV}$. The accelerator equipment will be installed in the existing Klystron Gallery after the existing shop and storage areas are relocated. A new pump building (625 GSF) will be required to house the new deionized water (DI) cooling loop that will provide cooling for the new accelerator equipment. There will be three exterior transformer pads, for a total of $700 \mathrm{ft}^{2}$. These changes are summarized in Table 6.1. There is no other CF scope for the Klystron Gallery.

Table 6.1. Klystron gallery upgrades

\begin{tabular}{lc}
\hline \multicolumn{1}{c}{ Space Type } & $\begin{array}{c}\text { Area } \\
\text { (NSF) }\end{array}$ \\
\hline Pump house & 650 \\
Transformer pads (exterior) & 700 \\
\hline
\end{tabular}




\section{Structural Systems}

The pump room will be a steel-framed structure with metal deck roof. The foundation will be a reinforced concrete slab with thickened edges (no isolated spread footings). The structure will be designed to meet PC-2 wind and seismic requirements. In addition to a 20 psf live load, the roof will be designed for a 50 psf collateral load and a suspended load of $3000 \mathrm{lb}$ (tank). The floor slab will be designed for a live load of $500 \mathrm{psf}$, and it will have floor drains and a sump.

\section{Building Enclosure Systems}

New construction will require the addition of a pump room building approximately $25 \times 25 \mathrm{ft}$, sandwiched between the east end of the existing Klystron Gap build-out project and the west end of the high-energy beam transport line service building (HEBT-SB). The pump room addition will be faced in a flat-faced insulated metal panel similar to other sections of the Klystron Gallery that are part of the original constructions. Special details will be required to interface with the deep rib panel of the HEBT-SB. Matching louvers will be provided as required to serve mechanical equipment.

The roof system will include a corrugated metal deck with R-30 insulation board and a built-up roof to match existing roofs. A highly reflective coating will be applied to meet DOE specifications for cool roof construction. Gutters and downspouts will provide drainage from the roof. Expansion will be provided between the new pump building and adjacent buildings.

New double doors will be cut into the south wall of the Klystron Gap building east of column line 9.7 to provide interior access into the existing Klystron Gap building. The existing single man door will be removed and the space filled in with matching metal panels. A $3 \mathrm{ft} \times 7 \mathrm{ft}$ man door in the south wall will provide access and egress to the exterior. A $12 \mathrm{ft}$ wide overhead door will also be provided in this wall.

The concrete slab on grade will receive an epoxy sealer for dust control and wear ability. There will be a floor drain and a sump within the room.

\section{Life Safety Requirements}

The new pump building will be separated from surrounding Klystron Gap and Klystron Gap infill buildings by a Underwriters Laboratories (UL)-listed $2 \mathrm{~h}$ partition. Egress will be provided directly to the outside via the man door in the south wall, in compliance with National Fire Protection Association (NFPA) 101, Life Safety Code.

\section{Mechanical Systems}

The section of the Klystron Gallery with new accelerator equipment will be heated and cooled with a rooftop-mounted chilled and heating water heating, ventilation, and air-condoning (HVAC) unit; the air will be distributed by galvanized ductwork. The cooling capacity of the HVAC unit will be 85 tons and 30,000 CFM.

The new pump room will be heated for freeze protection by a fan coil unit and ventilated by an exhaust fan. These HVAC schemes will be similar to those in the rest of the Klystron Gallery, except that better air distribution will be provided. 


\section{Piping Systems}

Chilled water and heating water lines will be routed to a new HVAC unit. Both utilities are available in the Klystron Gallery near the new unit. Alternatively, chilled water could be routed from the HEBT-SB.

Compressed air lines for utility air similar to existing systems in the Klystron Gallery will be routed to various locations for valve actuation, tool use, and other common uses.

Process water lines for makeup water similar to existing systems in the Klystron Gallery will be routed to technical equipment.

Tower water (supply and return) lines to will be routed to cool the new DI water pump skid. The tower water lines will be routed from the HEBT-SB.

A process waste sump and associated process drain lines will be installed to disposition HVAC condensate and other influent sources. Floor drains will also be routed to the sump.

\section{Fire Protection Systems}

Sprinkler system coverage will comply with NFPA 13, Standard for the Installation of Sprinkler Systems, where modifications are/ may be required

\section{Fire Alarm}

Very Early Smoke Detection Apparatus (VESDA) smoke detection will be extended into the east end of the Klystron Gallery from the Klystron Gallery extension (annex). Existing spot smoke detectors will be removed. Area notification appliances will be relocated as required for proper coverage requirements.

\section{Electrical Systems}

Adding new high-power radio frequency (RF) equipment and three high-voltage converter modulators (HVCMs) and operating the HEBT Ring-RTBT (ring to target beam transport line) magnets at higher current will significantly increase the SNS site electrical load. To support new Klystron Gallery electrical loads, three new $1500 \mathrm{kVA}$ transformers will be installed on new pads just south of the gallery structural build-out. These new transformers will support the new HVCMs on a one-to-one basis. A new interrupter switch will be required with each new transformer to provide isolation capability for the electrical distribution from the switchyard. Underground duct-banks will be provided from existing manholes to the new interrupter switches. A new feeder will be spliced into an existing feeder in manhole M20. Existing power distribution panels in the Klystron Gallery will supply power to new panels in the high-energy end of the gap area to provide for technical supporting systems-i.e., vacuum, controls, diagnostics, cooling loops, and so on.

Power will be provided for the new HVAC unit to be installed in the Klystron Gallery Gap Area Building.

New cable trays will be installed to distribute the power and communications cabling throughout the Klystron Gallery gap and build-out area.

Existing lighting fixtures in the Klystron Gallery will be replaced with fluorescent or light-emitting diode (LED) fixtures and relocated to accommodate installation of the technical equipment. 
Voice and data communications will be provided to convenient locations for phones and network connections.

Power, lighting, and communications will also be provided for the new pump skid building.

\section{Controls}

The HVAC unit will be controlled by the existing Experimental Physics and Industrial Control System (EPICS). Control sequences will be developed to support the operations of the technical equipment. Instrumentation and wiring will be provided to a new programmable logic controller (PLC). Programming and connection of the PLC to EPICS will be completed and the control system commissioned to ensure proper operation.

\section{Alternatives and Assumptions}

Some heat will be added to the remainder of the Klystron Gallery as a result of running at higher power levels. Currently, some Klystron Gallery equipment is running at a higher than desired temperature. It is assumed that the existing Klystron Gallery may need to run at a slightly higher temperature, and any equipment temperature issues will be addressed separately from STS by localized cooling and ongoing operational improvements.

\subsubsection{Central Helium Liquefier (CHL)}

No additional utilities or facilities will be required for the central helium liquefier or for the superconducting radio frequency annex for the accelerator to perform at the higher beam power that will result from this project. Additional shop and storage space will be provided for the cryo group, and that is detailed in a separate section.

\subsubsection{Radio Frequency Test Facility (RFTF)}

No additional utilities or facilities will be required in the existing RF test facility. Additional shop and storage space will be provided for the RF group; it is detailed in a separate section.

\subsubsection{Ring Service Building}

Additional heat loads will be introduced in the Ring Service Building (RSB), but no increase in HVAC is thought to be required. All scope at the RSB will be by other elements.

\subsubsection{Experiment Hall}

The existing mercury laboratory will be demolished, and that area will be converted to storage for activated samples to serve both FTS and STS, but this is not in the STS scope.

\subsubsection{Central Laboratory and Office Building}

No existing Central Laboratory and Office Building (CLO) scope is anticipated nor included at this time; however, it may be desirable to renovate the CLO to better match space utilization with the new CLO II. This will not be established until after additional study. 


\subsubsection{STS Buildings}

\subsubsection{STS Buildings Overview}

This overview provides a summary of the programmatic requirements of the STS project and key factors that affect the overall design. Subsequent sections refer to the specific buildings that make up the project. As a rule, building narratives include information that is unique to the particular building or an exception to the general information provided in Sect. 6.3.2.10, "Discipline Specific Overview."

The STS will fulfill the original SNS master plan, which was to support two target stations in conjunction with power upgrades to the existing accelerator. It will provide the capability of 22 additional potential experimental beam lines to the SNS research capabilities. The proposed STS complex will include a total of 380,000 GSF of new construction supporting a population of approximately 300 permanent staff and visiting users. Including the STS, the Chestnut Ridge campus will incorporate approximately $1,100,000 \mathrm{GSF}$ of space for a population of approximately 900 people.

The STS will be designed to conform to the current version of the International Building Code (IBC) and NFPA 101 codes and other codes and regulations defined in Sect. 6.3.4.7.

The STS consists of Target Building II, supporting tunnels and utility structures, four instrument buildings, the CLO II, and the Zeemans building. A fifth instrument building, the $30 \mathrm{M}$ instrument building, which will not be built as part of the STS project, is proposed for future construction. The STS will rely on various existing functions within the SNS complex that will not be duplicated, including user support, major conferencing, and food service. The STS building portion is organized by nine WBS items, which are summarized Table 6.2 and shown in Fig. 6.5.

Most of the STS will be constructed on a 12 acre site that was initially graded and partially surcharged with approximately $200,000 \mathrm{yd}^{3}$ of stockpiled earth from the initial construction of SNS. It is located directly east of the FTS within the existing elliptical road, Spallation Drive. Approximately 250 new parking spaces will be located further east on a currently wooded site. Approximately 50 additional spaces will be constructed adjacent to the new STS facilities for government vehicle parking. New shop space to support the STS will also be constructed on the south side of Los Alamos Drive in the vicinity of the Front End Building.

Table 6.2. STS program summary

\begin{tabular}{lr}
\hline \multicolumn{1}{c}{ Building } & $\begin{array}{r}\text { Area } \\
\text { (GSF) }\end{array}$ \\
\hline STS building overview & $\mathbf{3 8 8 , 1 1 0}$ \\
R2T2 tunnel and support building & 28,500 \\
Target building & 55,050 \\
Instrument buildings and tunnels & 114,520 \\
Zeemans building & 29,820 \\
Central Laboratory and Office II & 122,220 \\
Central Utility Building II & 9,100 \\
Central Exhaust Systems II & 200 \\
Shop building & 28,700 \\
Total Gross Area & $\mathbf{3 8 8 , 1 1 0}$ \\
\hline
\end{tabular}




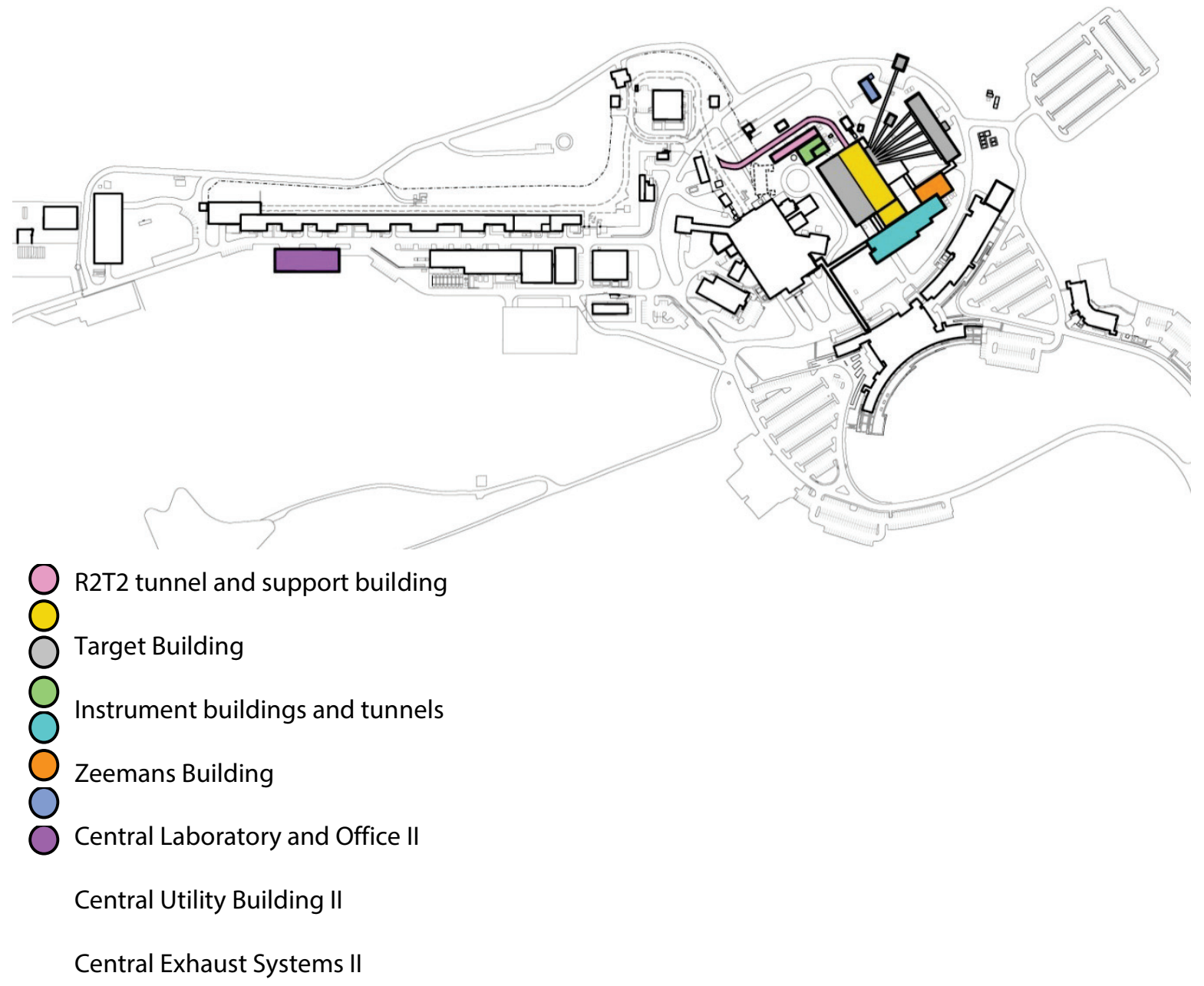

Shop building

Fig. 6.5. ORNL STS site overview.

STS staff, users, and visitors will generally occupy the CLO II and the instrument buildings, including the Zeemans building. Access to the target building, tunnels, and supporting utility structures will be limited to operational and maintenance personnel. General exterior pedestrian access will be from the new east parking area into a lobby located at the east end of the CLO II. Alternatively, pedestrians will enter the STS through the SNS CLO at the bridge level. The STS will include several bridges that, together with the existing SNS bridge, will link all the major research buildings at one floor level. Service access to all facilities will be from Spallation Drive at various locations and floor elevations that are described in more detail within the narrative for each building.

Maintaining ongoing operations of the SNS during STS construction is a key factor affecting the site design. During STS construction, the proposed future parking area will be developed to temporarily provide approximately 7 acres of construction staging and parking area. This space will subsequently be converted to permanent parking or be reforested. An STS access road will be developed behind the existing guest house to link the construction site with the primary SNS access drive, avoiding construction traffic within the operating SNS site. 
Soil removal and regrading of the STS site also affects the site design. A sufficient quantity of soil to backfill the STS site following the major construction activities will be stockpiled in the construction staging area. The remaining soil to be removed from the site will be placed near the west end of the campus along the south side of Los Alamos Drive, which will create a relatively flat ground surface suitable for additional parking or lightweight building construction. Figure 6.5 is an overview of the STS site.

\subsubsection{R2T2 Tunnel and Support Building}

\section{Programming}

The R2T2 tunnel and R2T2 support building will be two distinctly different but related structures that will house and support the new proton beam transport system transporting the beam from the existing RTBT beam line to the new STS target. Both the R2T2 tunnel and the R2T2 support building will be comparable, in purpose, to the existing RTBT tunnel and the RTBT support building. However, they will both be larger than the FTS structures; the R2T2 tunnel will be approximately 50\% longer than the RTBT tunnel. The R2T2 tunnel will house approximately 50 magnets, compared with 20 magnets in the RTBT, including large dipole magnets for multiple bends.

Table 6.3 summarizes the allocation of space for the R2T2 tunnel and support building program.

Table 6.3. R2T2 tunnel and support building program

\begin{tabular}{lcc}
\hline \multicolumn{1}{c}{ Space type } & Area (NSF) & \\
\hline Tunnel & 6,500 & \\
Egress stairs & 700 & \\
Total tunnel, net area & $\mathbf{7 , 2 0 0}$ & \\
Gross/net factor (80\% efficiency) & 1.25 & \\
Subtotal, tunnels & $\mathbf{9 , 0 0 0}$ & GSF \\
Tunnel power supplies & 4,000 & \\
Power control systems & 2,500 & \\
HVAC systems & 2,500 & \\
Pump systems & 2,500 & \\
Other systems & 2,500 & \\
Control room & 1,000 & \\
Total support building, net area & $\mathbf{1 5 , 0 0 0}$ & \\
Gross/net factor (75\% efficiency) & 1.3 & \\
Subtotal, support & $\mathbf{1 9 , 5 0 0}$ & GSF \\
Total, gross area & $\mathbf{2 8 , 5 0 0}$ & GSF \\
\hline
\end{tabular}

\section{R2T2 Tunnel}

The R2T2 tunnel will house the proton beam from the point where it splits from the primary beam, in the existing RTBT, to the point where it enters Target Building II. The overall tunnel will be approximately $500 \mathrm{ft}$ long. The R2T2 tunnel will be identical in width and height to the RTBT tunnel. Both tunnels will have $13 \times 13 \mathrm{ft}$ clear inside dimensions. A two-story enclosed stair, providing direct egress from the subgrade tunnel up to the exterior grade, is required at the end of the tunnel, adjacent to the target building. The grossing factor of 1.25 accounts for a tunnel and egress stair wall thickness of 18 in. The R2T2 tunnel will be constructed of regular-weight concrete; it will require a minimum of $15 \mathrm{ft} 6 \mathrm{in}$. of earth fill on all sides for shielding. 
In addition to housing and protecting the proton beam, the R2T2 tunnel will support the initial installation and ongoing maintenance of the beam components. The tunnel will be accessible only to trained personnel during periods when the accelerator is shut down. The tunnel will be accessible from the RTBT and the egress stairs. On-grade access from the exterior will be through the RTBT truck entrance, which will accommodate a tractor-trailer truck. The tunnel will be accessible only from Target Building II when temporary shielding blocks located above the tunnel are removed.

Approximately $280 \mathrm{ft}$ of the R2T2 tunnel will be sloped a total of $16 \mathrm{ft}$ vertically, or a $6 \%$ grade, to lower the beam as it enters Target Building II. This is necessary to optimize the overall siting of the STS with respect to the existing topography and to accommodate the STS feature of having neutron beam lines below grade.

The proton beam will be positioned asymmetrically in the tunnel to allow a circulation aisle for personnel and material. Movement of beam components will be accomplished with an overhead, 25 ton beam crane that will be similar to the crane in the RTBT. This crane will operate along the entire length of the tunnel, including the sloped section. Additionally, forklift access will be possible along the entire length of the tunnel in the circulation aisle.

Egress from the tunnel will be provided at the RTBT truck entrance and at the egress stairs located near the point where the tunnel enters Target Building II. Access to the egress stairs will be controlled; the construction of the egress stairs will be similar to the construction of existing egress stairs. During construction of the STS, when the FTS is in operation, temporary provisions will be needed to shield the R2T2 tunnel where it separates from the RTBT. During beam operation, radiation levels preclude personnel entry and require significant shielding for accident conditions.

\section{R2T2 Support Building}

The R2T2 support building will house the electrical power supplies; DI water cooling systems for the power supplies; and controls related to tunnel magnets, pumps, and other mechanical systems that serve the proton beam within the R2T2 tunnel. A separate adjacent building will also provide space for tunnel HVAC systems.

The program and area requirements for the R2T2 support building have been developed on a proportional basis, using comparison to the RTBT support building, which serves a similar function. The R2T2 tunnel will be approximately 50\% longer than the RTBT tunnel; this estimated size provided the basis for increasing the program requirements for the R2T2 support building. The HVAC, pump, and other systems will provide conditioning of both the R2T2 tunnel and the support building, in addition to supporting the proton beam.

The grossing factor of 1.3 is estimated based on comparable buildings and includes space for general building circulation, toilets, utility shafts, exterior wall, mechanical, electrical, plumbing, and other spaces not included in the estimated net program areas.

The R2T2 support building will be located in close proximity to the R2T2 tunnel to minimize the length of cabling, and the associated cost, between the power supplies and the proton beam magnets. However, the support building will be located beyond the $15 \mathrm{ft} 6 \mathrm{in}$. earth fill surrounding the tunnel to avoid the need for additional shielding. Subgrade, waterproofed tubes will provide cable routing paths between the support building and the tunnel.

To conserve space on the site, the R2T2 support building will be organized on two floors, with a partial third floor below grade. The upper floor will primarily house control systems. The intermediate floor, 
which will be approximately one level above the tunnel, will house power supplies. The upper floor will be accessible from the northern portion of Spallation Drive. The intermediate floor will be accessible from the courtyard between FTS and STS. Because of the access at both levels, elevators will not be required in the building. A floor hatch and a 3 ton overhead hoist will allow access to the cooling water pump systems located at the lower partial floor level.

Currently, the R2T2 support building is shown adjoined to the Zeemans building. These two structures will be functionally independent, although they may be integrated from the standpoint of life safety and building code requirements.

During subsequent STS design phases, it is anticipated that the following alternatives related to the R2T2 tunnel and support building, which may affect project scope or the design approach, will be further evaluated:

- The need for level sections along the sloped length of the R2T2 tunnel to support maintenance operations of the proton beam will be evaluated.

- The feasibility of omitting crane access in the sloped tunnel and relying only on forklift access will be evaluated.

- The space requirements to house the supporting utility systems will be confirmed.

- The need for on-grade access without relying on the RTBT truck entrance will be evaluated.

\section{Structural Systems}

The R2T2 tunnel is anticipated to be a buried concrete structure. It will be supported on friction piles in the vicinity of the Target Building and near the interface with the RTBT to avoid post-construction settlement, but otherwise it will be constructed on grade. The floor, walls, and roof will be reinforced normal-weight concrete. The tunnel floor will experience fork truck traffic. Water stops will be provided in construction joints to safeguard against water intrusion.

The tunnel will include grating covered trenches and sumps for water management, and channel strut embeds will be cast into the walls and ceiling/roof at $4 \mathrm{ft}$ spacing to allow the support of some mechanical and electrical systems.

The tunnel will meet PC-2 seismic performance requirements in accordance with DOE standards.

The R2T2 support building is anticipated to be conventional steel-framed building supported on spread footings. The below-grade elements (e.g., basement walls, retaining walls) will be constructed of reinforced normal-weight concrete. Water stops will be provided in construction joints to safeguard against water intrusion.

The R2T2 support building will share a common wall with the Zeemans support building. The roofing system will be supported on 1.5 in. galvanized metal roof deck on steel beams.

Elevated floors will be constructed of reinforced normal-weight concrete on composite metal deck (galvanized).

The slab on grade will be a minimum of 8 in. thick reinforced normal weight concrete slab. 
The R2T2 support building will meet PC-1 performance requirements in accordance with DOE standards. Because of the adjacency between the R2T2 support building and the Zeemans support building, the R2T2 support building may need to meet the higher performance requirements of the Zeemans support building. The extent to which these higher requirements are met will be based on a safety/hazard assessment that will occur in a subsequent phase of the project.

\section{Building Enclosure Systems}

The R2T2 tunnel will be constructed entirely of reinforced concrete, in a manner currently envisioned to be identical to the RTBT. Membrane waterproofing will be provided on all four sides of the tunnel. Foundation drainage tile will be placed along the entire perimeter of the tunnel structure and gravity drained to the site stormwater system. Egress stairs will also be constructed of reinforced concrete and similarly waterproofed and drained.

The R2T2 support building will be designed to be architecturally compatible with the existing SNS campus and other new buildings making up the STS. The exterior wall will be approximately $95 \%$ architectural grade, painted metal, insulated panels and 5\% low-E, insulated glass windows. The building will include two pedestrian entrances with overhead canopy protection and two service entrances with overhead canopy protection and depressed loading docks.

\section{Life Safety Systems}

The R2T2 tunnel will be defined as moderate-hazard factory industrial occupancy, similar to the RTBT. It will be considered an expansion of the RTBT that is within the allowable area limits of the occupancy type. The entire tunnel will be fully protected. The R2T2 tunnel will include one fully enclosed, $1 \mathrm{~h}$-rated egress stair exiting directly to the upper grade and will use the existing RTBT truck entrance as a second means of egress. The maximum travel distance in the R2T2 tunnel will be $400 \mathrm{ft}$.

The R2T2 support building will be also be defined as a moderate-hazard industrial occupancy factory, similar to the RTBT support building. The building will be fully protected, Type IIB, construction. The upper and intermediate floors of the R2T2 support building will each have two direct exits to grade. The partial lower floor will be connected to the intermediate level with two fully enclosed, $1 \mathrm{~h}$-rated egress stairs exiting directly to the lower grade level.

\section{Mechanical Systems}

\section{HVAC Equipment}

Heating and cooling for the conditioned spaces identified will be accomplished using air handling units (AHUs) located either on the roof, on the adjacent grade, or in a mechanical room. They are described in the following paragraphs. Conditioning of mechanical, electrical, and utility spaces will be accomplished using a heating and ventilation approach as described below.

\section{Control System}

The R2T2 tunnel and support building will be controlled by EPICS.

\section{Support Area Central Air Handling System}

The support areas of the R2T2 support building will include the ground-level utility space anticipated to house technical equipment control racks and the second subterranean level expected to house power 
supplies. Spaces on these two levels will be served by a variable air volume (VAV) AHU dedicated to each level. The supply air to these areas will be returned to the units. The AHUs serving the R2T2 support building will be located on the roof of the support building.

The AHU serving the R2T2 tunnel will be located in a grade-mounted mechanical building located on the north side of the proposed tunnel. As part of the HVAC system, a grade-mounted exhaust fan will be provided to permit manual smoke exhaust from the tunnel. The exhaust fan will connect to the return duct of the HVAC system, and control dampers will be provided to close off the air return to the HVAC unit and direct the tunnel airflow to the exhaust fan. A control damper in the HVAC system will also cause the HVAC unit to go to $100 \%$ outside air operation.

The AHUs will be designed as heating-cooling, single-zone, constant-volume type to provide from minimum outside air to $100 \%$ outside air economizer. Relief air will be discharged from the building through barometric relief dampers. The units will operate $24 \mathrm{~h}$ per day, 365 days per year. Supply fans will be plug type, and return fans will be plug type, arranged in a multi-fan array. AHU supply air temperature will be modulated as required to suit building load.

\section{Equipment and Materials}

The units will consist of the following components in the direction of air flow:

- $\quad$ MERV 8 (30\%) efficient prefilter bank

- MERV 14 (95\%) efficient filter bank

- Air-side economizer mixing damper section

- Heating water heating coil

- Chilled water cooling coil

- Supply fans

- Isolation/smoke damper

- Variable-frequency drives to modulate fan air flows

\section{Heating and Ventilation Equipment}

Conditioning of lower-level utility space housing the technical cooling water systems will be accomplished using a heating and ventilation approach. Ventilation will be achieved through the use of roof-mounted exhaust fans and an associated areaway mounted intake air louver with motorized dampers. Heating for these spaces will be accomplished through the use of heating water unit heaters located within the space.

\section{Chilled Water System}

The chilled water system for the building will be supplied from CUB II. Chilled water will be routed to the cooling coil in all AHUs. Based on an estimated flow of 230 GPM to this building, the chilled water branch line size to this building is expected to be 4 in.

\section{Technical Cooling Water DI Systems}

Technical cooling water systems will be required to support technical equipment to be included in this building, as well as in the R2T2 tunnel. The technical cooling water system will provide DI water with a resistivity in the range of 1 to $3 \mathrm{M} \Omega / \mathrm{cm}$, using a slip stream polishing loop. The pumps, heat exchanger, conditioners, sterilizers, and filter components will be located in a lower level mechanical room. One technical cooling water system will support the power supplies, and a second system will be used to cool 
the magnets in the tunnel. Both systems will reject heat to cooling tower water. The power supply cooling loop will support a $200 \mathrm{~kW}$ load using $320 \mathrm{GPM}$ of cooling tower water. The magnet cooling loop will support a $1000 \mathrm{~kW}$ load using $600 \mathrm{GPM}$ of cooling tower water. Pipe sizes for the power supply and the magnet cooling loops are estimated at 6 in. and 8 in., respectively. Tower water piping supplied to the building from the site distribution system is estimated to be 8 in.

The technical cooling water piping system will use all stainless-steel piping. All piping and equipment on the DI cooling water pump skids will be stainless steel. Pipe insulation will not be provided given the operating water temperature. The system will include the following equipment:

- base-mounted, end-suction, centrifugal pumps

- pump variable-frequency drives.

- tower water plate frame heat exchanger

- mixed bed deionizers

- resin trap filtration

- ultraviolet sterilizer

- air separator and expansion tank

- makeup water assembly

- appropriate valving and piping specialties

Two variable-speed technical cooling water pumps will be sized to provide $100 \%$ of the design flow rate each. If one of the booster pumps should fail, the other pump would provide $100 \%$ redundancy.

\section{Heating Water Systems}

The heating water system for the building will be supplied from CUB II. Based on an estimated flow of 40 GPM to this building, the heating water branch line size to this building is expected to be 2 in. The building's heating water distribution system will serve the water heating coil for the AHU and the heating water fan coil units within the building.

\section{Equipment Schedules}

The R2T2 tunnel and service building mechanical system requirements are summarized in Table 6.4. 
Table 6.4. RT2T tunnel and support building mechanical equipment schedules

\begin{tabular}{|c|c|c|c|c|c|}
\hline Equipment & Manufacturer & $\begin{array}{c}\text { Size } \\
\text { (each) }\end{array}$ & Quantity & $\begin{array}{c}\text { No. on } \\
\text { emergency } \\
\text { power }\end{array}$ & Remarks \\
\hline AHU - R2T2 tunnel & Trane & 20,000 CFM & 1 & 1 & $\begin{array}{l}20 \mathrm{hp}-\text { Total of all } \\
\text { motors }\end{array}$ \\
\hline AHU - R2T2 SB ground level & Trane & $8,000 \mathrm{CFM}$ & 1 & 0 & $\begin{array}{l}15 \mathrm{hp}-\text { Total of all } \\
\text { motors }\end{array}$ \\
\hline $\begin{array}{l}\text { AHU - R2T2 SB underground } \\
\text { level } 1\end{array}$ & Trane & $8,000 \mathrm{CFM}$ & 1 & $\mathbf{0}$ & $\begin{array}{l}15 \mathrm{hp}-\text { Total of all } \\
\text { motors }\end{array}$ \\
\hline Exhaust fans & Cook & Varies & $\begin{array}{l}\text { Estimated } \\
\text { at } 10\end{array}$ & 0 & $\begin{array}{l}\text { Roof mounted, some } \\
\text { with } 1 \text { phase and some } \\
\text { with } 3 \text { phase power }\end{array}$ \\
\hline Heating water unit heaters & Trane & Varies & $\begin{array}{l}\text { Estimated } \\
\quad \text { at } 4\end{array}$ & 0 & $\begin{array}{l}\text { Supplied with heating } \\
\text { water }\end{array}$ \\
\hline $\begin{array}{l}\text { Technical cooling water } \\
\text { distribution pumps (power } \\
\text { supplies) }\end{array}$ & Goulds & $\begin{array}{l}320 \text { GPM @ } \\
100 \text { psig } \\
\text { TDH }\end{array}$ & 2 & 0 & $\begin{array}{l}1 \text { standby, } \\
30 \mathrm{hp} \text { motor each }\end{array}$ \\
\hline $\begin{array}{l}\text { Technical cooling water } \\
\text { distribution pumps (tunnel } \\
\text { magnets) }\end{array}$ & Goulds & $\begin{array}{l}600 \text { GPM @ } \\
100 \text { psig } \\
\text { TDH }\end{array}$ & 2 & 0 & $\begin{array}{l}1 \text { standby, } \\
100 \text { hp motor each }\end{array}$ \\
\hline
\end{tabular}

$\mathrm{TDH}=$ total dynamic head

\section{Piping Systems}

\section{R2T2 Tunnel Foundation Drainage}

Perimeter foundation drainage system will be provided. Groundwater collected by the foundation drainage system will be drained by gravity into the site storm sewer. Provisions will be made to monitor the storm sewer flow for radioactivity.

\section{R2T2 Tunnel Interior Drainage System}

The interior of the tunnel will be provided with a trench system to collect interior water from DI water system leakage. The trench will contain a sump at the low end of the tunnel that will house a sump pump. The sump pump discharge will be piped to a storage tank located on the lowest level of the R2T2 support building. Any water collected will be sampled and then manually pumped to either the site sanitary sewer system or to a transport vehicle for offsite treatment, as appropriate.

\section{Fire Protection Systems}

Fire sprinkler systems for the R2T2 tunnel and support building will be hydraulically designed to provide water densities that meet the requirements for Ordinary Hazard, Group 2, protection throughout the facilities. Interior hose connections fed from the fire sprinkler system will also be provided in the R2T2 tunnel. 


\section{Electrical Systems}

\section{Exterior Power Distribution}

$480 \mathrm{Y} / 277 \mathrm{~V}$ electrical supply to the R2T2 tunnel and support building will be provided by connecting to a 2500 A circuit breaker in the outdoor unit substation located on the east side of the R2T2 support building. A $2500 \mathrm{~A}$ feeder bus duct will be routed overhead from the substation to the switchgear located in the electrical room.

$480 \mathrm{Y} / 277 \mathrm{~V}$ electrical supply to the makeup air structure will be provided by connecting to a $400 \mathrm{~A}$ circuit breaker in the substation. A 400 A duct bank will be routed from the substation to a panel board located on a wall of the mechanical room.

\section{Interior Power Distribution}

From the $2500 \mathrm{~A}, 480 \mathrm{Y} / 277 \mathrm{~V}$ switchgear, power will be distributed to CF loads, including lights and receptacles and mechanical equipment, as well as to technical loads, including the power supplies for the magnets located in the tunnel.

All panels and distribution panels will be located in the support building and makeup air structure. Only branch circuits will be routed to the tunnel. Empty conduit duct banks will be provided between the power supplies in the support building and the tunnel. Cable tray will be provided in the tunnel to support the power supply circuits.

\section{Emergency Power Distribution}

Emergency power to the tunnel, support building, and makeup air structure will be provided by connecting to the generator located on the east side of the support building. Separate duct banks will be routed from the generator to a disconnect switch in the support building electrical room and to a disconnect switch on the wall of the mechanical room in the makeup air structure.

\section{Fire Alarm System}

The fire alarm system for the R2T2 tunnel and support building will include VESDA and area heat and smoke detection as part of the means to provide redundant fire protection for these facilities.

\subsubsection{Target Building II}

\section{Programming}

Target Building II will house a second, lower power target at SNS. The STS target will be a solid tungsten target, not a liquid mercury target like the one employed in the FTS. Unlike the FTS, Target Building II will be separated from the instrument halls and will not be accessible to the scientific users and researchers. Access to the building will be limited to trained personnel with responsibilities for operation and maintenance of the target systems. A summary-level program for Target Building II is given in Table 6.5. 
Table 6.5. Target Building II program summary

\begin{tabular}{|c|c|c|}
\hline Space type & Area (NSF) & \\
\hline Target cooling loops $1-4$ & 3,500 & \\
\hline Target cooling loops DI water & 1,900 & \\
\hline Nitrogen/helium control panel & 200 & \\
\hline Tritium/inert gas glove box & 200 & \\
\hline Hot off-gas (HOG) system & 1,000 & \\
\hline LLLW tank vaults & 300 & \\
\hline LLLW pump valve vault & 200 & \\
\hline LLLW waste sampling glove box & 150 & \\
\hline Process waste pumping station & 150 & \\
\hline Change rooms/toilets & 200 & \\
\hline PCE and HOG HEPA filter train & 900 & \\
\hline SCE HEPA filter train & 1,200 & \\
\hline Communications room & 150 & \\
\hline Janitor's closet & 200 & \\
\hline Water loop and CF controls ${ }^{a}$ & 1,400 & \\
\hline General utility area & 2,000 & \\
\hline Truck bay hatch & 200 & \\
\hline Service cell & 1,600 & \\
\hline Manipulator gallery & 1,000 & \\
\hline Service gallery & 1,000 & \\
\hline Target hot shop & 600 & \\
\hline Moderator support building & 600 & \\
\hline Hydrogen equipment room & 400 & \\
\hline Hydrogen control room & 400 & \\
\hline Air handlers ${ }^{b}$ & 0 & \\
\hline Electrical distribution room $^{b}$ & 0 & \\
\hline Elevator/machine room ${ }^{b}$ & 0 & \\
\hline Neutron chopper lab/storage ${ }^{d}$ & 800 & \\
\hline Neutron tunnel support area & 2500 & \\
\hline Radiological maintenance shop & 500 & \\
\hline Support space, total net area & 23,250 & \\
\hline Gross $/$ net factor $(70 \% \text { efficiency })^{c}$ & 1.4 & \\
\hline Support space, total gross area & 32,550 & GSF \\
\hline Main bay & 22,500 & GSF \\
\hline Total gross area & 55,050 & GSF \\
\hline
\end{tabular}

${ }^{a}$ Utility corridor/water loop controls/RAD monitors/CF controls

${ }^{b}$ Included in grossing factor

${ }^{c} \mathrm{HVAC}$ space is included in $40 \mathrm{M}$ instrument hall

${ }^{d}$ Chopper space in Target Building II and CLO II equals 2000 NSF total

The main bay of Target Building II is a large, clear-span, open space served by one 50 ton bridge crane and one 10 ton bridge crane that will be used to construct and maintain the target and related systems. In addition to the initial construction, the bridge cranes will be used periodically to remove and replace multiple, interlaced layers of high-density concrete blocks that shield the proton beam line, target monolith, and radiating neutron beam lines within the footprint of Target Building II. The entire main bay of the target building will serve as a staging area for the movement of the shielding blocks and accelerator or target components that will be regularly moved in support of operating and maintaining the STS. The 
overall height of the main bay is driven by a required $30 \mathrm{ft}$ hook height above the top of the target monolith.

The area of the main bay has been estimated by the scientific design team in parallel with the development of the scientific requirements and assessment of operation and maintenance activities for the target. The elevation of the proton beam and target will be below the main bay floor level. The neutron beams that emanate from the target will also be below the main bay floor level. In order to access the neutron beam line components, pits will be located on either side of the monolith. Each pit will be covered with removable high-density concrete shielding planks. The pits will extend radially from the outside of the concrete monolith shield enclosure to a radius of approximately $40 \mathrm{ft}$. This arrangement will reduce the overall facility cost by eliminating the need for custom shielding for each beam line and by substantially reducing the amount of fixed monolith shielding. It will also fully load the target building foundation at startup, thus eliminating floor shifting which occurs if shielding is added piecemeal as new beam lines come on line.

The programmatic space requirements for Target Building II utility systems that support the target have been estimated using comparison to similar spaces that support the FTS. The space requirements for the STS systems will depend on detailed engineering that will occur in subsequent design phases. The service cell location and configuration are driven by requirements related to the operation of the target. The target building program area also includes the moderator support building that will house the helium compressor and other systems. It will be located on grade adjacent to the point where the R2T2 tunnel enters the target building.

A grossing factor of 1.5 is estimated, based on the grossing factor of the lower level of the FTS, and is applied only to the support space functions and not to the main bay of Target Building II. The grossing factor includes space for general building circulation, toilets, utility shafts, exterior wall, mechanical, electrical, plumbing, and other spaces not included in the net program areas.

Target Building II is positioned on the site, in accordance with the original SNS master plan, to optimize the overall siting of the STS with respect to existing buildings, ease of transporting beam to the new target, and roadway and topography, taking into account the geometry of the proposed instrument stations. The building will be two stories, including the basement floor utility support functions and the upper-level high bay space. The building will be accessed by personnel and tractor-trailer trucks at the main bay floor level from the east side. The building will also be accessible to personnel at the basement level adjacent to the tractor-trailer entrance into the $40 \mathrm{M}$ instrument hall. Alternative points of personnel connection between Target Building II, instrument tunnels, and instrument halls will be further evaluated in subsequent design phases.

During subsequent STS design phases, it is anticipated that the following alternatives related to Target Building II, which may affect project scope or the design approach, will be further evaluated:

- Space requirements to house the basement-level utility systems that support the target and related systems

- Optimum configuration for the subgrade beam array on the east side of Target Building II

- The feasibility of including instrument stations within Target Building II on beam lines 11 and 22

- Relative to the Life Safety Code and IBC, the consequences of defining Target Building II as a single building or integrating it with the CLO II, CUB II, and $30 \mathrm{M}$ and $40 \mathrm{M}$ instrument buildings 


\section{Structural Systems}

Target Building II is anticipated to be a hybrid of concrete and steel construction supported on micropiles down to rock. The below-grade elements (e.g., basement slab, basement walls, retaining walls) as well as the lower-level framing and columns will be constructed of reinforced normal-weight and heavyweight concrete. The upper level will use a beam and slab system to support a live load of 4,000 psf. A portion of the upper level will experience tractor-trailer loads. Water stops will be provided in construction joints of the perimeter walls and the floor slab to safeguard against water intrusion.

The monolith is planned as a large concrete and steel mass that will provide shielding consisting of both fixed and removable elements. The pit/monolith covers are planned as $540 \mathrm{yd}^{3}$ of removable heavyweight concrete covers, $70 \mathrm{yd}^{3}$ of removable normal-weight concrete covers, and $55 \mathrm{yd}^{3}$ of fixed normal-weight concrete covers.

The superstructure (the portion above the upper level) will be constructed as an industrial type building with stepped columns supporting a bridge crane. The roof will be constructed of deep trusses to provide a column-free space for crane operation and to provide access to the monolith and surrounding shielding. The steel columns will be encased on concrete fire protection to approximately $24 \mathrm{ft}$ above the lower level and gypsum board wrap to the bottom of the trusses. The concrete encasement will also protect the steel columns from damage.

The roof trusses will also be used to create moment frames for resisting lateral forces (wind and seismic). Concentric braced frames will be used in the long direction of the building to resist these loads.

Target Building II may share a common row of columns with the $40 \mathrm{M}$ instrument building. Its exterior wall system will be supported with steel girts. The roofing system will be supported on $1.5 \mathrm{in}$. galvanized metal roof deck on steel purlins.

Steel-framed platforms supporting galvanized steel grating are anticipated.

It is assumed that Target Building II will have to meet SDC-3 seismic requirements (current terminology for PC-3) in accordance with DOE standards. Structural materials and construction will be commissioned to meet the requirements of these performance categories (e.g., additional and higher levels of testing and inspection and documentation of the testing and inspection).

\section{Building Enclosure Systems}

The exterior design for Target Building II will conform to the requirements defined for the overall STS project. The exterior wall will be approximately $80 \%$ architectural-grade metal insulated panels and $20 \%$ low-E, insulated glass in a painted metal integrated strip window system. Currently, Target Building II and the $40 \mathrm{M}$ instrument hall (which houses instruments that are $\sim 40 \mathrm{~m}$ long) directly connected to it are envisioned as a single, visually integrated structure, although they will function as independent buildings. Target Building II will include four pedestrian entrances with overhead canopy protection and one gradelevel service entrance with overhead door clearance to allow tractor-trailer access into the building.

\section{Life Safety Systems}

Currently, Target Building II, along with two contiguous instrument buildings and the CLO II, is defined as a single, mixed-occupancy building comprising business, incidental assembly, and moderate-hazard factory industrial uses. The hydrogen utility room is defined as Hazardous Occupancy, Class I/Div I, and 
will be provided with the appropriate fire separation and safeguards as required by IBC and NFPA. The entire complex is envisioned as being fully sprinkler-protected Type IIA construction.

The definition of Target Building II as part of a larger single building, including the contiguous instrument buildings and CLO II, will be further evaluated through detailed fire hazard and code analysis that will occur in subsequent STS design phases.

\section{Mechanical Systems}

\section{HVAC Ventilation}

STS target and support areas will be designed with a minimum of eight air changes per hour at a virtual ceiling height of $10 \mathrm{ft}$, or $1.2 \mathrm{CFM} / \mathrm{ft}^{2}$. Instrument areas have been assumed at an average requirement of $20 \%$ outside air.

\section{Pressure Relationships}

All building areas containing potentially activated fluids will be exhausted using the primary or secondary exhaust systems and will be maintained at a negative pressure relative to the adjacent spaces.

\section{Control System}

Target Building II will be controlled by EPICS.

\section{The Target Building II Central Air Handling System}

Target Building II will be served by two constant-air-volume (CAV) AHUs. The units will be manifolded together and will share a common discharge plenum to provide partial redundancy in the event of a complete unit failure. The AHUs will be designed as heating-cooling, single-duct type. Each unit will also contain a reheat coil for use in a dehumidification control cycle. The units will operate $24 \mathrm{~h}$ per day, 365 days per year. Supply fans will be based on a multiple array, plenum fan type design. Variablefrequency drives will provide the capability of setback control of the supply fan volume.

The AHUs will be housed in the mechanical penthouse located above the instrument floor. Each unit will be provided with air-side economizer control. Relief air from the experimental area will be routed through the mechanical penthouse to provide a tempered environment within this space. Excess air in the penthouse will be relieved through wall-mounted louvers and associated barometric relief dampers.

The system will be designed for energy conservation adjustment. The potential to reduce air changes per hour will be accommodated. Changes in air changes per hour or unoccupied setbacks will be controlled by the building automation system (BAS).

The units will consist of the following components in the direction of air flow:

- $\quad$ intake isolation damper

- MERV 8 (30\%) efficient prefilter bank

- MERV 14 (90\%) efficient filter bank

- heating water preheat coil

- chilled water cooling coil

- heating water reheat coil

- $\quad$ supply fans (4-6 fan array) 
- isolation/smoke damper

- variable frequency drives to modulate fan air flows

The system consists of two AHUs. If one of the units should fail or need to be taken out of service for maintenance, the other will be able to provide $66 \%$ of the supply system design capacity.

\section{Tertiary Support Area Central Air Handling System}

The tertiary support areas of the building will be served by a single CAV AHU. The supply air to these areas will be returned to the unit. The AHU will be designed as heating-cooling, single-duct, reheat type to provide from minimum outside air to $100 \%$ outside air economizer. The unit will operate $24 \mathrm{~h}$ per day, 365 days per year. Supply fans will be plenum type, and return fans will be plenum type, arranged in a multi-fan array. Variable-frequency drives will provide the capability of simplified manual control of the supply fan volume. Variable-frequency drives will provide return fan volume control in response to a signal from air flow measuring stations used to establish volumetric offset between the return air and the supply air quantities.

The supply distribution system will consist of low-pressure ductwork with pressure-independent electrically actuated supply CAV air terminal devices, reheat coils, and low-pressure ductwork downstream of air terminals to diffusers. Sound attenuators at the air terminal devices will not be provided.

The units will consist of the following components in the direction of air flow:

- return/relief fans located within the AHU

- airside economizer mixing damper section

- MERV 8 (30\%) efficient prefilter bank

- MERV 14 (95\%) efficient filter bank

- chilled water cooling coil

- supply fans

- isolation/smoke damper

- variable-frequency drives to modulate fan air flows

\section{Primary and Secondary Support Area Make-up Air Handling System}

The primary and secondary support areas of the building will be served by two CAV AHUs. All air supplied to these spaces will be exhausted using the primary confinement exhaust (PCE) or secondary containment exhaust (SCE) systems.

The makeup AHUs will be designed as heating-cooling, single-duct, reheat type. The units will operate $24 \mathrm{~h}$ per day, 365 days per year. Supply fans will be plenum type, arranged in a multi-fan array. Variablefrequency drives will provide the capability of simplified manual control of the supply fan volume.

The supply distribution system will consist of low-pressure ductwork with pressure-independent electrically actuated supply CAV air terminal devices, reheat coils, and low-pressure ductwork downstream of air terminals to diffusers. Sound attenuators at the air terminal devices will not be provided.

The units will consist of the following components in the direction of air flow: 
- motorized intake isolation damper

- MERV 8 (30\%) efficient prefilter bank

- MERV 14 (95\%) efficient filter bank

- heating water preheat coil

- chilled water cooling coil

- heating water reheat coil

- supply fans

- isolation/smoke damper

- variable-frequency drives to modulate fan air flows

The system will consist of two AHUs. If one of the units should fail or need to be taken out of service for maintenance, the remaining unit will be able to provide $100 \%$ of the supply system design capacity.

\section{Primary and Secondary Support Area Supplemental Cooling System}

The primary and secondary support areas of the building that require supplemental cooling will be provided with fan coil units located within the spaces. To eliminate the need for treatment of potentially activated cooling coil condensate, the fan coil units will be supplied with sensible cooling chilled water that will be controlled to above the dew point temperature of the spaces.

\section{Hydrogen Utility Room HVAC System}

The hydrogen utility room will be designed with a $100 \%$ outside air, single pass HVAC concept. Outside air will be drawn in through a roof-mounted intake hood. The intake duct will be provided with a heating water heating coil and chilled water cooling coil to maintain the desired space temperature. All ventilation/purge air will be drawn from the space using roof-mounted exhaust fans.

\section{Moderator Support Building HVAC System}

The moderator support building will be conditioned using a heating and ventilation approach. Ventilation will be achieved through the use of a roof-mounted exhaust fan and associated wall-mounted intake air louver with motorized dampers. These spaces will be heated using heating water unit heaters located within the space.

\section{Primary and Secondary Support Area Exhaust Systems}

All makeup air supplied to these spaces will be exhausted using the PCE or SCE systems. A hot off-gas (HOG) exhaust system will also be provided for activated exhaust from equipment. The ductwork for these systems within the building will be constructed of all welded type 304L stainless steel. All air will be collected and routed to multistage nuclear-grade type HEPA filter banks. The PCE filtration system is expected to consist of two 304L stainless steel, three-high-filter banks. Each filter bank will have a butterfly isolation valve, prefilter, test section, HEPA filter section, test section, HEPA filter section, test section, and final butterfly isolation valve. The SCE filtration system is expected to consist of six sets of filter banks; two 304L stainless steel, three high-filter banks; and four 304L stainless steel, two-high-filter banks. Each filter bank will have a butterfly isolation valve, a prefilter, a test section, a HEPA filter section, a test section, and a final butterfly isolation valve. The HOG filtration system is expected to consist of two 304L stainless steel and two-high-filter banks. Each filter bank will have a butterfly isolation valve, a prefilter, a test section, a HEPA filter section, a test section, a HEPA filter section, a test section, and a final butterfly isolation valve. 


\section{Hydrogen Utility Room Exhaust System}

The exhaust system for the hydrogen utility room will consist of a single ventilation exhaust fan with an estimated capacity of approximately $300 \mathrm{CFM}$, as well as two roof-mounted purge exhaust fans with an estimated capacity of 8,000 CFM each. One purge fan will be stand-by. All exhaust fans will be of sparkfree construction and provided with explosion-proof motors.

\section{Secondary Technical Cooling Water Systems}

Two secondary technical cooling water systems will be required to support the four primary technical equipment cooling water systems to be included in this building by others. The four primary systems will be located in the target secondary containment support area and will serve the target, the inner reflector plug, moderators, and the proton beam window. The secondary technical cooling water systems will provide DI water with a resistivity in the range of 1 to $3 \mathrm{M} \Omega / \mathrm{cm}$, using a slip stream polishing loop. The pumps, heat exchanger, conditioners, sterilizers, and filter components will actually be located in a lowerlevel target support mechanical area of CLO II. One higher-temperature technical cooling water system will support the secondary DI water cooled by tower water, and a second system will be used to provide lower-temperature secondary DI water cooled by chilled water. The higher-temperature cooling loop will support a $530 \mathrm{~kW}$ load using 400 GPM of cooling tower water for the target, target shroud, and beam stop. The lower-temperature cooling loop will support a $70 \mathrm{~kW}$ load using $18 \mathrm{GPM}$ of chilled water and will serve the moderators. Pipe sizes for the 400 GPM higher-temperature and 18 GPM lower-

temperature cooling loops are estimated at 6 in. and 1.5 in., respectively. Tower water piping supplied to the building from the site distribution system is estimated to be 6 in.

The secondary technical cooling water piping systems will use all welded 304L stainless steel piping. All piping and equipment on the DI cooling water pump skids will be stainless steel. Pipe insulation will not be provided given the operating water temperature. The system will include the following equipment:

- base-mounted, end suction, centrifugal pumps

- pump variable-frequency drives

- tower water plate frame heat exchanger

- mixed bed deionizers

- resin trap filtration

- ultraviolet sterilizer

- air separator and expansion tank

- makeup water assembly

- appropriate valving and piping specialties

Two variable-speed technical cooling water pumps will be sized to provide $100 \%$ of the design flow rate each. If one of the booster pumps were to fail, the other would provide $100 \%$ redundancy.

\section{Chilled Water System}

The chilled water system for the building will be supplied from CUB II. Based on an estimated flow of 880 GPM to this building, which includes chilled water flow to the associated instrument areas except M2, the chilled water branch line size to this building is expected to be $8 \mathrm{in}$. Chilled water will be routed to cooling coils in all AHUs, as well as to HOG condensing heat exchangers. 


\section{Sensible Chilled Water System}

A sensible chilled water cooling system will be required to support fan coil equipment, where condensate is to be avoided, in this building. The sensible chilled water cooling system will consist of an independent pumped loop that will reject heat into the building chilled water through a plate frame heat exchanger. The system will be designed to operate at a supply temperature of $55^{\circ} \mathrm{F}$ to reduce the potential for condensation. The tank, pumps, heat exchanger, filters, and chemical treatment components will be located in a lower-level target support mechanical area of CLO II.

The sensible chilled water piping system will use copper pipe for pipe sizes up to $3 \mathrm{in}$. and grooved coupling or welded carbon steel piping for sizes above 3 in. Pipe insulation will not be provided, given the operating water temperature. The system will include the following equipment:

- base-mounted, end suction, centrifugal pumps

- $\quad$ pump variable-frequency drives

- chilled water plate frame heat exchanger

- water filtration

- automated chemical treatment system

- makeup water assembly

- $\quad$ appropriate valving and piping specialties

Two variable-speed process cooling water pumps will be sized to provide $100 \%$ of the design flow rate each. If one of the booster pumps were to fail, the remaining pump would provide $100 \%$ redundancy.

\section{Heating Water System}

The heating water system for the building will be supplied from CUB II. Based on an estimated flow rate of $120 \mathrm{GPM}$ to this building, the heating water branch line size to this building is expected to be $4 \mathrm{in}$. The building heating water distribution system will serve preheat and reheat heating water coils within the building.

\section{Equipment Schedules}

The new target building mechanical system requirements are summarized in Table 6.6.

Table 6.6. Target Building II preliminary mechanical equipment schedules

\begin{tabular}{|c|c|c|c|c|c|}
\hline Equipment & Manufacturer & $\begin{array}{l}\text { Size } \\
\text { (each) }\end{array}$ & Quantity & $\begin{array}{c}\text { No. on } \\
\text { emergency } \\
\text { power }\end{array}$ & Remarks \\
\hline AHU & Trane & $35,000 \mathrm{CFM}$ & 2 & $\mathbf{0}$ & $\begin{array}{l}\text { Located in the instrument } \\
\text { building M1 penthouse. } \\
40 \mathrm{hp} \text { (total of all motors). }\end{array}$ \\
\hline $\begin{array}{l}\text { AHU—target } \\
\text { support }\end{array}$ & Trane & $12,000 \mathrm{CFM}$ & 1 & 0 & $\begin{array}{l}\text { Located in the target support } \\
\text { area of the CLO II basement. } \\
15 \mathrm{hp} \text { (total of all motors). }\end{array}$ \\
\hline $\begin{array}{l}\text { Air handling } \\
\text { unit—-target } \\
\text { support makeup } \\
\text { air }\end{array}$ & Trane & $3,000 \mathrm{CFM}$ & 2 & $\mathbf{0}$ & $\begin{array}{l}\text { Located in the target support } \\
\text { area of the CLO II basement. } \\
5 \mathrm{hp} \text { (total of all motors). }\end{array}$ \\
\hline $\begin{array}{l}\text { Packaged fan coil } \\
\text { (sensible cooling) }\end{array}$ & Trane & $\begin{array}{l}1-2 \text { tons of } \\
\text { refrigeration }\end{array}$ & $\begin{array}{c}\text { Estimated at } \\
10 \\
\end{array}$ & 0 & $1.5 \mathrm{hp}$ motor each \\
\hline
\end{tabular}


Table 6.6. Target Building II preliminary mechanical equipment schedules (continued)

\begin{tabular}{|c|c|c|c|c|c|}
\hline Equipment & Manufacturer & $\begin{array}{c}\text { Size } \\
\text { (each) }\end{array}$ & Quantity & $\begin{array}{c}\text { No.on } \\
\text { emergency } \\
\text { power } \\
\end{array}$ & Remarks \\
\hline Exhaust fans & Cook & Varies & $\begin{array}{c}\text { Estimated at } \\
10\end{array}$ & 0 & $\begin{array}{l}\text { Roof mounted, some with } \\
1 \text { phase and some with } \\
3 \text { phase power }\end{array}$ \\
\hline $\begin{array}{l}\text { Heating water } \\
\text { unit heaters }\end{array}$ & Trane & Varies & $\underset{4}{\text { Estimated at }}$ & 0 & Supplied with heating water \\
\hline $\begin{array}{l}\text { Secondary } \\
\text { cooling water } \\
\text { distribution } \\
\text { pumps (tower } \\
\text { water) }\end{array}$ & Goulds & $\begin{array}{l}400 \text { GPM@ } \\
100 \mathrm{ft} \text { TDH }\end{array}$ & 2 & 0 & $\begin{array}{l}\text { Located in the target support } \\
\text { area of the CLO II basement. } \\
1 \text { Standby, } \\
20 \text { hp Motor each }\end{array}$ \\
\hline $\begin{array}{l}\text { Secondary } \\
\text { cooling water } \\
\text { distribution } \\
\text { pumps (chilled } \\
\text { water) }\end{array}$ & Goulds & $\begin{array}{l}20 \text { GPM@ } \\
100 \mathrm{ft} \text { TDH }\end{array}$ & 2 & 0 & $\begin{array}{l}\text { Located in the target support } \\
\text { area of the CLO II basement. } \\
1 \text { standby, } \\
5 \text { hp motor each }\end{array}$ \\
\hline $\begin{array}{l}\text { Sensible chilled } \\
\text { water distribution } \\
\text { pumps }\end{array}$ & Goulds & $\begin{array}{l}350 \text { GPM @ } \\
100 \mathrm{ft} \text { TDH }\end{array}$ & 2 & 0 & $\begin{array}{l}\text { Located in the target support } \\
\text { area of the CLO II basement. } \\
1 \text { standby, } \\
15 \mathrm{hp} \mathrm{motor} \mathrm{each}\end{array}$ \\
\hline $\begin{array}{l}\text { Instrument } \\
\text { sensible chilled } \\
\text { water distribution } \\
\text { pumps }\end{array}$ & Goulds & $\begin{array}{l}75 \text { GPM@ } \\
100 \mathrm{ft} \text { TDH }\end{array}$ & 2 & 0 & $\begin{array}{l}\text { Located in the target support } \\
\text { area of the CLO II basement. } \\
1 \text { standby, } \\
7.5 \mathrm{hp} \text { motor each }\end{array}$ \\
\hline
\end{tabular}

$\mathrm{TDH}=$ total dynamic head

\section{Fire Protection System}

Fire sprinkler systems will be hydraulically designed to provide water densities that meet the requirements for Ordinary Hazard, Group 2, protection throughout the basement and Extra Hazard, Group 1, protection throughout the main building. A separate standpipe system will also be provided for Target Building II.

\section{Electrical Systems}

\section{Exterior Power Distribution}

Target Building II, the $40 \mathrm{M}$ instrument bay, and the future $30 \mathrm{M}$ instrument bay buildings will be considered one building with one electrical power supply serving all three buildings. The one $480 \mathrm{Y} / 277 \mathrm{~V}$ electrical supply to the complex will be provided by connecting to a $3000 \mathrm{~A}$ circuit breaker in the outdoor unit substation located on the south side of the $40 \mathrm{M}$ instrument bay. A $3000 \mathrm{~A}$ feeder bus duct will be routed overhead from the substation to the switchgear located in the electrical room of the $40 \mathrm{M}$ instrument bay. Separate feeders will be routed to a technical distribution panel and to a CF distribution panel within Target Building II.

The moderator support building will be provided with two $4160 \mathrm{~V}$ supplies from the outdoor unit substation located on the north side of the moderator support building, one for each of the redundant helium compressors. It will also be provided with a $480 \mathrm{Y} / 277 \mathrm{~V}$ supply from a 400 A circuit breaker in the substation located on the east side of the R2T2 support building. 


\section{Interior Power Distribution}

From the technical distribution panel, power will be routed to technical branch circuit panels via conduit, cable trays, and step down transformers. Separate transformers and panels will be provided to serve sensitive electronic loads. From the CF distribution panel, power will be routed to 480 and $208 \mathrm{~V}$ branch circuit panels to serve lights and receptacles, mechanical equipment, and elevator(s).

Equipment and circuiting in the hydrogen utility room will be suitable for use in Class 1, Division 2, environments.

At the moderator support building, the $400 \mathrm{~A}, 480 \mathrm{Y} / 277 \mathrm{~V}$ distribution panel will serve mechanical equipment and will subfeed a lighting panel and a receptacle panel via a step down transformer.

\section{Emergency Power Distribution}

Emergency power to the STS, the $40 \mathrm{M}$ instrument bay, and the future $30 \mathrm{M}$ instrument bay will be provided by connecting to the generator located on the east side of the R2T2 support building. A duct bank will be routed from the generator to a generator power distribution panel board in the emergency power electrical room on the north side of the $40 \mathrm{M}$ instrument bay. Generator power will be distributed to an emergency automatic transfer switch and to one or more optional standby automatic transfer switches, which will in turn serve emergency and optional standby panels in the STS Building.

Emergency power to the moderator support building will be provided by connecting to generator GEN-E located on the east side of the R2T2 support building. A duct bank will be routed from generator GEN-E to a disconnect switch in the moderator support building.

\section{Fire Alarm System}

The fire alarm system for Target Building II will include area heat and smoke detection in the high bay spaces as part of the means to provide redundant fire protection for the facility.

A hydrogen detection system will be provided in the hydrogen utility room. Alarm and supervisory conditions will be reported to the fire alarm system.

\subsubsection{Instrument Buildings and Tunnels}

\section{Programming}

The instrument halls and the instrument tunnels will be distinctly different but related structures. The instrument halls will house one or more terminal instruments and related shop, laboratory, and office spaces that support user activities. The radiating instrument tunnels will house the neutron beam lines that will extend from Target Building II to the outlying instrument halls. A summary-level program for the STS instrument buildings and tunnels is shown in Table 6.7. 
Table 6.7. Instrument buildings and tunnels program summary

\begin{tabular}{|c|c|c|}
\hline Space type & Area (NSF) & \\
\hline $40 \mathrm{M}$ instrument hall (BL 2-10) & 22,500 & \\
\hline $40 \mathrm{M}$ instrument hall lab support & 1,500 & \\
\hline $40 \mathrm{M}$ instrument hall sample environment maintenance & 400 & \\
\hline $40 \mathrm{M}$ instrument hall chemical/waste storage & 900 & \\
\hline $40 \mathrm{M}$ instrument hall HVAC support ${ }^{a}$ & 12,000 & \\
\hline $30 \mathrm{M}$ instrument hall (BL 12-14) $)^{b}$ - future & 7,000 & \\
\hline $90 \mathrm{M}$ instrument hall (BL 15-19) & 12500 & \\
\hline $90 \mathrm{M}$ instrument hall lab support & 1,500 & \\
\hline $90 \mathrm{M}$ instrument hall sample environment maintenance & 700 & \\
\hline $90 \mathrm{M}$ instrument hall tunnel support & 2,500 & \\
\hline HRB (BL 20) & 1,500 & \\
\hline HRP hall (BL 21) & 2,500 & \\
\hline Total instrument building net area & 65,500 & \\
\hline Gross/net factor ( $70 \%$ efficiency) & 1.4 & \\
\hline Subtotal instrument building & 91,700 & GSF \\
\hline Tunnel BL 1 Zeemans & 600 & \\
\hline Tunnel BL 15 & 1,700 & \\
\hline Tunnel BL 16 & 1,600 & \\
\hline Tunnel BL 17 & 1,500 & \\
\hline Tunnel BL 18 & 1,500 & \\
\hline Tunnel BL 19 & 1,600 & \\
\hline Tunnel BL 20, HRB & 1,000 & \\
\hline Tunnel BL 21, HRP & 2,600 & \\
\hline Egress stairs & 2,200 & \\
\hline Tunnel commons & 2,000 & \\
\hline Total tunnels net area & 16,300 & \\
\hline Gross $/$ net factor ( $70 \%$ efficiency) & 1.4 & \\
\hline Subtotal, tunnels & 22,820 & GSF \\
\hline Total gross area & 114,520 & GSF \\
\hline
\end{tabular}

${ }^{a}$ Area to be confirmed

${ }^{b}$ Not included in STS project

\section{Instrument Buildings}

The STS currently is proposed to include four instrument buildings of varying sizes, excluding the Zeemans building, which is an additional instrument building with specialized requirements. One additional structure, the $30 \mathrm{M}$ instrument building, is proposed for construction after completion of the STS. The STS instrument buildings will be similar to the FTS instrument buildings, except that the STS instrument buildings will typically be farther away from the target, and the main floor level of the STS instrument buildings will typically be one level below grade. The STS instrument buildings are shown in Fig. 6.6. 


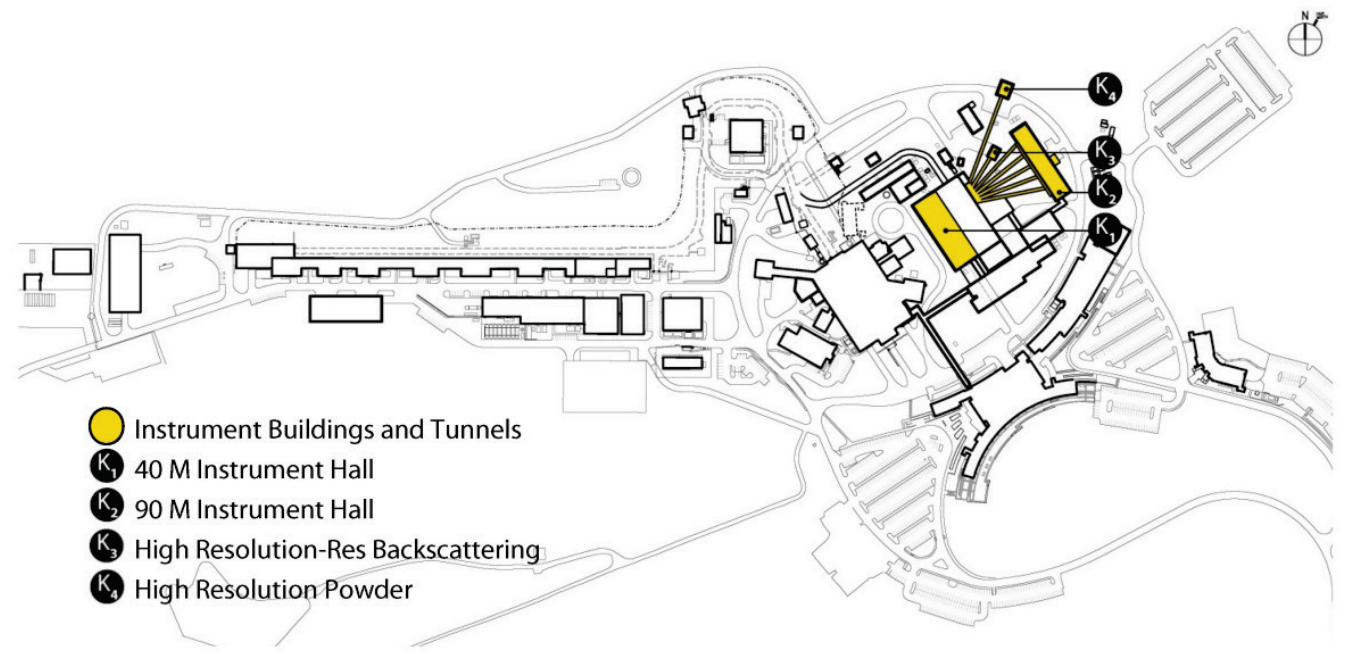

Fig. 6.6. Instrument building locations.

Space requirements for the instrument buildings have been established based on previous experience installing and operating the FTS instruments. The number of instruments planned for each building varies. The locations of the instruments relative to the target are designed to maximize the scientific potential of the STS while respecting the existing site conditions. The radial neutron beam lines within all of the instrument buildings will be shielded with movable, high-density concrete blocks. Each instrument building will include space that can be used as a staging area when it is necessary to remove the shielding blocks in order to make beam line modifications.

The instrument building grossing factor of 1.4 includes space for general building circulation, toilets, exterior wall, mechanical, electrical, and plumbing systems spaces that are not included in the net program areas.

\section{M Instrument Building}

The $40 \mathrm{M}$ instrument building, the largest of the five instrument buildings, will be located directly adjacent to Target Building II and will include a large high bay space for nine instruments. The entire space will be served by a 30 ton overhead bridge crane that will have a minimum hook height of $32 \mathrm{ft}$ above the instrument floor.

The $40 \mathrm{M}$ instrument building will have four floor levels including (1) instrument floor, (2) instrument floor mezzanine, (3) bridge to FTS and CLO, and (4) HVAC penthouse. The second and third floor levels will be relatively small in area, essentially serving to provide access within the building. The fourth floor level will be sized to house air-handling equipment for the $40 \mathrm{M}$ instrument building and Target 
Building II. It will partially cover the footprint of the instrument building. The remaining space at the fourth level will be used for exterior roof-mounted equipment.

The instrument floor for the $40 \mathrm{M}$ instrument building will be directly accessible by tractor-trailer from Spallation Drive, an arrangement that will require trucks to pass below the CLO II. Pedestrian access will be from the FTS or CLO at the existing bridge level, from the FTS/STS exterior courtyard (which will be one level above the instrument floor), or from Spallation Drive at the instrument floor level.

The building will be served by freight and passenger elevators that will provide access to the instrument, mezzanine, and bridge floor levels. The freight elevator will additionally provide access to the mechanical floor level.

\section{M Instrument Building (Future)}

The $30 \mathrm{M}$ instrument building, also to be directly adjacent to Target Building II, will include high bay space for three instruments. The space will be served by a 30 ton overhead bridge crane that will have a minimum hook height of $32 \mathrm{ft}$ above the instrument floor. It will have three floor levels: (1) instrument floor, (2) instrument floor mezzanine, and (3) bridge level. The second and third floor levels will be relatively small in area, essentially serving to provide access within the building.

The instrument floor for the $30 \mathrm{M}$ instrument building will be directly accessible at mezzanine level by tractor-trailer from Spallation Drive, an arrangement that will require trucks to pass below the bridge connecting CLO II with the $90 \mathrm{M}$ instrument building. Pedestrian access will be from the CLO II, FTS, or the CLO at the existing bridge level, from the exterior courtyard (approximately one level above the instrument floor), or Spallation Drive at the instrument floor level. It will be served by a freight elevator that will provide access to all three floor levels.

\section{M Instrument Building}

The $90 \mathrm{M}$ instrument building, the second largest of the five instrument buildings, will be approximately $200 \mathrm{ft}$ east of Target Building II and will include a large high bay space for five instruments. The entire space will be served by a 30 ton overhead bridge crane that will have a minimum hook height of $32 \mathrm{ft}$ above the instrument floor.

The building will have three floor levels: (1) instrument floor, (2) instrument floor mezzanine, and (3) bridge to CLO II, FTS, and CLO. The second and third floor levels will be relatively small in area, essentially serving to provide access within the building.

The instrument floor for the $90 \mathrm{M}$ instrument building will be directly accessible by tractor-trailer from Spallation Drive. Pedestrian access will be from the CLO II, FTS, or CLO at the existing bridge level, from the STS/instrument building 3 exterior courtyard (which will be approximately one level above the instrument floor), or from Spallation Drive at the instrument floor level. The building will be served by a freight elevator that will provide access to all three floor levels.

\section{High-Resolution Backscattering and High-Resolution Powder Instrument Buildings}

The HRB and HRP instrument buildings will be the smallest of the five instrument buildings. They will both be remotely located, approximately 120 and $320 \mathrm{ft}$, respectively, from Target Building II. Each building will include high bay space for one instrument. The space in both buildings will be served by a 30 ton overhead bridge crane that will have a minimum hook height of $32 \mathrm{ft}$ above the instrument floor. 
The HRB and HRP buildings will have two floor levels: (1) instrument floor and (2) instrument floor mezzanine. The second floor levels will be relatively small in area, essentially serving to provide access within the buildings.

The instrument floor levels of both buildings will not be directly accessible by tractor-trailer. Both buildings will require truck access from Spallation Drive at the mezzanine level because of the site topography. Pedestrian access for both buildings will also be at the mezzanine level from the exterior. Neither of the two buildings will have an interior pedestrian connection to other buildings in the SNS complex. They will each be served by a freight elevator that will provide access to both floor levels.

\section{Instrument Tunnels}

The STS will include multiple instrument tunnels of varying lengths. No equivalent to the STS instrument tunnels is associated with the FTS. The instrument building tunnels will all have $8 \times 8 \mathrm{ft}$ clear inside dimensions. At each end of the tunnels, adjacent to Target Building II and the $90 \mathrm{M}$ instrument building, the tunnels will be connected with an overhead mezzanine that will allow personnel movement between tunnels and egress. Stairs to the grade level above will be located as needed to provide egress from the tunnel areas. The connecting mezzanine adjacent to Target Building II will include a 5 ton overhead crane. The optimum configuration of the connecting mezzanine will be further evaluated in subsequent design phases.

The grossing factor of 1.4 accounts for a tunnel and for egress stair wall thickness of 18 in. In addition to housing and protecting the neutron beam lines, the instrument tunnels will provide space for ongoing maintenance of the beam line components. The tunnels will be accessible only to trained personnel during periods when the neutron beam is locked out. The tunnels will be accessible for maintenance only through the egress stairs. The tunnels will be accessible only from Target Building II or the instrument buildings when temporary shielding blocks, located at the points where the tunnels meet the buildings, are removed.

The instrument beam lines will be positioned asymmetrically in the tunnels to allow a circulation aisle for personnel and material passage. Beam line components or maintenance items will be moved using movable carts. Forklift access is not required in the instrument tunnels.

During subsequent STS design phases, it is anticipated that the following alternatives related to the instrument buildings, which may affect project scope or the design approach, will be further evaluated:

- the optimum configuration of the tunnel array adjacent to Target Building II

- the elevation of instrument floors relative to overall topography and proton beam slope

- the potential for CLO II and instrument building 1 to share freight and passenger elevators

\section{Structural Systems}

The $40 \mathrm{M}$ instrument building will abut Target Building II; it is anticipated to be a steel superstructure supported on thick concrete perimeter/foundation walls. The below-grade elements (e.g., basement walls, retaining walls) as well as the lower-level slab will be constructed of reinforced normal-weight concrete and will be supported on friction piles. Mezzanines constructed of steel framing and reinforced normal weight concrete on composite metal deck (galvanized) are planned. A portion of the lower level will experience tractor-trailer loads. Water stops will be provided in construction joints of the perimeter walls and the floor slab to safeguard against water intrusion.

The superstructure (the portion above Target Building II upper level) will be constructed as a mill or industrial building with stepped columns supporting a bridge crane. The roof will be constructed of deep 
trusses to provide a column-free space for crane operation and to provide access to the equipment in the lower level of the $40 \mathrm{M}$ instrument building. The steel columns will be encased in concrete fire protection to approximately $24 \mathrm{ft}$ above the lower level and gypsum board wrap to the bottom of the trusses. The concrete encasement will also provide protection from physical damage to the steel columns.

The roof trusses will also be used to create moment frame for resisting environmental events (wind and seismic). Concentric braced frames will be used in the long direction of the building to resist these loads.

A portion of the roof level (above the top chord of trusses) will be used to create a mechanical penthouse. A deck of normal-weight concrete on a composite metal slab is planned for the penthouse floor. Columns will be used to support a steel frame roof above the penthouse.

The $40 \mathrm{M}$ instrument building may share a common row of columns with the target building, and will support the exterior wall system with steel girt. The roofing system will be supported on 1.5 in. galvanized metal roof deck on steel purlins, except at the mechanical penthouse, where the roof deck will be supported on steel beams.

Platforms supporting galvanized steel grating are anticipated.

The instrument tunnels are expected to be buried concrete structures supported on friction piles. The floor, walls and roof will be reinforced normal-weight concrete. Water stops will be provided in construction joints to safeguard against water intrusion.

The remaining instrument buildings are expected to be constructed using methods similar to those used to build the $40 \mathrm{M}$ building, unless the building size is small enough to eliminate the roof trusses and use standard rolled steel beam shapes.

The instrument buildings and tunnels will meet PC-2 performance requirements in accordance with DOE standards. Owing to the adjacency of the $40 \mathrm{M}$ instrument building and Target Building II, the $40 \mathrm{M}$ building may need to meet the higher performance requirements of Target Building II. The extent to which these higher requirements are met will be based on a safety/hazard assessment that will occur in a subsequent phase of the project.

\section{Building Enclosure Systems}

With the exception of having a smaller cross section, the exterior design of the instrument tunnels will be comparable to that of the R2T2 tunnel. See Sect. 6.3.2.2.

\section{Life Safety Systems}

Currently, Target Building II, two contiguous instrument buildings, and the CLO II are defined as a single mixed-occupancy building consisting of business assembly and moderate-hazard factory industrial uses. The entire complex is envisioned as being fully protected Type IIA construction. The smaller instrument buildings will be classified as moderate-hazard factory industrial use and will be fully sprinkler-protected Type IIB construction. 


\section{Mechanical Systems}

\section{HVAC Ventilation}

Instrument buildings will be designed with a minimum of eight air changes per hour at a virtual ceiling height of $10 \mathrm{ft}$, or $1.2 \mathrm{CFM} / \mathrm{ft}^{2}$. Instrument areas have been assumed to have an average requirement of $20 \%$ outside air.

\section{Control System}

The instrument buildings and tunnels will be controlled by EPICS.

\section{M Instrument Building Central Air Handling System}

The $40 \mathrm{M}$ instrument and support areas of the building will be served by two CAV AHUs. The units will be manifolded together and will share a common discharge plenum to provide partial redundancy in the event of a complete unit failure. The AHUs will be designed as heating-cooling, single-duct type. Each unit will also contain a reheat coil for use in a dehumidification control cycle. The units will operate $24 \mathrm{~h}$ per day, 365 days per year. Supply fans will be based on a multiple array, plenum fan type design. Variable-frequency drives will provide the capability of setback control of the supply fan volume. The AHUs will be housed in the mechanical penthouse located above the instrument building. Each unit will be provided with air-side economizer control. Relief air from the experimental area will be routed through the mechanical penthouse to provide a tempered environment within this space. Excess air in the penthouse will be relieved through wall-mounted louvers and associated barometric relief dampers.

The system will be designed for energy conservation adjustment. The potential to reduce air changes per hour will be accommodated. Reductions in air changes per hour or unoccupied setback changes will be controlled by the BAS.

The units will consist of the following components in the direction of air flow:

- intake isolation damper

- MERV 8 (30\%) efficient prefilter bank

- MERV 14 (90\%) efficient filter bank

- heating water preheat coil

- chilled water cooling coil

- heating water reheat coil

- supply fans (4-6 fan array)

- isolation/smoke damper

- variable-frequency drives to modulate fan air flows

The system consists of two AHUs. If one of the units should fail or need to be taken out of service for maintenance, the remaining unit will be able to provide $66 \%$ of the supply system design capacity.

\section{M Instrument Building Central Air Handling System}

The $90 \mathrm{M}$ instrument and support areas of the building will be served by two CAV AHUs similar to those described for the $40 \mathrm{M}$ instrument area.

The AHUs will be housed in a grade-mounted mechanical room located adjacent to the instrument floor. Economizer relief air will be discharged through the mechanical room. 


\section{M Instrument Area Central Air Handling System}

The $30 \mathrm{M}$ instrument and support areas of the building will be served by two CAV AHUs similar to those described for the $40 \mathrm{M}$ instrument area. The AHUs will be housed in the mechanical penthouse located above the M1 instrument floor. Ductwork serving this space will be routed through the truss area of the target building and extend down the perimeter wall of the target building for entry into the instrument building. Return air and economizer relief air will be transferred through the target building for relief from the penthouse.

\section{High-Resolution Backscattering and High-Resolution Power Instrument Building Central Air Handling Systems}

The HRB and HRP instrument and support areas of these buildings will be served by two CAV AHUs similar to those described for the $40 \mathrm{M}$ instrument area. However, because of the small capacity of these units, it is anticipated that they can be mounted in the overhead of the experimental space, away from the sensitive instrumentation. Alternate locations could include the roof or a small-grade-mounted mechanical room.

\section{Instrument Building Exhaust Systems}

All instrument area buildings will be provided with a general exhaust system. The general exhaust system for the $40 \mathrm{M}$ and $30 \mathrm{M}$ instrument buildings will be located on the roof of the instrument area mechanical penthouse. The general exhaust system for the $90 \mathrm{M}$ instrument buildings will be located on the roof of the associated mechanical equipment room. The general exhaust for the HRB and HRP instrument buildings will be located directly on the roof of the instrument building. Each exhaust system will contain duplex vertical, radial dilution exhaust fans of spark-proof construction with bearings and motors out of the airstream (one fan will be a stand-by). Fans will have an air-side baked epoxy chemical-resistant coating. The common fan plenum and outside air intake dampers will be of galvanized steel construction with a chemical resistant coating. Each central exhaust system will consist of the following components:

- common exhaust plenum

- isolation damper at each fan inlet

- two vertical exhaust fans

- exhaust stacks

- bypass air inlet and bypass damper

- variable-frequency drives

Two fans will be provided to serve the common exhaust plenum, each sized at $100 \%$ of the load. If one of the fans fails, the remaining fan will be able to provide $100 \%$ of the common exhaust system's design capacity. All of the fans in the common exhaust plenum will be on emergency power to ensure continuous hazardous material containment, but they will be controlled to run at a reduced speed during a power outage to limit the negative pressure impact to the building.

All instrument buildings will also be provided with limited exhaust from the SCE and HOG systems. Ductwork will be stubbed into these spaces through extensions run down the neutron guide tunnels from Target Building II or will be direct connections from the site distribution into the $90 \mathrm{M}, \mathrm{HRB}$, and HRP instrument buildings. 


\section{Neutron Guide Tunnels}

The neutron guide tunnels will be cooled either through the use of fan coil cooling units located in the instrument buildings adjacent to the end of the tunnels or by using supply air from the associated instrument building AHU. The choice of the system will likely depend on the necessary supply air static pressure and the impact on fan energy usage. The fan coil unit or AHU will supply conditioned air into a duct that will extend down the length of the tunnel. Supply air registers along the length of the duct will distribute conditioned air throughout the tunnel. The air will then return through the tunnel into the instrument building and finally to either the fan coil or the AHU.

\section{Chilled Water System}

The chilled water system for the buildings will be supplied from CUB II. The estimated flows and chilled water branch line size for each building is shown in Table 6.8 .

Table 6.8. Estimated instrument buildings chilled water loads

\begin{tabular}{lcl}
\hline Instrument building number & $\begin{array}{c}\text { Estimated chilled } \\
\text { water flow } \\
\text { (GPM) }\end{array}$ & \multicolumn{1}{c}{ Estimated pipe size } \\
\hline $40 \mathrm{M}$ & 350 & Combined with Target Building \\
$90 \mathrm{M}$ & 190 & 4 in. \\
$30 \mathrm{M}$ & 115 & Combined with Target Building \\
$\mathrm{HRB}$ & 28 & 2 in. \\
HRP & 38 & 2 in. \\
\hline
\end{tabular}

Chilled water will be routed to cooling coils in all AHUs. Chilled water to the $40 \mathrm{M}$ and $30 \mathrm{M}$ instrument buildings will be supplied from chilled water piping extended into the target building. Chilled water will be supplied to the remote $90 \mathrm{M}$ HRB and HRP instrument buildings by a direct underground pipe connection to each building from the site distribution system.

\section{Sensible Chilled Water System}

A sensible chilled water cooling system will be required to support equipment cooling in each remote instrument building ( $90 \mathrm{M}, \mathrm{HRB}$, and HRP). The sensible chilled water cooling system will consist of an independent pumped loop that will reject heat into the building chilled water through a plate frame heat exchanger. The system will be designed to operate at a supply temperature of $55^{\circ} \mathrm{F}$ to reduce the potential for condensation. The tank, pumps, heat exchanger, filters, and chemical treatment components will be located in a mechanical support area of each instrument building. Sensible chilled water for the $40 \mathrm{M}$ and $30 \mathrm{M}$ instrument buildings will be served from a system in the target building. The sensible chilled water piping system will use copper pipe for pipe sizes up to $3 \mathrm{in}$. and grooved coupling or welded carbon steel piping for sizes above 3 in. Pipe insulation will not be provided given the operating water temperature. The system will include the following equipment:

- base-mounted, end-suction, centrifugal pumps

- pump variable-frequency drives

- chilled water plate frame heat exchanger

- water filtration

- automated chemical treatment system 
- makeup water assembly

- $\quad$ appropriate valving and piping specialties

Two variable-speed process cooling water pumps will be sized to provide $100 \%$ of the design flow rate each. If one of the booster pumps fails, the other will provide $100 \%$ redundancy.

\section{Heating Water System}

The heating water system for the building will be supplied from CUB II. The estimated flow to each building and the associated estimated heating water branch line size is shown in Table 6.9.

Table 6.9. Estimated instrument buildings heating water loads

\begin{tabular}{lcl}
\hline \multicolumn{1}{c}{ Instrument building number } & $\begin{array}{l}\text { Estimated heating } \\
\text { water flow (GPM) }\end{array}$ & \multicolumn{1}{c}{ Estimated pipe size } \\
\hline $40 \mathrm{M}$ & 65 & $\begin{array}{l}\text { Combined with Target } \\
\text { Building } \\
\end{array}$ \\
$90 \mathrm{M}$ & 34 & 2 in. \\
$30 \mathrm{M}$ & 20 & $\begin{array}{l}\text { Combined with Target } \\
\text { Building }\end{array}$ \\
$\mathrm{HRB}$ & & 1 in. \\
HRP & 6 & 1 in. \\
\hline
\end{tabular}

The heating water distribution system will serve heating water coils in all AHUs. Heating water to the $40 \mathrm{M}$ and $30 \mathrm{M}$ instrument buildings will be supplied from heating water piping extended into Target Building II. Supply to the remote 90 M, HRB, and HRP instrument buildings will be through direct underground pipe connections to each building from the site distribution system.

\section{Equipment Schedules}

The instrument building mechanical system requirements are summarized in Table 6.10.

Table 6.10. Instrument building and tunnels equipment schedules

\begin{tabular}{|c|c|c|c|c|c|}
\hline Equipment & Manufacturer & $\begin{array}{l}\text { Size } \\
\text { (each) }\end{array}$ & Quantity & $\begin{array}{l}\text { No. on } \\
\text { emergency } \\
\text { power }\end{array}$ & Remarks \\
\hline $\begin{array}{l}\text { AHU_ } 40 \mathrm{M} \\
\text { instrument building }\end{array}$ & Trane & $35,000 \mathrm{CFM}$ & 2 & $\mathbf{0}$ & $\begin{array}{l}40 \mathrm{hp} \text { - total of all } \\
\text { motors }\end{array}$ \\
\hline $\begin{array}{l}\text { AHU—90 M } \\
\text { instrument building }\end{array}$ & Trane & 20,000 CFM & 2 & 0 & $\begin{array}{l}30 \mathrm{hp} \text { - total of all } \\
\text { motors }\end{array}$ \\
\hline $\begin{array}{l}\text { AHU_-30 M } \\
\text { instrument building }\end{array}$ & Trane & $12,000 \mathrm{CFM}$ & 2 & 0 & $\begin{array}{l}15 \mathrm{hp} \text { - total of all } \\
\text { motors }\end{array}$ \\
\hline $\mathrm{AHU}-(\mathrm{HRB})$ & Trane & 2,800 CFM & 2 & 0 & 5 hp motor each \\
\hline $\mathrm{AHU}$ - (HRP) & Trane & $4,000 \mathrm{CFM}$ & 2 & 0 & $7.5 \mathrm{hp}$ motor each \\
\hline $\begin{array}{l}\text { General exhaust fan } \\
\text { systems - all } \\
\text { instrument buildings }\end{array}$ & MK Plastics & $3,000 \mathrm{CFM}$ & $\begin{array}{c}5 \text { systems } \\
\text { with } 2 \text { fans } \\
\text { each. }\end{array}$ & 5 & $\begin{array}{l}1 \text { system fan is back- } \\
\text { up. } \\
3 \mathrm{hp} \text { motor each. }\end{array}$ \\
\hline
\end{tabular}


Table 6.10. Instrument building and tunnels equipment schedules (continued)

\begin{tabular}{|c|c|c|c|c|c|}
\hline Equipment & Manufacturer & $\begin{array}{c}\text { Size } \\
\text { (each) }\end{array}$ & Quantity & $\begin{array}{c}\text { No.on } \\
\text { emergency } \\
\text { power }\end{array}$ & Remarks \\
\hline Utility exhaust fans & Cook & Varies & $\begin{array}{c}\text { Estimated at } \\
10\end{array}$ & 0 & $\begin{array}{l}\text { Roof mounted; some } \\
\text { with } 1 \text { phase, and } \\
\text { some with } 3 \text { phase } \\
\text { power }\end{array}$ \\
\hline $\begin{array}{l}\text { Sensible chilled water } \\
\text { distribution pumps }\end{array}$ & Goulds & $\begin{array}{l}\text { 25-100 GPM@ } \\
100 \mathrm{ft} \text { TDH }\end{array}$ & 6 & 0 & $\begin{array}{l}\text { Located in the } \\
\text { mechanical area of } \\
\text { the remote } 90 \mathrm{M} \\
\text { instrument building, } \\
\text { HRB, and HRP } \\
\text { instrument buildings. } \\
1 \text { standby pump, } \\
\text { up to } 3 \text { hp motor for } \\
\text { each system }\end{array}$ \\
\hline
\end{tabular}

\section{Fire Protection Systems}

Fire sprinkler systems for the instrument buildings will be hydraulically designed to provide water densities that meet the requirements for Extra Hazard, Group 1, protection throughout the facilities. Fire sprinkler systems for the tunnels will be hydraulically designed to provide water densities that meet the requirements for Ordinary Hazard, Group 2, protection throughout the tunnels.

\section{Electrical Systems}

\section{Exterior Power Distribution}

The $40 \mathrm{M}$ instrument bay, future $30 \mathrm{M}$ instrument bay, and the STS buildings will be considered one building with one electrical power supply serving all three buildings. The one $480 \mathrm{Y} / 277 \mathrm{~V}$ electrical supply to the complex will be provided by connecting to a 3000 A circuit breaker in the outdoor unit substation located on the south side of the $40 \mathrm{M}$ instrument bay. A $3000 \mathrm{~A}$ feeder bus duct will be routed overhead from the substation to the switchgear located in the electrical room of the $40 \mathrm{M}$ instrument bay. Separate feeders will be routed from this switchgear to the technical and CF distribution panels within the $30 \mathrm{M}$ instrument bay via Target Building II.

The $480 \mathrm{Y} / 277 \mathrm{~V}$ electrical supply to the $90 \mathrm{M}$ instrument bay will be provided by connecting to a 2,400 A circuit breaker in the outdoor unit substation located on the north side of the $90 \mathrm{M}$ instrument bay. A 2400 A feeder bus duct will be routed overhead from the substation to the switchgear located in the electrical room.

The $480 \mathrm{Y} / 277 \mathrm{~V}$ electrical supply to the HRB building will be provided by connecting to a $400 \mathrm{~A}$ circuit breaker in the substation located on the north side of the $90 \mathrm{M}$ instrument bay. A 400 A duct bank will be routed from the substation to a distribution panel located in the HRB.

The $480 \mathrm{Y} / 277 \mathrm{~V}$ electrical supply to the HRP building will be provided by connecting to a 400 A circuit breaker also in the substation located on the north side of the $90 \mathrm{M}$ instrument building. A $400 \mathrm{~A}$ duct bank will be routed from the substation to a distribution panel located in the HRP. 


\section{Interior Power Distribution}

In the instrument bays, power will be distributed to technical and CF branch circuit panels at the mezzanine level via cable tray routed around the perimeter and supported off the walls of the high bay spaces. Where beam line instruments are installed with the initial construction, a $480 \mathrm{Y} / 277 \mathrm{~V}$ technical branch circuit panel and two $208 \mathrm{Y} / 120 \mathrm{~V}$ technical branch circuit panels via separate step down transformers will be provided at the mezzanine level above each beam line. At beam lines for future instruments, a pull box and homerun conduit to the technical distribution panel will be provided to facilitate installation of future branch circuit panels.

Within the HRB and HRP buildings, the 400 A distribution panel will serve lighting panel, a receptacle panel via a step down transformer, a mechanical equipment panel, a $480 \mathrm{Y} / 277 \mathrm{~V}$ technical panel, and two $208 \mathrm{Y} / 120 \mathrm{~V}$ technical panels via separate step down transformers.

\section{Emergency Power Distribution}

Emergency power to the STS, the $40 \mathrm{M}$ instrument bay, and the future $30 \mathrm{M}$ instrument bay will be provided by connecting to the generator located on the east side of the R2T2 support building. A duct bank will be routed from the generator to a generator power distribution panel board in the emergency power electrical room on the north side of the $40 \mathrm{M}$ instrument bay. Generator power will be distributed to an emergency automatic transfer switch and to one or more optional standby automatic transfer switches, which will in turn serve emergency and optional standby panels in the $40 \mathrm{M}$ instrument bay.

Emergency power to the $90 \mathrm{M}$ instrument bay, and HRB and HRP instrument buildings will be provided by connecting to the generator located on the north side of the $90 \mathrm{M}$ instrument bay. Separate duct banks will be routed from the generator to a panel board in the $90 \mathrm{M}$ instrument bay electrical room and to a disconnect switch in the HRB and HRP instrument buildings.

\section{Fire Alarm System}

The first alarm system for the instrument buildings will include either VESDA or area heat and smoke detection as part of the means to provide redundant fire protection for these facilities.

\subsubsection{Zeemans Building}

\section{Programming}

The Zeemans neutron instrument will consist of a neutron instrument beam line that uses a hybrid superconducting/ambient-temperature high-field magnet (about 35 tesla) and a utility building to provide the substantial utilities required to operate the magnet. The instrument building will contain the high-field magnet, the components to bring neutrons to the sample position inside the magnet, and the neutron detectors and data collection array to allow experiments with the high magnetic fields.

The utilities building will provide electrical equipment to convert incoming ac power to about $12 \mathrm{MW}$ of high quality de power needed for the magnet. The magnet will be connected in series so the current flows through the superconducting and ambient temperature magnet coils. To cool the ambient-temperature portion of the magnet, approximately 1600 GPM of DI chilled water will be required at about 450 psi to overcome the large pressure drop across passages in the magnet coils. The DI water system will provide cooling for the magnet and power supplies and the conductors to the magnet. A separate lower-pressure set of chillers and cooling towers flowing at about 1600 GPM with heat exchangers will cool the highpressure DI water loop. A large insulated chilled water tank will be required to maintain relatively 
constant chilled water system temperature during changes in magnet operating parameters. A cryogenic helium system will provide cooling for the superconducting portion of the magnet. The heat from the cryogenic system will also be rejected to the cooling towers. A summary level program for the Zeemans instrument and support buildings is shown in Table 6.11 .

Table 6.11. Zeemans building and support buildings program summary

\begin{tabular}{lc}
\hline \multicolumn{1}{c}{ Space type } & Area (NSF) \\
\hline Chiller system & 4,500 \\
Compressor & 1,500 \\
Water treatment & 1,800 \\
Transformer I & 1,200 \\
DC power rectifier & 1,500 \\
Helium refrigeration i & 1,500 \\
Controls & 2,400 \\
Transformer II & 600 \\
Switchgear & 600 \\
Compensation & 600 \\
Low voltage electrical & 600 \\
Helium refrigeration ii & 1,500 \\
Instrument building & 2,500 \\
Instrument building support & 500 \\
External insulated chilled water tank & 0 \\
Total net area & $\mathbf{2 1 , 3 0 0}$ \\
Gross/net factor (70\% efficiency) & 1.4 \\
Total gross area & $\mathbf{2 9 , 8 2 0} \quad$ GSF \\
\hline
\end{tabular}

The space requirements for the Zeemans support building were derived from a similar, smaller facility located at the Hemholtz Centre in Berlin. Based on the known power requirements of the German facility and the anticipated power requirements of the Zeemans facility, a judgment was made to increase the supporting utility area requirements of the German facility by $20 \%$ to estimate the area required for the Zeemans facility. These assumptions will require further analysis and verification during subsequent STS design phases.

The space requirements for the Zeemans instrument building were established based on experience installing and operating the FTS instruments and will include additional space for ancillary office, laboratory, or shop space to support the instrument. The grossing factor of 1.4 includes space for general building circulation, toilets, exterior wall, mechanical, electrical, and plumbing systems spaces that are not included in the net program areas.

The Zeemans instrument building will be remotely located, approximately $80 \mathrm{ft}$ from Target Building II. It will include a high-bay space for one instrument and be served by a 30 ton overhead bridge crane that will have a minimum hook height of $32 \mathrm{ft}$ above the instrument floor. It will have two floor levels: (1) instrument floor and (2) instrument floor mezzanine. The second floor level will be relatively small in area, essentially serving to provide access within the building. 
The instrument floor will not be directly accessible by tractor-trailer. The building will require truck access from the FTS/STS courtyard at the mezzanine level because of the site topography. Pedestrian access to the building will also be at the mezzanine level from the exterior. The Zeemans building will not have an interior pedestrian connection to other buildings in the SNS complex.

To conserve space on the site, the Zeemans support building will be organized on three floors and will be closely integrated with the instrument building. All three floors will house utility systems. The lower floor will align with the instrument floor. The intermediate floor will be accessible by truck and pedestrians at the FTS/STS courtyard level. The upper floor will be accessible from the northern portion of Spallation Drive. The adjoined Zeemans instrument and support buildings will be served by a freight elevator that will provide access to all three floor levels.

Currently, the R2T2 support building is shown adjoined to the Zeemans building. These two structures will be functionally independent although they may be integrated from the standpoint of life safety and building code requirements.

During subsequent STS design phases, it is anticipated that the following alternatives related to the Zeemans buildings, which may affect project scope or the design approach, will be further evaluated:

- $\quad$ space requirements necessary to house support utility systems

- the consequences of separating the Zeemans support building, the Zeemans instrument building, and the R2T2 support building, or, alternatively, of combining all three buildings into a single structure

\section{Structural Systems}

The Zeemans support building is anticipated to be a conventional steel-framed building supported on friction piles. The below-grade elements (e.g., basement walls, retaining walls) will be constructed of reinforced normal-weight concrete. Water stops will be provided in construction joints to safeguard against water intrusion.

The Zeemans support building will share a common wall with the R2T2 support building unless it is determined in a subsequent phase of the project that isolation is required because of the science being performed in the Zeemans building. The exterior wall system will be supported with steel girts. The roofing system will be supported on 1.5 in. galvanized metal roof deck on steel beams.

Elevated floors are expected to be constructed of reinforced normal-weight concrete on a composite metal deck (galvanized). A portion of the floors will experience tractor-trailer loads, and these areas will be constructed using a bar-reinforced normal-weight concrete on metal deck (galvanized). Platforms supporting galvanized steel grating are anticipated.

The slab on grade will be a minimum of 8 in. thick reinforced normal-weight concrete slab.

A portion of the slab will be reinforced with non-magnetic stainless steel (A304LN) to limit the effects on a planned magnet in the Zeemans building.

The Zeemans support building will meet PC-2 performance requirements in accordance with DOE standards.

The R2T2 support building will likely be built before the adjacent Zeemans building. This construction phasing/sequence will need to be considered during the design of the Zeemans building. 


\section{Building Enclosure Systems}

The exterior design for the Zeemans building will conform to the requirements defined for the overall STS project. Below-grade walls of the Zeemans building will be constructed of waterproofed reinforced concrete. The exterior walls will be approximately $80 \%$ architectural-grade metal insulated panels and $20 \%$ low-E, insulated glass in a painted metal integrated strip window system. The building will include two pedestrian entrances with overhead canopy protection and one grade-level service entrance with overhead door clearance to allow tractor-trailer access up to the building. The building will include an exterior water tank, approximately $10 \mathrm{ft}$ in diameter and $40 \mathrm{ft}$ in height.

\section{Life Safety Systems}

The Zeemans building will be classified as moderate hazard factory industrial occupancy with incidental business use and will be fully sprinkler-protected Type IIB construction. Each of the three floor levels will have two means of egress, either through fully enclosed, 1 h-rated egress stairs or directly to the exterior grade.

\section{Mechanical Systems}

Mechanical systems required by the building including chilled water, condenser water, tower water, AHU supply and return air, and any exhaust systems will be contained within the building. Heating water and compressed air will be supplied from CUB II; however CUB II does not have capacity to provide other mechanical utilities. The building mechanical design will need to address the need for oxygen depletion hazard monitoring/alarm and purge exhaust to address a potential cryogenic release in either the support building or the instrument building.

\section{Fire Protection Systems}

The fire sprinkler systems for the Zeemans buildings will be hydraulically designed to provide water densities that meet the requirements for Extra Hazard, Group 1, protection for the instrument building and Ordinary Hazard, Group 2, protection throughout the support building.

\section{Electrical Systems}

\section{Exterior Power Distribution}

Four power supplies will be provided to the Zeemans facility, one at $13.8 \mathrm{kV}$, two at $4160 \mathrm{~V}$, and one at $480 \mathrm{Y} / 277 \mathrm{~V}$. The $13.8 \mathrm{kV}$ supply will be provided by a $600 \mathrm{~A}$ circuit breaker in the $15 \mathrm{kV}$ switchgear SWGR-C located on the east side of the Zeemans building. A 600 A duct bank will be routed from the switchgear to the magnet power supply disconnect. The two $4160 \mathrm{~V}$ supplies will be provided by the $4160 \mathrm{~V}$ outdoor unit substation located on the east side of the Zeemans building. A duct bank will be routed from the switchgear to each chiller disconnect. The $480 \mathrm{Y} / 277 \mathrm{~V}$ supply will be provided by connecting to a 3000 A circuit breaker in the $480 \mathrm{~V}$ outdoor unit substation located on the east side of the Zeemans building. A $3000 \mathrm{~A}$ feeder bus duct will be routed overhead from the substation to the switchgear located in the electrical room.

\section{Interior Power Distribution}

From the $3000 \mathrm{~A}, 480 \mathrm{Y} / 277 \mathrm{~V}$ switchgear, power will be distributed to the CF loads (including lights and receptacles, mechanical equipment, and an elevator) and to technical loads. Distribution panels will be located in the electrical room and will be dedicated to the types of loads they serve: CF or technical 
loads. These distribution panels will subfeed $480 \mathrm{Y} / 277 \mathrm{~V}$ branch circuit panels and $208 \mathrm{Y} / 120 \mathrm{~V}$ branch circuit panels via step-down transformers. Separate transformers and panels will be provided to serve sensitive electronic loads.

\section{Emergency Power Distribution}

Emergency power to the Zeemans building will be provided by connecting to a generator dedicated to the Zeemans facility; it will be located on the east side of the Zeemans building. Duct banks will be routed from the generator to a generator power distribution panel board in the electrical room. Generator power will be distributed to an emergency automatic transfer switch and to an optional standby automatic transfer switch to support the helium liquefaction system.

\section{Fire Alarm System}

The first alarm system for the Zeemans instrument building will include either VESDA or area heat and smoke detection as part of the means to provide redundant fire protection for this facility.

\subsubsection{Central Laboratory and Office Building II}

\section{Programming}

CLO II will house office space for STS staff and users, office support and conference spaces, laboratories and shop facilities for the entire STS facility. Table. 6.12 shows a summary-level program for the CLO II.

The CLO II office and office support spaces are sized to support an estimated population of 200 staff and users associated with the STS facility. The total net office area is based on the ORNL office planning standard of $20 \%$ private offices and $80 \%$ open workstations. The proposed laboratory and shop spaces are comparable in size and use to similar space supporting FTS. The programmatic requirements for the CLO II will require verification during subsequent design phases.

The grossing factor of 1.8 is typical for laboratory/office buildings and includes space for general building circulation; toilets; utility shafts; and exterior wall, mechanical, electrical, plumbing and other spaces not included in the net program areas. The CLO II program areas will be closely integrated with the CUB and utility support spaces for Target Building II that do not fit within the Target Building II footprint.

The CLO II is envisioned as a four-story building with an additional partial-floor penthouse level and partial basement. The CLO II will be centrally positioned among CLO, FTS, STS and CNMS. The linear building will have two primary entrance points. Pedestrian access for staff, users, and visitors who park in the existing parking areas will be through the existing CLO and across the existing bridge. There will be a second entrance at the east end of CLO II that will be used by staff and users who park in the new east parking lot. The CLO II will be linked by new elevated pedestrian bridges to FTS and the $40 \mathrm{M}$ and $90 \mathrm{M}$ instrument buildings. Shipping and receiving will be accommodated at the lower level of the CLO II from Spallation Drive. The CLO II is planned to allow emergency response vehicular access into the courtyard between FTS and STS and also to allow tractor-trailer access onto the $40 \mathrm{M}$ instrument building floor and Target Building II main bay floor. 
Table 6.12. Central Laboratory and Office Building II program summary

\begin{tabular}{|c|c|}
\hline Space type & Area (NSF) \\
\hline Offices (200 staff) & 18,200 \\
\hline Office support & 7,800 \\
\hline General laboratories (40 modules) ${ }^{a}$ & 11,000 \\
\hline General maintenance and support shops ${ }^{b}$ & 8,000 \\
\hline Neutron chopper lab ${ }^{c}$ & 600 \\
\hline Neutron chopper storage $^{c}$ & 600 \\
\hline Data acquisition and storage (DAS) lab & 1,600 \\
\hline DAS storage & 1,200 \\
\hline Detector fiber lab & 1,600 \\
\hline Detector fiber lab storage & 1,600 \\
\hline Detector $3 \mathrm{He}$ lab & 1,600 \\
\hline Detector $3 \mathrm{He}$ storage & 1,600 \\
\hline Electrical lab & 500 \\
\hline Electrical storage & 500 \\
\hline Maintenance shop/lab & 800 \\
\hline Maintenance storage & 1,200 \\
\hline Sample environment maintenance & 1,000 \\
\hline Sample receipt and storage lab & 900 \\
\hline New instrument staging and prep lab & 1,000 \\
\hline New instrument staging and prep storage & 200 \\
\hline Shipping/receiving & 800 \\
\hline General building storage & 1,000 \\
\hline Lobby (2) & 800 \\
\hline Bridges & 1,600 \\
\hline Total net area & 67,900 \\
\hline Gross/net factor (55\% efficiency) & 1.8 \\
\hline Total gross area & 122,220 \\
\hline
\end{tabular}

${ }^{a} 11 \mathrm{ft} \times 25 \mathrm{ft}$ modules

${ }^{b}$ Estimated area to be confirmed

${ }^{c}$ Chopper space in Target Building II and CLO II equals 2000 NSF total 
During subsequent STS design phases, it is anticipated that the following alternatives related to the CLO II Building, which may affect project scope or the design approach, will be further evaluated:

- The consequences of connecting the CLO II to the CNMS by bridge

- Relative to the Life Safety Code and IBC, the consequences of defining the CLO II as a single building or integrating it with Target Building II, CUB II, and the $40 \mathrm{M}$ and $30 \mathrm{M}$ instrument buildings

\section{Structural Systems}

The CLO II will be a conventional steel-framed laboratory/office building supported on spread footings. Concentric braced frames will be used for resisting environmental events (wind and seismic). The CLO II will be separated from the CUB II by an isolation joint to limit the transmission of vibration from equipment in CUB II to CLO II.

The below-grade elements (e.g., basement walls, retaining walls) will be constructed of reinforced normal-weight concrete. Water stops will be provided in construction joints to safeguard against water intrusion.

The CLO II will support the exterior wall system with steel girts. The roofing system will be supported on a 1.5 in. galvanized metal roof deck on steel beams.

Elevated floors are expected to be constructed of reinforced normal-weight concrete on a composite metal deck (galvanized) and will be designed to control footfall-induced vibrations to a vibration velocity below 2,000 $\mu \mathrm{in} . / \mathrm{s}$ (VC-A). Platforms supporting galvanized steel grating are anticipated.

The slab on grade will be a minimum of 8 in. thick reinforced normal-weight concrete slab.

The CLO II will meet PC-1 performance requirements in accordance with DOE standards.

\section{Building Enclosure Systems}

The CLO II will be designed to be architecturally compatible with the existing CLO and CNMS buildings. The exterior enclosure systems will be comparable in quality, material, color, and texture to the systems currently employed. The exterior wall will be approximately $50 \%$ architectural-grade metal insulated panels and 50\% low-E, insulated glass, painted metal curtain wall. Approximately $50 \%$ of the curtain wall area will include either fritted glass or external painted metal shading elements. Exterior doors will be compatible with the curtain wall system.

Five percent of the roof area will consist of an insulated, laminated, fritted glass skylight system comparable to the curtain wall system. The bridges linking the CLO II to adjacent buildings will be comparable to the existing bridge connecting the CLO with the FTS Target Building.

\section{Life Safety Systems}

Currently, CLO II, along with Target Building II and two contiguous instrument buildings, is being defined as a single mixed-occupancy building comprising business, incidental assembly, and moderatehazard factory industrial uses. The entire complex will be fully protected, Type IIA, construction. 
Laboratory space in CLO II will typically be separated from office space with rated construction as determined to be necessary via detailed fire hazard and code analysis that will occur in subsequent design phases. One-hour separations will be constructed between laboratories to maximize the number of allowable chemical control zones at each floor level within the CLO II. All vertical shafts within the CLO II will be $2 \mathrm{~h}$ rated construction.

The definition of the CLO II as part of a larger single building, including the target building and contiguous instrument buildings, for purposes of the life safety code will be further evaluated in subsequent STS design phases.

The CLO II will include two fully enclosed egress stairs. One is currently planned to be six stories in height, providing egress from all above-grade floor levels and the penthouse level. The second stair will be seven stories and will provide access to the basement and the penthouse roof. At the west end of the CLO II, adjacent to the bridge link to the FTS, an open monumental stair will connect the third and fourth floors of the CLO II through a double height space. A similar stair and double height space will connect the second and third floors of the CLO II at the east end of the building.

\section{Mechanical Systems}

\section{HVAC Ventilation}

Wet laboratory, dry/instrumentation laboratory, and laboratory support areas in CLO II will be designed for both occupied and unoccupied modes.

- Occupied: Minimum six to eight air changes per hour at a virtual ceiling height of $10 \mathrm{ft}$, or 1.0 to $1.2 \mathrm{CFM} / \mathrm{ft}^{2}$. Wet laboratories are assumed to be $100 \%$ outside air, whereas sample and instrument laboratories have been assumed to have an average requirement of $50 \%$ outside air.

- Unoccupied space will have a minimum of three air changes per hour depending on the temperature stability required.

In maintenance and support shop areas, occupied spaces will have a minimum six to eight air changes per hour at a virtual ceiling height of $10 \mathrm{ft}$, or 1.0 to $1.2 \mathrm{CFM} / \mathrm{ft}^{2}$.

Maintenance and support shop areas have been assumed to have an average requirement of $30 \%$ outside air with an unoccupied setting of a minimum of three air changes per hour, depending on the temperature stability required.

Office, conference, amenity, administrative support, and lobby areas are assumed to have the following requirements:

- Occupied: Minimum of four air changes per hour at a ceiling height of $10 \mathrm{ft}$, or $0.66 \mathrm{CFM} / \mathrm{ft}^{2}$

- Toilets: Code minimum of ten air changes per hour during hours of occupancy

\section{Fume Hood Performance Criteria}

The laboratory hoods will be VAV type for energy reduction and improved safety. The associated exhaust system will be sized with the capability to produce an average $100 \mathrm{ft} / \mathrm{min}$ face velocity (measured over the entire face) at an $18 \mathrm{in}$. sash height at the chemical fume hoods. These criteria correspond to the following exhaust rates at the listed fume hood types: 
- Six ft chemical fume hood (18 in. sash height) - 784 CFM.

- Eight ft chemical fume hood (18 in. sash height) - 1084 CFM.

The VAV hoods will be designed to provide at least $25 \mathrm{CFM} / \mathrm{ft}^{2}$ of working surface to ensure sufficient air dilution is maintained within the hoods.

\section{Other Laboratory Exhaust Devices}

Other exhaust devices in the building include

- canopy hoods: $100 \mathrm{CFM} / \mathrm{ft}^{2}$ depending upon application

- downdraft surfaces: $100 \mathrm{CFM} / \mathrm{ft}^{2}$ downdraft velocity at table surface for $100 \%$ smoke capture 12 in. above the table

- $\quad$ snorkel (6 in. \pm ) $150 \mathrm{CFM}$

- $\quad$ equipment vent (4 in. \pm ) $50 \mathrm{CFM}$

\section{Biosafety Cabinets}

Biosafety cabinet classifications are as follows:

- Class II, Type B2, biological safety cabinets (100\% exhaust) will require dedicated exhaust systems to accommodate a high static pressure of 1.4-2.0 in. WC for a $6 \mathrm{ft}$ unit or 1.0-1.4 in. WC for a $4 \mathrm{ft}$ unit. It is assumed there will be two $6 \mathrm{ft}$ II/B2 cabinets in the building.

- Class II, Type A2, biological safety cabinets (30\% exhaust) will require a canopy connected to the exhaust system. It is assumed there will be two $6 \mathrm{ft} \mathrm{II/A2} \mathrm{cabinets} \mathrm{in} \mathrm{the} \mathrm{building.}$

\section{Noise Criteria}

Average noise levels in the laboratory areas of CLO II are based on measurements taken $3 \mathrm{ft}$ in front of a $6 \mathrm{ft}$ fume hood. These noise levels do not include noise from equipment or personnel located within these spaces. Actual noise levels may exceed the design levels because of the actual type of equipment purchased, installation compromises, workmanship, and so on. The design will target the following average noise levels:

Laboratory areas with fume hoods

Laboratory areas without fume hoods

Laboratory support areas

Open offices

Private offices

Conference rooms

Circulation space, lobby

$$
\begin{aligned}
& \mathrm{NC}=50-55 \\
& \mathrm{NC}=45-50 \\
& \mathrm{NC}=50-55 \\
& \mathrm{NC}=40-45 \\
& \mathrm{NC}=45-50 \\
& \mathrm{NC}=45-50 \\
& \mathrm{NC}=40
\end{aligned}
$$

\section{Pressure Relationships}

Typical pressure relationships will be comparable to those maintained in similar facilities. Given the proposed use of supply and exhaust air valves in each pressure zone, it will be possible to modify pressure relationships between positive and negative. Pressure relationships will be maintained by offsets between supply and exhaust/return air flow rates. 
Building

Wet laboratories and shops

Corridors

Offices

Toilet rooms
Positive to ambient

Negative to adjacent spaces

Positive to laboratories and shops

Neutral or positive to adjacent spaces

Negative to adjacent spaces

\section{Control System}

CLO II will have a direct digital control (DDC) system designed around an open-protocol BACnet communication network. The basis of design is expected to be a JCI Metasys system. EPICS will control the mechanical systems serving the target environment and target support portions of the building.

\section{Outdoor Air Quality Control Methods}

Outdoor air intakes will be located to minimize cross-contamination between intakes and exhaust air streams. A wind tunnel computer simulation should be performed to define intake locations and determine minimum exhaust stack heights. Fume exhaust stacks will be a minimum of $10 \mathrm{ft}$ above the roof. Fume exhaust fans will be designed to be capable of exhausting a constant volume of air at a stack discharge velocity of approximately $3500 \mathrm{ft} / \mathrm{min}$. If wind analysis shows a lower stack velocity is acceptable, then provisions will be made within the control system to allow a lower stack velocity.

\section{Wet Laboratory and Shop Area Central Air Handling System}

Wet laboratory and shop support portions of CLO II will be served by three VAV AHUs. The units will be manifolded together and will share a common discharge plenum to provide partial redundancy in the event of a complete unit failure. The AHUs will be designed as heating-cooling, single-duct, reheat type. The units will operate $24 \mathrm{~h}$ per day, 365 days per year. Supply fans will be based on a multiple array, plenum fan type design. Variable-frequency drives will provide supply fan volume control in response to a signal from duct mounted static pressure sensors. The system will be designed to maintain minimum outside air quantities to meet exhaust air requirements, but will also include an air-side economizer control scheme.

The HVAC distribution system will be pressure-independent and VAV with a minimum and maximum number of air changes per hour established for laboratory spaces.

The system will be designed for energy conservation and pressurization adjustment. The potential to reduce air changes per hour, go to an unoccupied setback in a particular room (or rooms), or change pressurization will be accommodated. Reductions in air change per hour or changes in unoccupied setback or pressurization will be controlled by the BAS.

The potential to employ an energy recovery system will be evaluated by the design team. The energy recovery system probably would include either heat pipe energy recovery coil or a piped coil run-around loop extending between the exhaust air stream and the incoming outside air stream. Each energy recovery section would be provided with an upstream filter and by-pass damper to minimize fan static pressure when not in use during intermediate temperature periods.

All controls for this system will be DDC. Control to maintain pressure gradients will be accomplished by an active volumetric offset (tracking) control concept. 
All terminal boxes and reheat coil valves will be serviced from the laboratory space. All terminal units will be positioned for easy access with a minimum of $36 \mathrm{in}$. of service space on the panel/actuator side. All terminal reheat control valves serving the laboratory spaces will fail closed.

The supply distribution system will consist of low-pressure ductwork with pressure-independent, electrically actuated supply, VAV air terminal devices; reheat coils; low-pressure externally insulated ductwork downstream of air terminals; and diffusers. Sound attenuators at the air terminal devices will not be provided. The use of sound attenuating flexible duct at diffusers will be limited to $6 \mathrm{ft}$ in total length to minimize duct static pressure losses.

The units will consist of the following components in the direction of air flow:

- intake isolation damper

- MERV 8 (30\%) efficient prefilter bank

- MERV 14 (90\%) efficient filter bank

- heating water preheat coil

- chilled water cooling coil

- supply fans (4-6 fan array)

- isolation/smoke damper

- variable-frequency drives to modulate fan air flows

The wet laboratory and shop area air handling system consists of three AHUs. If one of the units should fail or have to be taken out of service for maintenance, the remaining two units would be able to provide $66 \%$ of the maximum supply system design capacity.

\section{Target Support Area Central Air Handling System}

The target support portions of CLO II will be served by two CAV AHUs. The units will be manifolded together and will share a common discharge plenum to provide partial redundancy in the event of a complete unit failure. The AHUs will be designed as heating-cooling, single-duct, reheat type. The units will operate $24 \mathrm{~h}$ per day, 365 days per year. Supply fans will be based on a multiple array, plenum fan type design. Variable-frequency drives will provide supply fan volume control in response to a signal from duct-mounted static pressure sensors. The system will be designed to maintain minimum outside air quantities to meet exhaust air requirements but will also include an air-side economizer control scheme.

The HVAC distribution system will be pressure-independent CAV control for all spaces. All terminal boxes and reheat coil valves will be serviced from the space. All terminal units will be positioned for easy access with a minimum of 36 in. of service space on the panel/actuator side. All terminal reheat control valves serving the target support spaces will fail closed.

The supply distribution system will consist of low-pressure ductwork with pressure-independent electrically actuated supply CAV air terminal devices, reheat coils, low-pressure externally insulated ductwork downstream of air terminals, and diffusers. Sound attenuators at the air terminal devices will not be provided. The use of sound attenuating flexible duct at diffusers will be limited to $6 \mathrm{ft}$ in total length to minimize duct static pressure losses.

The units will consist of the following components in the direction of air flow:

- intake isolation damper

- MERV 8 (30\%) efficient prefilter bank

- MERV 14 (90\%) efficient filter bank 
- heating water preheat coil

- chilled water cooling coil

- $\quad$ supply fans (4-6 fan array)

- isolation / smoke damper

- variable-frequency drives to modulate fan air flows

The target support area air handling system will consist of two AHUs. If one of the units should fail or have to be taken out of service for maintenance, the remaining unit will be able to provide $60 \%$ of the maximum supply system design capacity.

\section{Bridge Air-Conditioning Systems}

The bridges connecting to the CLO II will be heated and cooled through the use of fan coil cooling units located above the ceilings of the bridges. The fan coil unit will supply conditioned air into a duct that will extend the length of the bridge. Supply air diffusers along the length of the bridge will distribute conditioned air throughout the bridge. The air will then return through the bridge to a return air grille at the fan coil unit. Heating water and chilled water will be routed to the fan coil units from distribution in the CLO II.

\section{Wet Laboratory and Shop Area Central Fume/General Exhaust System}

The wet laboratory and shop spaces in CLO II will be served by one combined central exhaust system that will serve fume hood exhaust, snorkel exhaust, and general exhaust requirements. Combined fume and general exhaust duct risers from each floor will connect at a penthouse level to a roof-mounted common plenum exhausted by three vertical radial dilution fans.

The general exhaust and fume hood exhaust terminals served by the central exhaust system are variablevolume devices; therefore, the central exhaust system will operate at variable volume. To achieve operational flexibility, the system will incorporate both variable-frequency drive and outside air bypass control technologies.

Under outside air bypass operation, the system will have the ability to maintain a stack discharge velocity of approximately $3500 \mathrm{ft} / \mathrm{min}$. Under favorable wind conditions, the system will have the capability to close the outside air bypass damper and operate the fans under variable-frequency drive fan speed control, allowing the stack discharge velocity to drop. Minimum stack velocity will need to be confirmed by the wind tunnel test. A static pressure sensor in the exhaust ductwork will provide a control signal to adjust the speed of the exhaust fans, or to control an outside air bypass damper that allows makeup air into the fume exhaust plenum. Operation using constant-speed exhaust fans, in conjunction with the outside air bypass dampers, will produce higher stack discharge velocities during times of unfavorable wind speed or wind direction. To determine times of unfavorable wind conditions, the control system will need to interface with a building wind sensing (direction and speed) system.

The fume exhaust fan stacks for the combined fume and general exhaust fans will discharge at a minimum of $10 \mathrm{ft}$ above the roof or as determined by wind tunnel testing. The exhaust system will operate $24 \mathrm{~h}$ per day, 365 days per year.

Pressure-independent VAV exhaust air terminal devices will be provided to serve general exhaust grilles in lab areas. Pressure-independent variable-volume exhaust air terminal devices will be provided for the fume hoods to maintain face velocity at all sash positions. Low-pressure/low-velocity exhaust ductwork will be used between the exhaust air terminal devices and the central exhaust plenum. For all exhaust air from the fume hoods to the exhaust fans, the duct construction will be 304 stainless steel with fully 
welded seams and joints. General exhaust and local exhaust within laboratories can be galvanized steel construction up to the exhaust mains containing fumes. Sound attenuators at the air terminals will not be provided. Instead, sound attenuating flexible ductwork with woven nylon fabric type lining will be provided at the general exhaust grilles to control noise.

The vertical, radial dilution exhaust fans will be of spark-proof construction with bearings and motors out of the airstream. Fans will have a baked epoxy air-side chemical resistant coating. The common plenum and outside air intake dampers will be of galvanized steel construction with a chemical-resistant coating. The central exhaust system will consist of the following components:

- common exhaust plenum

- isolation damper at each fan inlet

- vertical exhaust fans

- exhaust stacks

- bypass air inlet and bypass damper

- variable-frequency drives

Three fans will be provided to serve the common exhaust plenum, each sized at $50 \%$ of the load. If one of the fans fails, the remaining fans will be able to provide $100 \%$ of the common exhaust system's design capacity. All of the three fans in the common exhaust plenum should be on emergency power to ensure continuous hazardous material containment, but they would be controlled to run at a reduced speed during a power outage to limit the negative pressure impact to the building.

\section{Target Support Area General Exhaust System}

Target support spaces in CLO II will be served by one combined central exhaust system serving general exhaust requirements. General exhaust duct risers from each floor will connect at a penthouse level to a roof-mounted common plenum exhausted by three vertical radial dilution fans.

The general exhaust terminals served by the central exhaust system will be constant-volume devices; therefore, the central exhaust system will operate at constant volume. The system will incorporate outside air bypass control technologies to maintain a stack discharge velocity of approximately $3500 \mathrm{ft} / \mathrm{min}$.

The general exhaust fans will discharge at a minimum of $10 \mathrm{ft}$ above the roof or as determined by wind tunnel testing. The exhaust system will operate $24 \mathrm{~h}$ per day, 365 days per year.

All general exhaust duct construction will be 304 stainless steel with fully welded seams and joints. The vertical, radial dilution exhaust fans will be of spark-proof construction with bearings and motors out of the airstream. Fans will have a baked epoxy air-side chemical resistant coating. The common plenum and outside air intake dampers will be of galvanized steel construction with a chemical resistant coating. The central exhaust system will consist of the following components:

- common exhaust plenum

- isolation damper at each fan inlet

- vertical exhaust fans

- exhaust stacks

- bypass air inlet and bypass damper

- variable-frequency drives 
Three fans will be provided to serve the common exhaust plenum, each sized at $50 \%$ of the load. If one of the fans fails, the remaining fans will be able to provide $100 \%$ of the common exhaust system's design capacity. All of the three fans in the common exhaust plenum should be on emergency power to ensure continuous hazardous material containment, but they would be controlled to run at a reduced speed during a power outage to limit the negative pressure impact to the building.

\section{Biosafety Cabinet Exhaust System}

Given the high static pressure loss of the HEPA filters associated with biosafety cabinets, an independent exhaust system will be provided to serve these loads. The design for this dual exhaust fan system will be similar to those described above, and two $100 \%$ capacity exhaust fans will be provided. Ductwork associated with this system will be all welded 304 stainless steel construction.

\section{Laboratory and Fume Hood Airflow Control Systems}

Fume hoods will be tested per ASHRAE Standard 110, Method of Testing Performance of Laboratory Fume Hoods. Fume hood face velocity will be based on the guidelines established by the American Conference of Governmental Industrial Hygienists in the 25th edition of Industrial Ventilation, which recommends an average face velocity of $100 \mathrm{ft} / \mathrm{min}$ with an $18 \mathrm{in}$. open sash for all fume hood applications. It is important that hoods be located in low-traffic areas away from doors or traffic ways.

Fume hoods will be of a variable-volume type with a horizontal sash. Each fume hood will be provided with a low-airflow alarm (audible and visual) by the fume hood manufacturer. Each fume hood will be provided with a variable-volume exhaust air valve and face velocity control system to achieve constant face velocity into the hoods.

The fume hood and laboratory airflow control system will consist of the following components:

- DDCs

- $\quad$ supply and exhaust air terminals

No redundancy will be provided for the control system. The control system will be on emergency power.

\section{Wet Laboratory, Shop and Target Support Area Return Air System}

Spaces within the shop and support areas of CLO II that are not using hazardous materials will be provided with return air capability. Combined laboratory and shop area return air duct risers from each floor will connect at a penthouse to three return/relief fans that will be manifolded together. Variablefrequency drives will provide return fan volume control in response to a signal from duct-mounted static pressure sensors. The return air terminals served by the return air system will be variable-volume devices; therefore, the return air system will operate at variable volume. The return air system will have the capability to either return the air from the spaces for recirculation or exhaust the air from the building as part of the air-side economizer operation. A separate return air system will be employed in the target support area for any spaces that are suitable for air return.

\section{Office, Conference, Amenity and Administrative Support Area Central Air-Handling System}

The office, conference, amenity, and administrative support areas of CLO II will be served by a single VAV air handler. The supply air to these areas will be returned to the unit. 
The AHU will be designed as a heating-cooling, single-duct, reheat type to provide outside air ranging from a minimum to $100 \%$ outside air economizer operation on a VAV basis. The unit will operate only during occupied periods of the day or cycle on as required to maintain night setback temperature settings. Supply fans and return fans will be plug type, arranged in a multi-fan array. Variable-frequency drives will provide supply fan volume control in response to a signal from a duct-mounted static pressure sensor.

Variable-frequency drives will provide return fan volume control in response to a signal from air flow measuring stations used to establish volumetric offset between the return air and the supply air quantities. AHU supply fan speed will be modulated as required by building load.

The control system for this $\mathrm{AHU}$ will include $\mathrm{CO}_{2}$ sensors located in high-occupancy spaces, such as conference or meeting rooms, to achieve demand-based ventilation control.

The supply distribution system will consist of low-pressure steel ductwork with pressure-independent electrically actuated supply VAV air terminal devices, reheat coils, low-pressure externally insulated ductwork downstream of air terminals, and diffusers. Sound attenuators at the air terminal devices will not be provided. Instead, sound attenuating flexible ductwork with a woven nylon fabric-type lining will be provided at the supply diffusers to control noise. The use of sound attenuating flexible ducts at diffusers and grilles will be limited to $6 \mathrm{ft}$ in total length to minimize duct static pressure losses.

The units will consist of the following components in the direction of air flow:

- return/relief fans located within the AHU

- MERV 8 (30\%) efficient prefilter bank.

- MERV 14 (95\%) efficient filter bank

- air-side economizer mixing damper section

- chilled water cooling coil

- supply fans

- isolation / smoke damper

- variable-frequency drives to modulate fan air flows

No provisions for redundancy will be made.

\section{Telecommunications Room and Electrical Equipment Room}

All telecommunications rooms and electrical equipment rooms in CLO II will be served by chilled watercooled fan coil units located within the spaces. Units will normally operate $24 \mathrm{~h}$ per day, 365 days per year. Provision will be made to provide some conditioned air from one of the constantly running AHUs as backup to the space should a fan coil unit fail.

\section{Data Center Equipment Room}

The data center equipment room in CLO II will be served by chilled-water computer-room AHUs located on the floor within the space. The space will be served by two units, each designed to meet $70 \%$ of the full load. Units will operate $24 \mathrm{~h}$ per day, 365 days per year and will be supplied with emergency power. Airflow will be designed for either overhead or under-floor distribution. An under-floor air distribution concept is recommended should the power density of the space reach $50 \mathrm{~W} / \mathrm{ft}^{2}$. 


\section{Chilled Water System}

The chilled water system for CLO II will be supplied from CUB II. Based on an estimated flow of 1200 GPM to the building, the chilled water branch line size is expected to be in the range of $8 \mathrm{in}$. Chilled water will be distributed to CLO II from CUB II using the interconnecting bridges. Chilled water will be routed to cooling coils in all AHUs and to the heat exchanger of the process chilled water system. To address laboratory equipment power usage beyond the heat rejection capacity provided by the AHUs, chilled water lines will be stubbed out onto each laboratory floor for use in fan coil units that could be installed in the future to provide additional cooling if required.

\section{Sensible Process Cooling Water System}

A process cooling water system will be required to support certain equipment to be included in CLO II. Laboratory equipment that can use water cooling will be cooled by the piped process cooling water system that will be extended throughout the laboratory floors. Equipment to be cooled will include such items as process and instrumentation equipment. The process cooling water system will consist of an independent pumped loop that will reject heat into the building chilled water through a plate frame heat exchanger. The system will be designed to operate at a supply temperature of $60^{\circ} \mathrm{F}$ to reduce the potential for condensation. To minimize back pressure at the equipment, if required, the system could be designed with an atmospheric tank and low-pressure loss return pipe system. The tank, pumps, heat exchanger, filters and chemical treatment components will be located in a lower-level mechanical room.

The process cooling water piping system will use copper pipe. Pipe insulation will not be provided, given the operating water temperature. The system will include the following equipment:

- base mounted, end suction, centrifugal pumps

- $\quad$ uump variable-frequency drives

- chilled water plate frame heat exchanger

- water filtration

- automated chemical treatment system

- makeup water assembly

- appropriate valving and piping specialties

Two variable-speed process cooling water pumps will be sized to provide $100 \%$ of the design flow rate each. If one of the booster pumps should fail, the other one would provide $100 \%$ redundancy. The process cooling water pumping system will be on emergency power since it must provide continuous service to critical laboratory equipment.

\section{Heating Water System}

The heating water system for CLO II will be supplied from CUB II. Based on an estimated flow of 240 GPM to CLO II, the heating water branch line size to this building is expected to be 4 in. The building heating water distribution system will serve preheat and reheat heating water coils and any unit heaters within the building.

\section{Equipment Schedules}

The CLO II mechanical system requirements are summarized in Table 6.13. 
Table 6.13. CLO II equipment schedules

\begin{tabular}{|c|c|c|c|c|c|}
\hline Equipment & Manufacturer & Size (each) & Quantity & $\begin{array}{c}\text { No. on } \\
\text { emergency } \\
\text { power }\end{array}$ & Remarks \\
\hline $\begin{array}{l}\text { AHU—wet lab and } \\
\text { shop areas }\end{array}$ & Trane & 18,000 CFM & 3 & 0 & $\begin{array}{l}\text { Total } 40 \text { motor } \\
\text { hp for each } \\
\text { AHU }\end{array}$ \\
\hline $\begin{array}{l}\text { AHU_target support } \\
\text { areas }\end{array}$ & Trane & 22,000 CFM & 2 & 0 & $\begin{array}{l}\text { Total } 50 \text { motor } \\
\text { hp for each } \\
\text { AHU }\end{array}$ \\
\hline $\begin{array}{l}\text { AHU w supply and } \\
\text { return fan-office } \\
\text { and admin support } \\
\text { areas }\end{array}$ & Trane & $25,000 \mathrm{CFM}$ & 1 & 0 & $\begin{array}{l}\text { Total } 40 \text { motor } \\
\text { hp for supply } \\
\text { fans } \\
\text { Total } 20 \text { motor } \\
\text { hp for return } \\
\text { fans }\end{array}$ \\
\hline $\begin{array}{l}\text { Lab and shop general } \\
\text { exhaust fans }\end{array}$ & MK Plastics & $15,000 \mathrm{CFM}$ & 3 & 3 & $\begin{array}{l}1 \text { fan is backup } \\
25 \mathrm{hp} \text { motor } \\
\text { each }\end{array}$ \\
\hline $\begin{array}{l}\text { Target support area } \\
\text { general exhaust fans }\end{array}$ & MK Plastics & $15,000 \mathrm{CFM}$ & 2 & 2 & $\begin{array}{l}1 \text { fan is back-up } \\
25 \mathrm{hp} \text { motor } \\
\text { each }\end{array}$ \\
\hline $\begin{array}{l}\text { Lab biosafety cabinet } \\
\text { exhaust fans }\end{array}$ & MK Plastics & $3,000 \mathrm{CFM}$ & 2 & 2 & $\begin{array}{l}1 \text { fan is backup } \\
3 \text { hp motor each }\end{array}$ \\
\hline $\begin{array}{l}\text { Lab, shop, and support } \\
\text { return fans }\end{array}$ & Cook & 7,500 CFM & 3 & 0 & 5 hp motor each \\
\hline $\begin{array}{l}\text { Target support area } \\
\text { return fans }\end{array}$ & Cook & $5,000 \mathrm{CFM}$ & 3 & 0 & $3 \mathrm{hp} \mathrm{motor} \mathrm{each}$ \\
\hline Packaged fan-coil & Trane & $\begin{array}{l}2-3 \text { tons of } \\
\text { refrigeration }\end{array}$ & $\begin{array}{c}\text { Estimated at } \\
12\end{array}$ & 0 & $\begin{array}{l}\text { Supplied with } \\
\text { chilled water }\end{array}$ \\
\hline Exhaust fans & Cook & Varies & $\begin{array}{c}\text { Estimated at } \\
10\end{array}$ & 0 & $\begin{array}{l}\text { Roof mounted, } \\
\text { some with 1- } \\
\text { phase and some } \\
\text { with 3-phase } \\
\text { power }\end{array}$ \\
\hline $\begin{array}{l}\text { Heating water unit } \\
\text { heaters }\end{array}$ & Trane & Varies & Estimated at 8 & 0 & $\begin{array}{l}\text { Supplied with } \\
\text { heating water }\end{array}$ \\
\hline $\begin{array}{l}\text { Process cooling water } \\
\text { distribution pumps }\end{array}$ & $B \& G$ & $\begin{array}{l}50 \text { GPM @ } \\
100 \mathrm{ft} \text { TDH }\end{array}$ & 2 & 0 & $\begin{array}{l}1 \text { standby, } \\
3 \text { hp motor each }\end{array}$ \\
\hline $\begin{array}{l}\text { Process cooling water } \\
\text { storage tank }\end{array}$ & Raven & 500 gal & 1 & N/A & FRP \\
\hline
\end{tabular}

$\mathrm{TDH}=$ total dynamic head

\section{Piping Systems}

\section{Potable Water Service}

Where possible, potable hot and cold water will be distributed throughout CLO II above the ceiling. 
A separate recirculating tempered water distribution system, including risers, will be provided to supply emergency safety equipment. Potable hot water will be generated at $140^{\circ} \mathrm{F}$ by two semi-instantaneous steam-fired water heaters located in CUB II. Each heater will be sized for $60 \%$ of the potable hot water demand. A thermostatic mixing valve will be provided to achieve $120^{\circ} \mathrm{F}$ distribution temperatures.

Hot water will be distributed through a separate recirculating system designed to provide hot water to the points of use.

Water velocity in distribution piping will not exceed $8 \mathrm{ft} / \mathrm{s}$ for cold water and $5 \mathrm{ft} / \mathrm{s}$ for hot water at or below $140^{\circ} \mathrm{F}$ and any softened water. Provisions will be made to arrest water hammer. Shock arrestors when installed will comply with PDI-WH201 or ASSE-1010.

\section{Non-potable Water Service (Laboratory Use)}

The laboratory-use water distribution system will provide cold and hot water to sinks, cup sinks, fume hoods, glass-washing equipment, and other laboratory-related equipment in lab areas. Laboratory hot water will be generated at $140^{\circ} \mathrm{F}$ by natural gas-fired water heaters located in CUB II. Each heater will be sized for $75 \%$ of the laboratory hot water demand. A thermostatic mixing valve will be provided to achieve $120^{\circ} \mathrm{F}$ distribution temperature. Laboratory hot water will be distributed through a separate recirculating system designed to provide hot water to the points of use.

Vacuum breakers will be provided for all laboratory faucets with serrated outlets. Vacuum breakers for fume hoods with serrated water outlets will be mounted high on the outsides of the fume hoods.

Design of laboratory-use cold and hot water will be based on fixture unit values and adjusted/increased to allow for projected water demand for laboratory-related equipment at the design stage.

Piping will be sized to maintain a minimum of $30 \mathrm{psi}$ at the most remote laboratory equipment connection.

Water velocity in distribution piping will not exceed $6 \mathrm{ft} / \mathrm{s}$. Provisions will be made to arrest water hammer. Shock arrestors when installed will comply with PDI-WH201 or ASSE-1010.

Approved pressure-reducing valve assemblies with strainers will be provided, if required, to limit the maximum water pressure to $80 \mathrm{psi}$ in the distribution piping.

The entire laboratory hot and cold water distribution system will be fully insulated.

\section{Laboratory Waste}

Laboratory waste from sinks, cup sinks, and other laboratory-related equipment will be collected and conveyed by a gravity system to the underground sanitary waste drainage system outside the building. Any hazardous waste will be bottled to be transported for processing.

Flow rates and pipe sizes will be calculated based on drainage fixture unit values and adjusted/increased to allow for projected wastewater discharge from various laboratory-related (and cage washing) equipment at the design stage.

Unless required for specific equipment, floor drains will not be provided in laboratory rooms. 
Complete accessibility will be provided to all cleanouts. Wall-type cleanouts will be used within the laboratory spaces.

Floor drains that do not receive regular use will be provided with trap primers. Primers will consist of an automatic trap primer valve that meters water based upon pressure fluctuation in the supply line.

\section{Compressed Air System}

High-pressure compressed air will be distributed throughout CLO II to supply compressed air to laboratory-related equipment. The compressed air system will be provided with local pressure-reducing valves to permit each piece of equipment to use air of an appropriate pressure.

Design compressed air flow at laboratory outlets will be 1 SCFM.

\section{Vacuum System}

Vacuum serving laboratory program spaces in CLO II will be supplied from a central system located in the mechanical room, complete with vacuum pumps, receiver, controls, accessories, and distribution piping designed to supply vacuum to laboratory and related inlets and equipment.

The design vacuum levels will be $24 \mathrm{in} . \mathrm{Hg}$ at the vacuum skid and $19 \mathrm{in} . \mathrm{Hg}$ at laboratory inlets.

Design vacuum flow at inlets will be $0.5 \mathrm{CFM}$.

Piping mains will be sized for the maximum calculated flow at the design stage and will limit velocities to $5,000 \mathrm{ft} / \mathrm{min}$ with a maximum pressure drop to the farthest inlet of not more than $5 \mathrm{in}$. $\mathrm{Hg}$ at estimated peak flow conditions.

The piping material will be type K copper with soldered joints.

\section{Specialty Gases}

Specialty gases (e.g., gaseous nitrogen, oxygen, helium, argon) will be supplied to CLO II program spaces from local cylinders and manifolds provided by the space owners. The pressure in the distribution system will be as required by the program. The piping material will be as required by the specific gas supplied.

\section{Fire Protection Systems}

The fire sprinkler system for CLO II will be hydraulically designed to provide water densities that meet the requirements for Ordinary Hazard, Group 2, protection throughout the facility. Combination sprinkler/standpipe risers will be located in the two main stairwells and will provide for floor control valves and fire department hose connections.

\section{Electrical Systems}

\section{Exterior Power Distribution}

The $480 \mathrm{Y} / 277 \mathrm{~V}$ electrical supply to CLO II will be provided by connecting to two 2400 A circuit breakers, one in each of the two outdoor unit substations located on the east side of CLO II. A 2400 A feeder bus duct will be routed overhead from each substation and its associated switchgear will be located in the electrical room. 


\section{Interior Power Distribution}

From the $2400 \mathrm{~A}, 480 \mathrm{Y} / 277 \mathrm{~V}$ switchgear, power will be distributed to lights and receptacles, laboratory equipment, mechanical equipment, and elevators. Distribution panels will be located in mechanical rooms and in each electrical closet on the lower level; stacked electrical closets will be provided on the upper levels. Distribution panels will be dedicated to the types of loads they serve: CF loads and technical loads.

The distribution panels at the bases of the stacked closets will subfeed $480 \mathrm{Y} / 277 \mathrm{~V}$ lighting panels on the upper floors, which will in turn subfeed $208 \mathrm{Y} / 120 \mathrm{~V}$ receptacle panels via step-down transformers. Technical distribution panels will subfeed $208 \mathrm{Y} / 120 \mathrm{~V}$ lab distribution panels via step-down transformers, which will in turn subfeed lab panels at the individual labs. Separate transformers and panels will be provided to serve sensitive electronic loads.

CLO II will provide power to the pedestrian bridges to the adjacent facilities.

\section{Emergency Power Distribution}

Emergency power to CLO II will be provided by connecting to the generator located on the east side of the building. A duct bank will be routed from the generator to a generator power distribution panel board in the electrical room. Generator power will be distributed to an emergency automatic transfer switch serving emergency loads, including lab exhaust fans. It will also be distributed to an optional standby automatic transfer switch serving the information technology (IT) system, its associated mechanical equipment, and any one of the elevators.

A $50 \mathrm{kVA}, 480 \mathrm{Y} / 277 \mathrm{~V}$ UPS system will be provided to support the IT system.

\section{Interior Lighting}

At the CLO II, office areas will be provided with a mix of fluorescent troffers and direct/indirect linear fluorescent lighting fixtures. Labs will be provided with direct/indirect linear fluorescent lighting fixtures. Lobbies and corridors will be provided with LED downlights. Daylight harvesting will be provided in areas with adequate levels of natural light.

\subsubsection{Central Utility Building II}

\section{Programming}

CUB II will house all central utilities except exhaust systems that are required to support the STS. A summary level program for the CUB II is shown in Table 6.14.

Table 6.14. Central Utility Building II program summary

\begin{tabular}{lcl}
\hline \multicolumn{1}{c}{ Space type } & Area (NSF) & \\
\hline Boiler systems & 2,000 & \\
Chiller systems & 2,000 & \\
Pump systems & 1,500 & \\
Compressed air system & 1,500 & \\
Total net area & $\mathbf{7 , 0 0 0}$ & \\
Gross/net factor (75\% efficiency) & 1.3 & \\
Total gross area & $\mathbf{9 , 1 0 0}$ & GSF \\
\hline
\end{tabular}


The net areas required to house the central utility systems have been estimated based on preliminary utility load estimates for the STS and parametric comparison to the FTS CUB.

The grossing factor of 1.3 is estimated based on comparable buildings and includes space for general building circulation, toilets, exterior wall, mechanical, electrical, plumbing, and other spaces not included in the net program areas.

The single-story CUB II will be located centrally between Target Building II, the instrument buildings, and CLO II to minimize utility distribution and operational costs. The cooling tower will be located so as to reduce the impact of cooling tower operation. The location of the CUB II and cooling tower with respect to the east parking area and pedestrian approach to the STS will be further evaluated in subsequent design phases.

The CUB II will be a double-height single-story building. A bridge linking the CLO II and $90 \mathrm{M}$ instrument hall will be integrated into the CUB II at an upper level, either overlooking the CUB II interior or on the roof above the interior space, depending on the floor elevation of the CUB II. Currently, the bridge is shown as being located on the roof above the CUB II. This relationship will be further evaluated during subsequent design phases.

\section{Structural Systems}

The CUB II will be a conventional steel-framed building supported on spread footings. Concentric braced frames will be used to resist environmental events (wind and seismic).

The below-grade elements (e.g., basement walls, retaining walls) will be constructed of reinforced normal-weight concrete. Water stops will be provided in construction joints to safeguard against water intrusion.

The CUB II will support the exterior wall system with steel girts. The roofing system will be supported on a 1.5 in. galvanized metal roof deck on steel beams.

Elevated floors will be constructed of a reinforced normal-weight concrete on a composite metal deck (galvanized).

The slab on grade will be a minimum of 8 in. thick reinforced normal-weight concrete slab.

The structure will be isolated from the CLO II to mitigate vibration between the two buildings.

The CUB II will meet PC-1 performance requirements in accordance with DOE standards.

\section{Building Enclosure Systems}

CUB II will be designed to be architecturally compatible with the existing SNS campus and other new buildings making up the STS. Walls below grade will be constructed of reinforced concrete. The exterior wall is expected to be $100 \%$ architectural-grade, painted metal insulated panels. The building will include two pedestrian entrances with overhead canopy protection and one service entrance with overhead canopy protection and a depressed loading dock. 


\section{Life Safety Systems}

Currently, Target Building II, two contiguous instrument buildings, and CLO II are being defined as a single mixed-occupancy building comprising business, incidental assembly, and moderate-hazard factory industrial uses. The entire complex is envisioned as being fully sprinkler-protected Type IIA construction. Currently, the plan is to include the CUB II in the mixed-occupancy building. Alternatively, CUB II could be separated from CLO II and defined as a moderate-hazard factory industrial occupancy. In this case, the building would be fully protected, Type IIB construction. The floor of the CUB II will have two direct exits to grade. The definition of the CUB II as part of a larger single building, including Target Building II, contiguous instrument buildings, and CLO II, will be further evaluated through detailed fire hazard and code analysis that will occur in subsequent STS design phases.

\section{Mechanical Systems}

\section{HVAC Ventilation}

HVAC will be provided to serve the control room and associated toilet/support space. Most of the spaces within the building will only be heated and ventilated. Toilets will have a code minimum of ten air changes per hour during hours of occupancy.

\section{Control System}

CUB II will be controlled by EPICS. A refrigerant alarm system will provided in accordance with the requirements of the International Mechanical Code.

\section{HVAC Equipment}

The conditioned spaces identified will be heated and cooled using a small AHU located on the roof of the CUB II. The AHU will be provided with an economizer mixing box, filtration, a heating water heating coil, and a chilled water cooling coil. The unit will be provided with an air-side economizer. Relief air will infiltrate through the roof using barometric dampers. Mechanical, electrical, and utility spaces will be conditioned using a heating and ventilation approach. Ventilation will be achieved through the use of roof-mounted exhaust fans and associated wall-mounted intake air louver with motorized electric dampers. These spaces will be heated using either electric or heating water unit heaters located within the spaces. The selected heating source will be determined based on a life cycle cost analysis performed during the preliminary design phase.

\section{Chilled Water System}

Based on parametric analysis, it is estimated that the chilled water plant capacity will be in the range of 1800 to 2400 tons of refrigeration. The chilled water system and all associated pumps will be located in a dedicated refrigeration machinery room. The chilled water plant is expected to consist of four watercooled centrifugal chiller units, four primary chilled water pumps, and up to four secondary chilled water pumps. Cooling towers, consisting of four Factory Mutual (FM) -approved factory-fabricated cross-flow cells and associated condenser water pumps will supply the chiller units. The entire chilled water plant will be designed in an $\mathrm{N}+1$ configuration, with one chiller, chilled water primary and secondary pumps, condenser water pump, and cooling tower cell being a standby unit. A primary-secondary pumping distribution system will be used for the chilled water distribution. Multiple pressure differential sensors in the chilled water loop at each major building will vary the variable-frequency drive pump speed of the secondary chilled water pumps to maintain the required pressure differential across the chilled water system. Chilled water from the building systems will be supplied to the AHUs at $44^{\circ} \mathrm{F}$. Return water 
temperature will be optimized, but it is expected to be operated with a 12 to $18^{\circ} \mathrm{F}$ temperature rise. Based on the minimum temperature differential stated above, the chilled water piping mains would be 16 in. in diameter. Chilled water will be routed to cooling coils in all AHUs and to the heat exchangers of the secondary chilled water process cooling water systems.

Condenser water will be supplied from four cooling tower cells dedicated to the chilled water plant. The cooling towers will be located on an elevated-grade, mounted steel support structure located to the east of CUB II. The cooling towers will flow into an underground sump located adjacent to the cooling towers. Four grade-mounted vertical turbine condenser water pumps, with one pump as a standby, will draw the condenser water from the sump and deliver it to the chillers in CUB II.

The chilled water plant will include the following equipment:

- four water-cooled centrifugal chillers (all with 800 tons of cooling)

- base-mounted, end suction, centrifugal primary chilled water pumps

- base-mounted, end suction, centrifugal variable-speed secondary chilled water pumps

- grade-mounted, vertical turbine condenser water pumps

- chilled water and condenser water pump variable-frequency drives

- appropriate valving and piping specialties

- expansion tank and air separator

- four 2400 GPM cooling tower cells

- cooling tower fan variable-frequency drives

- cooling tower and chilled water automated chemical treatment systems

Three chillers and three cooling tower cells have the capacity to meet the total estimated building load and a $15 \%$ allowance for future growth.

Three primary pumps, three variable-speed secondary chilled water pumps, and condenser water pumps will be sized to provide $100 \%$ of the design flow rate; a fourth pump will serve as a standby pump.

\section{Cooling Water System}

The technical equipment DI water cooling loops will reject heat to cooling tower water. Based on the technical equipment cooling load data provided, it is estimated that the cooling tower plant will need to supply 1500 GPM of tower water. The cooling tower water piping system will use copper or painted carbon steel for pipe up to 2 in. and grooved coupling or welded carbon steel pipes for sizes above 2 in. The cooling tower water system will include the following equipment:

- two (1200 GPM) cooling tower cell (with one acting as a standby cell)

- cooling tower fan variable-frequency drive

- two grade-mounted, vertical turbine tower water pumps (one acting as a standby)

- tower water pump variable-frequency drives

- appropriate valving and piping specialties

- cooling tower automated chemical treatment system

The cooling tower cells have the capacity to meet the total estimated technical equipment water load and a $15 \%$ allowance for future growth. 


\section{Heating Water System}

Preheat and reheat heating water will be generated using a new heating water system to be located in the boiler room of the CUB II. The heating plant is proposed to comprise three condensing boilers and heating water distribution pumps. The heating water system will serve the preheat and reheat loads of all buildings. Based on parametric analysis, it is estimated that the heating water plant capacity will be in the range of 14 to $18 \mathrm{MMBH}$. The proposed heating plant is expected to consist of three boilers at $10 \mathrm{MMBH}$ input ( 3 at $50 \%$ ), which will provide $\mathrm{N}+1$ redundancy for the facility. The maximum heating water supply temperature will be $140^{\circ} \mathrm{F}$ with a $40^{\circ} \mathrm{F} \Delta \mathrm{T}$ between supply and return heating water temperatures. The heating water supply temperature of the piping loop will be reset lower based on increases in the outside air temperature. Total heating water flow is estimated at $875 \mathrm{GPM}$, which would be provided by three 450 GPM pumps configured in an $\mathrm{N}+1$ arrangement.

A variable-flow pumping distribution system will be used to distribute the heating water to preheat and reheat coils. Three base-mounted variable-volume pumps will be used for the circulation loop, with one pump acting as a standby. A variable-frequency drive will be provided for each pump. The system will include the following equipment:

- three gas-fired condensing boilers

- base-mounted, end suction centrifugal pumps

- distribution pump variable-frequency drives

- expansion tank and air separator

- automated chemical treatment system

- makeup water assembly

- appropriate valving and piping specialties

Three distribution pumps will be sized at $100 \%$ of the design flow rate of each boiler, in an $\mathrm{N}+1$ configuration. If one pump fails, the remaining pumps will be able to provide $100 \%$ of the design capacity of the piped loop.

\section{Compressed Air}

The central compressed air system for the STS buildings will be located within the CUB II. Based on parametric evaluation of the existing system, it is estimated that the new compressed air system will have a required compressor capacity of 700 SCFM at 150 psig. The system will be located within a separate heated and ventilated space and will consist of two 420 SCFM water-cooled oil-free rotary screw compressors. Cooling water for the units will be provided from the cooling tower water system. The system will also be provided with duplex, dual-tower desiccant dryers designed to handle the capacity of each compressor and dry the air stream to a $-40^{\circ} \mathrm{F}$ pressure dew point (minimum). In addition, the system will be furnished with a series of moisture separators, prefilters, after filters, and final filters. An ASMErated and -stamped receiver tank with a capacity of 4,000 gal will be co-located near the compressor room. A pressure regulating valve will be installed in the compressed air main supply line to control the site-wide distribution pressure. A control panel with an interface to EPICS will provide status and alarm conditions. The system controls for the compressors and dryers will operate to alternate each compressor for even wear on the system.

The compressed air will be distributed throughout the site in coated black steel piping at a minimum of 100 psig. The new compressed air piping system will be looped and interconnected to the existing system in the existing Experiment Hall and tunnels. 
Each compressor will be sized to handle two-thirds of the load. Each one will serve as a partial backup in case of failure of a single compressor. The distribution piping serving each building will be sized for the current building program, with an additional $20 \%$ load provided to accommodate renovation flexibility.

\section{Equipment Schedules}

The CUB II mechanical requirements are summarized in Table 6.15.

Table 6.15. CUB II mechanical equipment schedules

\begin{tabular}{|c|c|c|c|c|c|}
\hline Equipment & Manufacturer & $\begin{array}{l}\text { Size } \\
\text { (each) }\end{array}$ & Quantity & $\begin{array}{c}\text { No. on } \\
\text { emergency } \\
\text { power }\end{array}$ & Remarks \\
\hline $\begin{array}{l}\text { Water-cooled } \\
\text { centrifugal } \\
\text { chiller unit }\end{array}$ & Trane & 800 ton & 4 & 0 & 1 chiller is backup \\
\hline $\begin{array}{l}\text { Primary chilled } \\
\text { water pumps }\end{array}$ & $\begin{array}{l}\text { B\&G end-suction, base } \\
\text { mounted }\end{array}$ & $\begin{array}{l}\text { Pumps-1600 } \\
\text { GPM @ } 40 \mathrm{ft}\end{array}$ & 4 & 0 & $\begin{array}{l}1 \text { pump for backup. } \\
20 \text { hp motor each. }\end{array}$ \\
\hline $\begin{array}{l}\text { Secondary } \\
\text { chilled water } \\
\text { pumps }\end{array}$ & $\begin{array}{l}\text { B\&G end-suction, base } \\
\text { mounted }\end{array}$ & $\begin{array}{l}\text { Pumps-1600 } \\
\text { GPM@75 ft }\end{array}$ & 4 & 0 & $\begin{array}{l}1 \text { pump for backup. } \\
50 \text { hp motor each. }\end{array}$ \\
\hline $\begin{array}{l}\text { Condenser } \\
\text { water pumps }\end{array}$ & $\begin{array}{l}\text { B\&G vertical turbine, } \\
\text { slab mounted }\end{array}$ & $\begin{array}{l}\text { Pumps-2400 } \\
\text { GPM@70 ft }\end{array}$ & 4 & 0 & $\begin{array}{l}1 \text { pump for backup. } \\
60 \mathrm{hp} \text { motor each. pumps } \\
\text { located at remote cooling } \\
\text { towers. }\end{array}$ \\
\hline $\begin{array}{l}\text { Cooling tower } \\
\text { water pumps }\end{array}$ & $\begin{array}{l}\text { B\&G vertical turbine, } \\
\text { slab mounted }\end{array}$ & $\begin{array}{l}\text { Pumps-1200 } \\
\text { GPM @ } 120 \mathrm{ft}\end{array}$ & 4 & 0 & $\begin{array}{l}1 \text { pump for backup. } \\
60 \text { hp motor each. } \\
\text { Pumps located at remote } \\
\text { cooling towers. }\end{array}$ \\
\hline $\begin{array}{l}\text { Cooling towers } \\
\text { (condenser } \\
\text { water) }\end{array}$ & $\begin{array}{l}\text { BAC cross flow cooling } \\
\text { tower }\end{array}$ & 1000 ton & 4 & 0 & $\begin{array}{l}1 \text { cooling tower for backup. } \\
40 \text { hp fan motor each. } \\
\text { Located remote from CUB } \\
\text { II. }\end{array}$ \\
\hline $\begin{array}{l}\text { Cooling towers } \\
\text { (tower water) }\end{array}$ & $\begin{array}{l}\text { BAC cross flow cooling } \\
\text { tower }\end{array}$ & $\begin{array}{l}600 \text { ton } \\
(2100 \mathrm{~kW})\end{array}$ & 2 & 0 & $\begin{array}{l}1 \text { cooling tower for back-up. } \\
30 \text { hp fan motor each. } \\
\text { Located remote from CUB } \\
\text { II. }\end{array}$ \\
\hline Boilers & $\begin{array}{l}\text { Cleaver Brooks } \\
\text { condensing - heating } \\
\text { water }\end{array}$ & $\begin{array}{l}3 \text { boilers }-1000 \\
\text { MBH input } \\
\text { each }\end{array}$ & 3 & 0 & 1 boiler is backup. \\
\hline $\begin{array}{l}\text { Primary heating } \\
\text { water pumps }\end{array}$ & $\begin{array}{l}\text { Primary-B\&G end- } \\
\text { suction, base mounted }\end{array}$ & $\begin{array}{l}\text { Pumps-450 } \\
\text { GPM@ } 970 \mathrm{ft}\end{array}$ & 3 & 0 & $\begin{array}{l}1 \text { pump for back-up. } \\
20 \text { hp motor each. }\end{array}$ \\
\hline Air compressors & $\begin{array}{l}\text { Ingersoll-Rand, } \\
\text { Kobelco }\end{array}$ & $\begin{array}{l}420 \text { SCFM } \\
\text { (each } \\
\text { compressor) }\end{array}$ & 2 & 0 & $\begin{array}{l}\text { Water-cooled oil-free rotary } \\
\text { screw. } \\
100 \text { hp motor each. }\end{array}$ \\
\hline $\begin{array}{l}\text { Packaged air- } \\
\text { handling unit }\end{array}$ & Trane - roof mounted & $\begin{array}{l}2--3 \text { tons of } \\
\text { refrigeration }\end{array}$ & 1 & 0 & $\begin{array}{l}\text { Supplied with chilled water } \\
\text { and heating water }\end{array}$ \\
\hline Exhaust fans & Trane & Varies & $\begin{array}{l}\text { Estimated } \\
\text { at } 5\end{array}$ & 0 & $\begin{array}{l}\text { Roof-mounted, some with 1- } \\
\text { phase and some with 3-phase } \\
\text { power }\end{array}$ \\
\hline $\begin{array}{l}\text { Heating water } \\
\text { unit heaters }\end{array}$ & Trane & Varies & $\begin{array}{c}\text { Estimated } \\
\text { at } 8\end{array}$ & 0 & Supplied with heating water \\
\hline
\end{tabular}




\section{Fire Protection}

The fire sprinkler system for the CUB II will be hydraulically designed to provide water densities that meet the requirements for Ordinary Hazard, Group 2, protection throughout the facility.

\section{Electrical Systems}

\section{Exterior Power Distribution}

The $480 \mathrm{Y} / 277 \mathrm{~V}$ electrical supply to CUB II will be provided by connecting to two $3000 \mathrm{~A}$ circuit breakers, one in each of the two outdoor unit substations located on the south side of CUB II. The $3000 \mathrm{~A}$ feeder bus ducts will be routed overhead from the two substations to their associated switchgear located in the electrical room.

The $480 \mathrm{Y} / 277 \mathrm{~V}$ electrical supply to the cooling towers will be provided by connecting to two $400 \mathrm{~A}$ circuit breakers, one in each of the two outdoor unit substations located on the south side of CUB II. The 400 A duct banks will be routed from the two substations to their associated panel board located in the variable-frequency drive/electrical room adjacent to the cooling towers.

\section{Interior Power Distribution}

With most of the major mechanical components being provided in groups of four in a $3+1$ redundant configuration, each of the $3000 \mathrm{~A}, 480 \mathrm{Y} / 277 \mathrm{~V}$ switchgear and $400 \mathrm{~A}$ panel boards will serve two of the four components. This will allow CUB II to operate at two/thirds capacity if one of the substations is out of service. A transfer switch will be provided to serve the loads that do not have a redundant component.

\section{Emergency Power Distribution}

Emergency power to CUB II will be provided by connecting to the generator located on the east side of CLO II. A duct bank will be routed from generator GEN-B to a disconnect switch in the electrical room.

\subsubsection{Central Exhaust Facility II}

\section{Programming}

Central exhaust facility II (CEF II) will manifold together primary, secondary, and tunnel exhaust systems serving Target Building II, the R2T2 tunnel, instrument halls, and tunnels at a single location. Exhaust for CLO II will be handled through separate systems located at the penthouse level of the CLO II. A summary-level program for CEF II is provided in Table 6.16.

Table 6.16. Central exhaust facility II

\begin{tabular}{ll}
\hline \multicolumn{1}{c}{ Space type } & \multicolumn{1}{c}{$\begin{array}{c}\text { Area } \\
\text { (GSF) }\end{array}$} \\
\hline Fan control building & 200 \\
Total gross area & $\mathbf{2 0 0}$ \\
Exterior fan systems & 6000 \\
\hline
\end{tabular}

CEF II is a grade-level exterior installation. A small controls building will be located in the vicinity of the exterior fan systems. The exhaust systems will be discharged through a single stack estimated to be $80 \mathrm{ft}$ 
in height. Refer to Sect. 6.4.5, "Mechanical Systems," for facilities served by the systems housed in CEF II.

\section{Structural Systems}

CEF II will be an exterior-grade level slab for support of equipment. The slab will be used as the foundation for the equipment. The slab on grade is anticipated to be a minimum of 8 in. thick reinforced normal-weight concrete slab. Where the slab/foundation is required to be thicker (e.g., the cantilevered primary stack) the slab will be thickened and isolated from the adjacent thinner slab.

A small building $\left(<200 \mathrm{ft}^{2}\right)$ with a cantilevered screen wall is planned; conventional steel construction will be used for this building. Concentric braced frames will be used to resist environmental events (wind and seismic). The building will support the exterior wall system with steel girts. The roofing system will be supported on a 1.5 in. galvanized metal roof deck on steel beams.

The CEF II will meet PC-1 performance requirements in accordance with DOE standards.

\section{Building Enclosure Systems}

The CEF II is primarily an exterior engineering system and associated structural support. A small controls building will be designed to be architecturally compatible with the existing SNS campus and other new buildings making up the STS. The exterior wall will be $100 \%$ architectural-grade painted metal insulated panels. The building will include one pedestrian entrance with overhead canopy protection. The exterior fan systems will be visually concealed with a $12 \mathrm{ft}$ high screen wall constructed of corrugated, perforated, painted metal panels attached to a painted structural steel frame.

\section{Life Safety Systems}

The controls building will be classified as moderate-hazard factory industrial occupancy. The small building will be fully protected and will be of Type IIB construction with a single exit to grade.

The exterior fan structures will meet the Life Safety Code and Occupational Safety and Health Administration requirements for industrial equipment access.

\section{Exhaust Equipment}

The project technical exhaust air streams are expected to consist of ventilation from the R2T2 tunnel, the Target Building II PCE and SCE systems, and Target Building II HOG exhaust. All exhaust fans will be located on an equipment pad located to the north of the STS Building. All fans will be constructed of coated steel. All fan systems will be arranged in a duplex configuration with one fan in each set acting as a full standby. Each fan will be provided with a shutoff damper on the suction side and a motorized shutoff damper on the fan discharge. All aboveground ductwork will be painted carbon steel except the HOG system, which will be 304 SS. All underground ductwork will be constructed of HDPE plastic.

The R2T2 tunnel exhaust duct will connect to the tunnel near its connection point to Target Building II. System capacity is expected to be in the range of 2,000 to 3,000 CFM. Ductwork is estimated to be 16-18 in. in diameter.

The Target Building II PCE will collect exhaust from the potentially highly activated areas of Target Building II. System capacity is expected to be in the range of 3,000 to 4,000 CFM. Ductwork is estimated to be $18-20$ in. in diameter. 
The Target Building II SCE will collect exhaust from the potentially activated areas of Target Building II. System capacity is expected to be in the range of 10,000 to 12,000 CFM. Ductwork is estimated to be 3034 in. in diameter.

The Target Building HOG exhaust will collect exhaust from highly activated areas of the Target Building II system. System capacity is expected to be in the range of 300 to 400 CFM. Ductwork is estimated to be 6 in. in diameter

An exhaust header manifold will be used to combine the exhaust streams of the four fan systems. The manifold is expected to range in size from 34 to $40 \mathrm{in}$. The manifold will extend into a vertical stack that will also be mounted on the equipment pad. The stack will be $40 \mathrm{in}$. or less, as required to achieve a $3,000 \mathrm{ft} / \mathrm{min}$ stack discharge velocity. The height of the stack is anticipated to range from 40 to $80 \mathrm{ft}$. Final stack height will be based on input from the Environment, Safety, and Health Directorate.

\section{Equipment and Materials}

- two PCE exhaust fans with variable-frequency drives

- two SCE exhaust fans with variable-frequency drives

- two HOG exhaust fans with variable-frequency drives

- two R2T2 tunnel exhaust fans with variable-frequency drives

- ductwork manifold (34-40 in. 304L SS)

- $\quad$ vertical stack (40-80 ft high)

- fan isolation dampers

- controls and instrumentation

Each exhaust fan system will be designed in an $\mathrm{N}+1$ configuration, with two full-capacity fans. Should one fan fail or be shut down for maintenance, the second fan will be capable of meeting the full airflow requirement. All exhaust fans will be served by emergency power.

Control System: CEF II will be connected to EPICS.

\section{Equipment Schedules}

The CEF-II mechanical requirements are summarized in Table 6.17.

Table 6.17. CEF II equipment schedules

\begin{tabular}{|c|c|c|c|c|c|}
\hline Equipment & Manufacturer & $\begin{array}{l}\text { Size } \\
\text { (each) }\end{array}$ & Quantity & $\begin{array}{c}\text { No. on } \\
\text { emergency } \\
\text { power }\end{array}$ & Remarks \\
\hline $\begin{array}{l}\text { Primary confinement } \\
\text { exhaust fans }\end{array}$ & Spencer Blower & $\begin{array}{l}4000 \text { CFM @ } \\
40 \text { in. WG }\end{array}$ & 2 & 2 & $\begin{array}{l}1 \text { blower is backup. } \\
50 \mathrm{hp} \text { motor each }\end{array}$ \\
\hline $\begin{array}{l}\text { Secondary confinement } \\
\text { exhaust fans }\end{array}$ & Twin City Fan & $\begin{array}{l}12000 \text { CFM @ } \\
20 \text { in. WG }\end{array}$ & 2 & 2 & $\begin{array}{l}1 \text { fan for backup. } \\
60 \mathrm{hp} \text { motor each }\end{array}$ \\
\hline $\begin{array}{l}\text { Hot-off gas confinement } \\
\text { exhaust fans }\end{array}$ & Spencer Blower & $\begin{array}{l}400 \text { CFM @ } \\
60 \text { in. WG }\end{array}$ & 2 & 2 & $\begin{array}{l}1 \text { blower is backup. } \\
10 \text { hp motor each }\end{array}$ \\
\hline $\begin{array}{l}\text { R2T2 tunnel exhaust } \\
\text { fans }\end{array}$ & Twin City Fan & $\begin{array}{l}3000 \text { CFM@ } \\
5 \text { in. WG }\end{array}$ & 2 & 2 & $\begin{array}{l}1 \text { fan for backup. } \\
5 \mathrm{hp} \mathrm{motor} \text { each }\end{array}$ \\
\hline
\end{tabular}




\section{Electrical Systems}

\section{Exterior Power Distribution}

The $480 \mathrm{Y} / 277 \mathrm{~V}$ electrical supply to CEF II will be provided by connecting to a 400 A circuit breaker in the outdoor unit substation located on the north side of the $90 \mathrm{M}$ instrument bay. A 400 A duct bank will be routed from the substation to a disconnect switch located in the variable-frequency drive/electrical room.

\section{Interior Power Distribution}

A 400 A automatic transfer switch will be provided to serve the entire facility.

From the $400 \mathrm{~A}, 480 \mathrm{Y} / 277 \mathrm{~V}$ emergency distribution panel, power will be distributed to lights and receptacles, exhaust fans, and their associated mechanical support equipment and control systems.

\section{Emergency Power Distribution}

Emergency power to CEF II will be provided by connecting to the generator located on the north side of to $90 \mathrm{M}$ instrument bay. A duct bank will be routed from the generator to a disconnect switch in the variable-frequency drive/electrical room.

The entire CEF II will be provide with emergency power, including the exhaust fans and all supporting mechanical systems and controls.

\subsubsection{Shop Building}

\section{Programming}

The shop building will house multiple shop functions necessary to support the STS and required upgrades to the proton beam. New shop space is needed to replace existing space in the klyston gallery. It will be used to house additional klystron equipment related to the accelerator upgrade and to maintain the increased amount of additional technical equipment that will be associated with the STS project. This space also will be used as assembly area during the accelerator upgrade period. A summary-level program for the shop building is given in Table 6.18.

Table 6.18. Shop building programming summary

\begin{tabular}{lc}
\hline \multicolumn{1}{c}{ Space type } & Area (NSF) \\
\hline Cryogenics shop & 6,000 \\
Pump shop & 2,500 \\
RF shop & 6,000 \\
Modulator shop & 6,000 \\
Gross/net factor & 1.4 \\
Total net area & $\mathbf{2 0 , 5 0 0 ~ G S F}$ \\
\hline
\end{tabular}

The net areas required for shop space have been estimated based on parametric comparison to the FTS shop space with input from ORNL staff supporting current shop operations. The grossing factor of 1.4 is estimated based on comparable buildings and includes space for general building circulation, toilets, exterior wall, mechanical, electrical, plumbing and other spaces not included in the net program areas. 
The shop building will be a high-bay one story building with partial mezzanine areas, located adjacent to the Klystron Gallery on the south side of Los Alamos Drive. Proximity to the accelerator is required for the new shop building. It will be accessible by tractor-trailer and will include two 20 -ton bridge cranes. The mezzanine floor levels will be constructed with modular pre-fabricated systems and will be connected with the primary floor by convenience stairs.

\section{Structural Systems}

The shop building will be a conventional steel-framed building supported on friction piles because of the anticipated poor soil at the proposed location on the ORNL site. Concentric braced frames will be used to resist environmental events (wind and seismic).

The shop building will support the exterior wall system with steel girts. The roofing system will be supported on a 1.5 in. galvanized metal roof deck on steel beams.

Mezzanine floors will be constructed of reinforced normal-weight concrete on a composite metal deck (galvanized).

The slab on grade will be a minimum of 8 in. thick reinforced normal-weight concrete slab.

The shop building will meet PC-1 performance requirements in accordance with DOE standards.

\section{Building Enclosure Systems}

The shop building will be designed to be architecturally compatible with the existing SNS campus and other new buildings making up the STS. The exterior wall will be approximately $80 \%$ architectural-grade metal insulated panels and 20\% low-E insulated glass in a painted metal integrated strip window system. The building will include six pedestrian entrances with overhead canopy protection and two grade-level service entrances with protected overhead door clearance to allow tractor-trailer access in the general vicinity of the building and small truck access into the building.

\section{Life Safety Systems}

The shop building will be classified as moderate-hazard factory industrial occupancy. The building will be fully protected Type IIB construction. The single story building will have a minimum of two direct exits to grade in addition to four convenience entrance/exits. Convenience stairs will connect the two floors.

\section{Mechanical Systems}

\section{HVAC Ventilation}

Shop areas will have a minimum of eight air changes per hour at a virtual ceiling height of $10 \mathrm{ft}$ or 1.2 $\mathrm{CFM} / \mathrm{ft}^{2}$. Shop areas have been assumed to have an average requirement of $20 \%$ outside air.

\section{Control System}

The shop building will be controlled by EPICS. 


\section{HVAC Equipment}

The conditioned spaces identified above will be heated and cooled using packaged rooftop airconditioning units located on the roof of the shop building. The air-conditioning units will be provided with an economizer mixing box, filtration, condensing unit section, and direct-expansion cooling coil. Each unit will be provided with an air-side economizer. Relief air will infiltrate through the roof via barometric dampers.

Mechanical, electrical, and utility spaces will be conditioned using a heating and ventilation approach. Ventilation will be achieved through the use of roof-mounted exhaust fans and associated wall-mounted intake air louver with motorized dampers. These spaces will be heated by heating water unit heaters located within the space. Exhaust fans for toilet areas, exhaust hoods, or process exhaust will be provided.

\section{Shop Area Air-Conditioning System}

The shop areas of the building will be served by two VAV, factory-fabricated packaged air-conditioning units. The air-conditioning units will be designed as a cooling-only, single-duct type. The unit will operate during hours of occupancy or as required to maintain night setback control temperatures. A variable-frequency drive will provide the capability of setback control of the supply fan volume, based on the supply ductwork static pressure set point.

The units will be provided with air-side economizer control. Relief air from the shop areas will be routed through wall-mounted louvers and associated barometric relief dampers.

The supply distribution system will consist of low-pressure ductwork with pressure-independent, electrically actuated supply VAV air terminal devices, heating water reheat coils, and low-pressure ductwork downstream of air terminals to duct-mounted air diffusers. One VAV air terminal will be provided for each major shop area.

The packaged air-conditioning units will be of painted steel construction. The units will consist of the following components in the direction of air flow:

- $\quad$ MERV 8 (30\%) efficient prefilter bank

- MERV 14 (90\%) efficient filter bank

- direct-expansion cooling coil

- supply fan

- isolation/smoke damper

- variable-frequency drives to modulate fan air flow

- integral refrigerant condensing unit

No provisions will be made for reserve capacity and redundancy.

\section{Heating Water System}

The heating water system for the building will be supplied from the existing CUB. The estimated heating water flow rate to the building is expected to be approximately 14 GPM. Based on this estimated flow, the anticipated heating water branch line sizes to the building are expected to be $1.5 \mathrm{in}$.

\section{Equipment Schedules}

The mechanical requirements for the shop building are summarized in Table 6.19. 
Table 6.19. Shop building preliminary mechanical equipment schedule

\begin{tabular}{|c|c|c|c|c|c|}
\hline Equipment & Manufacturer & $\begin{array}{c}\text { Size } \\
\text { (each) }\end{array}$ & Quantity & $\begin{array}{c}\text { No. on } \\
\text { emergency } \\
\text { power }\end{array}$ & Remarks \\
\hline $\begin{array}{l}\text { AHU_-shop bldg. } \\
\text { areas }\end{array}$ & Trane & $\begin{array}{l}35 \text { ton unit, } \\
14,000 \mathrm{CFM}\end{array}$ & 2 & $\mathbf{0}$ & MCA-87 A $(480 / 3)$ \\
\hline Exhaust fans & Cook & Varies & Estimated at 4 & 0 & $\begin{array}{l}\text { Roof-mounted, some with } \\
\text { 1-phase and some with 3- } \\
\text { phase power }\end{array}$ \\
\hline $\begin{array}{l}\text { Heating water unit } \\
\text { heaters }\end{array}$ & Trane & Varies & Estimated at 6 & 0 & $\begin{array}{l}\text { Supplied with heating } \\
\text { water }\end{array}$ \\
\hline
\end{tabular}

\section{Fire Protection Systems}

The fire sprinkler system for the shop building will be hydraulically designed to provide water densities that meet the requirement for Ordinary Hazard, Group 2 protection throughout the facility.

\section{Electrical Systems}

\section{Exterior Power Distribution}

The $480 \mathrm{Y} / 277 \mathrm{~V}$ electrical supply to the shops will be provided by connecting to a $600 \mathrm{~A}$ spare circuit breaker in existing unit substation KL-SS1 located on the south side of the Klystron Gallery. A 600 A duct bank will be routed from the substation KL-SS1 to a distribution panel in the shops electrical room.

\section{Interior Power Distribution}

From the $600 \mathrm{~A}, 480 \mathrm{Y} / 277 \mathrm{~V}$ distribution panel, power will be distributed to a lighting panel, two equipment and receptacle panels, and a mechanical equipment panel.

\section{Emergency Power Distribution}

Emergency power will be provided by connecting to an existing emergency panel in the Klystron Gallery.

\subsubsection{Discipline-Specific Overview}

The following criteria are applicable to all buildings in the previous sections.

\section{Structural Systems Overview}

Table 6.20 summarizes the structural standards for the STS. 
Table 6.20. STS structural standards summary

\begin{tabular}{ll}
\hline \multicolumn{1}{c}{ Item } & \multicolumn{1}{c}{ Remarks } \\
\hline Codes & IBC 2012, ASCE 7-10, ASCE 4-98, DOE 1020-2012 \\
Wind loads & ASCE 7 Risk Category II-IV \\
& IW = 1.0, Vult = 120 mph, Exposure C \\
Seismic criteria & ASCE 7 Seismic Design Category (SDC) 1-3 (assumed) \\
Foundations & Spread footings (assumed allowable bearing 2,500 lb/ $\mathrm{ft}^{2}$ ), friction piles, and micropile to rock \\
Separation joints & $\begin{array}{l}\text { Separation joints will occur between the superstructure of some buildings and bridges in an } \\
\text { effort to isolate buildings and allow differential movement and/or limit the transmission of }\end{array}$ \\
& $\begin{array}{l}\text { vibration from equipment } \\
\text { Project delivery }\end{array}$ \\
& $\begin{array}{l}\text { Means and methods sequencing and logistics will affect settlement (including rebound). The } \\
\text { tie-in to the existing tunnel is one such item. Water and groundwater management will impact } \\
\text { project delivery and costs }\end{array}$ \\
Geotechnical report titled Report of Phase III Geotechnical Exploration-The Spallation \\
Gasis of Design & $\begin{array}{l}\text { Neutron Source Oak Ridge Tennessee prepared by LAW/LAWGIBB group dated June 30, } \\
\text { 2000. }\end{array}$ \\
\hline
\end{tabular}

\section{Building Enclosure Systems Overview}

The exterior architectural design of the STS will complement the existing Chestnut Ridge campus in form, scale, material, color, and texture. Portions of the complex that support the general staff and user populations will be developed with amenities, finishes, and landscaping comparable to that of the SNS facility. Exterior utility and service areas will be developed to provide long-term durability and minimal maintenance requirements.

Below-grade walls of the STS buildings and tunnel structures will be constructed of waterproofed, normal-weight reinforced concrete. All subgrade construction that encloses interior space will be constructed to ensure permanent watertightness, filtered exterior drainage, granulated backfill, and wall protection. Exterior drainage will be a gravity-based system unless that is not feasible; if not, pumping systems will be provided. Drainage systems will include groundwater monitoring. Interior subgrade space will be provided with floor drains. Tunnels will be waterproofed on all four sides.

Exterior wall systems will be comparable to those of existing campus facilities and will vary depending on the use and exposure of the building. Generally, the exterior material systems include architecturalgrade concrete and various grades of insulated composite metal panel systems. The ratio of glazing to solid exterior wall is described in more detail within the narrative for each building. Exterior doors will be industrial-grade painted metal similar to existing doors.

Roofs throughout the complex will meet the requirements of the Cool Roof Rating Council Program, mandated by DOE, including a minimum R-30 insulation value, minimum 3-year aged solar reflectance of 0.55 , minimum 3-year aged thermal emittance of 0.75 , or a minimum 3-year aged solar reflectance index of 64 in accordance with ASTM Standard E1980-01.

Unless established otherwise on the basis of use, the STS will be designed to achieve a performance level of LEED Gold and meet the requirements of the DOE High Performance Sustainable Buildings Guiding Principles. 


\section{Life Safety Systems Overview}

The STS buildings will generally be classified by IBC as moderate-hazard factory industrial occupancy (Target Building II, supporting tunnels and utility structures, instrument buildings, and the Zeemans building) and as business occupancy (CLO II). Incidental occupancies of other classifications will exist within these general categories, as identified in the individual building sections of this report,

The minimum construction type for the STS buildings will be Type IIB as defined by the IBC. Where required because of the area and/or height of a specific building, Type IIA construction may be used to meet IBC requirements.

Fire barrier locations and corresponding ratings will be provided as is determined to be necessary via detailed fire hazard and code analysis that will occur in subsequent design phases. Hazardous material control areas will be provided in facilities containing laboratories and/or using significant quantities of hazardous materials.

Means of egress from the STS buildings, including the supporting tunnels, will be provided in accordance with the requirements of NFPA 101, Life Safety Code, and the IBC. An appropriate number of exits will be provided for the STS buildings so that the travel distance to an exit does not exceed $300 \mathrm{ft}$ for business occupancy areas and $400 \mathrm{ft}$ for the moderate hazard industrial occupancy areas.

Emergency lighting will be provided in the means of egress and other key areas of the buildings and structures. Emergency light units will be powered by an emergency generator. Means of egress and exits from the buildings will be marked with approved exit signs. Emergency lights and exit signs will typically be powered by emergency generators.

\section{Mechanical Systems Overview}

\section{Indoor Temperature and Humidity Design Conditions for Spaces Served with HVAC.}

The design conditions for spaces supplied with HVAC and for unconditioned space (e.g., mechanical rooms, electrical rooms, and utility rooms) are given in Tables 6.21 and 6.22.

Table 6.21. Indoor design conditions for conditioned spaces

\begin{tabular}{lc}
\hline Summer dry bulb temperature & $74^{\circ} \mathrm{F} \pm 2^{\circ} \mathrm{F}$ \\
Winter & $70^{\circ} \mathrm{F} \pm 2^{\circ} \mathrm{F}$ \\
Relative humidity & $<60 \%$ \\
\hline
\end{tabular}

Table 6.22. Indoor design conditions for unconditioned spaces

\begin{tabular}{lc}
\hline Summer dry bulb temperature & $100^{\circ} \mathrm{F}$ maximum \\
Winter & $55^{\circ} \mathrm{F}$ minimum \\
Relative humidity & No requirement \\
\hline
\end{tabular}

Active humidity control will not be provided for any building. Therefore, HVAC units will not have a humidification capability. The maximum relative humidity should not exceed $60 \%$ as a result of moisture removal at the cooling coils. 


\section{Design Calculations}

The building heating loads have been sized using parametric data to establish a block heating load for the spaces, outside air loads, and process (reheat) loads, plus $20 \%$ extra capacity for future flexibility.

The cooling loads have been sized using parametric data to establish a block load plus $20 \%$ extra capacity.

\section{Pressure Relationships}

Most air-conditioned buildings will be designed to be positive to ambient pressure. Pressure relationships within the buildings will be positive, negative, or neutral to suit the functional requirement. Buildings that are conditioned using unit heaters and ventilation exhaust fans will be negative to ambient pressure.

\section{AHU Construction and Component Sizing}

Each unit will be custom, factory- fabricated, and constructed with 2 in. thick double walls and will be constructed of painted steel.

Maximum allowable nominal face velocities for all AHU components are as follows:

- air intake louvers (through free area): $350 \mathrm{ft} / \mathrm{min}$

- heating water coils: $500 \mathrm{ft} / \mathrm{min}$

- cooling coils: $400 \mathrm{ft} / \mathrm{min}$

- filters: $400 \mathrm{ft} / \mathrm{min}$

\section{Indoor Air Quality Control Methods}

Indoor air quality will be addressed by the following principal means:

- Minimum filtration-MERV 8 (30-35\%) prefilters and MERV 14 (90-95\%) final filters at the building AHU.

- No fibrous media exposed to the airstream will be allowed in the ductwork downstream of any AHU's final filter bank. Sound-attenuating flexible ducts at the office and support area diffusers will have woven nylon fabric type lining.

\section{Smoke Safety Considerations}

Smoke or fire/smoke dampers will be provided at the following locations in conformance with code requirements:

- penetrations of area or occupancy separation walls

- in air systems serving multiple floors with a capacity greater than $15,000 \mathrm{CFM}$, to isolate the air handling equipment 


\section{Ductwork Materials and Construction}

The supply distribution system will consist of low-pressure galvanized steel ductwork to the supply air diffusers. Ductwork that is routed through unconditioned spaces, ceiling spaces, or the exterior will be insulated. Ductwork routed through conditioned spaces will be uninsulated.

Ductwork will be constructed in accordance with SMACNA standards, and duct leakage will not exceed $2 \%$ for low-pressure ductwork.

\section{Chilled Water and Heating Water Piping Materials}

The chilled water piping system within the building will use copper or carbon steel for pipe up to 2 in. and grooved coupling or welded carbon steel piping for sizes above 2 in. Insulation for interior piping will be closed-cell elastomeric foam. Underground chilled water piping serving the building will be distributed in a pre-insulated, factory-fabricated piping system.

The heating water system will use copper pipe within the building. The pipe insulation will be rigid glass fiber. Underground heating water piping serving the building will be distributed in a pre-insulated, factory fabricated piping system.

\section{Seismic Criteria}

Seismic bracing will be in compliance with the ASCE 7-10.

Equipment mounted on isolators will be seismically braced using loose cables, telescoping pipes or box sections, angles or flat plates used as limit stops, or snubbers, either integral to or separate from the isolators. Nonrotating, fixed equipment will be bolted directly to floors or the structure.

\section{Control Systems}

Two control system networks will be included as part of the STS project. All new buildings, with the exception of CLO II, will be connected to an expansion of EPICS. Instrumentation and wiring will be provided and connected to a new EPICS PLC in each building. Programming and connection of PLCs to EPICS will be completed and the control system commissioned to ensure proper operation.

The mechanical and process support systems in CLO II will be controlled and monitored through a DDCbased BAS with distributed processing at the local level.

Electric actuation will be used for all control valves and dampers on both systems so that instrument air is not required. All control systems will be on UPS and emergency power. System software and firmware will provide the following functions:

- Control sequences will be developed to support the operation of the technical equipment.

- Proportional, integral, and derivative (PID) control to allow faster and closer control to system set points.

- Adaptive tuning to adjust PID loop constants to ensure that control system response remains accurate and reliable over a wide range of dynamic operating conditions. 
- Monitoring to read the value of measured variables; to read control loop set points, to monitor control signals to actuators; and to indicate status of equipment, alarms, and overrides.

- Energy management including optimum start/stop, variable air change rates in laboratories, duty cycling, supply air temperature reset, supply air static pressure reset, demand limiting, time totalization, and so on.

- Data management, including continuous database updating, alarm reporting, trend logging, and report generation.

- System programming to add delete or change points, set points, schedules, control algorithms, report formats, and so on.

- System software will allow building operators to graphically monitor and control building operations and provide the functions listed. Graphics will include site plans, overall building plans, floor plans and individual system graphics.

\section{Piping Systems Overview}

\section{Perimeter Foundation Piping Material}

A perimeter foundation drainage system will be provided. The groundwater collected by the foundation drainage system will be drained by gravity into the interior stormwater system, or outside the building into the campus storm sewer. Piping material will be flexible perforated PVC or PE (polyethylene) tubing.

\section{Building Piping Systems}

Piping mains will be sized for the maximum calculated flow at the design stage plus $10 \%$ for future expansion. Piping connections to equipment will be arranged so that a minimal amount of piping must be removed for servicing of equipment. Valves will be provided at each piece of equipment for service isolation of the equipment from the system. Water service will be supplied from the underground campus potable water distribution system. Service will be sized for the maximum calculated flow at the design stage. Piping will be sized to maintain a minimum of $30 \mathrm{psi}$ residual pressure at the most remote toilet flush valve or emergency shower. Water velocity in distribution piping will not exceed $8 \mathrm{ft} / \mathrm{s}$. Provisions will be made to arrest water hammer. Shock arrestors, where installed, will comply with PDI-WH201 or ASSE-1010.

\section{Domestic Water System}

The entire cold water distribution system will be insulated. Piping material interior above-grade piping will be type L copper with wrought copper fittings and lead-free solder joints.

\section{Sanitary System}

Sanitary waste from general use mechanical equipment floor drains will be conveyed by gravity to the campus sanitary sewer system. Flow rates and pipe sizes will be calculated to allow for projected wastewater discharge from various items of mechanical equipment at the design stage. Floor drains that do not receive regular use will be provided with trap primers. 
Below grade, gravity bell-and-spigot cast iron soil pipe will be used. Above grade, gravity no-hub cast iron soil pipe will be used.

\section{Stormwater System}

Rainwater from roof areas will be conveyed by a separate gravity system into the site storm drainage system. The roof drainage system will be designed for a 100 year storm return frequency and a 60 minute period of duration/concentration. The emergency drainage system will be designed for a 100 year storm frequency and a 60 minute time of duration/concentration. The emergency roof drainage system will discharge above grade and will be exposed to view. Insulation 1 in. thick with a vapor barrier will be provided for all horizontal rainwater conductors, including elbows, roof drain basins, and vertical piping on the underside of the roof.

Below grade, bell-and-spigot service weight cast iron soil pipe with push-on joints will be used. Above grade, no-hub cast iron soil pipe will be used.

\section{Compressed Air System.}

Compressed air at 115 psi will be distributed throughout the site to support maintenance operations. It will be provided with local pressure-reducing valves to permit the use of air of various pressures. Piping mains will be sized for the maximum calculated flow at the design stage and will limit air velocities to $5,000 \mathrm{ft} / \mathrm{min}$ with a maximum pressure drop to the farthest outlet of not more than $5 \mathrm{psig}$ at estimated peak flow conditions.

Piping material will be type K copper with brazed joints.

\section{Elevator Sump Pumps}

Elevator hoistway pit sumps will be provided with a sump pump. The pump will be a simplex unit with an oil minder switch that will not allow the pump to operate if oil is detected. The pump will be located in a grated, covered sump pit in the elevator hoistway. An isolation valve and check valve will be installed in the discharge piping. The pump discharge will be directed to the building sanitary system. The elevator sump pump will be controlled with a float level switch and will have a simple plug-in power cord.

Elevators required for firefighter emergency operation will have a 50 GPM sump pump in accordance with ANSI A17.1.

\section{Plumbing Fixtures and Specialties}

All plumbing fixtures will be institutional-grade vitreous china or stainless steel as required.

Floor sinks and floor drains in laboratory areas (if required) will be stainless steel, flush with the finished floor, with a minimum 3 -in. outlet.

Hub drains and similar unsanitary fixtures will not be provided in laboratory spaces.

Water closets will be of wall-hung, siphon-jet type, high-efficiency 1.28 gal per flush maximum with hard-wired infrared sensor operation.

Urinals will be of wall-hung, siphon-jet type, high efficiency 0.125 gal per flush maximum with hardwired infrared sensor operation. 
Lavatories will be wall-hung or countertop type units with a 0.5 gal per minute maximum with hard-wired infrared sensor-operated faucets.

Showers will be high-efficiency units with a 1.5 gal per minute maximum.

Safety showers, eyewashes, and combination units with have a 30 psi minimum.

Electric water coolers with be recessed and self-contained.

Interior hose bibs will be chrome plated in finished areas and rough brass in mechanical rooms. They will be wall-mounted, will be furnished with vacuum breakers, and will have $0.75 \mathrm{in}$. hose threaded outlets.

Wall hydrants will be freeze-resistant in recessed boxes with 0.75 in. hose threaded outlets.

\section{Fire Protection Systems Overview}

\section{Buildings}

The STS buildings will be provided with complete automatic fire protection systems as required by the IBC and/or DOE Order 420.1C, Facility Safety. The design, installation, and acceptance testing of automatic sprinkler protection will be in accordance with NFPA 13, Standard for the Installation of Sprinkler Systems and DOE-STD-1066-2012, Fire Protection.

Fire sprinkler systems will be hydraulically designed to provide water densities that meet the requirements for Ordinary Hazard, Group 1 protection in office areas and general corridors. Ordinary Hazard, Group 2 protection will be provided in laboratories, shop areas, supporting tunnels, and utility structures. Extra Hazard, Group 1 protection will be provided in Target Building II and the instrument buildings.

Standpipe systems will be provided in the STS buildings as required by the IBC and/or DOE Order 420.1C, Facility Safety. The design, installation, and acceptance testing of standpipe systems will be in accordance with NFPA 14, Standard for the Installation of Standpipe and Hose Systems and DOE-STD1066-2012, Fire Protection.

Fire water service will be supplied from the underground campus potable water distribution system. Service will be sized for the maximum calculated flow at the design stage.

Portable fire extinguishers will be provided throughout the STS buildings in accordance with the IBC and NFPA 10, Portable Fire Extinguishers.

\section{Electrical Systems Overview}

The following electrical requirements are typical for all buildings or facilities. Refer to the electrical systems paragraph associated with each specific building section for electrical requirements unique to that building or facility.

\section{Power Distribution}

In general, the interface between the site distribution system and the building distribution system occurs at the load-side terminals of the feeder circuit breaker within the exterior unit substations. 
Where $480 \mathrm{~V}$ switchgear is provided, it will consist of electrically operated draw-out power circuit breakers with solid state trip units in accordance with UL 1558.

\section{Emergency Power Systems}

Emergency loads include egress lighting and exit signs, fire alarm and public address systems, instrument safety and control systems, and the security systems. Where provided, optional standby loads include select pieces of technical equipment necessary to prevent damage to equipment and/or loss of long-term experiments.

Generators will be located outdoors in reach-in, sound-attenuated, weatherproof enclosures with $24 \mathrm{~h}$ subbase fuel tanks. Five generators will be provided to support the STS facilities.

One generator will support the R2T2 support building, the makeup air structure, the STS and $40 \mathrm{M}$ instrument buildings, and the moderator support building. It will be rated at $300 \mathrm{~kW}$ at $480 \mathrm{Y} / 277 \mathrm{~V}$ and will be provided with four output circuit breakers to serve each of the four facilities.

A second generator will support the $90 \mathrm{M}$ instrument bay, HRB, HRP, and CEF II. It will be rated at $350 \mathrm{~kW}$ at $480 \mathrm{Y} / 277 \mathrm{~V}$ and will be provided with four output circuit breakers to serve each of the four facilities.

A third generator will be dedicated to the Zeemans facility. It will be rated at $500 \mathrm{~kW}$ at $480 \mathrm{Y} / 277 \mathrm{~V}$ and will be provided with a single output circuit breaker.

A fourth generator will support CLO II and CUB II. It will be rated $250 \mathrm{~kW}$ at $480 \mathrm{Y} / 277 \mathrm{~V}$ and will be provided with two output circuit breakers to serve the two buildings.

A central UPS will not be provided except at CLO II (see CLO II narrative). If select pieces of equipment need UPS backup, small point-of-use UPS units can be provided with the instruments.

\section{Grounding}

Each facility will be provided with a grounding electrode system that will consist of building steel, foundation rebar (UFER ground), a \#4/0 direct buried counterpoise around the perimeter of each facility, and metal underground supply pipes. Ground rods will be located at each corner and at increments not exceeding $100 \mathrm{ft}$. The grounding grids of adjacent facilities will be interconnected.

The power system grounding will be provided in accordance with the National Electrical Code. Separate green equipment grounding conductors will be provided with all feeder and branch circuits. Ground buses will be provided throughout the facilities for grounding of instruments. The telecommunication system will be grounding in accordance with Electronic Industry Alliance/Telecommunications Industry Alliance (EIA/TIA) standards.

A complete lightning protection system will be provided.

Neither active nor passive cathodic protection will be provided. Ferrous piping and/or pipe jacketing will be coated.

\section{Exterior Lighting}

Pole-mounted LED light fixtures will be provided along roadways, drives, and parking lots. 
Building-mounted LED light fixtures will be located around the perimeter of the each building at vehicular circulation areas, walkways, and building exits.

Exterior lighting will be provided with photocell and time clock controls.

\section{Interior Lighting}

Interior lighting will be provided in accordance with Illuminating Engineering Society recommendations and ASHRAE 90.1 energy budgets.

Lighting will generally be provided by $\mathrm{T} 8$ or T5HO fluorescent lamps or LED lamps, depending on the space and application.

Utility spaces will generally be provided with pendant-mounted heavy-duty industrial reflector-type fluorescent fixtures.

High bay spaces will be provided with high bay LED fixtures.

All spaces will be provided with some type of automatic control to comply with ASHRAE 90.1.

\section{Telecommunication Systems}

A complete pathways and cabling voice over IP telecommunication system will be provided consisting of a telecommunication room, racks, cable trays, conduits, back boxes, copper cables, fiber optic cables, connectors, cover plates, terminal blocks, cross connect cables, patch panels and other accessories in accordance with EIA/TIA standards.

Each facility will be provided with a separate telecommunication service from the site telecommunications distribution system.

\section{Fire Alarm System}

The fire alarm system will be a complete manual and alarm notification system. The system will be noncoded, addressable, and microprocessor-based with initiating devices, notification appliances, and monitoring and control devices. Initiating and notification circuits will be Class B. For compatibility, all new equipment will match the existing campus system (Edwards Technology, Inc.). The fire alarm system will be installed and acceptance-tested in accordance with NFPA 72, National Fire Alarm Code.

The fire alarm system will report all fire, supervisory, and trouble alarms to the ORNL Fire Department and the Laboratory Shift Superintendent via fiber optics network.

Duct smoke detectors will be provided in air systems with a capacity greater than 2,000 CFM.

\section{Public Address System}

A public address system will provided and used for "mass notifications" and will be connected to the existing ORNL public address system.

\section{Security System}

The security system will consist of access controls. Central equipment will be located in the telecommunications room. 
For compatibility, all new equipment will match the existing ORNL system. Supervisory and alarm conditions will be reported to the central monitoring station.

\subsection{SITE UTILITIES}

\subsubsection{Power Distribution}

The power distribution for the STS campus will originate at the existing Tennessee Valley Authority (TVA)/SNS Switch House, Building 8912. Two existing circuit breakers will be used to provide electrical service to the proposed buildings. One circuit will feed the STS facilities and one circuit will feed the Zeemans facilities.

The feeders will consist of concrete-encased duct banks buried underground starting near the Switch House and proceeding along the south side of the site along Spallation Drive. The feeders will continue around Argonne Drive and connect with the existing duct banks on the northwest side in manhole M26. Duct banks will also spur off the loop toward equipment locations close to the associated buildings. Additional manholes will be installed at convenient locations for pulling and dispersing the $15 \mathrm{kV}$ cables.

Three separate switchgear units will be located at strategic locations across the STS to distribute the power from the loop into dedicated unit substations. Each unit substation will include a transformer to reduce $13.8 \mathrm{kV}$ power to the required voltage for the associated facility.

A separate feeder will be provided from the Switch House through the existing duct banks on the north side of the FTS site to provide additional reliability and flexibility during outages.

\section{Temporary Electrical Service}

Temporary electric service with distribution will be provided for construction of this project. The temporary service will be located in the construction/AE/CM trailer/laydown/parking area.

\subsubsection{Communications System}

The following functions will require interconnecting cabling between the new STS buildings and the existing CLO:

- IT

voice over IP, business computing, experimental data, and similar services

Telephone (copper cables)

Public address

- Fire alarm system

- Building access control and security

- Building automation and control systems

These systems will require fiber optic cable connectivity between CLO IT rooms J-114 and J-115 to the new STS buildings. Existing spare conduits will be used in the existing underground communications duct bank system to connect to the existing CLO through manhole 17. The communications system duct bank will be extended east of manhole 17 to serve the new STS buildings. The duct banks will continue 
around Argonne Drive and connect to the existing communications duct bank for the northwest side at manhole M25.

The IT systems networking equipment and cabinets will be supplied by the SNS IT organization. This system will be compatible with the existing SNS systems. The STS project will provide interconnecting fiber optic cabling between existing and new facilities.

The fire alarm system will be compatible with the existing Edwards EST3 Emergency Communications Platform and will report to the ORNL LSS office.

Building access and security for the STS facilities will be compatible with the existing ORNL building access system.

\subsubsection{Instrument and Control Distribution Description}

The following functions will require interconnecting cabling between the new STS buildings and the existing CLO:

- $\quad$ EPICS

- electrical power monitoring and controls

- personnel protection system

- machine protection system

- $\quad$ target protection system (two conduits)

These systems will use fiber optic cabling routed through the communications duct banks, as indicated in Sect. 6.3.2.

\subsubsection{Ventilation Exhaust}

Site ventilation exhaust will consist of tunnel ventilation from the R2T2 tunnel, Target Building II PCE and SCE, and Target Building II HOG exhaust. All exhaust fans will be located on an equipment pad located to the north of the STS building. All underground ductwork will be constructed of HDPE plastic and will be routed approximately as indicated on the exhaust utility distribution site layout, with estimated duct sizes as indicated. Ductwork that is crossing the neutron guide tunnels should be evaluated to ensure that there are no radiation issues.

\subsubsection{Mechanical Systems}

Site mechanical hydronic piping will include chilled water, heating water, condenser water and tower water piping. Chilled water and heating water will originate at the CUB II and be distributed to several buildings in underground piping as shown on the Proposed Hydronics System Layout. Condenser water and tower water will originate at the cooling towers to the east of CUB II. Condenser water will be distributed to CUB II for heat rejection from the chiller plant, and tower water will be distributed to most technical buildings for equipment cooling systems. Underground chilled water and heating water piping serving the buildings will be distributed in pre-insulated, factory-fabricated piping systems. Tower water and condenser water serving the buildings will be distributed in factory-coated, black steel piping systems. Estimated pipe sizes for each piping system are also indicated on the site plan.

Site compressed air piping will be distributed to several buildings in underground piping as shown on the Proposed Site Compressed Air layout. Compressed air serving the buildings will be distributed in a 
factory-coated black steel piping system. Estimated pipe sizes for compressed air piping are also indicated on the site plan.

\subsubsection{Sanitary Sewer}

Sanitary sewer service will be provided to the proposed STS, associated buildings, and Zeemans facility by connecting to the existing SNS wastewater collection system. The proposed STS, CLO II, and Zeemans instrument sanitary flow will be routed to the main SNS site pump station. The CUB II, $90 \mathrm{M}$ instrument bay, HRB, HRP, and CEF II wastewater flow will be routed to the CNMS grinder pump station. The shop building located on the west side of the SNS campus will connect to an adjacent force main via a proposed pump station and force main.

The existing sanitary sewer collection system was designed with enough capacity to serve the proposed STS and associated buildings. Most of the SNS water usage is for cooling and thus is not returned to the wastewater collection system. The current measured sewer flow is around 16,000 gal/day, which is significantly less than the calculated design rate of 63,000 gal/day. The SNS pump station also has a higher measured capacity than the design parameters (300-350 GPM measured versus 200 GPM design capacity). The sanitary sewer system would have adequate capacity to handle additional flows from the STS. If flow from the proposed CUB II, $90 \mathrm{M}$ instrument bay, HRB, HRP, and CEF II exceeds the CNMS grinder pump station design flow, the pump station will need to be upgraded with larger pumps. The flow parameters will be determined as facility buildings are refined, although these buildings are not heavy wastewater producers. It is also assumed that the sanitary sewer will exit the STS at the same elevation as the FTS. If the exit elevation is at a lower elevation, pumping from the building will be required.

The SNS main pump station removes sanitary sewer flow from the SNS site and pumps it into the ORNL main campus sanitary system at manhole M618. The sanitary sewer line downstream from manhole M618 is 6 in. PVC pipe and is currently flowing at capacity when the SNS pump station is active. It is recommended that this 1,200 linear foot section between manhole 618 and manhole 608 be upsized to at least $8 \mathrm{in}$. in in diameter to convey the flow. This line may need to be larger than 8 in. depending on future developments planned in the 7000 area.

Low-pressure sewers and pumps at the laydown area should be considered for temporary use during construction.

\subsubsection{Fire Protection and Potable Water System}

\section{System Components}

A water booster station withdraws water from a 24 in. water line on the east side of the SNS site and pumps that water to an elevated water storage tank. The pump station has a firm capacity (capacity with one pump out of service) of 1,100 GPM. A dedicated transfer line connects the pump station and storage tank. The multileg elevated storage tank has a storage capacity of 300,000 gal. The storage tank feeds the site water distribution piping and fire protection system, which distributes water by gravity to all of the buildings on the site at the required flow and pressure.

The potable water system serves several purposes. It provides (1) drinking water, water for sanitary purposes, and miscellaneous process water (e.g., for cleaning, filling of systems) for the Chestnut Ridge employees and visitors; (2) makeup water for the site cooling towers; and (3) water for fire suppression. The largest single system demand is for fire suppression. 


\section{Existing System Design Capacity}

The distribution system piping system is sized to provide the design flows (potable, process, cooling water, and fire) at the required residual pressures. The potable, process, and cooling water demand capacity was designed for a peak day water system demand of 640 GPM. Fire suppression flows, required only during fire events, are not included in the normal peak day flow but are included in system design. The fire flow design rate provides 1440 GPM for a $2 \mathrm{~h}$ duration. It should be noted that the existing water system was designed using system demands that included the installation of a second target station in the future.

The 1,100 GPM pump station capacity was based on (1) the normal peak day flow, (2) the flow rate calculated to refill the storage tank fire storage volume in $8 \mathrm{~h}$, and (3) a 10\% design contingency. The 300,000 gal storage tank is the sum of the following volumes: (1) tank operating volume, which provides for adequate starting and stopping of pump station pumps; (2) a fire suppression volume, which is equal to the highest fire flow rate (1,440 GPM) for a duration of $120 \mathrm{~min}$; and (3) an emergency storage volume, which provides the design potable water, cooling tower, and process flows for a period of $3 \mathrm{~h}$. The target station fire flow requirements govern the tank elevation to provide the required residual pressure.

\section{Historical System Demand Data}

Peak water flow demands are influenced by operation of the accelerator and seasonal building skin heating. Therefore, the highest water usage occurs when the accelerator is running during the summer. Records from summer 2013 were examined and show that the maximum usage was 310,616 gal on September 24, 2013. The existing water system has historically delivered all system demands with adequate flow and pressure based on the 2009 STS study. All fire flow tests to date have been acceptable. The only concern expressed has been the limited $(3 \mathrm{~h})$ emergency storage volume in the elevated water storage tank.

There have been no water system modifications since the initial system installation which would significantly affect the water system's capacity to deliver the design water demands to the site.

\section{Future Demand Projections}

The future system demand would be influenced by the addition of the STS, CLO II, and instrument buildings and tunnels, as well as the Zeemans instrument. The anticipated demands will require $69,062 \mathrm{gal} /$ day of additional flow.

Coupling these projected demands with the historical peak day demand results in a peak day flow of $379,678 \mathrm{gal} /$ day. Applying the peak flow over a $10 \mathrm{~h}$ day results in a peak flow rate of $633 \mathrm{GPM}$ (compared with the design flow rate of $640 \mathrm{GPM}$ ). These changes result in a 107 GPM safety factor (approximately 11\%) at the pump station. The elevated storage tank has an emergency storage time of $3 \mathrm{~h}$. The limited emergency storage volume leaves SNS vulnerable if water delivery is interrupted.

The booster pump station can remain in its current configuration as long as its location is not affected by other operations at the STS. The pump station has three pumps. Two of the pumps are required to fill an empty tank and the third is a dedicated standby. The third pump is locked out to prevent its starting while the other two are in operation. Operating three pumps simultaneously can result in low pump suction pressure and deenergize all of the pumps. The Utilities Division has a fourth pump in inventory in case one of the three pumps must be removed from service. One pump can generally meet the daily operational demand (potable, process and cooling tower makeup water), and the pump cycle time is dependent on the 
tank settings. For the purposes of this report, an additional pump was included in the improvements. Additional hydraulic modeling will be required before adding more pumps to the booster station to verify low suction pressures will not occur.

ORNL is supplied water from the City of Oak Ridge via a single 24 in. cast iron water line. There is no redundancy on this line; therefore, should delivery be interrupted, ORNL and SNS will be left with limited water for fire protection and domestic usage. There is also no redundancy in the water feed line from the booster pump station to the elevated storage tank. The limited elevated tank volume is an item of concern at the SNS site. Two 1.1 million gal ground storage tanks are planned as part of a separate project that will provide additional emergency water storage on Chestnut Ridge. Water will be withdrawn from the 24 in. water line upstream of the existing booster pump station to fill the ground storage tanks. A separate pipe will return water to the $24 \mathrm{in}$. water line upstream of the existing booster pump station. The planned ground storage tanks should be constructed before or during construction of the STS. The ground storage tanks are not included in the STS scope but will be constructed as a separate ORNL project.

It should be noted that the existing booster pump station can exceed the required fire protection pressures with the elevated storage tank out of service. Operating the system with the elevated tank out of service is currently a manual exercise. Control panel modifications should be made to automate this process to minimize overpressurizing of the fire protection system. For the purposes of this report, a standby fire pump was included in the improvements. Additional hydraulic modeling will be required before adding more pumps to the booster station to verify low suction pressures will not occur. The pump and a redundant elevated tank supply line may need to be installed with suction directly from the ground storage tanks to minimize suction pressure problems.

Modifications to the underground water supply system will be of the looped grid type providing multiple flow paths, with sectional valves installed and arranged to provide alternate flow paths to any point in the system. Fire mains will be at least 8 in. in diameter, except that those supplying a single fire hydrant may be 6 in. in diameter. Water supply mains will be sized to supply the largest expected fire suppression flow demand plus the largest potable water domestic demand. The design and installation of all combined potable and fire water supply system components will be in accordance with the requirements of NFPA 24, Standard for the Installation of Private Fire Service Mains and Their Appurtenances.

Water will be provided to the STS, CLO II, CUB II, CEF II, R2T2 tunnel and support building, Zeemans facility shop building, cooling tower, and neutrino facility from existing water line loops around the site. The fire protection, potable water demands, and line sizes will be determined as the facility buildings are further developed. Additional demands are expected to total approximately 70,000 gal/day.

Beam line 22 construction and site grading will conflict with two water lines near Argonne Drive. These water lines should be relocated on top of the beam line tunnel before each line is taken out of service. The first line is the dedicated feed line from the booster pump station that fills the elevated storage tank. An adjacent distribution line parallel to this line should be relocated in conjunction with beam line 22 . The beam line construction and grading should be phased to minimize disruptions to the Chestnut Ridge water system. The size and depth of the beam line would otherwise cut off all water to the elevated storage tank. After a portion of the beam line has been constructed and backfilled, the water lines (including the critical tank feed line) can be constructed over the beam line. Once these water lines are operational, the beam line construction may proceed to the north. The existing lines would then be abandoned and could be removed during excavation of the beam line. Sufficient clearance and shielding should be provided between the water lines and the beam line.

The current STS parking lot plan is designed so that the 24 in. water line that feeds the main ORNL campus will not be impacted significantly. The parking lot entrance grading will place some fill over this 
line. The depth of cover on the 24 in. water line currently cannot exceed $11 \mathrm{ft}$, and proposed grading operations consider this requirement. Should the fill exceed $11 \mathrm{ft}$ or additional protection be desired because of construction traffic, special pipe bedding and/or encasement can be provided on the affected section to prevent disruption of water service on this vital line. The parking lot plan should not infringe on the water pump station, nor should the elevated storage tank be relocated from its current position. Because the elevated storage tank will not be relocated, it is recommended that the STS be set at the same elevation as the FTS, or lower, to develop adequate fire flow residual pressure.

Temporary construction water should be provided from the SNS distribution system rather than from the 24 in. ORNL transmission main.

The existing unit substation at the booster pump station should be adequate to serve the additional fire pump. However, the existing generator will be replaced with a $300 \mathrm{~kW}, 480 \mathrm{Y} / 277 \mathrm{~V}$ generator. The generator will serve the existing pumps and the new fire pump.

\subsubsection{Zeemans}

The Zeemans instrument facility will be served via proposed water lines from the STS. Given the proposed service tie-in location of the STS, only a small extension to the water and sewer lines will be necessary to serve the Zeemans facility.

Because it will have a large power demand, dedicated power will have to be routed from the existing Switch House to the Zeemans facilities. The feeders will be distributed through conduits in concrete encased duct banks.

Communication and controls cabling will also be extended from CLO II to the Zeemans facility through duct banks connected along the loop road.

\subsection{ASSUMPTIONS/ALTERNATIVES/RISKS/FURTHER DEVELOPMENT}

During subsequent STS design phases, it is anticipated that the following alternatives, which may impact project scope or design approach, will be further evaluated:

\subsubsection{Assumptions}

- An ORNL Institutional General Plant Project (IGPP) will install two large ground-based water tanks on Chestnut Ridge to serve ORNL.

- An ORNL IGPP will replace the undersized sanitary sewer line at the main ORNL campus

- A programmatic GPP will fund a Chestnut Ridge conditioned warehouse to accommodate current storage needs. No new conditioned warehouse needs have been identified beyond those served by the new shop.

- No new HVAC system is needed for existing Klystron Gallery because of STS power upgrades in any area, except for the area of the Klystron Gallery that will contain the new RF equipment to support the new cryomodules. Existing Klystron Gallery cooling issues will be resolved by cooling the equipment, not by adding to the building HVAC system. 
- No new HVAC is needed in the Ring Service Building because of increased heat loads from new equipment required for the STS accelerator upgrades.

- The existing TVA/SNS substation has adequate capacity to supply the total power usage requirements for the STS project. A study will validate this assumption.

\subsubsection{Alternatives}

- Evaluate the provision of a second elevated water storage tower will be evaluated as an alternative to the direct-feed automatic fire pump being provided for system redundancy and the additional potable water pump being provided to meet the Zeemans potable water demand.

- Evaluate the feasibility of routing PCE, SCE, and HOG systems that serve Target Building II through the building vs underground across the neutron guide area.

- Conduct a study to determine if the resonance control cooling system chilled water load must be served by a dedicated system rather than the central system to provide the required cooling and stability for STS operation.

- Optimize the location, size, and shape of parking areas.

\subsubsection{Risks}

The following are risks in the design which were identified for consideration and development in subsequent design phases:

- The connection of the R2T2 tunnel with the RTBT tunnel to ensure continuity of operations

- The capability to run the site utilities to the $120 \mathrm{~m}$ instrument building

- The higher power requested by the Zeemans program

- The timing and location of the neutrino facility

- An unfavorable outcome of the geotechnical report

- Space allocation for the Zeemans building being insufficient

\subsubsection{Further Development}

The following is a list per building of items to be considered in subsequent design phases. These items are duplicated in each individual section.

\section{General}

- Evaluate seismic concepts once preliminary safety analysis is complete.

- Evaluate access to neutron tunnels.

- Confirm all programming assumptions to ensure proper space requirements.

- Evaluation optimum location of CLO II in relation to Target Building II.

- Evaluate the optimum elevation of major buildings.

\section{R2T2 Tunnel and Support Building}

- Confirm the estimated space requirements necessary to house the power and controls systems necessary to support the proton beam within the R2T2 tunnel. 
- Evaluate the design consequences of constructing the R2T2 service building and the Zeemans building at separate times in the event the Zeemans building is not constructed with the STS project.

\section{The Target Building II}

- Confirm the estimated space requirements necessary to house the basement-level utility systems that support the target and related systems.

- Evaluate the optimum configuration of the subgrade beam array on the east side of Target Building II.

- Evaluate the feasibility of including instrument stations within Target Building II on beam lines 11 and 22 .

- Relative to the Life Safety Code and IBC, comparatively evaluate the consequences of defining Target Building II as a single building or integrating it with the CLO II, CUB II and $30 \mathrm{M}$ and $40 \mathrm{M}$ instrument buildings.

\section{Instrument Buildings and Tunnels}

- Evaluate the optimum configuration of a tunnel array adjacent to Target Building II at the basement level.

- Evaluate the elevation of instrument floors relative to the overall topography and proton beam slope.

- Evaluate the potential for CLO II and the $40 \mathrm{M}$ instrument building to share freight and passenger elevators.

- Evaluate the egress from the neutron tunnels

\section{Zeemans Building}

- Confirm area requirements necessary to house support utility systems.

- Evaluate the consequences of separating the Zeemans support building, Zeemans instrument building, and R2T2 support building or, alternatively, of combining all three buildings into a single structure.

- Evaluate and define utility requirements especially with regard to electrical demand.

\section{Central Laboratory and Office II}

- Connect the CLO II to the CNMS by bridge.

- Relative to the Life Safety Code and IBC, comparatively evaluate the consequences of defining the CLO II as a single building or integrating it with Target Building II, CUB II, and the $30 \mathrm{M}$ and $40 \mathrm{M}$ instrument buildings.

- Relocate CNMS functions and use CNMS space for future programmatic needs. 


\section{Central Utility Building II}

- Confirm the estimated space requirements necessary to house the utility systems.

- Evaluate the optimum location of the CUB II cooling towers with respect to pedestrian movement and tower off-spray.

\section{Central Exhaust Facility II}

- Evaluate the optimum stack height for the exhaust stacks.

\section{Shop Building}

- Evaluate the feasibility of the shop building's being a two story, rather than a single story building. 



\section{INTEGRATED CONTROLS}

The integrated control system (ICS) for the STS will include controls for all of the new or upgraded accelerator, target, instrument beam line, and conventional subsystems. In general, these subsystems will be controlled in the same manner as or in a similar manner to existing subsystems already in use at the SNS. Therefore, for the most part, the control system design is "more of the same." This section describes the scope associated with managing the control system elements of this project.

\subsection{FIRST TARGET STATION CONTROLS}

The FTS integrated controls scope described in this section covers systems that are global in nature, like the accelerator systems leading to the FTS.

\subsubsection{First Target Station Global Control Systems}

\subsubsection{Integrated Control System Network and Computing Systems}

This includes network and computing needs that will be handled by expanding the existing SNS infrastructure. The communication services that will be required include Ethernet/IP, terminal service [e.g., to input/output (I/O) controllers (IOCs)], and remote reboot service. Three major categories of "global communications" will be required for the STS.

1. Ethernet Network

2. Timing System

3. Machine Protection System (MPS)

Hardware design and procurements are covered under their respective work sections. However, as it is more efficient to install all communication cables together, the cable design and installation costs for all three communication categories were rolled up in this work package.

The existing server infrastructure is sufficient to handle the FTS upgrades. The following are the computing systems activities included in the cost estimate.

- three Klystron Gallery Experimental Physics and Industrial Control System (EPICS) operator interfaces (OPIs) for equipment checkout and start-up in the field

- technical support for new ICS network nodes (e.g., interfaces to terminal servers and remote rebooters, network switch diagnostics and monitoring, new device registering, and IOC boot infrastructure and monitoring)

\subsubsection{Timing System}

The FTS timing requirements will be handled by expanding the existing SNS timing system infrastructure. Timing system equipment required for the FTS includes IOC timing receiver boards, timing link fanouts, and IOC fiber optic transmitter boards. The design will meet detailed timing system requirements for production and delivery of FTS and STS beam pulses. Timing equipment and quantities will be based on these requirements.

The installation of timing-system-related field cable is covered under Network Installation. (It was deemed to be more efficient to design and install all communication cables together.) Because of obsolescence, some of the timing hardware cannot be replicated and needs to be redesigned. 


\subsubsection{Machine Protection System}

The FTS machine protection requirements will be handled by expanding the existing SNS MPS. Equipment required includes MPS IOCs (MIOCs) and associated cables and termination hardware. The design will meet detailed MPS system requirements for production and delivery of FTS and STS beam pulses. The preliminary design includes assessing detailed MPS requirements for FTS equipment.

\subsubsection{First Target Station Linac Control System Requirements}

\subsubsection{Linac Radio Frequency Controls}

This includes implementing the following radio frequency (RF) control functions.

- EPICS interface to the new high-voltage converter modulators. Existing SNS controls will be duplicated. This covers IOC hardware and EPICS software development.

- $\quad$ EPICS interface to the new RF transmitters. Existing SNS controls will be duplicated as much as possible. Updates will be needed to accommodate the new cryomodule cavity tuner, coupler bias, and coupler cooling control system design. This covers the IOC hardware, programmable logic controller (PLC), and EPICS software development.

- $\quad$ EPICS interface to low-level RF systems. Existing SNS low-level RF controls will require extensive modifications to accommodate both FTS and STS pulses.

- Superconducting linac (SCL) cavity tuner motor controls. This is another area where a new design will be implemented. The existing design uses custom hardware that would take considerable effort to reproduce. PLC-based commercial-off-the-shelf hardware was successfully used at the SNS RF test facility to perform the same function, and that is the approach planned for the new FTS tuner motor controls.

- SCL coupler bias power supply controls. This includes hardware and software required to monitor and control coupler bias voltage and current.

- SCL coupler cooling water controls. For the most part, existing controls will be copied. As a PLC will be in place to handle tuner motor controls, that same PLC will be used to handle coupler cooling water controls. (The existing SCL coupler cooling water controls are handled by a Beckhoff remote I/O system directly monitored and controlled by an EPICS IOC.) Installation of the cables associated with the SCL cavity tuner, coupler bias power supply, and coupler cooling water controls is provided for in the FTS Linac Cryomodule Controls work section.

\subsubsection{First Target Station Linac Cryomodule Controls}

The FTS cryogenic control system will closely mirror the existing high beta cryomodule cryogenic control system. The scope begins at the controls racks in the Klystron Gallery and stops at the ends of the cables in the linac tunnel. The STS cryomodule design is based on that developed for the spare cryomodule and differs from the original SNS cryomodules. From the controls point of view, the major cryomodule differences are the supply and return end-can silicon diode connections. The primary and secondary Joule-Thomson (JT) valves are in a different location, which will reduce the length of the cables needed to connect to these valves. The FTS cryomodule control system will take advantage of the improvements in the design. 
The major design change for the FTS cryomodule controls is the tunnel trunk cable termination on the beam line interface (BLI) and the wiring from the BLI to connectors on the cryomodule. The original SNS construction required about 6 person weeks of labor per cryomodule to route the cables in the tunnel and terminate them on the BLI. Repeating this for this project will not be possible because of ALARA concerns and lack of tunnel access time to do this work.

The FTS cryogenic control system includes the following.

1. Equipment racks

2. PLC hardware

3. IOC hardware

4. Level meters

5. Pressure sensors

6. Power supplies

7. Terminal strips

8. Other equipment mounted in the racks and marshalling panel

9. Rack equipment mounting and wiring

10. Cables from the racks in the Klystron Gallery to the linac tunnel, including the cables needed for WBS 3.05.03.02

11. Junction boxes and terminal strips in the tunnel (BLI)

12. Cables and connectors from the BLI to connectors on the cryomodule

13. Engineering design, drawings, procurement, fabrication, software, installation, calibration, and acceptance testing of all components

14. Updates to the feasibility study document

15. Power supply and wiring for coupler cooling fan

It does NOT include

1. Valves

2. Temperature sensors

3. Heater elements

4. Liquid helium level probe

5. Connectors and wiring attached to the cryomodule

6. Components attached to the cryomodule or transfer lines

7. Components attached to the cooling water system 
8. Vacuum gauges and other components

9. RF instrumentation and wiring

The cryogenic control systems will be powered from the uninterruptible power supply (UPS) circuit all the time. An automatic transfer switch (ATS) will be installed in each rack group and marshaling panel to provide a connection to "normal" power when the UPS circuit is turned off. These are described below.

1. IOC and PLC rack

a. one 20 A UPS circuit and one 20 A normal power circuit with ATS for PLC, IOC, and instrument power

b. three 20 A UPS power circuits and three 20 A normal power circuits with three ATS units for the window and cavity heater programmable power supplies

c. one normal power circuit for rack fans and portable equipment connection

2. Marshaling panel
a. one 20 A UPS circuit and one 20 A normal power circuit with ATS for PLC and instrument power
b. one normal power circuit for rack fans and portable equipment connection

The EPICS software scope is as follows.

1. Add three new IOCs to the EPICS system

2. Generate the EPICS database files for each of the three new IOCs

3. Update the Visual Database Configuration Tool files for the new cryomodules

4. Update the following screens to add the new cryomodules
a. cryogenic system overview
b. linac distribution system
c. SCL 2 K overview
d. SCL shield overview
e. SCL primary JT valves
f. SCL primary JT valve setup
g. SCL secondary JT valves
h. SCL secondary JT valve setup
i. SCL primary return valves
j. SCL primary return valve setup
k. SCL shield control valves
1. SCL shield control valve setup
m. SCL cool down valves
n. SCL cool down valve setup
o. high beta cryomodule status
p. high beta cavity heaters 
q. high beta window heaters

r. SCL alarm set points

5. Update the following sequence logic software
a. heater control
b. $2 \mathrm{~K}$ pump-down
c. $2 \mathrm{~K}$ trip
d. JT off
e. cryoconditioning

6. Update the archive request files for the new cryomodule signals

7. Update the alarm system for the new cryomodule signals

The PLC software scope is as follows.

1. Revise $\mathrm{I} / \mathrm{O}$ configuration for replacement (1756-OB8) discrete output modules

2. Update PLC tag names for the added cryomodules

3. Update PanelView screen

4. Update control logic for new interlocks

The installation of cables for project RF and cryomodule signals will be coordinated because signals from these two packages share connectors on the cryomodule.

Design of a new BLI has to be completed. Some design details were developed to verify the preliminary design concepts. Junction boxes similar to the ones needed for the new BLI design were successfully fabricated at a reasonable price. It was verified that the connectors could be installed on the cables and then pulled from the gallery into the tunnel. This type of cable with connector installation has been used several times in the FTS Experiment Hall.

Because connectors will be installed before the conduit is pulled through, the connector size and number of conductors in a cable are important. Based on past experience, it was determined that the largest particle size for a connector that could be pulled through the conduit would allow the termination of an eight-shielded twisted-pair cable. This changed the number and types of cables to be installed. It also forces the revision of most of the cryomodule control system drawings.

\section{Known Issues}

1. Tunnel access time required for cable installation, termination, and cryomodule checkout must be greatly reduced from the amount required for original SNS construction. A new design is proposed for the installation of cryomodule control system tunnel wiring to address this issue.

2. Several of the cable types used in the original design are no longer available or are very difficult (expensive) to obtain. Replacement cable types need to be identified and routed for approval (electrical, fire protection, radiation resistance) for use at SNS.

3. Valve and heater controls and interlocks need to be upgraded to support the latest Federal Emergency Management Agency requirements. This may require significant wiring changes.

4. More PanelView functionality is needed. 
5. Allen-Bradley 1756-OB8EI is no longer available and the replacement, 1756-OB8, requires substantial modification to the marshaling panel design and wiring.

6. Power supply power cord wiring (120 Vac) needs to include a fuse for every supply.

7. ATS needs to be added to PLC, IOC rack, and marshaling panel design.

8. Relays for valve direction must be updated.

9. Power supplies for pressure transmitters have changed.

10. The connections for the primary supply and surge tank silicon diodes were changed in the new cryomodule design. An interface box and cables would need to be designed and fabricated to allow for an "old" SNS cryomodule to be placed in a new STS project slot.

\section{Advice}

1. Make sure needed cables and connectors are available.

2. Remember to use radiation-compatible components for anything installed in the linac tunnel.

3. Get the 0.5 in. instrument air supply tube moved out of the way of the BLI tray.

4. Make sure the cryomodule pressure sensors and signal conditioners are calibrated in pairs and that calibration and serial number information are maintained for all components.

5. Build cryomodule simulator boxes to allow quick tunnel checkout of all cables. Include switches/pots on the simulator boxes to allow verification of all interlocks.

6. If at all possible, standardize the location of all junction boxes with respect to the center of the cryomodule. This will keep tunnel cable lengths more consistent.

\subsubsection{First Target Station Linac Cryomodule Fabrication}

The FTS cryomodule controls design will closely mirror the existing SNS high beta cryomodule cryogenic control design. The controls scope begins at the sensor in the cryomodule and stops at the vacuum-side connector on the vacuum feedthrough. It includes the three cryomodule pressure transducers. Cryogenic personnel will mount all instrumentation. The controls scope includes design, specification of instrumentation, and installation of wiring.

\subsubsection{First Target Station Linac Water System Controls}

EPICS and PLC controls will be provided to control the new deionized water system (DIWS) pump loop KL06. The new facilities PLC CPU chassis will be installed in klystron building communication room 6 and will control new pump loop KL06 and new air handler AH-KL-06. Separate I/O panels local to KL06 and AH-KL-6 and the new I/O panels will be connected to the new PLC CPU chassis via Ethernet.

\subsubsection{First Target Station Linac Beam Line Vacuum Control System}

This subsystem provides vacuum controls for the new cryomodules in slots 24-32, the warm sections, and the high-energy differential pumping (HEDP) section. These controls will 
- monitor and control vacuum components (valves, gauges, pumps)

- $\quad$ provide interlock signals to low-level RF systems and MPSs

Preliminary work includes development of cable block diagrams and field cable lists. Implementation of the vacuum controls will be complicated by the need to modify an operational system while maintaining nearly continuous vacuum pumping.

The existing SCL vacuum controls will be duplicated for this project. The last two zones of the existing system handle the vacuum controls for the drift sections that will be replaced by STS/FTS cryomodules. An additional control zone will be added for STS/FTS and the existing cryomodule controls redistributed as follows:

- Zone 7 will be modified to cover CM24-27

- Zone 8 will be modified to cover CM28-30

- new Zone 9 will cover CM31-32 and the HEDP section

Because of the importance of maintaining SCL vacuum pumping, ac power for SCL vacuum controls is typically fed by two circuits (one UPS and one normal) through an ATS. The new Zone 9 will include this same method of power distribution.

\subsubsection{First Target Station Linac Insulating Vacuum System Controls}

This subsystem provides vacuum controls for pumping the insulating jackets of cryomodules $17-32$. The existing insulating vacuum controls for cryomodules $1-16$ will be duplicated for the FTS. The existing design of a single SNS insulating vacuum system handled pumping for eight cryomodules. Plans are to continue with this grouping. Preliminary design work included development of cable block diagrams and field cable lists.

Because of the importance of maintaining the SCL insulating vacuum, ac power for SCL insulating vacuum controls is typically fed by two power circuits (one UPS and one normal) through an ATS. This method of power distribution will be continued for the FTS insulating vacuum systems.

\subsubsection{First Target Station Klystron Gallery Facility Process System Controls}

EPICS and PLC controls will be provided to control the new air handler AH-KL-6. As stated previously, the new facilities PLC CPU chassis in klystron building communication room 6 will control both the new pump loop KL06 and the new air handler AH-KL-6. Separate I/O panels for KL06 and AH-KL-06 will be connected to the new PLC CPU chassis via ControlNet. (Instrumentation is covered under the facilities WBS.)

\subsubsection{First Target Station Ring Global Control System Requirements}

\subsubsection{First Target Station Ring Timing System}

The FTS ring timing infrastructure requirements will be handled by expanding the existing SNS ring timing system infrastructure. The timing system equipment required for the FTS includes IOC timing receiver boards, timing link fanouts, and IOC fiber optic transmitter boards. The design will meet detailed timing system requirements for production and delivery of FTS and STS beam pulses. Timing equipment and quantities will be based on these requirements. Preliminary design included assessing detailed timing system requirements for control equipment. 
The costs associated with installation of timing-system-related field cable are covered under "Network Installation." (It was deemed to be more efficient to design and install all communications cables together.)

\subsubsection{First Target Station Ring Machine Protection System Controls}

It is assumed that the number of extraction kickers will be increased from 14 to 16 . The following steps will be taken to accommodate this transition.

- The MIOC that handles the extraction kicker charged inputs has two extra channels, so no additional MIOCs are required by FTS for this function. (However, two cables will need to be installed within the extraction kicker control racks.)

- The extraction kicker waveform monitoring system will have to be upgraded to support the STS pulses. This upgrade will provide capability to support the changes required for the FTS work. The new system will have the capability of monitoring 16 channels (i.e., it will accommodate the addition of two kickers for the FTS). Current transformers will be added as part of the upgrade, and 16 cables will be pulled (i.e., two extra to accommodate the FTS).

\subsubsection{Ring Primary and Secondary Stripper Foil System Controls}

EPICS and PLC controls will be provided to accommodate modifications to the primary and secondary stripper foil mechanism. The existing EPICS IOC-based system will be replaced by a PLC-based stepper motor control system. The existing Ring Service Building racks will house the new equipment. Existing cables from the service building to the tunnel will be reused and new cables installed as required. All modifications to the junction boxes, wiring, and connectors in the tunnel will be designed to reduce the amount of time required for installation to meet ALARA guidelines.

\subsubsection{Ring Water System Controls}

EPICS and PLC controls will be provided to accommodate modifications to the existing DIWS RN03 and implementation of the new DIWS RN04. The existing PLC system CF_RN:PLC8541 will be used to provide the processing power. A new I/O panel (CF_RN:PLC8541B) will be installed in the Ring Service Building basement.

\subsection{SECOND TARGET STATION INTEGRATED CONTROL SYSTEM REQUIREMENTS}

These systems include the control system effort required for the new equipment in the STS, the new ring to target two beam transfer line, and the new STS instruments.

\subsubsection{Second Target Station Global Control System Requirements}

The scope described in this section covers global controls not addressed in the earlier power upgrade project effort, which was constrained to accelerator improvements. This includes efforts in the new target and beam lines. This may be combined with the effort described in Sect. 7.1.1.

\subsubsection{Integrated Control System Network and Computing Systems}

The STS network and computing needs will be handled by adding to the existing SNS infrastructure. The communication services that will be required include Ethernet/IP, terminal service (e.g., to IOCs), and remote reboot service. Three major categories of global communications will be required for the STS. 
1. Ethernet Network

2. Timing System

3. MPS

Hardware design and procurements were to be covered under their respective work sections. However, as it is more efficient to install all communication cables together, the cable design and installation costs for all three communication categories were rolled up under this work section.

New servers and OPIs will be added to handle STS requirements. Computing systems additions will include

- a new server for the second target station

- $\quad$ STS control room EPICS OPIs

- target facility local OPIs for equipment checkout and start-up in the field

- target facility instrument hall coordinator OPIs

New communication room equipment in the STS building will include

- new equipment racks for communication hardware

- fiber termination panels

- fiber and copper Ethernet patch panels

- fiber and copper network switches

- media converter,

- terminal servers and remote rebooters

- network monitoring and diagnostic hardware

Design work will include assessing detailed communications requirements for control and operations equipment. Communications equipment will be specified accordingly. Specifications, drawings, test plans, and other documents will be prepared to detail the requirements for completing this activity.

As the global controls network infrastructure cabling will be routed to the same locations as the timing and MPS systems cabling, installation of the cabling for the timing and MPS systems will be included in this work package to reduce installation time and costs.

\subsubsection{Timing System}

The primary function of the timing master is to produce all of the relevant information necessary to synchronize systems that are responsible for the production of each beam pulse and coordinating beam line data acquisition systems. Synchronization is accomplished by broadcasting a unique series of identification numbers to accelerator and target subcomponents over the event link. The current accelerator and PTS consume about $40 \%$ of these values. An additional $20 \%$ utilization is expected to support the STS requirements and is well below levels of concern. Dynamic configuration information is broadcast over the real-time data link and is currently operating at $20 \%$ utilization. The additional requirements needed to support the STS are expected to increase this demand to $50 \%$ to resolve the changes in machine configuration from pulse to pulse.

The existing timing master hardware platform was designed around the FTS only and is not capable of multiple target station operations. The next-generation hardware has already been developed to address obsolescence concerns with the original installed equipment and is capable of operating both target stations simultaneously; however, a significant amount of software development and testing effort will 
still be required to accommodate the STS configuration. The existing timing system subcomponents and distribution hardware do not require any changes to accommodate the STS.

\subsubsection{Machine Protection System}

The MPS will not be capable of handling the STS without a redesign of a portion of the front-end electronics. The standard field components do not require changes, but they do contain large numbers of obsolete items of equipment that will pose significant challenges for manufacturing additional assemblies. Changes required on front-end electronics are mostly directed toward handling the new machine modes necessary for operating the target stations independently or simultaneously. Additional measures will also have to be in place to ensure each target station is receiving its intended beam pulse. Deficiencies with the existing hardware platform will be resolved under the MPS AIP. The redesign of this hardware addresses concerns with STS integration and resolves manufacturability problems.

\subsubsection{Ring to Target Two Beam Transfer Line-Magnet Power Supply Control Systems}

The ring to target two (R2T2) beam transfer line magnet power supply controls will provide the same functionality and capabilities as the existing magnet power supply controls for the FTS RTBT(RTBT1) magnets. The controls will be updated to include more modern hardware.

The controls for the R2T2 kickers will be a new design based on existing system functionality. Appropriate interface signals and hardware will be provided. These kicker controls will exchange signals with the MPS and timing systems.

\subsubsection{Ring to Target Two Beam Transfer Line-Vacuum Control Systems}

The R2T2 vacuum controls will provide the same functionality and capabilities as the existing vacuum controls for the RTBT1 vacuum system. The controls will be updated to include more modern hardware.

\subsubsection{Second Target Station Target Control Systems}

\subsubsection{Second Target Station Target Protection System}

The target protection system (TPS) is assumed to be similar to that of the FTS. The TPS will not use a PLC for control logic but rather will use hardwired relay logic and comparators to monitor the target parameters and interrupt the beam should any condition occur that warrants doing so. Some process information will be conveyed to the ICS and EPICS displays via secondary devices.

\subsubsection{Second Target Station Target Utility Cooling Loop Controls}

The utility cooling loop control system is assumed to be similar to that of the FTS. There will be four independent cooling loops to cool the target, shroud, proton beam window, and infrastructure. Each loop will use a separate PLC to monitor process conditions such as flow, temperature, and pressure and to control field devices like pumps and valves. The controllers will set alarms and provide automatic actions should the process parameters exceed the desired or allowable limits. These loop configurations and controls will be similar in design and operation to those in the current SNS cooling loops.

\subsubsection{Second Target Station Target Reflector System Controls}

The target reflector system is assumed to be similar to that of the FTS. The reflector system controls will use a PLC to monitor temperature, flow, and pressure conditions and relay that information via the 
network to other PLCs, operator control stations, and the data archiving system. Automatic actions or operator commands will be acted on by the PLC and output to the reflector system field devices.

\subsubsection{Second Target Station Target Vessel System Controls}

This system is assumed to be similar to that of the FTS. The vessel system controls will use a PLC to monitor temperature, flow, and pressure conditions and relay that information via the network to other PLCs, operator control stations, and the data archiving system. Automatic actions or operator commands will be acted on by the PLC and output to any vessel system field devices.

\subsubsection{Second Target Station Target Remote Handling System Controls}

The target and target infrastructure will be installed and removed using remote handling equipment. This equipment will be automated by a separate controller commanding the drives and solenoid devices. The controller will monitor positions and loads and prevent collisions and undesirable configurations via permissive logic and interlocks. This controller will not be integrated into the PLC network.

\subsubsection{Second Target Station Target Process Instrumentation}

Similar to the current target process instruments control system, the STS process instrument control system will monitor process parameters such as pressure, temperature, and flows and convey this information to the operator stations and data archiving system.

\subsubsection{Second Target Station Target Moderator Cryogenic System Controls}

The requirements for the STS target moderator controls will be essentially the same as for the FTS moderator controls. Control hardware and software similar to that deployed for the existing FTS moderator will be used for the STS moderator system.

\subsubsection{Second Target Station Front-End Control System}

The front-end control system will address the requirements related to separate control of the chopping pulse formation. The chopping pulse formation is different between the FTS and STS pulses. The requirements for these controls are to be determined.

\subsubsection{Personnel Protection Systems}

Safety systems include credited and defense-in-depth controls with the primary function of mitigation of postulated accident scenarios that could otherwise result in harm to people or the environment. Hazards and accident scenarios will be addressed throughout the project, culminating in major revisions to the Proton and Neutron Facility Safety Assessment Documents (FSADs). The revised FSADs will describe the credited controls, including protection systems, that form the basis for a new accelerator safety envelope. The FSADs are the primary drivers for safety system performance requirements.

Existing safety systems include the TPS, target bay differential pressure monitoring system (TBDPMS), personnel protection system (PPS), oxygen deficiency hazard monitoring (ODH), and central stack monitoring system. The PPS are further divided into accelerator PPS, target PPS, and instrument PPS. The STS project will include modifications to the accelerator PPS to accommodate the energy upgrade as well as to integrate new STS systems and functions with the existing infrastructure. 
New safety systems for the STS are functional duplicates of the TBDPMS, target PPS, instrument PPS, $\mathrm{ODH}$, and central exhaust stack monitoring system 2 . The STS TPS will be functionally similar to the FTS TPS, with necessary changes to address the tantalum target.

The primary function of safety systems is mitigation of significant hazards addressed through DOE Order 420.2C, Safety of Accelerator Facilities (2011), and the associated guidance 420.1-2A (DOE 2014). Critical devices are used to prevent beam transport to an area that is not in a safe configuration. For example, disabling the DH13 RTBT1 dipole prevents beam transport to the FTS target when the target or any of the instrument lines are not safely configured for beam. RTBT2 elements include both horizontal and vertical dipoles suitable for use as credited controls to protect the STS.

The new PPS will include the use of independent testing laboratory certified safety controls similar to those used in the most recent FTS instrument PPS systems.

\section{Graded Approach}

Adding another beam termination point increases the complexity of machine operating modes. The safety system design criteria include maximizing each target station's availability by limiting faults that result in simultaneous beam shutdown to both user facilities. Similarly, the safety systems will have the capability for quick recovery so that, in the event of a beam shutdown to both facilities, the faulted target segment can be quickly isolated in a safe configuration and the machine reconfigured for beam operation to the other target.

\section{New Hazards}

Introduction of the STS introduces new postulated accident scenarios, the most serious of which is misdirection of high-power FTS beam to the lower-power STS target.

1. Errant direction of FTS high power beam structure $(50-60 \mathrm{~Hz} \mid 40 \mathrm{kJpp})$ to the STS target

2. Errant operation of FTS $50 \mathrm{~Hz}, 2 \mathrm{MW}$ beam structure at $60 \mathrm{~Hz}, 2.4 \mathrm{MW}$

3. Errant direction of STS $0.5 \mathrm{MW}$ pulse to the FTS $(10+50 \mathrm{~Hz}, 2.5 \mathrm{MW}$ beam structure

4. Errant direction of STS high-intensity pulse $(47 \mathrm{kJpp})$ to the FTS target at the FTS repetition rate $(2.8 \mathrm{MW}$ at $60 \mathrm{~Hz})$

5. Errant operation of STS high-intensity pulse (47 kJpp) at the FTS repetition rate $(2.8 \mathrm{MW}$ at $60 \mathrm{~Hz})$

6. Errant direction of the STS full power beam (0.5 MW) to the RTBT tune-up dump

First-order protection against the scenarios mentioned is handled in the timing and MPS systems by disallowing a pulse/accumulation cycle if conditions are not correct for the beam mode. However, the consequences of unmitigated failure may be severe enough to warrant robust protection systems to detect and mitigate those and other failure modes. Allocation of these protection functions as credited, defensein-depth, or machine protection functions will be a deliverable during the preliminary design and hazard analysis project phases.

Many of the faults discussed require detection of two cycles of errant beam before a protection system can take action to shut down the beam. Therefore, the targets must be able to handle at least two errant pulses. Further thermodynamic studies of both the FTS and STS targets may be required to verify this 
criterion, e.g., two $40 \mathrm{~kJ}$ pulses spaced at $16.67 \mathrm{~ms}$ to the STS target or two $47 \mathrm{~kJ}$ pulses spaced at 16.67 ms to the FTS target.

The safety system scope and deliverables also include the following:

- System level operation, certification, and maintenance documentation

- Expenses for a minimum of two external design reviews

- Safety-system-specific, system-level networking and interconnection infrastructure

- Hardware/software required to interface the PPS systems to EPICS, e.g., "mail-box" PLC

- Safety-system-specific EPICS screens and archiver functions

\section{Assumptions}

- Accelerator Safety Order-related activities such as FSAD and ASE development are outside the scope of the protection system work section.

- The RTBT2 will be accessible only when RTBT1 is in an access state.

- RTBT2 and RTBT1 will be separate PPS segments with a PPS access point at the junction between the two areas.

- $\quad$ The FTS target will not be damaged by a minimum of two consecutive STS pulses or 2 seconds at 2.5 MW.

- The STS target will not be damaged by a minimum of two consecutive FTS pulses with an inter-pulse period of $16.67 \mathrm{~ms}(60 \mathrm{~Hz})$.

- Existing EPICS IOC(s) will be used for data collection and archiving.

\section{Project Risks}

Technical risks are minimal given that the safety system architecture will be based on existing SNS designs and associated management practices. Lessons learned from other projects show that obsolescence is the major technological risk for large DOE construction projects. This risk will be mitigated by continuity of funding and by making just-in-time equipment/material purchases.

\subsubsection{First Target Station Personnel Protection System Requirements}

The PPS design for the FTS includes interfaces to the additional RF systems in the Klystron Gallery (four modulators and six RF transmitters). Components of this work section include the following:

- material cost and installation: PLC components, cable, conduit, RF interface panels

- management and integration with project team and field contractors

- PLC programming, testing, certification, and documentation for safety system

\section{Other notes}

- This work involves only the linac PPS and the new RF systems in the Klystron Gallery.

- No other segments of the PPS were involved.

- No other components (e.g., chipmunks or magnet power supply interfaces) were included.

The scope and deliverables of the protection systems for this project include all lifecycle activities required to develop and implement the systems specific to the STS. Each element in the following list includes design, documentation, quality assurance activities, and installation. 
- Modify/extend existing protection systems

- Central Control Room equipment

- PPS controls to establish the new machine operating configurations of the accelerator and two target stations

- PPS controls for the new RTBT2 tunnel segment and critical devices

- PPS controls for the STS target PPS

$\circ \quad$ STS TPS remote monitoring and controls

- Modify front end controls

- PPS interface to MPS/timing system

- PPS/TPS interface to front end

$\circ$ Intensity monitor at the front end

- Add linac PPS interlocks

- PPS interlocks for the linac power upgrade RF modulators and klystrons

- Modify ring PPS

- Modifications to PPS access controls to add the ring-RTBT2 interface

- TPS/PPS interlocks for kicker and septa magnets

$\circ$ PPS interlocks for beam intensity

- $\quad$ Modify RTBT1

- Modifications to PPS interface to DH13

- Stack monitoring

- Upgrades to the flow monitoring

- Upgrades to the radiation monitoring

\subsubsection{Second Target Station Personnel Protection System Requirements}

\section{STS Ring to Target Two Beam Transfer Line Personnel Protection System}

Requirements to be addressed

- New PPS access control segment

- Interface to critical devices equivalent to DH13 (TBD)

- Interface to

- Ring PPS

- Front-end PPS controls

\section{STS Target Personnel Protection System}

A separate, credited engineering control PLC will handle monitoring and control of the air pressure zones within the building. Instruments will monitor the pressures and configurations within the zones and notify the operator of current conditions. This control system will also activate evacuation alarms should the conditions exceed allowable limits or dangerous conditions exist.

The scope includes

- $\quad$ Target Protection System 
- Radiation monitoring

- Target cooling and integrity monitoring

- Dedicated TPS link to critical devices and front-end PPS controls

- Transfer Bay 2 access control system

- Radiation monitoring

- Access control system

- STS target bay differential pressure monitoring and alarm system

- Radiation monitoring

- Pressure monitoring

- Alarm system

- Second target PPS

- Radiation monitoring

- Centralized instrument PPS link to RTBT2 and front-end PPS controls

- Second target instrument PPS for five instrument lines

- Radiation monitoring

- Access controls

- Oxygen deficiency monitoring

- Other safety functions identified in the individual instrument hazard assessment

- Central exhaust monitoring system 2

- Radiation monitoring - internal and baseline

- Air flow monitoring

\subsubsection{Second Target Station Instrument Controls}

No instrument controls have been defined, so estimates are based on experience with the FTS instruments. Similar type systems will be deployed on the STS to leverage the lessons learned and systems already deployed in this area. 



\section{RESEARCH AND DEVELOPMENT}

Much of the STS design involves the use of existing or standard technologies. For example, the accelerator energy upgrade calls for use of cryomodules of the same design as the spare cryomodule in present operation. There are some exceptions however, that require development to establish a firm design basis. A key development area is the target (see Sect. 8.1), with the cladding being a primary concern. The solid target has many advantages, and developing the cladding design basis is a critical step in validating this approach. There are a few accelerator issues related to the higher beam intensity, as discussed in Sect. 8.2.

\subsection{TARGET SYSTEMS}

\subsubsection{Tungsten Target Plates}

Spallation targets made of tungsten and cooled by water have been successfully operated at several neutron source facilities. These include the two ISIS sources at Rutherford Appleton Laboratory, the Lujan source at Los Alamos Neutron Science Center, and the former KENS at KEK (High Energy Accelerator Research Organization) in Japan. This experience provides reason to be confident that a successful STS tungsten target design can be developed, and lessons learned from those facilities are highly valuable to the STS. However, their experience and difficulties have also demonstrated that there are technical risks to overcome to achieve reliable high-performing target operations.

Bare tungsten is vulnerable to aggressive corrosion in water-cooled spallation targets,[1,2] as is exemplified by post-irradiation examination of the Lujan MK 0 target seen in Fig. 8.1. Irradiated tungsten particles and other spallation products will contaminate the coolant, making normal water loop maintenance hazardous; and the tungsten plates can crumble, leaving voids in the beam interaction region. ISIS and the current Lujan target use a protective cladding of tantalum to avoid such effects. If developed and installed properly, a tantalum cladding will protect the tungsten from corrosion and may mitigate tungsten vaporization in the event of loss-of-coolant events.

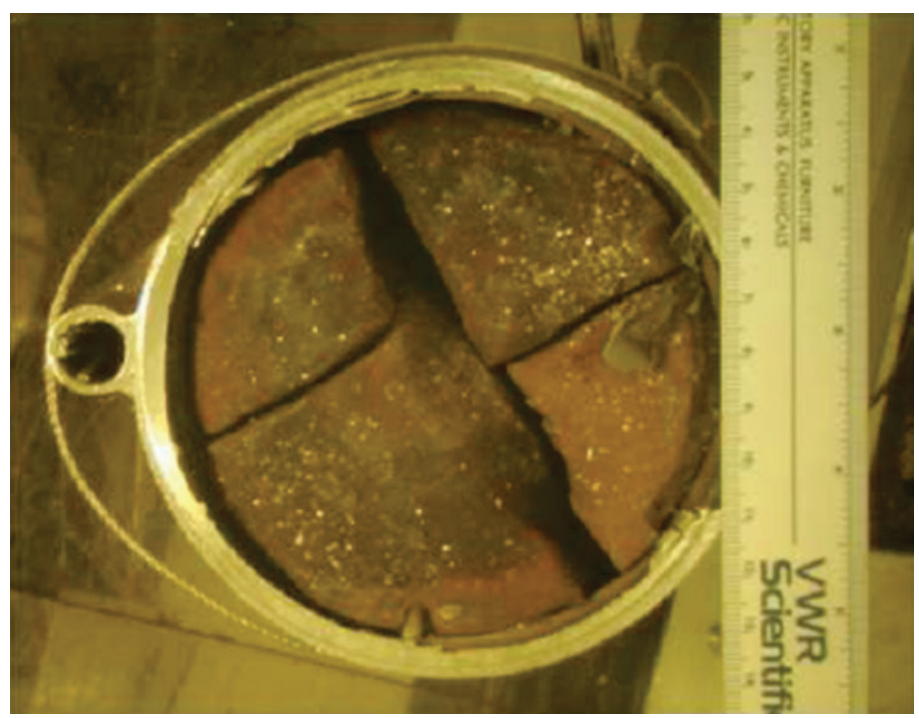

Fig. 8.1. Photograph of the upper portion of the opened MK 0 Lujan target following service, as viewed from the impinging beam [1]. 
Cladding tantalum on tungsten is not a standard industrial process. ISIS, Lujan, and KENS invested in the development of processing techniques to achieve robust bonding with the tungsten and joining of clad seams. Their approaches use the hot isostatic press (HIP) method but with notable differences in process temperature. Critical process parameters include material surface preparation (finish, cleaning) and oxidation control; the use of a HIP furnace with a molybdenum element, rather than graphite, appears essential; but suitable molybdenum-equipped furnaces are uncommon. An intimate bond between cladding and plate is required to ensure that heat will be conducted out of the plate with minimal interface resistance. Plate temperature and thermal stress must be controlled in the high-brightness STS target. Robust seam joints in the clad will limit the escape of volatile spallation products from the plate into the coolant.

A detriment of tantalum for this application is its relatively high and long-lived decay heat. This is important for operational and safety considerations. Minimizing the amount of tantalum employed in the target is desirable from this point of view; but very thin cladding may be less robust against erosion, thermal stress, or leaks.

The ISIS TS- 1 target uses a rather thick cladding of about $2 \mathrm{~mm}$, whereas the ISIS TS- 2 cylindrical target cladding is $1 \mathrm{~mm}$. The MK III Lujan target uses a cladding only $0.25 \mathrm{~mm}$ thick. It has been reported that in the latter two cases, there is evidence of clad fracture by way of elevated activity in cooling water. ISIS engineers suspect a clad failure at a joint near the beam entrance end of the target.

A clear understanding of plate and clad stresses, material requirements, and life-limiting mechanisms is needed to develop the optimized target design for the high neutron brightness needed to meet STS performance goals. In addition to steady state operating conditions, working stresses must consider cyclic stresses from beam trips and pulses, and residual stress from manufacturing processes. Design criteria (limits) for these stresses need to be established; those used at ISIS and LANSCE should be reviewed carefully along with other data for application to the STS target.

The research and development (R\&D) plan for tungsten plate production has the following aims.

1. Develop clad application processes for the STS target plate design that provide robust and intimate bonding between cladding and tungsten substrate and robust joining of cladding seams with acceptable residual stresses and tantalum grain growth size.

2. Develop inspection techniques to verify bond and weld integrity.

3. Demonstrate fabrication and inspection methods on prototype design clad plates.

4. Evaluate the need for direct temperature measurement within plates (like ISIS TS-1) and demonstrate fabrication methods if necessary.

5. Develop engineering analysis techniques to adequately estimate target plate and cladding operating stresses, including production residual stresses.

6. Benchmark production residual stress predictions with a neutron scattering experiment (or suitable substitute).

7. Formulate plate and cladding structural design criteria.

8. Employ analysis techniques to guide design and clad application development. 
9. Define material specification requirements for tungsten and tantalum.

10. Identify primary and alternate providers of tungsten target plates properly clad with tantalum.

11. Identify primary and alternate suppliers of tungsten and tantalum.

Although the STS target design concept calls for using $0.5 \mathrm{~mm}$ thick cladding, it has many similarities to the design of the ISIS TS-1 (Fig. 8.2) target; and exploiting ISIS experience is an attractive option. After years of subcontracting target production to industrial vendors, ISIS has assembled the equipment and shop capabilities to produce targets for TS-1 and TS-2 internally (see Fig. 8.3). Collaboration with and/or subcontracting to ISIS engineering for development of STS plate cladding may be an expedited path to achieving R\&D goals. In preliminary discussions, ISIS staff have indicated receptiveness to our development purposes. However, ISIS management are reluctant for ISIS to be a production supplier for STS target plates. This issue may be solvable. In any case, arrangements with ISIS for assistance with our development objectives will be pursued.

An alternative but unproven (for spallation target application) technique for applying a protective tantalum coating is a chemical vapor deposition process called Tantaline. Typically, the process applies a coating thickness of only $50 \mu \mathrm{m}$, and the company represents the process as having a number of advantageous properties with appeal for the tungsten spallation target application. An intimate bond to the substrate is claimed, and residual stress from application should be low relative to HIP application of a clad layer. A few sample pieces of tungsten were treated with Tantaline multiple times to create a coating layer of about $0.3 \mathrm{~mm}$ as part of an earlier STS Laboratory Director's Research and Development project. Examination of the layer revealed a rough and apparently porous coating that did not instill confidence that the process would protect a target plate well (see Fig. 8.4). A publication on this research is under preparation. It may be that after further work with the company, a suitable process for this application could be developed; however, plans currently do not envision that that will be the case.
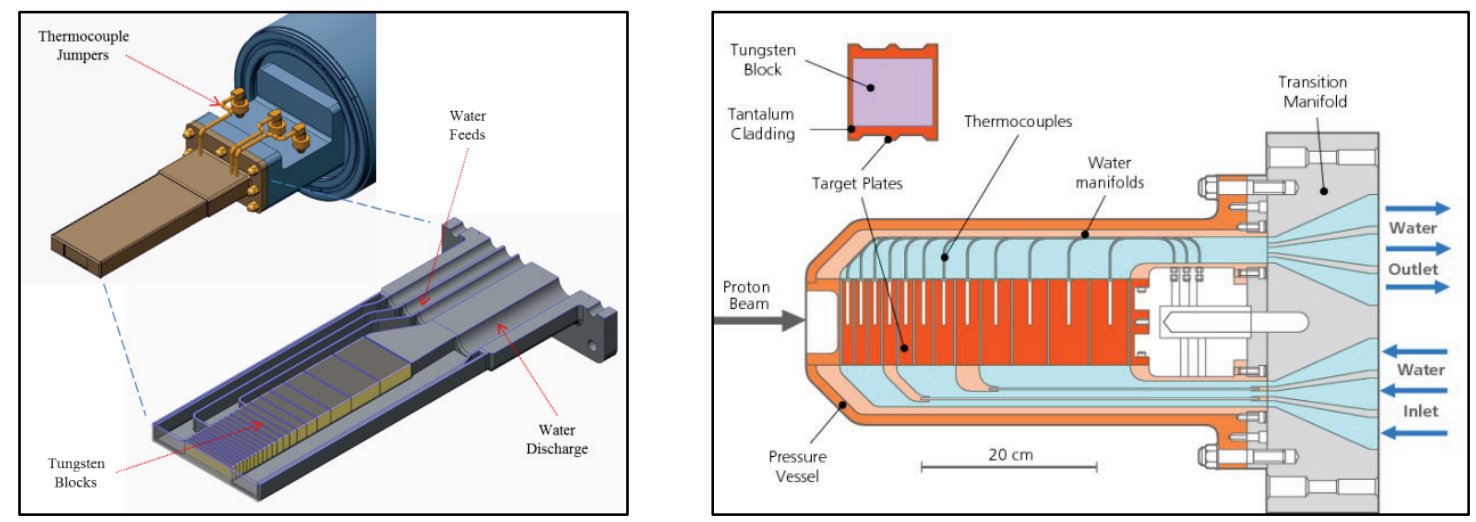

Fig. 8.2. STS tungsten target design concept (left) has similarities to the ISIS TS-1 target design (right). 


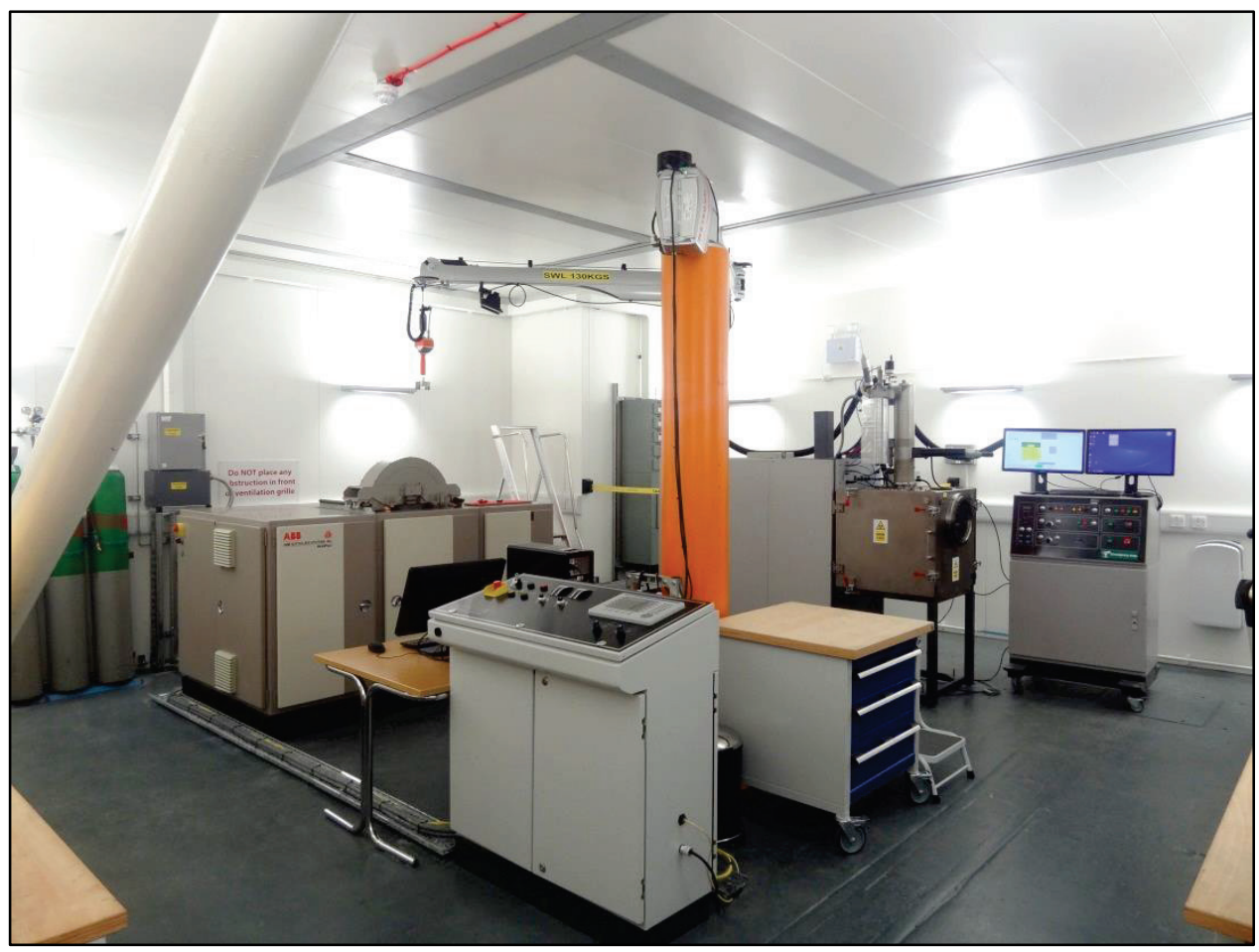

Fig. 8.3. ISIS shop for tungsten target cladding application.

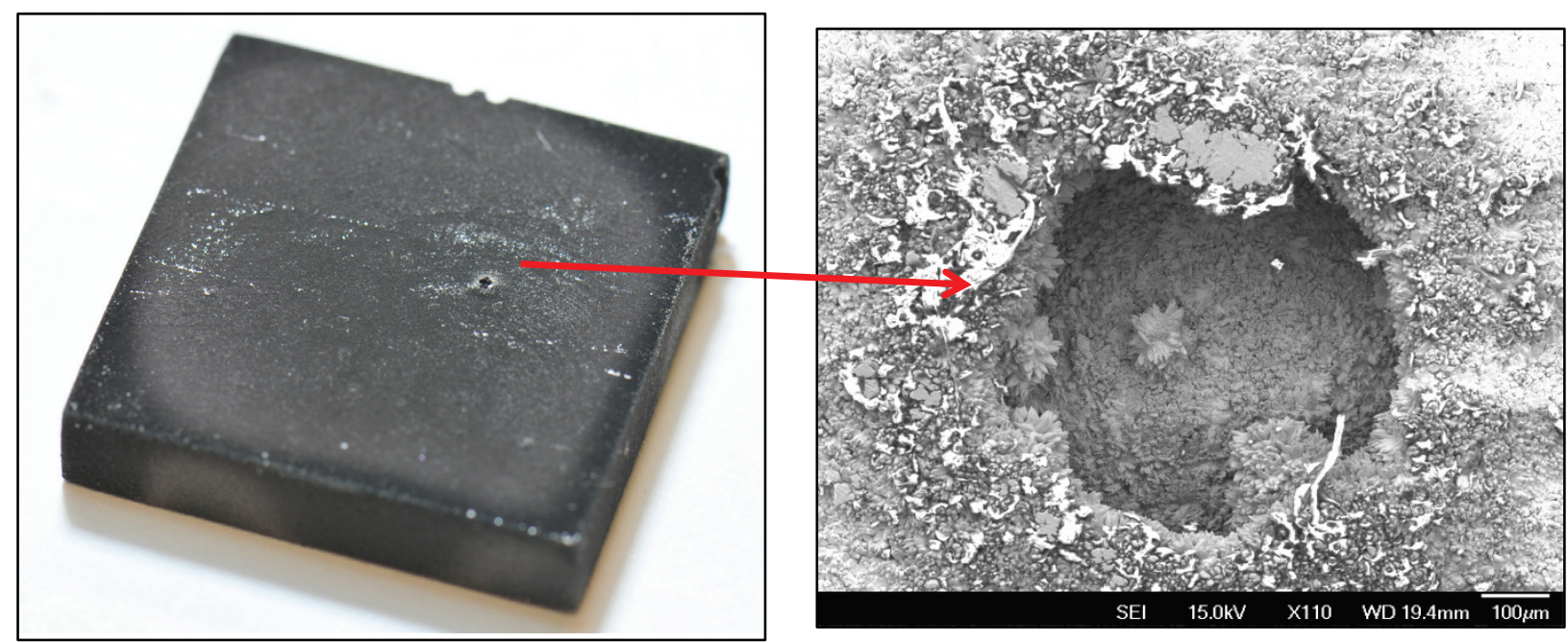

Fig. 8.4. Photo of a Tantaline treated tungsten specimen (left) with dimensions of $0.9 \times 0.9 \times 0.175 \mathrm{in}^{3}$. It was requested that this specimen have 200 microns of coating applied, but only 141 microns was achieved. A micrograph of a pit in the coating is shown on the right. 


\subsubsection{Tungsten Vaporization Tests}

Experimental studies by Greene and Frinfrock[4,5] demonstrated that at temperatures above $700^{\circ} \mathrm{C}$ and under certain moist air conditions, tungsten would vaporize and could be transported away from the solid tungsten. The phenomenon is relevant to a contemplated loss-of-water-coolant accident scenario that is to be addressed in the course of evaluating the STS safety basis. Target decay heat would drive the temperature rise if the target were not cooled by other means. At worst, the activated tungsten and spallation products might be transported offsite. Safety experts will conduct a detailed analysis regarding any contemplated accident. The required safety-credited features of the STS could have significant impact on design decisions, neutron performance, operational constraints, and cost.

No test data are known to be available that assess the mitigation of vaporization by virtue of a cladding such as tantalum. This R\&D effort aims to provide data that safety experts could use in evaluating the facility safety basis. Researchers envision assembling an apparatus with capabilities duplicating those of the one used by Greene and Frinfrock (shown schematically in Fig. 8.5) and repeating their tests on bare tungsten rods to benchmark the experimental procedures. Tests with clad tungsten would then begin and the extent of vapor reduction would be quantified. In a next stage, a similar assessment would be made of clad tungsten with a deliberate crack or fractured weld in the cladding. This step could establish some tolerance for less-than-perfect cladding, which should be considered in view of ISIS TS-2 and Lujan MK III target experience.

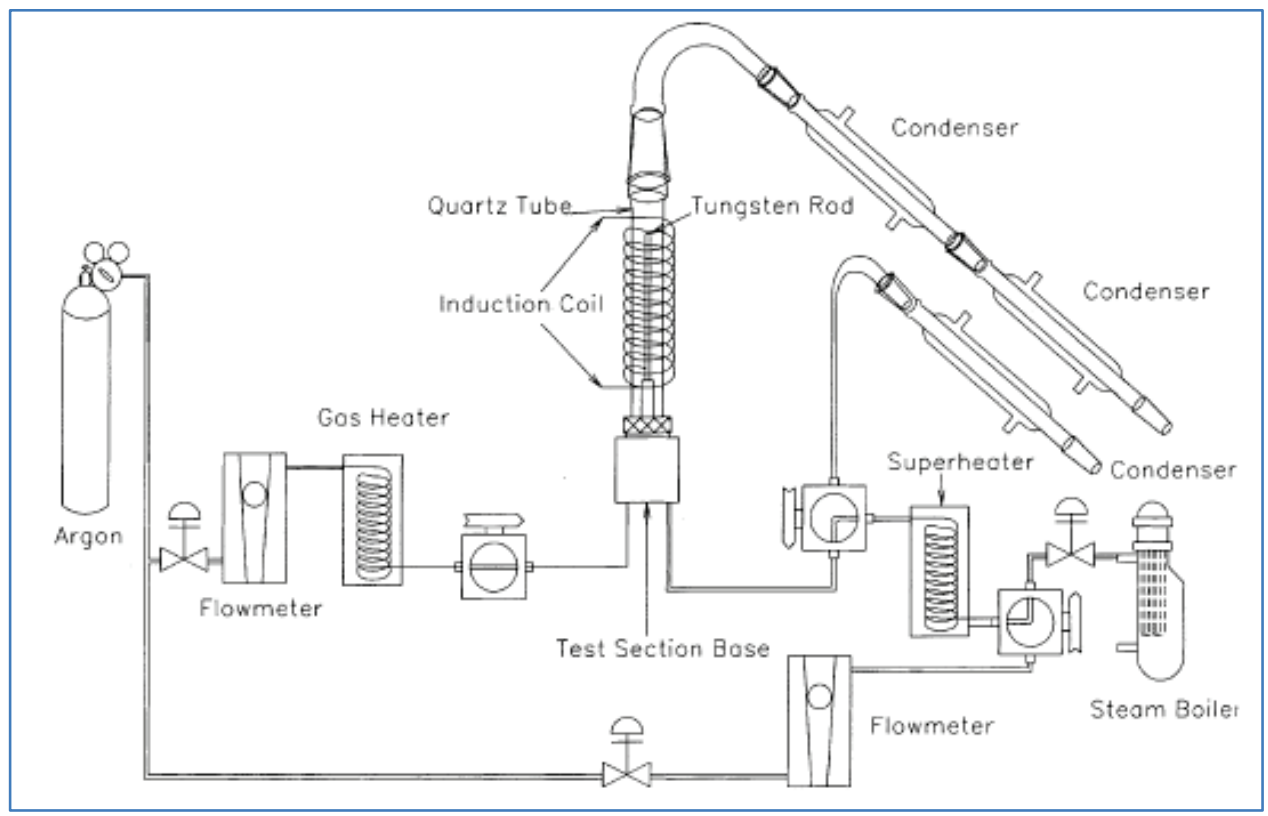

Fig. 8.5. Schematic of experimental facility used by Greene and Frinfrock. Adapted from ref. [4].

Coordination with a facility safety expert is necessary but has not yet taken place. A clear understanding of what data will be useful for making the needed safety judgments will guide the test scope.

\subsubsection{Target Cooling Loop Natural Convection Tests}

Water coolant flow through the target and water loop during normal (pumped) operation can be adequately modeled with contemporary computational fluid dynamics and pipe flow simulation codes. In 
the case of a pump operation failure with water still in the target and loop, natural convection might be exploited to provide enough heat removal capacity for the target decay heat. This R\&D task aims to simulate and experimentally measure natural convection in a water test loop with a mockup target with a heating capability adequate to simulate target decay heat. The results could render credited backup water pumps for this accident scenario unnecessary. To accomplish this research effort properly, the test loop and target should be configured prototypically to the STS design.

Coordination of this R\&D effort with a facility safety expert is necessary. A cost estimate has not been established for this effort.

\subsubsection{Gas Bubble Injection}

R\&D for the mercury target vessel of the FTS aims to provide solutions to increased rates of beaminduced cavitation erosion and pressure wave-induced vessel cyclic strain. Cavitation is expected to be a more critical issue at the $2 \mathrm{MW}$ power levels expected as a result of the SNS accelerator upgrade. Aggressive erosion has been consistently observed in SNS targets (Fig. 8.6). Target lifetime average power has only recently exceeded $1 \mathrm{MW}$. The worst erosion has been on an interior wall that has also become fractured in all but two targets. We note that there is a secondary outer wall for mercury containment. As power increases, the erosion and fracture may eventually lead to greater outer wall damage, putting target life at greater risk. Earlier research has indicated an erosion rate dependence on beam power by power law dependence with an exponent between 2 and 4 . This simplistic scaling overlooks many interacting mechanisms relevant to beam-induced cavitation erosion, but observations based on post-irradiation examination of used targets, and correlation of the powers at which they operated, are consistent with greater damage rates at higher power.
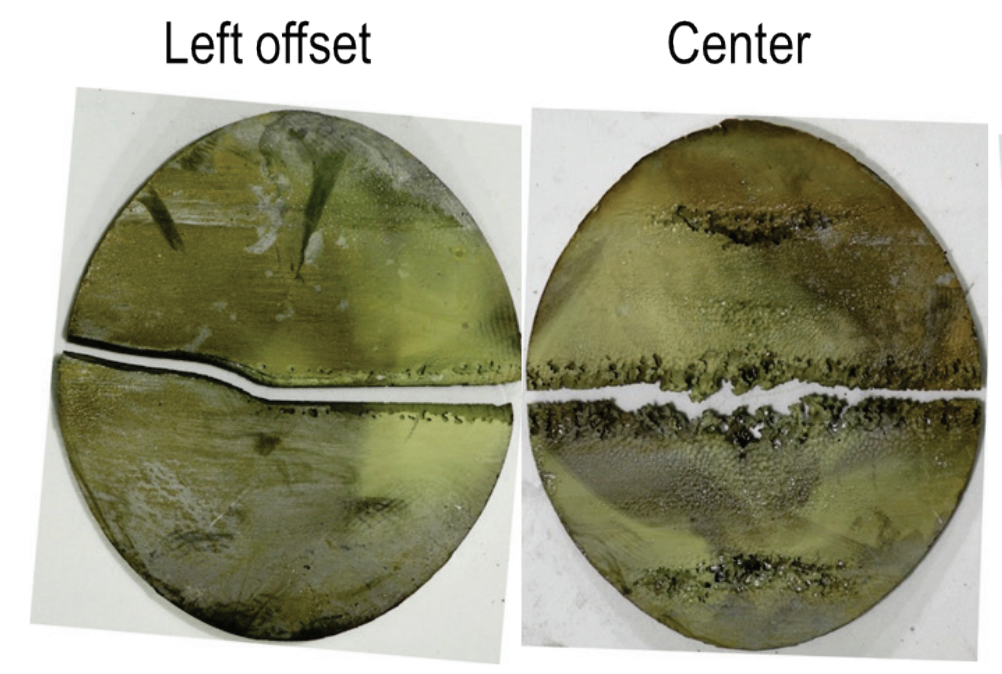

\section{Right offset}

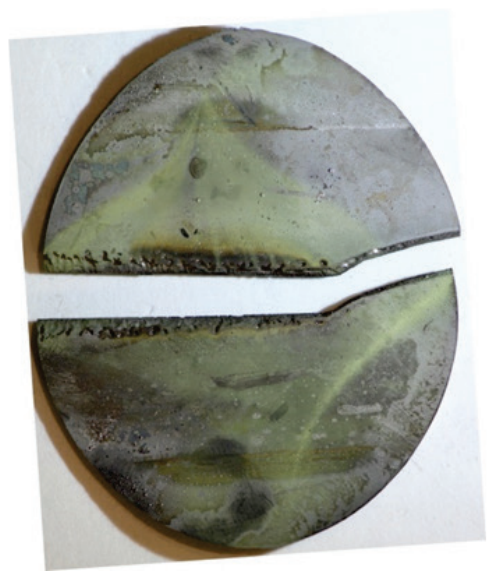

Fig. 8.6. Target 4 inner wall disk specimens show typical heavy central erosion and cross-width fracture. These disks are $\sim 60 \mathrm{~mm}$ in diameter. Target 4 was subject to an average power of $782 \mathrm{~kW}$ and it accepted $3252 \mathrm{MW}-\mathrm{h}$ of accelerator beam without leaking. 


\subsection{ACCELERATOR SYSTEMS}

\subsubsection{High-Voltage Modulator Systems}

Currently, two areas of development are associated with the modulator systems required to support the STS. The first area involves modifying the superconducting linac high-voltage converter modulator (HVCM) boost transformer to achieve a higher system voltage gain, while maintaining the leakage inductance necessary to maintain the present insulated gate bipolar transistor resonant conditions. The modification will permit reliable operation at a reduced dc bus voltage. Simultaneously with this effort, the current system will be tested at higher dc bus voltages to characterize the system reliability and assess whether the boost transformer changes are necessary. The second area of development requires a similar approach for the normal conducting linac HVCM systems to produce the additional modulator voltage necessary to drive the klystrons to higher power levels. This area will also be addressed with a redesign / optimization of the boost transformers and/or boost capacitors. Both of these development activities will be followed by a multi-month run period to assess the impact of these changes on system reliability.

\subsubsection{Ion Source}

As noted in Sect. 3.1, the average beam current will be increased by $\sim 45 \%$ relative to present operation. Much of the increase will be accomplished by replacing the present production radio frequency quadrupole to reestablish design transmission levels, and by using "smart" chopping scenarios. However, there is still a need for an increase of $\sim 10 \%$ in the peak ion source current. This increase should be achieved through ongoing ion source development efforts using the ion-source test stand. Past experience shows improvements of this level require 2-3 years of development work. Areas presently being pursued include

- optimization of the convertor surface shape and material properties

- optimization of apertures of the various source components

- optimization of the longitudinal magnetic field profiles (dump field)

- an increase in the radio frequency power applied to the plasma, for example with the external antenna source

\subsubsection{Ring Issues}

Maintaining beam stability is especially a concern, in light of the record beam intensities required for STS. As was discussed in Sect. 3.1, impedance-driven instabilities should not be a problem; however the electron-proton (e-p) instability onset is difficult to predict accurately. As a precautionary measure, a broadband damper feedback system is being developed to counter e-p transverse instability issues that may arise.

Section 3.1 also highlighted the charge exchange foil concerns associated with doubling the ring beam intensity. In addition to the issue of foil survivability from a heating standpoint, issues such as foil holder robustness against reflected convoy electrons and against mechanically driven fluctuations (thermal transient-driven and/or charge buildup-driven) are concerns. These issues are being investigated using the foil test stand and as part of ongoing efforts to support operations at $1.4 \mathrm{MW}$. It is expected that these efforts will continue under the operational support function. 


\subsection{REFERENCES}

1 A. T. Nelson, J. A. O’Toole, R. A. Valicenti, et al., "Fabrication of a tantalum-clad tungsten target for LANSCE," Journal of Nuclear Materials 431, 172-184 (2012).

2 S. A. Maloy, R. S. Lillard, W. F. Sommer, et al., "Water corrosion measurements on tungsten irradiated with high energy protons and spallation neutrons," Journal of Nuclear Materials 431, 140146 (2012).

3 “Tantaline - The material for hot acid," http://www.tantaline.com/, accessed October 2014.

4 G. A. Greene and C. C. Finfrock, "Vaporization of tungsten in flowing steam at high temperatures," Exp. Thermal and Fluid Science 25, 87-99 (2001).

5 G. A. Greene, C. C. Finfrock, "Behavior of high temperature tungsten and Inconnel in spallation target applications," Exp. Thermal and Fluid Science 25, 917-929 (2001). 


\section{FIRST TARGET STATION OPERATION AT 1.3 GEV AND 2 MW}

The SNS FTS was primarily designed around $2 \mathrm{MW}$ and $1.0 \mathrm{GeV}$ operation. Target systems include the mercury target module, mercury process systems, water and cryogenic hydrogen moderator systems, water, gas and related utilities, proton beam window, proton beam flight tube, source shielding, shutter systems, neutronics for all systems, and source terms for instruments. A notable exception for $2 \mathrm{MW}$ operation is the mercury target vessel, which is designed only for $1.4 \mathrm{MW}(1.0 \mathrm{GeV})$ operation. Its power capacity was de-rated because it was necessary to use thickened vessel beam entrance walls to increase the target lifetime against beam-induced cavitation damage. The target water shroud design is rated for 2.0 MW. The original (and still in service) inner reflector plug (IRP) was also power de-rated to $1.4 \mathrm{MW}$ because the fit-up between a hydrogen moderator vacuum vessel and an IRP receptacle is less than specified, and the poor fit inhibits vessel cooling.

Increasing the mercury vessel power capacity will require research and development and design changes. By the time SNS accelerator upgrades can provide for $2 \mathrm{MW}$ power at the FTS, the IRP power limit will be resolved with the deployment of a next-generation IRP. However, for all systems, operation with $1.3 \mathrm{GeV}$ protons will require updated neutronics and power deposition data. Indeed, the higher energy will affect heating, radiation damage rate, and shielding throughout the target systems and will require review and updating of system performance and lifetime assessments.

\subsection{FTS NEUTRONICS ANALYSES}

Neutronics analysis will evaluate 2.0 MW, 1.3 GeV nuclear heating, radiation damage, and shielding analysis for all relevant target systems components, including the target module, proton beam window, bulk shielding, core vessel, inner and outer reflector plugs, shutters, shine shield and pan shielding, water systems, and gas generation rates. Radiation damage lifetimes of components, e.g., the outer reflector plug, must be reassessed.

New source terms for each moderator must be provided to instrument scientists.

Radionuclide characterization of the system mercury, target, and proton beam window will be performed for safety case review and waste disposal.

\subsection{FTS TARGET SYSTEMS AT 2 MW AND 1.3 GEV}

Aside from the target module exception, the design basis for the FTS target systems was $2.0 \mathrm{MW}$ at $1.0 \mathrm{GeV}$. Operation at $1.3 \mathrm{GeV}$ will have little impact on some systems, but the higher energy will lead to deeper energy deposition in hardware at relatively close proximity to the target. Heating of the core vessel, internal components, and bulk monolith shielding will change somewhat. Adequate cooling in affected regions must be verified and necessary changes identified.

System investigation and engineering analysis will be necessary for the following target systems /issues with the potential for adverse effects from $1.3 \mathrm{GeV}$ and $2 \mathrm{MW}$ power.

- Increased heating of core vessel, internal components, and core vessel support cylinder will alter operating temperature distributions, thermal stress, and deflections.

- Higher temperatures in the core vessel support cylinder could adversely affect the alignment of the neutron guide systems. 
- Changes in bulk monolith shielding heating should be analyzed, as there are no provisions for cooling.

- Increased heating at the front of the target carriage, which also is uncooled, will require thermal and thermal stress analysis updates.

- New heat deposition in moderators should be checked and engineering analyses should be updated, if needed, for the moderator vessels and for loads on the liquid hydrogen cryogenic system.

- For Inconel and aluminum proton beam window options, new or updated heating, thermal, and thermal stress analyses are needed for the proton beam window, core vessel near the proton beam window, and IRP structure around the proton beam.

- Core vessel insert heating limits must be reviewed.

A mercury target module design for $2.0 \mathrm{MW}$ and $1.3 \mathrm{GeV}$ is necessary. This topic is covered in Sect. 9.3.

Operation at $2.0 \mathrm{MW}$ - whether at 1.0 or $1.3 \mathrm{GeV}$ - is cause to review target systems design basis calculations and compare those predictions with current high-power operating behavior where possible. Upgrading beyond 2.0 MW is not the intent; but the validation of design basis calculations and extrapolation to 2.0 MW to assess power margins will be useful and necessary, in some cases, to revalidate set point limits.

The mercury pump has been operating at less than the design basis nominal speed $-400 \mathrm{rpm}-$ because of concerns regarding pump seal longevity that arose as a result of issues with early bearing grease seals and helium gas seals. The mercury flow rate and pump speed must be increased to the nominal speed to keep mercury temperature, target temperature and thermal stress, and mercury process piping thermal stress within limits. The risk for reduced pump life upon a return to nominal speed is unquantified.

Heat removal systems will be evaluated, including all the water utility loops and the mercury heat exchanger. The margin for the cryogenic moderator systems will be estimated. The capacities of the mercury off-gas treatment system and hot off-gas ventilation systems at $2.0 \mathrm{MW}$ will be reexamined.

Some of these evaluations have already been partially completed as a part of normal FTS operation. Thorough reviews and documentation of the evaluations are appropriate for FTS operation at $2.0 \mathrm{MW}$ and $1.3 \mathrm{GeV}$.

\subsection{MERCURY TARGET DESIGN FOR 2 MW AND 1.3 GEV}

A mercury target vessel design for $2.0 \mathrm{MW}$ and $1.3 \mathrm{GeV}$ operation must satisfy design criteria for the higher steady state power and altered energy deposition field. By the time of its deployment, an increase in the radiation damage limit of the water-cooled shroud is expected as a result of current efforts in target post-irradiation examination. This increase will permit higher integrated beam energy (MW'h) and beam pulses over the life of the target, provided cavitation damage or high-cycle fatigue from pulse pressure waves do not shorten target life. Research and development is required to resolve these challenges. The research and development suggested for gas bubble mitigation is discussed in Sect. 8.1.4.

The early leak in operating target T10 provides an opportunity to assess the damage mitigation efficacy of the jet-flow target design to protect the inner target wall. In any case, prospects for sufficient damage reduction as a result of the jet-flow design are not certain at $2 \mathrm{MW}$. Gas injection techniques-protective gas walls and small gas bubble injection - are viewed as necessary. It is envisioned that both of those 
techniques will be incorporated in some combination into the jet-flow design. Either approach will require a process gas injection or recirculation system.

To prepare for deployment of these damage mitigation techniques, several development steps are envisioned, including

- gas recirculation system development with flow rate to be determined

- small gas bubble injection modeling and experiments in the water test loop and in the target test facility

- gas wall injection modeling and experiments in the water test loop and target test facility

High-cycle fatigue stress due to beam pulsing is increasingly a concern because of its importance to an adequate target life at higher power. The beam-induced pressure waves that drive cavitation also drive dynamic vessel stress. A small amount of erosion or a weld or material defect could act as a fatigue crack initiator. With cyclic loading (on the order of $10^{9}$ pulses are experienced in a target life), these events could lead to fatigue failure and leakage, as was the case with the defective welds in T6 and T7. Small bubble gas injection can reduce pressure wave-driven vessel stress, as well as cavitation intensity. The Japan Spallation Neutron Source (JSNS) has adopted this approach for its mercury target, where on-line vibration monitoring indicates reduced stress. The present gas injection rate at the JSNS is 7 SLPM (standard liters per minute), and JSNS staff are preparing for 10 SLPM, which corresponds to an injected gas volume ratio of $1 \%$ (JSNS has half the mercury flow rate of the SNS). The corresponding gas rate for SNS would be approximately 20 SLPM. The long-term damage protection benefit for the JSNS target has yet to be confirmed, as its target average power and operating life lag that of the FTS. SNS staff are observing the JSNS operating experience closely before committing to a gas rate.

The SNS mercury vessel currently features a center baffle that was incorporated late in the SNS construction project. The baffle was added out of concern regarding the pulse rate excitation of a mode shape that was revealed by coupled acoustic-structural analysis of the vessel without a baffle at $73 \mathrm{~Hz}$. Although the analysis technique contained considerable uncertainties, no better approach could be found, and the $60 \mathrm{~Hz}$ beam pulse rate was thought to be close enough to possibly drive the vessel to resonance. The addition of the center baffle increases the mode shape frequency at the expense of higher beam pulse vessel stress and high thermal stress in the baffle (it is directly in the beam). Post-irradiation examination of FTS mercury vessels has revealed substantial cavitation erosion of the front end of the baffle and a lengthwise through-wall crack extending its full length, both illustrated in Fig. 9.1 (from Target 9). Its benefit against modal resonance is in question, as the baffle offers stiffness in compression but not in tension. Removal or modification of the baffle and other design changes will be needed for $2 \mathrm{MW}$ operation.

Narrowing or shortening the front of the target could increase this mode frequency if the center baffle were removed; this issue will be explored by simulation. Shortening the target may also have neutronics performance benefits. Off-line experimental verification is not perceived as useful because a major uncertainty is the reduction of mercury sound speed (and consequent lowering of mode frequency) if an unknown population of cavitation vapor bubbles, or injected helium gas bubbles, is present. A useful online experiment could be conducted with a target instrumented with strain or vibration measuring sensors, if suitable techniques could be found and if SNS operating time were approved for this purpose. A more direct option might be trial operation of an un-instrumented, modified production target with the center baffle cut lengthwise to remove part or all of it. If it were approved, operation for a week or two during a maintenance period would be sufficient to confirm whether resonance is an issue with such a 
design change. If it did survive, a design path forward would be confirmed and the target could be left in for neutron production.
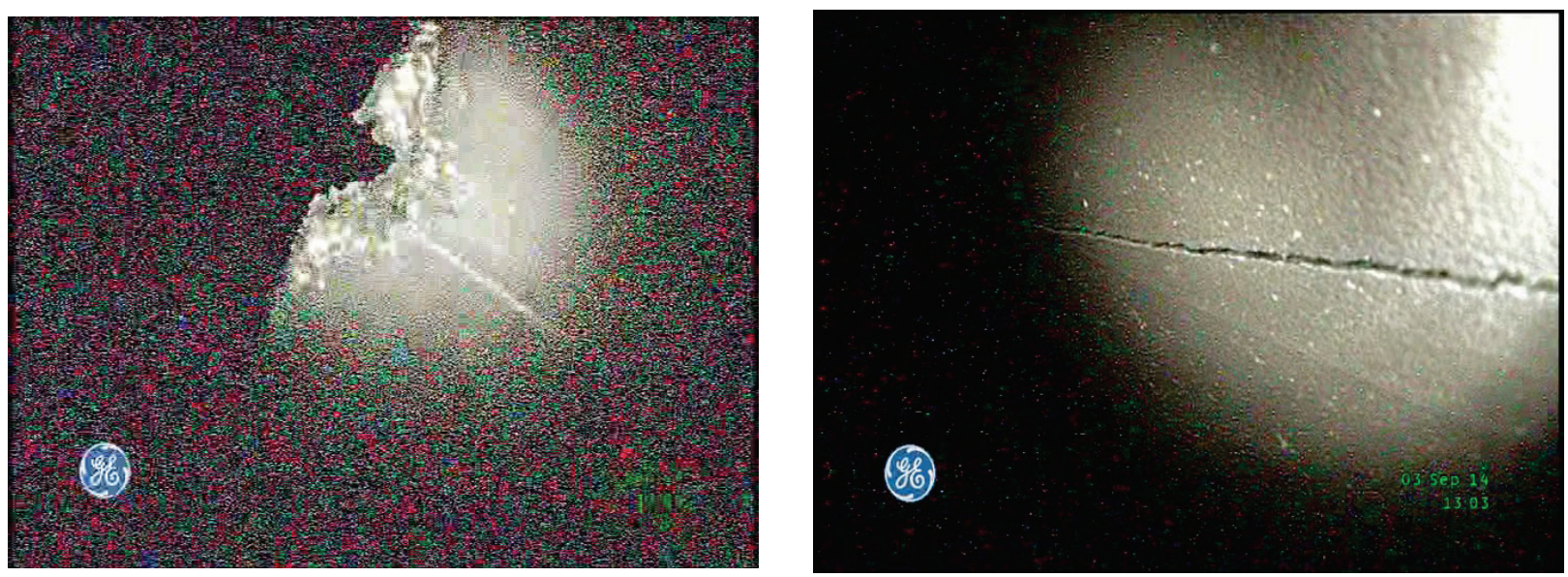

Fig. 9.1. Target 9 center baffle cavitation erosion at its forward edge is shown on the left; a through-wall crack is apparent in the image on the right, from the mid-length of the center baffle. Target 9 average lifetime power was $1.03 \mathrm{MW}$, and it accepted $4195 \mathrm{MW} \cdot \mathrm{h}$ of beam energy. 


\section{SAFETY ASSESSMENT}

\subsection{NATIONAL ENVIRONMENTAL POLICY ACT IMPACT}

In examining the National Environmental Policy Act documentation for construction and operation of SNS, approval for the project and/or activity was granted by the "Record of Decision (ROD) for the Construction and Operation of the Spallation Neutron Source," issued June 30, 1999 [1]. The respective ROD was based on the analysis contained in Ref. [2] (Final Environmental Impact Statement for the Construction and Operation of the Spallation Neutron Source).

As described in the final environmental impact statement (FEIS), the proposed action was to construct and operate SNS, a state-of-the-art, short-pulsed spallation neutron source comprising an ion source, linear accelerator (linac), proton accumulator ring, liquid mercury target, and suite of neutron scattering instruments. Furthermore, the facility would be designed to operate at a proton beam power of $1 \mathrm{MW}$ yet be economically upgradeable to $4 \mathrm{MW}$. The respective upgrades were identified as follows:

1. Adding a second target station with its own set of instrumentation

2. Increasing the proton beam power to $2 \mathrm{MW}$ by doubling the ion source output

3. Increasing the proton beam power to $4 \mathrm{MW}$ by adding a second ion source, modifying the linac, and adding a second proton accumulator ring

It was recognized that implementation of the upgrades would depend on the need for the upgrades and the availability of future funding. However, the FEIS analyzed both the base $1 \mathrm{MW}$ facility and the fully upgraded $4 \mathrm{MW}$ configuration. The $4 \mathrm{MW}$ case is a bounding case beyond the scope of the upgrade proposed here.

Based on analysis found in Ref. [2], DOE decided to proceed with construction and operation of a stateof-the-art spallation neutron source facility at the preferred location, Oak Ridge National Laboratory in Oak Ridge, Tennessee, and the respective "ROD for the Construction and Operation of the Spallation Neutron Source" was issued in the Federal Register on June 30, 1999 [1]. Thus construction and operation of the STS has been addressed in the FEIS and ROD for SNS. If supplemental National Environmental Policy Act documentation is required to support ancillary facilities and/or access roads, that documentation will be prepared at the appropriate time.

\subsection{RADIOACTIVE CONTAMINATION OF GROUNDWATER}

In the FEIS, it was recognized that operation of SNS might result in neutron activation of soils in the shielding berm surrounding the linac and accumulator ring. The resulting contamination of berm soils by radionuclides would create the potential for infiltrating water to transport radionuclides from the berm to saturated groundwater zones nearby. The stakeholders expressed concern about this potential. In response to this concern, SNS implemented engineering controls and a site-specific groundwater monitoring program.

\subsubsection{Engineering Controls}

SNS implemented engineering controls focusing on both facility design and construction features to minimize the mobility of activation products in the site hydrologic system. Specifically, the shielding berm (Fig. 10.1) was designed and constructed to isolate radionuclide contamination generated by the SNS particle beam and to provide radiation protection for outside areas around the beam and ring tunnel. 
The activation of berm soils was minimized by beam loss control and passive shielding. Furthermore, the berm was constructed of compacted native soils and was engineered to isolate activation products by minimizing the amount of water infiltrating the berm. The construction of the berm using compacted indigenous clay rendered it relatively impermeable to water (The Darcy permeability of the compacted clay was much less than $1 \mathrm{e}-05 \mathrm{~cm} / \mathrm{s}$ ). In addition, a flexible membrane liner coupled with a drainage net was included in the design to provide an additional degree of control against water penetration.

These same engineering controls (or equivalent ones) will be incorporated into the design and operation of the STS.

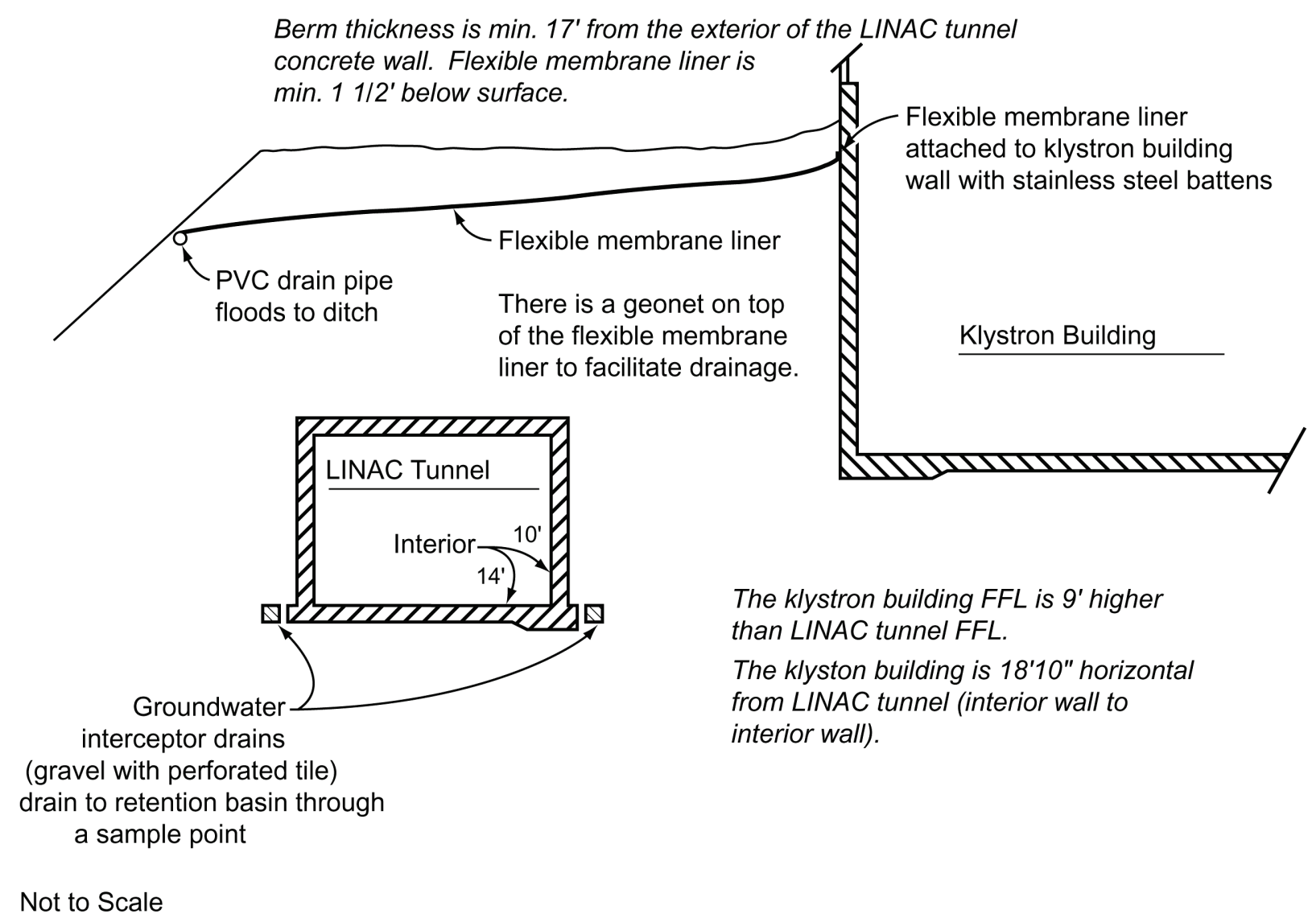

Fig. 10.1. Typical berm cross section.

\subsubsection{Groundwater Monitoring Program}

In addition to the use of engineering controls, a groundwater monitoring program was implemented to ensure that any releases of radiological contaminants from the facility would be detected, tracked, and evaluated to identify any unacceptable impacts to groundwater or surface water. 
The SNS site is located atop Chestnut Ridge, northeast of the main ORNL facilities. The site slopes to the north and south, and small stream valleys, populated by springs and seeps, lie on the ridge flanks. Surface water drainage from the site flows into Bear Creek to the north and White Oak Creek to the south.

The site is a hydrologic recharge area underlain by geologic formations that form karst geologic features. Groundwater flow directions at the site are based on the generally observed tendency for groundwater to flow parallel to geologic strike (parallel to the orientation of the rock beds) and via karst conduits that break out at the surface in springs and seeps located down gradient of the SNS site. A sizable fraction of infiltrating precipitation (groundwater recharge) flows to springs and seeps via the karst conduits.

The ability to accurately model the fate and transport of neutron activation products generated by beam interactions with the engineered soil berm is complicated by multiple uncertainties resulting from a variety of factors, including hydraulic conductivity differences in earth materials found at depth, the distribution of water-bearing zones, the fate and transport characteristics of neutron activation products produced, diffusion and advection, and the presence of karst geomorphic features found on the site. These uncertainties led to the initiation of the groundwater surveillance monitoring program at the SNS site.

A total of seven seeps/springs and surface water sampling points (seeps/springs S-1, S-2, S-3, S-4, S-5, and SP-1 and surface-water point SW-1) are routinely monitored as analogues to, and in lieu of, groundwater monitoring wells. Locations were chosen based on hydrogeological factors and proximity to the beam line. Figure 10.2 shows the locations of the specific monitoring points sampled as part of the monitoring program.

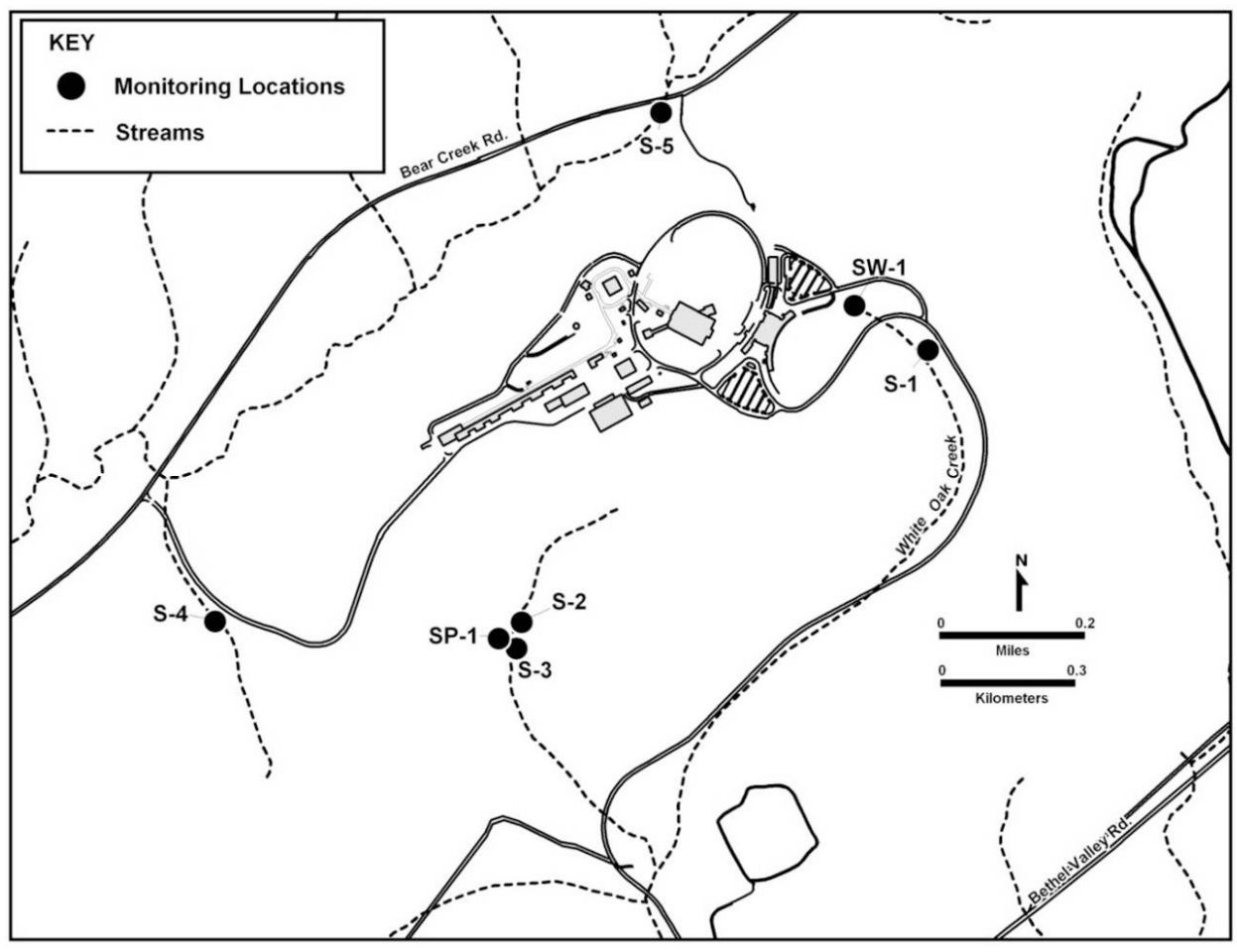

Fig. 10.2. Groundwater monitoring locations at the SNS site. 
The SNS groundwater monitoring program was implemented in 2004. From 2004-2006, a two-year baseline program was conducted before commencement of accelerator operations. Subsequently, with the commencement of accelerator operations in April 2006, the baseline groundwater monitoring program was completed and an operational groundwater monitoring program commenced. As of 2014, there have been no adverse impacts to groundwater quality as a result of accelerator operations at SNS. Furthermore, the current monitoring program is robust and more than adequate to support construction and operation of the STS.

\subsection{RADIOACTIVE EMISSIONS TO THE ATMOSPHERE}

The SNS STS will release short-lived, accelerator-produced radionuclides into the atmosphere. STS air emissions will be permitted by the Tennessee Department of Environment and Conservation, as delegated by the US Environmental Protection Agency (EPA).

Radioactive releases from DOE facilities are regulated under 40 CFR 61, National Emission Standards for Hazardous Air Pollutants, Subpart H, which are also incorporated in Tennessee Air Pollution Control Regulation 1200-3-11-.08, "Emission Standards for Emissions of Radionuclides Other than Radon from Department of Energy Facilities" [3,4]. Subpart H $§ 61.92$ states that "Emissions of radionuclides to the ambient air from Department of Energy facilities shall not exceed those amounts that would cause any member of the public to receive in any year an effective dose equivalent of $10 \mathrm{mrem} /$ year." Subpart $\mathrm{H}$ \$61.93(a) requires the following: "To determine compliance with the standard, radionuclide emissions shall be determined and effective dose equivalent values to members of the public calculated using EPAapproved sampling procedures, computer models CAP-88 [Clean Air Act Assessment Package-1988] ...."

It is assumed that STS radioactive emissions will be comparable with those currently discharged by SNS. However, a specific source term will be developed, and a CAP- 88 determination will be performed for the modeled STS releases in accordance with Subpart H §61.93(c)(4)(f):

To determine whether a release point is subject to the emission measurement requirements of paragraph (b) or (c) of this section, it is necessary to evaluate the potential for radionuclide emissions for that release point. In evaluating the potential of a release point to discharge radionuclides into the air for the purposes of this section, the estimated radionuclide release rates shall be based on the discharge of the effluent stream that would result if all pollution control equipment did not exist, but the facilities operations were otherwise normal.

Such releases without pollution control equipment are referred to as unabated releases, while those with pollution control equipment are called abated releases. Based on preliminary calculations and process knowledge, unabated releases from the STS have the potential to exceed an effective dose equivalent of $10 \mathrm{mrem} /$ year [5]; therefore, Subpart H $§ 61.93(\mathrm{e})$ requires the following:

Radionuclide emission measurements in conformance with the requirements of paragraph (b) or (c) of this section shall be made at all release points that have a potential to discharge radionuclides into the air in quantities that could cause an effective dose equivalent in excess of $1 \%$ of the standard. All radionuclides that could contribute greater than $10 \%$ of the potential effective dose equivalent for a release point shall be measured. With prior EPA approval, DOE may determine these emissions through alternative procedures. For other release points that have a potential to release radionuclides into the air, periodic confirmatory measurements shall be made to verify the low emissions. 
The STS emission monitoring requirement will be met under Subpart H §61.93(c).

(1) Effluent flow rate measurements shall be made using the following methods:

(i) ANSI/HPS N13.1-1999, Sampling and Monitoring Releases of Airborne Radioactive Substances from the Stacks and Ducts of Nuclear Facilities, shall be used to determine velocity and volumetric flow rates for stacks and large vents [6].

(iii) The frequency of the flow rate measurements shall depend upon variability of the effluent flow rate. For variable flow rates, continuous or frequent flow rate measurements shall be made. For relatively constant flow rates only periodic measurements are necessary.

(2) Radionuclides shall be directly monitored using the following methods:

(i) ANSI/HPS N13.1-1999 shall be used to select monitoring or sampling sites.

(ii) The effluent stream shall be directly monitored continuously with an in-line detector ... following the guidance presented in ANSI/HPS N13.1-1999.

(iii) Radionuclides shall be collected and measured using procedures based on principles of measurement described in appendix B, Method 114, of this part.

STS emission monitoring will be conducted by in-line, gross gamma counting as prescribed in Appendix B, EPA Method 114, Method G-4, "Gross Gamma Counting."

Principle. The sample is counted with a gamma detector, usually a thallium activated sodium iodine crystal. The detector is coupled to a photomultiplier tube and gamma rays above a specific threshold energy are counted.

Applicability. Gross gamma measurements may be used to measure emissions of specific radionuclides only when it is known that the sample contains a single radionuclide or the identity and the isotopic ratio of the radionuclides in the effluent stream are well known. When gross gamma measurements are used to determine emissions of specific radionuclides, periodic measurements using methods G-1 or G-2 should be made to demonstrate that the gross gamma measurements provide reliable emissions data. This method may be applied to analysis of gaseous radionuclides directly in an effluent stream by placing the detector directly in or adjacent to the effluent stream.

The STS will demonstrate that gross gamma measurements provide reliable radionuclide-specific emissions data using EPA Method 114, Method G-1, "High-Resolution Gamma Spectrometry."

Principle. The sample is counted with a high resolution gamma detector, usually either a Ge (Li) or a high purity Ge detector, connected to a multichannel analyzer or computer. The gamma emitting radionuclides in the sample are measured from the gamma count rates in the energy regions characteristic of the individual radionuclide. Corrections are made for counts contributed by other radionuclides to the spectral regions of the radionuclides of interest.

Applicability. This method is applicable to the measurement of any gamma emitting radionuclide with gamma energies greater than $20 \mathrm{keV}$. It can be applied to complex mixtures of radionuclides. ... The method may also be applied to the analysis of gaseous gamma emitting radionuclides directly in an effluent stream. 
The STS radioactive release requirements under the National Emission Standards for Hazardous Air Pollutants will be met using an in-line detector [3]. The STS releases are short-lived, gaseous or gaseouslike radionuclides that are best monitored by an in-line detector. The source term will be developed and modeled based on known accelerator conditions.

\subsection{WASTE MANAGEMENT}

The STS will generate numerous types of waste from normal operations and maintenance activities. According to Refs. [7] and [8], planning shall be performed to address the entire life cycle for all waste streams before generation. A waste management plan will be developed for the STS that will address the entire life cycle of all waste streams foreseen to be generated as the result of SNS/STS operations and maintenance. Furthermore, based on process knowledge, all waste streams envisioned to be generated by the STS have identified paths to disposal.

\subsubsection{Disposition Mapping}

To address the entire life cycle of STS operational and maintenance waste streams, the STS Waste Management Plan shall incorporate the use of disposition mapping [9]. "Disposition" is an inclusive term that refers to "those activities that follow generation of a waste and which constitute completion of the life cycle of management of the waste, including, but not limited to, stabilization, deactivation, disposal, decommissioning, dismantlement, and/or reuse" (adapted from DOE O 430.1B) [10].

DOE Headquarters (DOE-HQ) has addressed planning for low-level waste (LLW) and mixed low-level waste (MLLW). The Record of Decision for the Department of Energy's Waste Management Program: Treatment and Disposal of Low-Level Waste and Mixed Low-Level Waste; Amendment of the Record of Decision for the Nevada Test Site was issued February 18, 2000 [11]. The decisions contained therein serve as the programmatic bases for the current master LLW and MLLW disposition maps maintained at DOE-HQ. There were four decisions addressing LLW treatment and disposal and MLLW treatment and disposal, respectively.

1. DOE decided that each site will perform minimal treatment on its LLW, although each site may perform additional treatment as would be useful to decrease overall costs. However, it was noted that this decision does not preclude DOE's use of commercial treatment facilities, consistent with DOE regulations and policies.

2. DOE decided to establish regional LLW disposal at two DOE sites: the Hanford Site and the Nevada Test Site (NTS). In addition to disposing of their own LLW, the Hanford Site and NTS will receive and dispose of LLW that is generated and shipped (by either truck or rail) by other sites that meets the waste acceptance criteria. Although the decision acknowledged that DOE will continue, to the extent practicable, on-site disposal at the Oak Ridge Reservation (ORR), current disposition maps show the majority of ORR-generated LLW going to NTS.

3. DOE decided to conduct regional MLLW treatment at numerous sites, inclusive of ORR, as would be consistent with current site treatment plans. This decision does not preclude DOE's use of commercial treatment facilities, consistent with DOE regulations and policies.

4. DOE decided to establish regional MLLW disposal operations at two DOE sites: the Hanford site and the NTS. In addition to managing their own MLLW, the Hanford site and NTS will receive and dispose of MLLW generated and shipped (by truck or rail) by other sites, consistent with permit conditions and other applicable requirements. Once again, this decision does not preclude DOE's use of commercial disposal facilities, consistent with current DOE regulations and policies. 
Hazardous waste planning is also addressed by DOE-HQ. However, the treatment and/or disposal of hazardous waste is well understood, and numerous commercial treatment and/or disposal options are available. Therefore, in the Record of Decision for the Department of Energy's Waste Management Program: Treatment of Non-wastewater Hazardous Waste, issued August 5, 1998 [12], DOE states its decision to continue use of off-site facilities for the treatment of major portions of the nonwastewater hazardous waste generated at DOE sites. Concurrently, DOE recognized that the ORR may treat some of its own nonwastewater hazardous waste on-site, where capacity is available in existing facilities and where treatment is economically favorable. This decision serves as the programmatic basis for the current master hazardous waste disposition map maintained at DOE-HQ.

Sanitary/industrial waste is typically not included in DOE-HQ planning initiatives because treatment and/or disposal of sanitary/industrial waste is well understood and occurs at all DOE facilities. Typically, the treatment and/or disposal of sanitary/industrial waste is regulated by state programs administered by the state of the respective DOE facility. Sanitary/industrial waste generated on the ORR consists of recyclable materials, such as metals, and nonrecyclable materials such as glass, paper, and wood. Nonrecyclable materials are disposed of in state-permitted land disposal units (sanitary/industrial landfills).

Regardless of waste categorization, the STS will aggressively implement pollution prevention principles to prevent and/or reduce the amount of waste generated. As delineated in the Pollution Prevention Act of 1990, the STS will employ the following four principles with respect to all operations: (1) pollution should be prevented and reduced at the source whenever feasible, (2) pollution that cannot be prevented should be recycled in an environmentally safe manner whenever feasible, (3) pollution that cannot be prevented or recycled should be treated in an environmentally safe manner whenever feasible, and (4) disposal or other release to the environment should be employed only as a last resort and should be conducted in an environmentally safe manner. Furthermore, the STS embraces the ORNL Pollution Prevention Policy whereby "we are committed to providing a safe and healthful working environment for all staff, guest scientists and engineers, and visitors; protecting the general public and the environment from unacceptable environment, safety, and health (ES\&H) risks; operating in a manner that protects and restores the environment; and integrating pollution prevention into planning and decision-making." As such, the STS will follow ORNL pollution prevention guidelines.

\subsubsection{Radioactive Low-Level Waste}

Low-level radioactive waste is radioactive waste that is not high-level radioactive waste, spent nuclear fuel, transuranic waste, by-product material [as defined in Section 11(e)2 of the Atomic Energy Act of 1954 (42 U.S.C. 2011 et seq)], or naturally occurring radioactive material (adapted from the Nuclear Waste Policy Act of 1982, as amended). Furthermore, "[r]adioactive waste produced as a result of operations of DOE accelerators is low-level waste and shall be managed in accordance with DOE O 435.1, Radioactive Waste Management, and [DOE M 435.1-1], and all applicable Federal or State requirements" (DOE M 435.1-1, p. IV-1) [7,8]. Typical examples of STS waste streams in this category include dry active waste (e.g., air filters, plastic cables, hoses and piping, floor sweepings, mops, wipes), radioactive scrap metal (e.g., metal filters, motors, pumps, and piping), resins and trapping materials (i.e., filters and ion-exchange resins), remote-handled miscellaneous solids (e.g., dry active waste, irradiated hardware, radioactive scrap metal, and resins/trapping material), wastewaters (condensate and cooling waters), and organic liquids (oils) generated during STS maintenance and operations.

At present, dry active waste and radioactive scrap metal generated by STS will be transported directly to commercial facilities and/or the Nevada National Security Site (NNSS) for ultimate disposition. Radioactive scrap metal may undergo recycle and reuse when feasible. Resins/trapping material and remote-handled LLW may be temporarily stored for decay and/or transported directly to commercial 
and/or NNSS for storage for decay and/or disposal. STS process wastewaters will undergo on-site treatment at either the ORNL Wastewater Treatment Complex or the LLW Evaporator Facility. LLW organic liquids will be managed as MLLW.

The STS LLW management approach is consistent with the February 18, 2000, Record of Decision for DOE's Waste Management Program: Treatment and Disposal of Low-Level Waste and Mixed Low-Level Waste; Amendment of the Record of Decision for the Nevada Test Site [11] and is in agreement with the current waste disposition practices at ORNL.

\subsubsection{Mixed Low-Level Waste}

MLLW comprises both hazardous and low-level radioactive waste. It is managed according to requirements established under the Resource Conservation and Recovery Act (RCRA) for hazardous waste and the Atomic Energy Act of 1954 for its radioactive components. The hazardous component of MLLW is subject to either EPA or state hazardous waste regulations promulgated under RCRA. The STS will not generate any hazardous and low-level radioactive waste for which the hazardous component is regulated under the Toxic Substances Control Act (TSCA).

STS operations and maintenance will typically generate four MLLW waste streams (1) miscellaneous solids, (2) organic liquids, (3) remote-handled miscellaneous solids, and (4) remote-handled organic liquids. At present, both miscellaneous solids and organic liquids will be transported to commercial facilities and/or NNSS for treatment and/or disposal. At present, the NNSS has a mixed waste disposal unit located within the Radioactive Waste Management Complex in Area 5 of the NTS. In addition, there are commercial facilities that can support treatment and/or disposal of MLLW.

The STS MLLW management approach is consistent with the February 18, 2000, Record of Decision for the Department of Energy's Waste Management Program: Treatment and Disposal of Low-Level Waste and Mixed Low-Level Waste; Amendment of the Record of Decision for the Nevada Test Site [9] and is in agreement with current waste disposition practices at ORNL.

\subsubsection{Hazardous Waste}

Hazardous waste comprises nonradioactive waste exhibiting the characteristics of ignitability, corrosivity, reactivity, or toxicity, as defined by the EPA's regulations implementing RCRA, or waste that the EPA has listed under RCRA as hazardous waste. In addition, DOE manages some state-regulated hazardous wastes and hazardous wastes regulated under TSCA as hazardous wastes. STS maintenance and operations should not generate TSCA waste; therefore, the management of TSCA-hazardous waste is not addressed in this plan. Conversely, STS maintenance and operations will generate RCRA-hazardous waste. Typical examples of waste streams in this category include miscellaneous solids (i.e., lamps and wipes) and miscellaneous liquids (i.e., oils, plating solutions, and solvents). STS hazardous waste disposition will focus on use of commercial treatment and/or disposal.

The commercial treatment and/or disposal of hazardous waste is consistent with DOE's August 5, 1998, Record of Decision for the Department of Energy's Waste Management Program: Treatment of Nonwastewater Hazardous Waste to continue use of off-site facilities for treatment of major portions of nonwastewater hazardous waste generated at DOE sites [12]. However, DOE does acknowledge that ORR may treat some of its own nonwastewater hazardous waste on site where capacity is available in existing facilities and where such treatment is economically favorable. Thus, the STS hazardous waste management approach is consistent with DOE's decision and current waste disposition practices at ORNL. 


\subsubsection{Sanitary/Industrial Waste}

Sanitary/industrial waste comprises both liquid and solid wastes. Liquid wastes typically consist of industrial and/or sanitary wastewaters that are routed to the ORNL sanitary sewer system. All such wastewaters comply with the respective waste acceptance criteria for the sewage treatment plant. The STS cooling tower waters will be discharged to an on-site sediment/retention pond, which discharges to the headwaters of White Oak Creek. All liquid discharges, both those routed to the sewage treatment plant and those routed to the on-site sediment/retention pond, are permitted under existing National Pollutant Discharge Elimination System permit(s).

The solid wastes are nonhazardous, nonradioactive solid wastes from areas or systems free of potential activation or surface contamination. Typical examples of waste streams in this category include dry solids (e.g., air supply filters, plastic cables, hoses and piping, floor sweepings), resins and trapping materials (ion-exchange resins), and nonrecyclable scrap metal (potentially aerosol cans) generated during STS maintenance and operations. In addition to potentially nonrecyclable scrap metal, a significant quantity of recyclable scrap metal will result from maintenance and operations.

At present, the state of Tennessee regulates a subcategorization of sanitary/industrial waste known as "special wastes." Special wastes include sludges, bulky wastes, pesticide wastes, medical wastes, industrial wastes, liquid wastes, exempted hazardous wastes, friable asbestos wastes, combustion wastes, and other solid wastes that are difficult or dangerous to manage. Presently, approved special waste streams that could be disposed of in the reservation landfill include empty hazardous material containers, empty aerosol cans, fiberglass, glass, and empty pesticide containers. Special waste streams requiring evaluation and approval include ion-exchange resins and filters from the Central Utilities Building's deionized water supply, filters from the tunnel confinement or purge ventilation exhaust system, filters and ion-exchange resins from the target cooling loop deionized water supplies, and used oil filters from nonactivated systems. Miscellaneous maintenance materials that are not contaminated with oils or solvents from the front-end system, warm linac, or Klystron Gallery are also in this category. Process knowledge and/or sampling and analysis may be used for categorizing and characterizing solid waste.

\subsubsection{Facility Safety}

The STS will have a safety evaluation in accordance with DOE G 420.2-1, Accelerator Facility Safety Implementation Guide for DOE 420.2B, Safety of Accelerator Facilities [13]. A preliminary safety analysis document and final safety analysis document will be developed during preliminary and final design. As was done for the FTS, a systematic evaluation will be performed using safety experts and the design teams to identify hazards, credible impacting events, initiators of events, assumptions used in estimating impacts, consequences of events, and the controls required to reduce risks. This will be done for the accelerator upgrades, operation of the FTS at up to $2 \mathrm{MW}$, and operation of the STS with new instruments at up to $500 \mathrm{~kW}$. One of the principal differences between the FTS and the STS is the proposed use of a compact tungsten target. Such a target has the decay heat concentrated in a small volume that can reach elevated temperatures and has a potential release mechanism by the vaporization of the tungsten in steam. A preliminary evaluation of the radiological consequences of releases for the conceptual tungsten target will be prepared.

Furthermore, the Accelerator Readiness Review (ARR) program delineates a structured approach for ensuring that commissioning and/or routine operations are conducted safely. The program reflects requirements of DOE O 420.2C, Safety of Accelerator Facilities [14]. According to the order, an accelerator safety program must include the following elements: an approved accelerator safety envelope; a safety assessment document; clearly defined roles and responsibilities for accelerator activities, including those for training and procedures; an unreviewed safety issue process; an ARR program; and a 
current listing/inventory of accelerators under this order (inclusive of exemptions or equivalencies under the order). As the STS moves forward, the ARR program will be fully implemented.

\subsubsection{Natural Phenomenon Hazards}

DOE Standard 1020-2012, Natural Phenomena Hazards Analysis and Design Criteria for DOE Facilities [15], provides criteria and guidance for the analysis and design of facility structures, systems, and components (SSCs) that are necessary to implement the requirements of DOE O 420.1C, Facility Safety [16], and to ensure that the SSCs will be able to effectively perform their intended safety functions under the effects of natural phenomena hazards (NPHs). This standard also provides criteria and guidance for the use of industry building codes and voluntary consensus standards in the NPH analysis and design of SSCs in DOE facilities. In particular, it provides criteria and guidance for the following:

Establishing the performance requirements for SSCs in terms of parameters that define failure of their safety functions (e.g., design basis flood water level relative to the location of an SSC that is vulnerable to inundation, the state of SSC deformation under various NPH loads, and limit states under dynamic loads) and grading the SSCs into more than one NPH design category, based on the consequences of SSC failure when subjected to NPH events:

- Calculating NPH demands on SSCs resulting from NPH events in terms of parameters that define failure of their safety functions

- Designing (or, for existing facilities, design evaluation) SSCs to ensure their ability to maintain required functionality when subjected to demands of NPH events

This standard will be used for the analysis and design of the STS. At present, the assumptions listed in Table 10.1 are used here to categorize the current accelerator/target facilities.

Table 10.1. Seismic design categories used for the STS buildings

\section{Seismic design categories for new facilities associated with STS}

1. Lab/Office/Maintenance/Experiment Support Facility

PC-1 Risk

2. Central Utility Bldg. (including cooling towers)

PC-1

3. R2T2 Support Bldg.

PC-1

4. Modulator Support

PC-1

5. Central Exhaust Facility

PC-1

6. R2T2

$\mathrm{PC}-2$

7. STS (e.g., monolith, hot cell)

$\mathrm{PC}-3$

8. STS Instrument Hall

$\mathrm{PC}-2$

9. Satellite and 90M Instrument Bldgs. (including tunnels)

PC-2 
Table 10.1. Seismic design categories used for the STS buildings (continued)

\begin{tabular}{lccc}
\hline \multicolumn{1}{c}{ Building } & $\begin{array}{c}\text { Seismic design } \\
\text { category (SDC) }\end{array}$ & Limit state & $\begin{array}{c}\text { ASCE 7-10 risk } \\
\text { category }\end{array}$ \\
\hline Lab/Office/Maintenance/Experiment Support Facility & SDC-1 & A (?) & II \\
Central Utility Bldg. (including cooling towers) & SDC-1 & A (?) & II \\
R2T2 Support Bldg. & SDC-1 & A (?) & II \\
Modulator Support & SDC-1 & B (?) & II \\
Central Exhaust Facility & SDC-1 & B (?) & II \\
R2T2 & SDC-2 & C (?) & IV \\
STS (e.g., monolith, hot cell) & SDC-3 & D & n/a \\
STS Instrument Hall & SDC-2 & C (?) & IV \\
Satellite and 90M Instrument Bldgs. (including tunnels) & SDC-2 & C (?) & IV \\
\hline
\end{tabular}

\subsection{RADIATION SAFETY}

The SNS facility places the safety of the public and workers above all other considerations. Direct radiation resulting from beam operations and residual radiation emitted by activated materials are two of the most important nonindustrial safety hazards at the SNS site. A major consideration in the design and operation of the STS will be to reduce radiation exposures to the public, to facility and co-located workers, and to the environment to levels as low as reasonably achievable (ALARA).

In compliance with 10 CFR 835.1001, Design and Control, and 10 CFR 835.1002, Facility Design and Modification, the STS facility design objective is to limit prompt radiation in continuously occupied areas to less than $0.25 \mathrm{mrem} / \mathrm{h}$ and as far below that level as is reasonably achievable [17,18]. Iterations of extensive modeling calculations based on conservative beam-loss predictions will be used to optimize shielding. The adequacy of installed shielding and the magnitude and characteristics of radiation produced by routine operations will be assessed to the extent practical during initial commissioning and operations. Fault studies will be conducted as part of commissioning. Areas that are not occupied full-time, yet have dose rates above the nominal design objective, might have restricted access at an appropriate level. A high-integrity automatic protective system, the Personnel Protection System, will exclude workers from areas with dangerous radiation levels in accordance with DOE O 420.2B, Safety of Accelerator Facilities [19]. During normal operations, real-time radiation monitors and ongoing periodic radiation surveys will ensure that radiation levels in accessible areas are well characterized and within expected ranges and that access is controlled in accordance with 10 CFR 835. Active real-time radiation detectors with the capability to shut off beam through the Personnel Protection System will be placed in potentially occupied areas where credible beam-spill accidents could produce unacceptable radiation levels.

\subsubsection{Shielding Policy}

SNS minimizes radiation exposure to ALARA levels and well below regulatory limits, and this same philosophy will be implemented for the STS. Engineered controls will be employed as the primary method for maintaining radiation exposures in occupied areas to ALARA levels, in accordance with 10 CFR 835.1001 and 10 CFR 835.1002. Administrative procedures, appropriate area classifications and restrictions, and compliance with ORNL Standards Based Management System subject areas will be additional components in the STS defense-in-depth strategy to further limit radiation exposures. In addition to normal operating conditions, fault and accident conditions will be considered for shielding design and selection. Bulk shielding surrounding the proton and neutron beam confinements (including 
concrete, iron, soil, and other materials) will be designed to maintain radiation exposures at an ALARA level in all areas, considering the expected occupancy levels. Additional physical barriers (e.g., locked doors, fences) will be incorporated in areas surrounding the shielding to ensure that occupancy in these areas is controlled as necessary. ALARA design reviews will serve as a foundational element for the approval process needed for major changes in design or operations. Trained personnel will confirm the continuing adequacy of the workplace radiological controls as part of periodic area radiological surveillances.

Shielding designs (drawings and calculations), including proposed major modifications to existing area shielding, will be reviewed and approved by both management and technical representatives. The STS facility shielding will be under a configuration control procedure, and movable shielding will be locked or labeled as appropriate to prevent unauthorized changes that might significantly reduce worker safety. Visual inspections will be used to confirm proper installation of approved shielding and barriers. Controlled low-intensity beam and fault studies will be used as appropriate to verify the effectiveness of area shielding designs.

The effectiveness of the SNS/STS shielding and access control policy will be demonstrated by achievement of the following performance goals:

- Radiation exposures at the SNS facility will be ALARA, as implemented by the SNS ES\&H director.

- The annual radiation dose from normal operations at the SNS site boundary will not exceed 25 mrem from all pathways.

- Beam faults at the SNS will not lead to an integrated dose greater than 25 mrem per year at the site boundary.

- No members of the public or untrained visitors will unintentionally access a radiological area.

- No one who is not trained as a radiological worker will receive more than 100 mrem in any calendar year while on the SNS site.

Most worker dose is expected to result from exposure to activated materials. Materials included in designs will be chosen to minimize activation and the generation of hazardous/mixed waste. The upgraded accelerator and associated systems will be designed and operated to minimize activation and worker exposure to activated materials. Shielding will be employed as necessary to reduce the dose received from activated equipment and items. Personnel exposure to liquid and gaseous activated materials (including cooling water, dust, air, and mercury) will be minimized through appropriate combinations of confinement, differential pressure, holding for decay, flushing, local shielding, and protective equipment. Personnel who will potentially be exposed to radiation will wear appropriate dosimetry, including extremity and neutron dosimeters as appropriate.

Some activated structures and materials being removed from beam-accessible areas or awaiting repair may be stored for decay. These items will be shielded so that dose rates in adjoining areas will meet the SNS/STS dose rate criteria. It is also important to protect materials stored outdoors from deterioration so that activated components do not develop removable contamination, so it is SNS policy that radioactive components and materials stored outside are protected from the elements by covers or paint.

When the beam is off, a permit-access system will be employed that controls entry to the beam tunnel and other areas containing potentially highly activated equipment and materials. Radiological work permits for work in radiological areas will ensure proper authorization and training of personnel before access. 
Most users and visiting scientists will be restricted to experimental areas of the STS and adjacent publicaccess areas of the facility.

Configuration control will play a prominent role in ensuring radiation safety at the STS. Shielding configurations, monitor locations, and Personnel Protection System access will be carefully controlled by procedures and will be approved at an appropriate level. Periodic radiation surveys will be used to assess shielding effectiveness. Active radiation monitors considered important to safety will be placed in accordance with supporting calculations and will be removed only with an equivalent level of supporting information.

\subsection{FIRE PROTECTION SAFETY}

The identification and control of fire-related hazards are well-defined processes, both within industry and the DOE complex. Extensive standards have been established through the National Fire Protection Association's NFPA 101: Life Safety Code [20], state and federal building codes, and DOE. In general, the codes focus on elimination of ignition sources, minimization of combustible/flammable materials, establishment of early warning fire detection, confinement of higher-hazard areas, and installation of fire suppression systems based on the value of the facility and the safety of the respective occupants.

Based on operational experiences of accelerators throughout the DOE complex, most fires in accelerator facilities are electrically initiated, typically by failure of components. However, in addition to evaluation of this risk, additional sources of fire will be considered in the design, construction, and operation of the STS. These include the combustibility of building construction materials, accumulation of combustible materials by occupants, use of pyrophoric and/or reactive materials, improper storage and/or use of flammable materials, lightning storms, and static discharge.

At SNS, a comprehensive fire safety program has been implemented that addresses response to fire and management of fire protection for emergencies; prevention and control of fire hazards (experimental and temporary flammable gas systems, facility natural gas service, flammable atmospheres, cutting and welding, materials of construction and transient fire loads, and ignition control); maintaining the integrity of facility fire safety features; and fire hazards analyses and evaluations. This comprehensive approach will be implemented for design, construction, and operation of the STS.

\subsection{CONSTRUCTION SAFETY}

All construction activities on Chestnut Ridge in support of the STS shall comply with the SNS ES\&H Plan [21]. The safety of all personnel is recognized as a primary concern to all participants at ORNL. Unsafe conditions and unsafe behavior can result in injuries and deaths, as well as impact schedules, cause financial losses, and damage professional reputations. As such, the goal is that all project participants plan, manage, and execute their respective operations with the ultimate goal of conducting their operations injury-free on a daily basis.

It is the responsibility of the respective contractor to adhere to the requirements of the SNS ES\&H Plan [21]. Each contractor shall incorporate safety into the planning of each task, ensure the safety of its personnel, provide all safety devices necessary for its employees, establish a safe and drug-free work environment, and confirm that its equipment meets applicable safety standards. Each contractor is responsible for any actions of its personnel that could endanger or otherwise expose other participants to potential hazards on the project site.

The Integrated Safety Management System shall be used to achieve these goals. The system is a practical approach to the prevention of accidents with an emphasis on line management responsibility for safety. A 
central premise is that work planning starts with a focus on the nature of the job to be performed and assessment of the hazards involved in each step. Through the use of self-assessment and feedback from the line organizations, continuous improvement in each contractor's safety process is expected. Project participants are required to supervise and direct activities using their best management skills and technical expertise. Each contractor will be solely responsible for all work means, methods, techniques, sequences, and procedures. This includes all safety precautions and programs in connection with the work, as well as coordinating all portions of the work. Each lower-tier subcontractor is likewise required to be responsible for all safety precautions and programs in connection with the work under the subcontractor's contractual agreement.

Furthermore, contractors will be selected based on past documented performance indicators. Contractors not demonstrating exceptional past performance in safety will not be invited to participate in STSsponsored work. A copy of the SNS ES\&H Plan will be provided to all contractors and subcontractors [21].

\subsection{REFERENCES}

1. "Record of Decision for the Construction and Operation of the Spallation Neutron Source," DOE/EIS-0247, US DOE, Federal Register, Vol. 64, p. 35140, June 1999.

2. Final Environmental Impact Statement for the Construction and Operation of the Spallation Neutron Source, SNS FEIS, DOE/EIS-0247, US DOE, April 23, 1999.

3. 40 CFR 61, National Emission Standards for Hazardous Air Pollutants, Subpart H.

4. Rules of Tennessee Department of Environment and Conservation, Bureau of Environment, Division of Air Pollution Control, §1200-3-11-.08, "Emission Standards for Emissions of Radionuclides Other than Radon from Department of Energy Facilities," May 2006.

5. S. M. Trotter, R. W. Leggett, A. L. Sjoreen, and K. F. Eckerman, Dose Calculations for Determination of Sampling Procedures for Routine Emissions of Radionuclides from the Spallation Neutron Source, Oak Ridge National Laboratory, 2007.

6. ANSI/HPS N13.1-1999, Sampling and Monitoring Releases of Airborne Radioactive Substances from the Stacks and Ducts of Nuclear Facilities, Health Physics Society, 2011.

7. DOE O 435.1, Radioactive Waste Management, US DOE, January 2007.

8. DOE M 435.1-1, p. IV-1, Radioactive Waste Management Manual, US DOE, January 2007.

9. S. M. trotter and J. R. DeVore, Spallation Neutron Source Waste Management Plan, SNS 102030000-TR0002-R02, Oak Ridge National Laboratory, March 2006.

10. DOE O 430.1B, Real Property and Asset Management, US DOE, September 2003.

11. "Record of Decision for the Department of Energy's Waste Management Program: Treatment and Disposal of Low-Level Waste and Mixed Low-Level Waste; Amendment of the Record of Decision for the Nevada Test Site,” DOE/EIS-0200, US DOE, Federal Register, Vol. 65, p. 10061, February 2000 . 
12. "Record of Decision for the Department of Energy's Waste Management Program: Treatment of Non-wastewater Hazardous Waste," US DOE, Federal Register, Vol. 63, No. 150, August 5, 1998 (issued on July 30, 1998).

13. DOE G 420.2-1A, Accelerator Facility Safety Implementation Guide for DOE O 420.2C, Safety of Accelerator Facilities, August 2014.

14. DOE O 420.2C, Safety of Accelerator Facilities, July 2011.

15. DOE Standard 1020-2012, Natural Phenomena Hazards Analysis and Design Criteria for Department of Energy Facilities, August 2012.

16. DOE O 420.1C, Facility Safety, December 2012.

17. 10 CFR 835.1001, Design and Control, January 2014.

18. 10 CFR 835.1002, Facility Design and Modifications, January 2011.

19. DOE O 420.2B, Safety of Accelerator Facilities, May 2008.

20. NFPA 101: Life Safety Code, National Fire Protection Association, 2014.

21. Oak Ridge National Laboratory/Chestnut Ridge Facilities Project Environmental, Safety, and Health Plan, SNS 102030000-ES0007-R05, Oak Ridge National Laboratory, Oak Ridge, Tennessee, September 2012. 



\section{ALTERNATIVES}

The present STS design adopts a number of different design choices relative to earlier STS approaches, which are addressed here. A significant change in the STS design is adoption of the short pulse (SP) option over the long pulse (LP) option. The evolution from long pulse to short pulse choice is explained in Sect. 11.1. Alternative site locations evaluated in 2007 for the target are presented in Sect. 11.2. In the accelerator area, the energy increase will be accomplished with a more aggressive superconducting radiofrequency (SRF) approach, which is discussed in Sect. 11.3. Finally, the target design approach is always a major consideration in high-power proton beam applications. Several target concepts were evaluated, with the final choice being the stationary solid target. The alternative target designs are discussed in Sect. 11.4.

Design choices made in an effort to minimize cost are presented in Sect. 11.5. Although these are discussed throughout the report, they are collected here for convenience.

\subsection{LONG PULSE OPTION}

Initial consideration of a second target at SNS began in 2002, resulting in a joint Argonne National Laboratory-SNS proposal (generally referred to as the Long Wavelength Target Station Report) [1]. In addition to describing how the number of available neutron scattering instruments would be doubled, the report highlights the advantages of providing long wavelength cold neutrons This report called for using one in six of the $60 \mathrm{~Hz}$ SNS accelerator pulses for the long wavelength target station.

An additional target study took place in 2006-2007, resulting in a design concept for an LP neutron source driven by a $1 \mathrm{MW}, 20 \mathrm{~Hz}$ proton beam providing $1 \mathrm{~ms}$ long proton beam pulses [2]. The instruments proposed for this source also emphasized long wavelength. This proposal called for a highpower 1 MW STS proton beam (upgradeable to $1.5 \mathrm{MW}$ with significant accelerator rework and cost), which is a significantly higher beam power than proposed here. This high power level was necessary for the LP option to be competitive with an SP option.

In 2012, the LP proposal was reevaluated, and a study was conducted for a reduced-power SP option [3]. A key element of this study was optimizing the peak usable neutron flux at the moderator faces. By considering reduced proton drive beam power (below $0.5 \mathrm{MW}$ ), it is possible to engineer a more compact target/moderator configuration with respect to heat removal and fault condition issues. This compact geometrical arrangement allows for a higher peak neutron flux than the earlier STS proposal. In addition, it is possible to provide the $500 \mathrm{~kW}$ proton beam power for the present STS concept with only a $10 \mathrm{~Hz}$ repetition rate, minimizing "pulse stealing" from the FTS. The reduced repetition rate also provides more intra-pulse spacing, which is useful for resolving low-energy neutron arrival times and which increases the available neutron wavelength bandwidth without resorting to neutron chopping decimation schemes required in the LP case.

Figure 11.1 shows a comparison of neutron flux for a coupled SP and an LP station for equal proton intensities (same number of neutrons), for two different wavelengths. The SP option has from 3 to 16 times higher peak intensity, which is beneficial for instruments that rely on time-of-flight techniques. As stated in Ref. [4], "Time-of-flight instruments that depend on time-averaged moderator brightness will perform about as well on either option, while instruments that rely more on peak brightness will perform better on the SP-STS." Furthermore, instruments that are optimized toward higher time-averaged neutron flux are likely to be better sited at HFIR, which has $~ 10$ times the time-averaged cold neutron brightness of an SP STS or $\sim 5$ times the time-averaged brightness of a $20 \mathrm{~Hz}$ LP STS. The 2013 ORNL Neutron Sciences Strategic Plan [5] calls for placement of instruments that favor high time-averaged flux at HFIR. 

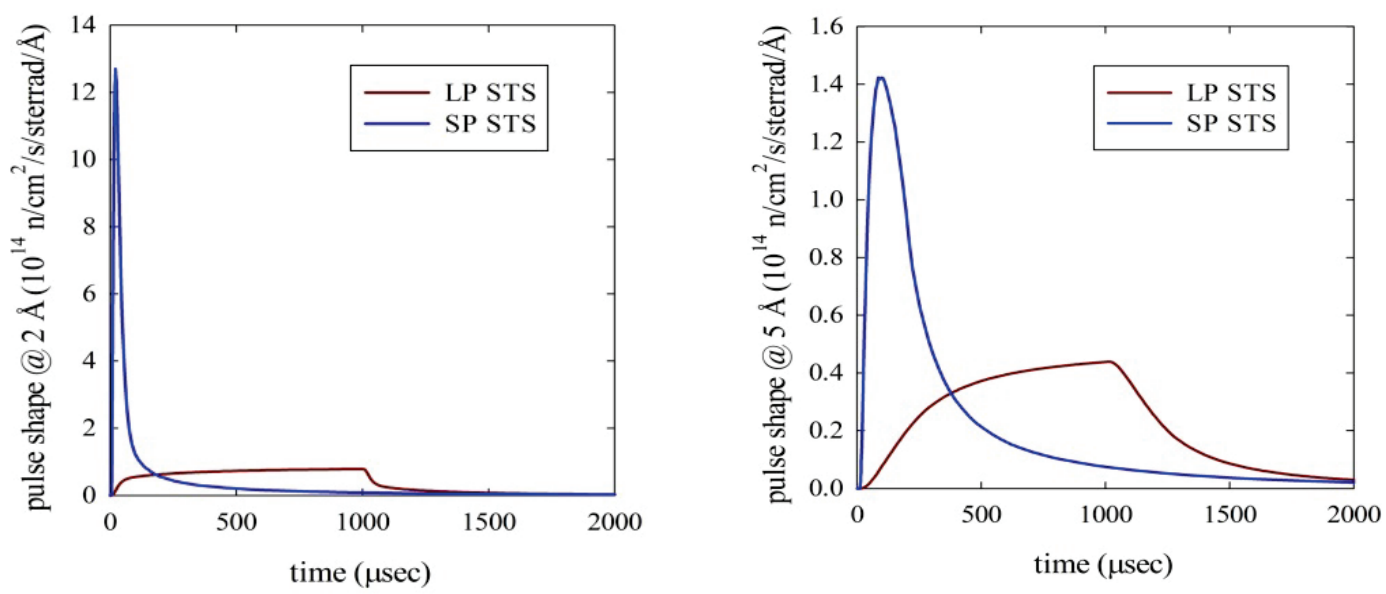

Fig. 11.1. Comparison of neutron flux between a coupled SP source (left) and an LP source (right) for the same neutron intensity, for $2 \AA$ (left) and $5 \AA$ (right) wavelength neutrons.

Note that at $5 \mathrm{MW}$, the baseline European Spallation Source (ESS) design would provide $\sim 3$ times taller and $\sim 3$ times broader pulses than those shown for the LP STS in Figure 2. The ESS neutron scattering instrument suite would significantly outperform comparable instruments on an SNS LP STS. However, by taking advantage of the existing SNS accumulator ring, an SP STS could produce comparable or significantly higher peak pulse heights, benefiting large classes of neutron scattering instruments even with an order of magnitude lower proton beam power.

Some of the high-level machine parameters for the present SP design and the 2007 LP design are compared in Table 11.1.

Table 11.1. Comparison of high-level machine parameters of the present design and the 2007 LP option

\begin{tabular}{lrr}
\hline \multicolumn{1}{c}{ Parameter } & Present design & 2007 LP design \\
\hline FTS/STS beam power $(\mathrm{MW})$ & $2.0 / 0.47$ & $1.33 / 1.0$ \\
FTS/STS target repetition rate $(\mathrm{Hz})$ & $50 / 10$ & $40 / 20$ \\
Second target pulse length $(\mu \mathrm{s})$ & $<1$ & 1000 \\
Target type & Tungsten & Mercury \\
Average linac macro-pulse current $(\mathrm{mA})$ & 41 & 38 \\
\hline
\end{tabular}

In summary, the decision to use an SP design was based on several considerations:

- Target/moderator/instrument optimization design studies identified a high-performance lower proton power option [3] (see Sect. 4 of this report).

- A comprehensive strategic plan [5] was formulated in which instruments favoring high time-averaged brightness will be implemented at HFIR and instruments favoring high peak brightness will be implemented at the STS.

- The risk of delivering a high-power SP proton beam is considerably less now than in 2007, based on operational experience gained in the interim. 
- The LP options for avoiding use of the accumulator ring (a major advantage of the LP option) were prohibitively expensive [6].

These considerations are discussed in more detail in the following subsections.

\subsubsection{Target/Moderator Optimization}

For the 2007 LP STS, the primary driver for neutron intensity was proton beam power. A target concept similar to the FTS was adopted (mercury target), so heat removal was not a major consideration. This was a rather "brute-force" approach toward neutron flux maximization. Since 2007, studies have offered a different approach to high-brightness sources with reduced power requirements, namely, compact sources at reduced power with higher brightness per unit of proton beam power. A key element is the compact design that lends well to high neutron flux. However, the compact design becomes increasingly difficult to engineer for higher absolute beam powers (e.g., at $1 \mathrm{MW}$ ). Heat removal becomes a limiting factor. The approach adopted here takes advantage of the compact design to increase the neutron brightness per unit proton by a factor of 2.5 to 5 compared with the FTS.

\subsubsection{Strategic Plan}

Another major factor in the decision to adopt the SP STS approach is consideration of a global strategy for providing neutrons across all three ORNL neutron sources. The current SP STS design does have a reduced time-averaged brightness relative to the 2007 LP option. However, following the logic outlined in the 2013 ORNL Neutron Sciences Strategic Plan [5], instruments requiring high time-averaged flux will be placed at HFIR, and instruments favoring a high peak flux will be used at the STS.

\subsubsection{Short Pulse Risk}

In 2007 when the LP approach was adopted, consideration of providing a 1 MW SP beam in addition to the $2 \mathrm{MW}$ beam to be delivered to the FTS was daunting. The operational proton beam power in 2007 was $100-200 \mathrm{~kW}$. Consideration of an option that minimized the required beam injected into and stored in the ring certainly reduced technical risk. At that time it was not obvious how the $1.4 \mathrm{MW}$ design goal of the FTS design would be attained. Now with $1.4 \mathrm{MW}$ operation demonstrated, extrapolation to the STS $2.5 \mathrm{MW}$ beam power is a much smaller step ( $80 \%$ power increase vs. a factor of 20 for the 2007 period). The required power increase is especially modest when considering that $\sim 40 \%$ of the increase is accommodated by straightforward beam energy increase.

Avoiding the ring for beam delivery to the STS was considered advantageous so that equipment in the ring could be repaired while still delivering beam to the STS. This advantage has proved to be minimal, however, in that linac issues dominate the actual fraction of accelerator downtime; ring downtimes contribute less than $6 \%$ of the accelerator downtime in the most recent 9 month run period in FY 2014.

\subsubsection{Site/Conventional Facility Issues}

Although many of the building concerns are similar for the two designs, a major difference is the possibility to provide a direct proton beam transport line from the linac to the STS for the LP option. The LP option allows consideration of

1. an alternative STS site, which is not necessarily near the ring exit

2. avoiding having the proton beam pass through the ring, even if the STS is located as originally planned near the ring exit 
These issues were evaluated in 2009 [6]. None of the alternative sites identified for the STS was attractive. Also, the option of bypassing the ring for the nominal STS site was found to be prohibitively expensive, so the proposed site selection was to place the STS as originally planned and to use the ring as a portion of the transport line to the STS. Thus the potential flexibility of an LP option did not come to fruition.

\subsection{ALTERNATIVE TARGET SITES}

Three possible sites for the STS target and instrument hall were identified on the SNS Chestnut Ridge site and are shown in Fig. 11.2. Alternative site 1 is the original proposed location of the STS, positioned to extract beam from the existing transport line from the ring to the first target. Alternative site 2 has the advantage of being relatively near the existing Central Laboratory and Office Building, and the proton transport line would be relatively short. However, it has the major drawback that the proton beam line would intersect major utility lines through the SNS campus, and construction would be a major imposition to operations. The impact on site utilities is likely an unacceptable operational risk.

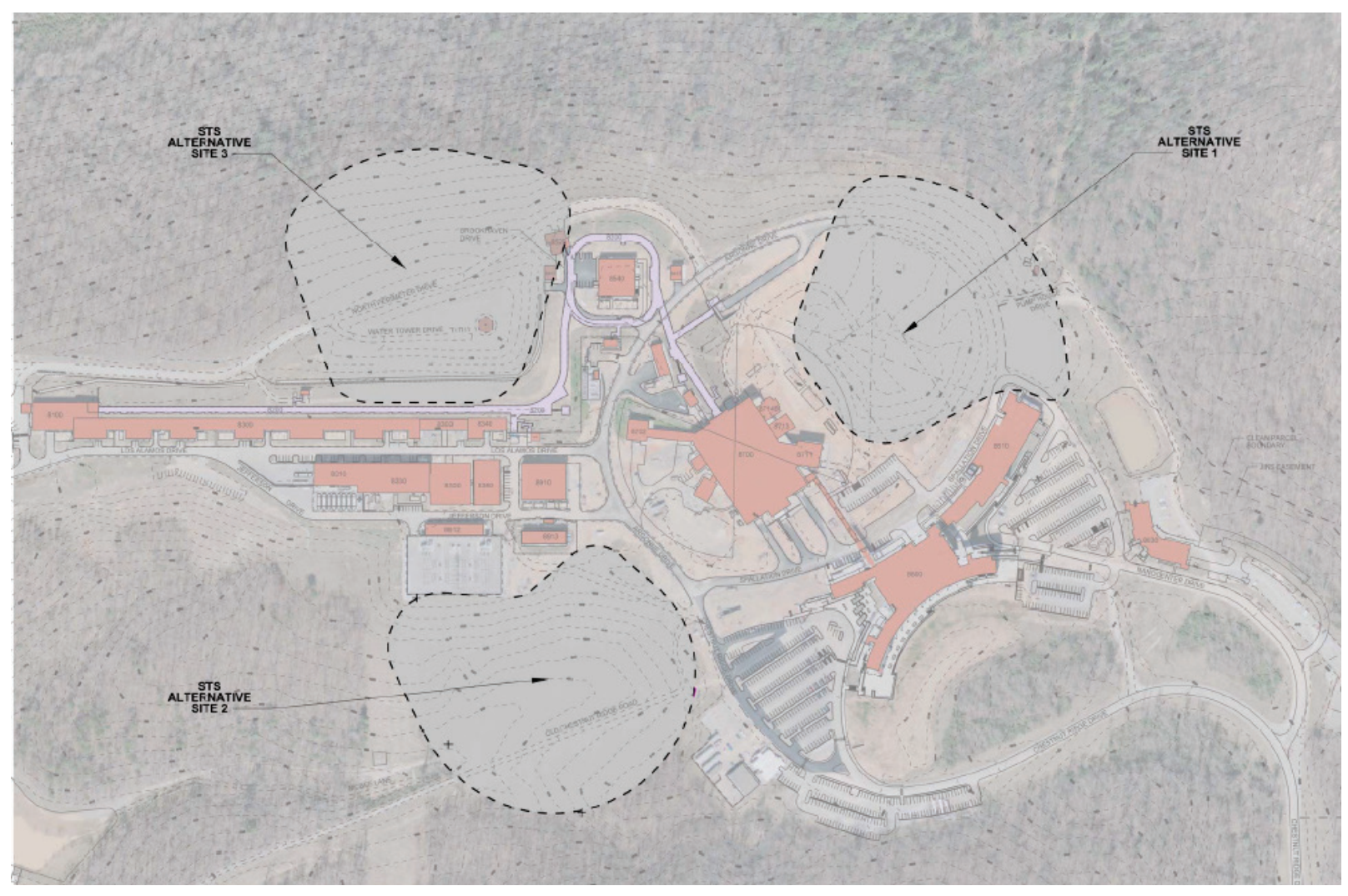

Fig. 11.2. The three sites considered for the STS target/instrument hall. STS alternative site 1 is the baseline site.

Alternative site 3 (north of the linac) has the advantage of a relatively short proton transport beam line. However, this option requires relocation of the site water tower and has the major disadvantage of being physically distant from the FTS. This would hinder sharing of resources such as sample environments and hall coordinators, which would have a negative science impact.

The comparative analysis in [6] proposes retaining the present STS site location. 


\subsubsection{Proton Beam Line to STS}

Even with the STS site situated at the original location (alternative site 1), the LP option offers the possibility of a direct path from the linac to the STS, obviating the need to use the ring. Figure 11.3 shows the four possible paths considered (including two that partially use the ring). Alternate path 1, north of the ring, has the disadvantage that the path is quite long, disrupts the access road north of the ring (important for construction), and requires a large retaining wall to avoid disrupting the Bear Creek watershed (a site criterion). In addition, the site water tower would have to be moved. Alternate path 3 has the major disadvantage of intersecting many utility lines, intersecting the existing ring to target beam transfer line, and disrupting the roads through the heart of the SNS campus (discussed previously). Although alternate paths 2 and 4 do not have major impacts on utilities and other site features, they use the ring as a transport line. Thus there are no attractive transport line paths that avoid some use of the ring as a transport system, which eliminates the potential independence of the STS from the ring systems.

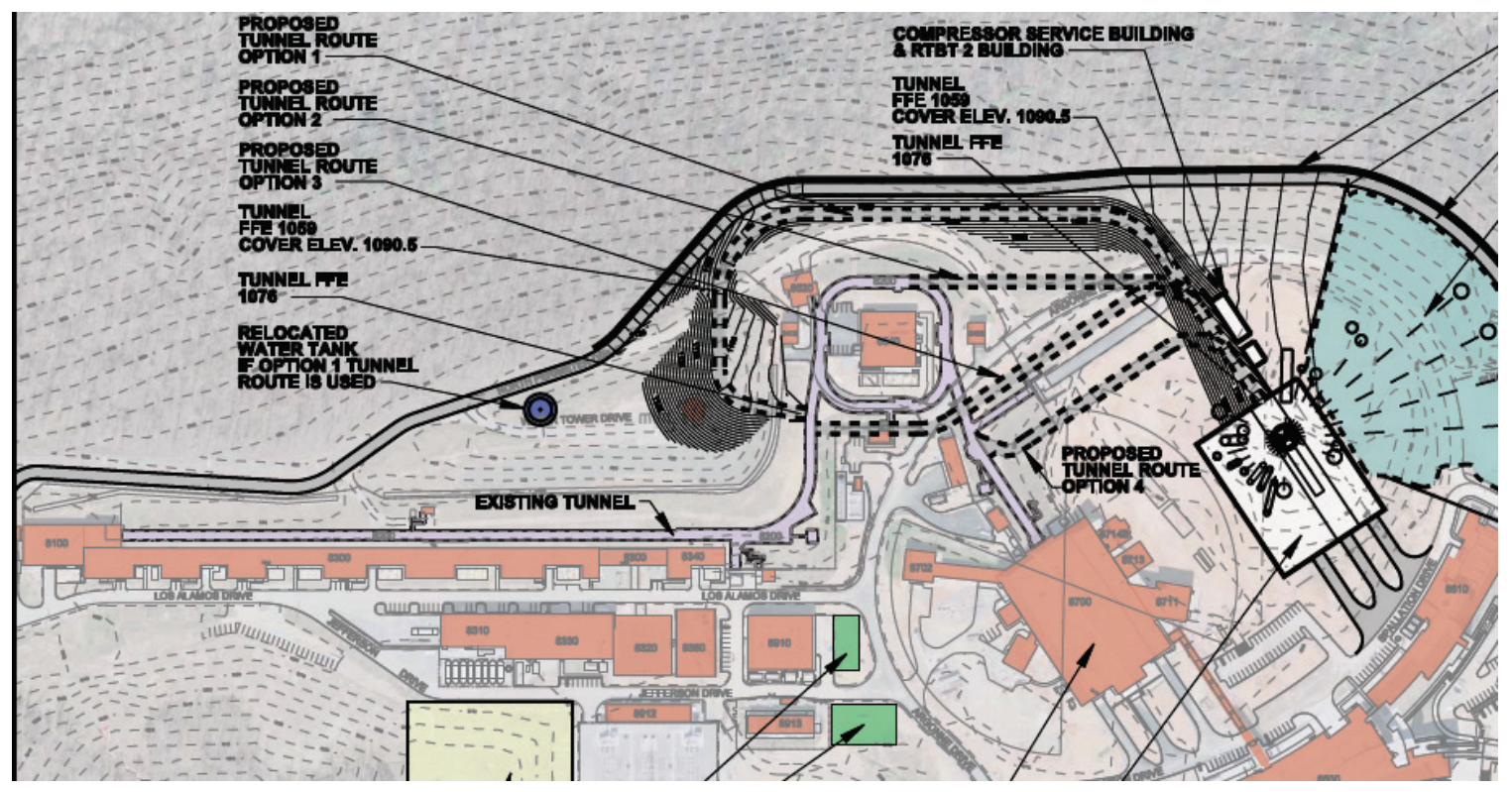

Fig. 11.3. Proposed alternate paths for the proton beam line to the STS for an LP option.

\subsection{PUP ENERGY UPGRADE PLAN}

The Power Upgrade Project (PUP) covered the energy upgrade portion of the accelerator power upgrade. The plans for an energy upgrade from the original Conceptual Design Report in 2008 [7] through the final plan just before project cancellation [8], called for 9 additional cryomodules (36 cavities) to provide the energy increase to $1.3 \mathrm{GeV}$. The nine cryomodule upgrade (which included one spare) in these proposals required a rather conservative $13.7 \mathrm{MV} / \mathrm{m}$ cavity gradient to reach $1.3 \mathrm{GeV}$ and resulted in a peak beam loading of $\sim 550 \mathrm{~kW}$ (see Fig. 11.4). The original PUP approach is rather conservative and is not adopted here for the following reasons:

- Gradients of $16 \mathrm{MV} / \mathrm{m}$ have been operationally demonstrated with the spare high-beta cryomodule built at SNS. Lessons learned from building the spare are incorporated in the STS design. The spare cryomodule fabrication, installation, and operation had not been done at the time of the PUP. The $16 \mathrm{MV} / \mathrm{m}$ gradient baseline for the STS is also modest relative to other SRF linac projects ongoing now (ESS, Proton Improvement Plan-II, Facility for Rare Isotope Beams). A comparison of the PUP 
gradients with other ongoing SRF projects is shown in Table 11.2 and indicates that the present STS cavity requirements are relatively modest compared with other ongoing projects.

- Even with the higher $16 \mathrm{MV} / \mathrm{m}$ gradient, the RF power requirements are not severe. Spare superconducting linac klystrons being purchased as spares already have $700 \mathrm{~kW}$ ratings (sufficient for the STS), and the new coupler design for $700 \mathrm{~kW}$ is a small variation of the existing fundamental power coupler (see Sect. 3.2).

- Requiring only seven additional cryomodules offers flexibility in the installation plan. There are a number of "chases" between the Klystron Gallery and the tunnel that contain significant amounts of cables (which should not be there) in the energy upgrade buildout space. These chases are not suitable for installing RF waveguides. Moving the cables to the proper chases would take considerable effort. Allowing the most egregious "full chases" to remain as is would greatly simplify the installation challenge with an operational machine. Also, having fewer required RF stations in the buildout area alleviates space demands in the Klystron Gallery.

- Using only seven additional cryomodules does place a somewhat higher burden on the high power supply for the RF (high-voltage convertor modulator). However, the development progress in this area over the past 8 years has opened several possible solutions, as discussed in Sect. 3.3.

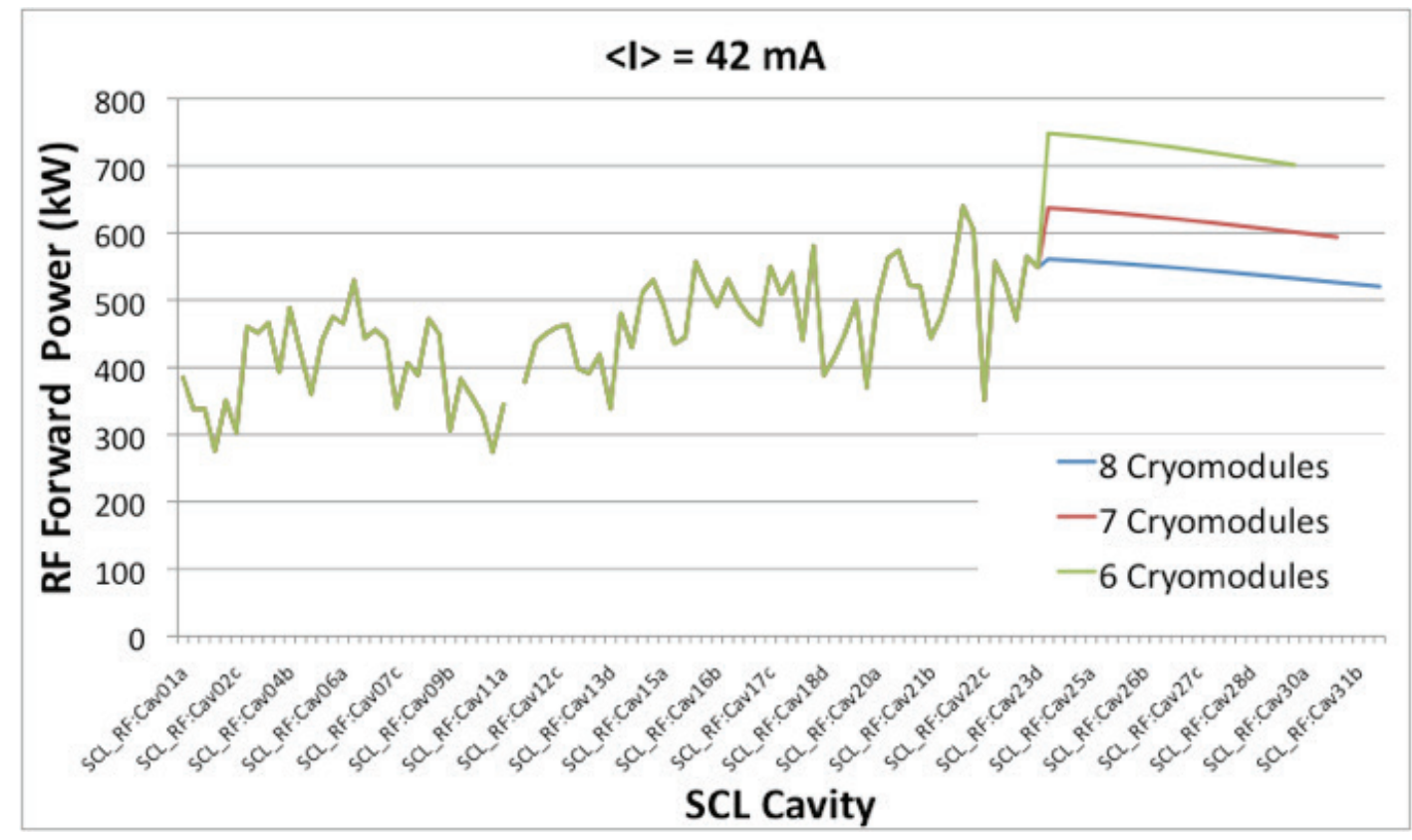

Fig. 11.4. Power requirements for the superconducting linac from the PUP for different numbers of new cavities and for beam loading similar to the STS requirement [8]. 
Table 11.2. Comparison of high-level SRF cavity characteristics for STS and other ongoing SRF projects

\begin{tabular}{lcccccc}
\hline \multicolumn{1}{c}{ Project } & $\begin{array}{c}\text { Frequency } \\
\text { (MHz) }\end{array}$ & $\begin{array}{c}\text { Geometric } \\
\text { beta }\end{array}$ & $\begin{array}{c}\text { Accelerating } \\
\text { gradient } \\
\text { (MV/m) }\end{array}$ & $\begin{array}{c}\text { Peak electric } \\
\text { field (MV/m) }\end{array}$ & $\begin{array}{c}\text { Peak } \\
\text { magnetic } \\
\text { field (mT) }\end{array}$ & $\begin{array}{c}\text { RF duty } \\
(\%)\end{array}$ \\
\hline SNS STS & 805 & 0.81 & 16 & 35.2 & 76 & 8 \\
SNS FTS $_{\mathrm{PIP}-I I^{a}}$ & 805 & 0.61 & 10.2 & 27.5 & 59 & 8 \\
$\mathrm{ESS}^{b}$ & 650 & 0.6 & 16.5 & 37.5 & 70 & 12 \\
$\mathrm{SPL}^{b}$ & 704 & 0.67 & 16.7 & 45 & 80 & 5 \\
CEBAF-upgrade $^{c}$ & 704 & 0.65 & 19 & 57 & 95 & 8 \\
\hline
\end{tabular}

${ }^{a}$ Proton Improvement Plan-II (FermiLab).

${ }^{b}$ Superconducting Proton Linac (CERN).

${ }^{c}$ Continuous Electron Beam Accelerator Facility (Jefferson Laboratory).

\subsection{ALTERNATIVE TARGETS}

A water-cooled solid target was selected for the proposed STS based primarily on the successful operation of similar targets at existing facilities over many years. However, since the STS will operate at a higher power than those facilities, several backup options have been considered for use if unanticipated technical problems arise during the development phase.

\subsubsection{Rotating Target}

A water-cooled solid, rotating disk target is a feasible alternative for the STS. The rotating disk concept was studied in depth as part of the FY 2010 STS study because of the lifetime advantages derived from distributing the radiation damage and heat of large areas and masses. Confidence in the concept was ensured though detailed neutronics, computational fluid dynamics, and structural studies and the construction and successful operation of a full-scale, mechanical mockup for more than 5,000 $\mathrm{h}[9,10]$, as shown in Fig. 11.5.

The 2010 STS feasibility study analyses and layouts of a 1.5 MW LP rotating target were based on parameters established early in the preconceptual design. First among these was the selection of a watercooled, tantalum-clad tungsten block assembly enclosed in a stainless steel shroud as the fundamental configuration of the STS rotating target. The target blocks would be segmented into pie-shaped sections to facilitate fabrication and accommodate thermal cycling from the distribution of beam heating. The clad tungsten blocks would be contained in a 316L stainless steel housing designed for structural stability, radiation tolerance, and cooling water containment. A rotational speed range of 30 to $60 \mathrm{rpm}$ would distribute the $30 \mathrm{~Hz}$ proton beam pulses without overlap on the face of the target. Coordinated neutronic, thermal, and structural studies were undertaken based on this initial concept. The resulting target disk design is shown in Fig. 11.6.

Practical factors ultimately defined the proposed disk size. Basically, the $25 \mathrm{~cm}$ solid target combined with a hub of adequate size to uniformly distribute water throughout the target disk results in an overall diameter of $\sim 120 \mathrm{~cm}$. This diameter also provides space for the reflector around the upper moderators and provides for reasonable forward neutron beam channels. 


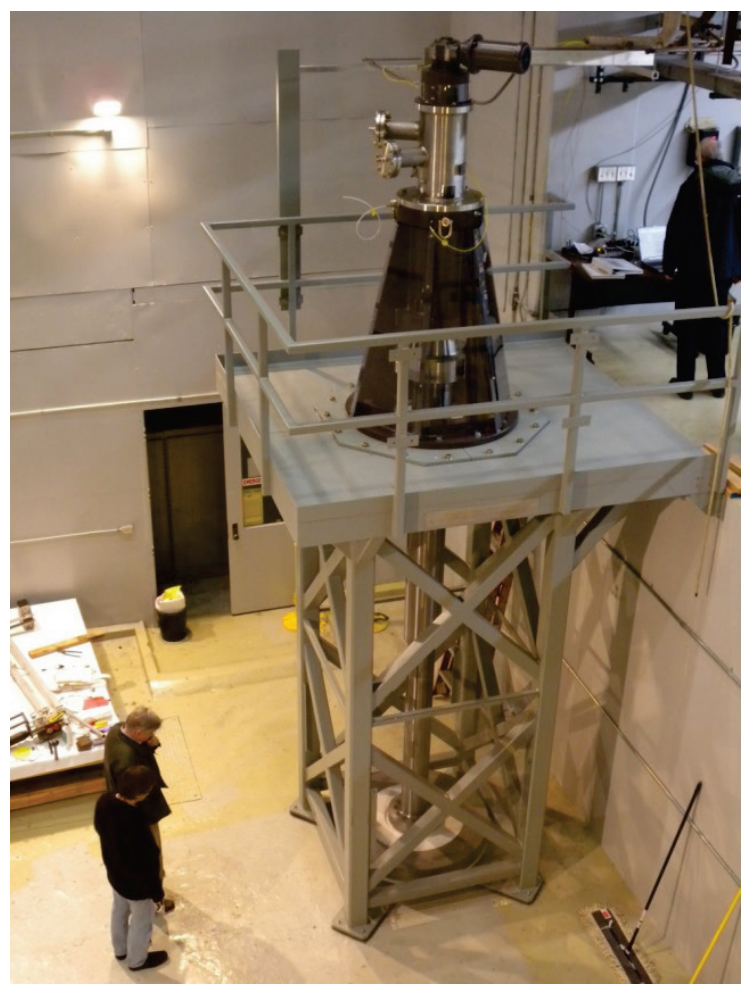

Fig. 11.5. Full-scale $3 \mathrm{MW}$ rotating target mockup.

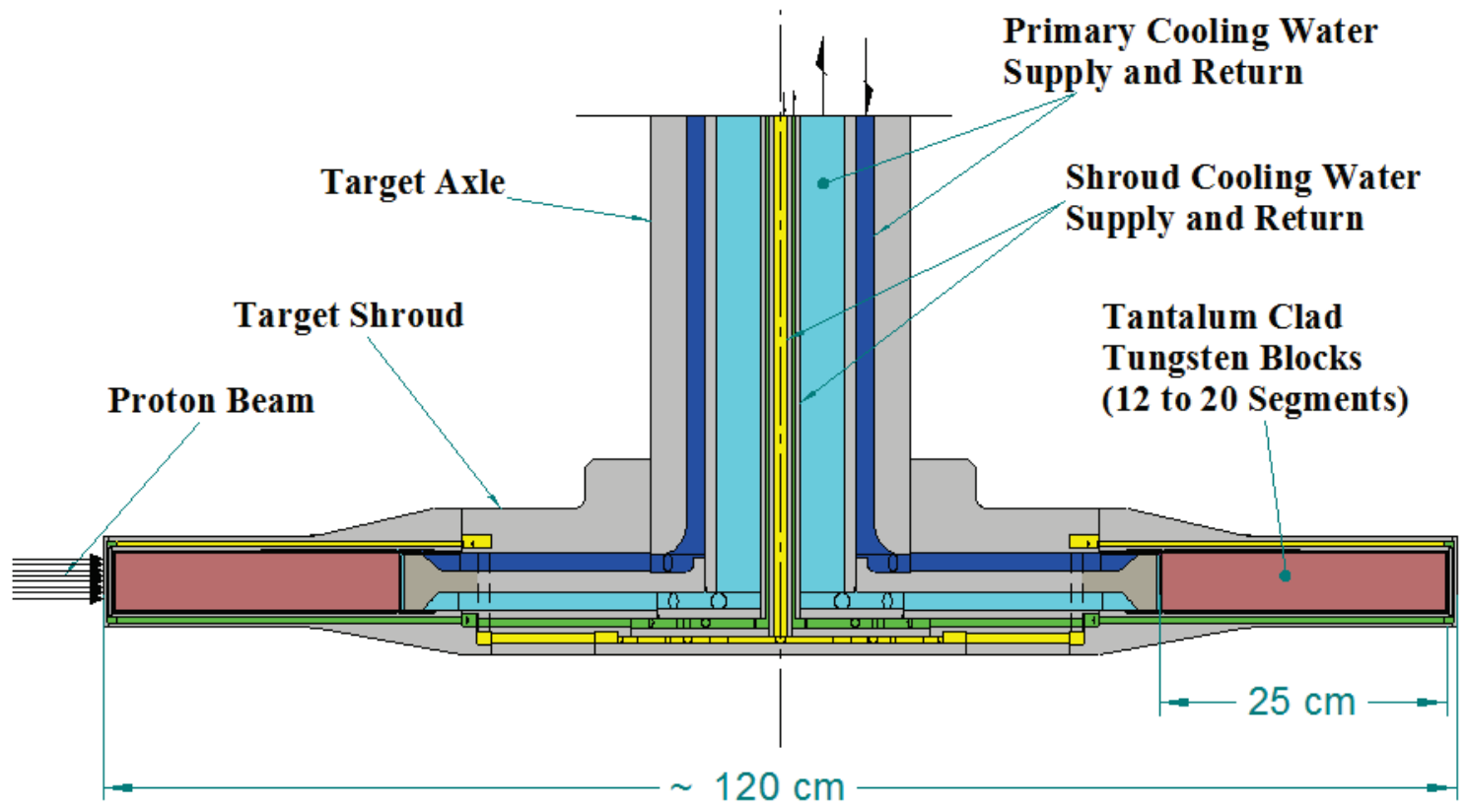

Fig. 11.6. Conceptual rotating target cross section.

For the proposed SP STS application at $500 \mathrm{~kW}$ and $10 \mathrm{~Hz}$ with a $30 \mathrm{~cm}^{2}$ proton beam, the thickness of the tungsten blocks could be the same as for the compact, fixed target: $4.5 \mathrm{~cm}$. The quasi steady-state thermal response for $500 \mathrm{~kW}$ operation with a $3.5 \mathrm{~cm}$ high beam by $8.6 \mathrm{~cm}$ wide is shown in Fig. 11.7 with a peak of $\sim 80^{\circ} \mathrm{C}$. 


\section{2 m Diameter Target Temperature}

Water cooled top, bottom and front surfaces

$\mathrm{h}=2.6 \mathrm{E} 4 \mathrm{~W} / \mathrm{m}^{\wedge} 2-\mathrm{K}$

$500 \mathrm{~kW}$ beam operation

$3.5 \mathrm{~cm}$ beam height

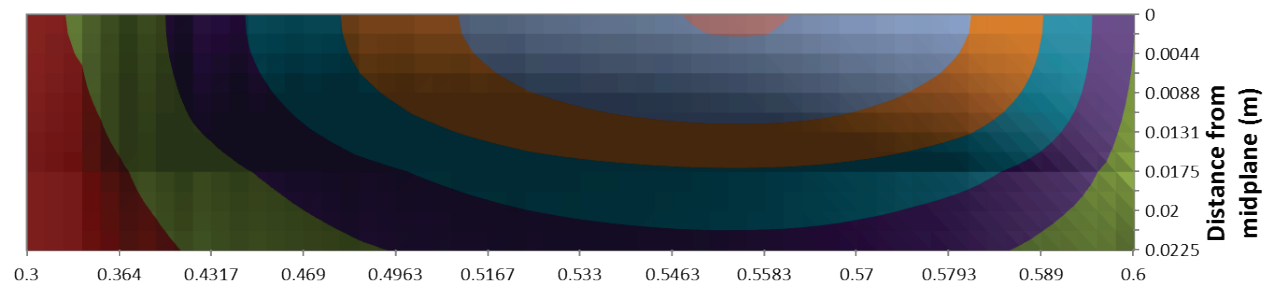

Radius (m)

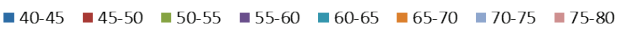

Fig. 11.7. Quasi steady-state rotating target thermal response (degrees Celsius).

Distribution of the incident proton beam around the perimeter of a rotating target would extend the ideal radiation damage life to more than 10 years while providing large steady-state thermal margins. The large area of the disk would also be able to passively dissipate decay heat to the surrounding shielding during loss-of-cooling accidents. However, handling and maintenance issues are a concern. The large disk and axle assembly effectively block access to the lower moderators. Thus it is likely that the rotating target, along with a large amount of shielding, would have to be removed each time the moderators are changed. The size of the components and the complexity of the operations would likely be much greater than for the baseline horizontal target with correspondingly higher costs.

Thermal shock stresses were estimated in a rotating target block in response to a microsecond pulse and were found to reach $\sim 70 \mathrm{MPa}$ in the tungsten and $39 \mathrm{MPa}$ in the cladding, which might be acceptable. A lack of well-defined stress limits for irradiated clad tungsten targets inhibits design optimization, an issue affecting both rotating and fixed target options.

Thermal stresses from an SP $500 \mathrm{~kW}$ beam have not been estimated for the rotating target. Temperature cycles will be larger than in a fixed target, as rotation allows for more cooldown between pulses. Fatigue from thermal cycles has the potential to adversely affect target lifetime, which is less tolerable in a rotating target than in a fixed target because the rotating target's higher cost and difficult installation demand greater lifetime payoff than a fixed target. Design and manufacturing that ensure a much longer lifetime than a fixed target will be necessary for a rotating target.

A rotating target might be desirable for high-power operation; however, at $500 \mathrm{~kW}$ the simpler, compact target proposed for the STS is believed to have cost, development, and operational advantages. 


\subsubsection{Mercury Target}

A flowing liquid mercury target is a feasible STS target option based on the successful operation in the FTS and an established safety basis case. Radiation damage to the stainless steel vessel is expected to set the changeout life of the target at 9 to 12 months. A separate water-cooled shroud would be needed as on the FTS to contain mercury leaks. The peak pulse intensity for the STS solid target is more than four times that of the FTS mercury target for the same number of protons per pulse because of a more compact incident beam used to maximize neutron brightness. Pressure pulses - and resultant cavitation intensitywould be much greater as a result. A gas injection technology is believed necessary for the FTS at 2 MW, but cavitation damage mitigation efficacy for the STS will be even more critical even though its pulse repetition rate is lower. The beam spot size might have to be increased compared with the solid target to reduce the peak pulse pressures. The combination of the additional shroud and a larger beam spot would reduce the neutronic performance.

A flowing liquid mercury target would have the same basic configuration and layout as at the FTS. The mercury process equipment would be installed in a shielded service bay downstream of the monolith. A target designed specifically for the STS would be mounted on a horizontal cart that could be retracted into the service bay for changeout. Some process system improvements, such as the use of an electomagnetic pump, might need to be adopted; however, no significant changes are proposed. Consequently, a mercurybased STS service bay would be similar in size to the FTS bay with some changes incorporated to improve operations. As with the FTS service bay, a highly dexterous manipulator system with sophisticated monitoring and containment systems would be required to maintain the process equipment. It should be noted that the Telerob manipulator used in the FTS is no longer available.

Conventional systems such as the mercury off-gas absorbers and waste-handling equipment would also be very similar to those at FTS. Moderators and the inner reflector plug would be handled remotely in the high bay. At this time it appears that the baseline concept would give better neutronic performance and have significant advantages in cost and ease of maintenance of the primary cooling system, which would be "hands-on" for water cooling.

\subsubsection{Eutectic Target}

Any liquid eutectic target would be subject to the same cavitation damage issue as the mercury target. This was demonstrated with a lead-bismuth eutectic target during the FTS SNS research and development program. Substantial research and development would be needed for the cavitation damage issue and for material compatibility issues with no significant advantages and more potential problems. Maintaining a molten state adds considerable engineering difficulties; management of radiotoxic polonium would be a new hazard to deal with. There would also be substantial additional costs for remote handling and remote process pumping systems. There does not appear to be any justification for pursuing this approach for the STS.

\subsubsection{Vertical Target}

Preliminary neutronic analysis of an STS configuration based on a vertical target indicates that there are potential performance gains for the smaller proposed neutron beams (i.e., $3 \times 3 \mathrm{~cm}$ ). A companion study of the mechanical aspects of this arrangement also showed that there could be operational and operating advantages. For example, it could be relatively easy to arrange the target, moderator, and reflector in concentric vertical plugs sized to correspond to the respective change-out requirements. Without the need for a horizontal target cart, the service cell could be moved away from the monolith and configured for use with the vertical plugs. Further, since the target would be handled vertically and the proton beam 
would be injected from below, theoretically the full perimeter of the monolith could be available for neutron beam lines.

However, a vertical target would require significant reconfiguration of Target Building II. The proton beam would be need to be routed more than $10 \mathrm{~m}$ lower than the currently proposed beam to accommodate the vertical turn below the monolith. This change introduces several significant construction, installation, and handling issues. Most important of these is the difficulty of maintenance access and handling of the vertically positioned, final alignment magnets. Also, it is not obvious that the number of neutron beam lines could be increased despite the enlarged radial coverage available because of practical limitations in the shape of the moderators and space constraints on the STS site.

The vertical target option will have to be evaluated in conjunction with the next generation of proposed STS instruments to determine whether this configuration will provide improvements in instrument performance.

\subsubsection{Helium-Cooled Solid Target}

Cooling the proposed STS stationary solid target with helium instead of water was considered. One advantage of helium cooling would be that tantalum cladding would not be required from the water corrosion perspective. This could simplify fabrication and reduce the long-term decay heat significantly, which could mitigate active cooling requirements for spent targets. A preliminary thermal analysis indicates that this approach is feasible. However, there are development requirements and foreseen neutronics penalties that make this option less attractive than water cooling, which has an established history of success (ISIS at Rutherford Appleton Laboratory and the Lujan Center at the Los Alamos Neutron Science Center).

A preliminary thermal analysis was performed assuming the same plate and flow geometry as used for the STS baseline water-cooled configuration. In that case each flow channel was $4.5 \mathrm{~cm}$ high by $1.5 \mathrm{~mm}$ deep with a length of $10 \mathrm{~cm}$. The optimized plate thicknesses gave about the same heat input in each channel except toward the back, where thick plate temperatures would reduce the size and heat input. The target model had 19 channels with $\sim 12.5 \mathrm{~kW}$ per channel. For helium cooling with this heat load and geometry, the flow rates and heat transfer parameters for an assumed Mach number of 0.5 were found. Two

differential pressures were evaluated: 2 and 5 bar. A summary of the results is given in Table 11.3.

Table 11.3. Thermal analysis results for helium cooling of solid target

\begin{tabular}{lcc}
\hline \multicolumn{1}{c}{ Helium pressure } & 2 bar & 5 bar \\
\hline Power per channel $(\mathrm{kW})$ & \multicolumn{2}{c}{12.5} \\
T inlet $\left({ }^{\circ} \mathrm{C}\right)$ & \multicolumn{2}{c}{40} \\
Mach \# & \multicolumn{2}{c}{0.5} \\
Bulk temperature rise $\left({ }^{\circ} \mathrm{C}\right)$ & 260 & 90 \\
Mass flow per channel $(\mathrm{g} / \mathrm{s})$ & 9.26 & 26.75 \\
Total mass flow $(\mathrm{g} / \mathrm{s})$ & 176 & 508 \\
Flow velocity $(\mathrm{m} / \mathrm{s})$ & 603 & 533 \\
Heat transfer coefficient & 3035 & 6833 \\
$\mathrm{~h},=\mathrm{Nu} / \mathrm{De}\left(\mathrm{W} / \mathrm{m}^{2} \mathrm{~K}\right)$ & & \\
Film temperature rise $\left({ }^{\circ} \mathrm{C}\right)$ & 458 & 203 \\
T tungsten surface max $\left({ }^{\circ} \mathrm{C}\right)$ & 758 & 333 \\
Friction pressure drop $(\mathrm{bar})$ & .34 & .75 \\
\hline
\end{tabular}


For the 5 bar case, the peak tungsten temperature on the surface is about $333^{\circ} \mathrm{C}$ (steady-state average). The heat transfer coefficient is about an order of magnitude lower than with water. With $10 \mathrm{~Hz}$ operation, at the peak heating location, the temperature fluctuates about $10^{\circ} \mathrm{C}$ above and below the average and is $\sim 20^{\circ} \mathrm{C}$ higher on the mid-plane. The large temperature differences within a plate are likely to cause a thermal stress problem in the tungsten, which is expected to behave as a brittle material after irradiation. Changes in target configuration can be made to ameliorate this problem; however, all the available options would likely degrade the neutronic performance of the target. For example, thinner plates would decrease thermal stress but would also decrease the effective density and consequently the neutron brightness. Pelletized tungsten might have potential, but again with a probable decrease in brightness. Helium inlet and return conduits would be larger than for water, thus taking away reflector material close to the target. Also, it should be noted that for the plate configuration, the target structure would have to be designed for at least 6 bar, resulting in a thicker structure compared with the water-cooled assembly.

Heat removal from the helium would have to be accomplished with high assurance that water could not leak into the helium loop. Such a leak could lead to a tungsten-steam interaction for loss-of-cooling events, with high tungsten temperatures resulting from decay heat. This would require either a doublewalled heat exchanger or an intermediate loop to prevent mixing. Even with helium cooling, seismic protection for core components might be needed to prevent failures, which would release hydrogen and water and allow air into the vessel. It should also be noted that simply containing helium in a large, activated loop would also be a design and operating challenge.

At this time the helium option appears to require a relatively complicated target and process system design; and although the impact on moderator performance is not known, it is likely to be negative because of thicker structures and removal of heavy water next to the target, which acts as reflector material. Nevertheless, with development and engineering - and tolerance for some neutronic penaltyhelium cooling might be a workable alternative.

\subsection{COST SAVINGS EFFORTS}

Incorporating cost-effective approaches has been a driver in the STS design presented here. The preceding sections describe some of these savings and the uses of lessons learned from operation over the past seven years. These savings are summarized here.

\subsubsection{Target Systems}

- Adoption of a solid water-cooled target eliminates complications and safety issues introduced with a mercury target.

- Maintenance operations savings include the use of existing SNS remote-handling tools and procedures for the inner reflector plug and proton beam window (Sect. 4.9).

- Elimination of primary shutters offers design simplification and more efficient use of cold neutrons produced in the moderator.

- The utility systems are based on the proven FTS system and the use of existing filter and ionexchange column designs and tooling (Sect. 4.8).

- $\quad$ The proton beam window design is a virtual copy of the FTS design (Sect. 4.5.3).

- Incorporation of the building foundation into the neutron beam line shielding reduces shielding cost, improves alignment, simplifies operations, and will ultimately decrease the cost of instruments. 


\subsubsection{Accelerator Systems}

- Use of the demonstrated $16 \mathrm{MV} / \mathrm{m}$ cavity performance reduces the number of required cryomodules for the energy upgrade from 9 to 7 and reduces the amount of support RF equipment needed.

- Use of 9 and 10 klystrons per modulator requires only 3 high-voltage modulator systems (compared with 4 in the previous PUP proposal).

- Incorporation of smart chopping reduces the requirement for peak ion source current for the power upgrade. This results in only a modest $\sim 10 \%$ increase over present source performance and eliminates the need to develop a dual ion source front end.

\subsubsection{Conventional Facilities}

- Placing instruments with comparable beam line lengths adjacent to each other offers the possibility of dual use of buildings.

- Separating the instrument hall and target halls offers the possibility of simpler instrument hall building requirements.

\subsection{REFERENCES}

1 J. M. Carpenter and T. E. Mason, Technical Concepts for a Long Wavelength Target Station for the Spallation Neutron Source, ANL-02/16, ORNL/SNS-TM-2001/163, Oak Ridge National Laboratory, Oak Ridge, Tennessee, November 2002.

2 A Second Target Station for the Spallation Neutron Source, SNS 100000000-TR0029-R00, Oak Ridge National Laboratory, Oak Ridge, Tennessee, Oct. 2007.

3 F. Gallmeier, Moderator Studies for a SNS Short-Pulse Second Target Station, STS03-31-TR0004R00, Oak Ridge National Laboratory, Oak Ridge, Tennessee, May 2013.

4 J. K. Zhao et al., "Instrument performance study on the short and long pulse options of the second Spallation Neutron Source target station," Review of Scientific Instruments 84, 105104 (2013).

52013 ORNL Neutron Sciences Strategic Plan, Oak Ridge National Laboratory, Oak Ridge, Tennessee, October 2013.

6 SNS Second Target Station Alternate Site Evaluation Study, SNS 108000000-RA0002, Oak Ridge National Laboratory, Oak Ridge, Tennessee, September 2009.

7 Preliminary Design Report for the Spallation Neutron Source Power Upgrade MIE Project, PUP0100-DC0001-R00, Oak Ridge National Laboratory, Oak Ridge, Tennessee, January 2007.

8 Preliminary Design Report for the Spallation Neutron Source Power Upgrade MIE Project, PUP0100-DC0001-R05, Oak Ridge National Laboratory, Oak Ridge, Tennessee, February 2011.

9 M. Rennich et al., "Design and testing of a prototype Spallation Neutron Source rotating target assembly," ICANS XIX, 19th Meeting of the Collaboration on Advanced Neutron Sources, March 812, 2010, Grindelwald, Switzerland. 
10 M. Rennich et al., "Prototype Spallation Neutron Source rotating target assembly final test report," AccApp'11, Tenth International Topical Meeting on Nuclear Applications of Accelerators, April 3-7, 2011, Knoxville, Tennessee. 